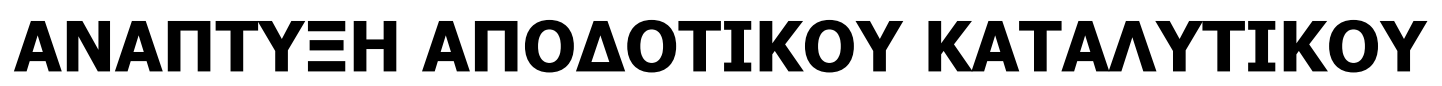

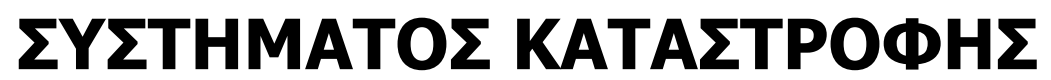

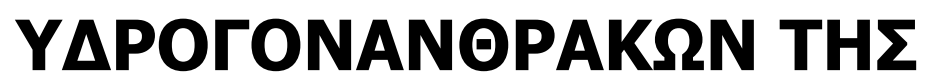

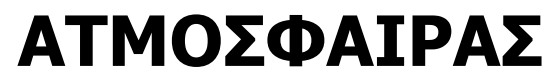

\section{$\triangle$ IDAKTOPIKH $\triangle$ IATPIBH}

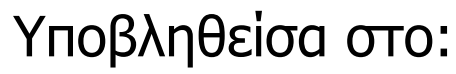

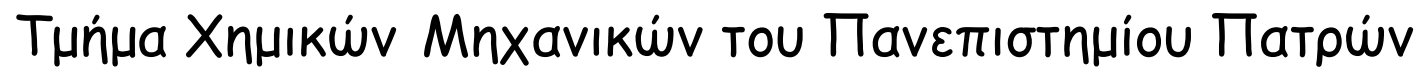

$$
Y_{\pi o ́}
$$

Saleh Sager tou Mohammed

Гıa tnv atióktnon tou tít^ou tou

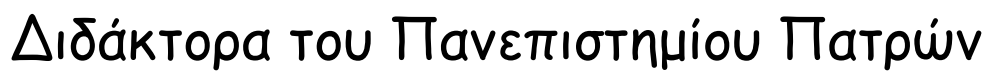

ПАТTPA, 2009 


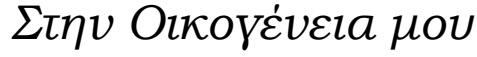

$\Sigma \tau \eta v ~ \Pi а \tau \rho i \delta a ~ \mu о \nu ~$ 


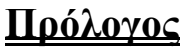

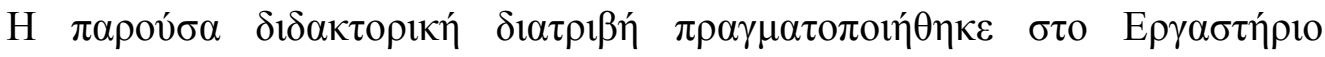

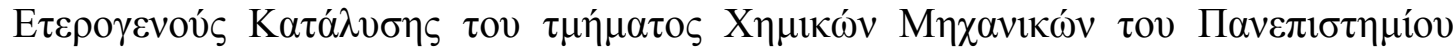

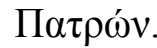

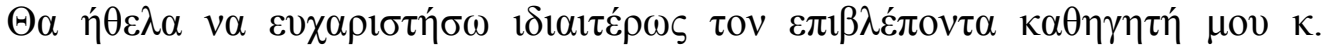

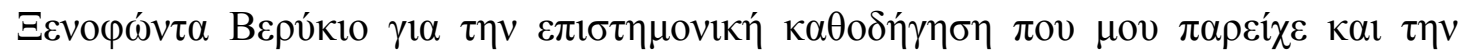

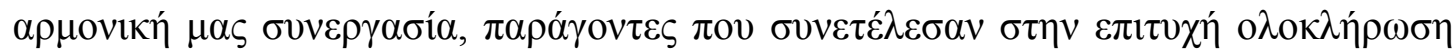

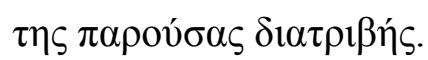

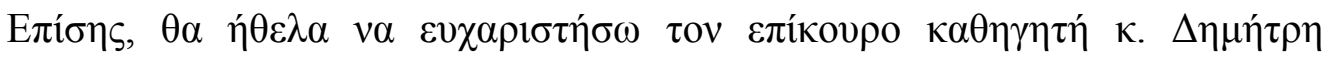

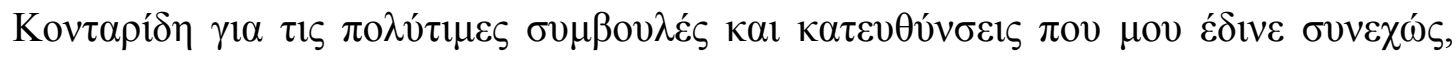

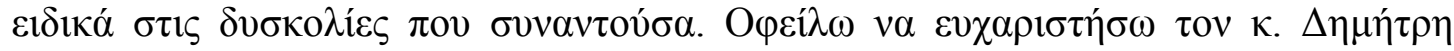

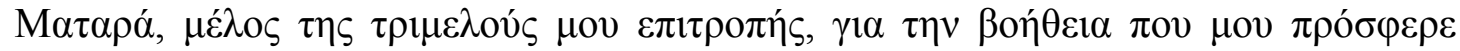

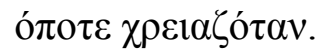

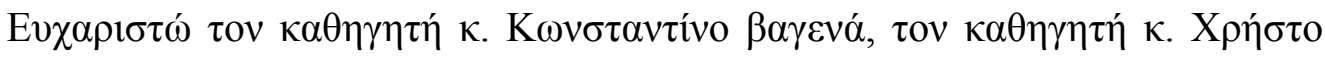

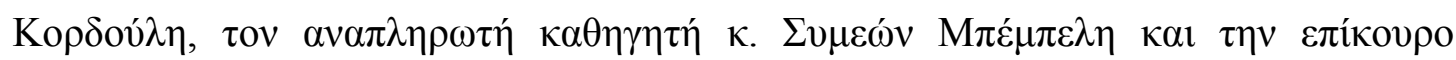

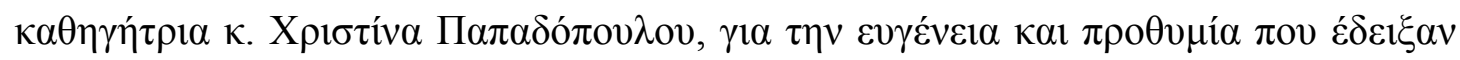

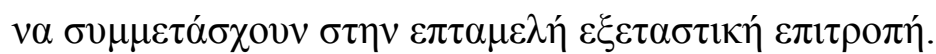

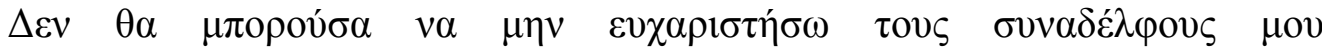

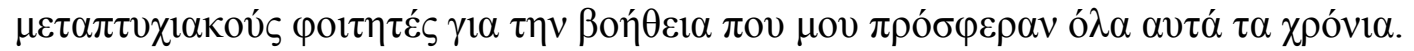

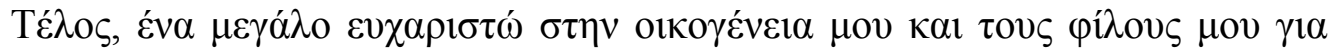

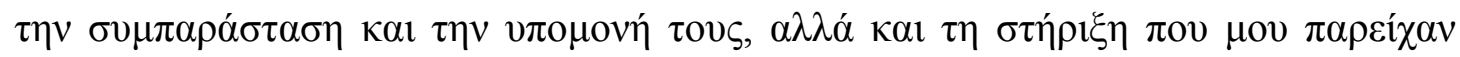

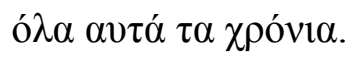

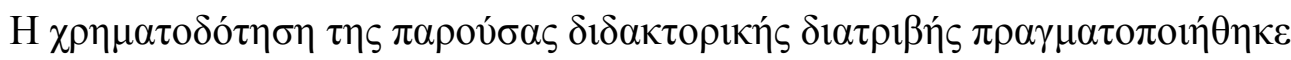

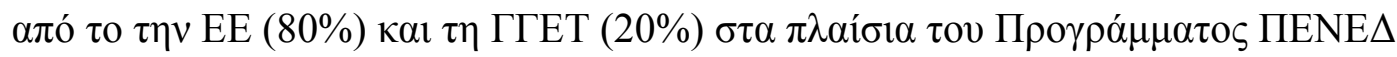
$2003(03 \mathrm{E} \Delta 257)$. 


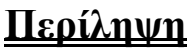

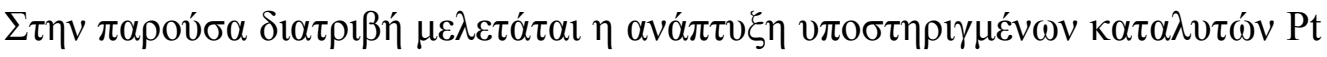

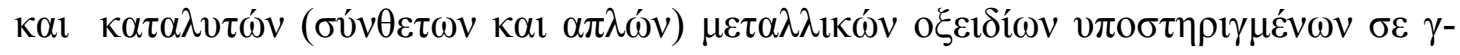

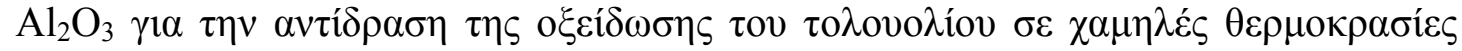

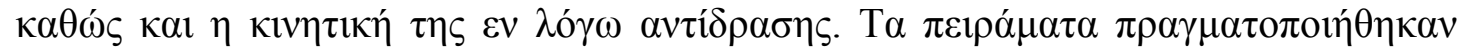

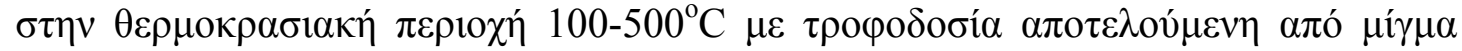
$0.1 \% \mathrm{C}_{7} \mathrm{H}_{8} \sigma \varepsilon \alpha \varepsilon \dot{\varepsilon} \rho$.

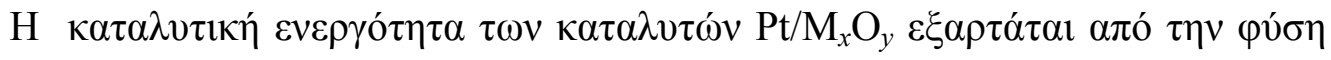

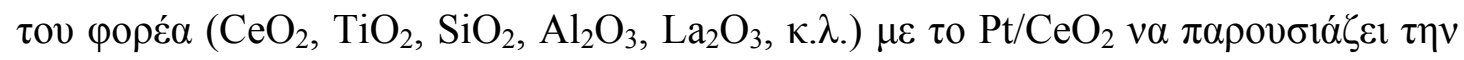

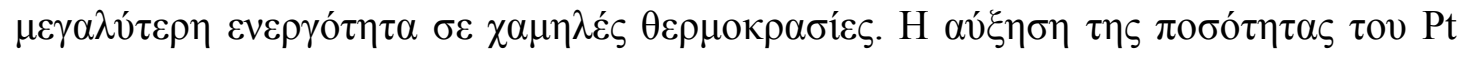

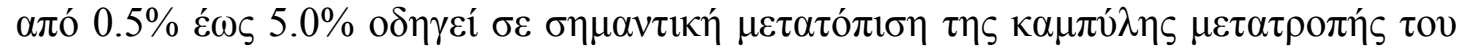

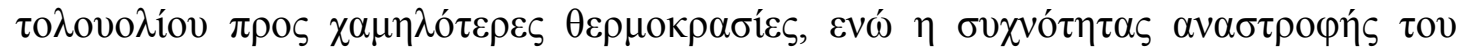

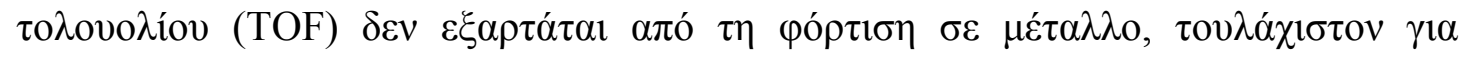

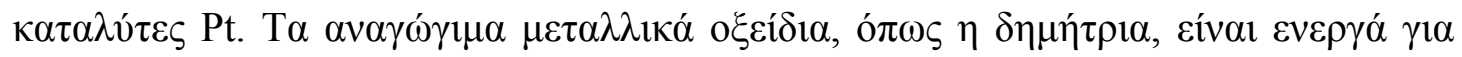

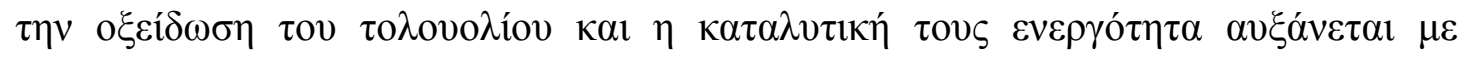

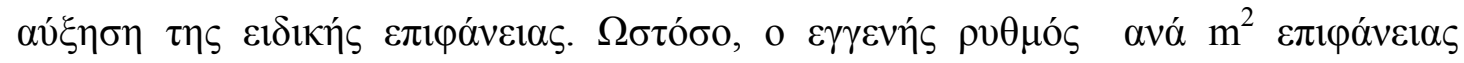

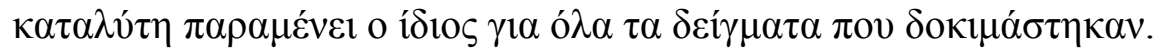

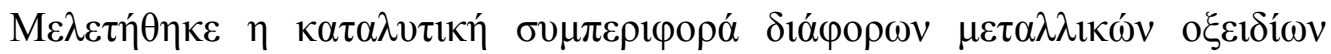

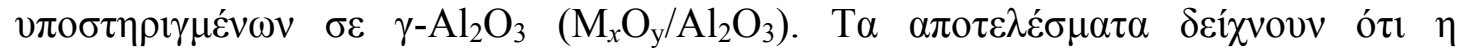

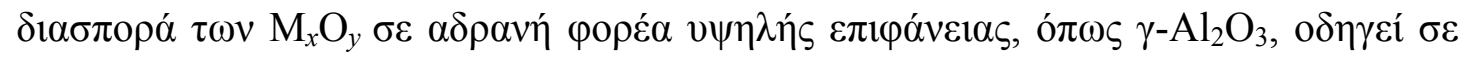

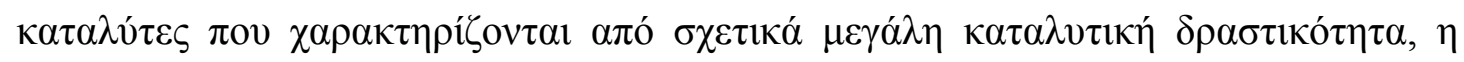

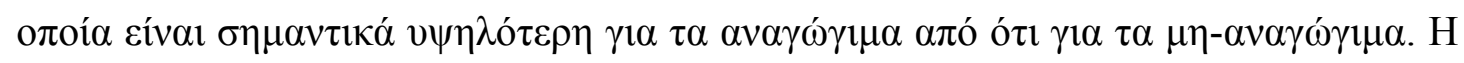

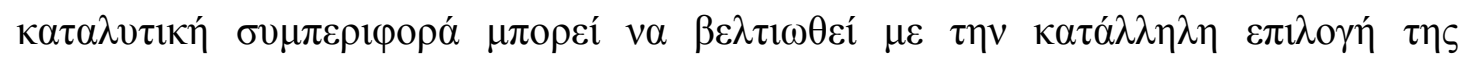

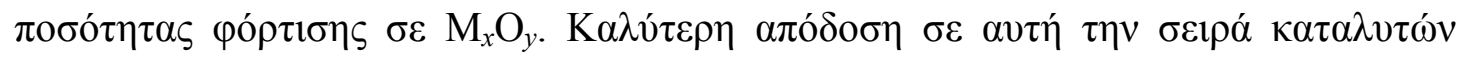

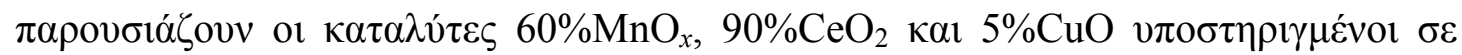

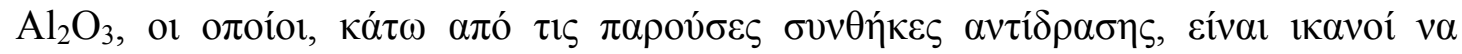

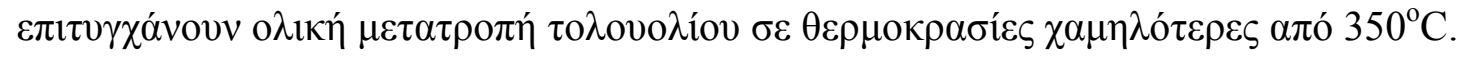

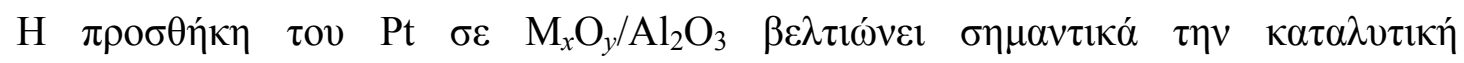

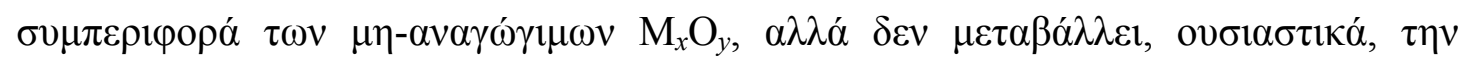

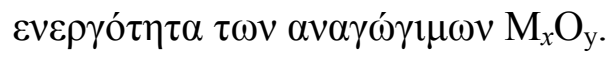

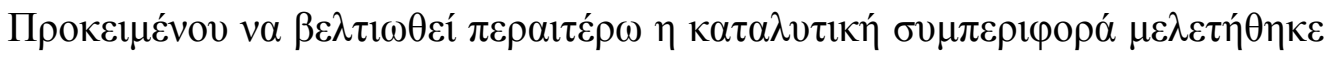

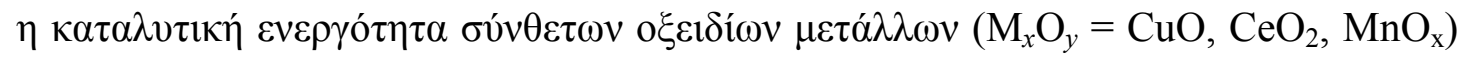




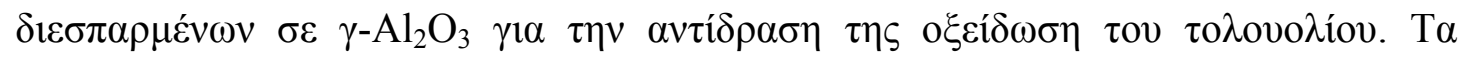

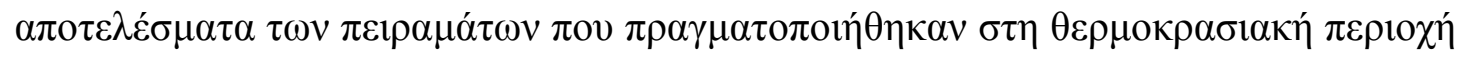

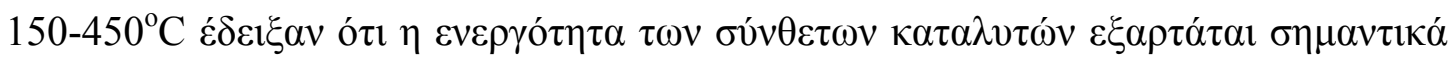

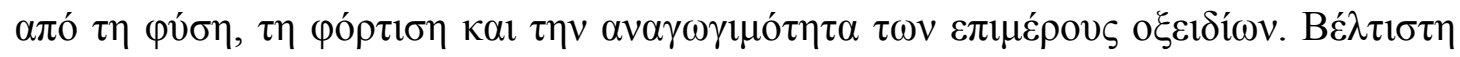

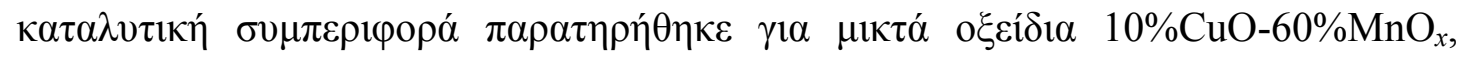

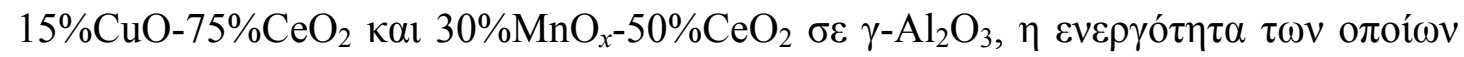

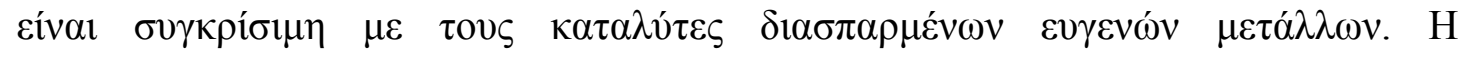

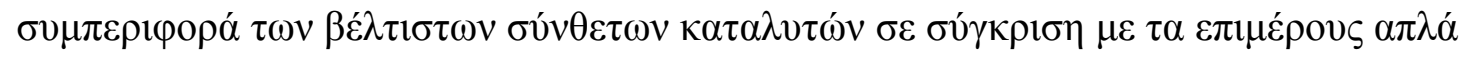

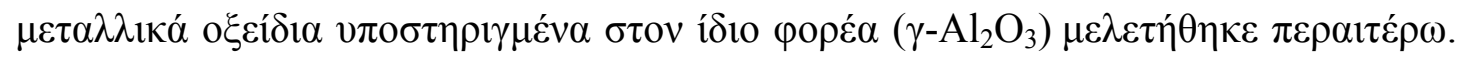

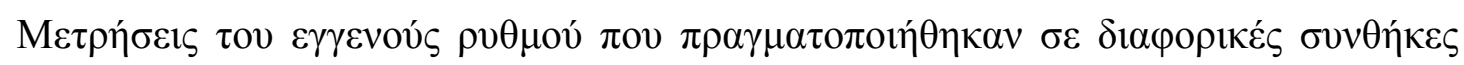

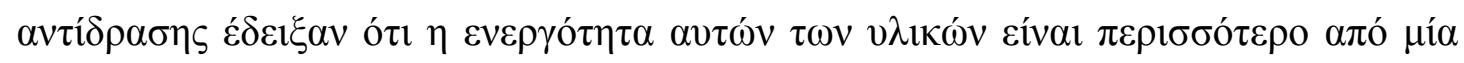

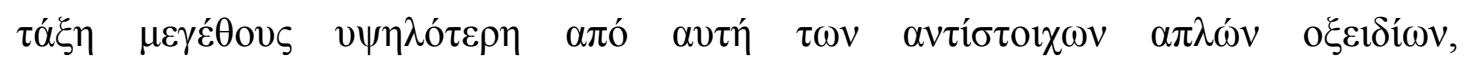

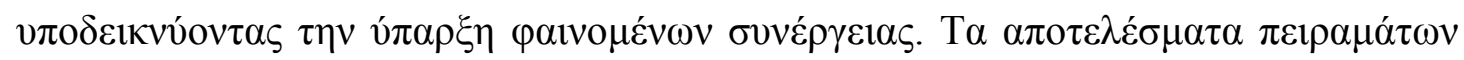

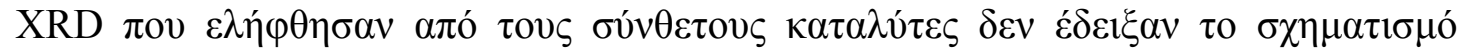

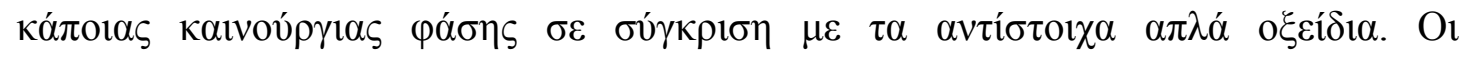

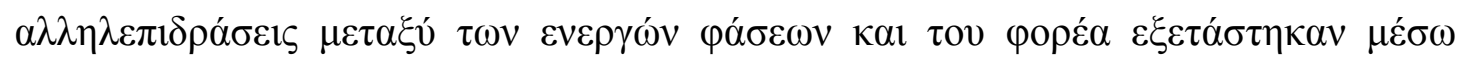

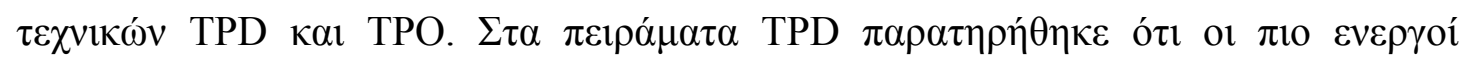

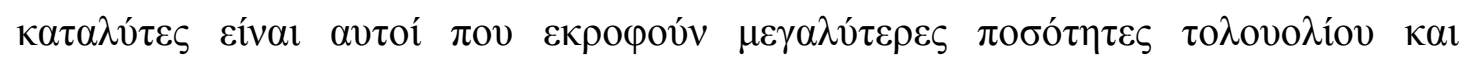

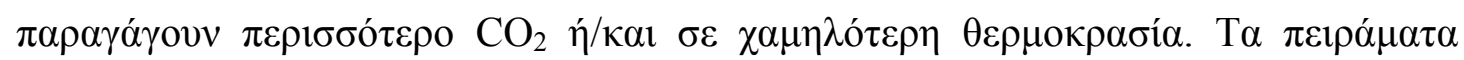

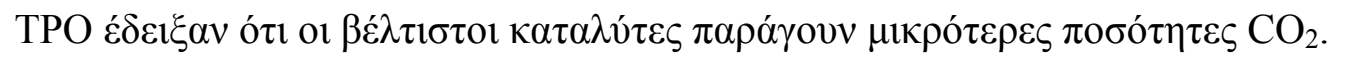

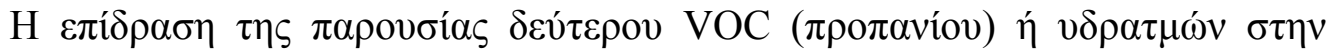

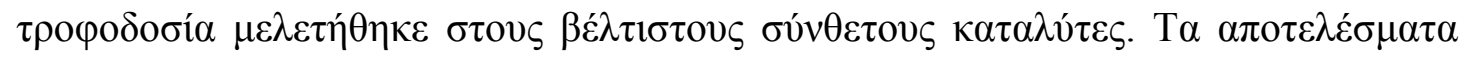

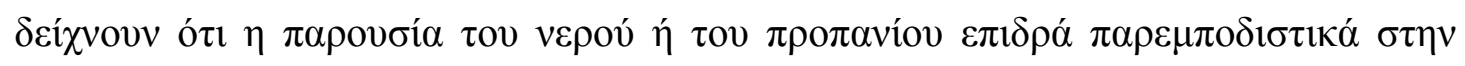

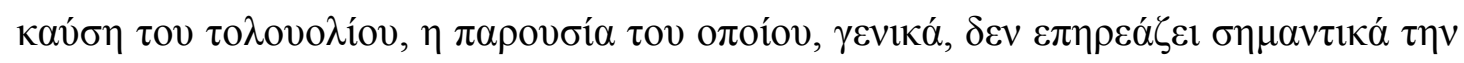

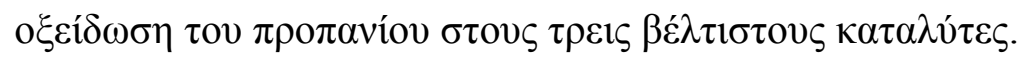

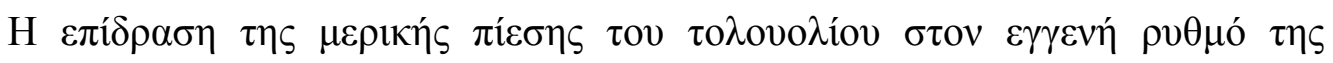

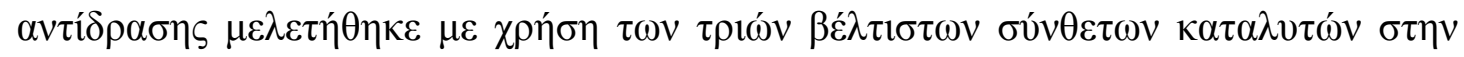

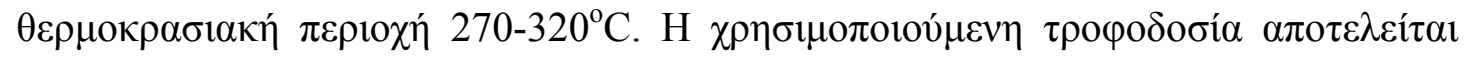

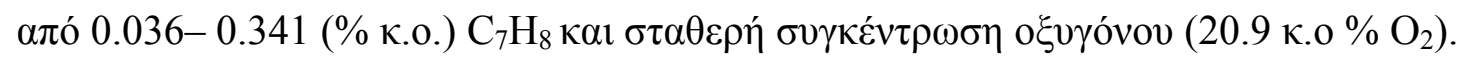

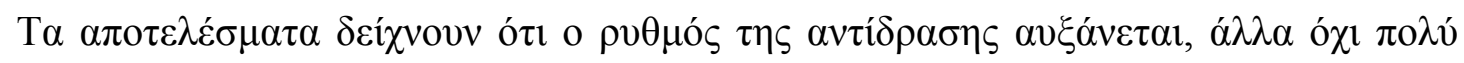

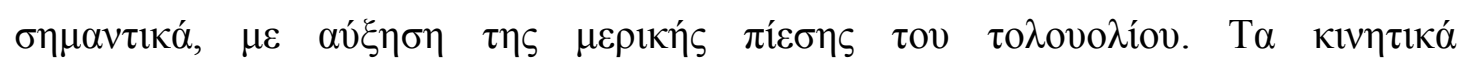

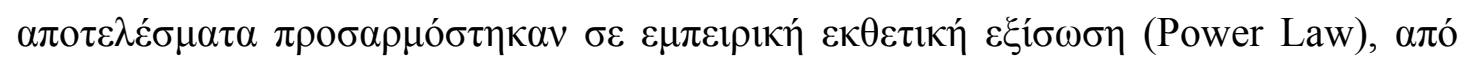

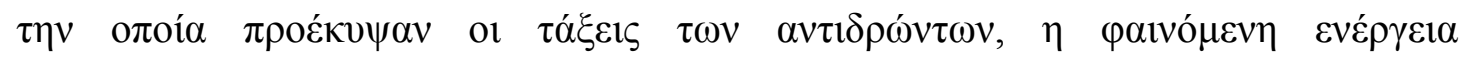

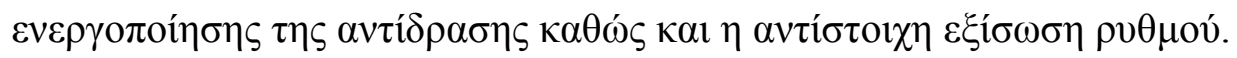




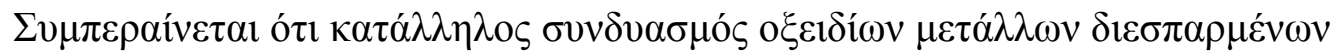

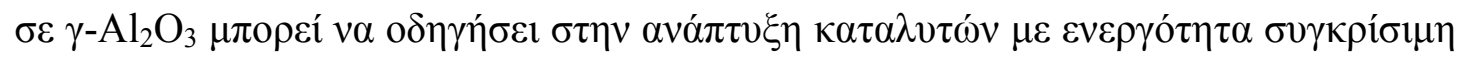

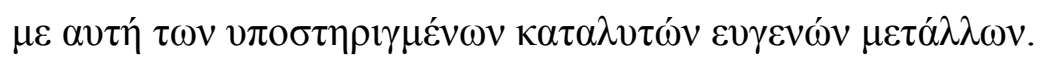




\begin{abstract}
$\underline{\underline{\text { Abstract }}}$
Volatile organic compounds (VOCs) present at low concentrations in industrial waste streams are considered as significant air pollutants due to their toxic and malodorous nature, as well as their contribution to the formation of photochemical smog. Catalytic combustion over supported noble metal catalysts provides an effective method for the elimination of VOCs in exhaust gases and this technology seems to be able to satisfy strict emission standards. Efforts in this field are currently directed toward the development of cheaper, noble metal-free catalytic materials characterized by high activity at low temperatures and long-term stability under reaction conditions.

In the present thesis, oxidation of toluene has been investigated over supported platinum catalysts as well as over single and mixed metal oxide $\left(\mathrm{M}_{x} \mathrm{O}_{y}\right)$ catalysts dispersed on high surface $\gamma-\mathrm{Al}_{2} \mathrm{O}_{3}$. Catalysts were characterized with respect to their specific surface area (BET), metal dispersion (selective chemisorption of $\mathrm{CO}$ ), phase composition and $\mathrm{M}_{x} \mathrm{O}_{y}$ crystallite size (XRD) and reducibility $\left(\mathrm{H}_{2}-\mathrm{TPR}, \mathrm{CO}-\mathrm{TPR}\right)$. Catalytic performance for the title reaction was investigated in the temperature range of $100-500^{\circ} \mathrm{C}$, using a feed composition consisting of $0.1 \%$ toluene in air. For $\mathrm{Pt} / \mathrm{M}_{x} \mathrm{O}_{y}$ catalysts, it has been found that catalytic performance depends on the nature of the support, with $\mathrm{Pt} / \mathrm{CeO}_{2}$ being the most active catalyst at low temperatures. The intrinsic reaction rate per surface platinum atom does not depend on Pt loading (0.5-5 wt.\%), at least for $\mathrm{Pt} / \mathrm{Al}_{2} \mathrm{O}_{3}$ catalyst, but the global reaction rate increases with increase of exposed metallic surface area. Reducible metal oxides, such as ceria, are active for the title reaction and catalytic performance is improved significantly with increase of specific surface area (SSA). However, the intrinsic reaction rate per unit surface area is the same regardless of SSA. Dispersion of $\mathrm{M}_{x} \mathrm{O}_{y}$ on high surface inert supports, such as $\mathrm{Al}_{2} \mathrm{O}_{3}$, results in materials with relatively high catalytic activity, which is considerably higher for reducible, compared to irreducible metal oxides. Catalytic performance of $\mathrm{M}_{x} \mathrm{O}_{y} / \mathrm{Al}_{2} \mathrm{O}_{3}$ catalysts can be optimized by proper selection of $\mathrm{M}_{x} \mathrm{O}_{y}$ loading. Best performing catalysts of this series include $60 \% \mathrm{MnO} x$, $90 \% \mathrm{CeO}_{2}$ and $5 \% \mathrm{CuO}$ on $\mathrm{Al}_{2} \mathrm{O}_{3}$ which, under the present experimental conditions, are able to completely convert toluene toward $\mathrm{CO}_{2}$ at temperatures lower than $350^{\circ} \mathrm{C}$. Dispersion of $\mathrm{Pt}$ on $\mathrm{M}_{x} \mathrm{O}_{y} / \mathrm{Al}_{2} \mathrm{O}_{3}$ catalysts improves significantly the catalytic
\end{abstract}


performance of irreducible $\mathrm{M}_{x} \mathrm{O}_{y}$ but does not alter appreciably activity of reducible $\mathrm{M}_{x} \mathrm{O}_{y} / \mathrm{Al}_{2} \mathrm{O}_{3}$ catalysts.

The catalytic oxidation of toluene has been investigated also over single and composite metal oxide catalysts supported on $\gamma-\mathrm{Al}_{2} \mathrm{O}_{3}$. Catalysts were synthesized with the impregnation method and were characterized with respect to their specific surface area (BET method), crystalline mode and mean crystallite size (XRD technique), as well as with respect to their reducibility (temperature programmed reduction with $\mathrm{H}_{2}$ or $\mathrm{CO}$ ). The effects of the nature, loading and composition of catalytic materials on their performance for VOC combustion has been investigated. Optimal results were obtained over $\mathrm{Al}_{2} \mathrm{O}_{3}$-supported $\mathrm{CuO}, \mathrm{CeO}_{2}, \mathrm{MnO}_{2}$ catalysts and their mixtures. For certain metal oxide combinations, e.g., $10 \% \mathrm{CuO}-60 \% \mathrm{MnO}_{x}$, $15 \% \mathrm{CuO}-75 \% \mathrm{CeO}_{2}$ and $30 \% \mathrm{MnO}_{x}-50 \% \mathrm{CeO}_{2}$, activity was found to be comparable to that of supported noble metal catalysts. Measurements of reaction rates under differential reaction conditions showed that specific activity of these materials was up to one order of magnitude higher, compared to that of the corresponding single metal oxides, implying that synergistic effects are operable. Results of XRD experiments did not show formation of new phases, but mixed oxide catalysts were found to exhibit a higher reducibility compared to catalysts consisting of the corresponding single metal oxides. The synergic effect of metal oxides interaction on the oxidation reaction was studied employing TPD and TPO techniques. The more active catalyst, the higher the amount of desorbed toluene and the higher the amount of $\mathrm{CO}_{2}$ production in the in the TPD experiments. The TPO experiments indicate that the optimized composite catalysts produce lower amounts of $\mathrm{CO}_{2}$ at lower temperature, compared to the corresponding single metal oxides. The influence of the presence of a second VOC (propane) or of water on the oxidation of toluene was also investigated. Results showed that the presence of water or propane in the feed results in a decrease of catalytic activity, while the presence of toluene doesn't have any influence in the catalytic oxidation of propane over the optimized composite catalysts.

The effect of partial pressure of toluene on the kinetic reaction rate has been investigated over the optimum composite catalysts in the $270-320^{\circ} \mathrm{C}$ range using a feed stream consisting of $0.036-0.341$ vol $\% \mathrm{C}_{7} \mathrm{H}_{8}$ and a constant concentration of oxygen $\left(20.9\right.$ vol\% $\left.\mathrm{O}_{2}\right)$. Result showed that increasing the partial pressure of toluene leads to an increase of the reaction rate. 
The orders of the reaction with respect to reactants for the optimized catalysts were determined by fitting the experimental data to an empirical power-law rate expression according to which the reaction rate is given by the following relationships:

$$
\begin{aligned}
& r=k P_{\text {tol. }}^{a} p_{O_{2}}^{b} \\
& k=k_{0} \cdot e^{E_{a} / R . T}
\end{aligned}
$$

Results of the present study show that the catalytic performance of certain $\mathrm{Al}_{2} \mathrm{O}_{3}$-supported composite metal oxide catalysts is comparable to that of conventional supported noble metal catalysts. These materials could provide the basis for the development of cost-effective catalysts for combustion of VOCs present in waste gas streams. 


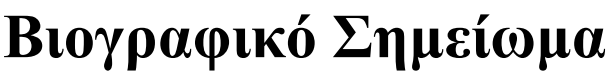

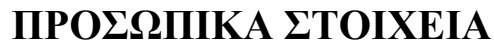

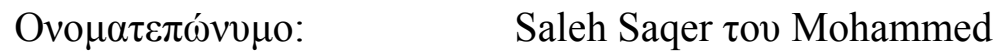

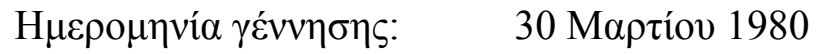

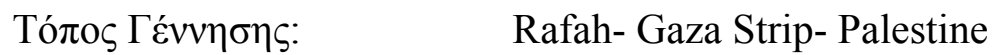

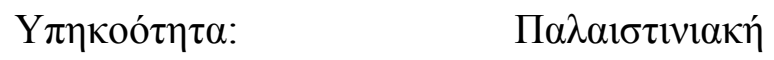

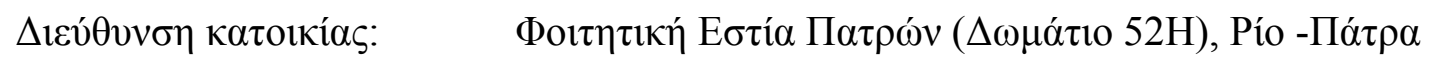

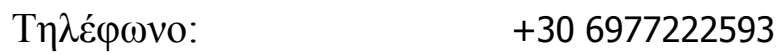

E-mail: $\quad$ salehsaqer@gmail.com

\section{$\Sigma \Pi O Y \Delta E \Sigma$}

1998:

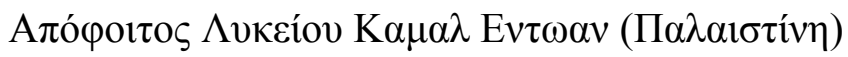

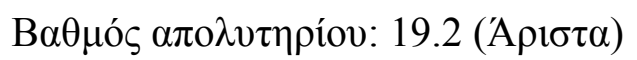

2005:

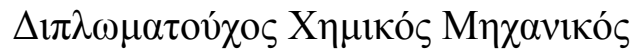

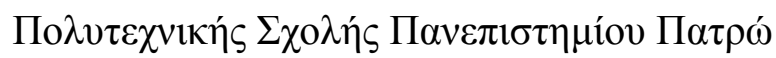

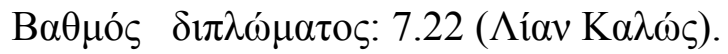

2005-2009

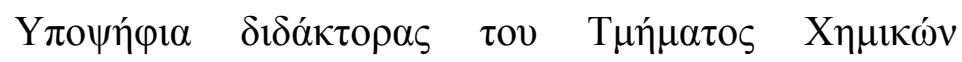

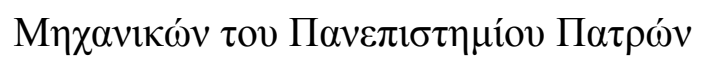

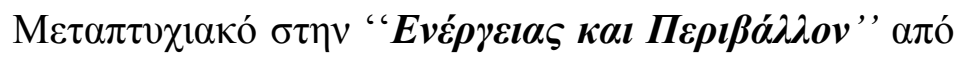

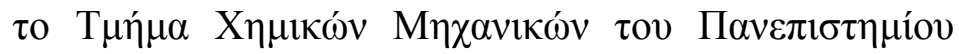

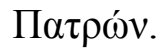

\section{ЕПАГГЕАМАТІКН ЕМПЕIРІА}

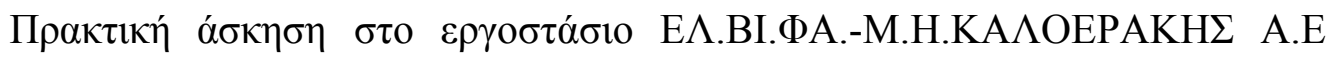

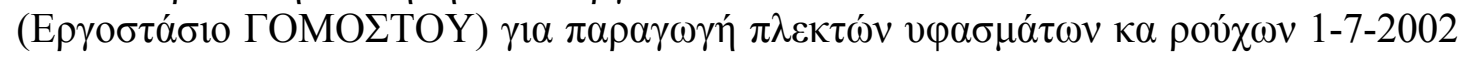

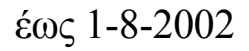




\section{МIAAKTIKH EMIEIPIA}

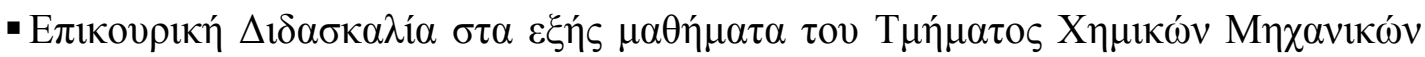

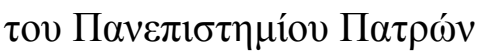

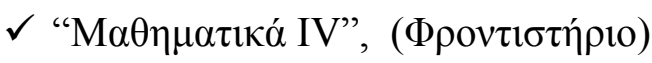

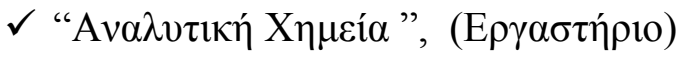

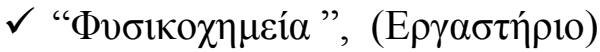

\section{ГYMМЕТОХН ГЕ ЕРЕYNHTIКА ПРОГРАММАТА}

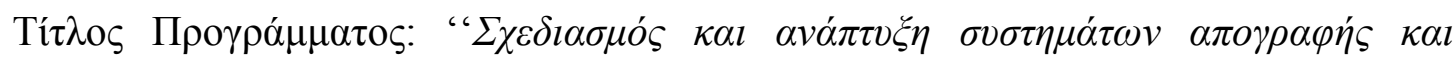

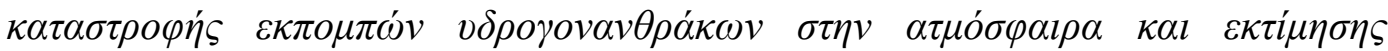

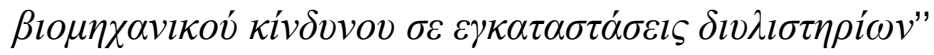

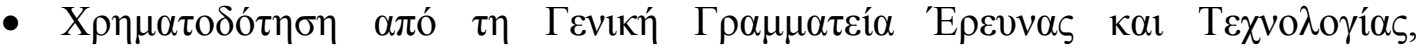

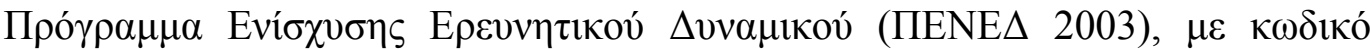

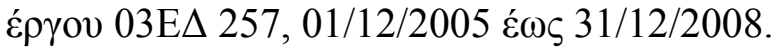

\section{IEMINAPIA}

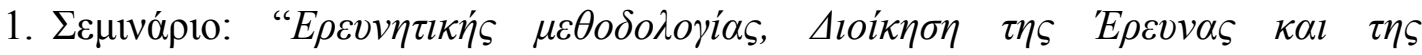

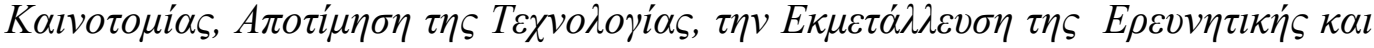

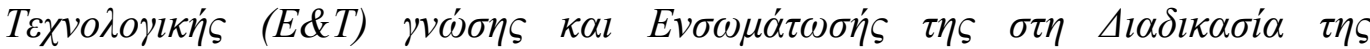

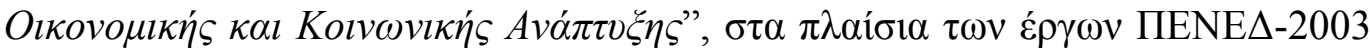

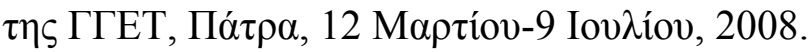

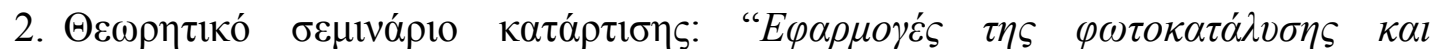

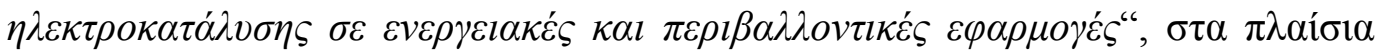

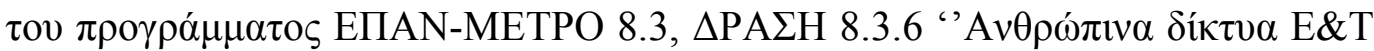

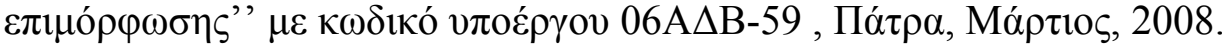

\section{YПОТРОФІА}

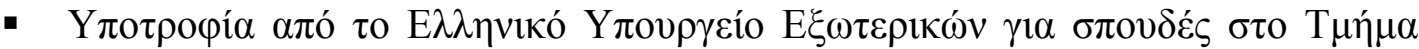

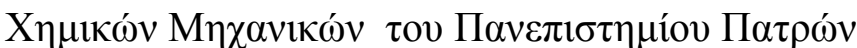




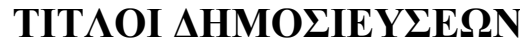

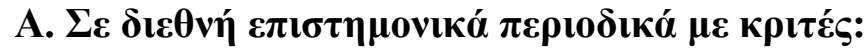

1. Saleh M. Saqer, D. I. Kondarides, X.E. Verykios, "Catalytic activity of supported platinum and metal oxide catalysts for toluene oxidation', Topics in Catalysis 52 (2009) 517-527.

2. Saleh M. Saqer, D. I. Kondarides, X.E. Verykios, "Effect of deposition two metal oxides ( $\left.\mathrm{CuO}, \mathrm{CeO}_{2}, \mathrm{MnO}_{2}\right)$ on $\mathrm{Y}^{-} \mathrm{Al}_{2} \mathrm{O}_{3}$ support for toluene total oxidation', (in preperation)

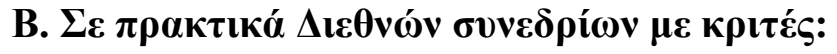

1. Saleh M. Saqer, Dimitris I. Kondarides, Xenophon E. Verykios, (2009), "Effect of deposition two metal oxides ( $\left.\mathrm{CuO}, \mathrm{CeO}, \mathrm{MnO}_{2}\right)$ on $\gamma-\mathrm{Al}_{2} \mathrm{O}_{3}$ support for toluene total oxidation", $5^{\text {th }}$ International Conference of Enviromental Catalysis', September 2009, Belfast, Northern Ireland, United Kingdom.

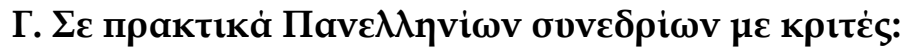

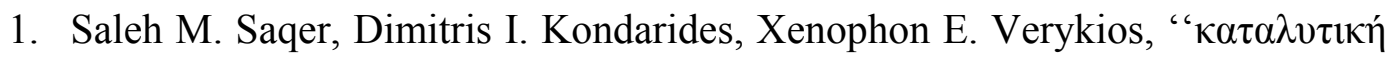

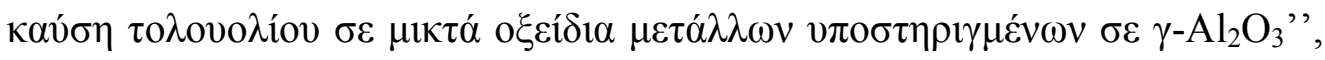

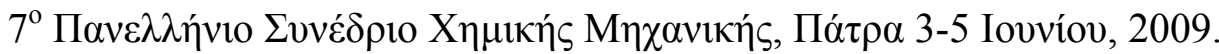

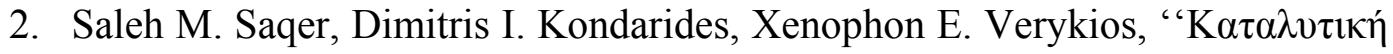

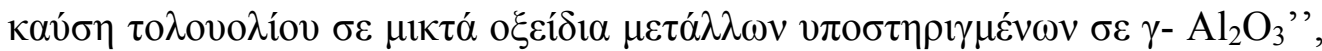

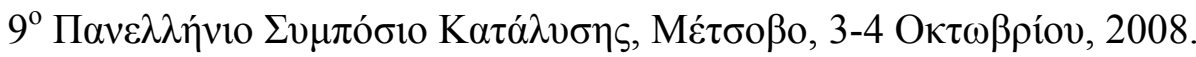

3. Saleh M. Saqer, A. Kotsifa, Dimitris I. Kondarides, Xenophon E. Verykios,

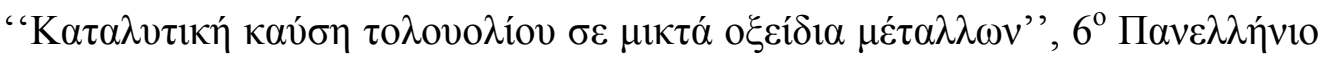

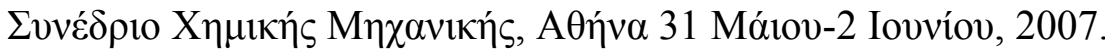

4. Saleh M. Saqer, A. Kotsifa, Dimitris I. Kondarides, Xenophon E. Verykios,

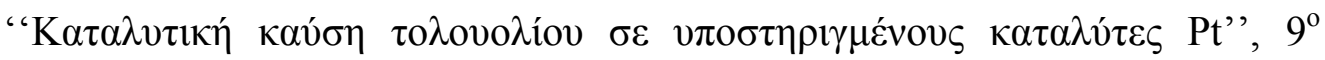

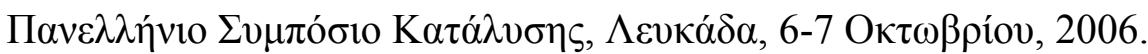

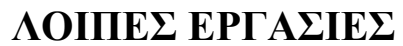

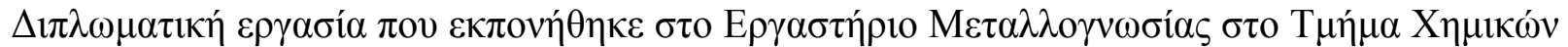

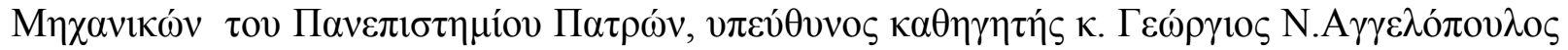




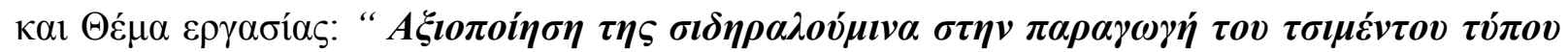
Portland".

\section{छENE $\Sigma \Gamma \Lambda \Omega \Sigma \Sigma E \Sigma$}

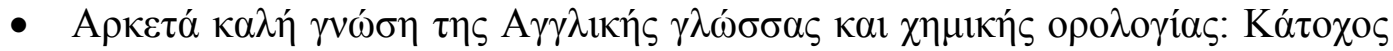
Certificate of Competency in English of University of Michigan

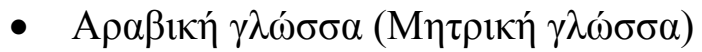

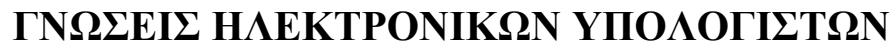

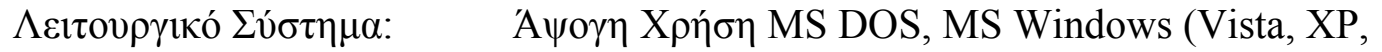
2000, NT, 98)

$\Lambda о \gamma \imath \sigma \tau 1 \kappa \alpha ́ ~ П \alpha \kappa \varepsilon ́ \tau \alpha$

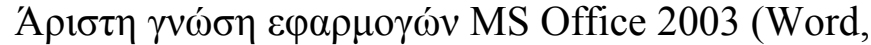
Excel, PowerPoint)

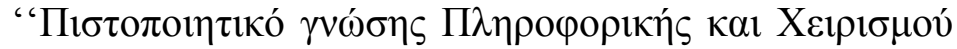

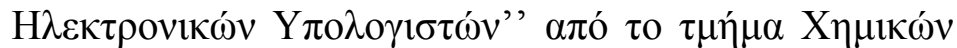

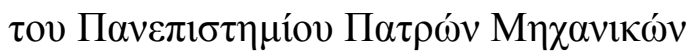

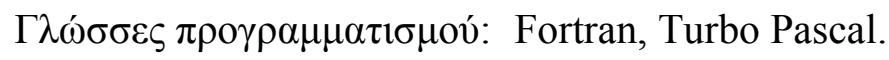




\section{ПEPIEXOMENA}

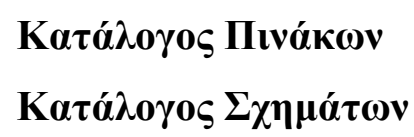

$\underline{\text { KEDAAAIO } 1^{\circ}}$

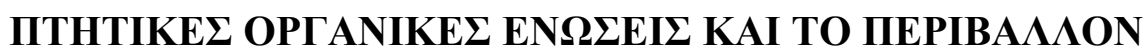

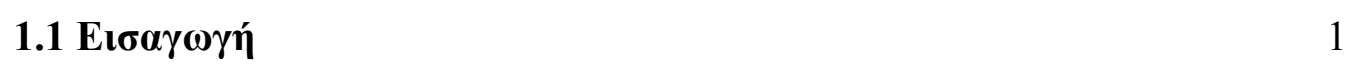

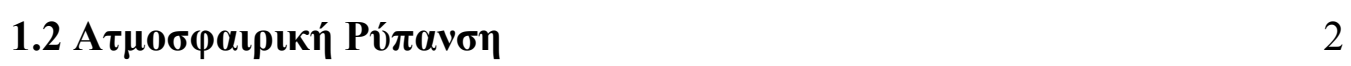

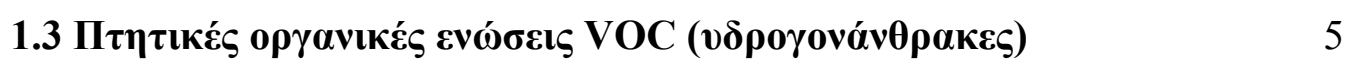

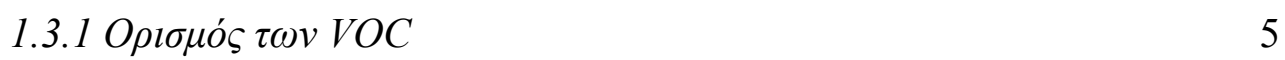

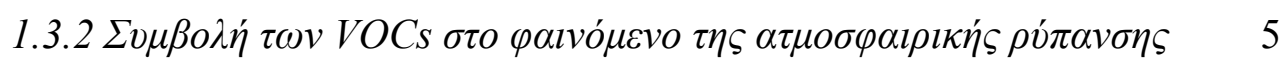

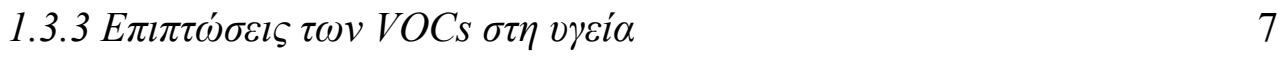

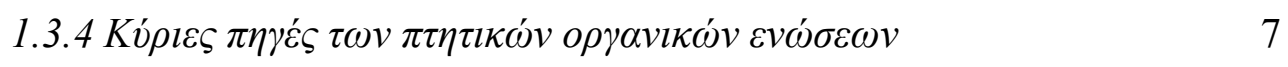

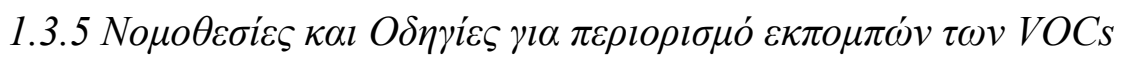

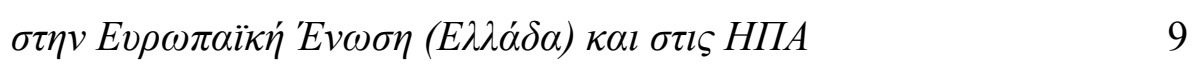

1.3.6 O

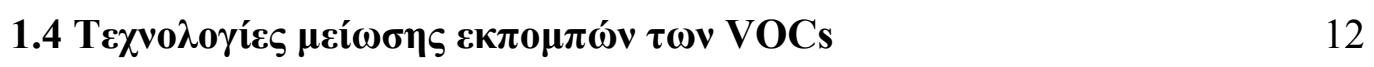

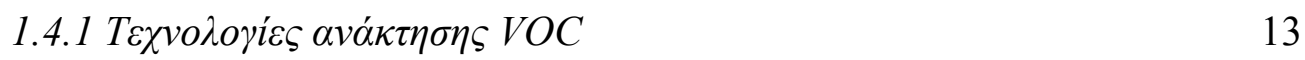

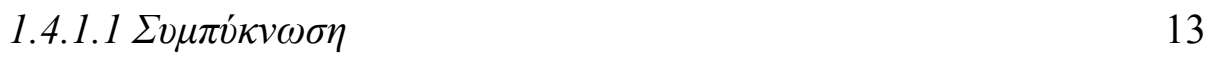

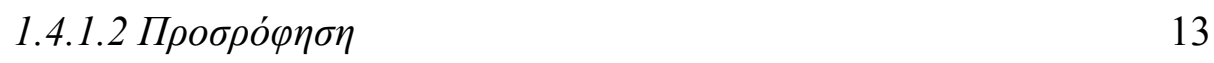

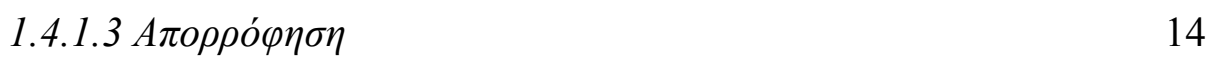

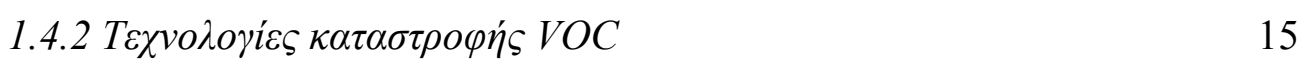

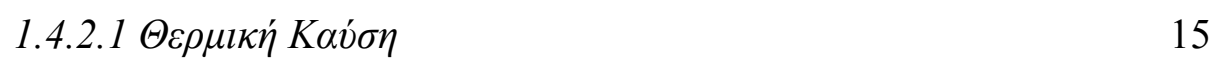

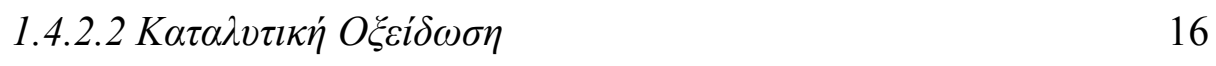

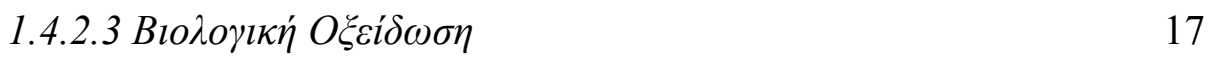

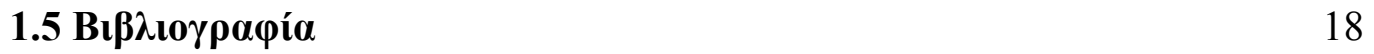

$\underline{K E \Phi A \Lambda A I O 2^{o}}$

KATAАYTIKH OEEI $\triangle \Omega \Sigma H$ VOCS

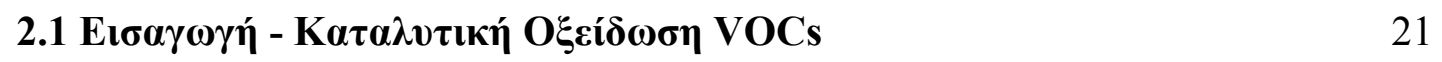

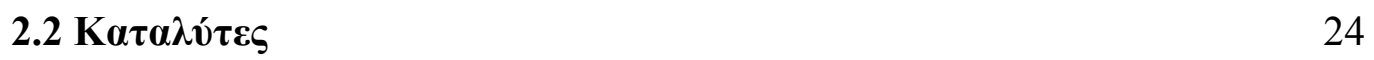




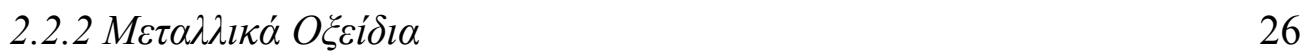

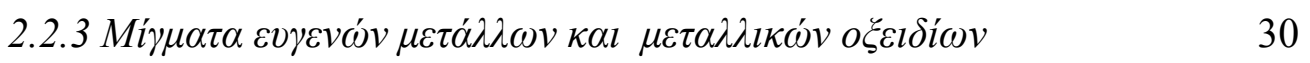

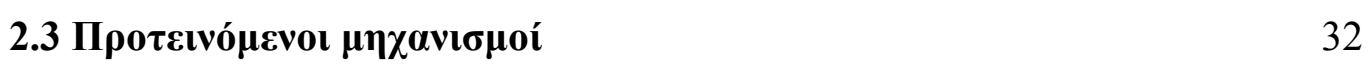

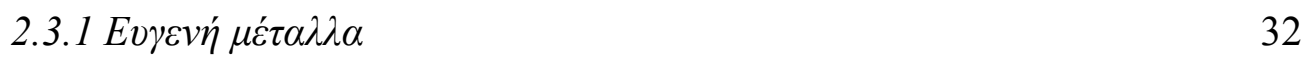

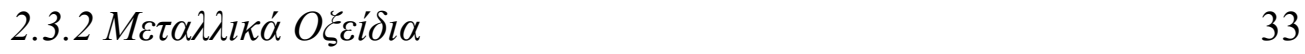

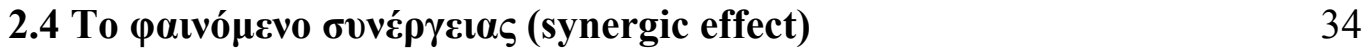

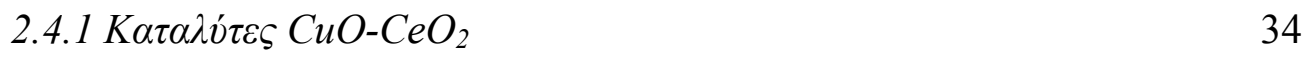

2.4.2 $\mathrm{K \alpha \tau \alpha \lambda \nu} \tau \varepsilon \varsigma \mathrm{MnO}_{\chi^{-}} \mathrm{CeO}_{2}$

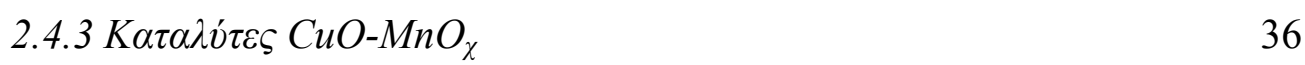

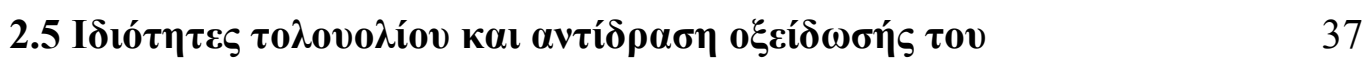

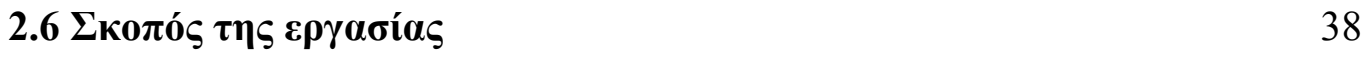

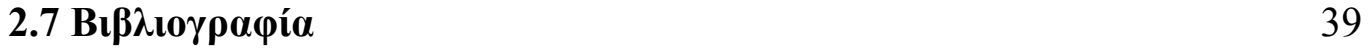

${\underline{K E \Phi A \Lambda A I O ~} 3^{\circ}}^{\circ}$

ПЕIРАМАТІКО МЕРОГ

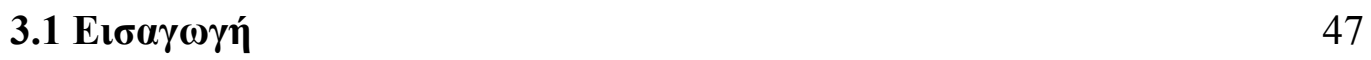

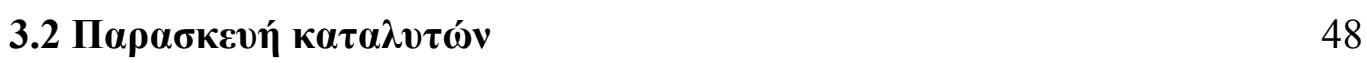

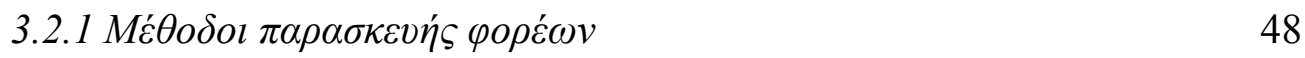

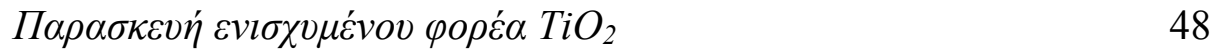

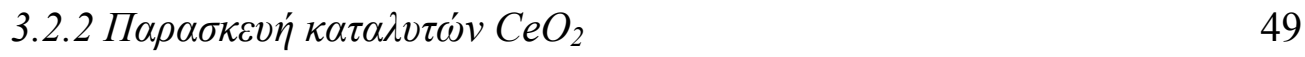

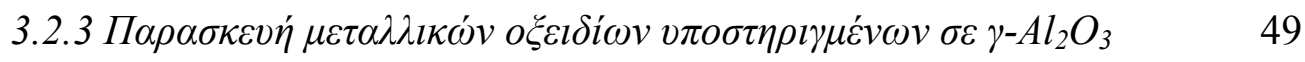

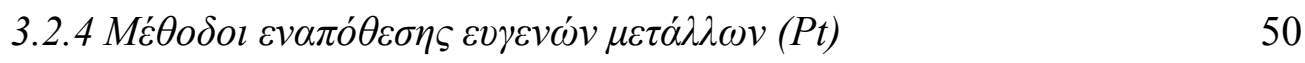

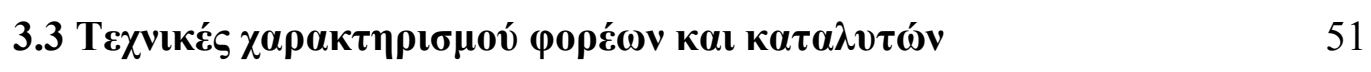

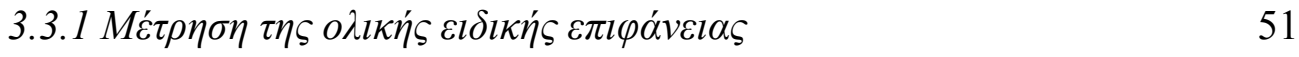

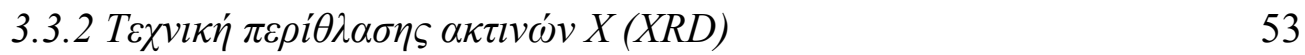

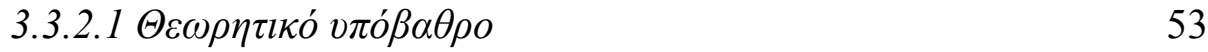

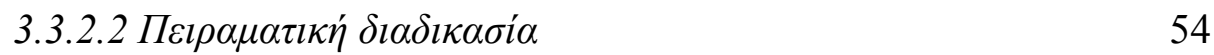

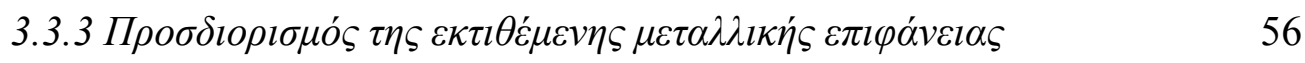

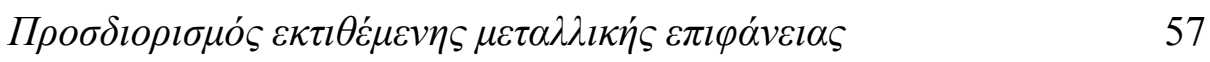

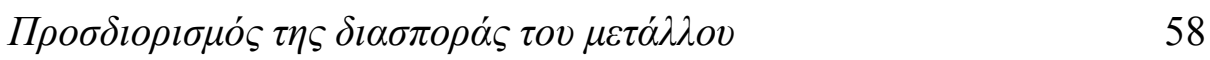

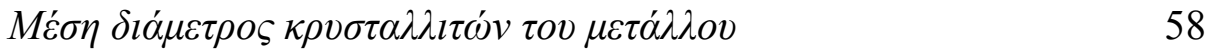

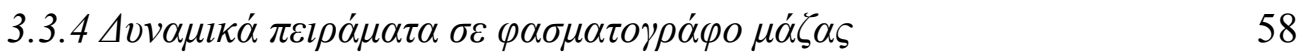




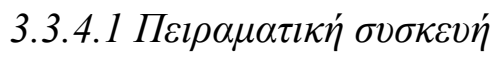

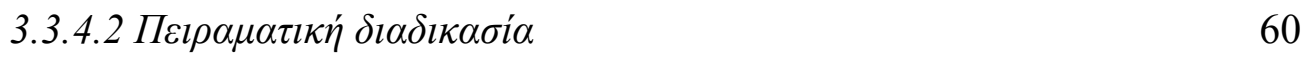

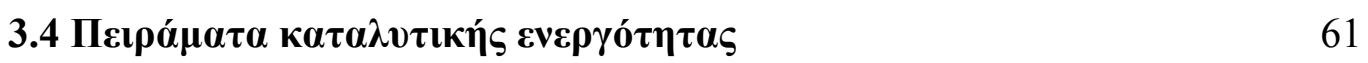

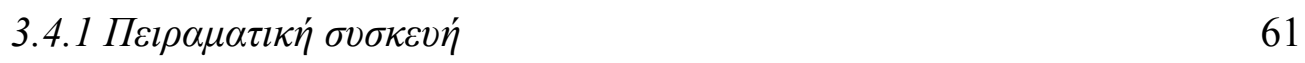

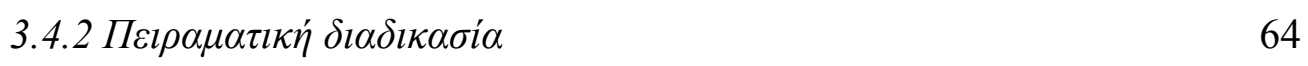

3.5 Вıрдıорафía 66

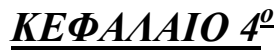

H ANTI $\triangle P A \Sigma H ~ O E E I \Delta \Omega \Sigma H \Sigma$ TOY TOLOYOAIOY $\Sigma E$

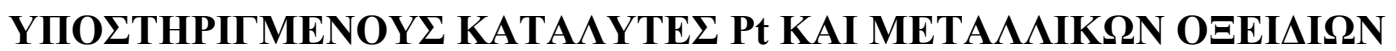

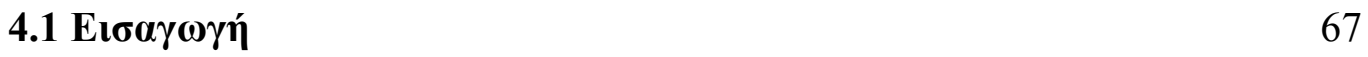

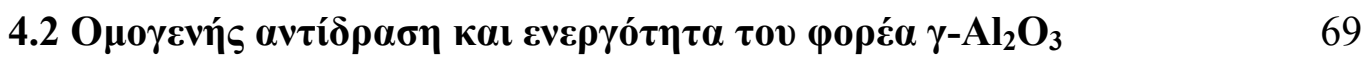

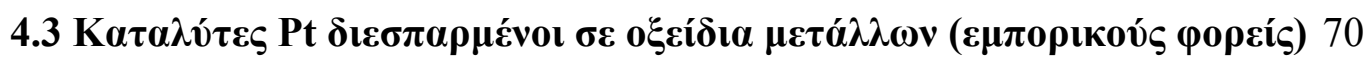

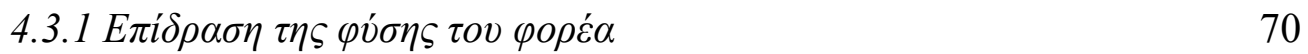

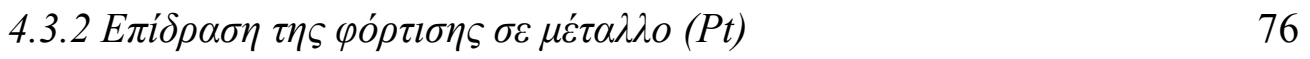

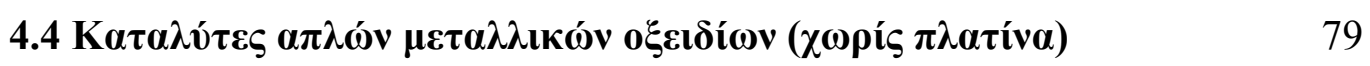

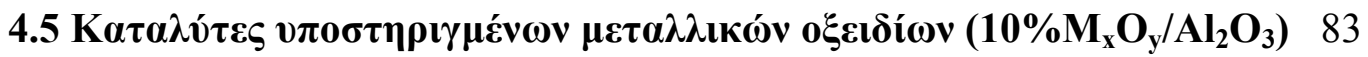

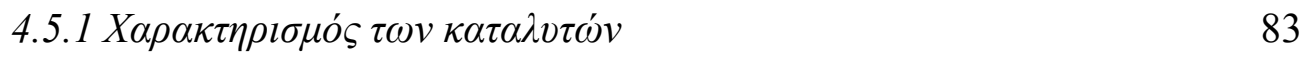

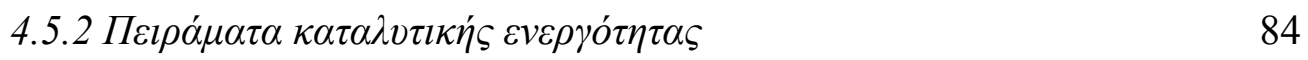

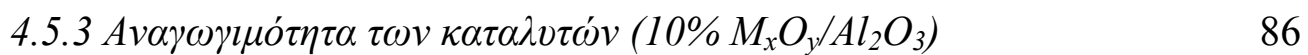

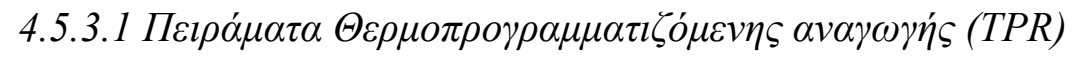

$\mu \varepsilon \mathrm{H}_{2}$

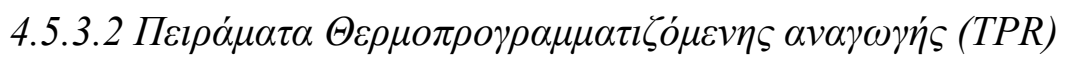
$\mu \varepsilon \mathrm{CO} \quad 88$

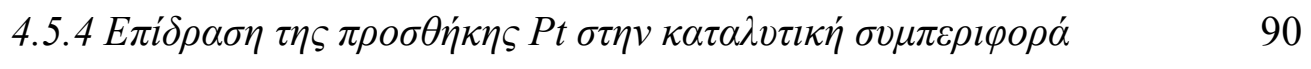

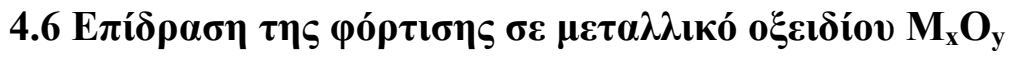

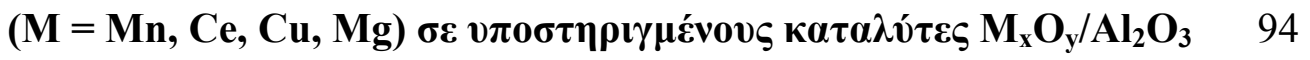

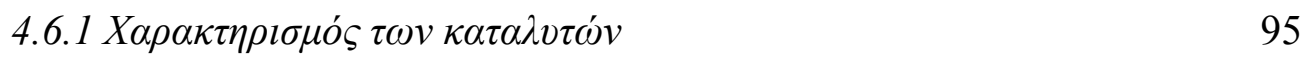

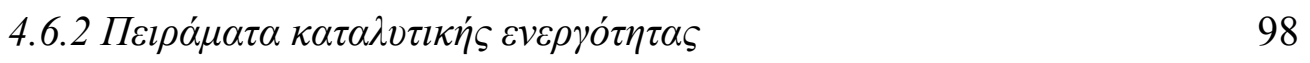

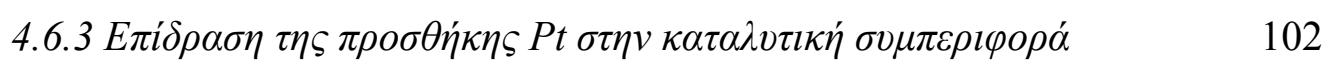

$\begin{array}{ll}4.7 \Sigma v \mu \pi \varepsilon \rho \alpha ́ \sigma \mu \alpha \tau \alpha & 106\end{array}$

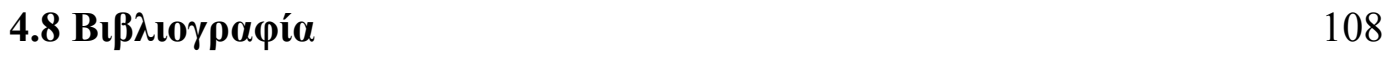


$\underline{\text { KEФAAAIO }}^{\circ}$

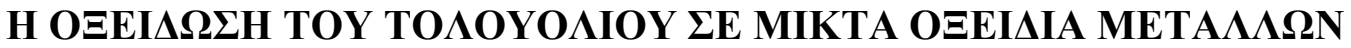

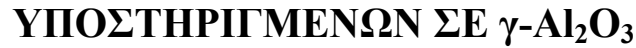

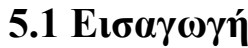

111

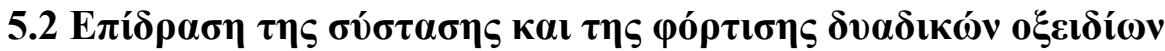

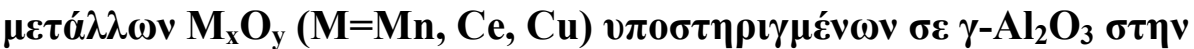

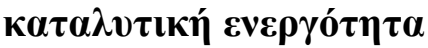

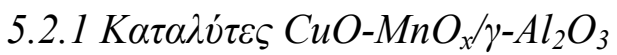

5.2.2 $\mathrm{K \alpha} \tau \alpha \lambda \dot{v} \tau \varepsilon \varsigma \mathrm{MnO}_{x}-\mathrm{CeO}_{2} / \mathrm{Al}_{2} \mathrm{O}_{3}$

5.2.3 $\mathrm{K \alpha} \tau \alpha \lambda v i \tau \varepsilon \varsigma \mathrm{CuO}-\mathrm{CeO}_{2} / \mathrm{Al}_{2} \mathrm{O}_{3}$

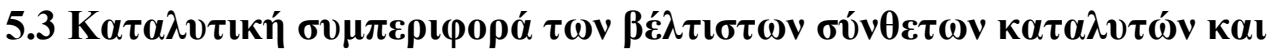

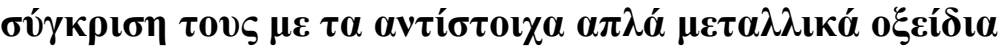

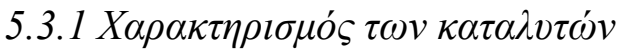

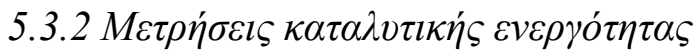

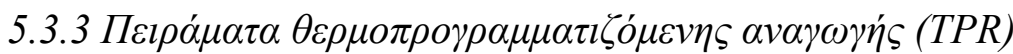

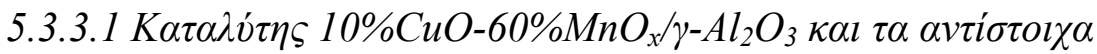
$\alpha \pi \lambda \dot{\alpha} o \xi \varepsilon i \delta l \alpha$

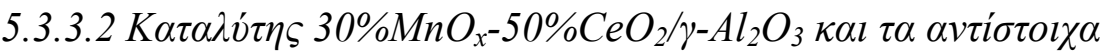
$\alpha \pi \lambda \dot{\alpha} o \xi \varepsilon i \delta \delta \alpha$

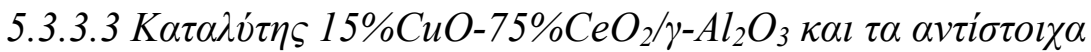
$\alpha \pi \lambda \dot{\alpha} o \xi \varepsilon \dot{\varepsilon} \delta \imath \alpha$

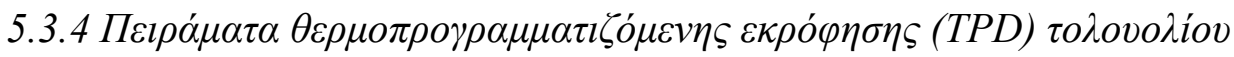

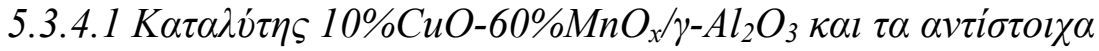
$\alpha \pi \lambda \dot{\alpha} o \xi \operatorname{ci} \delta \imath \alpha$

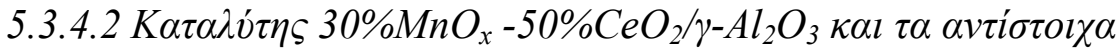
$\alpha \pi \lambda \dot{\alpha} o \xi \varepsilon i \delta \delta \alpha$

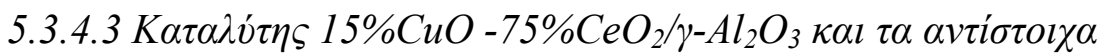
$\alpha \pi \lambda \alpha \dot{o} o \xi \varepsilon i \delta l \alpha$

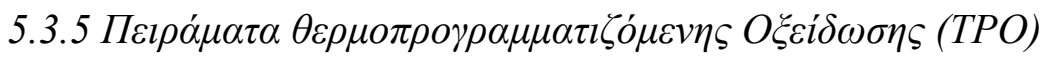

$5.4 \Sigma v \mu \pi \varepsilon \rho \alpha ́ \sigma \mu \alpha \tau \alpha$

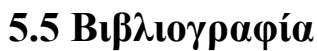


$\underline{K E \Phi A \Lambda A I O ~}^{\mathbf{o}}$

KINHTIKH MEАETH KAI ФAINOMENA ПАРEMПO

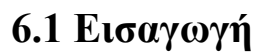

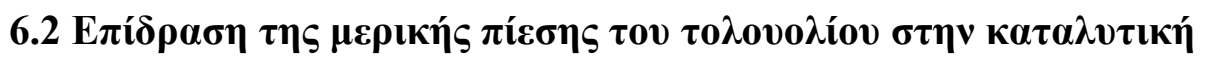

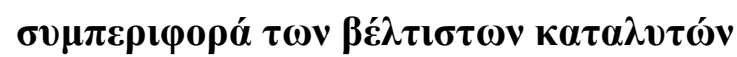

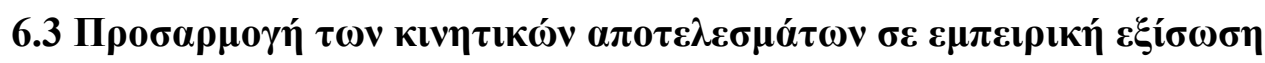
power-law

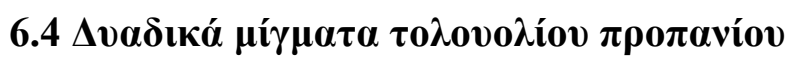

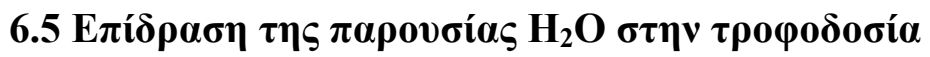

$6.6 \Sigma v \mu \pi \varepsilon \rho \alpha ́ \sigma \mu \alpha \tau \alpha$

$\underline{\operatorname{KE\Phi } A \Lambda A I O 7^{\circ}}$

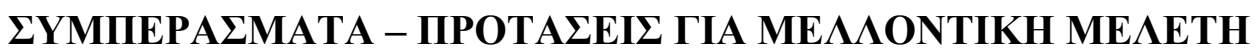

$7.1 \Sigma v \mu \pi \varepsilon \rho \alpha ́ \sigma \mu \alpha \tau \alpha$

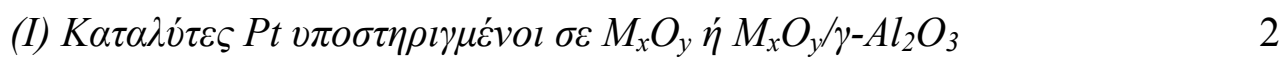

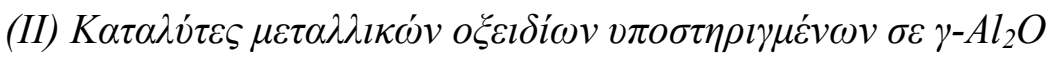
$\left(\mathrm{M}_{x} \mathrm{O}_{y} / \gamma-\mathrm{Al}_{2} \mathrm{O}_{3}\right) \quad 2$

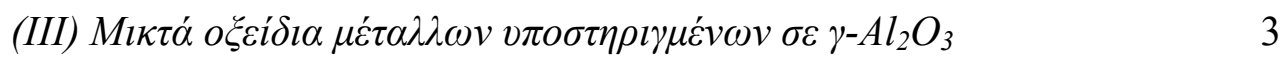

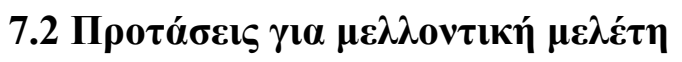




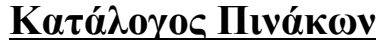

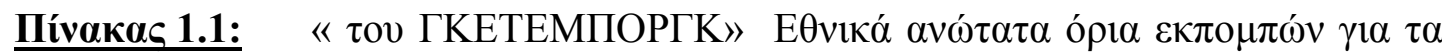

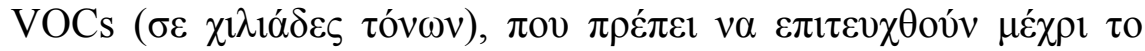
2010 .

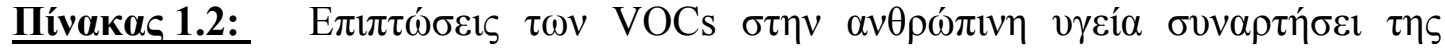

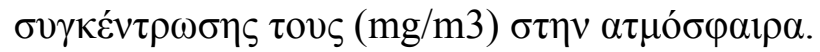

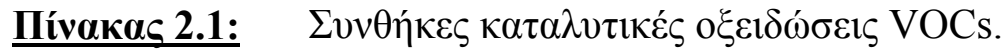

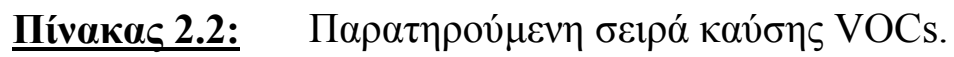

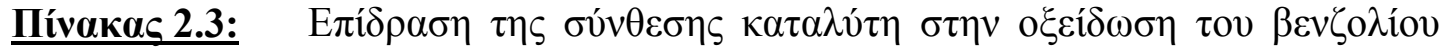

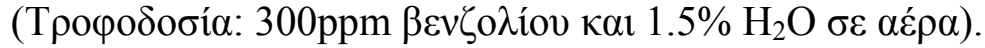

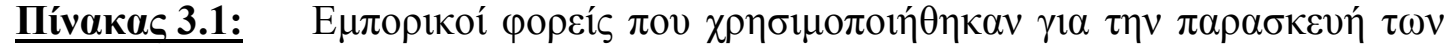
$\kappa \alpha \tau \alpha \lambda v \tau \omega ́ v$.

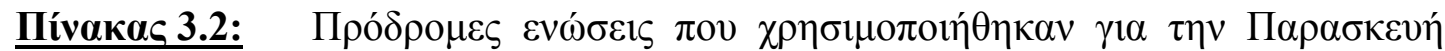
$\mu \varepsilon \tau \alpha \lambda \lambda \iota \kappa \omega ́ v ~ o \xi \varepsilon เ \delta i ́ \omega v$.

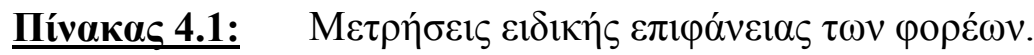

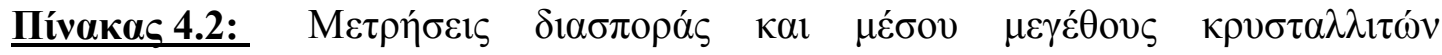

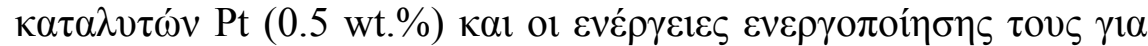

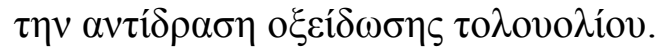

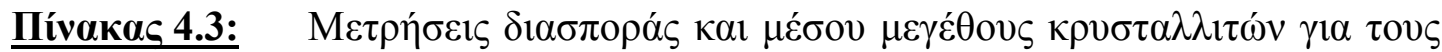
$\kappa \alpha \tau \alpha \lambda \hat{\tau} \tau \varepsilon \zeta \mathrm{Pt}\left(0.5-5\right.$ wt.\%)/ $/ \mathrm{Al}_{2} \mathrm{O}_{3}$.

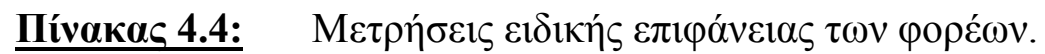

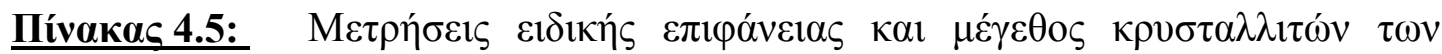

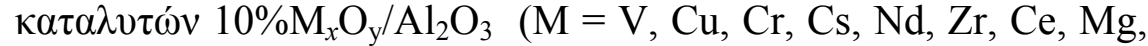
$\mathrm{Mn})$.

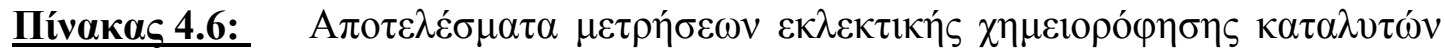

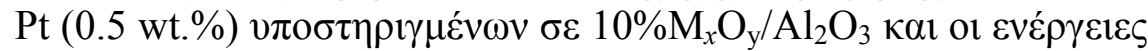

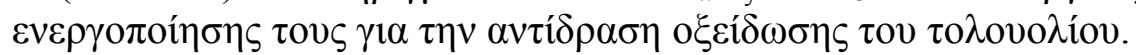

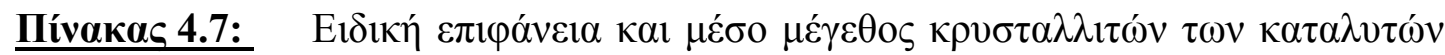
$\mathrm{M}_{x} \mathrm{O}_{\mathrm{y}} / \mathrm{Al}_{2} \mathrm{O}_{3}(\mathrm{M}=\mathrm{Cu}, \mathrm{Mn}, \mathrm{Ce}, \mathrm{Mg})$.

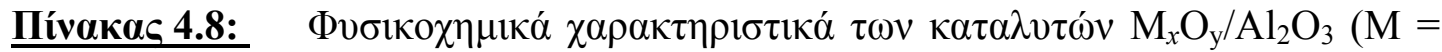
$\mathrm{Cu}, \mathrm{Mn}, \mathrm{Ce}, \mathrm{Mg})$. 


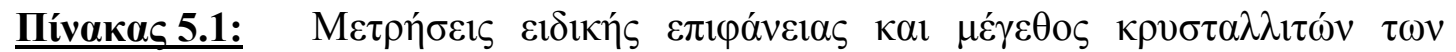

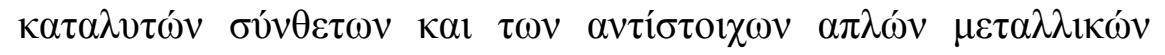
o $\xi \varepsilon 1 \delta i ́ \omega v$.

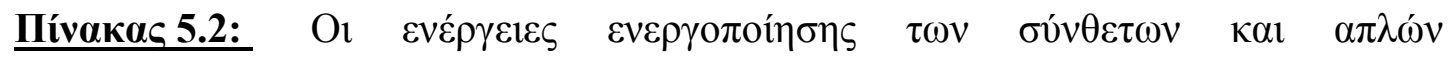

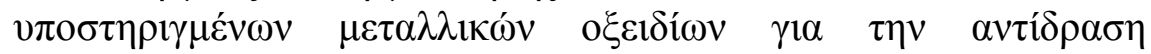

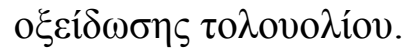

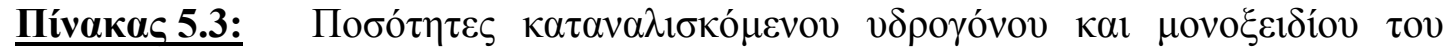

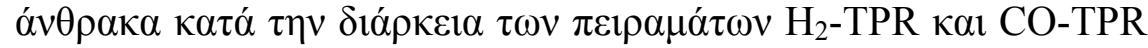

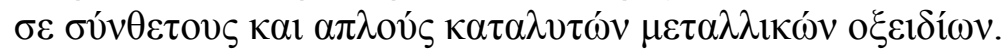

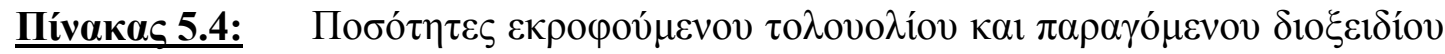

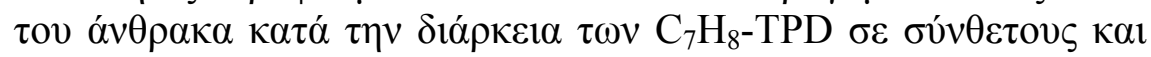

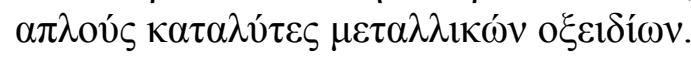

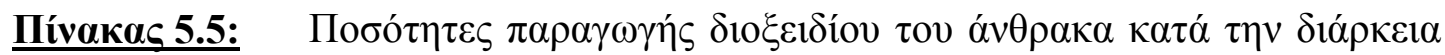

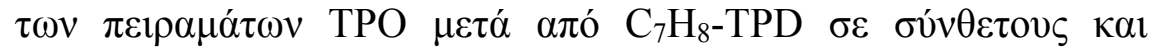

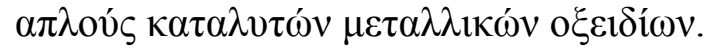

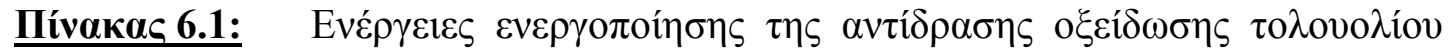

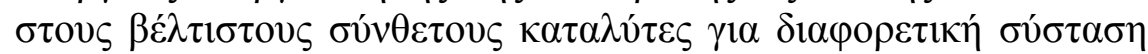

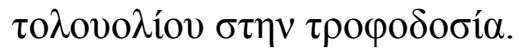

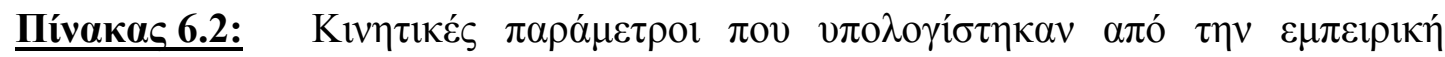

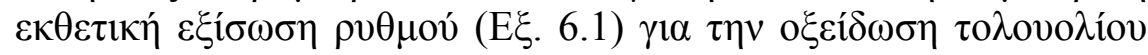
$\sigma \varepsilon \sigma u ́ v \theta \varepsilon \tau o v \varsigma \kappa \alpha \tau \alpha \lambda v ́ \tau \varepsilon \varsigma$.

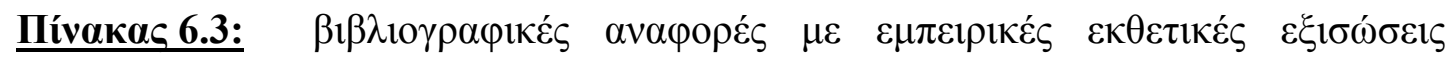
$v \theta \mu \omega ́ v$ Power-Law) $\gamma 1 \alpha \tau \eta v$ o $\xi \varepsilon i ́ \delta \omega \sigma \eta \tau \omega v$ VOC.

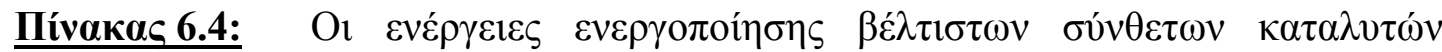

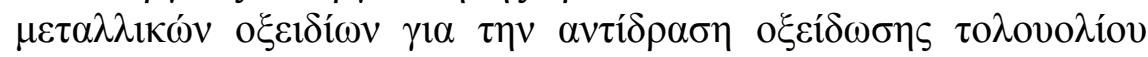

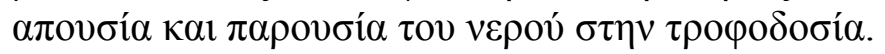




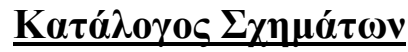

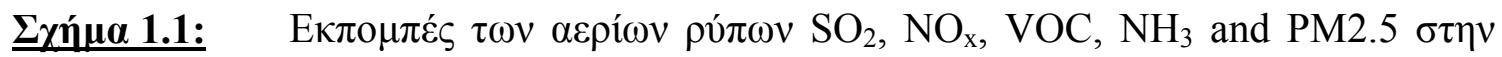
EU-25 "With climate measures" projection, relative to year 2000 [= $100 \%]$

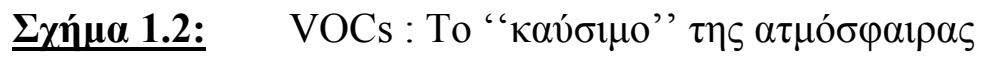

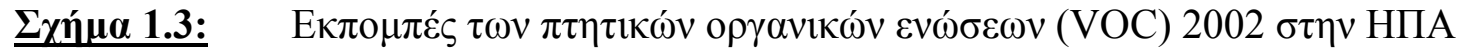

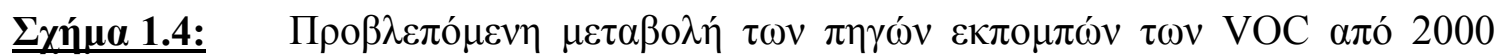

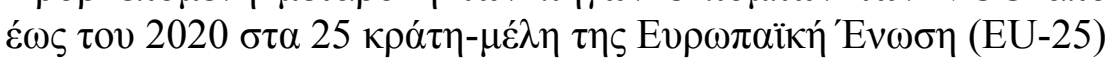

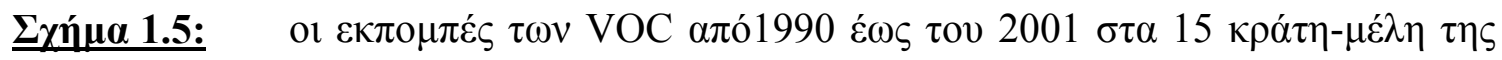

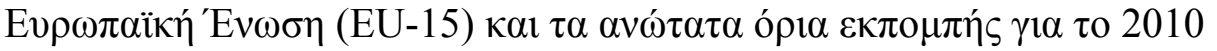

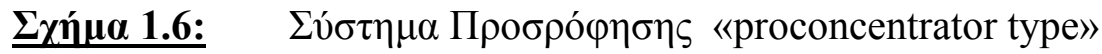

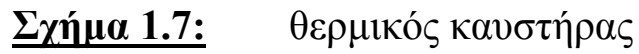

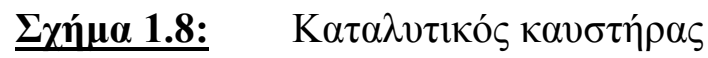

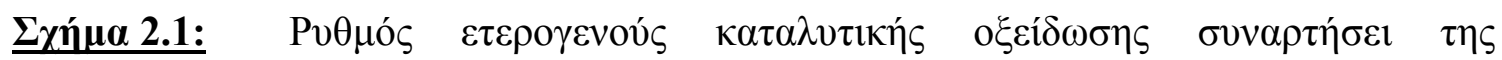
$\theta \varepsilon \rho \mu о к \rho \alpha \sigma i ́ \alpha$

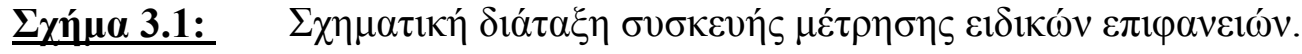

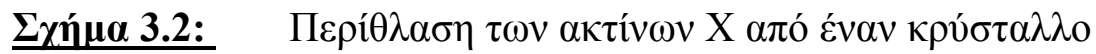

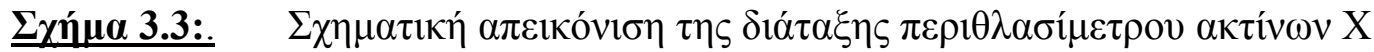

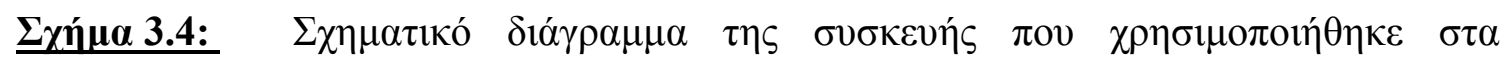

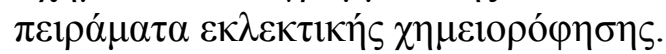

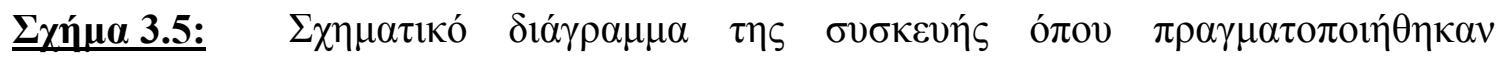

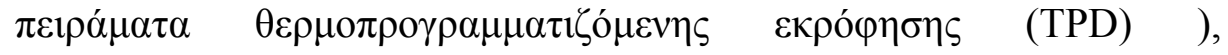

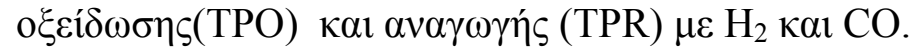

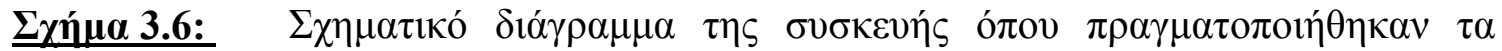

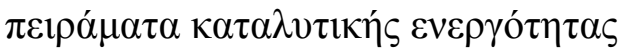

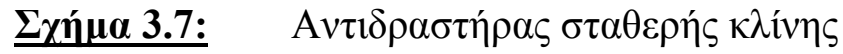

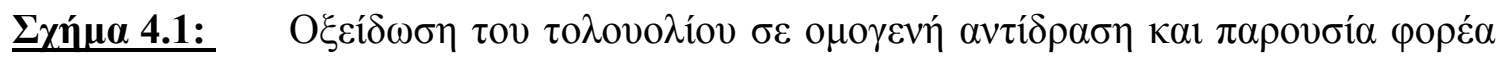
$\gamma-\mathrm{Al}_{2} \mathrm{O}_{3}$

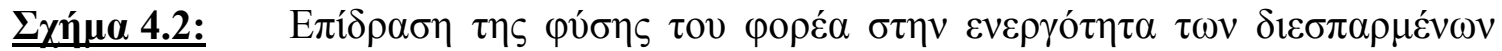
$\kappa \alpha \tau \alpha \lambda \nu \tau \omega ́ v \operatorname{Pt}(0.5$ wt.\%). 


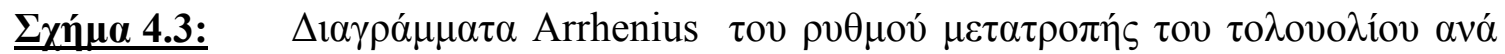

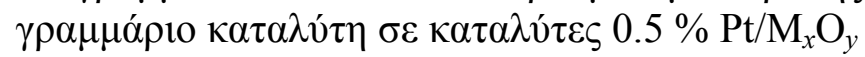

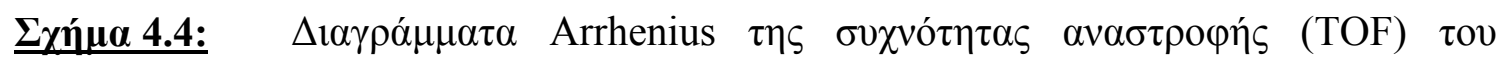

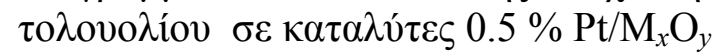

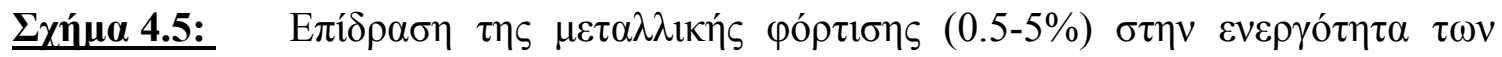
$\kappa \alpha \tau \alpha \lambda v \tau \omega ́ v \mathrm{Pt} / \mathrm{Al}_{2} \mathrm{O}_{3}$.

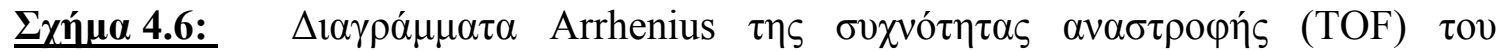

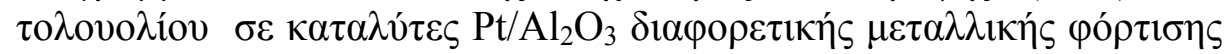
$(0.5-5 \%)$

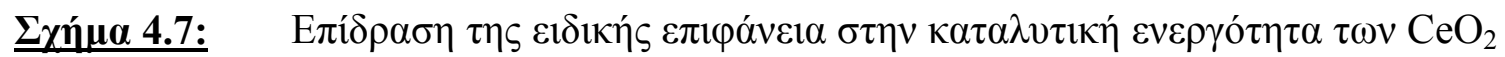
$\kappa \alpha \tau \alpha \lambda v \tau \omega ́ v$

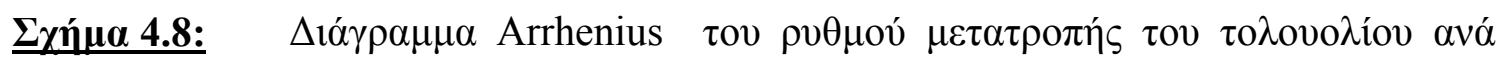

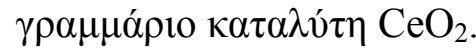

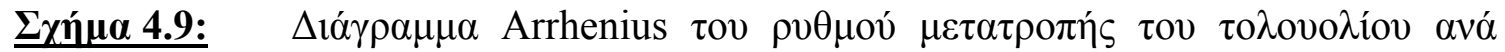
$\mu o v \alpha ́ \delta \alpha \varepsilon \pi i \varphi \alpha ́ v \varepsilon 1 \alpha \varsigma \kappa \alpha \tau \alpha \lambda u ́ \tau \eta \mathrm{CeO}_{2}$.

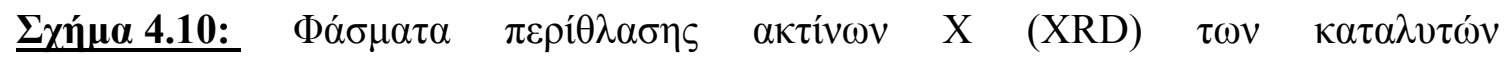
$10 \% \mathrm{M}_{x} \mathrm{O}_{\mathrm{y}} / \mathrm{Al}_{2} \mathrm{O}_{3}(\mathrm{M}=\mathrm{V}, \mathrm{Cu}, \mathrm{Cr}, \mathrm{Cs}, \mathrm{Nd}, \mathrm{Zr}, \mathrm{Ce}, \mathrm{Mg}, \mathrm{Mn})$.

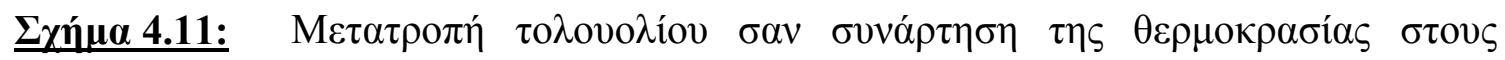

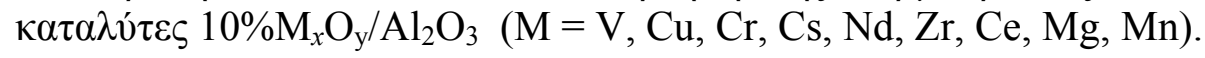

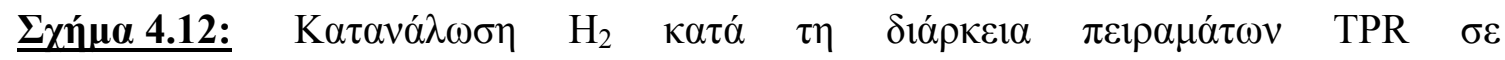
$\pi \rho \circ \xi_{\varepsilon} \delta \omega \mu \varepsilon ́ v o v \varsigma \kappa \alpha \tau \alpha \lambda v ́ \tau \varepsilon \varsigma 10 \% \mathrm{M}_{x} \mathrm{O}_{\mathrm{y}} / \mathrm{Al}_{2} \mathrm{O}_{3}$.

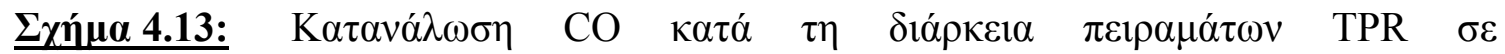
$\pi \rho \circ o \xi \varepsilon 1 \delta \omega \mu \varepsilon ́ v o v \varsigma \kappa \alpha \tau \alpha \lambda v ́ \tau \varepsilon \varsigma 10 \% \mathrm{M}_{x} \mathrm{O}_{\mathrm{y}} / \mathrm{Al}_{2} \mathrm{O}_{3}$

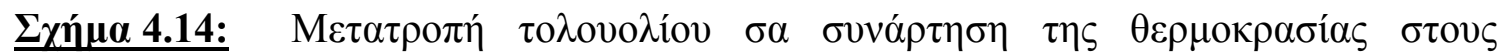

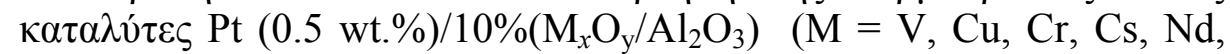
$\mathrm{Zr}, \mathrm{Ce}, \mathrm{Mg}, \mathrm{Mn})$.

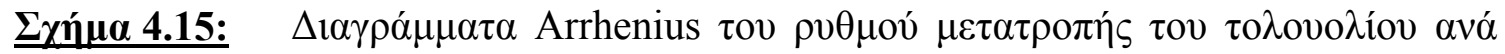

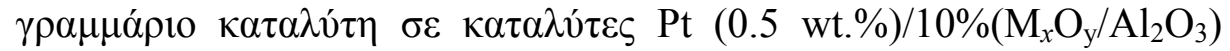
$(\mathrm{M}=\mathrm{V}, \mathrm{Cu}, \mathrm{Cr}, \mathrm{Cs}, \mathrm{Nd}, \mathrm{Zr}, \mathrm{Ce}, \mathrm{Mg}, \mathrm{Mn})$.

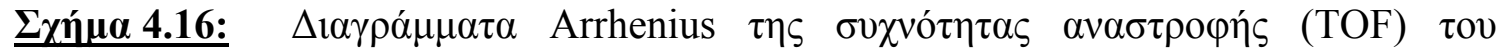

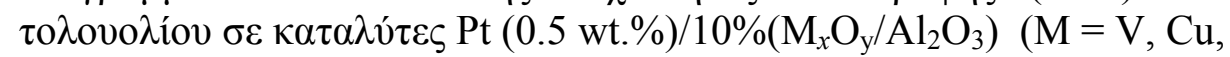
$\mathrm{Cr}, \mathrm{Cs}, \mathrm{Nd}, \mathrm{Zr}, \mathrm{Ce}, \mathrm{Mg}, \mathrm{Mn})$.

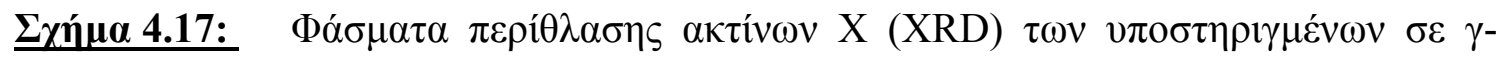

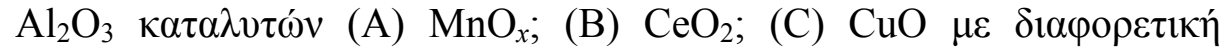

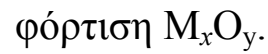




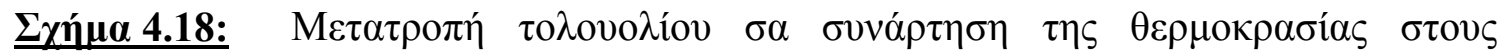

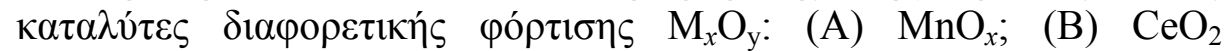

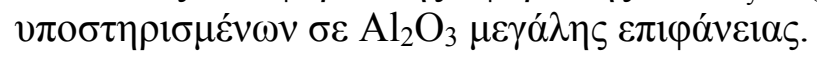

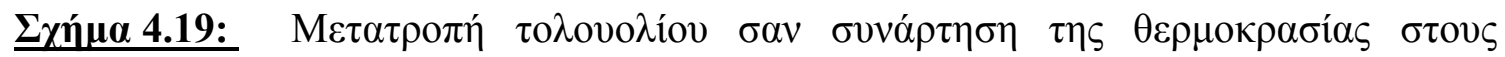

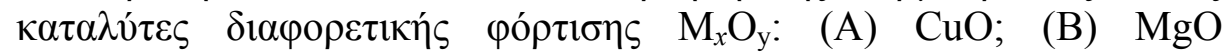

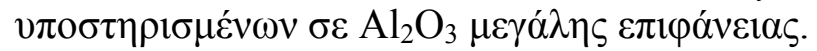

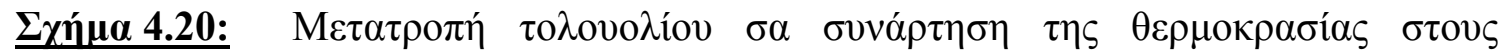

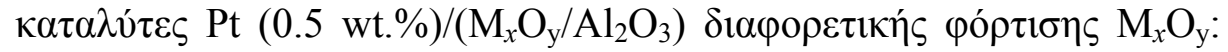
(A) $\mathrm{MnO}_{x} ;$ (B) $\mathrm{CeO}_{2}$.

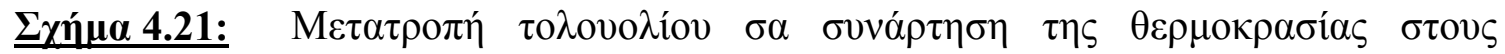

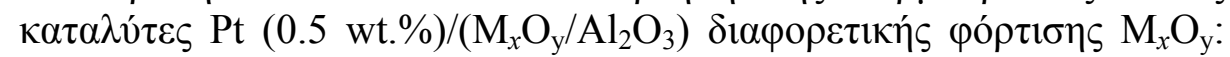
(A) $\mathrm{CuO}$; (B) $\mathrm{MgO}$.

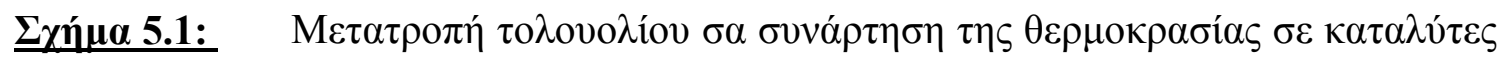

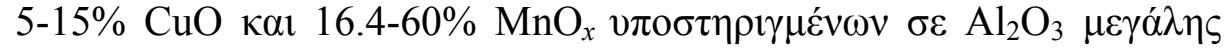

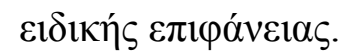

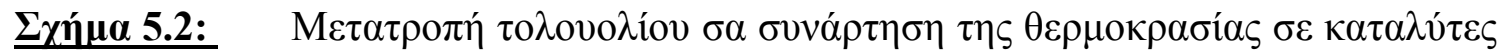

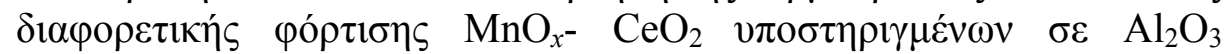

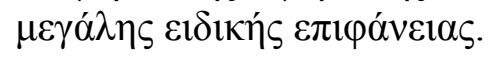

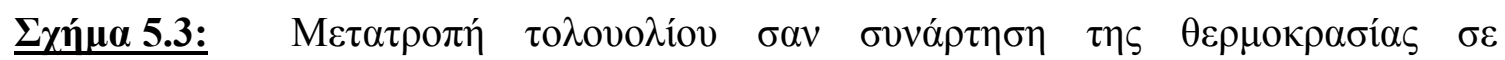

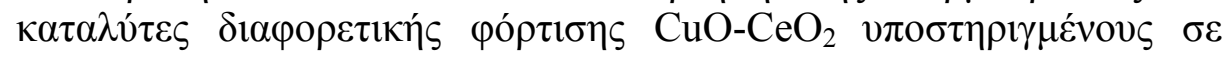

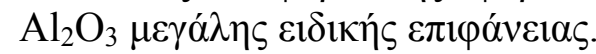

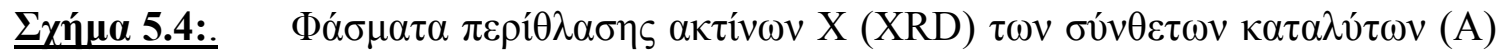

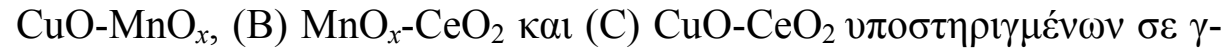

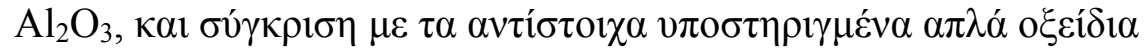

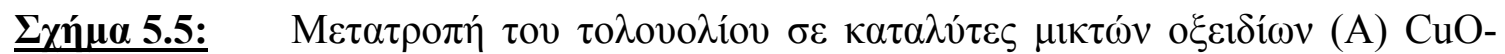

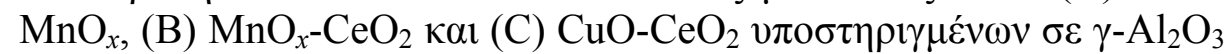

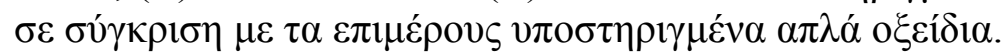

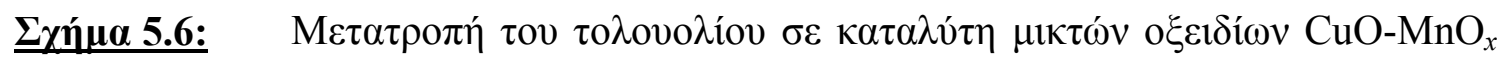

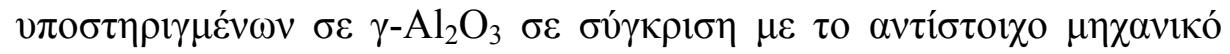

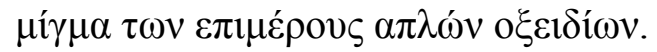

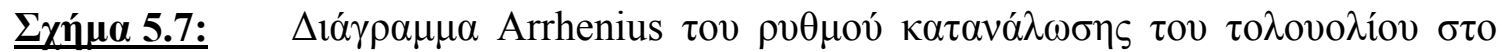

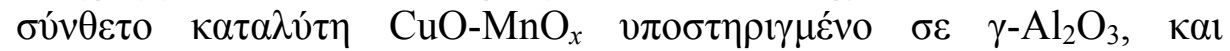

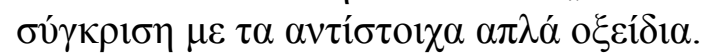

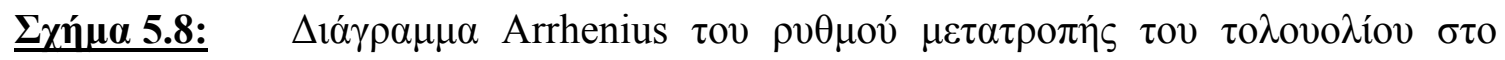

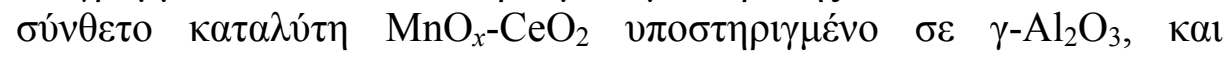

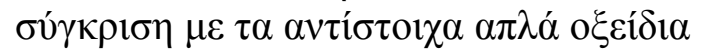




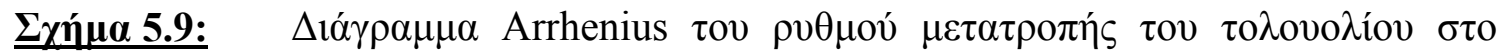

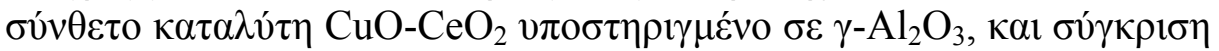

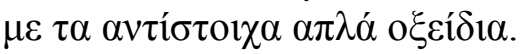

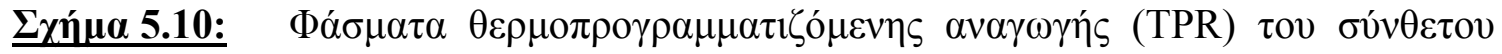

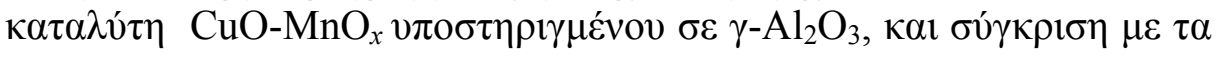

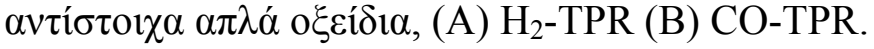

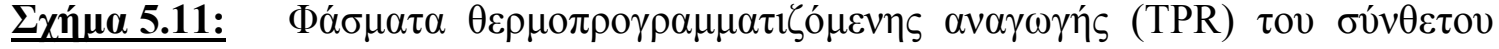

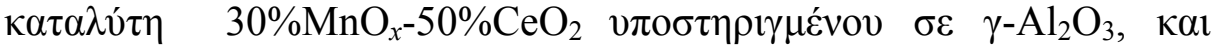

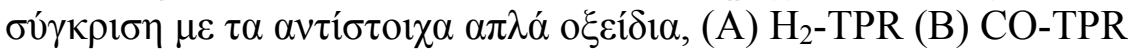

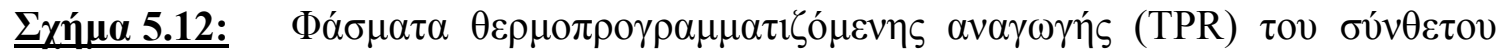

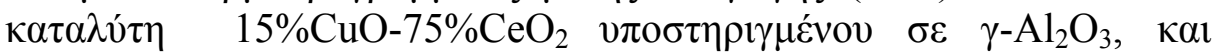

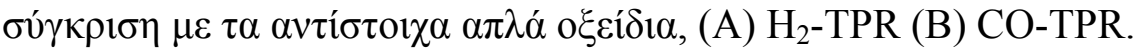

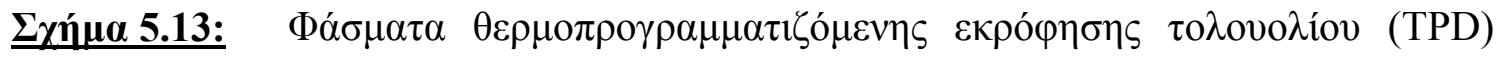

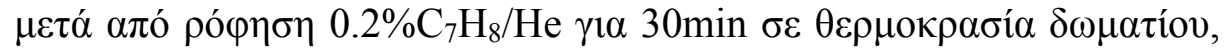

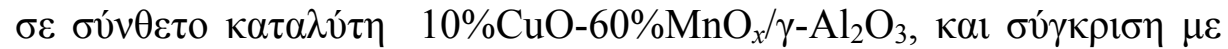

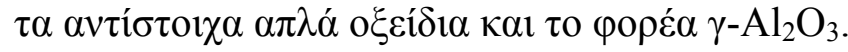

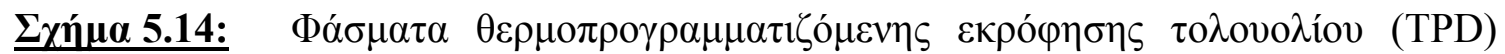

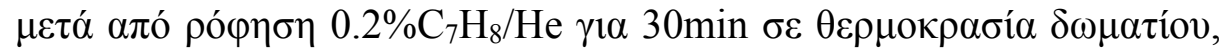

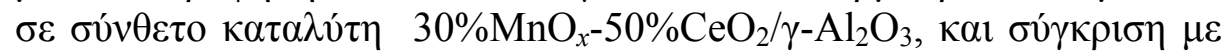

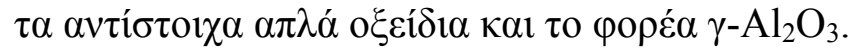

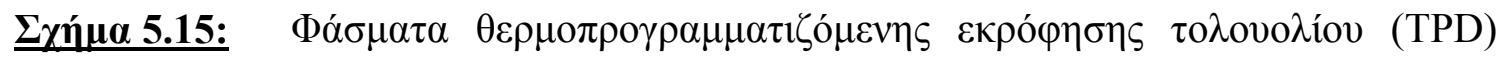

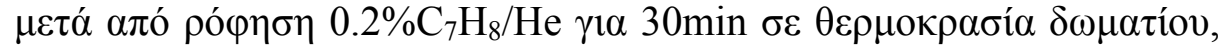

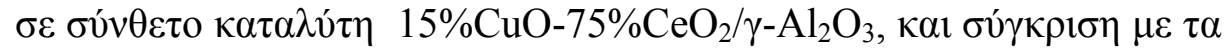

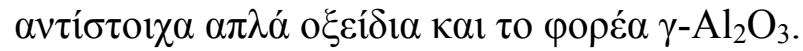

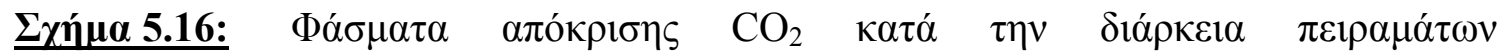

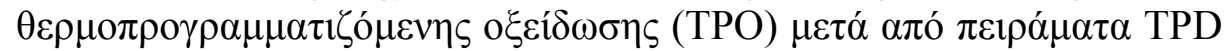

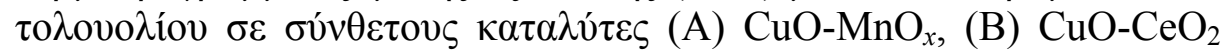

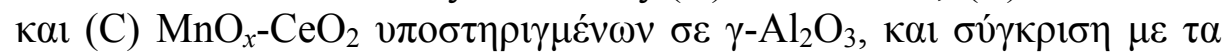

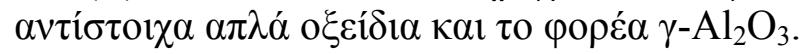

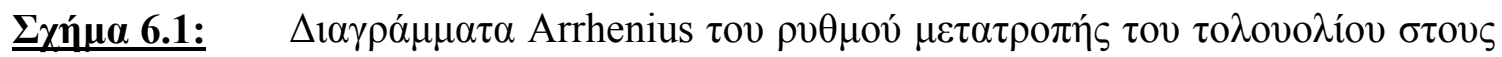

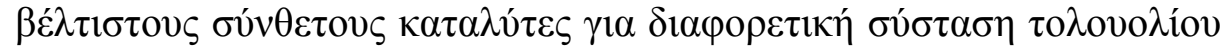
$\sigma \tau \eta v \tau \rho о \varphi о \delta о \sigma i ́ \alpha$.

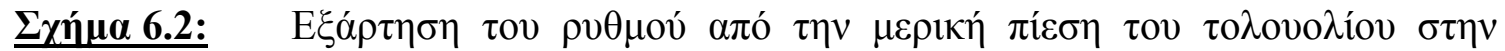

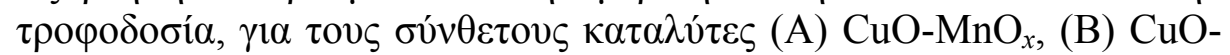

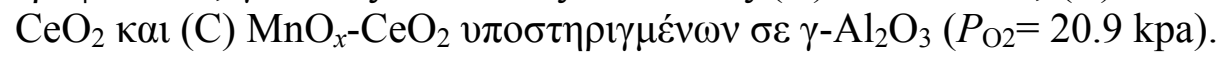

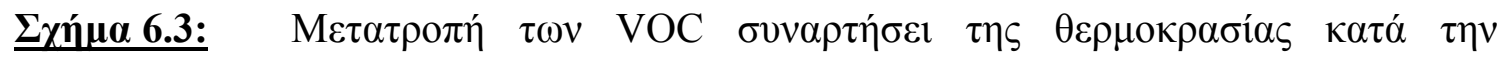

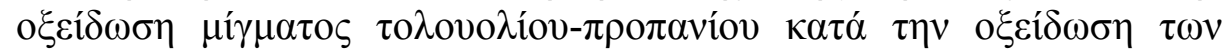

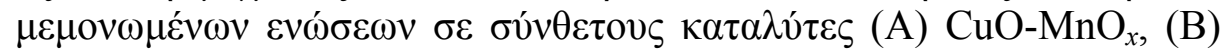
$\mathrm{CuO}-\mathrm{CeO}_{2} \kappa \alpha \imath(\mathrm{C}) \mathrm{MnO}_{x}-\mathrm{CeO}_{2} v \pi \mathrm{o} \tau \tau \rho \imath \gamma \mu \varepsilon \dot{v} \omega \nu \sigma \varepsilon \gamma-\mathrm{Al}_{2} \mathrm{O}_{3}$. 


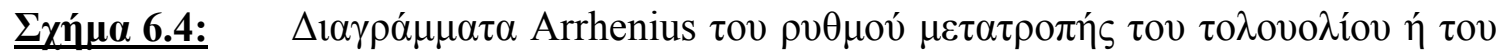

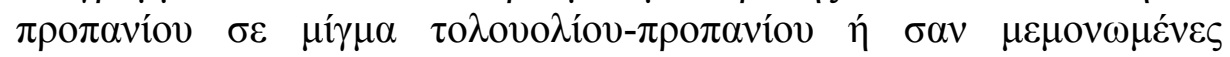

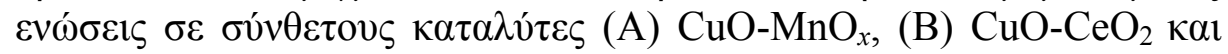

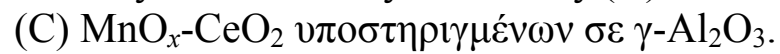

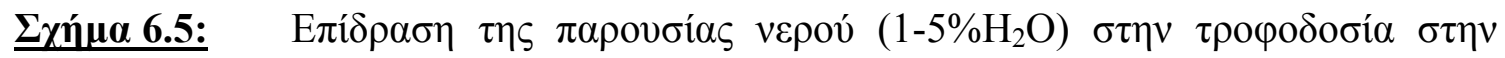
$\kappa \alpha \tau \alpha \lambda v \tau \iota \kappa \eta ́ ~ \varepsilon v \varepsilon \rho \gamma o ́ \tau \eta \tau \alpha ~ \tau \omega v ~ \sigma u ́ v \theta \varepsilon \tau \omega v ~ \kappa \alpha \tau \alpha \lambda v \tau \omega ́ v ~(A) ~ C u O-\mathrm{MnO}_{x},(\mathrm{~B})$

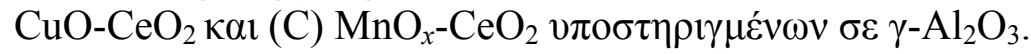

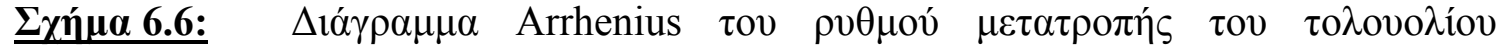

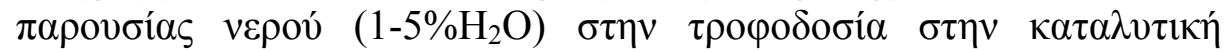

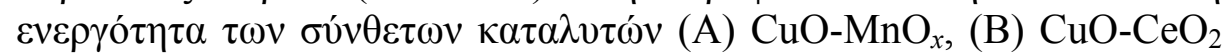

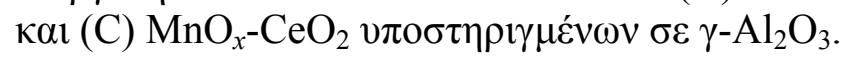

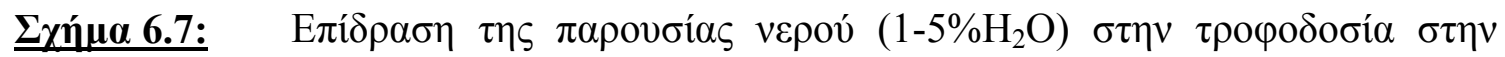

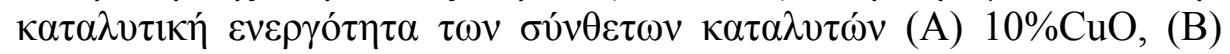

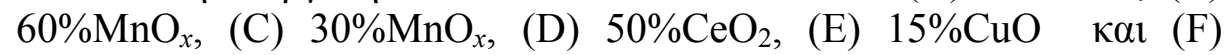
$75 \% \mathrm{CeO}_{2} v \pi 0 \sigma \tau \eta \rho \imath \mu \varepsilon ́ v \omega v \sigma \varepsilon \gamma-\mathrm{Al}_{2} \mathrm{O}_{3}$. 


\section{КEФA $А$ AIO $1^{\circ}$}

\section{ПTHTIKЕ $\Sigma$ OPГANIKE $\Sigma$ EN $\Omega \Sigma E I \Sigma$ KAI TO ПЕРIBA $\Lambda \Lambda$ ON}

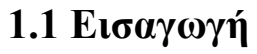

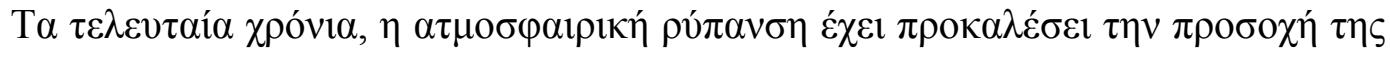

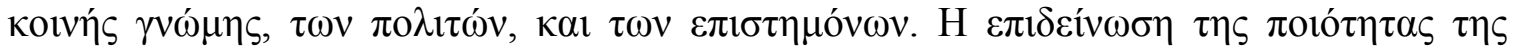

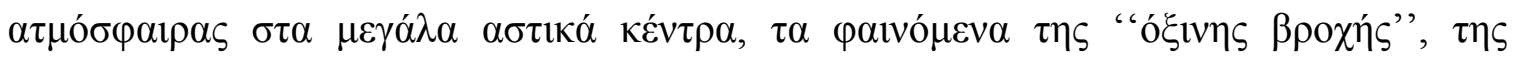

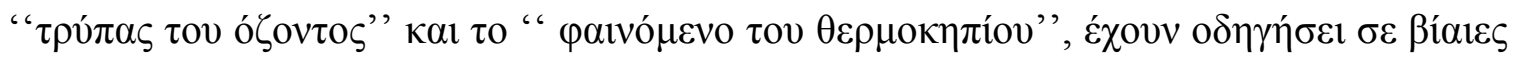

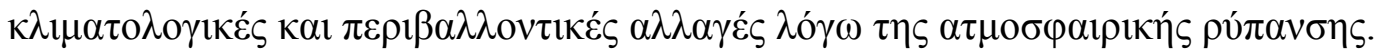

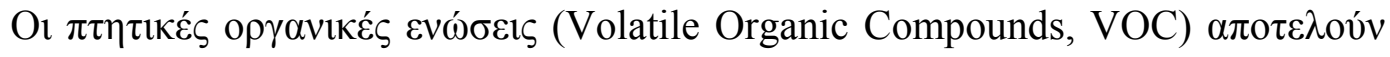

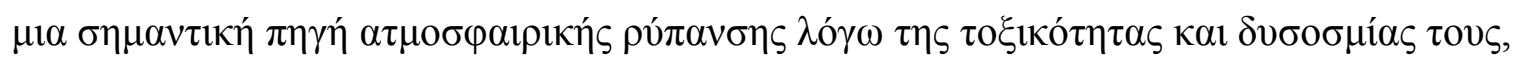

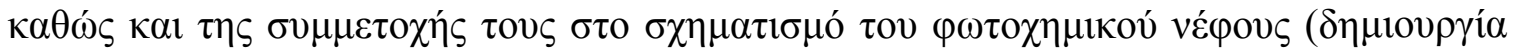

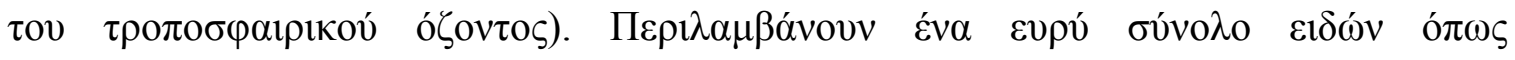




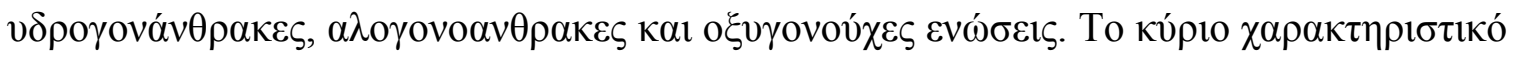

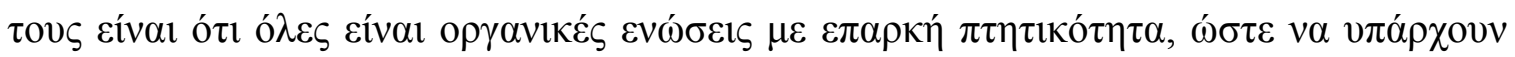

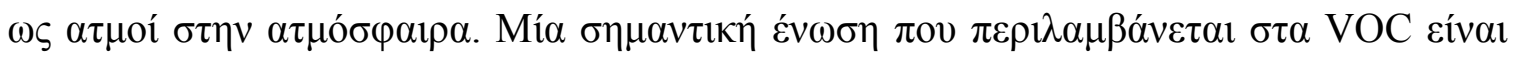

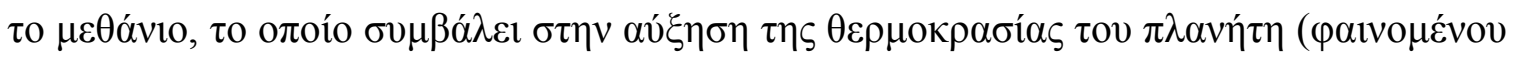

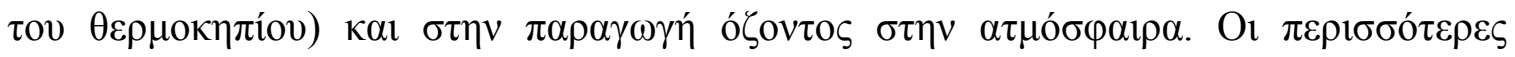

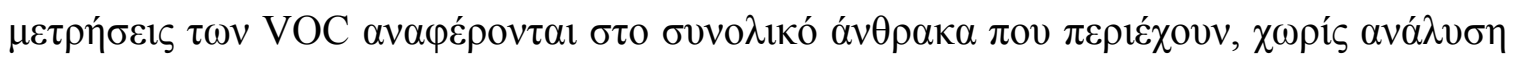

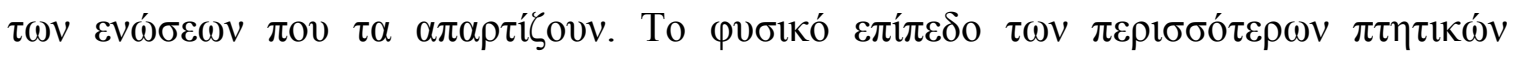

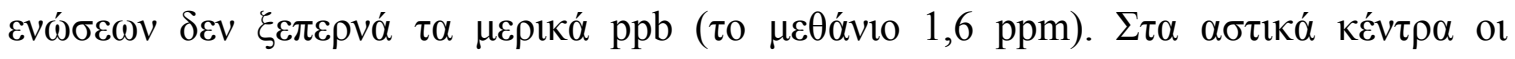

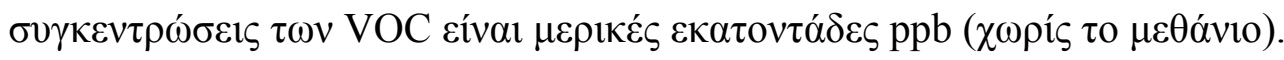

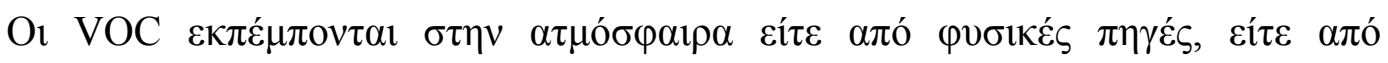

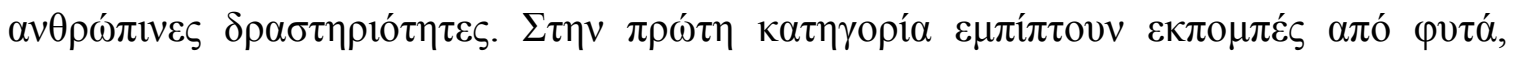

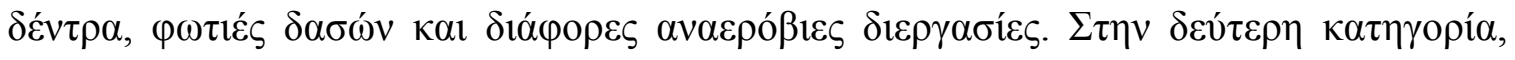

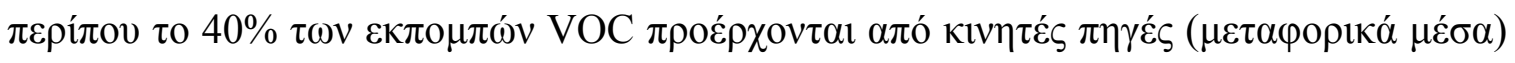

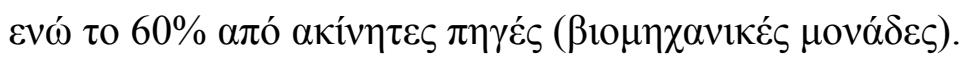

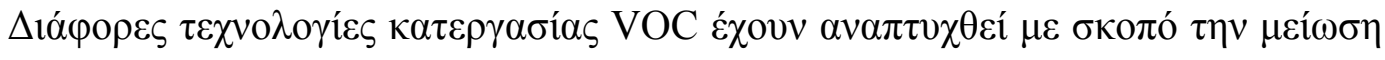

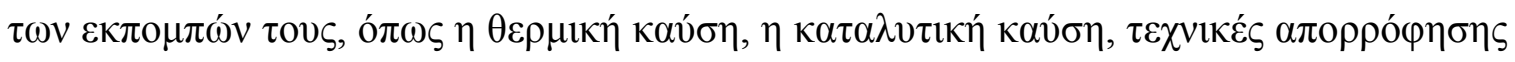

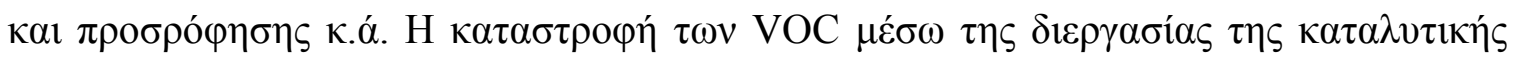

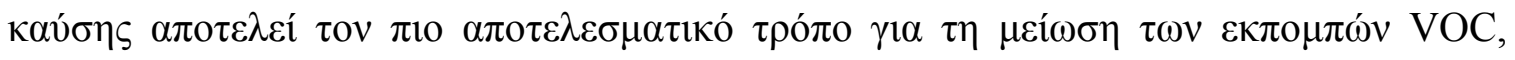

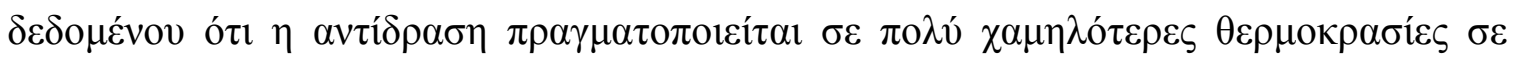

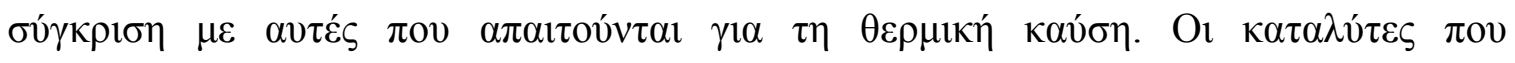

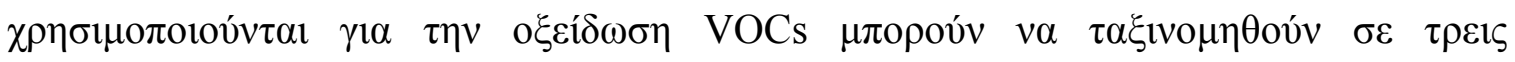

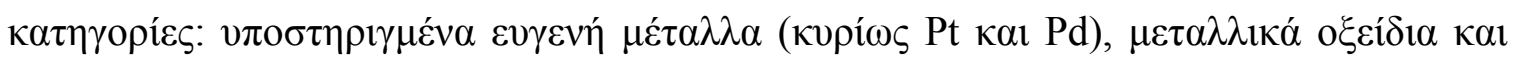

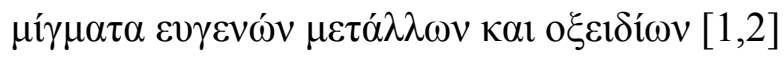

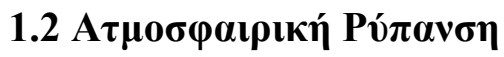

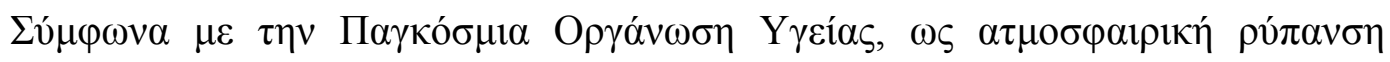

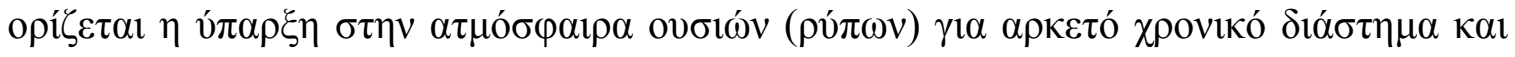

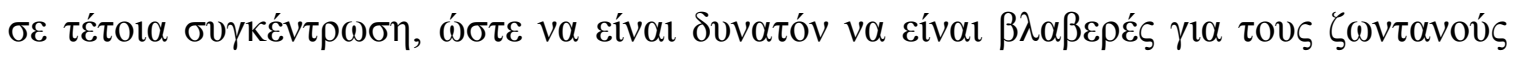

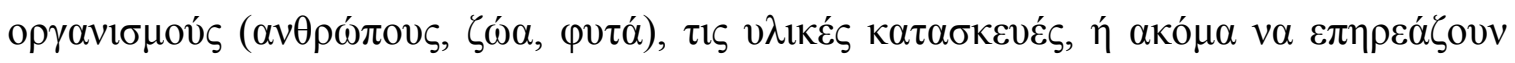

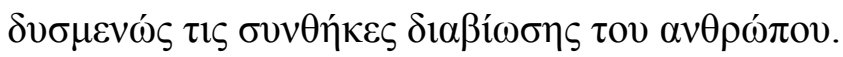




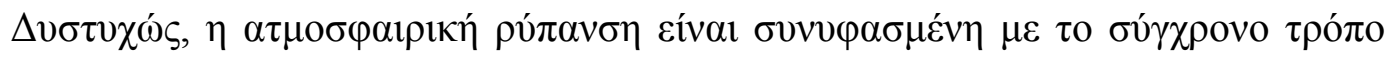

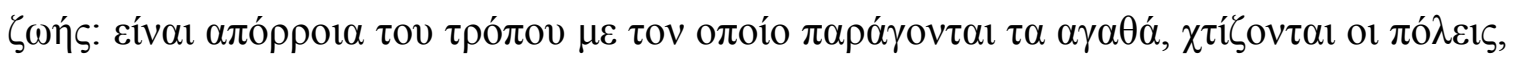

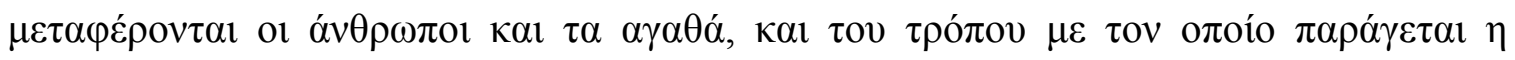

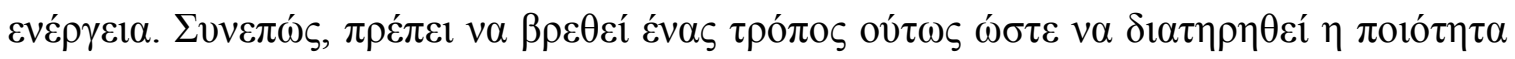

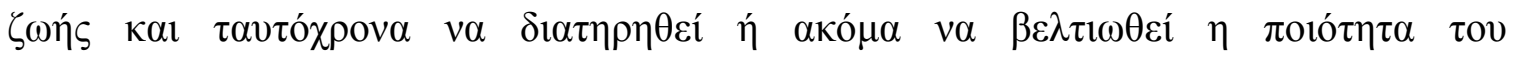

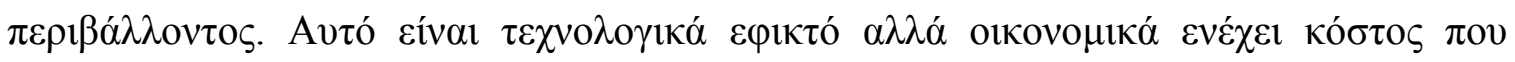

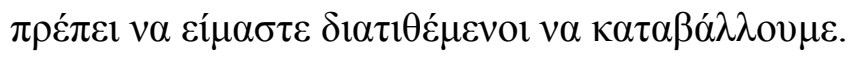

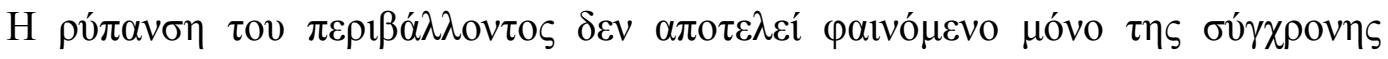

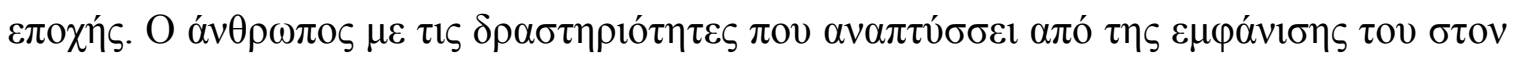

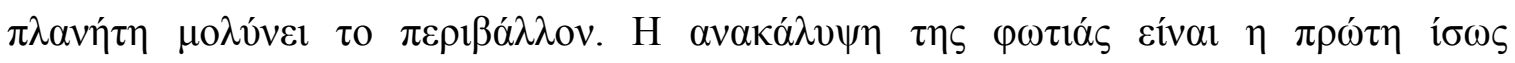

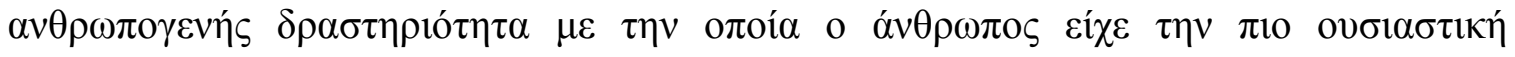

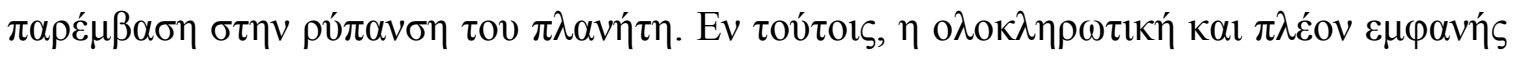

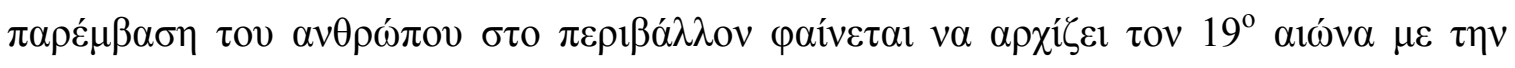

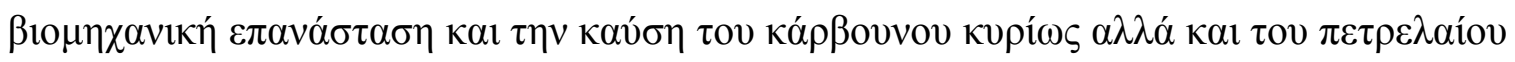

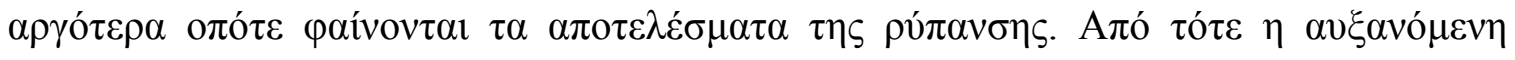

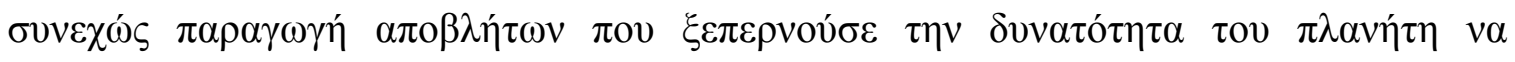

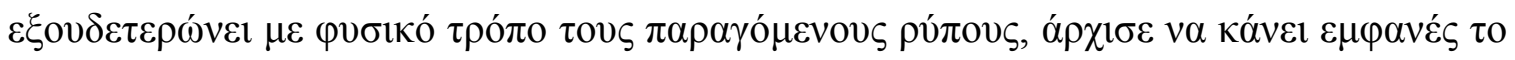

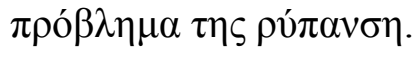

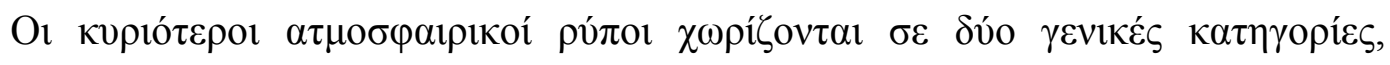

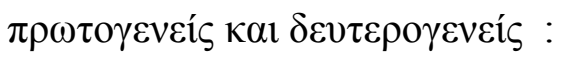

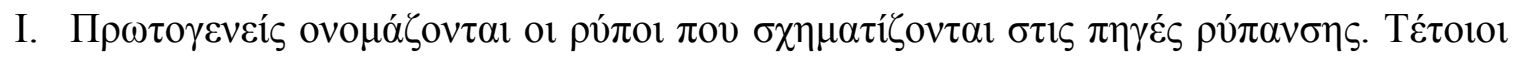

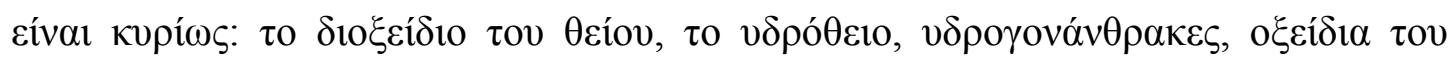

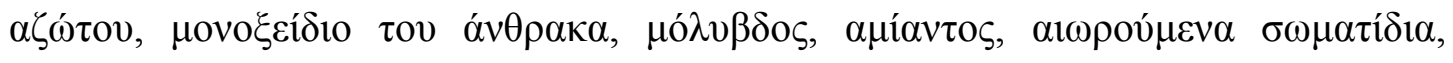
калvós.

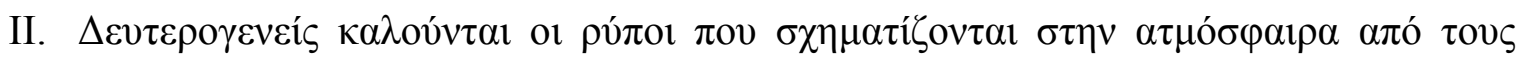

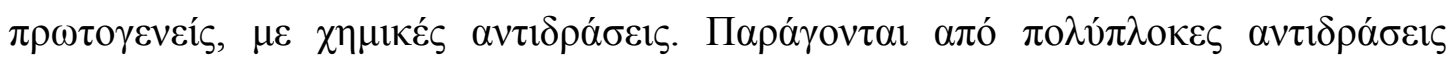

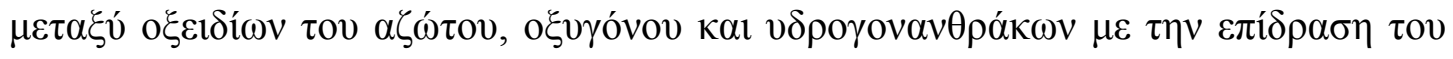

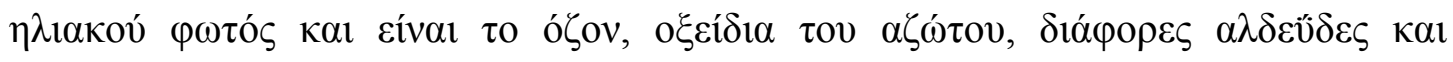

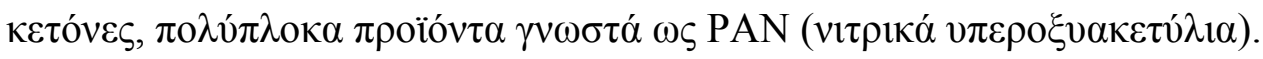

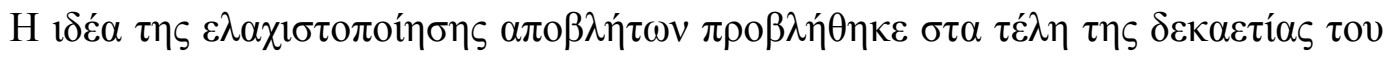

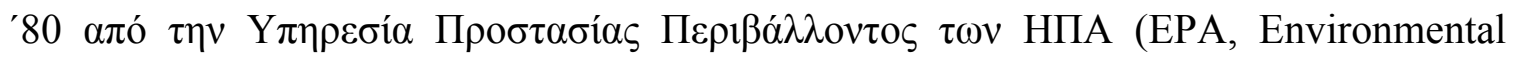




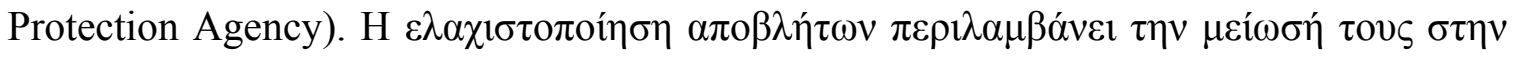

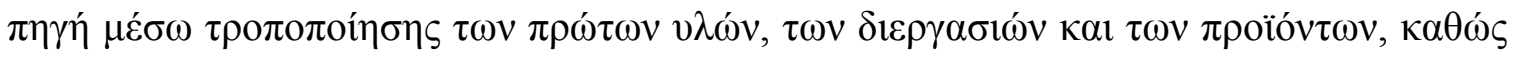

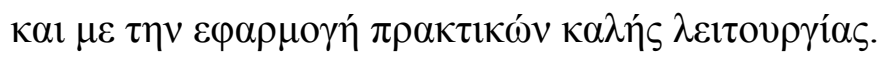

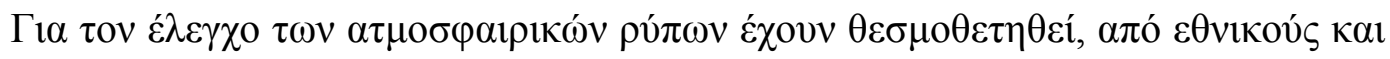

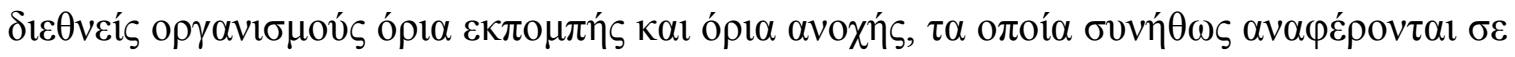

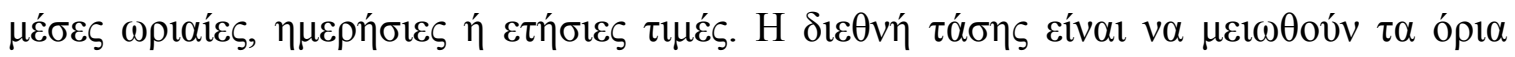

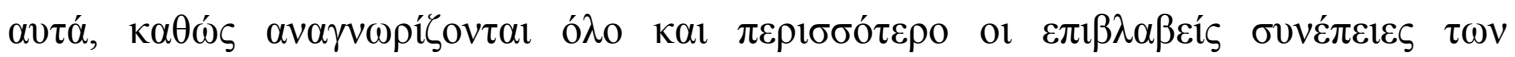

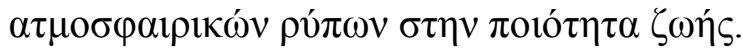

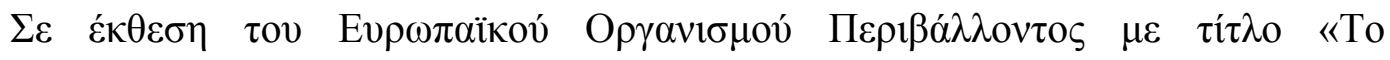

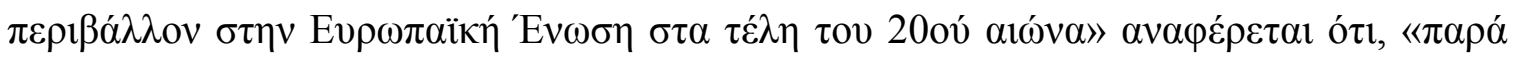

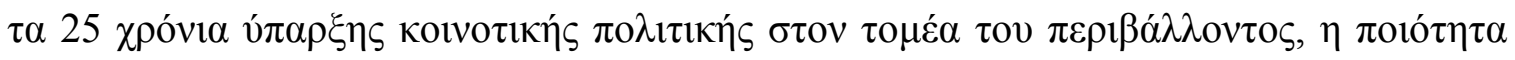

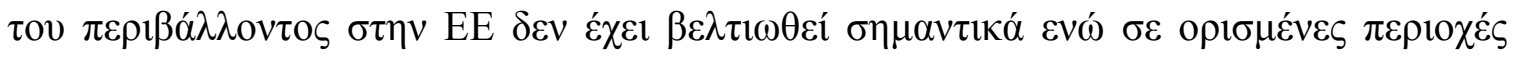
$\varepsilon \pi \imath \delta \varepsilon เ v \omega ́ v \varepsilon \tau \alpha \imath »$.

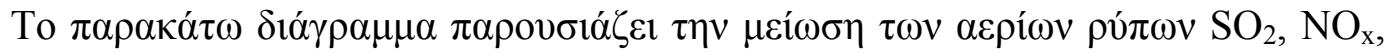

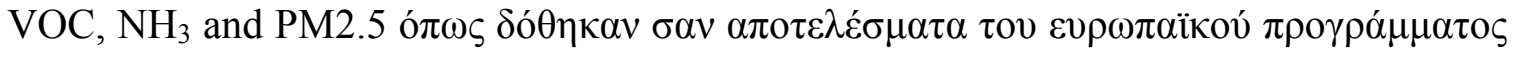
«Baseline projections of European air quality up to 2020» [3].

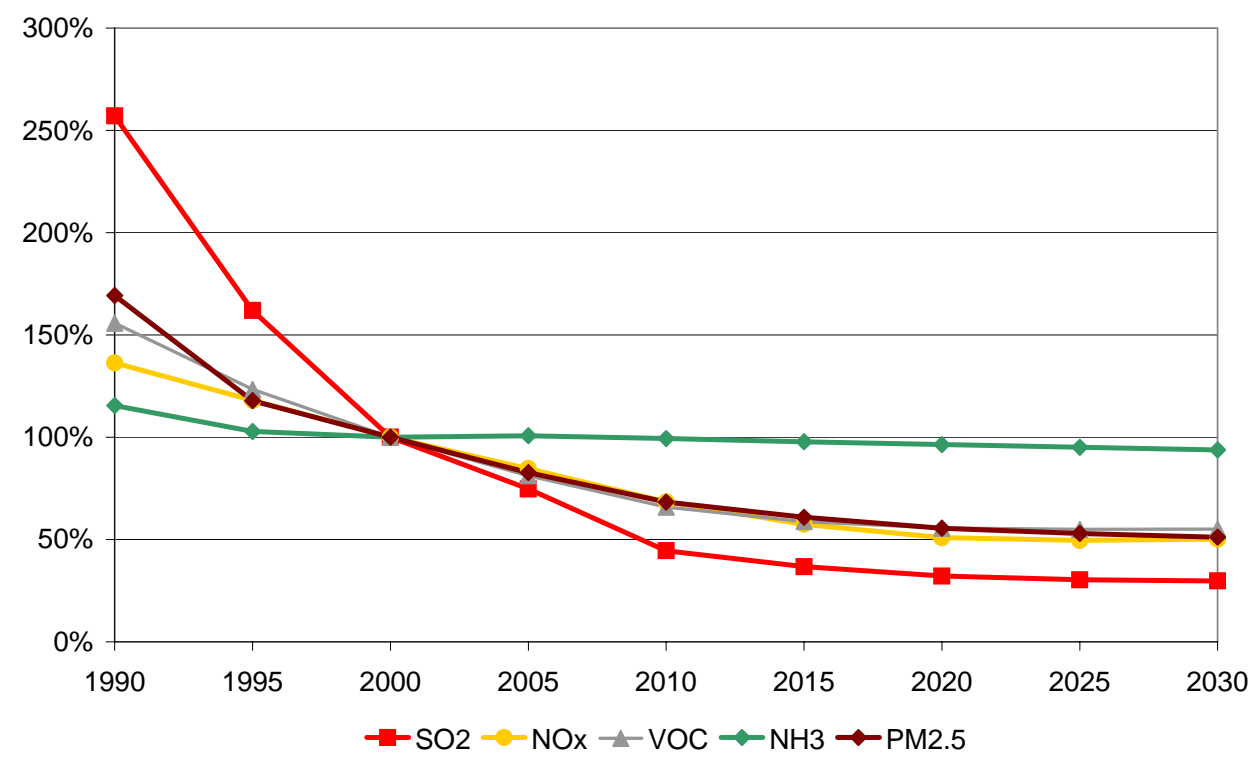

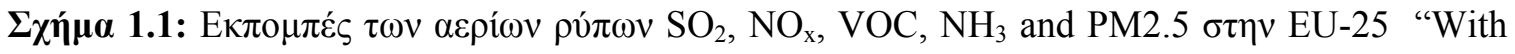
climate measures" projection, relative to year $2000[=100 \%]$ 


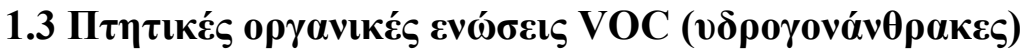

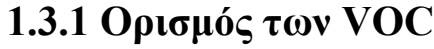

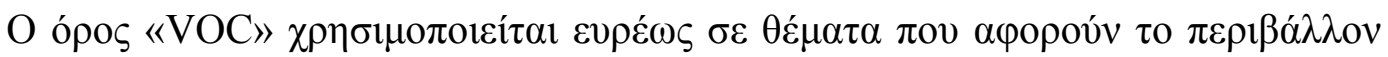

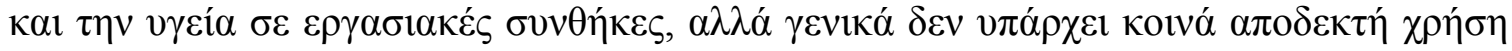

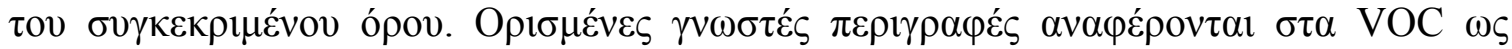

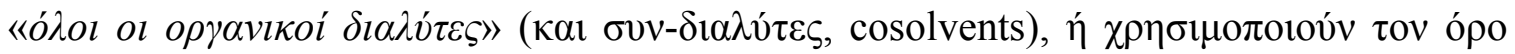

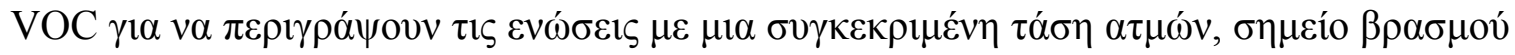

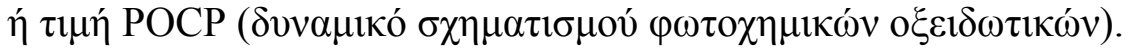

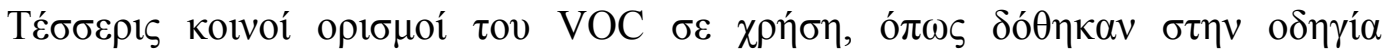

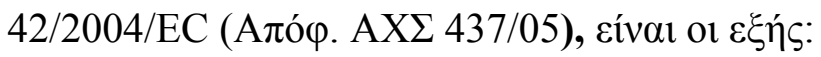

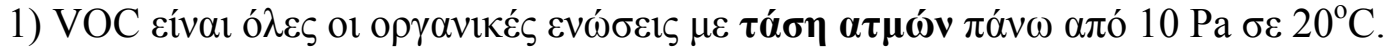

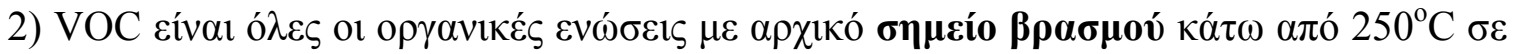
$1 \mathrm{~atm}$.

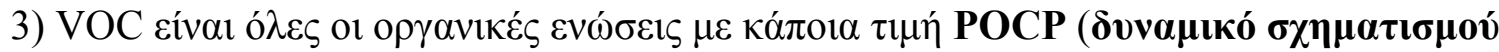

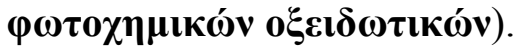

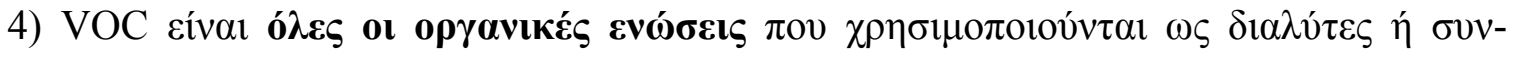
$\delta 1 \alpha \lambda \hat{\tau} \varepsilon \varsigma[4]$.

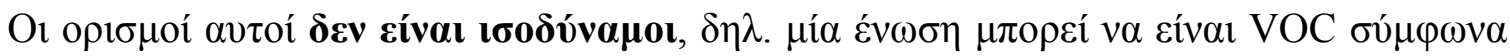

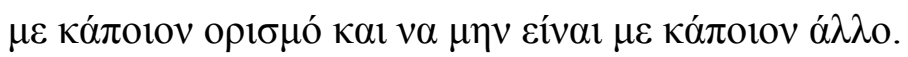

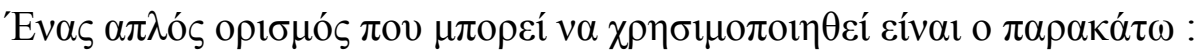

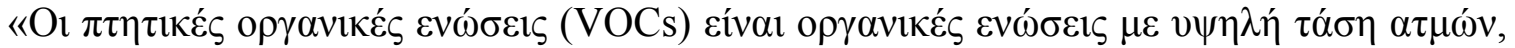

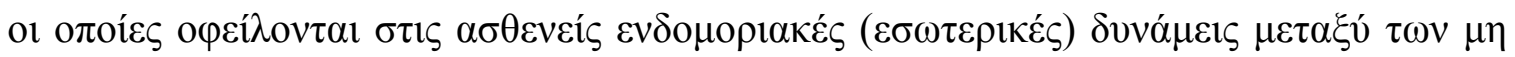

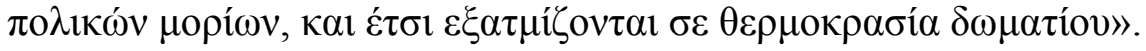

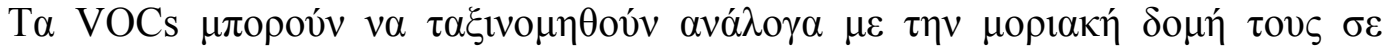

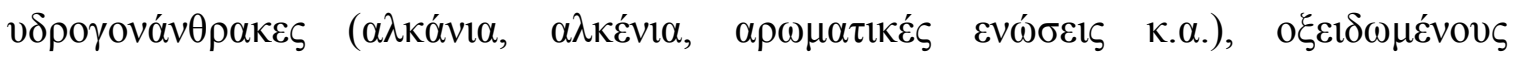

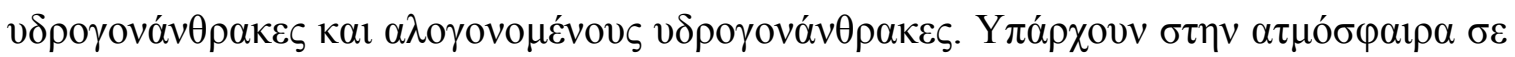

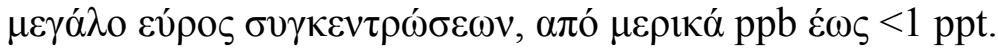

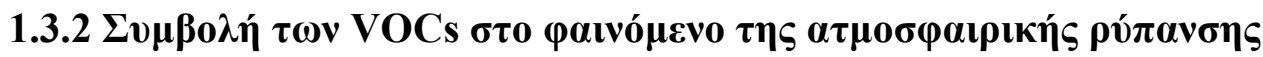

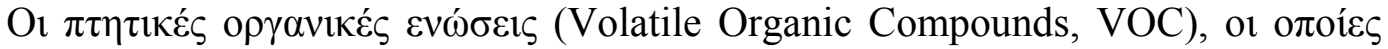

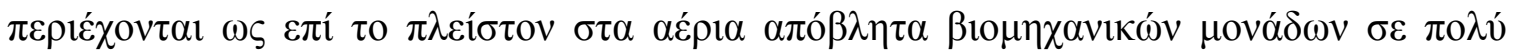




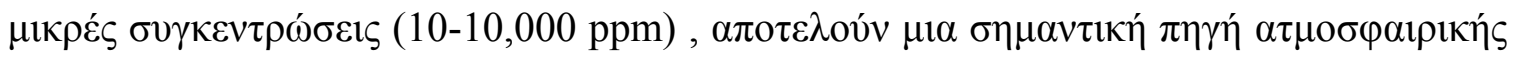

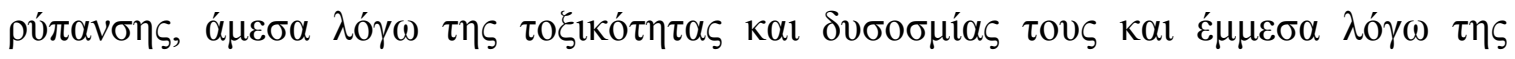

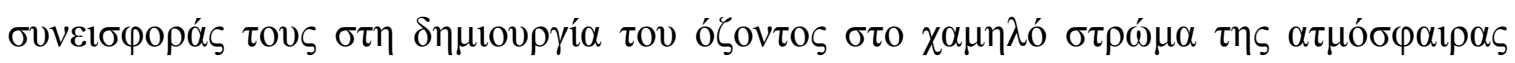

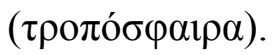

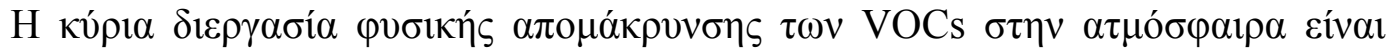

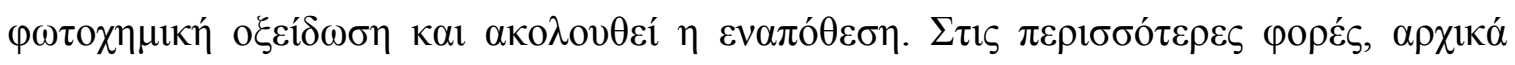

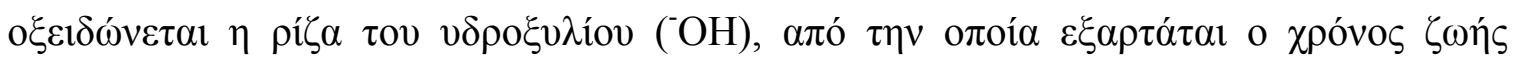

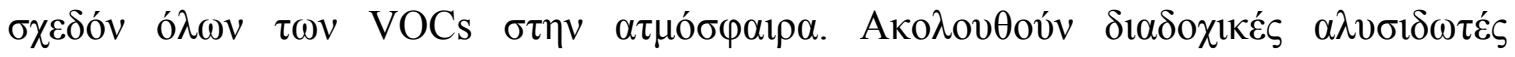

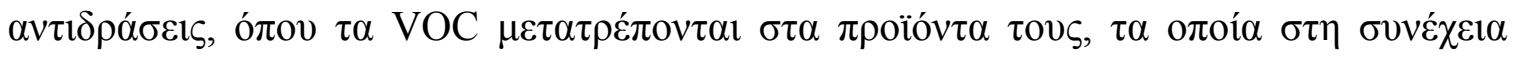

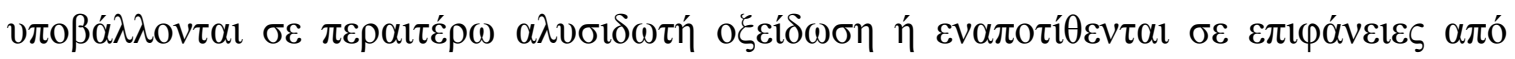

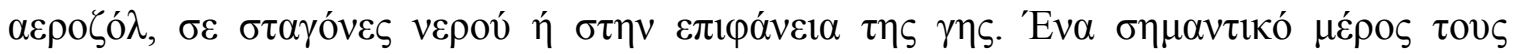

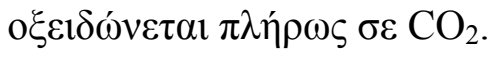

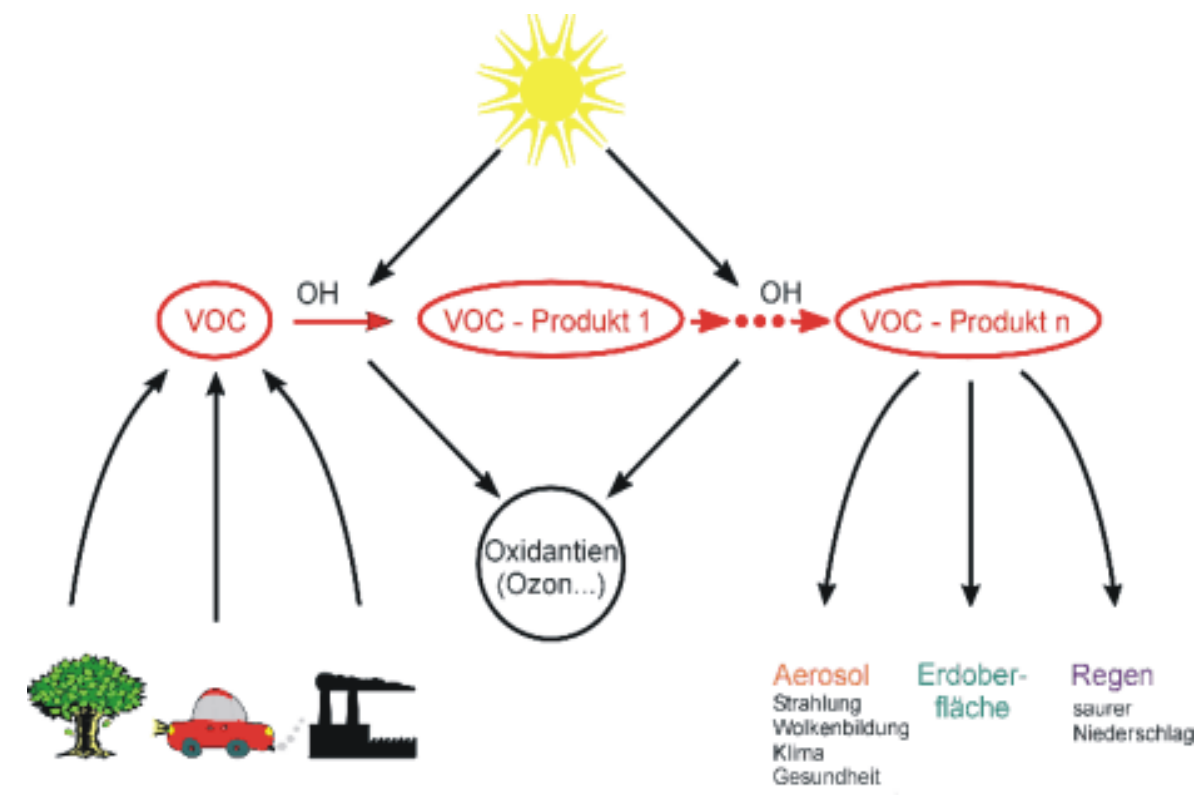

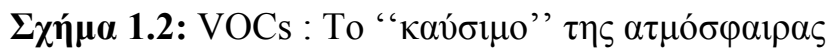

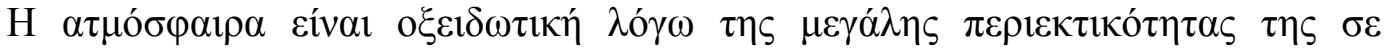

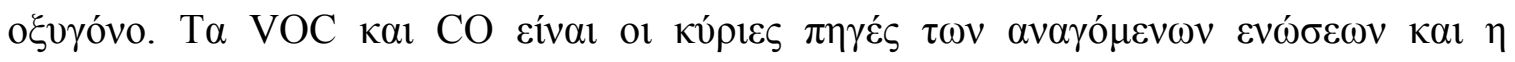




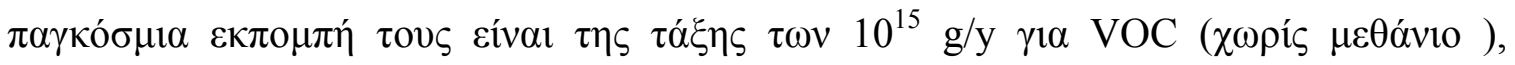

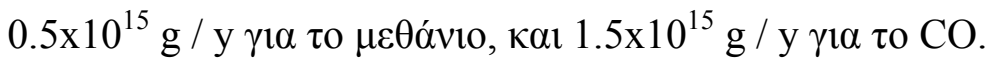

$$
\text { VOCs }+\mathrm{NO}^{-}+\text {sunlight } \rightarrow \longrightarrow \mathrm{O}_{3}+\mathrm{HNO}_{3}+\text { organic compounds }
$$

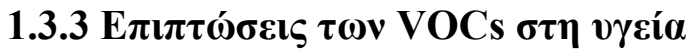

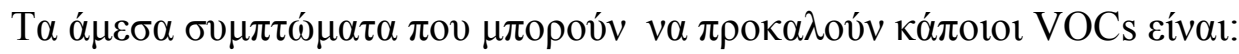

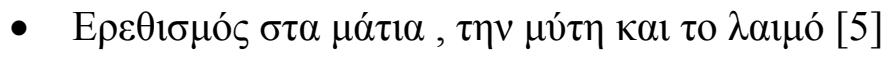

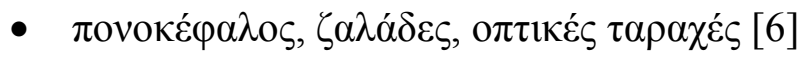

- $\varepsilon \xi \alpha \sigma \theta \varepsilon ́ v \eta \sigma \eta \mu \nu \eta \dot{\mu \eta}$ [7]

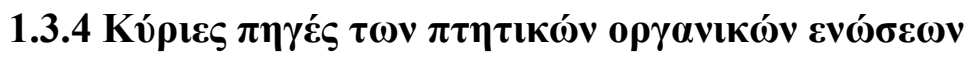

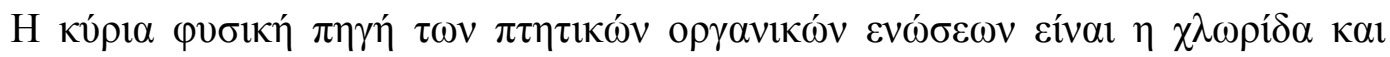

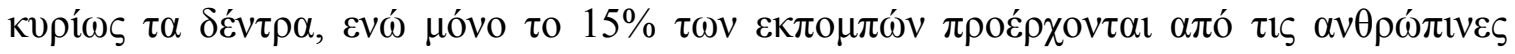
$\delta \rho \alpha \sigma \tau \eta \rho$ เó $\eta \tau \varepsilon \varsigma$.

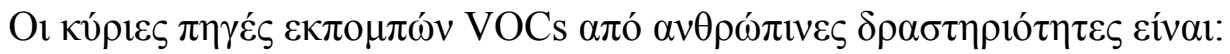

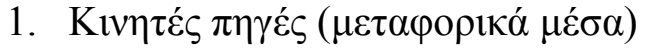

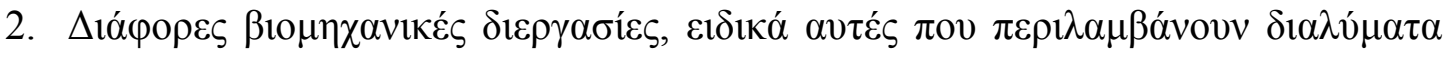

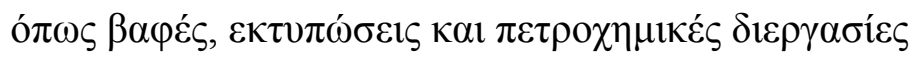

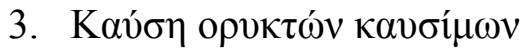

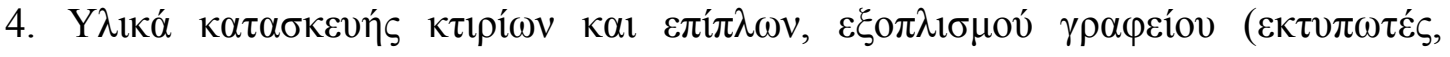

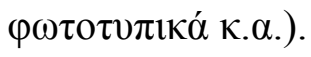

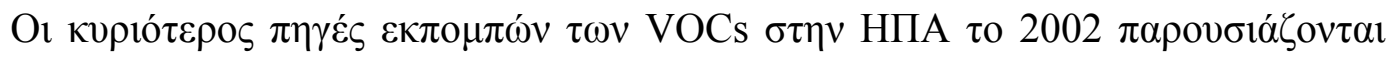

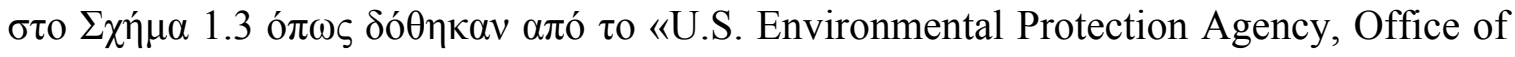
Air and Radiation». 


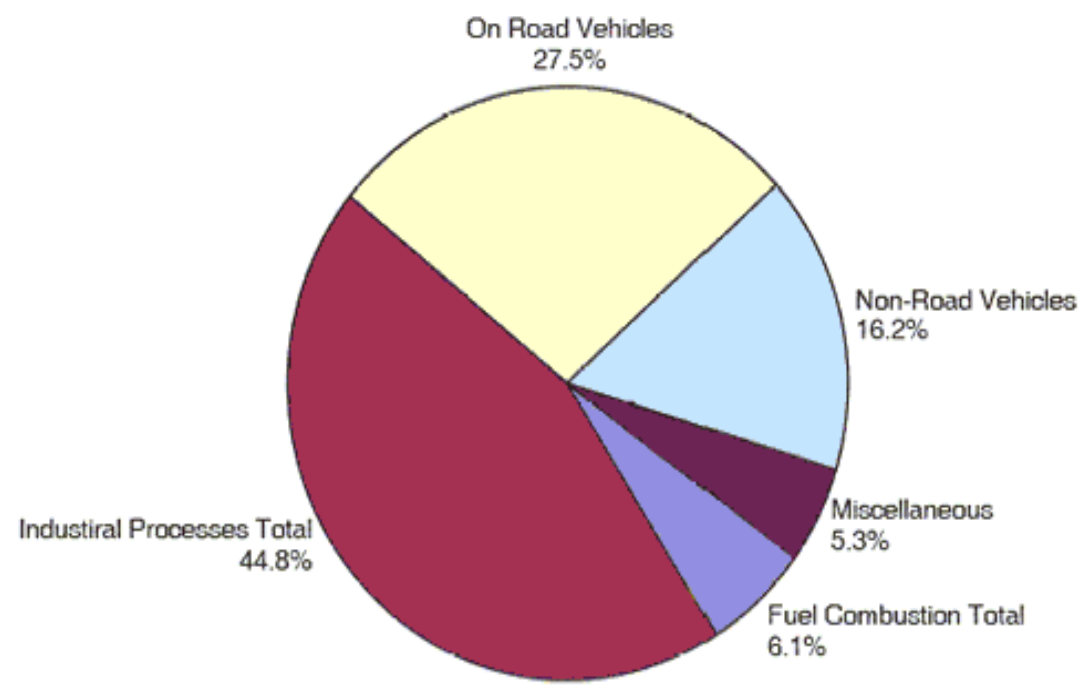

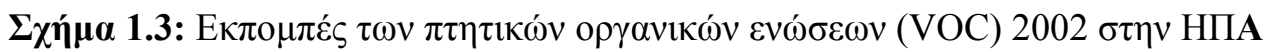

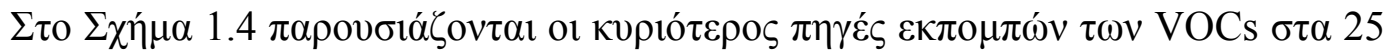

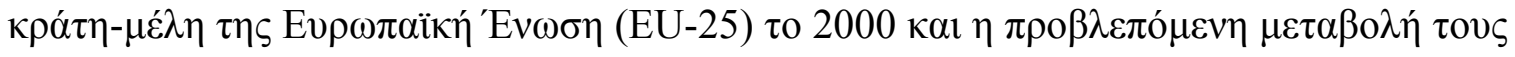

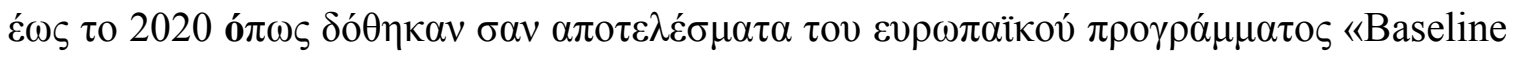
projections of European air quality up to 2020»

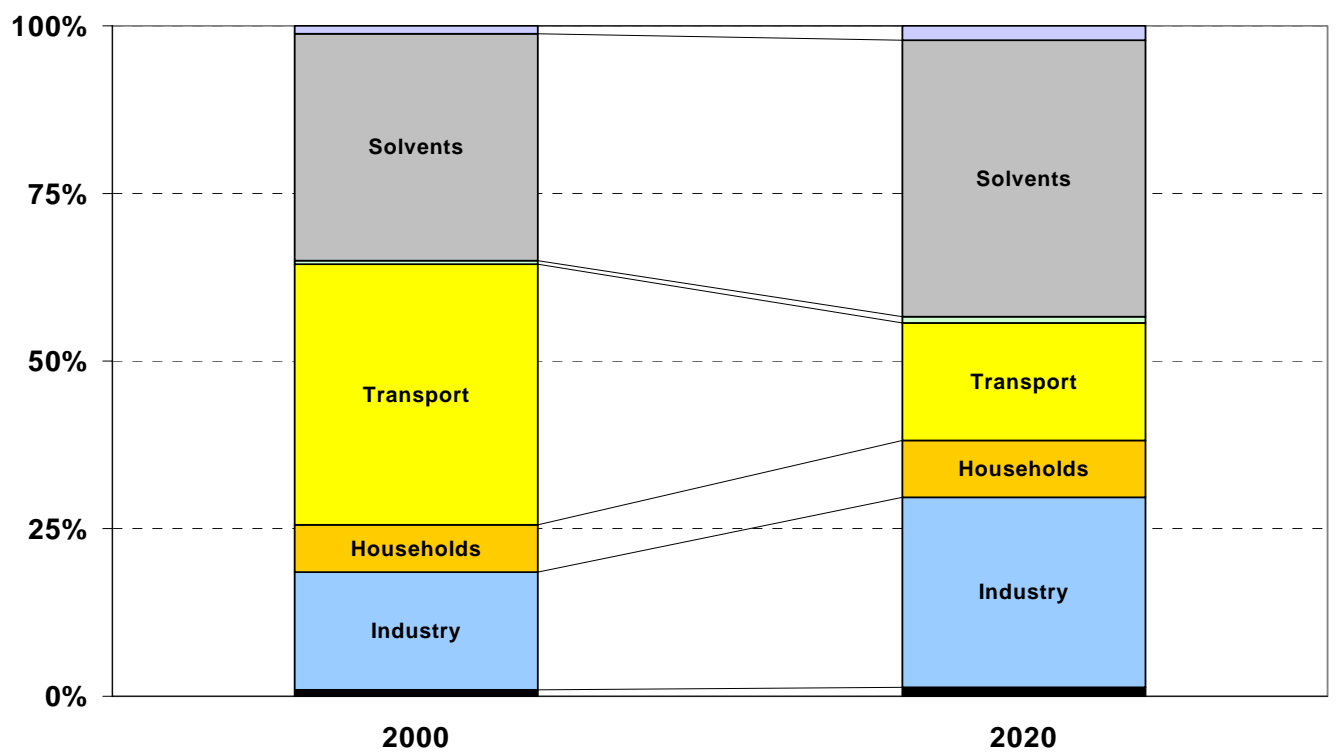

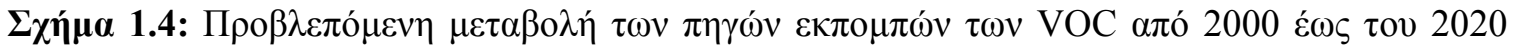

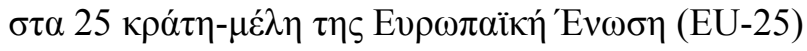




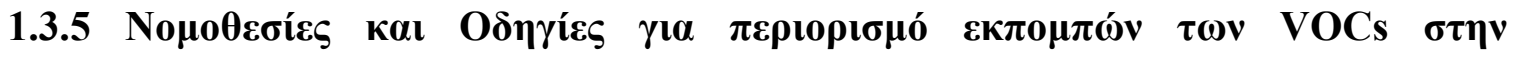

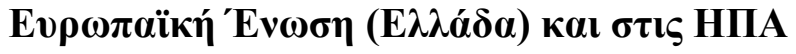

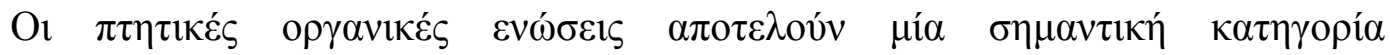

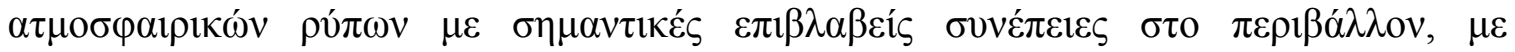

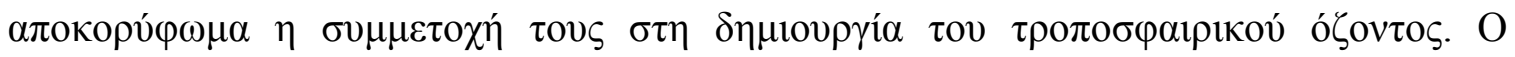

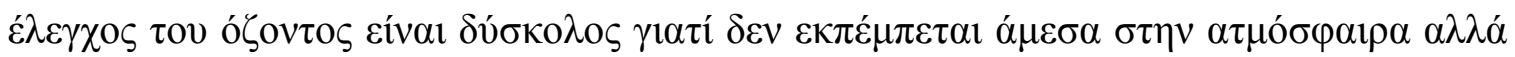

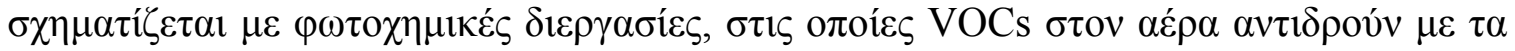

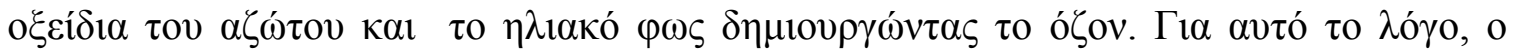

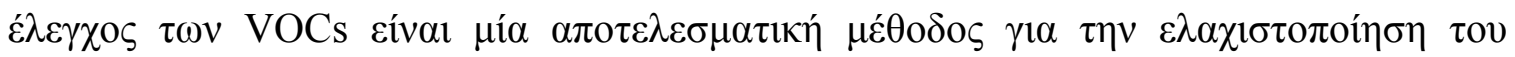

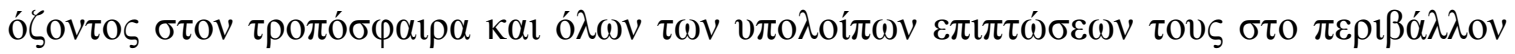

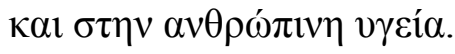

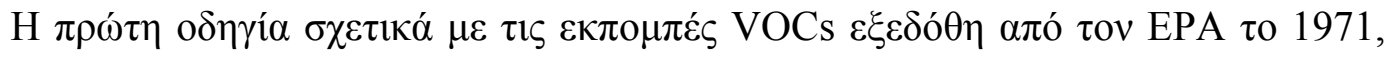

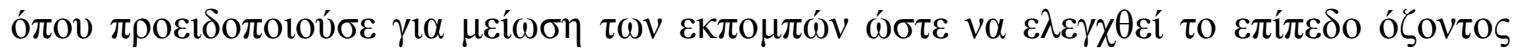

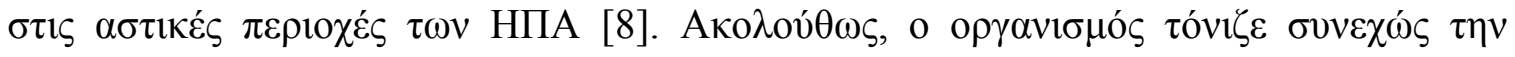

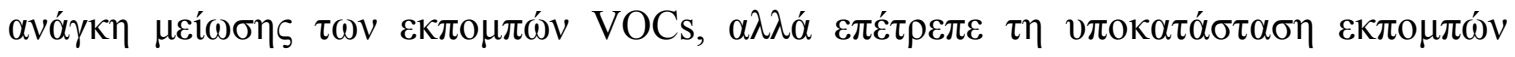

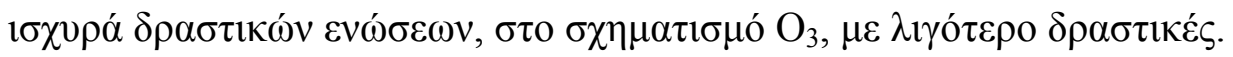

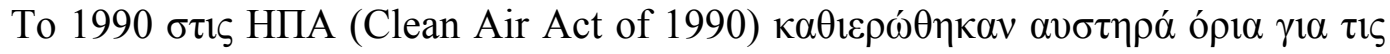

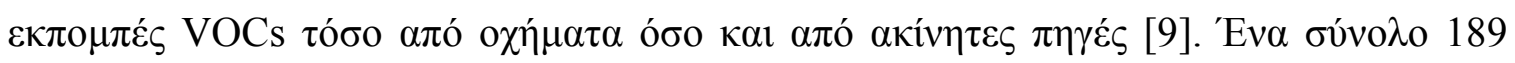

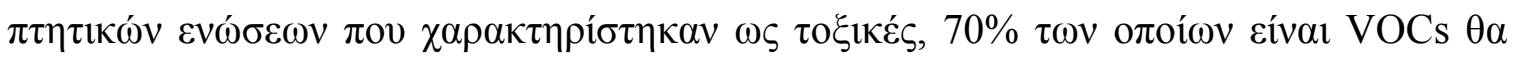

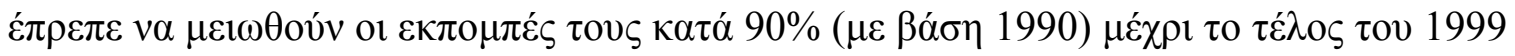
[10].

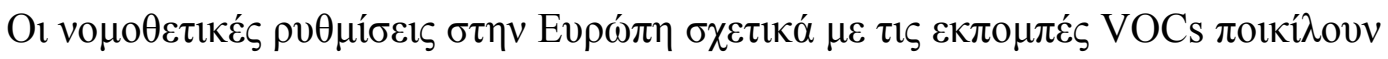

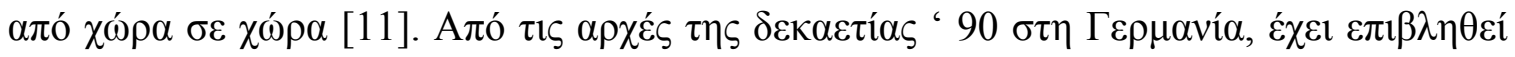

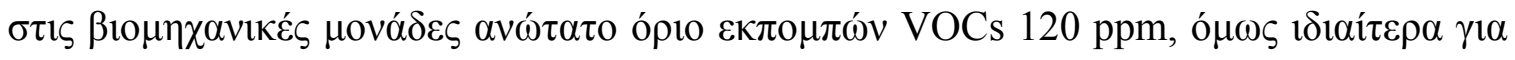

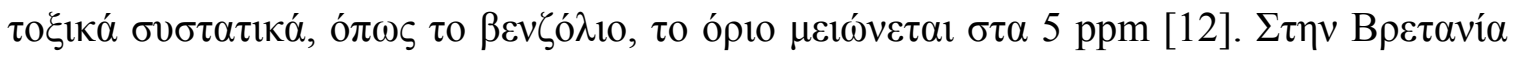

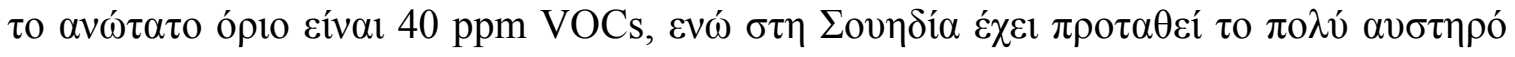
ópıo $\tau \omega v 15$ ppm [13].

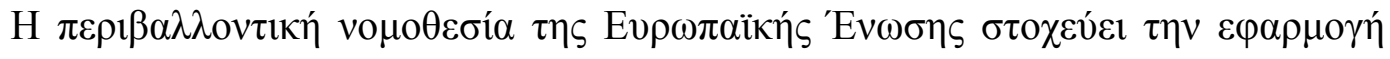

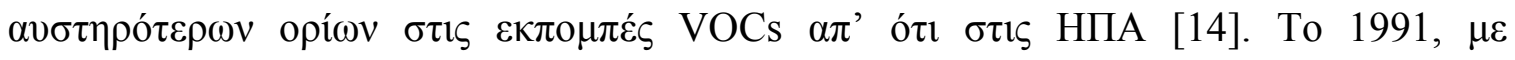

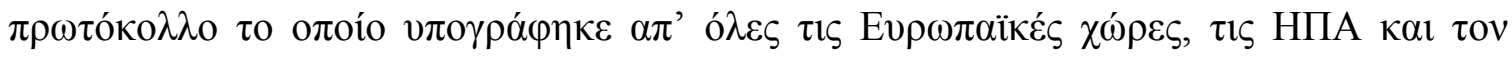




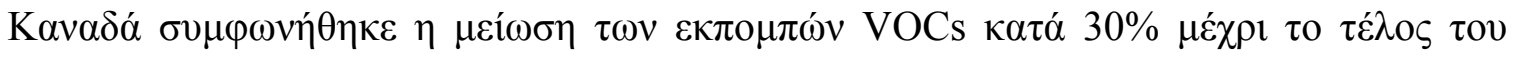

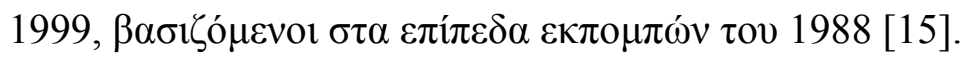

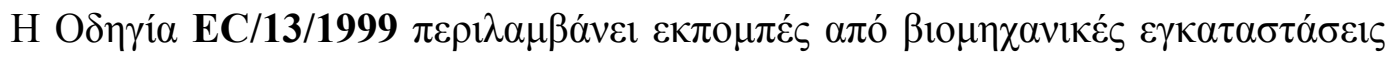

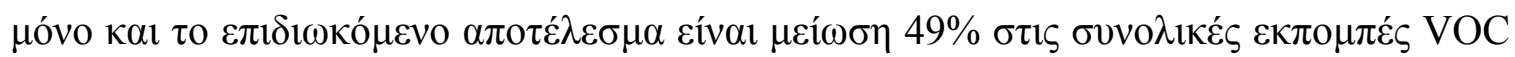

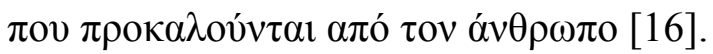

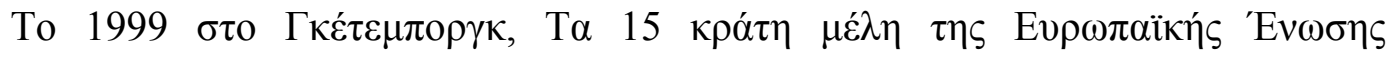

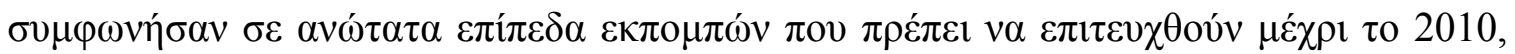
Пıvó́каs 1.1, [17].

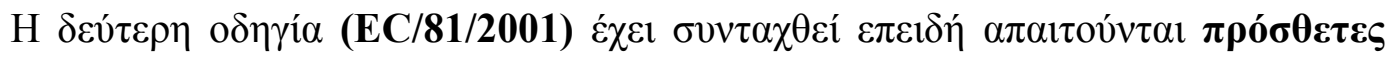

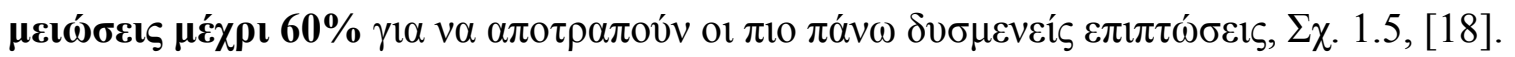

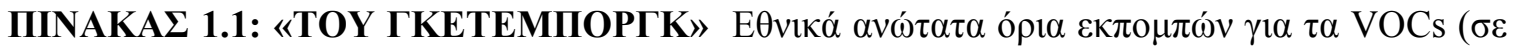

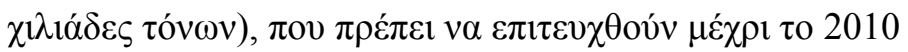

\begin{tabular}{|c|c|}
\hline 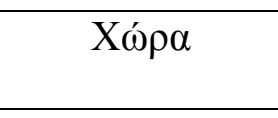 & 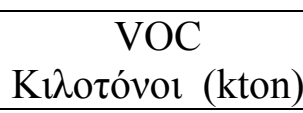 \\
\hline 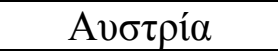 & 159 \\
\hline B $\varepsilon ́ \lambda \gamma 10$ & 144 \\
\hline$\Gamma \alpha \lambda \lambda i \alpha$ & 1100 \\
\hline 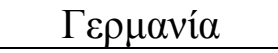 & 995 \\
\hline$\Delta \alpha v i ́ \alpha$ & 85 \\
\hline$E \lambda \lambda \alpha \dot{\alpha} \delta \alpha$ & 261 \\
\hline 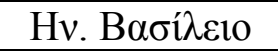 & 1200 \\
\hline Ip & 55 \\
\hline Iб $\pi \alpha v i ́ \alpha$ & 669 \\
\hline I $\tau \alpha \lambda i ́ \alpha$ & 1159 \\
\hline 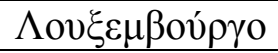 & 9 \\
\hline 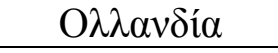 & 191 \\
\hline Порто $\alpha \lambda i \alpha^{\alpha}$ & 202 \\
\hline 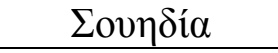 & 241 \\
\hline$\Phi ı v \lambda \alpha v \delta i ́ \alpha$ & 130 \\
\hline EC 15 & 6600 \\
\hline
\end{tabular}




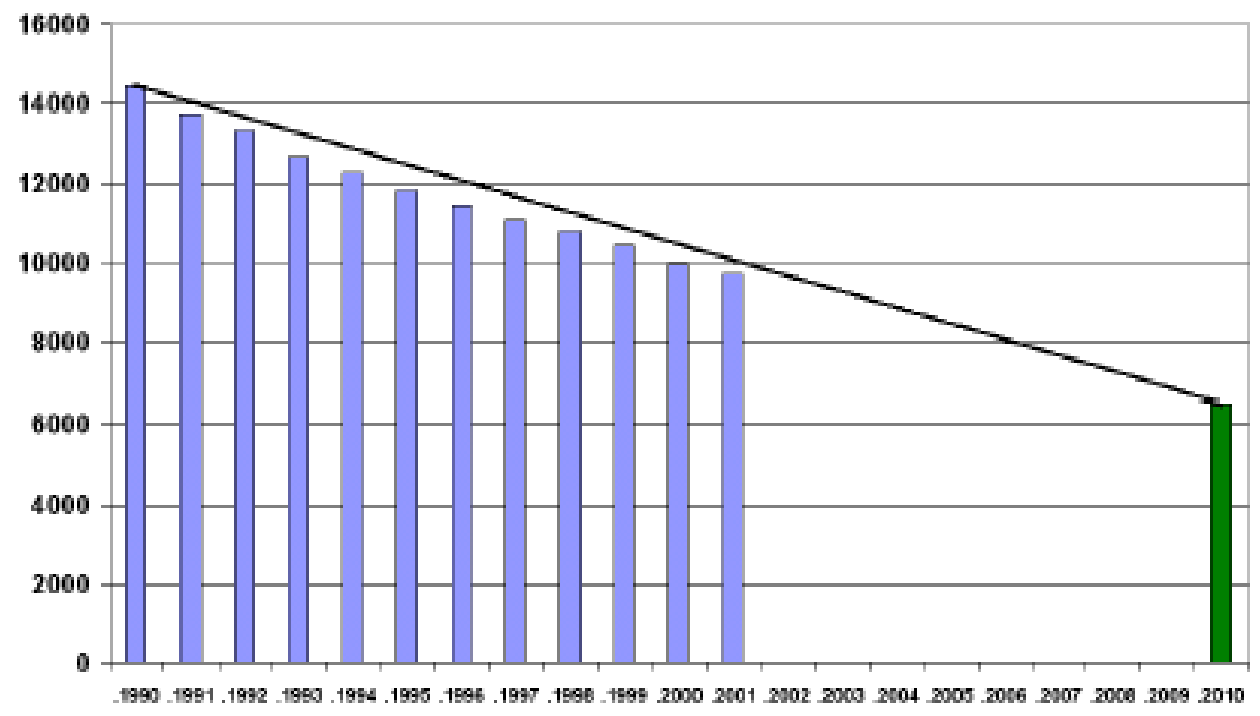

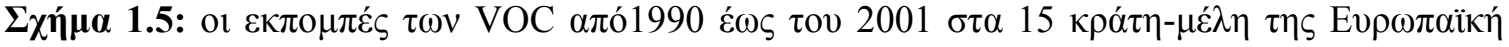

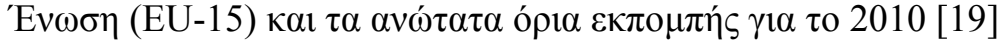

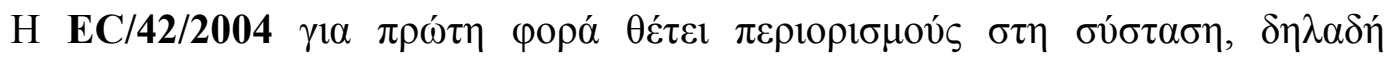

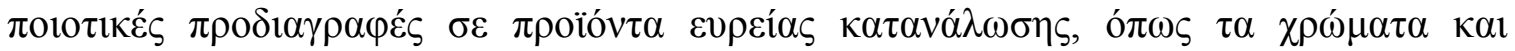

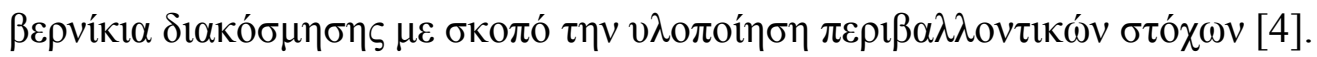

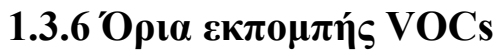

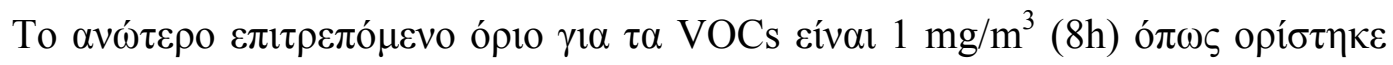

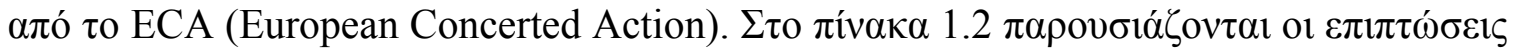

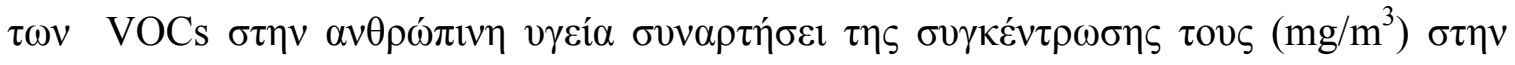

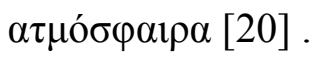




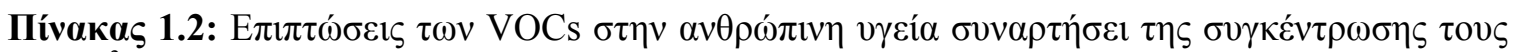
$\left(\mathrm{mg} / \mathrm{m}^{3}\right) \sigma \tau \eta \nu \alpha \tau \mu o ́ \sigma \varphi \alpha \iota \rho \alpha$.

\begin{tabular}{|c|c|c|}
\hline 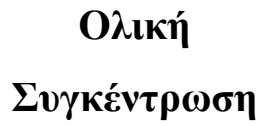 & 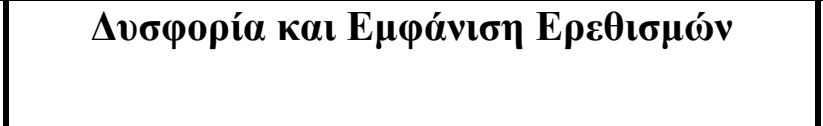 & 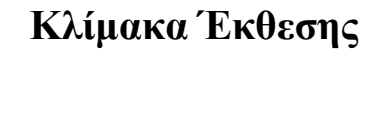 \\
\hline$<0,2 \mathrm{mg} / \mathrm{m}^{3}$ & 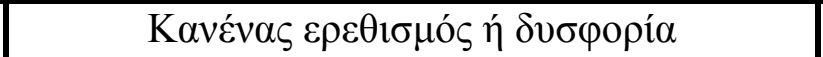 & 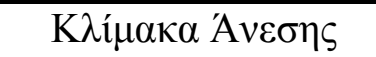 \\
\hline $0,2-3,0 \mathrm{mg} / \mathrm{m}^{3}$ & 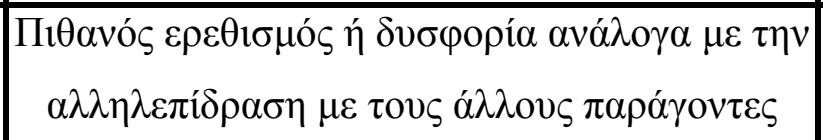 & $\begin{array}{l}\mathrm{K} \lambda \dot{\mu} \mu \alpha \kappa \alpha^{\prime} \mathrm{E} \kappa \theta \varepsilon \sigma \eta \varsigma \sigma \varepsilon \\
\pi \circ \lambda \lambda \circ{ }^{\prime} \varsigma \pi \alpha \rho \alpha ́ \gamma о \nu \tau \varepsilon \varsigma\end{array}$ \\
\hline $3,0-25 \mathrm{mg} / \mathrm{m}^{3}$ & 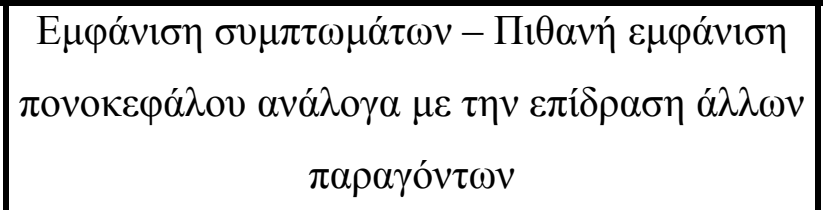 & 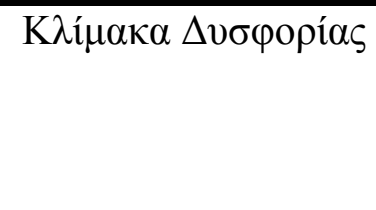 \\
\hline$>25 \mathrm{mg} / \mathrm{m}^{3}$ & 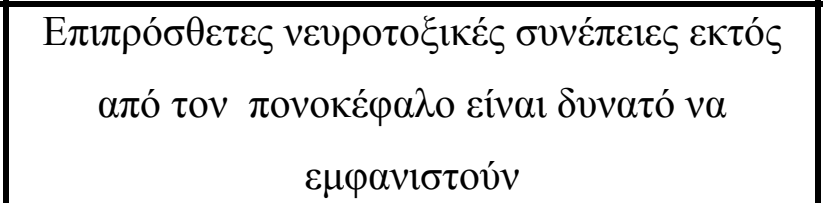 & 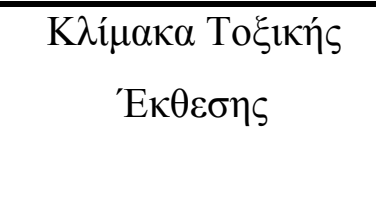 \\
\hline
\end{tabular}

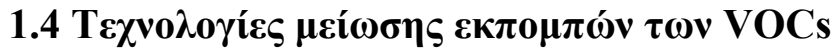

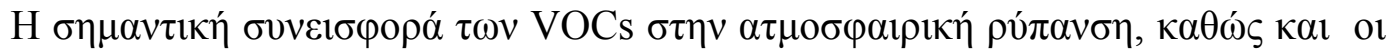

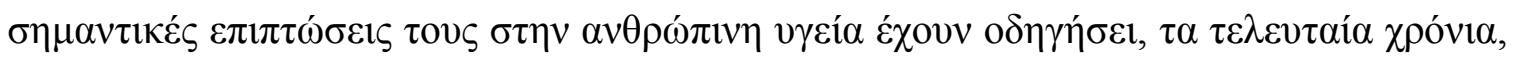

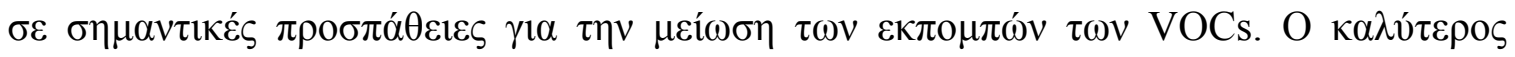

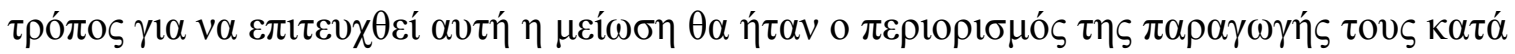

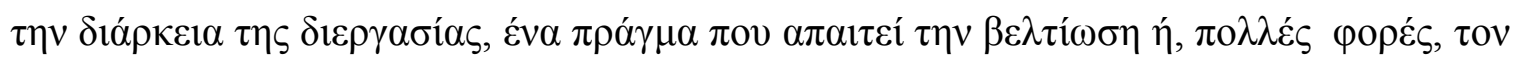

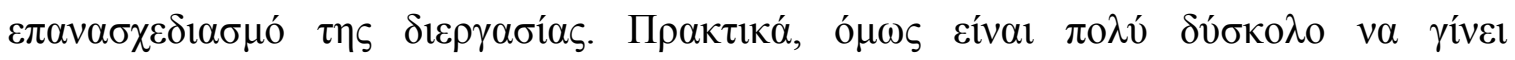

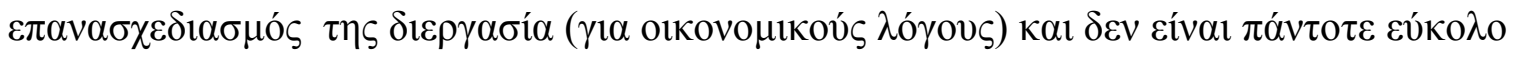

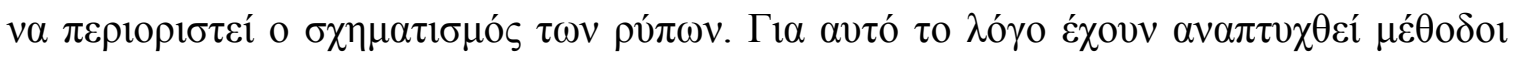

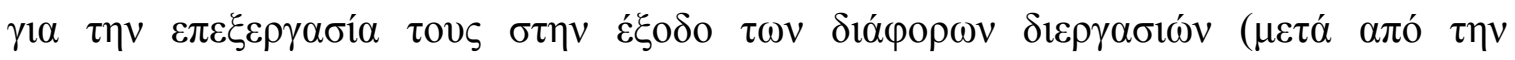

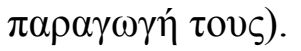

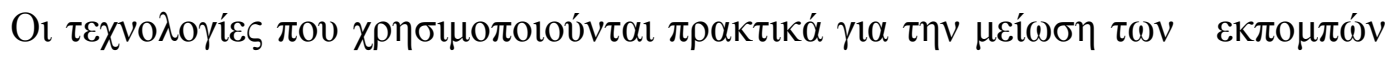

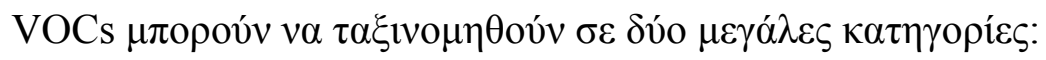

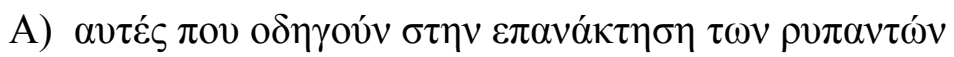

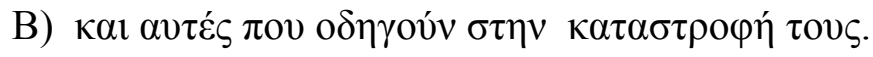




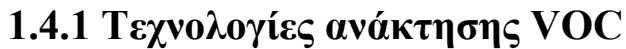

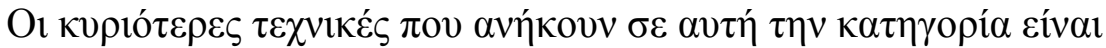

1. $\Sigma v \mu \pi v ́ \kappa v \omega \sigma \eta$

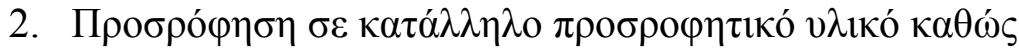

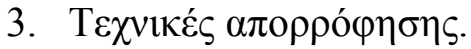

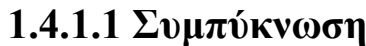

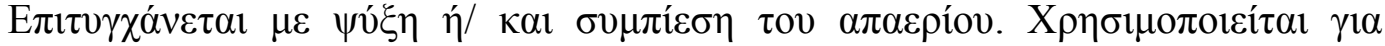

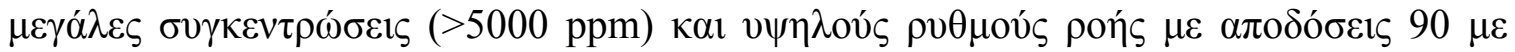

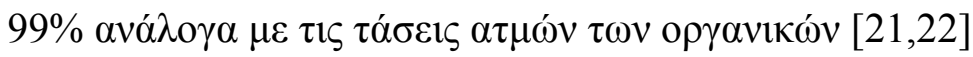

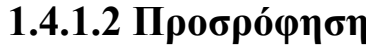

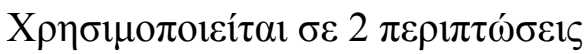

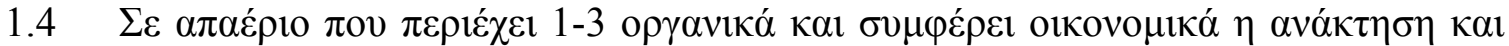

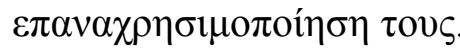

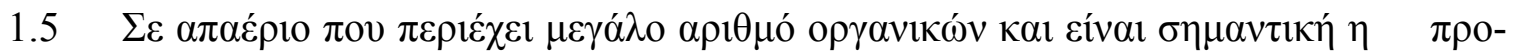

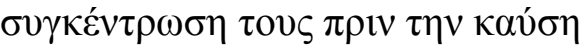

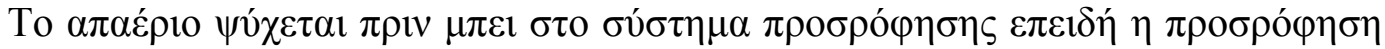

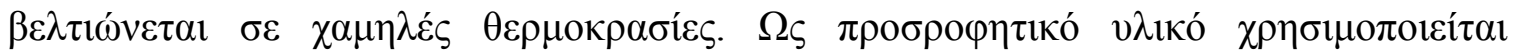

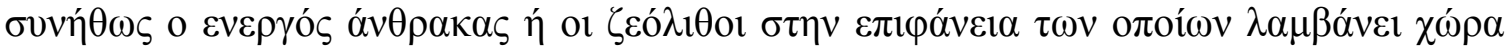

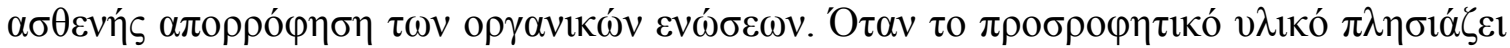

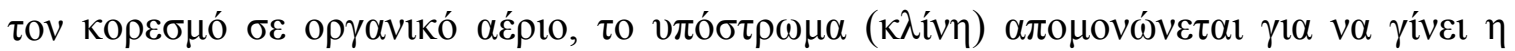

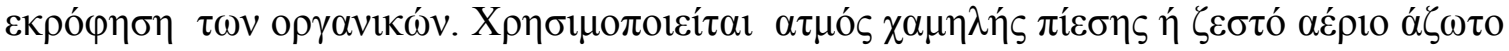

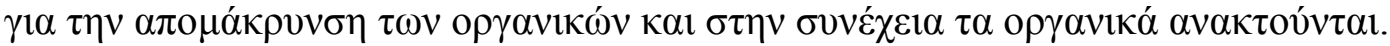

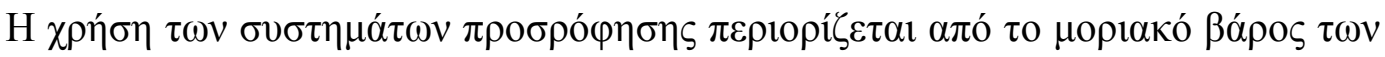

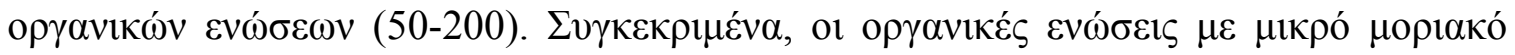

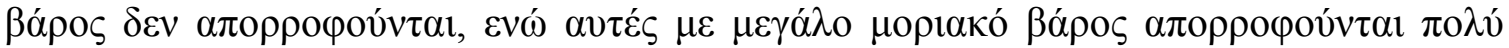

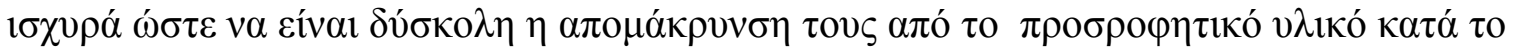

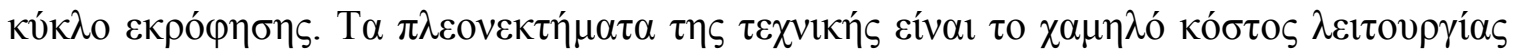

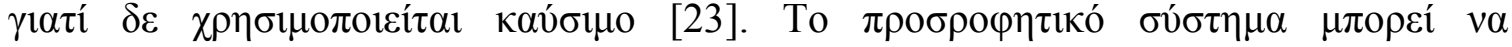

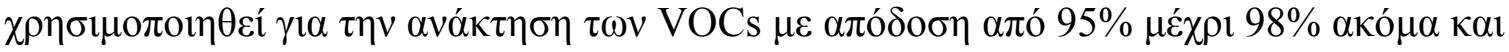




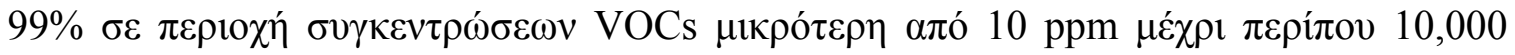
ppm.

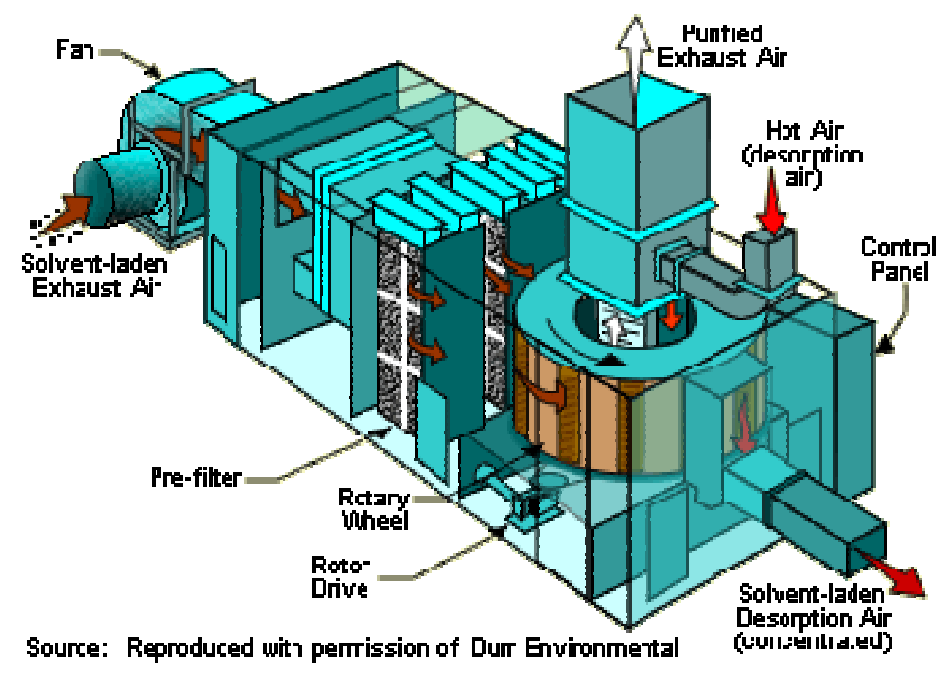

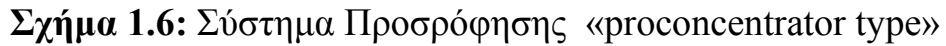

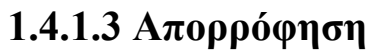

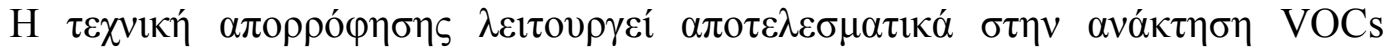

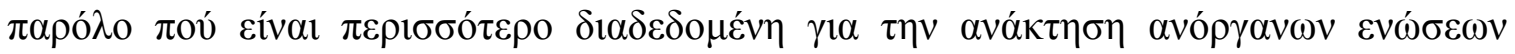

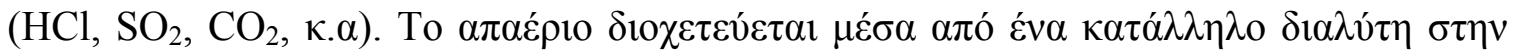

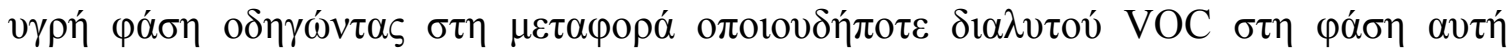

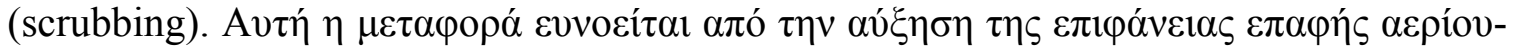

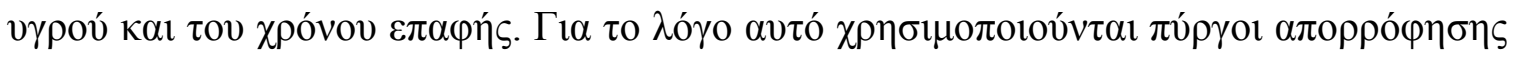

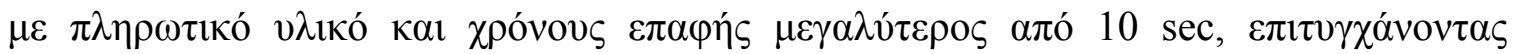

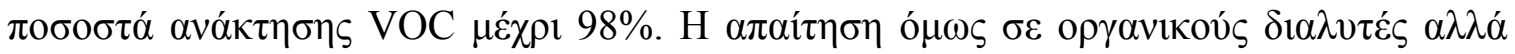

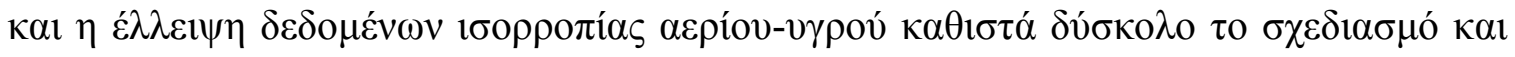

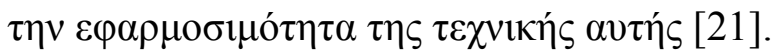

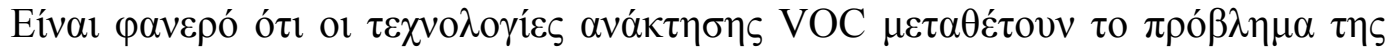

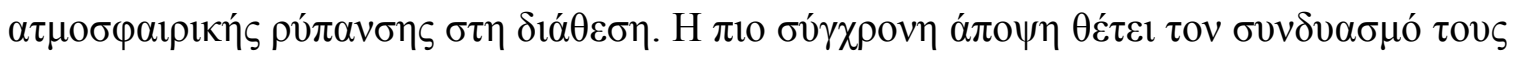

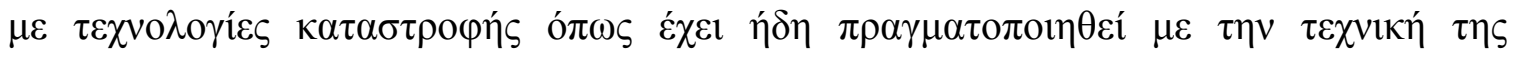




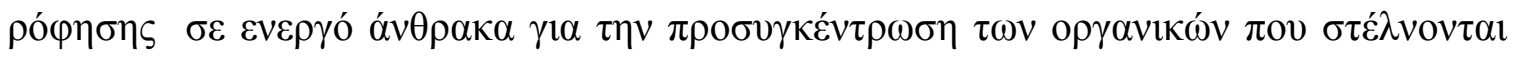
$\gamma 1 \alpha \kappa \alpha v ́ \sigma \eta[10]$.

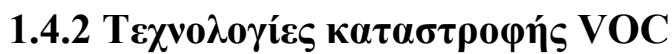

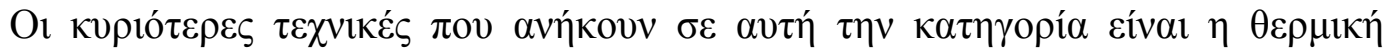

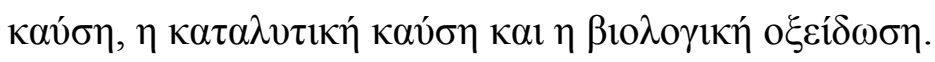

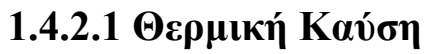

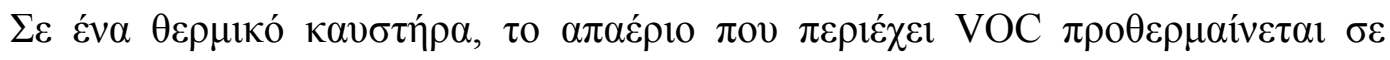

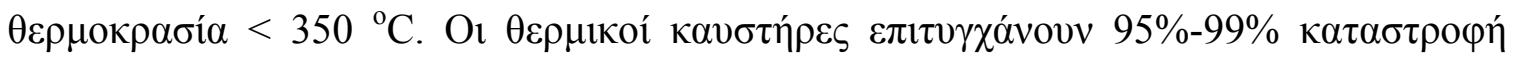

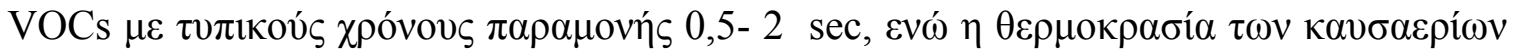

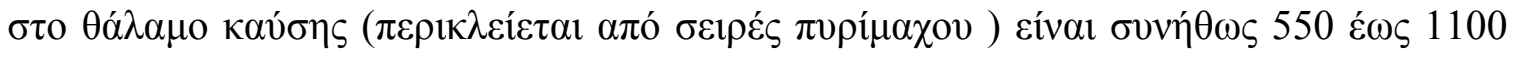
${ }^{\circ} \mathrm{C}$.

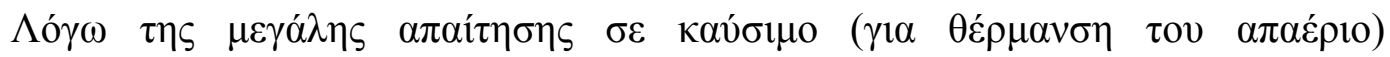

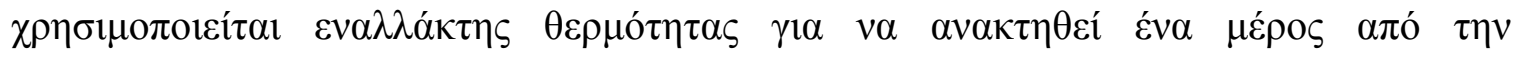

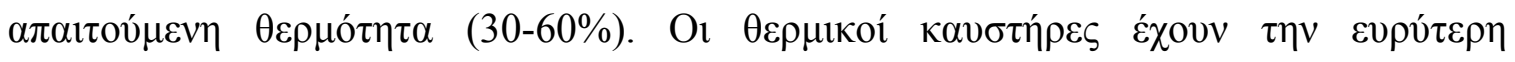

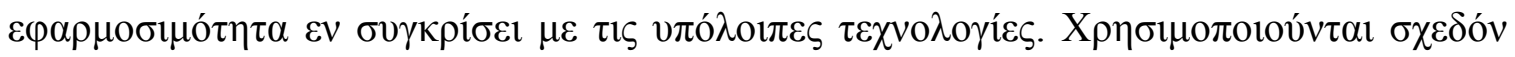

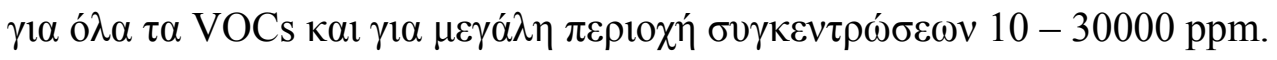

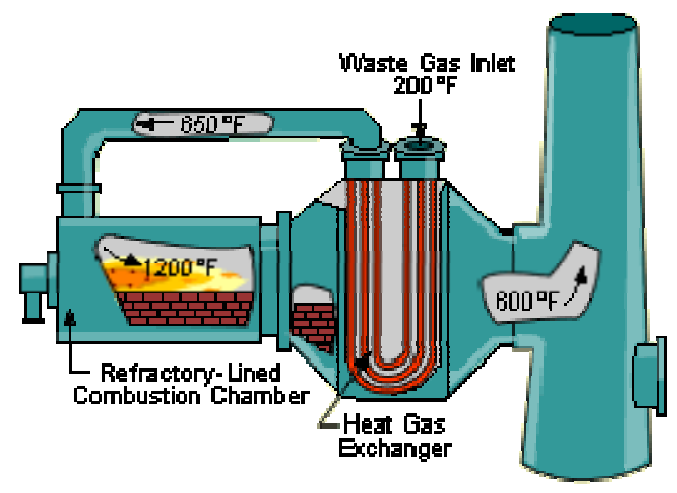

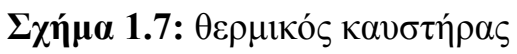




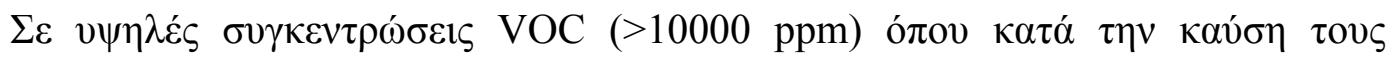

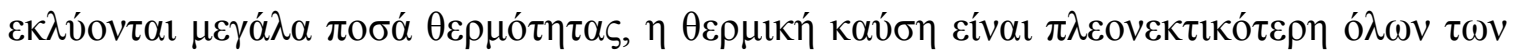
$\alpha \dot{\lambda \lambda \lambda \omega \nu \tau \varepsilon \chi v \imath \kappa \omega ́ v . ~}$

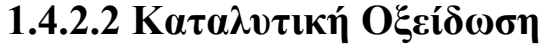

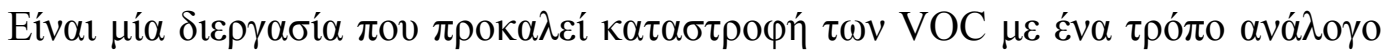

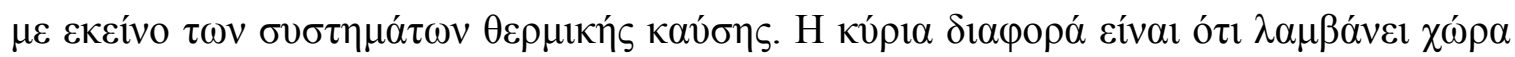

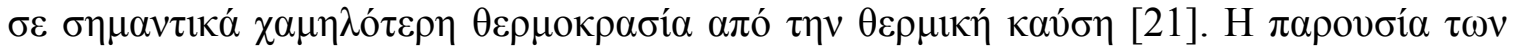

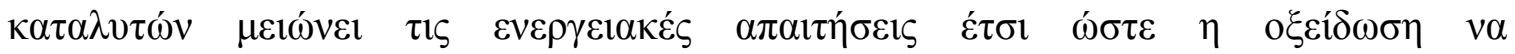

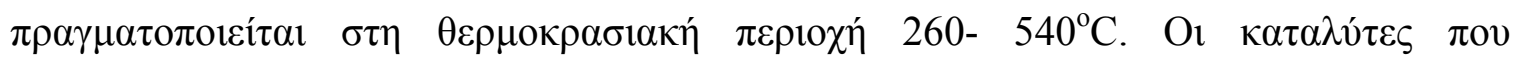

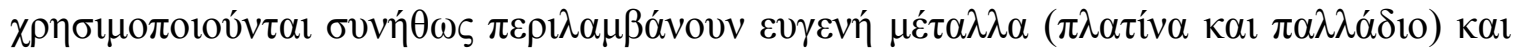

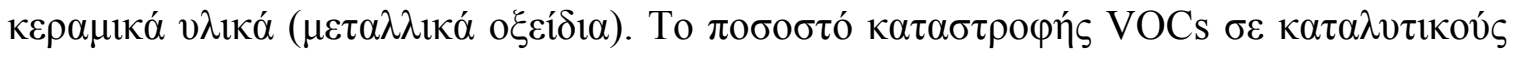

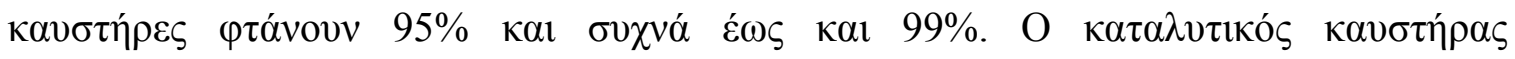

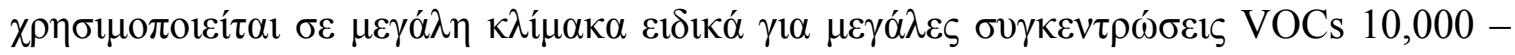
20,000 ppm

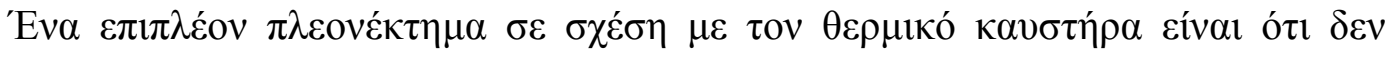

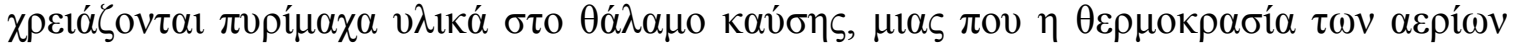

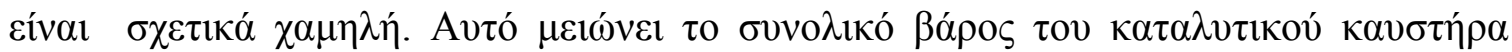

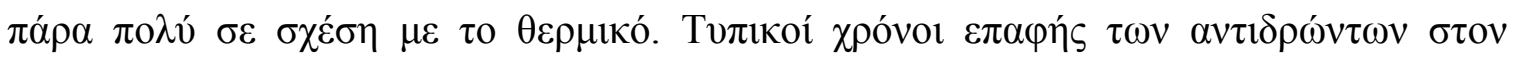

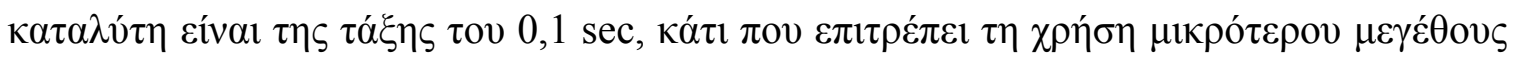

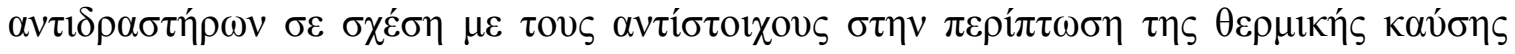
$[24,25]$.

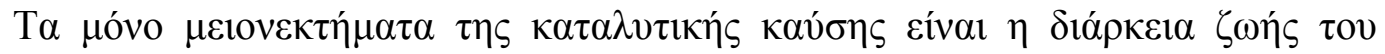

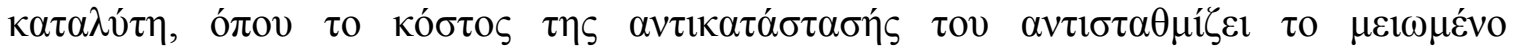

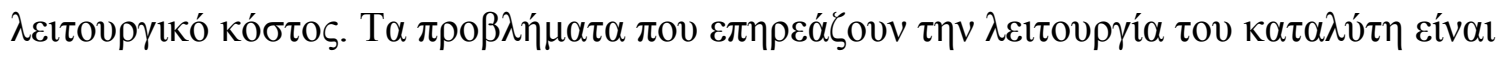

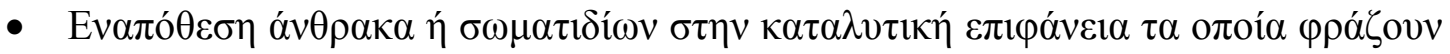

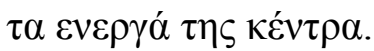

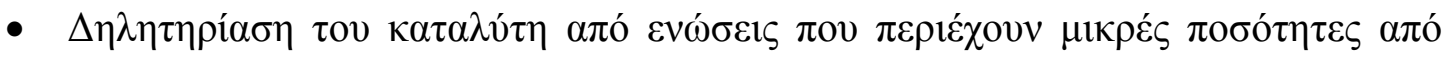

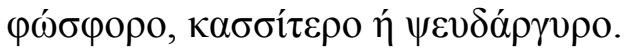

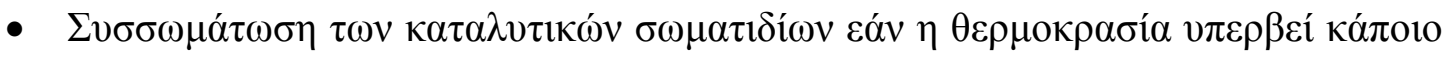
ópio. 


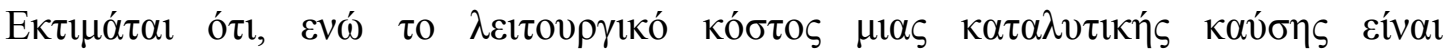

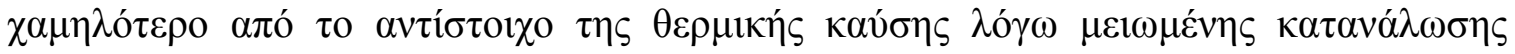

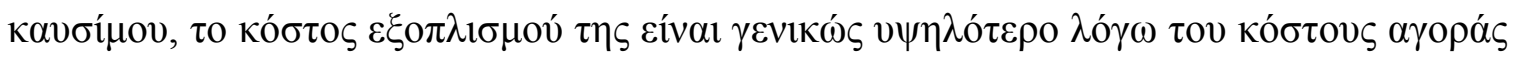

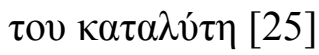

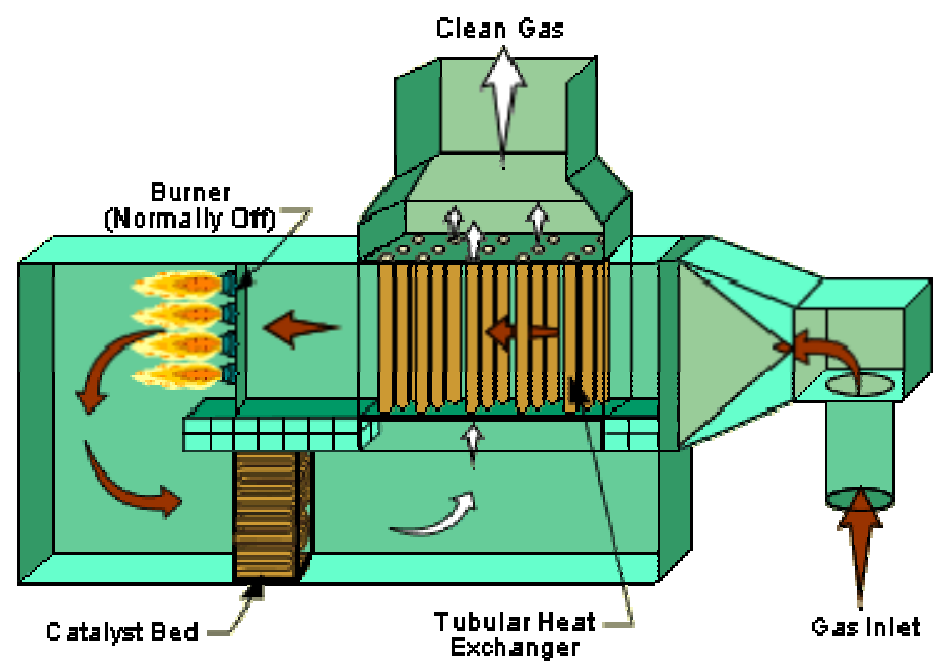

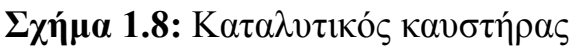

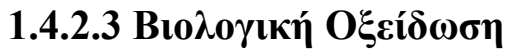

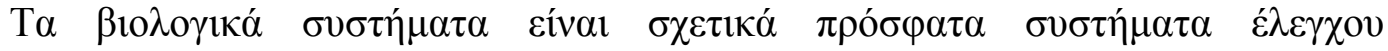

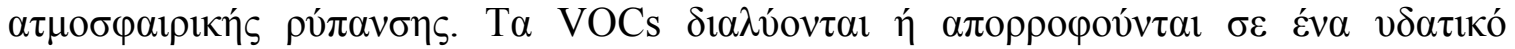

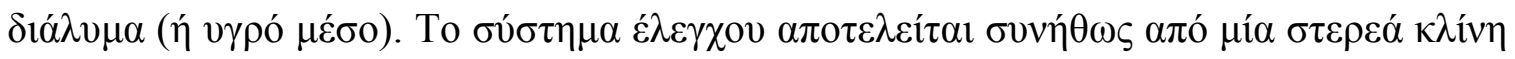

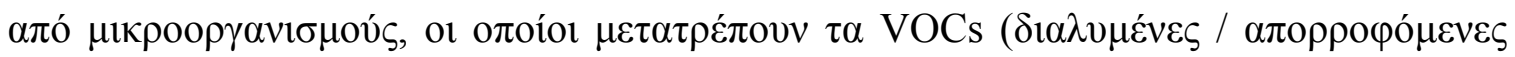

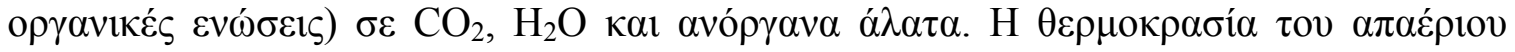

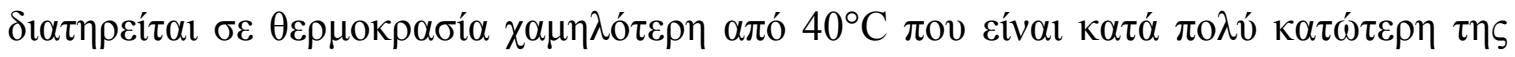

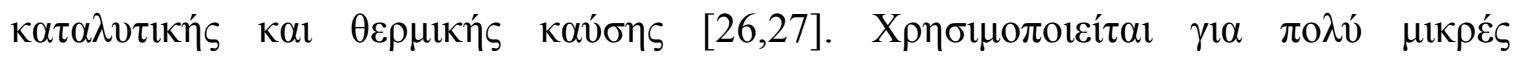

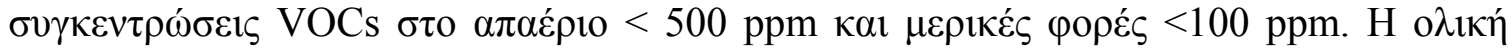

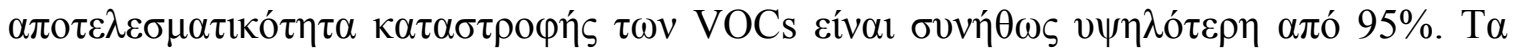

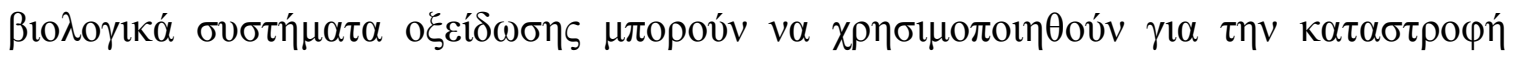




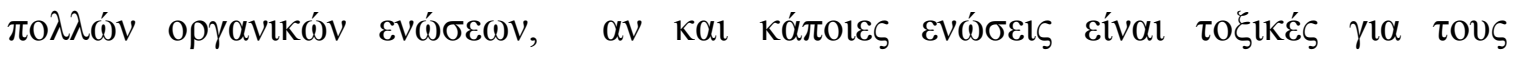

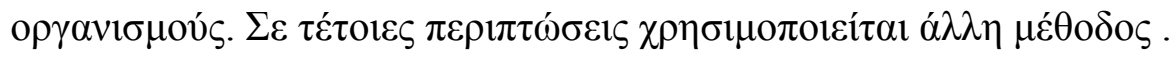

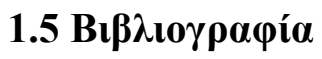

[1] P. Papaefthimiou, T. Ioannides, X.E. Verykios, Appl. Catal. B: Environmental, 13 (1997) 175.

[2] P. Papaefthimiou, T. Ioannides, X.E. Verykios, Appl. Catal. B: Environmental, 15 (1998) 75 .

[3] M. Amann, I. Bertok, et al. “ Baseline emission projections and scope for further reductions in Europe up to 2020', Results from the CAFE analysis

[4] Council Directive 2004/42/CE: European Parliament and of the Council of 21 April 2004, Official Journal L 143, 30/04/2004 P. 0087 - 0096

[5] L.Molhave, G.C. Jensen, S. Larsen. Atmospheric Environment 25A (1991) 1283.

[6] T.Godish Journal of Environment Health 53(3) (1990) 34.

[7] T.Godish Journal of Environment Health 44(3) (1981) 116.

[8] Dimitriades , B., J. Air Waste Manage. Assoc., 46 (1996) 963.

[9] Horsley, J. A., «Catalysis for the elimination of Volatile Organic Compounds: Nonhalogenated Compounds», Catalytica Environmental Report No. E4, 1993.

[10] Parkinson, G., Chem. Eng., July 1991, 37.

[11] Chynoweth, E., Schoenmakers, J., Chem. Week, 1991, 149(16), 42.

[12] Mullin, R., Chem. Week, 1993, 152(2), 22.

[13] Chynoweth, E., Schoenmakers, J., Chem. Week, 1993, 152(7), 15.

[14] European Chem. News 57 (1991) 28.

[15] Chemical Industry 23 (1991) 855.

[16] COUNCIL DIRECTIVE 1999/13/EC: European Parliament and of the Council of 11 March 1999, Official Journal L 85, 29.3.1999, p. 1.

[17] Council Directive on national emission ceilings for certain atmospheric pollutants (COM(1999) 125 - C5-0047/1999 - 1999/0067(COD)) (report by Riitta Myller)

[18] COUNCIL DIRECTIVE 1999/13/EC: European Parliament and of the Council of 11 March 1999, Official Journal L 85, 29.3.1999, p. 1. 
[19] Vigdis Vestreng, Review and revision: Emission data reported to CLRTAP. EMEP MSC-W, Note 1/2003, Report July 2003.

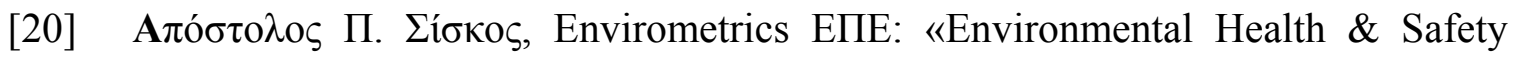

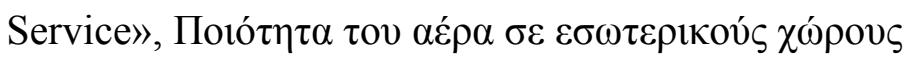

[21] Ruddy, E.N. and Carroll, L.A., Chem. Eng. Prog., July 1993, 28.

[22] Martin, A.M., Nolen, S.L., Gess, P.S., Baesen, T.A., Chem. Eng. Prog., 1992, 88(12),53.

[23] Morretti, E.C. and Mukhopadhyay, N. Chem. Eng. Prog., July 1993, 20

[24] Kosusko, M., Munez, C.M., J. Air Waste Manage. Assoc., 40 (1990) 254.

[25] Van der Vaart, D.R., Vatavuk, W.M., Wehe, A.H., J. Air Waste Manage. Assoc., 4 (1991) 92.

[26] Bohn, H., Chem. Eng. Prog., A

[27] Leson, G. and Winer A.M., J. Air Waste Manage. Assoc., 41 (1991) 1045. 


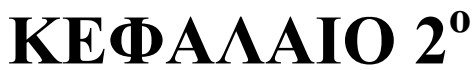

\section{KATA $\Lambda$ YTIKH O $\Xi E I \Delta \Omega \Sigma H$ VOCs}

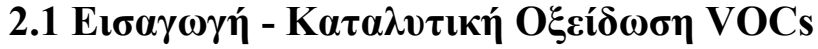

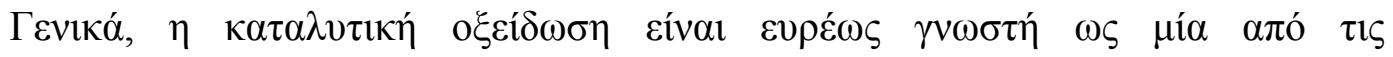

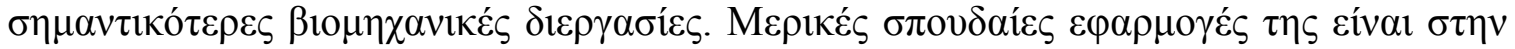

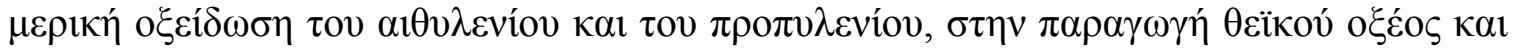

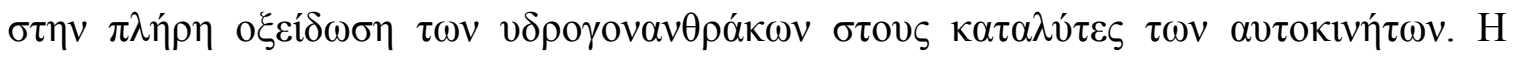

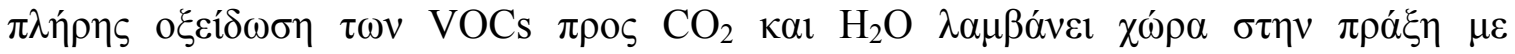

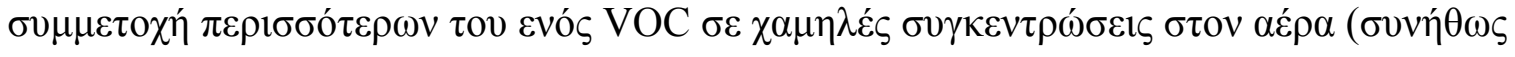

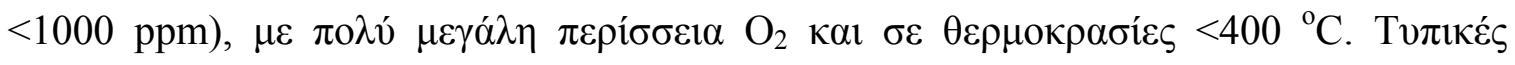

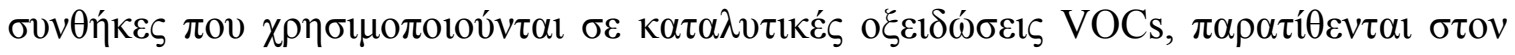

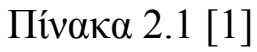




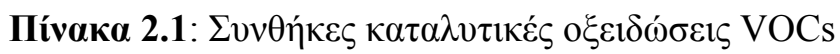

$\Theta \varepsilon \rho \mu о к \rho \alpha \sigma i ́ \alpha$

$25-400{ }^{\circ} \mathrm{C}$

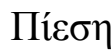

$1 \mathrm{~atm}$

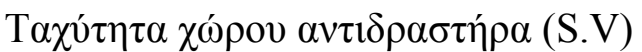

$10^{3}-10^{5} \mathrm{~h}^{-1}$

$\Sigma v \gamma \kappa \varepsilon ́ v \tau \rho \omega \sigma \eta \mathrm{VOC}$

$10^{2}-10^{3} \mathrm{ppm}$

$\mathrm{O} \xi \varepsilon 1 \delta \omega \tau \imath \kappa o ́$

$\mathrm{O}_{2} \sigma \varepsilon \alpha \varepsilon \dot{\rho} \alpha$

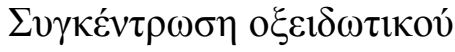

$21 \%$ к.о.

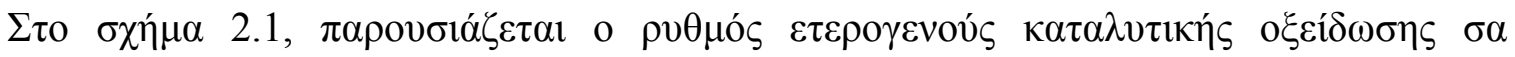

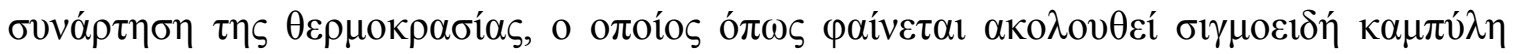

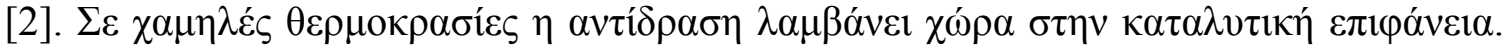

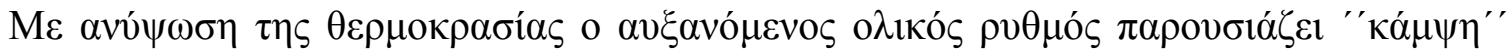

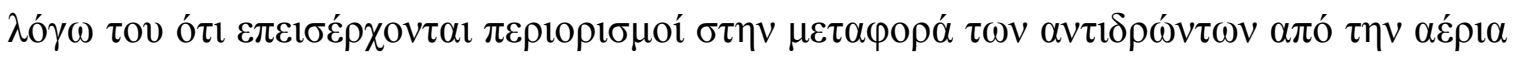

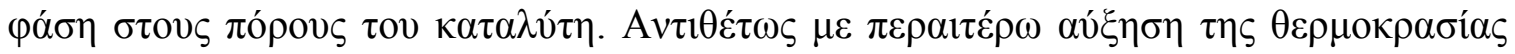

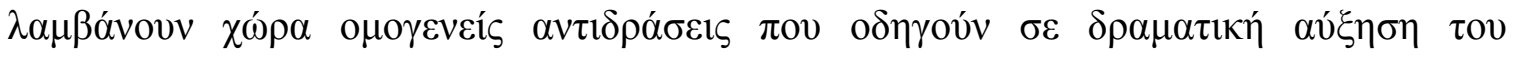
$\pi \alpha \rho \alpha \tau \eta \rho o v ́ \mu \varepsilon v o v \rho v \theta \mu o v ́$.

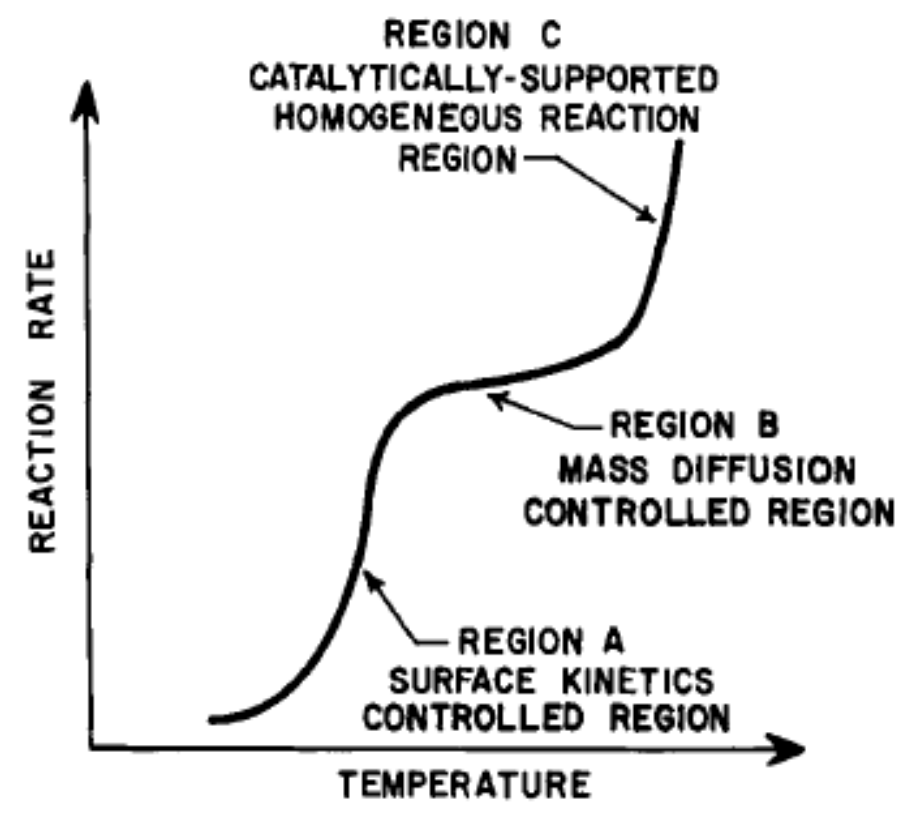

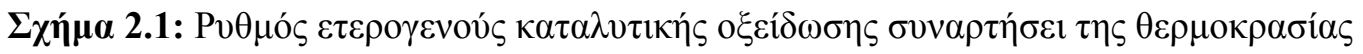




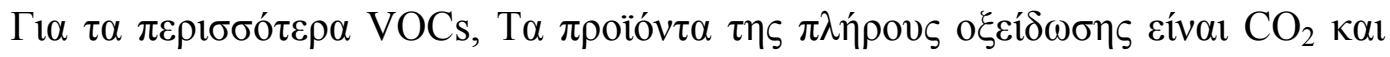

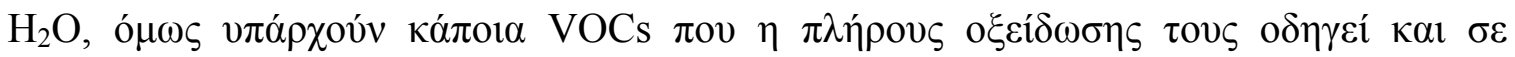

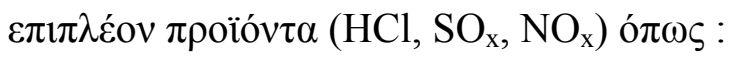

VOC

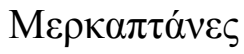

Auíves

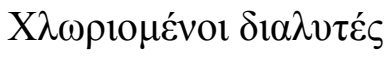

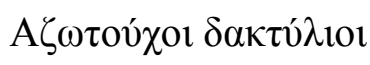

\section{Хๆ}

$\mathrm{R}-\mathrm{SH}$

$\mathrm{R}-\mathrm{NH}_{2}$

$\mathrm{R}-\mathrm{Cl}_{\mathrm{x}}$

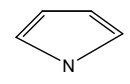

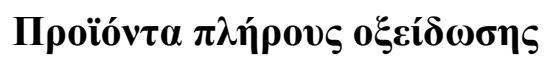

$$
\mathrm{SO}_{\mathrm{x}}+\mathrm{CO}_{2}+\mathrm{H}_{2} \mathrm{O}
$$$$
\mathrm{NO}_{\mathrm{x}}+\mathrm{CO}_{2}+\mathrm{H}_{2} \mathrm{O}
$$

$\mathrm{HCl}+\mathrm{CO}_{2}+\mathrm{H}_{2} \mathrm{O}$

$\mathrm{NO}_{\mathrm{x}}+\mathrm{CO}_{2}+\mathrm{H}_{2} \mathrm{O}$

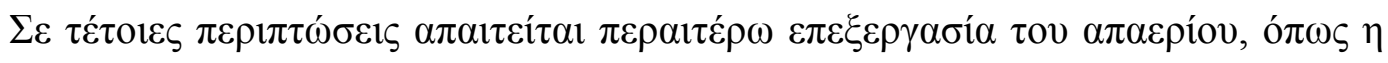
$\alpha v \alpha \gamma \omega \gamma \eta \dot{~} \tau \omega \nu \mathrm{NO}_{\mathrm{x}} \eta \dot{\alpha} \alpha \pi \rho \rho \rho o ́ \varphi \eta \sigma \eta \tau \omega \nu \mathrm{SO}_{\mathrm{x}} \kappa \alpha \iota \mathrm{HCl}$ (Scrubbing)

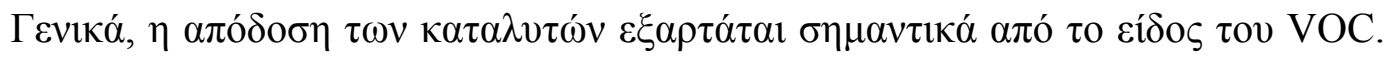

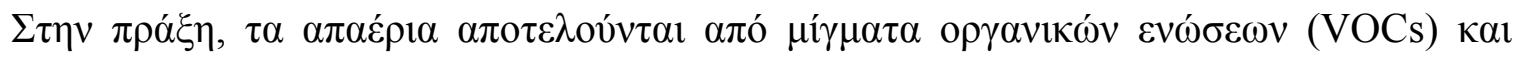

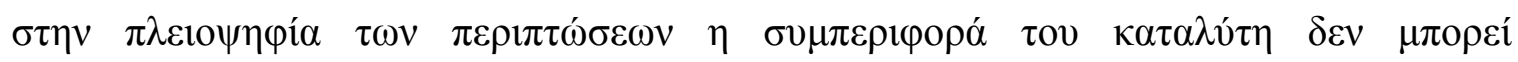

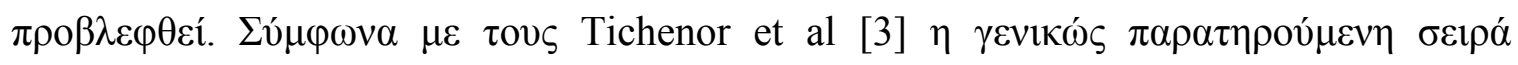

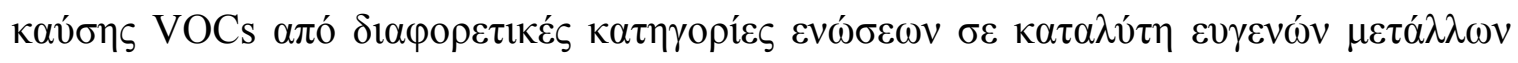
Pt-Pd, é́vaı $\eta \alpha \kappa o ́ \lambda o v \theta \eta$ (Пív. 2.2) :

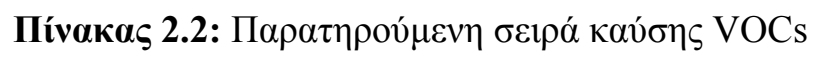

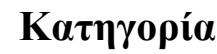

$\mathrm{A} \lambda \kappa о o ́ \lambda \varepsilon \varsigma$

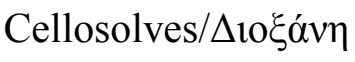

$\mathrm{A} \lambda \delta \varepsilon \hat{v i \delta \varepsilon \varsigma}$

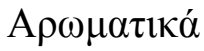

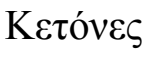

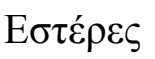

A $\lambda \kappa \alpha ́ v i \alpha$

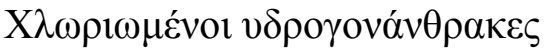

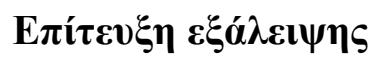

Y $\psi \lambda \lambda \eta$ 


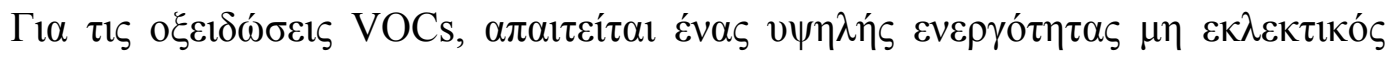

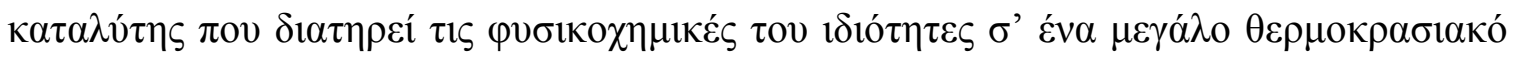

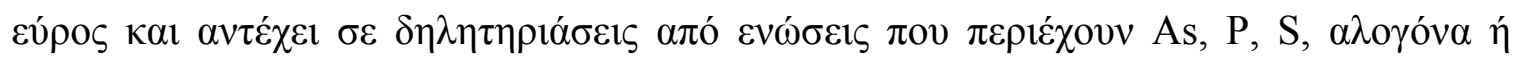

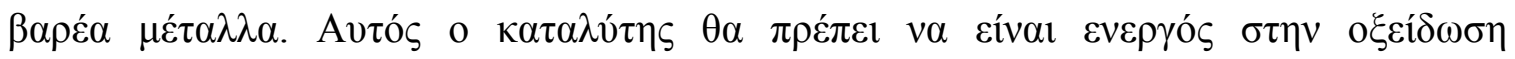

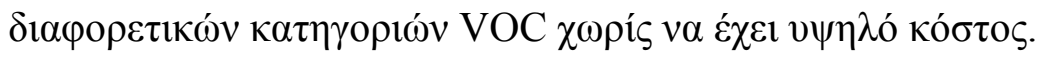

\section{$2.2 \operatorname{K\alpha \tau \alpha \lambda v́\tau \varepsilon \varsigma ~}$}

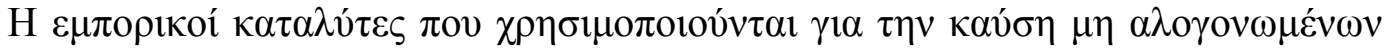

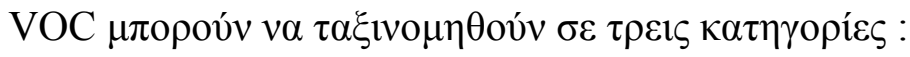

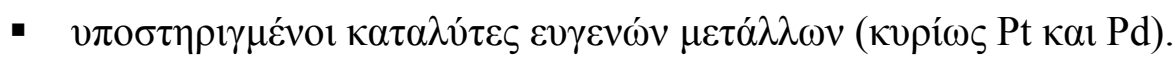

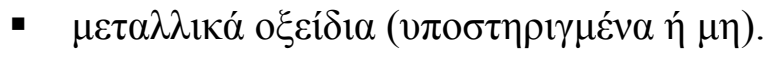

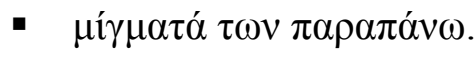

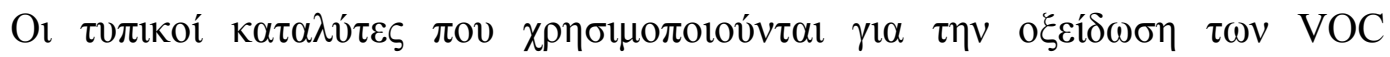

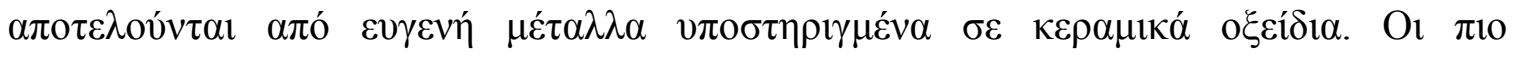

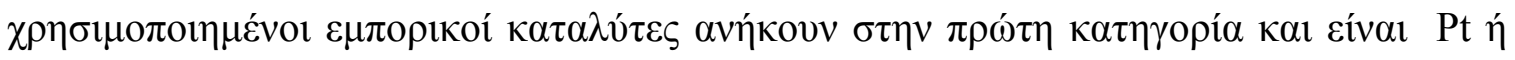

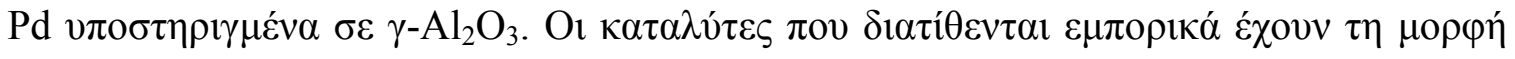

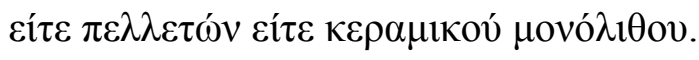

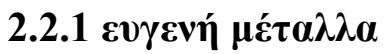

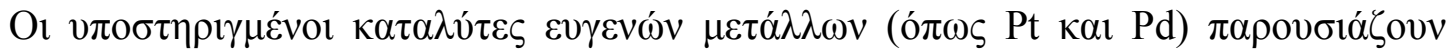

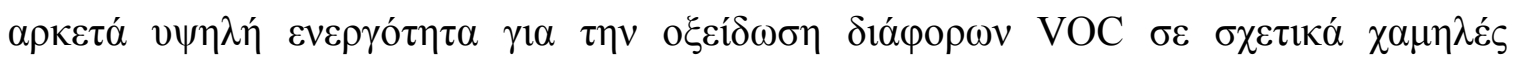

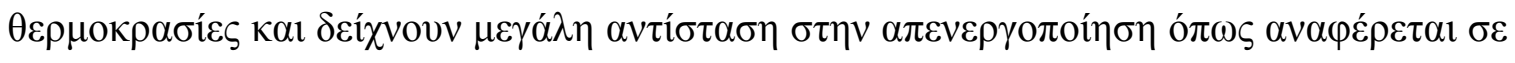

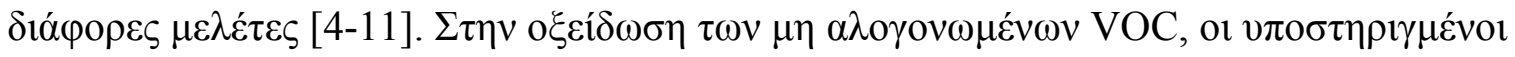

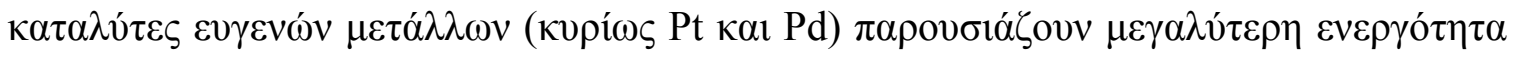

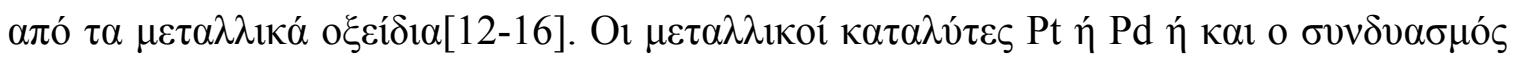

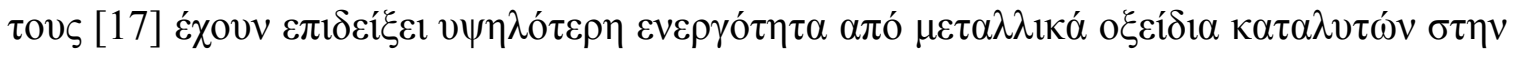

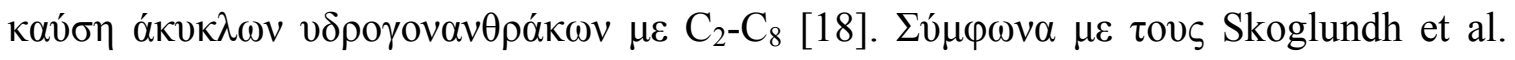

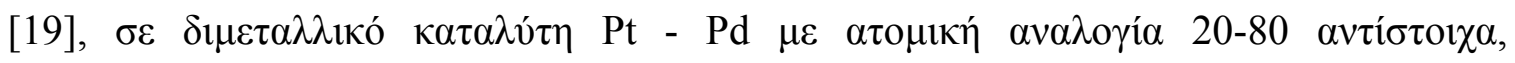

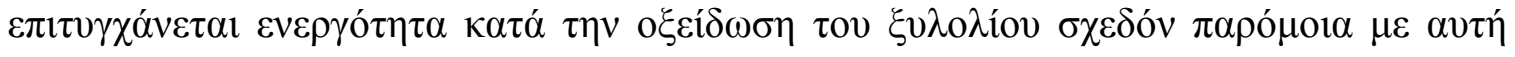

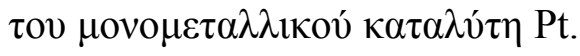




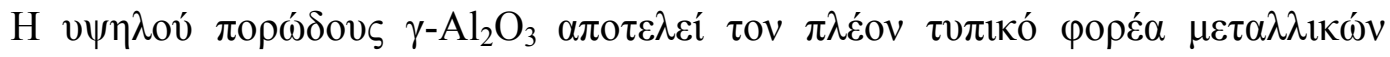

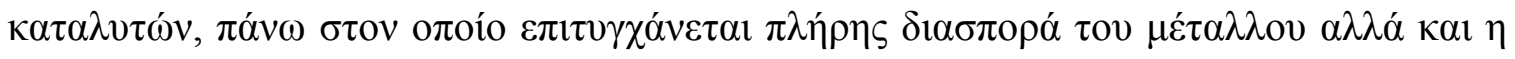

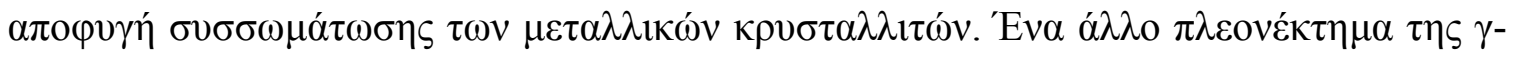

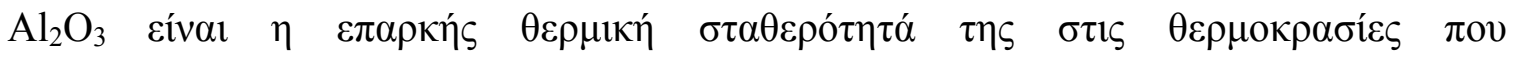

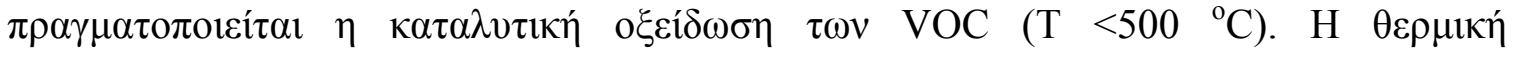

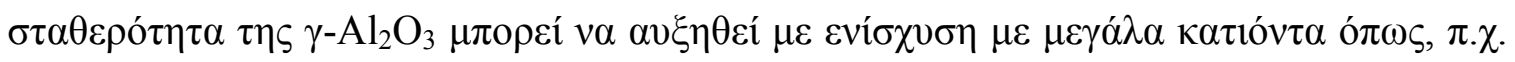

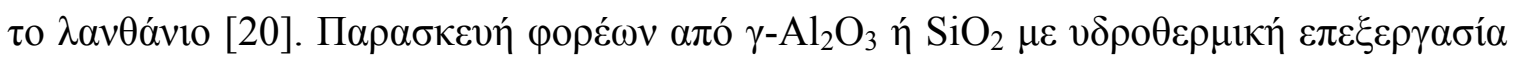

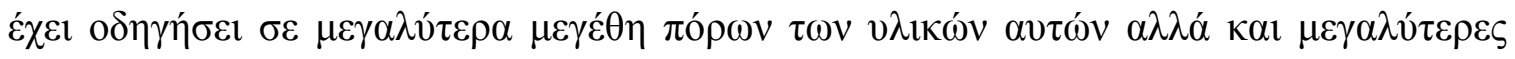

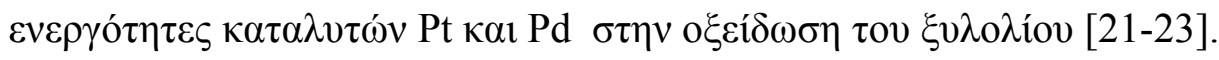

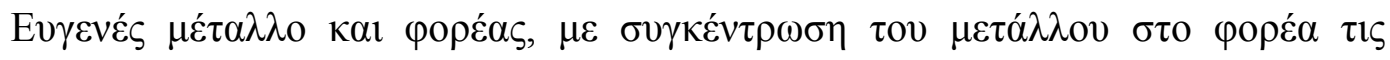

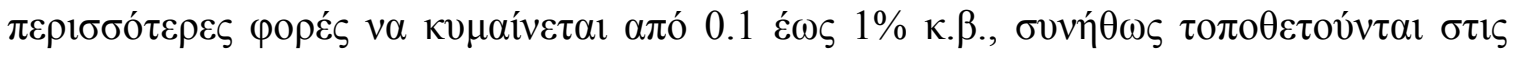

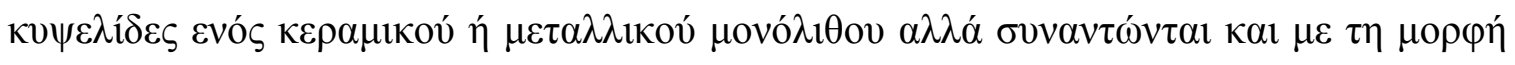

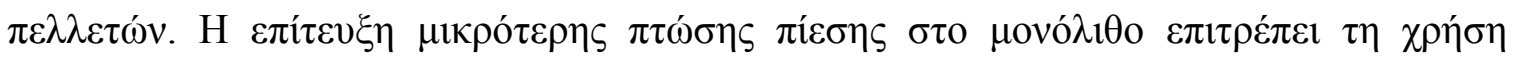

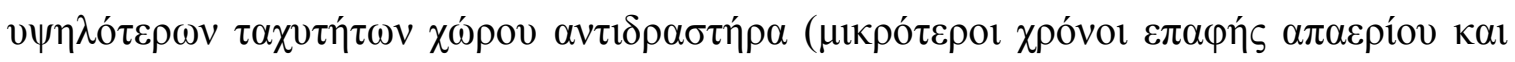

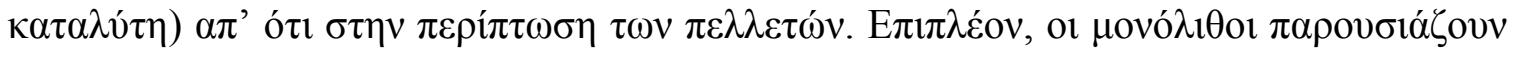

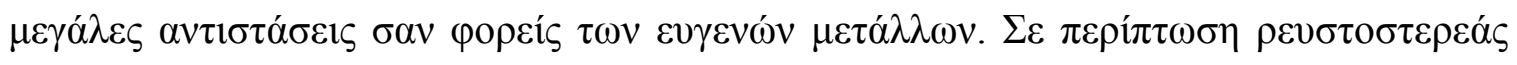

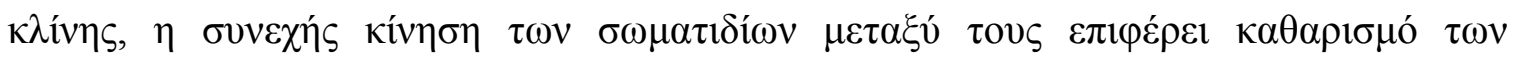

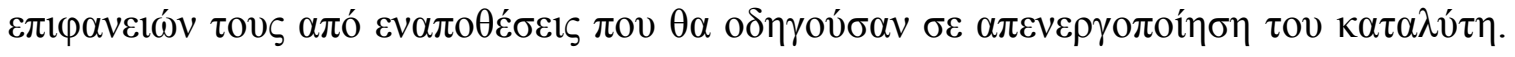

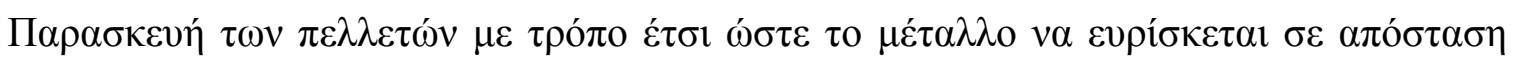

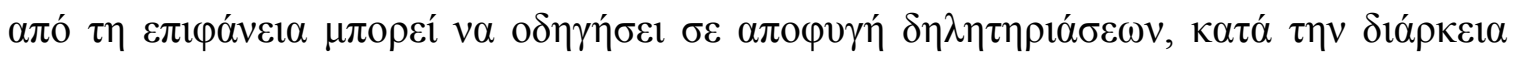

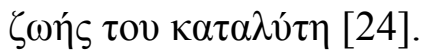

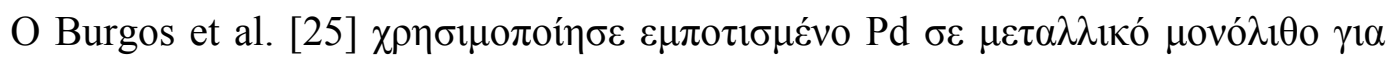

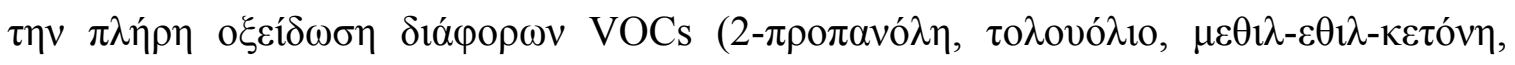

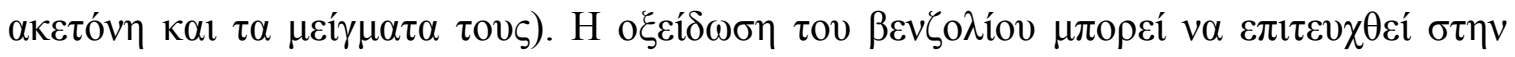

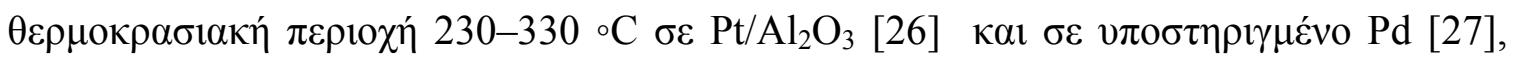

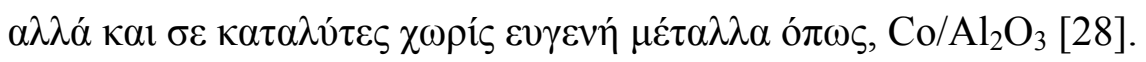

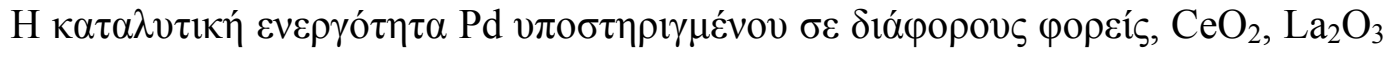

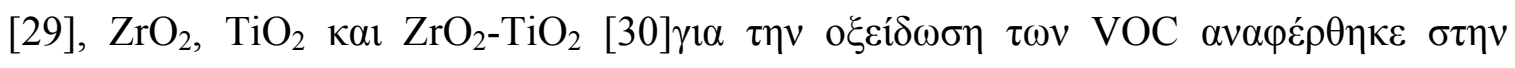

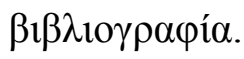

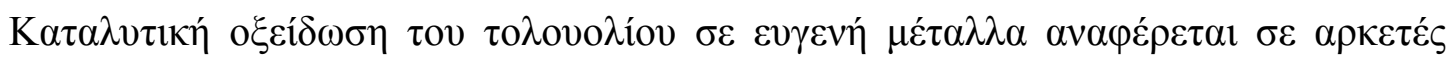

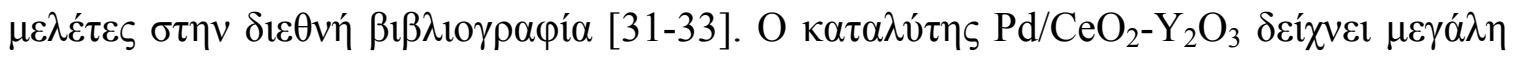




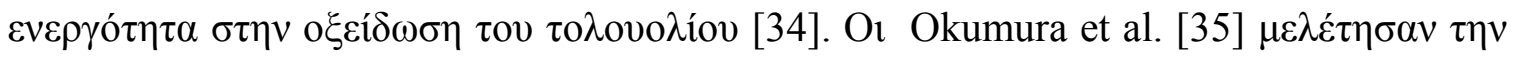

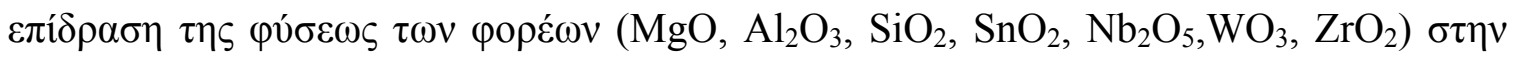

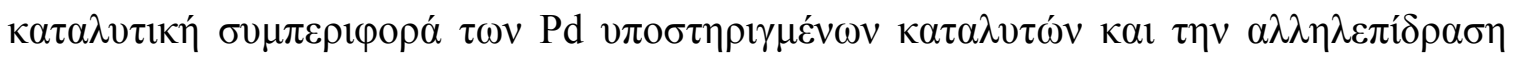

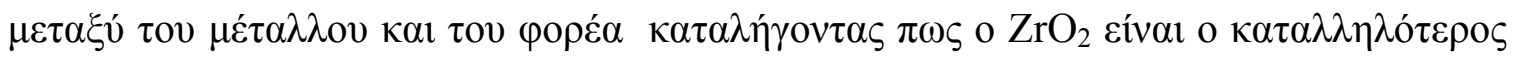

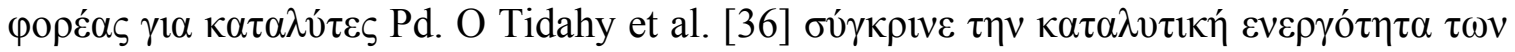

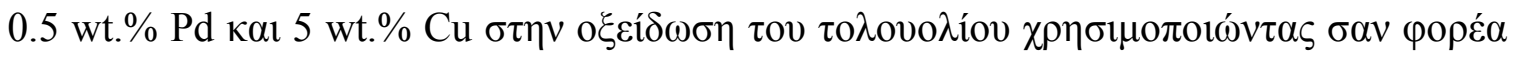

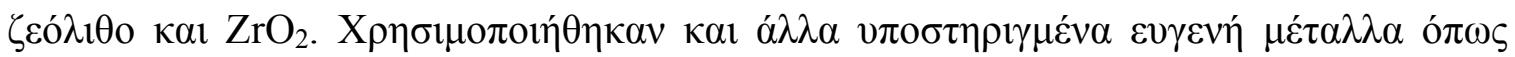

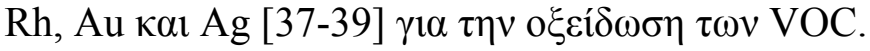

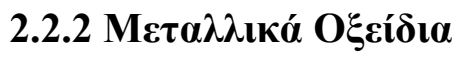

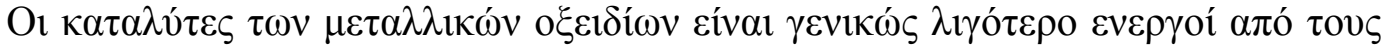

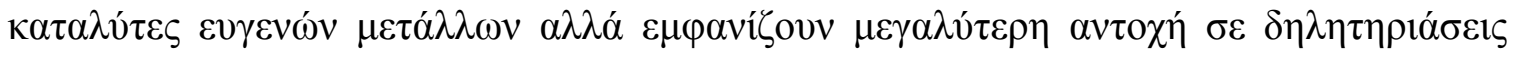

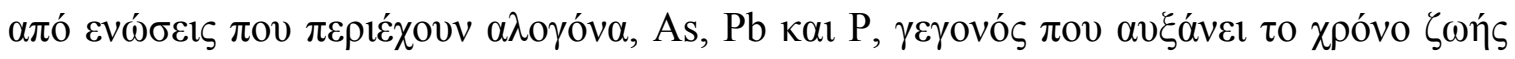

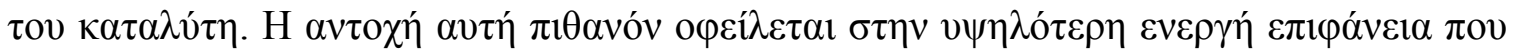

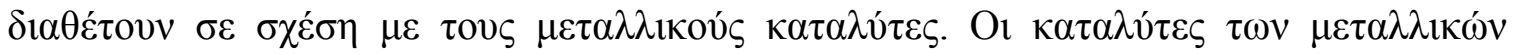

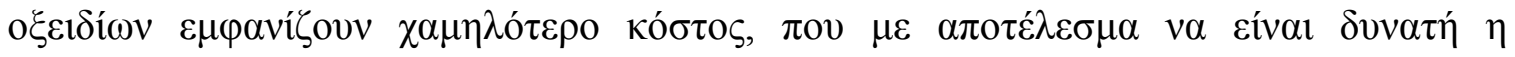

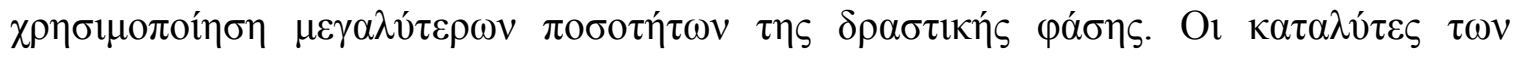

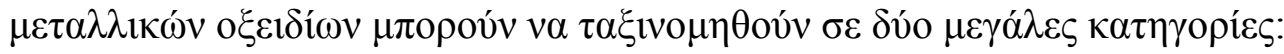

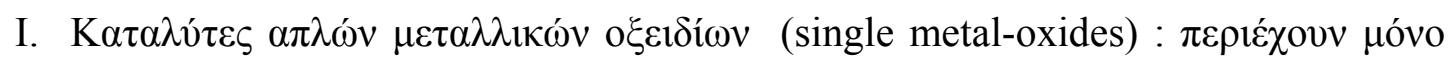

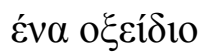

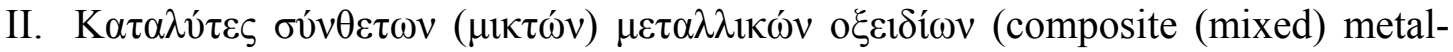
oxides)

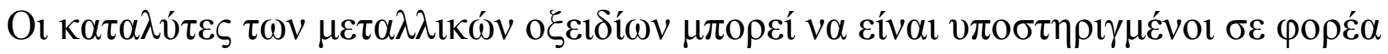

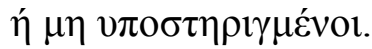

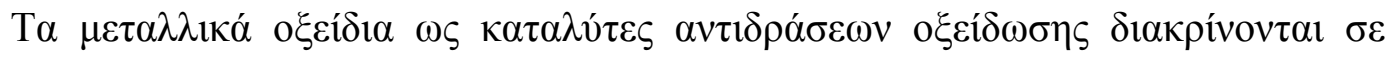

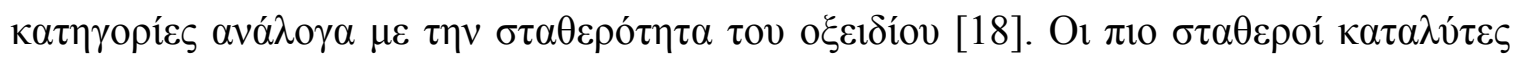

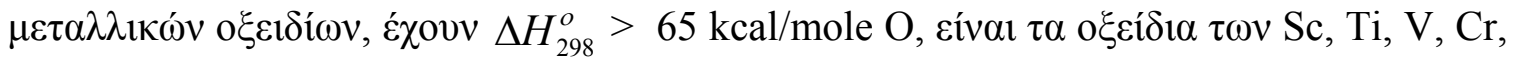

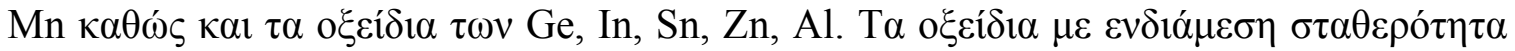

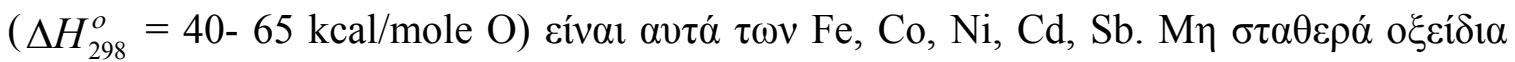

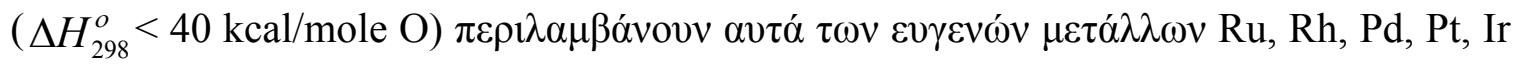




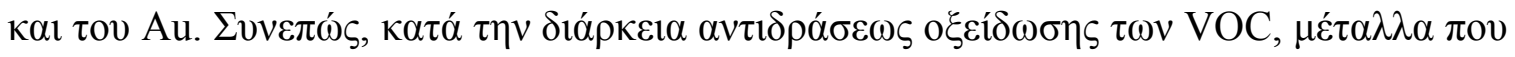

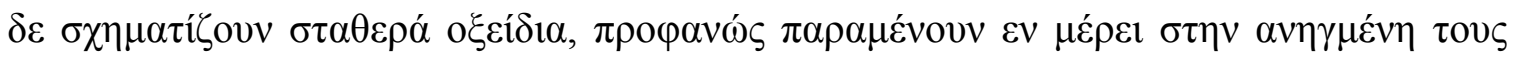

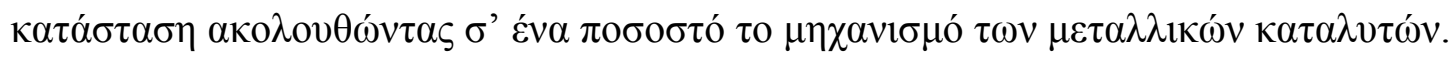

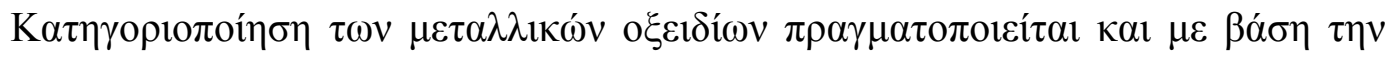

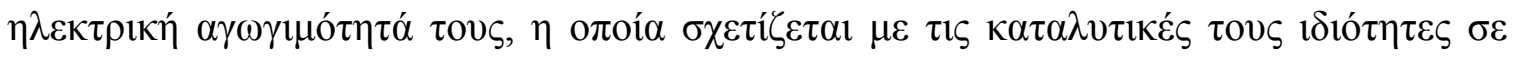

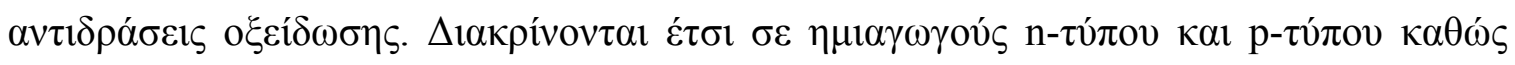

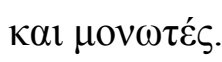

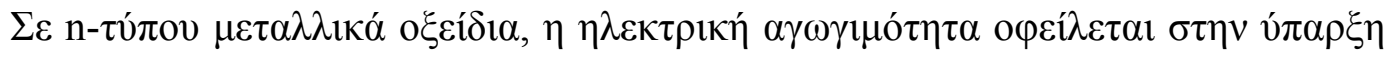

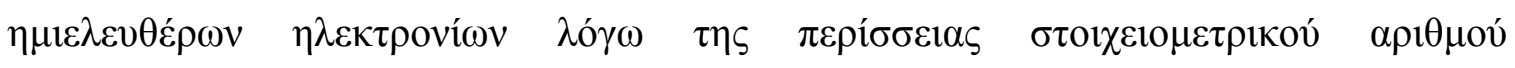

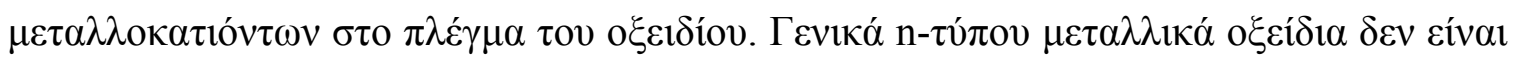

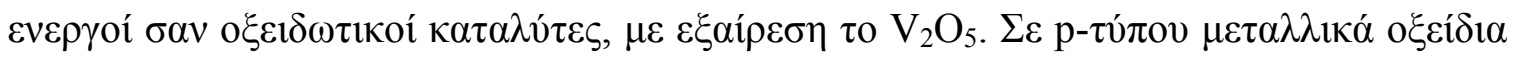

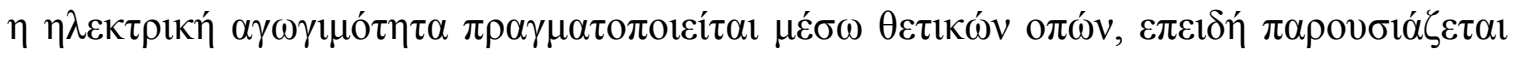

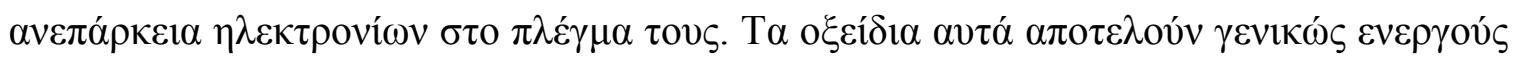

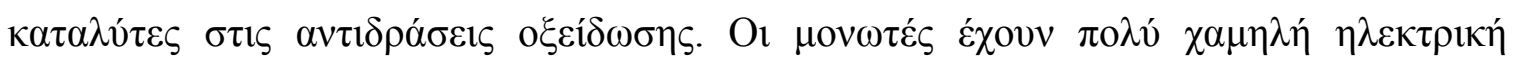

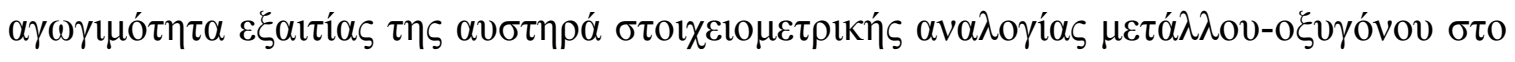

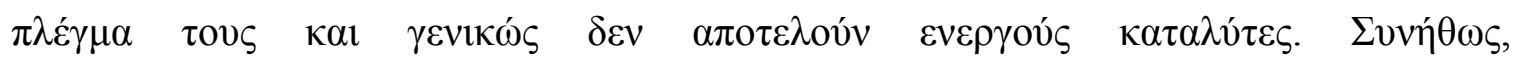

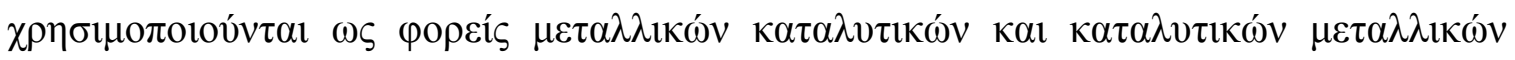
o $\xi \varepsilon 1 \delta i ́ \omega v\left(\pi \cdot \chi \cdot \mathrm{Al}_{2} \mathrm{O}_{3}, \mathrm{SiO}_{2}\right)$.

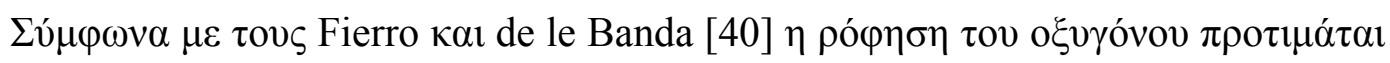

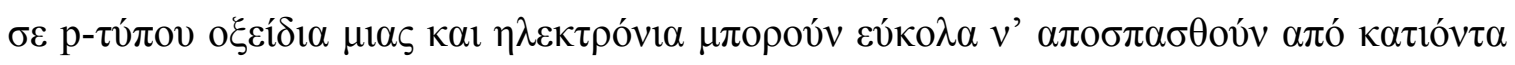

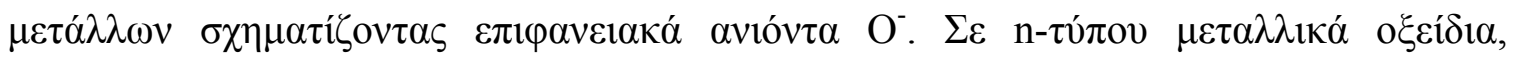

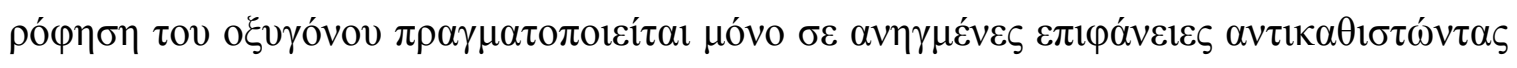

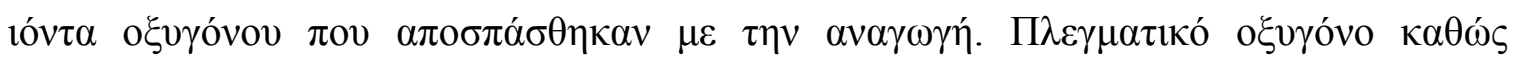

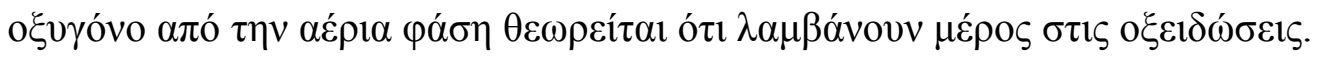

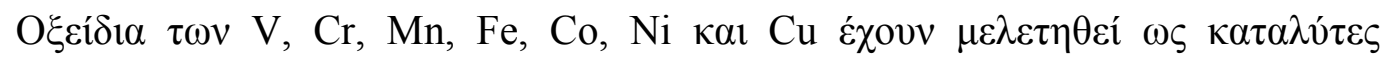

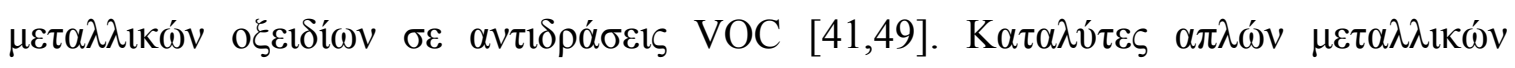

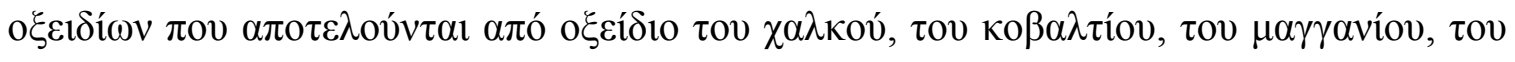

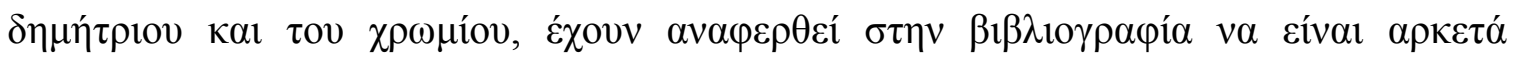

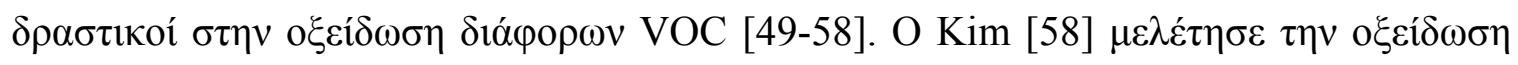

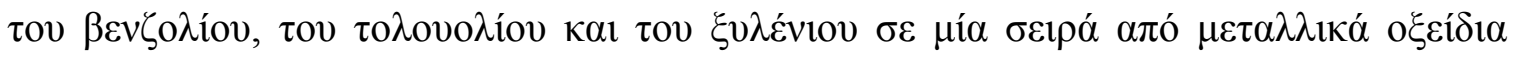

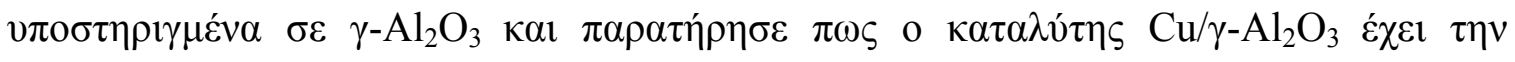




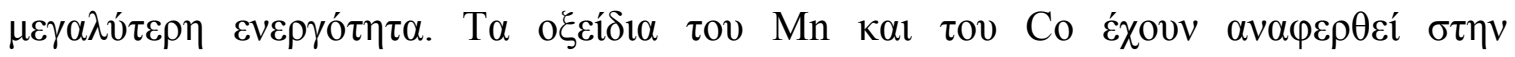

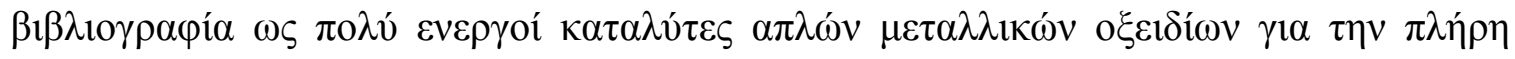

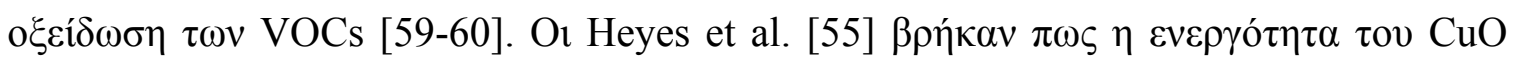

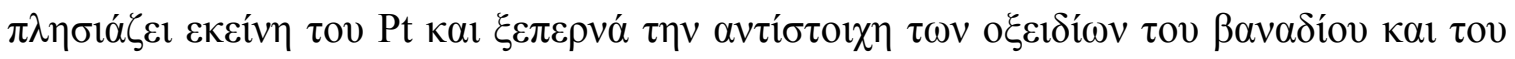

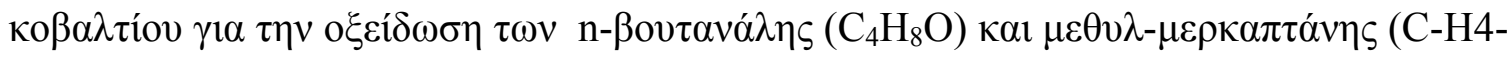

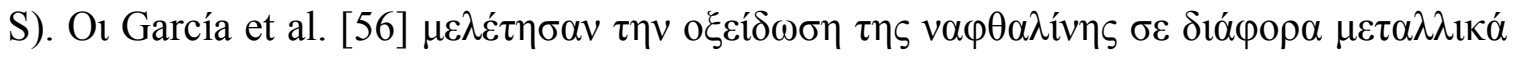

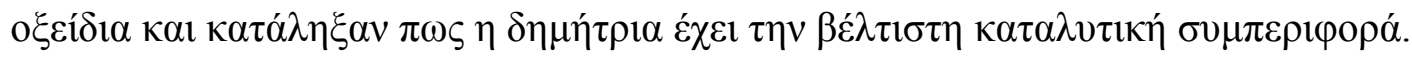

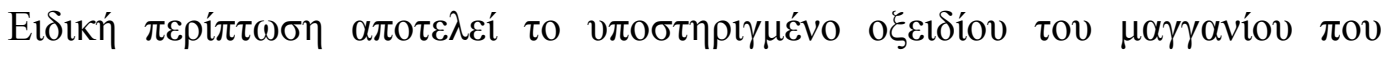

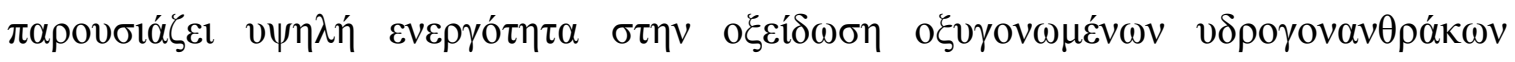

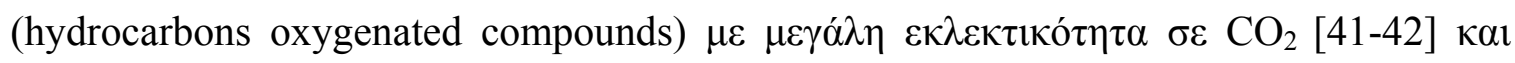

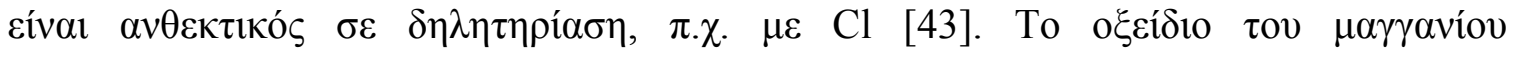

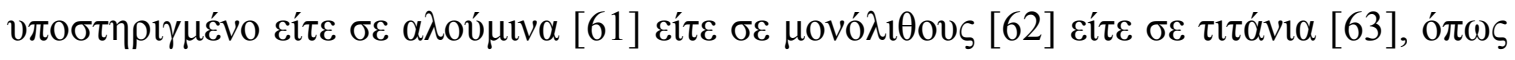

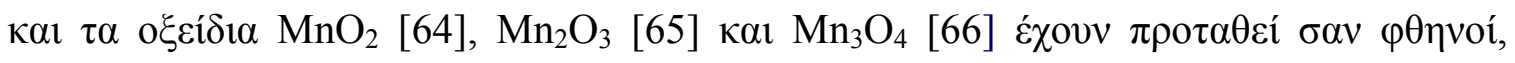

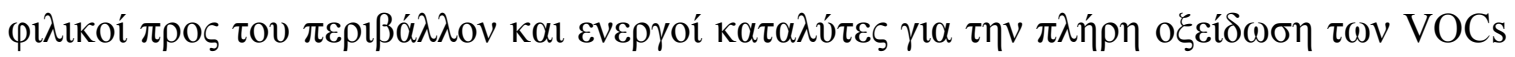

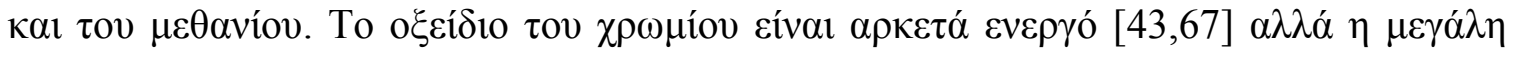

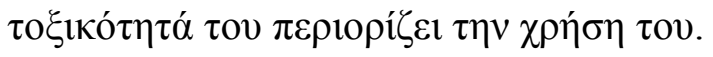

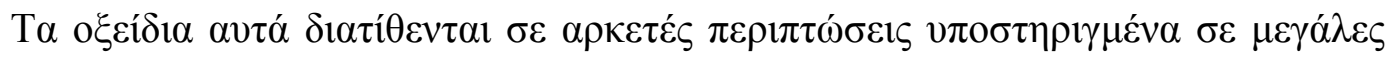

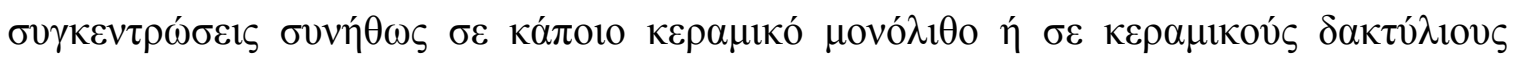

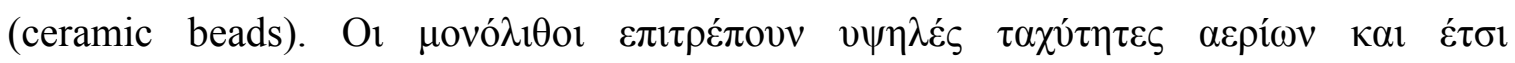

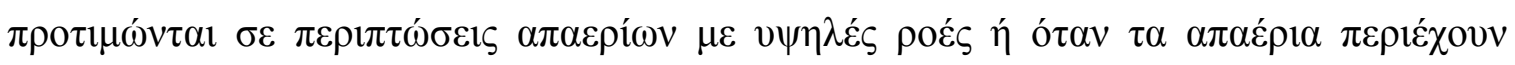

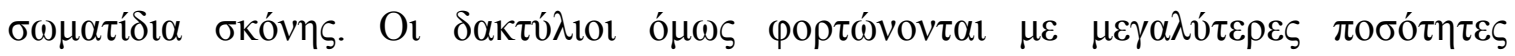

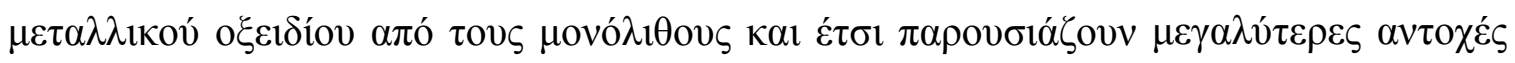

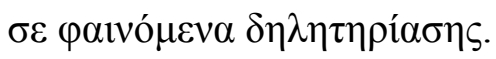

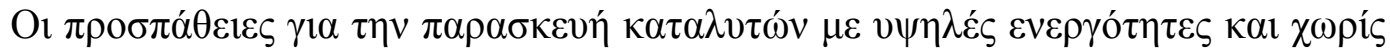

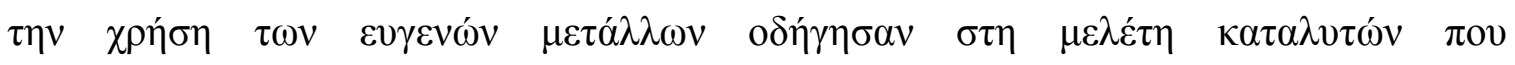

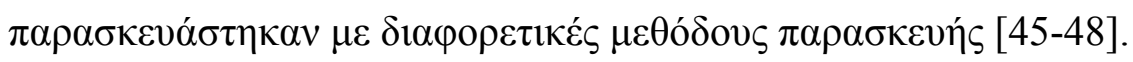

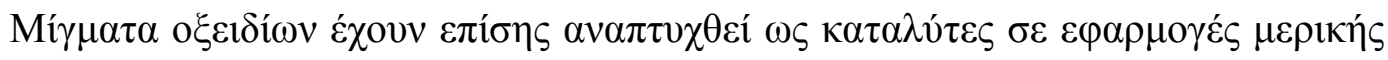

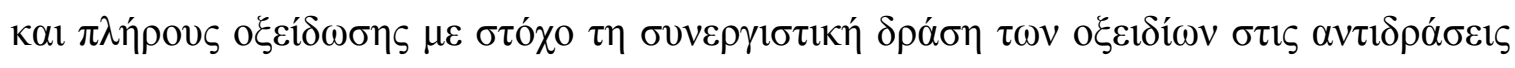

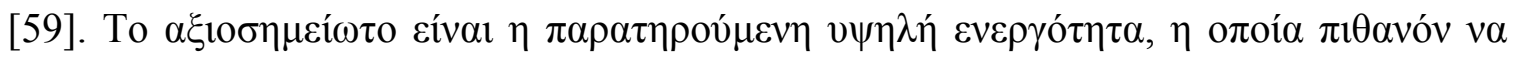

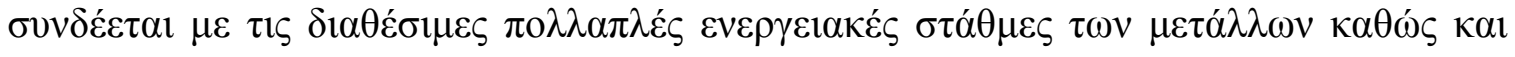

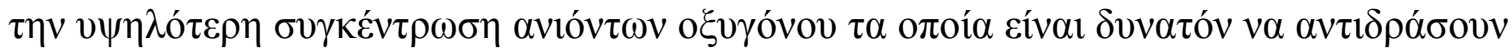




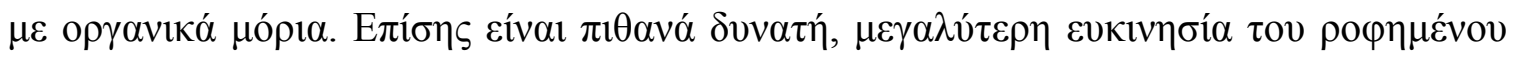

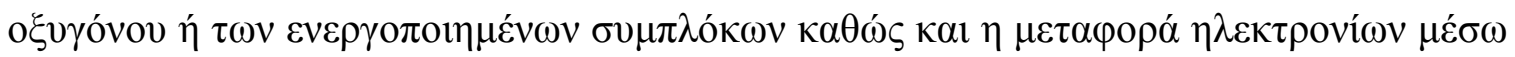

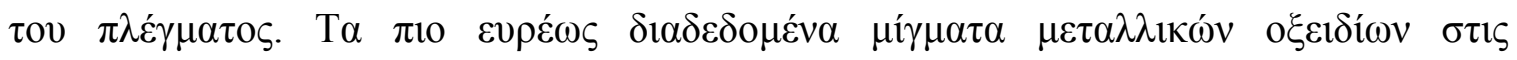

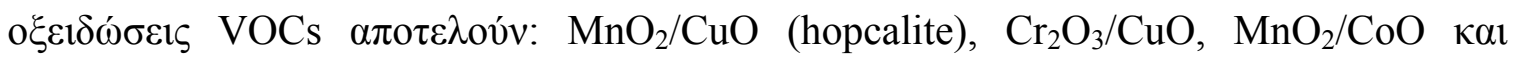
$\mathrm{MoO}_{3} / \mathrm{CoO}[68]$.

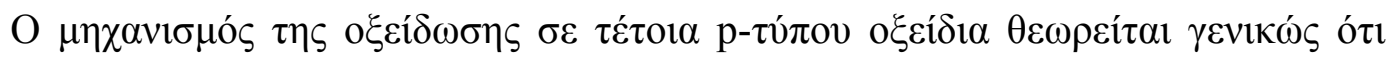

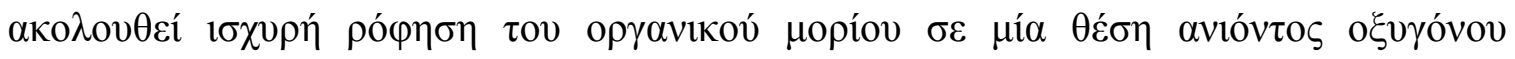

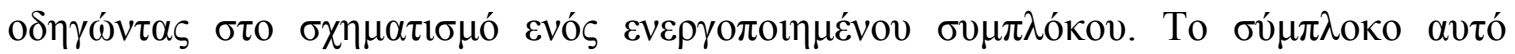

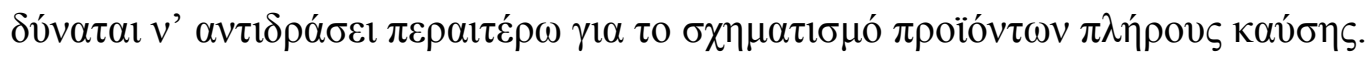

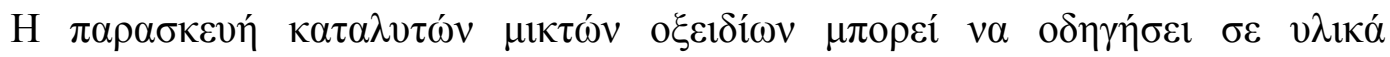

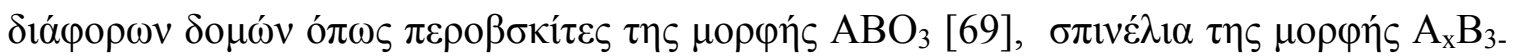

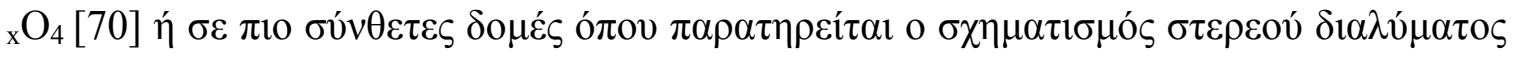

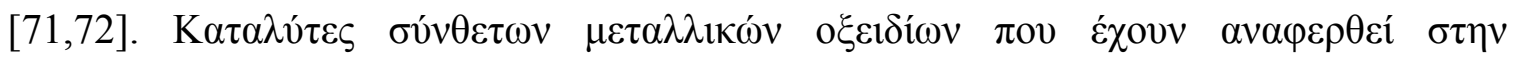

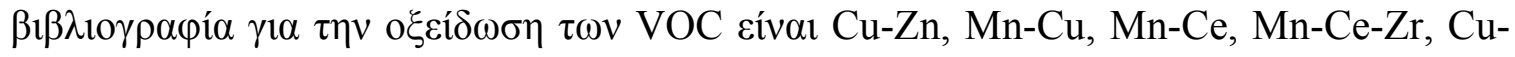
Ce кal $\mathrm{Mn}-\mathrm{Cu}[73-78]$.

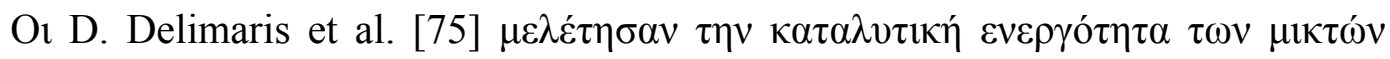

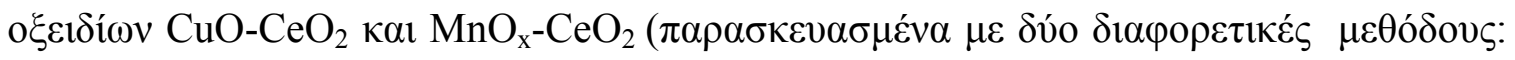

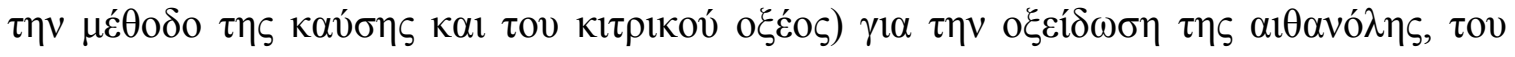

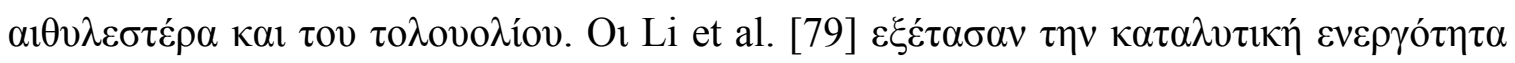

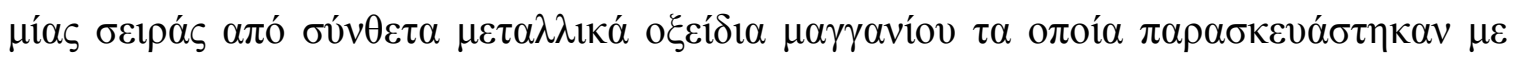

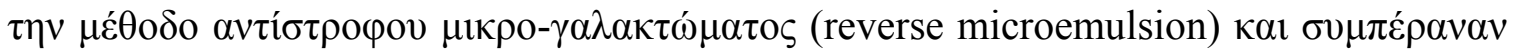

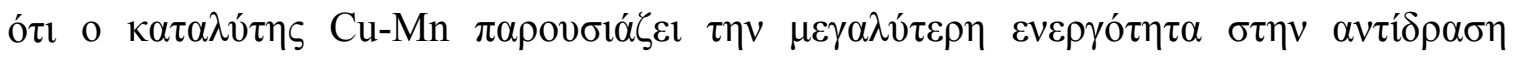

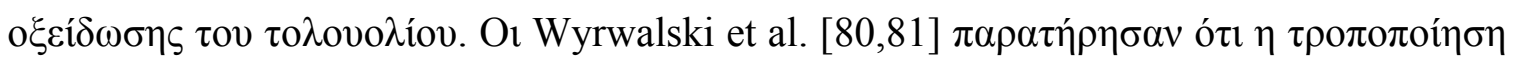

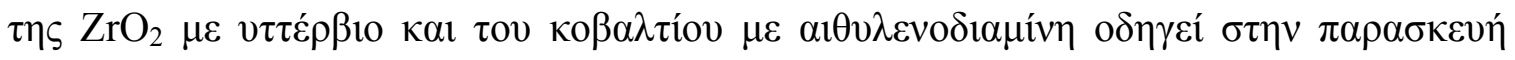

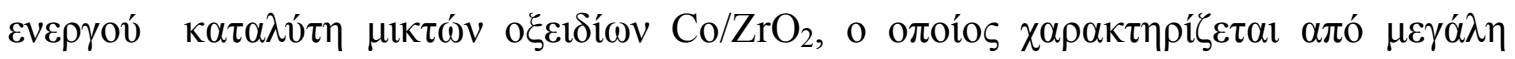

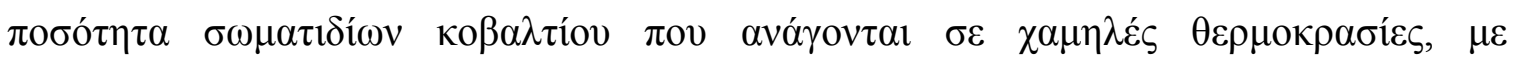

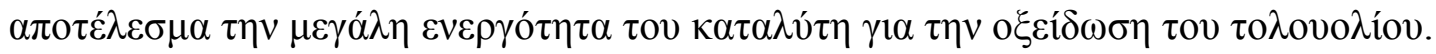

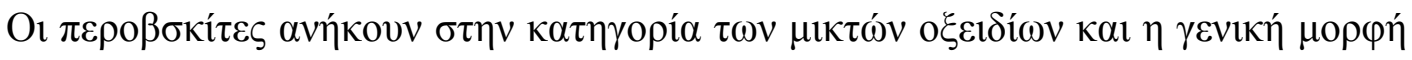

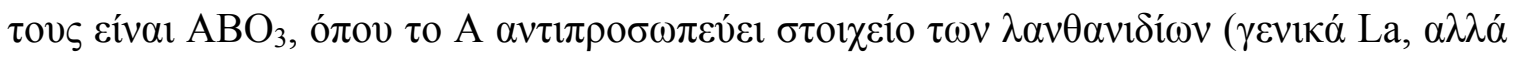

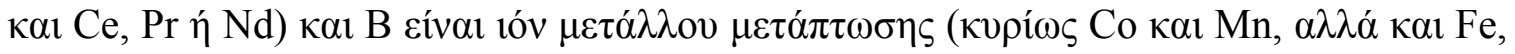

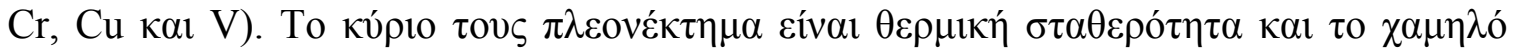




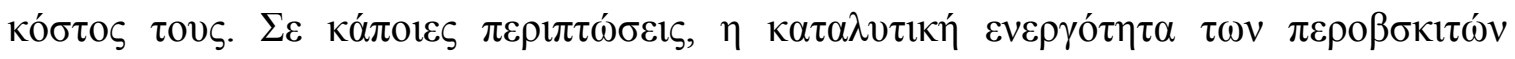

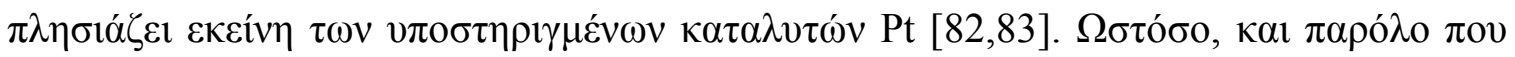

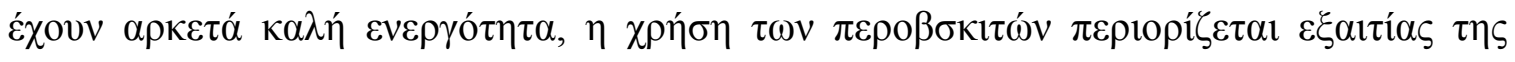

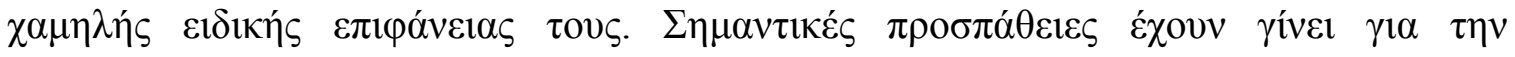

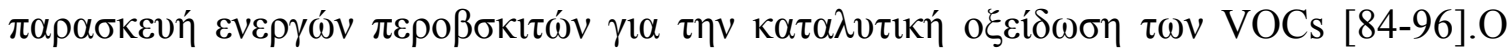

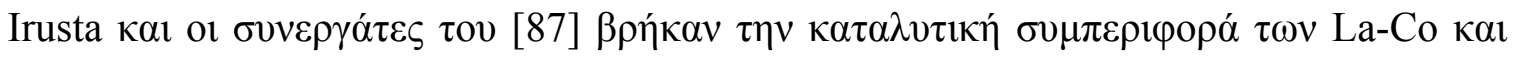

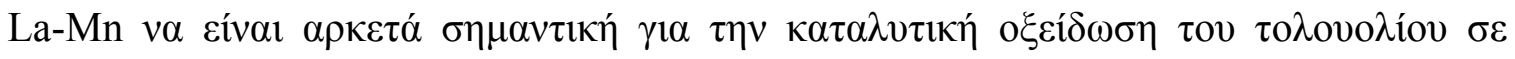

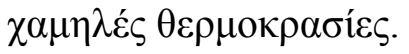

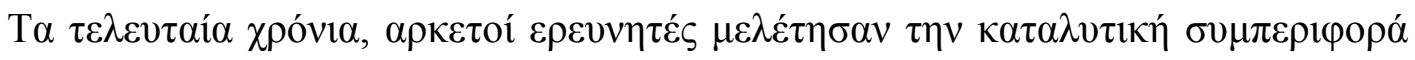

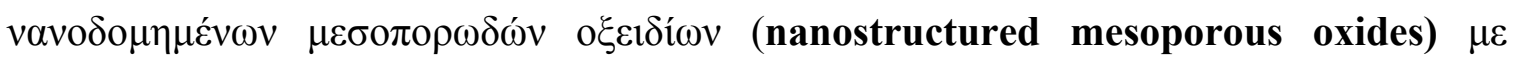

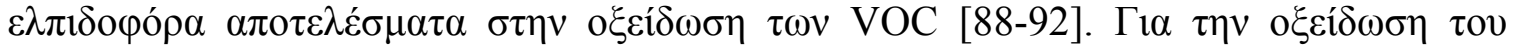
$\tau o \lambda$ ovo $\lambda$ íov $\sigma \varepsilon \mu \varepsilon \sigma o \pi о \rho \omega ́ \delta \eta ~ v \lambda \iota \kappa \alpha ́$ (mesoporous materials), or Sinha et al. [93]

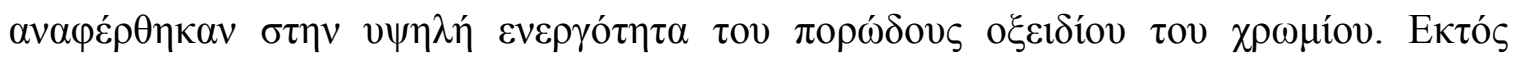

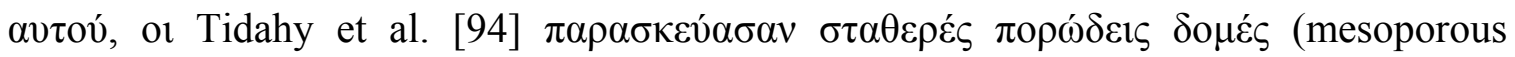

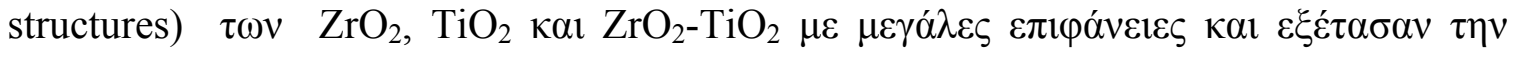

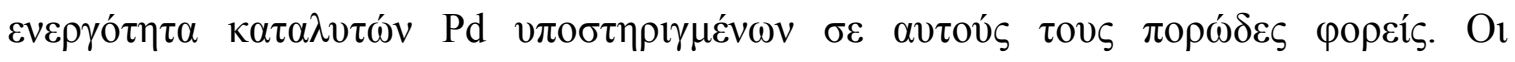

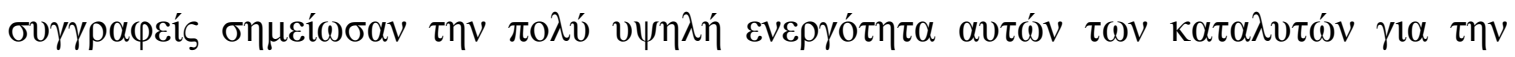
$\pi \lambda \eta ́ \rho \eta$ o $\xi \varepsilon i ́ \delta \omega \sigma \eta \tau$

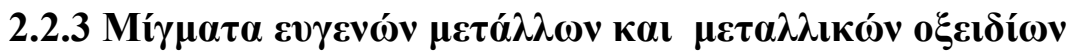

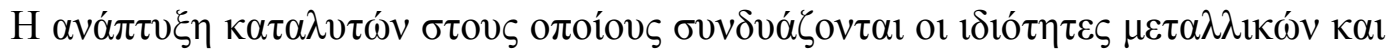

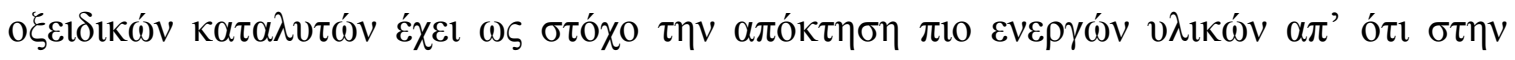
$\pi \varepsilon \rho i ́ \pi \tau \omega \sigma \eta \tau \omega \nu \mu \varepsilon \mu o v \omega \mu \varepsilon ́ v \omega \nu ~ \kappa \alpha \tau \alpha \lambda v \tau \omega ́ v . ~ M \varepsilon \lambda \varepsilon ́ \tau \varepsilon \varsigma \tau \omega \nu$ Vassileva et al. [95] $\sigma \varepsilon \kappa \alpha \tau \alpha \lambda \nu ́ \tau \eta$

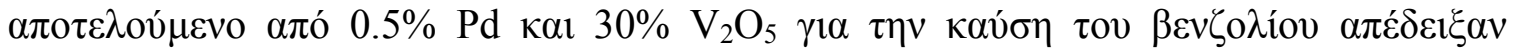

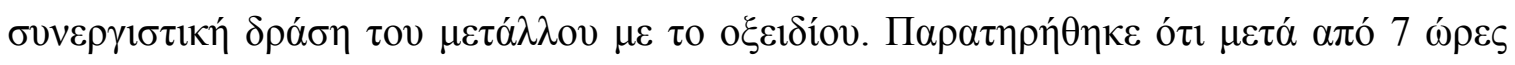

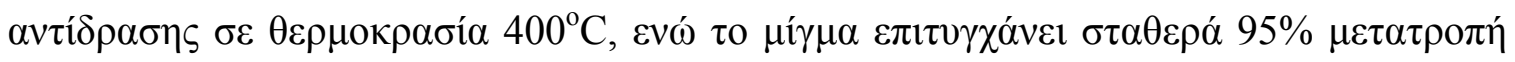

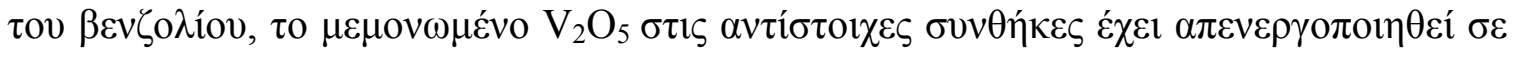

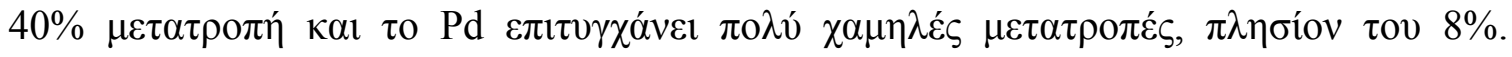

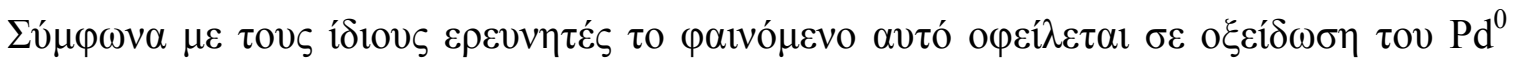

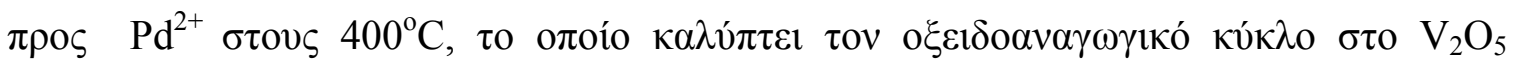




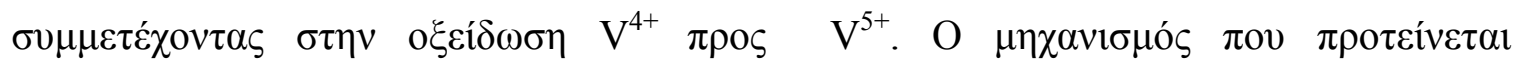

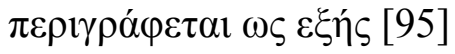

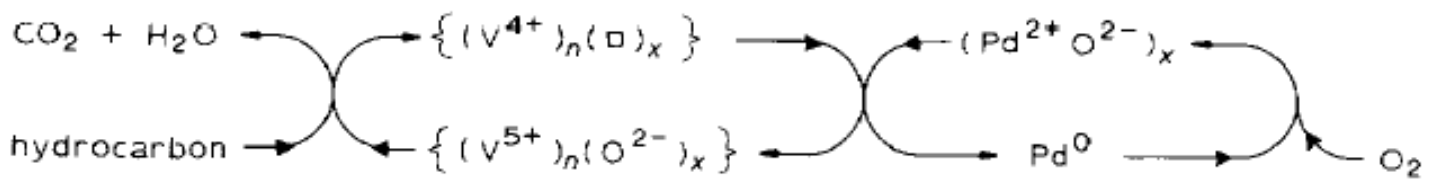

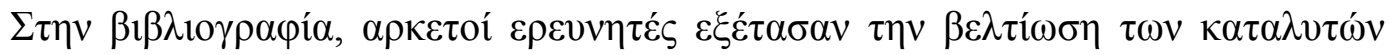

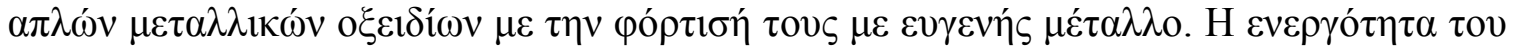

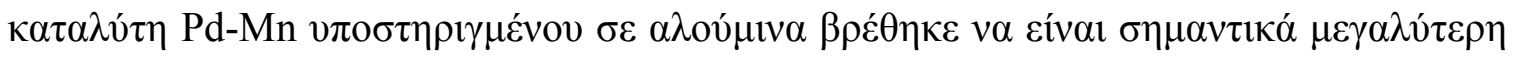

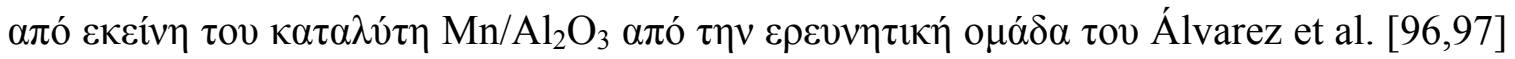

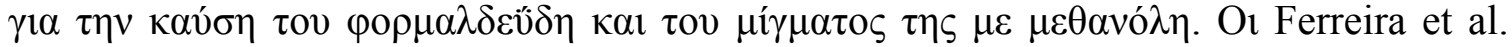

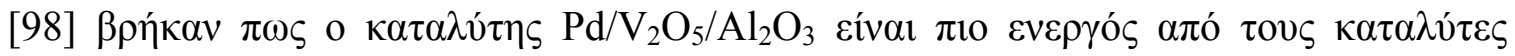

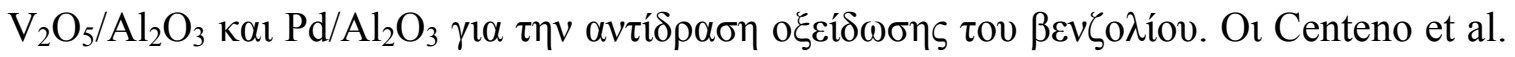

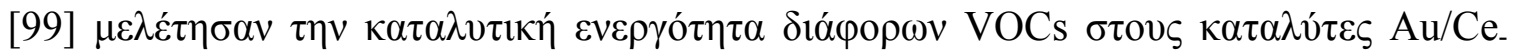

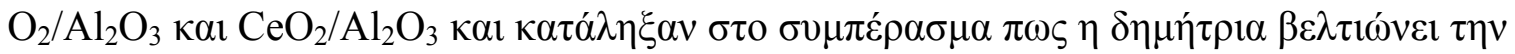

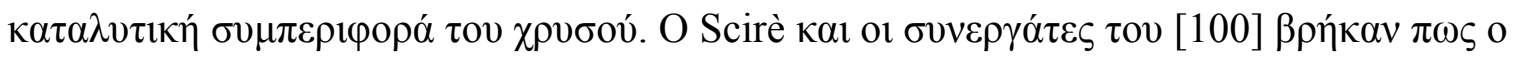

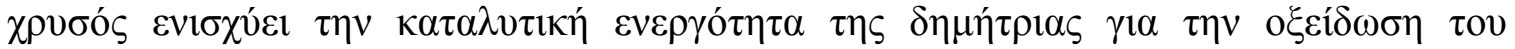

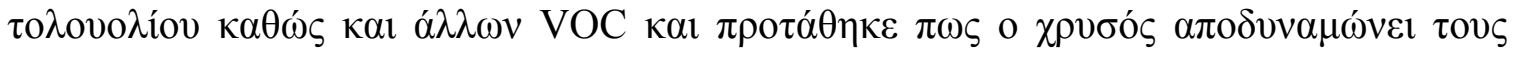

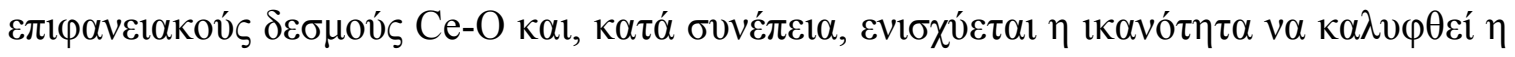

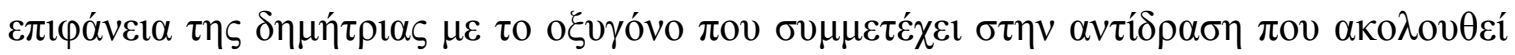
$\mu \eta \chi \alpha v i \sigma \mu o ́$ Mars-van Krevelen.

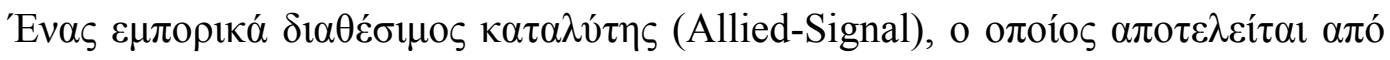

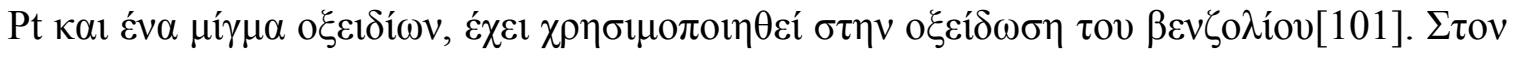

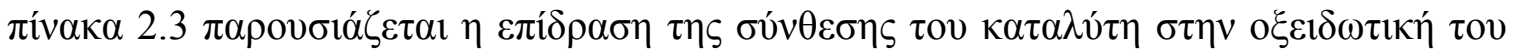

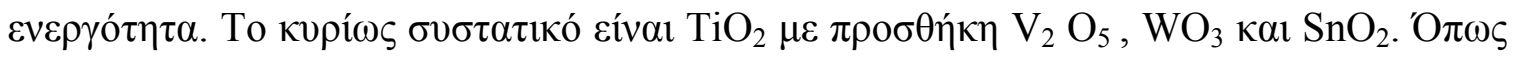

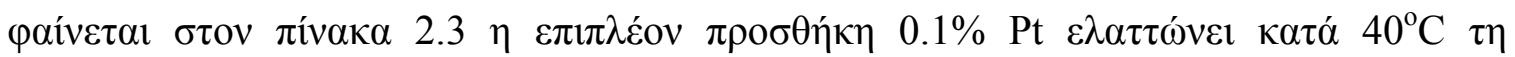

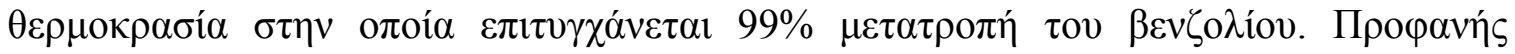

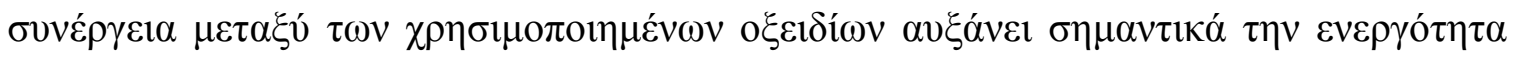

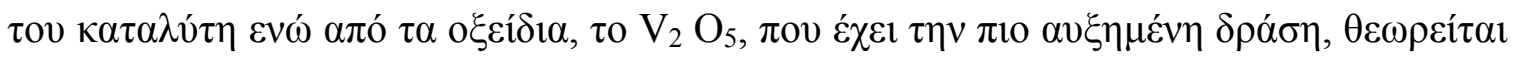

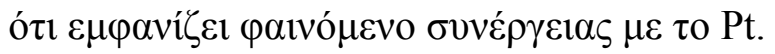




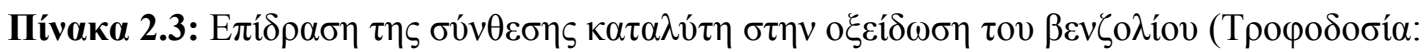

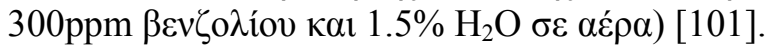

\begin{tabular}{|c|c|c|c|}
\hline \multirow{2}{*}{$\Delta \varepsilon i ́ \gamma \mu \alpha$} & \multicolumn{3}{|c|}{ 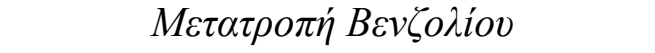 } \\
\hline & T-99\% & T-90\% & $\mathrm{T}-\mathbf{5 0} \%$ \\
\hline $\mathrm{TiO}_{2}$ & - & 575 & 390 \\
\hline $\mathrm{TiO}_{2} / \mathrm{V}_{2} \mathrm{O}_{5}$ & 404 & 355 & 355 \\
\hline $\mathrm{TiO}_{2} / \mathrm{WO}_{3}$ & - & 475 & 292 \\
\hline $\mathrm{TiO}_{2} / \mathrm{V}_{2} \mathrm{O}_{5} / \mathrm{WO}_{3} / \mathrm{SnO}_{2}$ & 355 & 316 & 270 \\
\hline $\mathrm{TiO}_{2} / \mathrm{V}_{2} \mathrm{O}_{5} / \mathrm{WO}_{3} / \mathrm{SnO}_{2} / \mathrm{Pt}$ & 305 & 268 & 235 \\
\hline
\end{tabular}

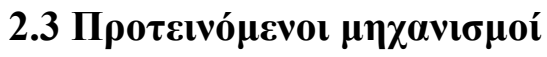

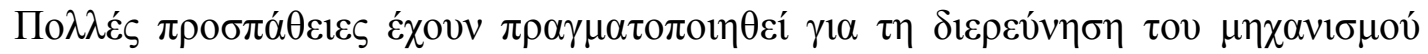

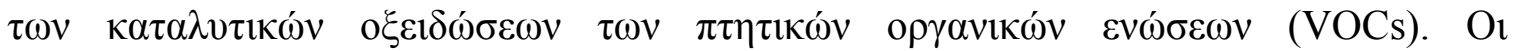

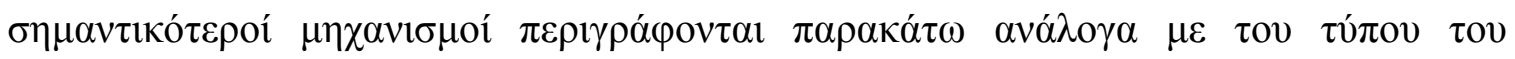
$\kappa \alpha \tau \alpha \lambda u ́ \tau \eta$.

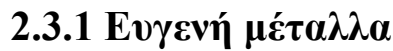

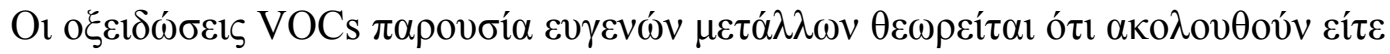

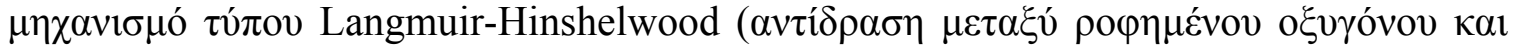

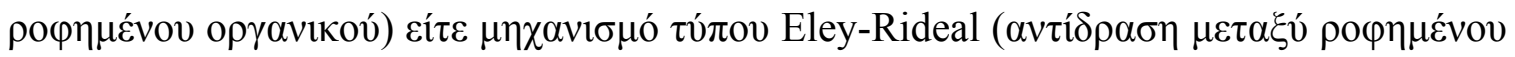

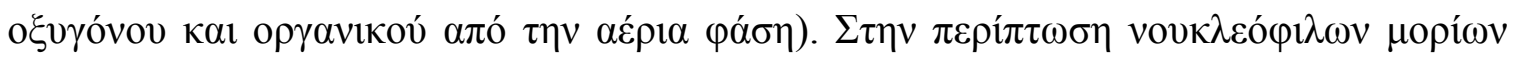

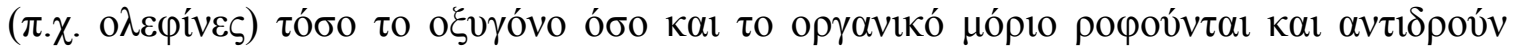

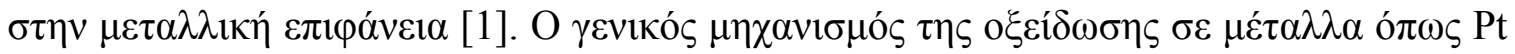

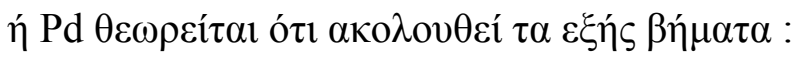

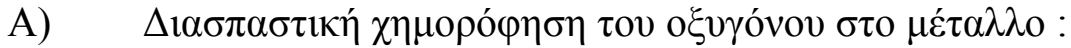

$$
\mathrm{O}_{2}+[] \rightarrow\left[\mathrm{O}_{2}\right] \tau \alpha \chi \dot{\varepsilon} \omega \varsigma 2[O]
$$

ó

B)

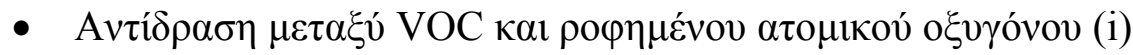

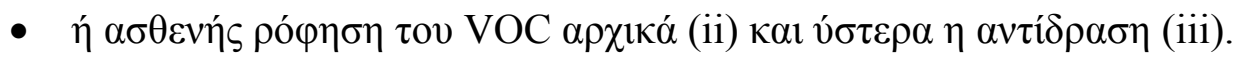




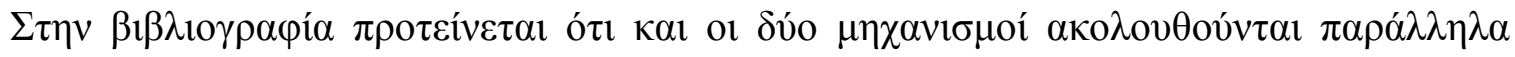
$\sigma \tau ı \varsigma \pi \varepsilon \rho ı \sigma \sigma o ́ \tau \varepsilon \rho \varepsilon \varsigma \pi \varepsilon \rho ı \tau$ $\sigma \varepsilon ı \varsigma ~[18,102]$

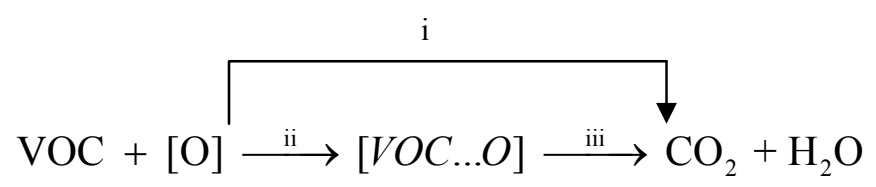

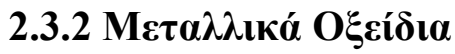

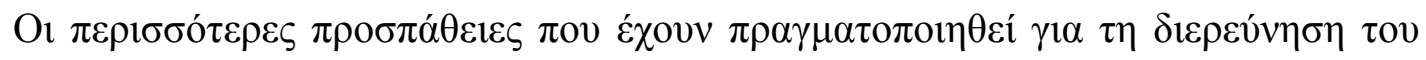

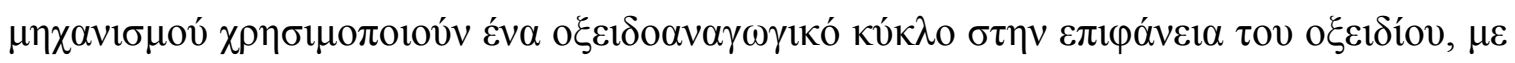

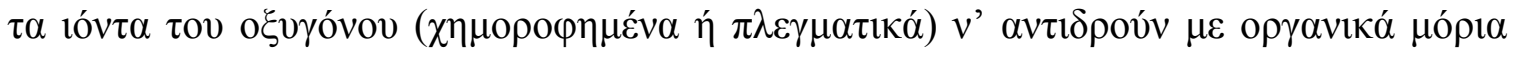

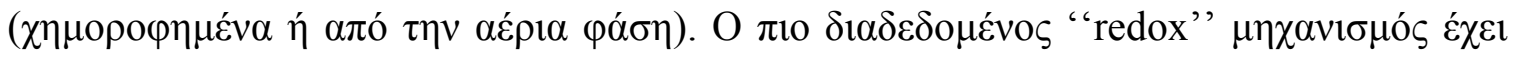

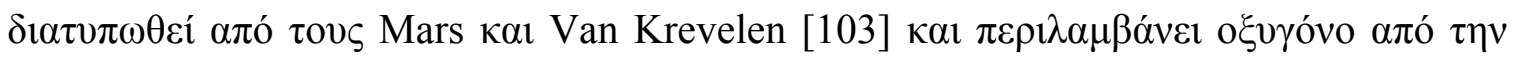

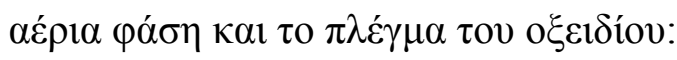

$$
\begin{aligned}
& \mathrm{MeO}+R \rightarrow R O+\mathrm{Me} \\
& 2 \mathrm{Me}+\mathrm{O}_{2} \rightarrow 2 \mathrm{MeO}
\end{aligned}
$$

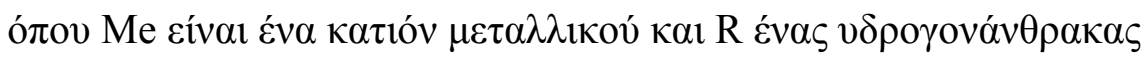

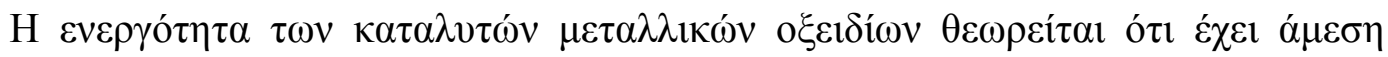

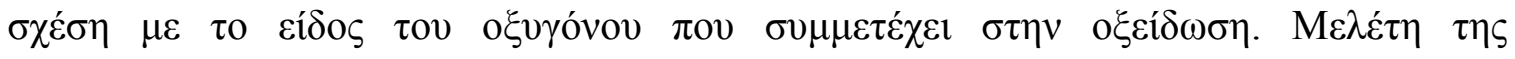

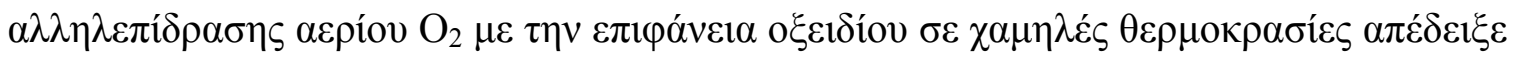
$\tau \alpha \varepsilon \xi \eta \dot{\varsigma} \beta \beta \dot{\eta} \mu \alpha \alpha$ [104]:

$$
\begin{aligned}
& O_{2(g)}+e^{-} \rightarrow O_{2}^{-} \\
& O_{2}^{-}+e^{-} \rightarrow 2 O^{-} \\
& O^{-}+e^{-} \rightarrow O^{2-}
\end{aligned}
$$

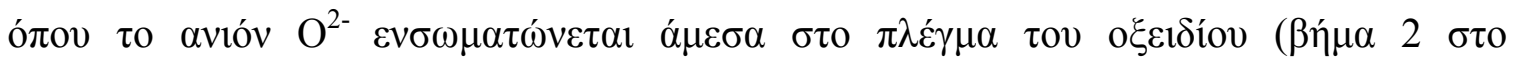

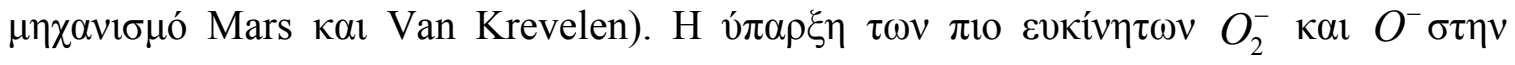

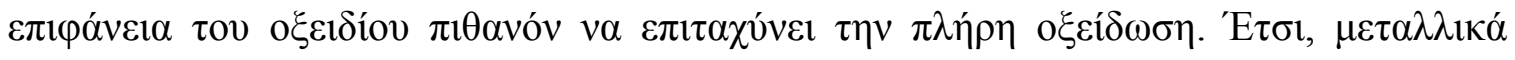

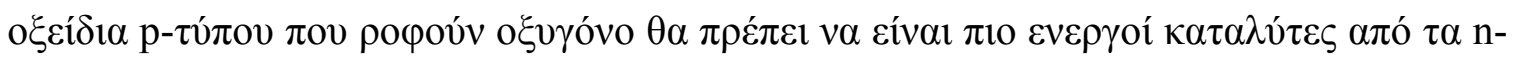

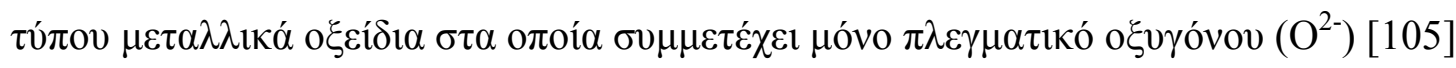




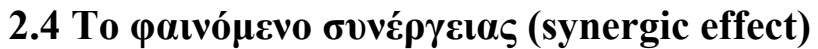

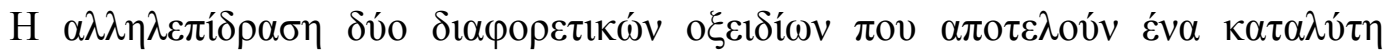
$\varepsilon \xi \alpha \rho \tau \alpha ́ \tau \alpha \iota$ a

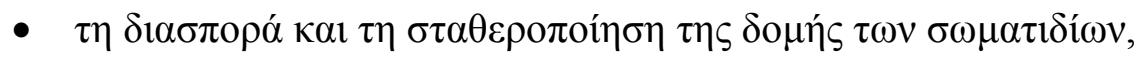

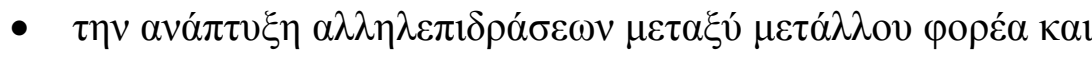

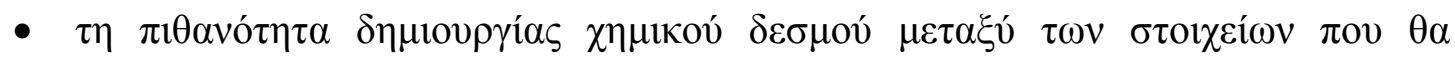

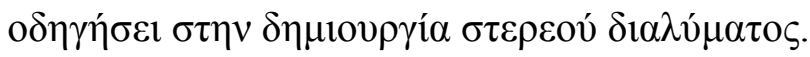

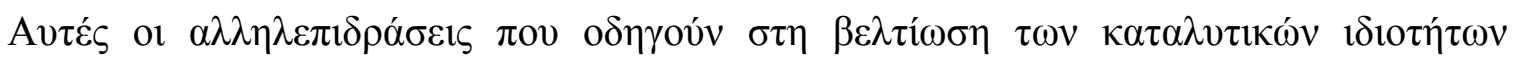

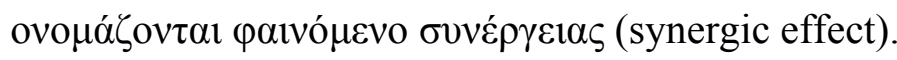

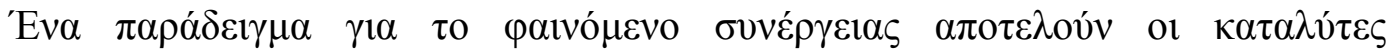
$\mu \varepsilon \tau \alpha \lambda \lambda \iota \kappa \omega ́ v ~ o \xi \varepsilon ı i ́ \omega v ~ C u O-\mathrm{CeO}_{2}, \mathrm{MnO}_{2}-\mathrm{CeO}_{2} \kappa \alpha \imath \mathrm{CuO}-\mathrm{MnO}_{2}$.

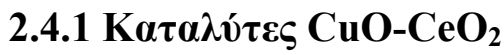

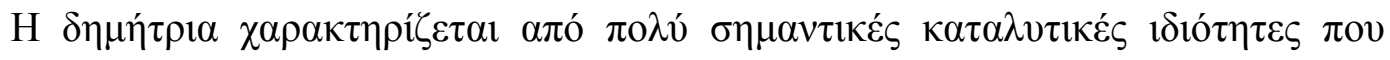

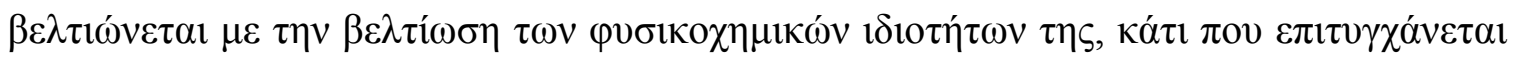

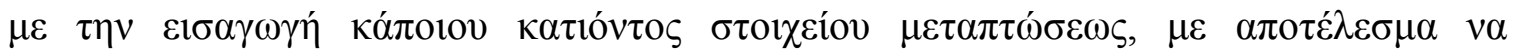

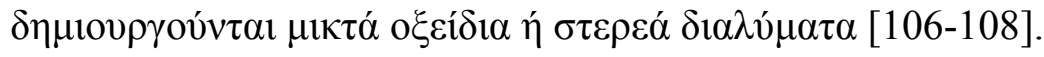

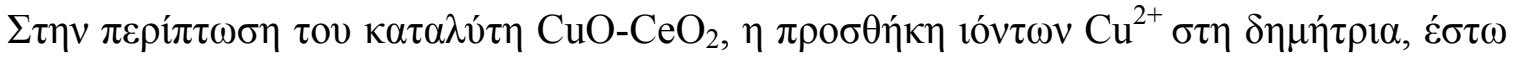

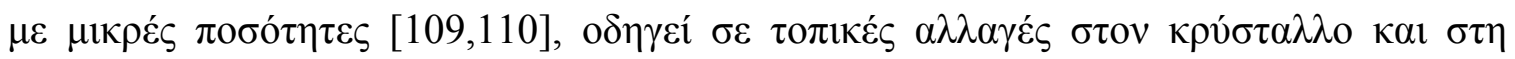

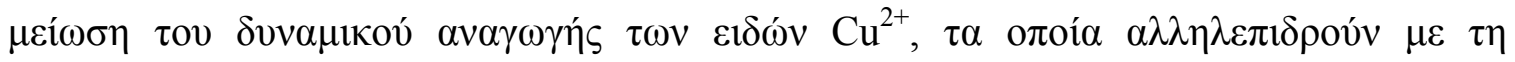

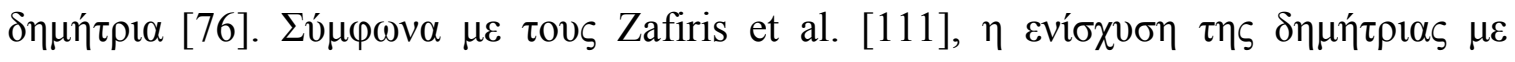

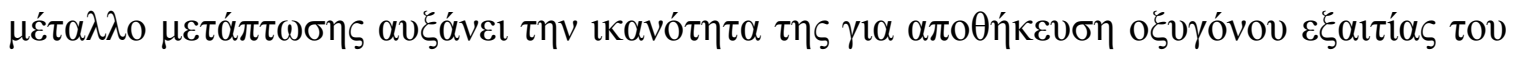

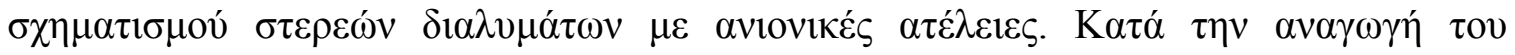

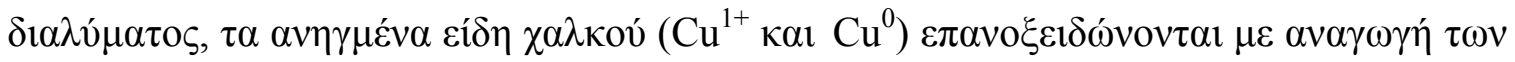

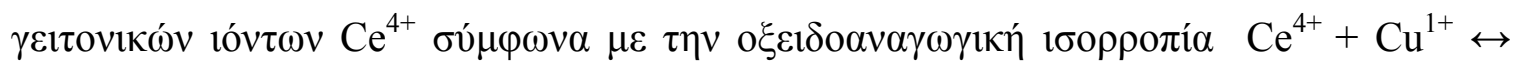

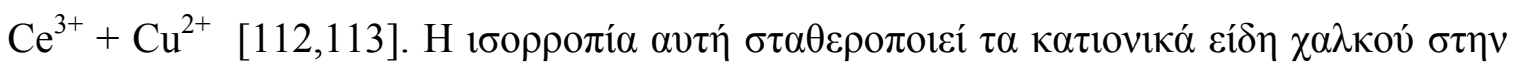

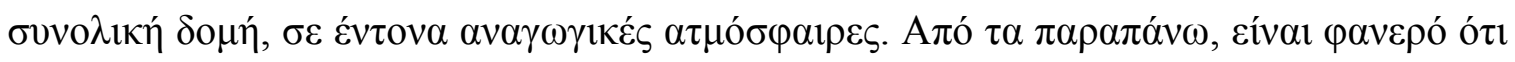

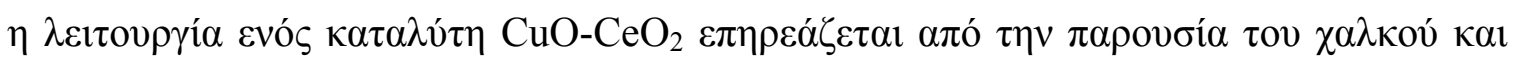

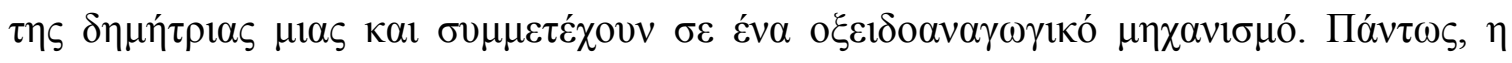

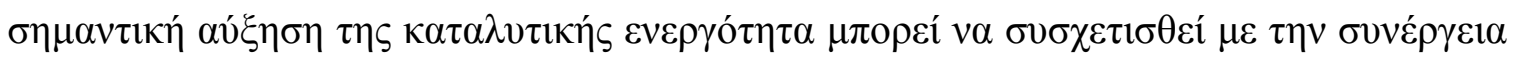

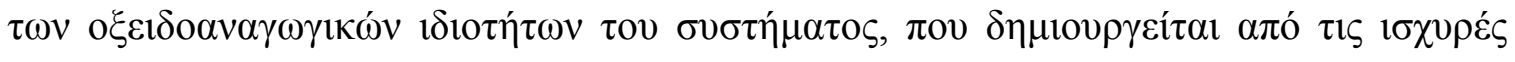




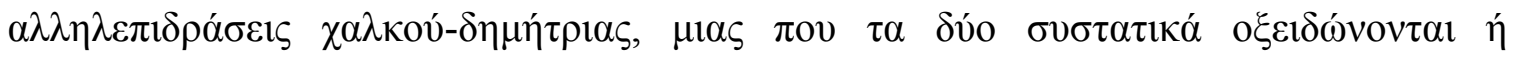

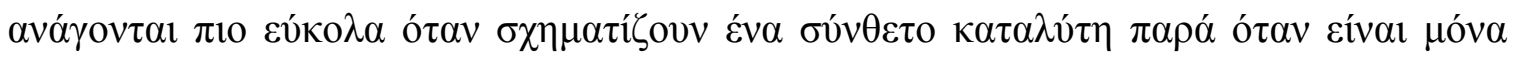

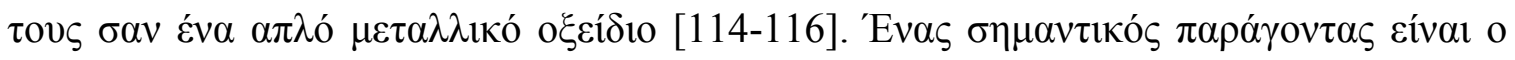

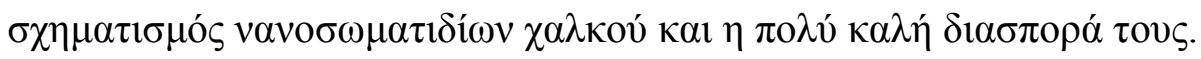

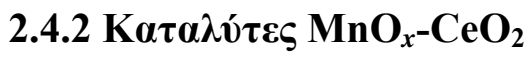

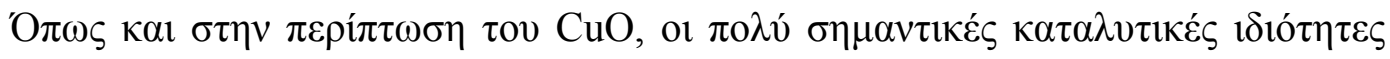

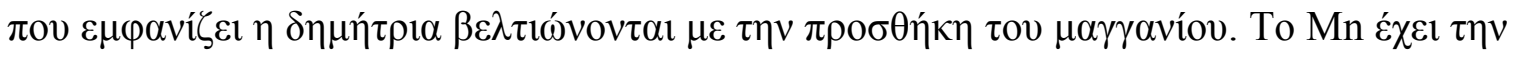

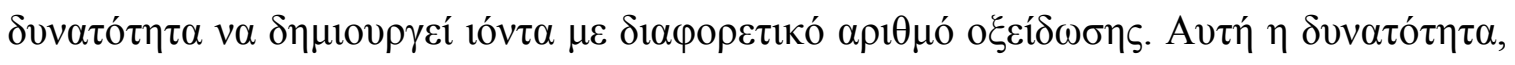

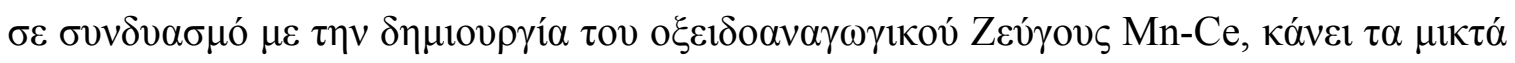

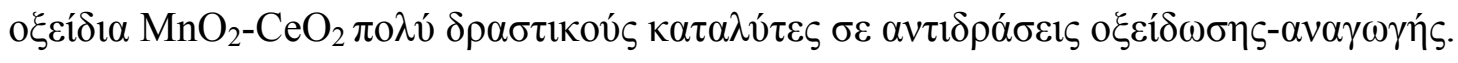

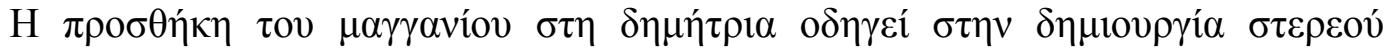

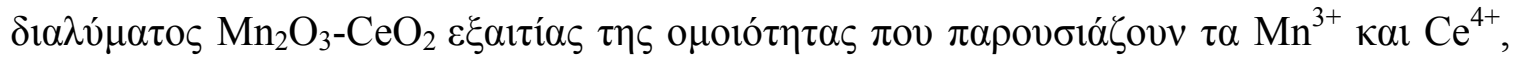

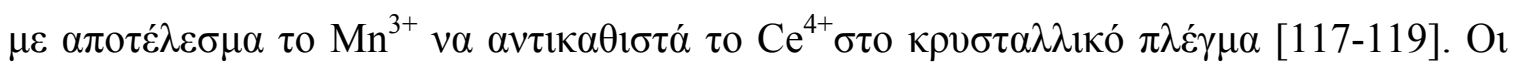

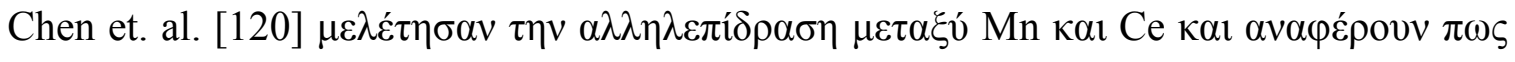

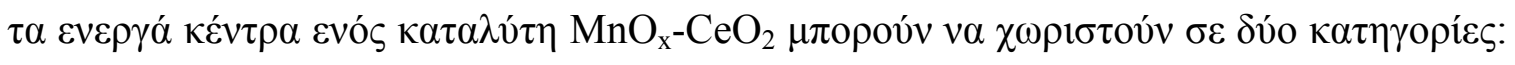

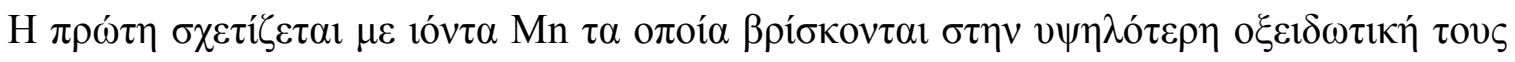

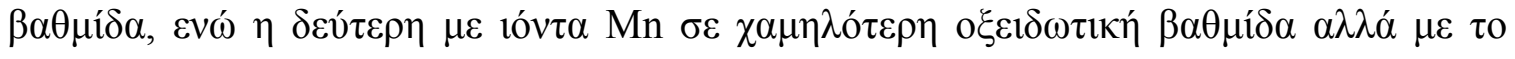

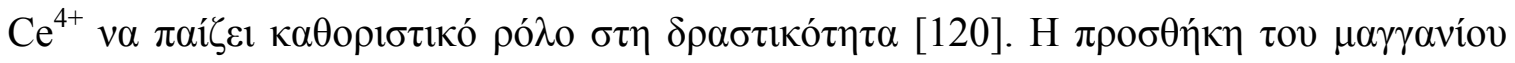

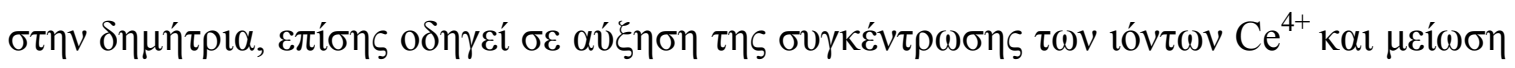

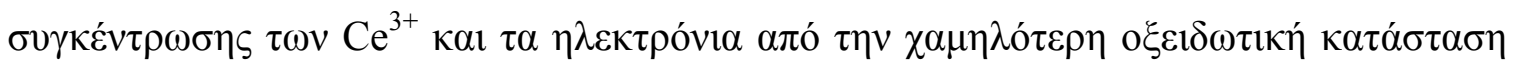

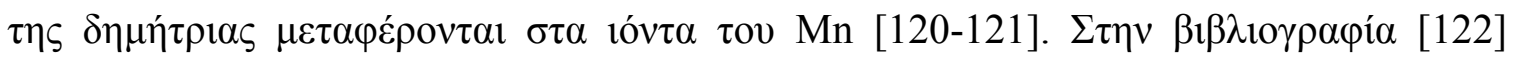

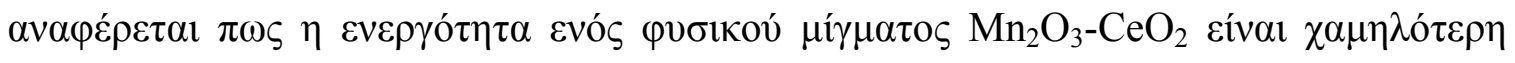

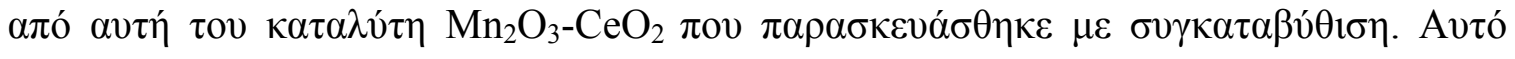

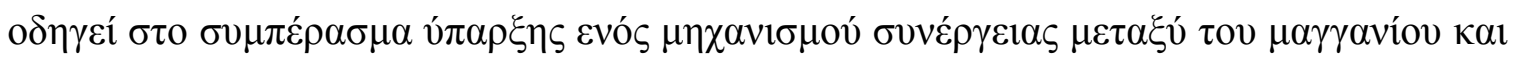

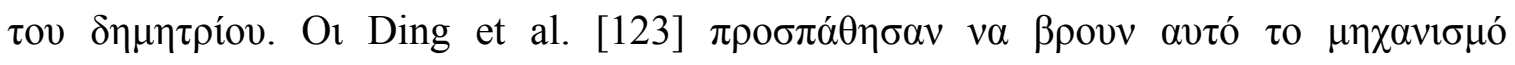

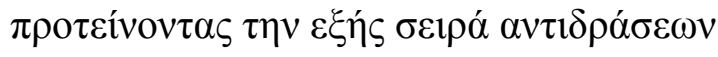

$$
\begin{aligned}
& 2 \mathrm{MnO}_{2} \rightarrow \mathrm{Mn}_{2} \mathrm{O}_{3}+\mathrm{O} \\
& \mathrm{Mn}_{2} \mathrm{O}_{3}+2 \mathrm{CeO}_{2} \rightarrow 2 \mathrm{MnO}_{2}+\mathrm{Ce}_{2} \mathrm{O}_{3} \\
& \mathrm{Ce}_{2} \mathrm{O}_{3}+1 / 2 \mathrm{O}_{2} \rightarrow 2 \mathrm{CeO}_{2}
\end{aligned}
$$




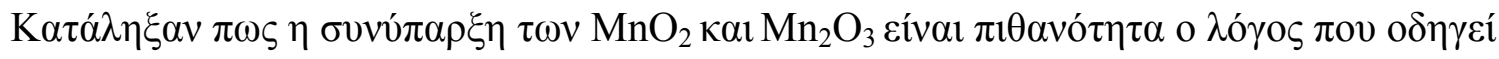

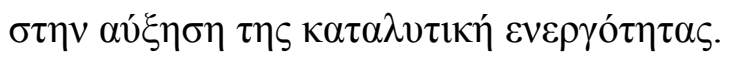

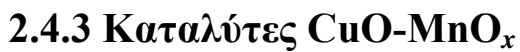

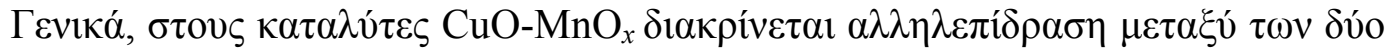

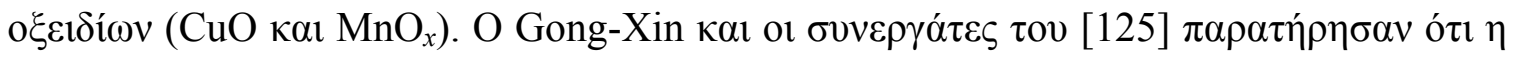

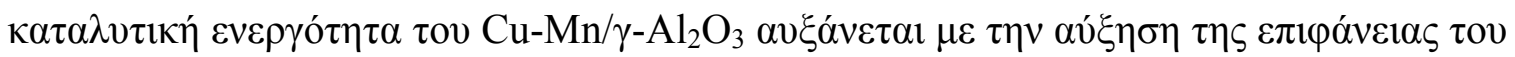

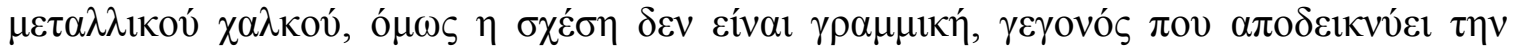

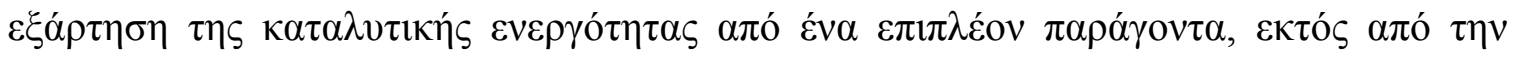

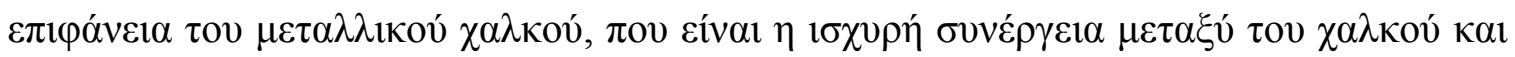

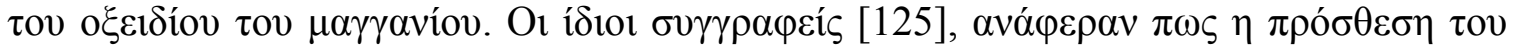

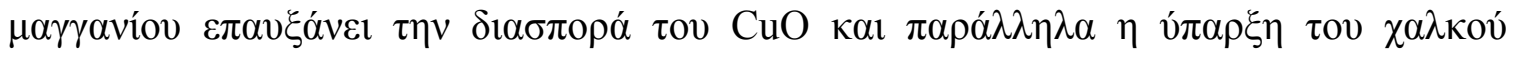

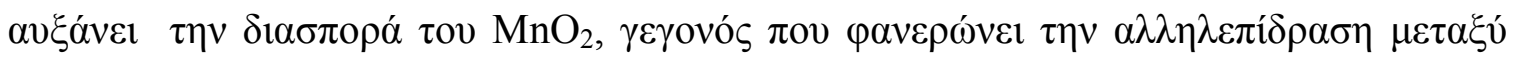

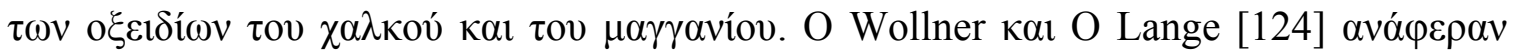

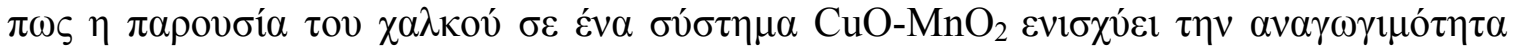

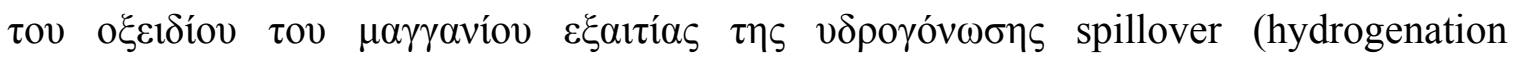

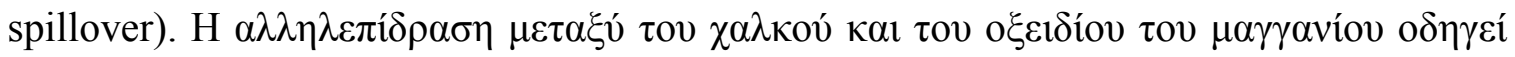

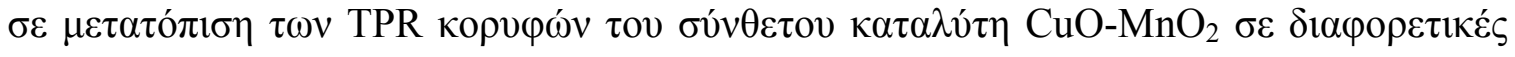
$\theta \varepsilon \rho \mu о \kappa \rho \alpha \sigma i ́ \varepsilon \varsigma$ a

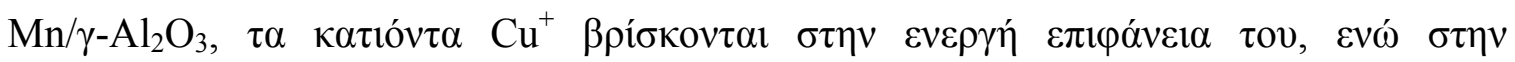

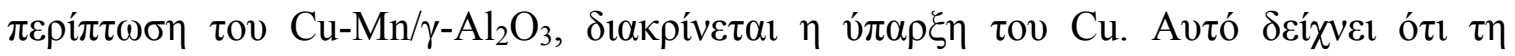

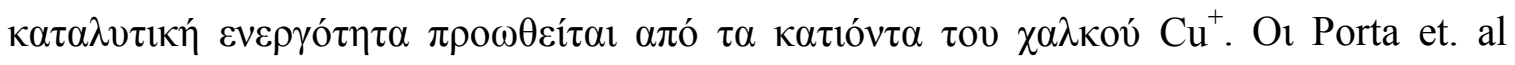

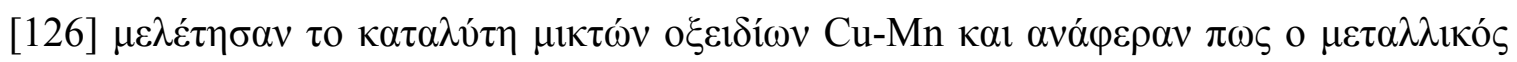

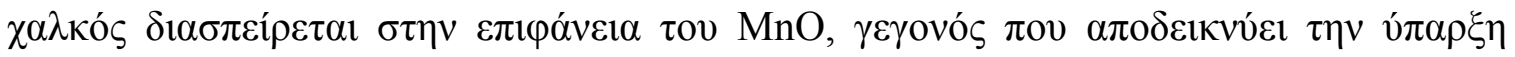

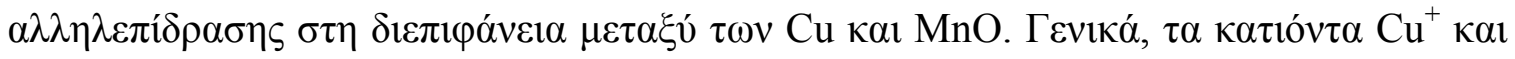

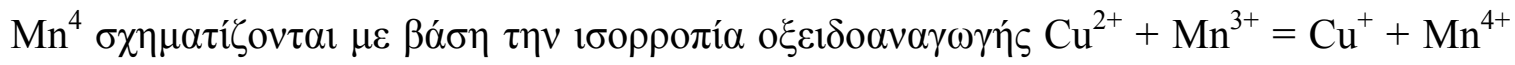

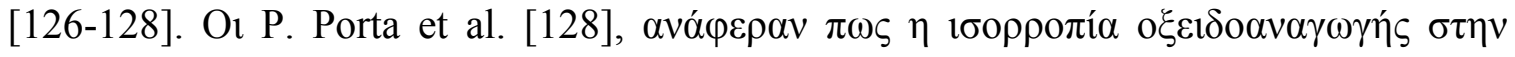

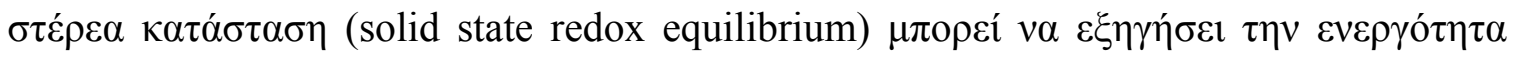

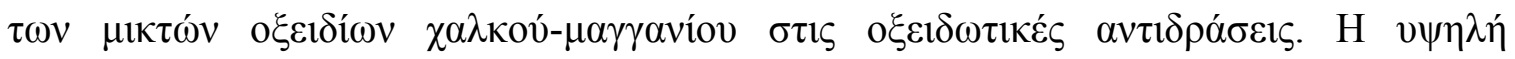

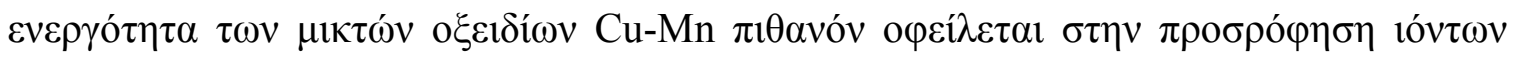

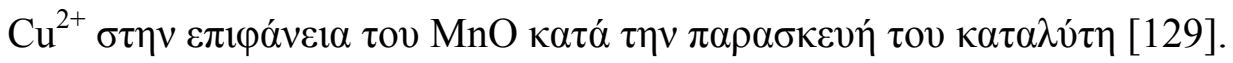




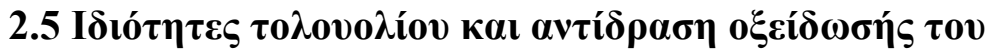

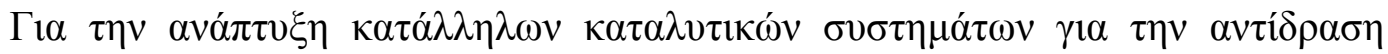

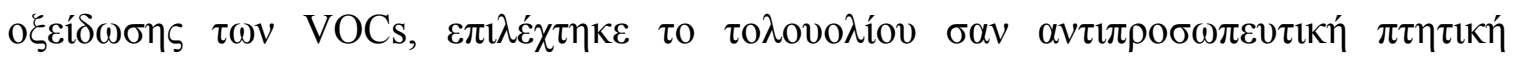

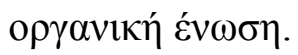

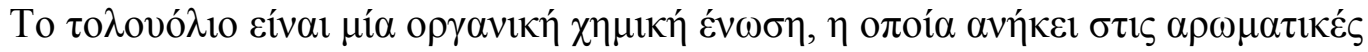

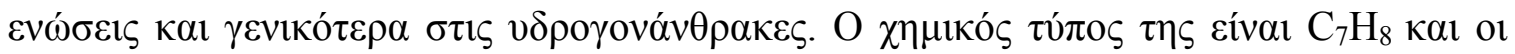

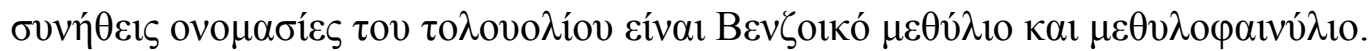

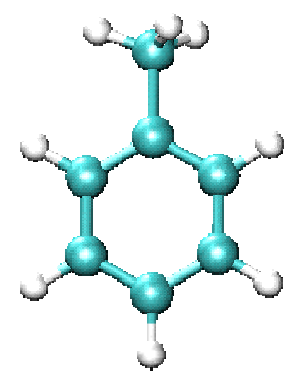

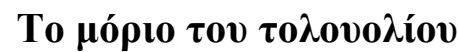

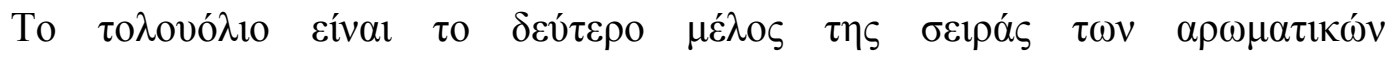

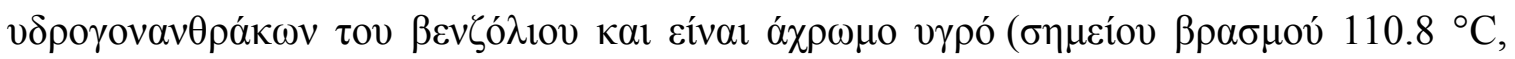

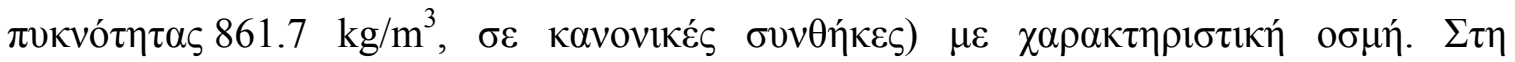

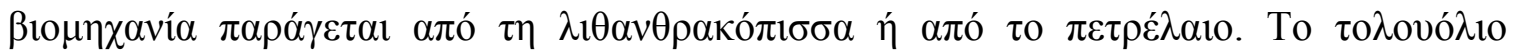

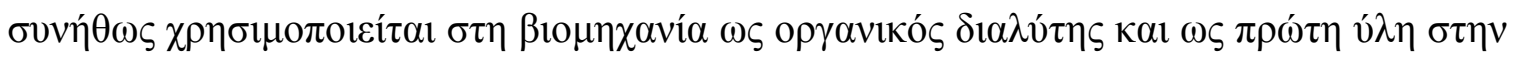

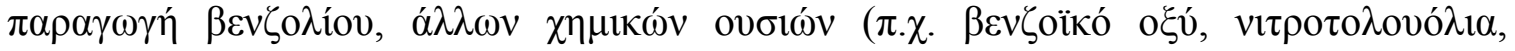

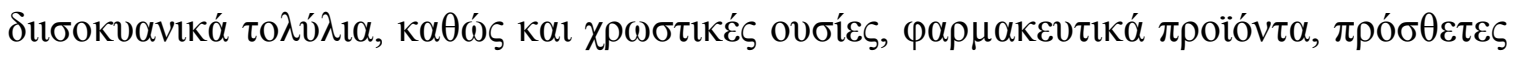

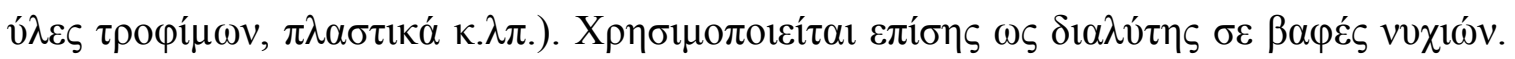

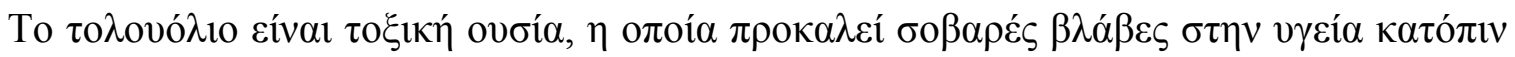

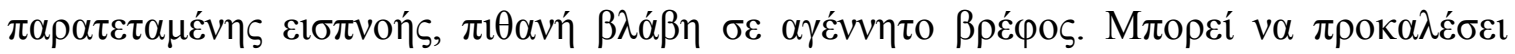

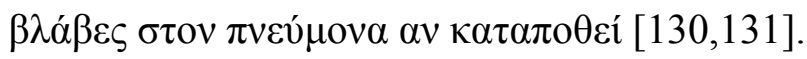

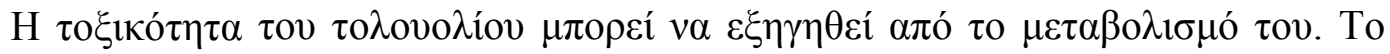

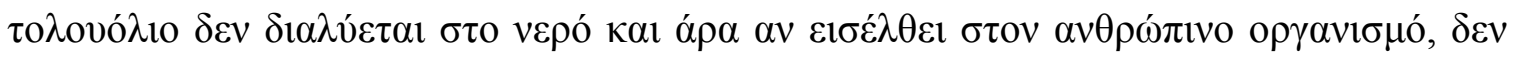

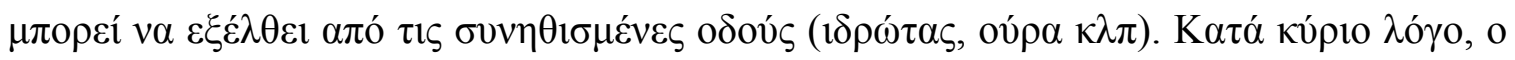

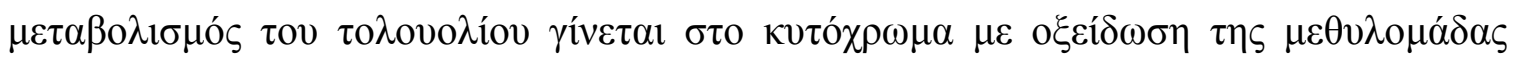

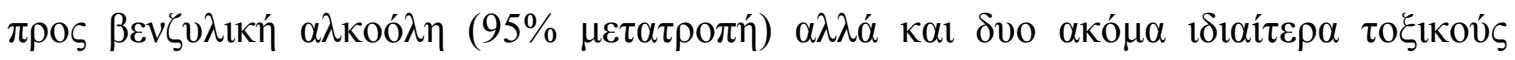




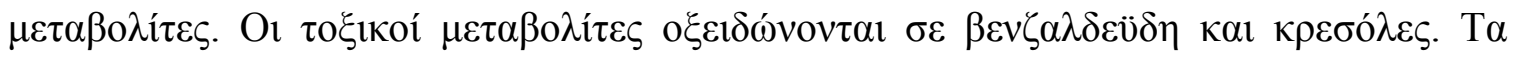

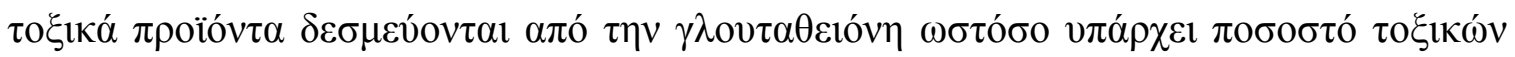

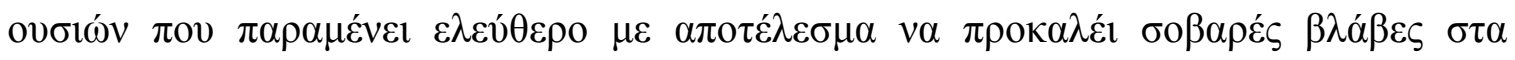

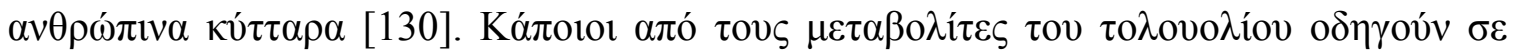

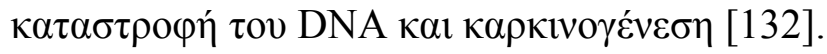

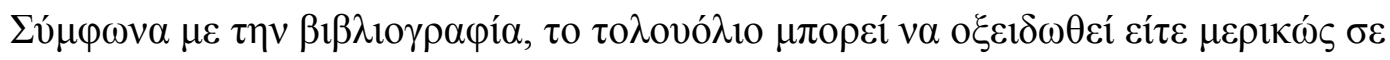

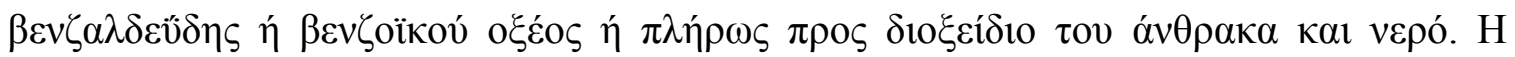

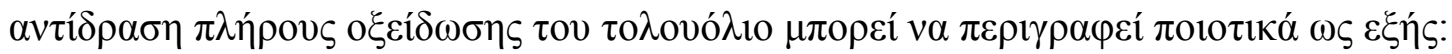

$$
\mathrm{C}_{7} \mathrm{H}_{8}+9.5 \mathrm{O}_{2} \rightarrow 7 \mathrm{CO}_{2}+4 \mathrm{H}_{2} \mathrm{O}
$$

\section{$2.6 \Sigma \kappa о \pi o ́ \varsigma \tau \eta \varsigma ~ \varepsilon \rho \gamma \alpha \sigma i ́ \alpha \varsigma$}

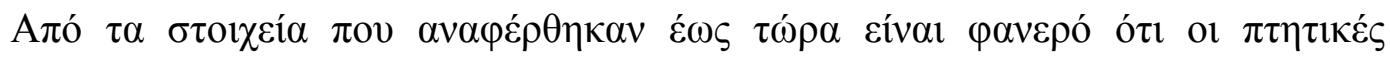

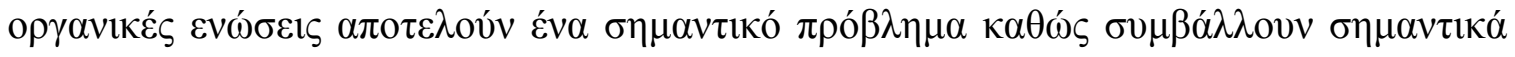

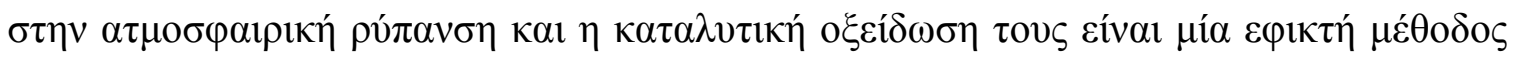

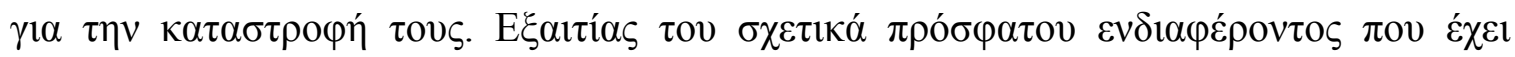

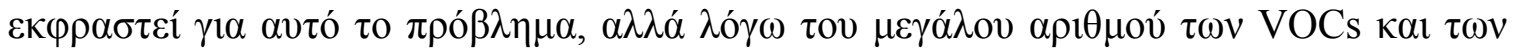

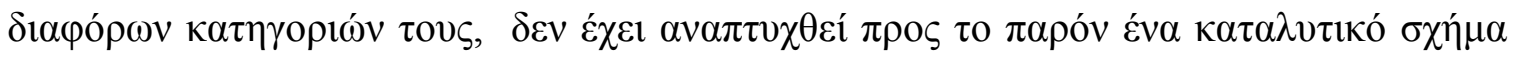

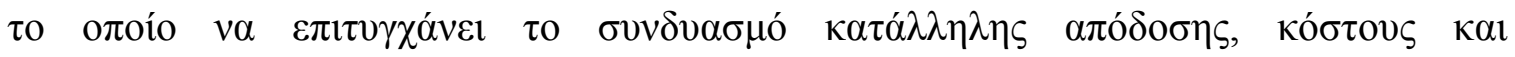

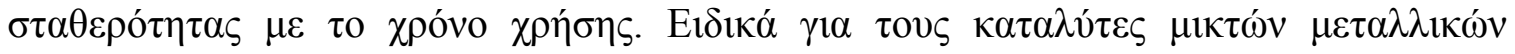

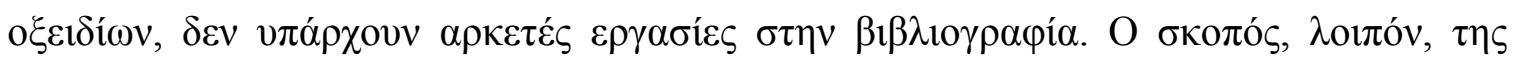

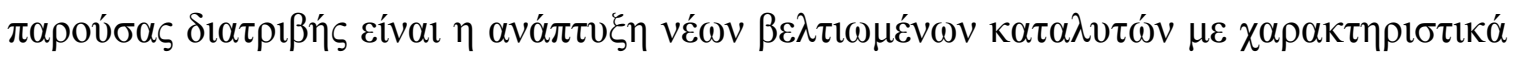

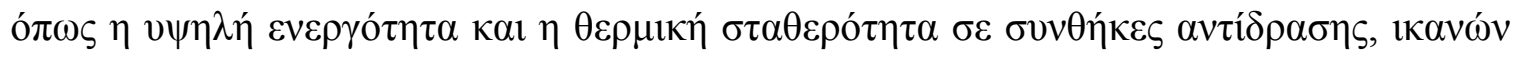

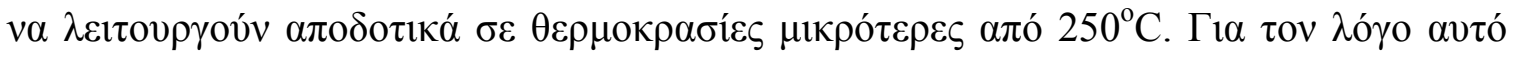

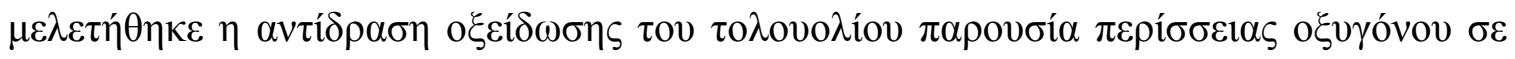

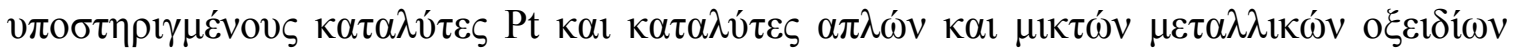

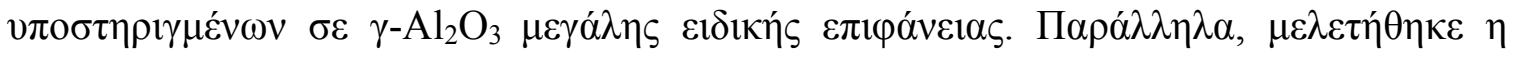

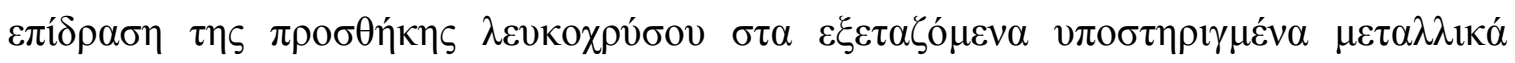

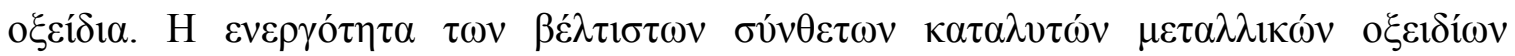
$\left(10 \% \mathrm{CuO}-60 \% \mathrm{MnO}_{2} / \gamma-\mathrm{Al}_{2} \mathrm{O}_{3}, 15 \% \mathrm{CuO}-75 \% \mathrm{CeO}_{2} / \gamma-\mathrm{Al}_{2} \mathrm{O}_{3} \kappa \alpha 130 \% \mathrm{MnO}_{2}-50 \% \mathrm{CeO}_{2} / \gamma-\right.$

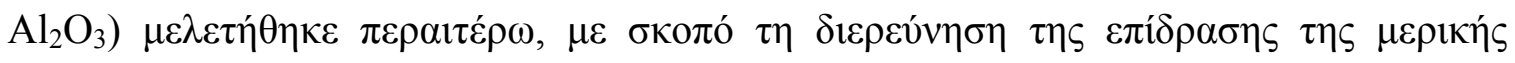

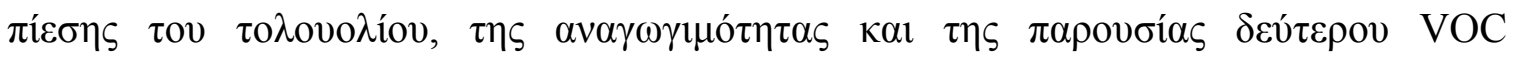




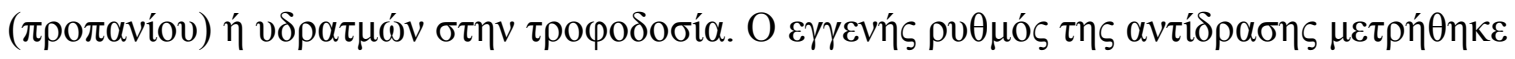

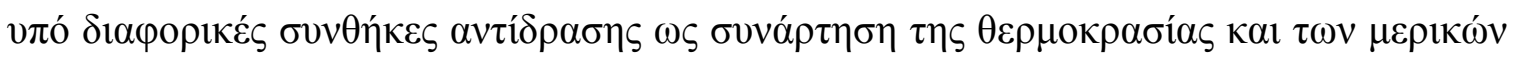

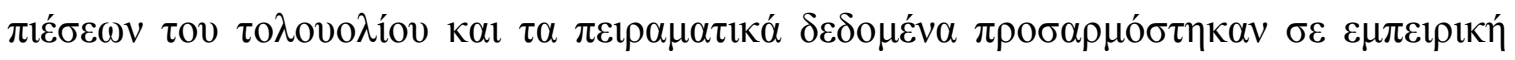

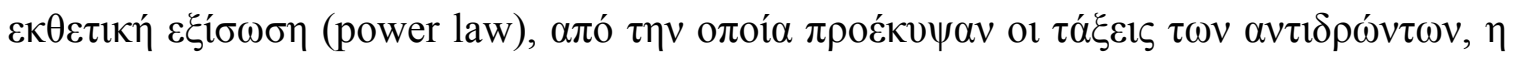

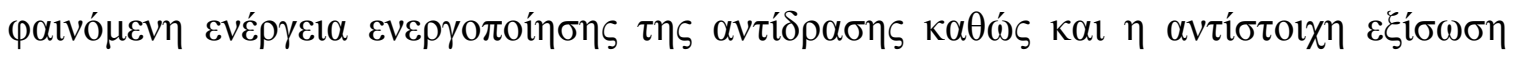

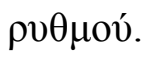

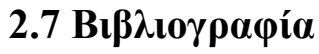

[1] Spivey, J.J., Ind. Eng. Chem. Res., 26 (1987) 2165.

[2] Prasad, R., Kennedy, L.A., Ruckenstein, E., Catal. Rev.-Sci. Eng., 26(1984) 1.

[3] Tichenor, B.A., Palazzolo, M.A., Env. Prog., 6 (Aug. 1987) 172.

[4] P. Papaefthimiou, T. Ioannides, X.E. Verykios, Catal. Today, 54 (1999) 81.

[5] S. Scirè, S. Minicò, C. Crisafulli, C. Satriano, A. Pistone, Appl. Catal. B 40 (2003)43.

[6] T. Takeguchi, S. Aoyama, J. Ueda, R. Kikuchi, K. Eguchi, Top. Catal. 23 (2003) 159.

[7] R. Brayner, D. dos Santos Cunha, F. Bozon-Verduraz, Catal. Today 78 (2003) 419.

[8] T.-K. Tseng, H. Chu, T.-H. Ko, L.-K. Chung, J. Hazard. Mater. B 122 (2005) 155.

[9] A. Janbey, W. Clark, E. Noordally, S. Grimes, S. Tahir, Chemosphere 52 (2003) 1041.

[10] J. Tsou, L. Pinard, P. Magnoux, J.L. Figueiredo, M. Guisnet, Appl. Catal. B 46 (2003) 371 .

[11] C. Zhang, H. He, K. Tanaka, Catal. Commun. 6 (2005) 211.

[12] J. Carpentier, J.F. Lamonier, S. Siffert, E.A. Zhilinskaya, A. Aboukaïs, Appl. Catal. A 234 (2002) 91.

[13] M. Paulis, H. Peyrard, M. Montes, J. Catal. 199 (2001) 30.

[14] J.C.S. Wu, T.-Y. Chang, Catal. Today 44 (1998) 111.

[15] Changbin Zhang, Hong He, Ken-ichi Tanaka, Catal. Commun. 6 (2005) 211. 
[16] Son- Ki Ihm, Young-Du Jun, Dae-Chul Kim, Kwang-Eun Jeong Catalysis Today 93-95 (2004) 149.

[17] Hyoung Sik Kim, Tae Won Kim, Hyoung Lim Koh, Seo Ho Lee, Byoung Ryul Min, Appl. Catal A: General 280 (2005) 125.

[18] Golodets, G.I., “ Heterogeneous Catalytic Reaction Involving Molecular Oxygen', Elsevier, New York, 1983.

[19] Skoglundh, M., Lowendahl, L.O., Ottersted, J. Appl. Catal., 77 (1991) 9.

[20] S. Arnone, G. Busca, L. Lisi, F. Milella, G. Russo, M. Turco, Proceedings of the 22th International Symposium on Combustion, The Combustion Institute, Pittsburgh, 1998, p. 2293.

[21] Evaldsson, L., Lowendahl, L., Ottersted, J.E., Appl. Catal., 48 (1977) 123.

[22] Lowendahl, L.O., Ottersted, J.E. Appl. Catal., 59 (1990) 89.

[23] koglundh, M., Lowendahl, L.O., Menon, et. al. Catal. Lett., 13 (1992) 27.

[24] Hegedus, L.L.,Summers, J.C., J. Catal. 48 (1977) 345.

[25] N. Burgos, M. Paulis, M.M. Antxustegi, M. Montes, Appl. Catal. B 38 (2002) 251.

[26] T.F. Garetto, C.R. Apesteguia, Appl. Catal. B: Environ. 32 (2001) 83.

[27] M.-W. Ryoo, S.-G. Chung, J.-H. Kim, Y.S. Song, G. Seo, Catal. Today 83 (2003) 131.

[28] T. Ataloglou, C. Fountzoula, K. Bourikas, J. Vakros, A. Lycourghiotis, C. Kordulis, Appl. Catal. A: Gen. 288 (2005) 1.

[29] G. Pecchi, P. Reyes, T. López, R. Gómez, J. of Non-Cryst. Sol. 345\&346 (2004) 624

[30] H.L. Tidahy, S. Siffert, J.-F. Lamonier, E.A. Zhilinskaya, A. Aboukaïs, Z.-Y. Yuan, A. Vantomme, B.-L. Su, X. Canet, G. De Weireld, M. Frère, T.B. N'Guyen, J.-M. Giraudon, G. Leclercq, Appl. Catal A: General 310 (2006) 61.

[31] J. Carpentier, J.F. Lamonier, S. Siffert, E.A. Zhilinskaya, A. Aboukaïs, Appl. Catal. A 234 (2002) 91.

[32] M. Paulis, H. Peyrard, M. Montes, J. Catal. 199 (2001) 30.

[33] J.C.-S. Sheng, T.-Y. Chang, Catal. Today 44 (1998) 111.

[34] M.-F. Luo, M. He, Y.-L. Xie, P. Fang, L.-Y. Jin, Appl. Catal. B 69 (2006) 213. 
[35] K. Okumura, T. Kobayashi, H. Tanaka, M. Niwa, Appl. Catal. B 44 (2003) 325.

[36] H.L. Tidahy, S. Siffert, F. Wyrwalski, J.-F. Lamonier, A. Aboukaïs, Catal. Today 119 (2007) 317

[37] McCarty JG, Gusman M, Lowe DM, Hildenbrand DL, Lau KN., Catal Today 47 (1999)5-17.

[38] Gılin P, Primet M., Appl Catal B: Environ. 39 (2002) 1.

[39] M. Koddeini, S. Siffert, H.L. Tidahy, R. Cousin, J.-F. Lamonier, A. Aboukais, A. Vantomme, M. Roussel, B.-L. Su Catalysis Today 122 (2007) 391.

[40] Fierro, J.L.G., de le Banda, J.F.G., Catal. Rev., 28 (1986) 301.

[41] J.M. Gallardo Amores, T. Armaroli, G. Ramis, E. Finocchio, G. Busca, Appl. Catal. B: Environ. 22 (1999) 249.

[42] M.A. Peluso, E. Pronsato, J.E. Sambeth, H.J. Thomas, G. Busca, Appl. Catal. B: Environ. 78 (2008) 73.

[43] E. Finocchio, R.J. Willey, G. Busca, V. Lorenzelli, J. Chem. Soc., Faraday Trans. 93 (1997) 175.

[44] E. Finocchio, M. Baldi, G. Busca, C. Pistarino, G. Romezzano, F. Bregani, G.P. Toledo., Catal. Today 59 (2000) 261.

[45] Lu CY, Wey MY, Chen LI., Appl Catal A: 325 (2007) 163.

[46] Tavares Figueiredo R, Lopez Granados M, Fierro JLG, Vigas L, Ramvrez de la Piscina P, Homs N., Appl Catal A:Gen. 170 (1998) 145.

[47] Moretti E, Storaro L, Talon A, Patrono P, Pinzari F, Montanari T, et al., Appl Catal A: Gen 344 (2008)165.

[48] Han Y, Li W, Zhang M, Tao K., Chemosphere 72 (2008) 53.

[49] J.G. Carriazo, M.A. Centeno, J.A. Odriozola, S. Moreno, R. Molina, Appl. Catal. A: Gen. 317 (2007) 120.

[50] E.M. Cordi, P.J.O’Neill, J.L. Falconer, Appl. Catal. B: Environ. 14(1997) 23.

[51] L. Lamaita, M.A. Peluso, J.E. Sambeth, H.J. Thomas, Appl. Catal. B: Environ. 61(2005) 128.

[52] H. Rajesh, U.S. Ozkan, Ind. Eng. Chem. Res. 32 (1993) 1622.

[53] C. Lahousse, A. Bernier, P. Grange, B. Delmon, P. Papaefthimiou, T. Ioannides, X. Verykios, J. Catal. 178 (1998) 214. 
[54] T.K. Tseng, H. Chu, Sci. Total Environ. 275 (2001) 83.

[55] C.J. Heyes, J.G. Irwin, H.A. Johnson, R.L. Moss, J. Chem. Technol. Biotechnol. $32(1982) 1025$.

[56] T. García, B. Solsona, S. Taylor, Appl. Catal. B 66 (2006) 92.

[57] C. Hettige, K.R.R. Mahanama, D.P. Dissanayake, Chemosphere 43 (2001) 1079.

[58] S.-C. Kim, J. Hazard. Mater. B91 (2002) 285.

[59] R. Prasad, L.A. Kennedy, E. Ruckenstein, Rev.-Sci. Eng. 26 (1984) 1.

[60] M.F.M. Zwinkels, S.G. Jaras, P.G. Menon, T.A. Griffin, Catal. Rev.-Sci.Eng. 35 (1993) 319.

[61] U.S. Ozkan, R.F. Kueller, E. Moctezuma, Ind. Eng. Chem. Res. 29 (1990) 1136.

[62] J. Carno, M. Ferrandon, E. Bjornbom, S. Jaras, Appl. Catal. A: Gen. 155 (1997) 265.

[63] J.M. Gallardo-Amores, T. Armaroli, G. Ramis, E. Finocchio, G. Busca, Appl. Catal. B: Environ. 22 (1999) 249.

[64] G.K. Boreskov, B.I. Popov, V.N. Bibin, E.S. Kozishnikova, Kinet. Katal. 9 (1968) 796.

[65] J.E. Germain, R. Perez, Bull. Soc. Chim. France (1972) 541.

[66] M. Baldi, E. Finocchio, F. Milella, G. Busca, Appl. Catal. B 16 (1998) 43.

[67] A.K. Sinha, K. Suzuki, Appl. Catal. B: Environ. 70 (2007) 417.

[68] Spivey, J.J. and Butt, J.B., Catal. Today, 11 (1992) 465.

[69] H.G. lintz, K. Wittstock, Catal. Today 29 (1996) 457.

[70] J. Papavasiliou, G. Avgouropoulos, T. Ioannides, Catal. Commun. 6 (2005) 497.

[71] P. Bera, K. R. Priolkar, P.R. Sarode, M.S Hegde, S.Emura, R. Kumashiro. N.P. Lalla, Chem. Mater. 14 (2002) 3591

[72] J. Xiaoyuan, Lu Guanglie, Zhou Renxian, Mao Jianxin, Chen Yu, Zheng Xiaoming, Appl. Surf. Sci. 173 (2001) 208

[73] W. Mokwa, D. Kohl, G. Heiland, Anal. Chem. 314 (1983) 315.

[74] M.R. Morales, B.P Barbero, L.E. Cadus, Fuel 87 (2002) 1177.

[75] D. Delimaris, T. Ioannides, Appl. Catal. B 84 (2008) 303.

[76] T. Rao, M. Shen, L. Jia, J. Hao, J. Wang, Catal. Commun. 8 (2007) 1743.

[77] P.O. Larsson, A. Andersson, Appl. Catal. B: Environ. 24 (2000) 175. 
[78] X. Tang, Y. Li, X. Huang, Y. Xu, H. Zhu, J. Wang, W. Shen, Appl. Catal. B: Environ. 62 (2006) 265.

[79] W.B. Li, W.B. Chu, M. Zhuang, J. Hua, Catal. Today 93-95 (2004) 205.

[80] F. Wyrwalski, J.-F. Lamonier, S. Siffert, A. Aboukaïs, Appl. Catal. B 70 (2006) 393.

[81] F. Wyrwalski, J.-F. Lamonier, S. Siffert, L. Gengembre, A. Aboukaïs, Catal. Today 119 (2007) 332.

[82] H. Arai, T. Yamada, K. Eguchi, T. Seiyama, Appl. Catal. B 26 (1986) 265.

[83] D. Klvana, J. Kirchnerova, J. Chaouki, J. Delvar, W. Yaïci, Catal. Today 47 (1999) 115.

[84] R. Burch, P.J.F. Harris, C. Pipe, Appl. Catal. A 210 (2001) 63.

[85] I. Rosso, E. Garrone, F. Geobaldo, B. Onida, G. Saracco, V. Specchia, Appl. Catal. B 34 (2001) 29.

[86] H. Fujii, N. Mizuno, M. Misono, Chem. Lett., (1987) 2147.

[87] S. Irusta, M.P. Pina, M. Menéndez, J. Santamaría, J. Catal. 179 (1998) 400.

[88] V. Choudhary, K. Mantri, Micropor. Mesopor. Mater. 40 (2000) 127.

[89] V. Choudhary, K. Mantri, Micropor. Mesopor. Mater. 46 (2001) 47.

[90] Y.-H. Chu, H.-J. Kim, K.-Y. Song, Y.-G. Shul, K.-T. Jung, K. Lee, M.-H. Han, Catal. Today 74 (2002) 249.

[91] Ch. Subrahmanyam, B. Louis, B. Viswanathan, A. Renken, T.K. Varadarajan, Appl. Catal. A 282 (2005) 67.

[92] V. Boonamnuayvitaya, C. Tayamanon, S. Sae-ung, W. Tanthapanichakoon, Chem. Eng. Sci. 61 (2006) 1686.

[93] A.K. Sinha, K. Suzuki, Appl. Catal. B 70 (2007) 417.

[94] H.L. Tidahy, S. Siffert, J.-F. Lamonier, E.A. Zhilinskaya, A. Aboukaïs, Z.-Y. Yuan, A. Vantomme, B.-L. Su, X. Canet, G. De Weireld, M. Frère, T.B. N'Guyen, J.-M. Giraudon, G. Leclercq, Appl.Catal. A. 310 (2006) 61.

[95] M. Vassileva, A. Andreev, S. Dancheva, N. Kotsev, Appl. Catal., 49 (1989) 125

[96] M.C. Álvarez-Galván, V.A. de la Peña O’Shea, J.L.G. Fierro, P.L. Arias, Catal. Commun. 4 (2003) 223. 
[97] M.C. Álvarez-Galván, B. Pawalec, V.A. de la Peña O’Shea, J.L.G. Fierro, P.L. Arias, Appl. Catal. B 51 (2004) 83.

[98] R.S.G. Ferreira, P.G.P. de Oliveira, F.B. Noronha, Appl. Catal. B 50 (2004) 243.

[99] M.A. Centeno, M. Paulis, M. Montes, J.A. Odriozola, Appl. Catal. A 234 (2002) 65.

[100] S.Scirè, S.Minicò, C.Crisafulli, C. Satriano, A. Pistone, Appl. Catal. B 40 (2003) 43.

[101] Horsley, J. A., «Catalysis for the elimination of Volatile Organic Compounds: Nonhalogenated Compounds», Catalytica Environmental Report No. E4, 1993.

[102] Germain, J.E., “Catalytic Conversion of Hydrocarbons”, Academic, New York, 1969.

[103] Mars, P., Van Krevelen, D.W., special supplement to Chem. Eng. Sci. 1954, 3.

[104] Kon, M. Ya., Schvest, V.A., Kazanskii, V.B., Kinet. Katal. 13 (1972) 635.

[105] Bond, G.C., "Heterogeneous Catalysis: Principles and Applications' Oxford University Press: Oxford, UK, 1974.

[106] Y. Zhang, S. Andersson, M. Muhammed, Appl. Catal. B: Environ. 6 (1995) 325.

[107] P. Fornasiero, G. Balducci, R. Di Monte, J. Kašppar, V. Sergo, G. Gubitosa, A. Ferrero, M. Graziani, J. Catal. 164 (1996) 173.

[108] A.E.C. Palmqvist, E.M. Johansson, S.G. Järås and M. Muhammed, Catal. Lett. 56 (1998) 69.

[109] W. Liu, M. Flytzani-Stephanouplos, J. Catal. 153 (1995) 304.

[110] W. Liu, M. Flytzani-Stephanouplos, J. Catal. 64 (1996) 283.

[111] G.S. Zafiris, R.J. Gorte, J. Catal. 143 (1993) 86.

[112] C. Lamonier, A. Ponchel, A. D’Huysser, L. Jalowiecky-Duhamel, Catal. Today, 50 (1999) 247.

[113] P. Bera, S. Mitra, S. Sampath, M.S Hegde, Chem. Commun. (2001) 927

[114] W. Liu, A.F, Sarofim, M. Flytzani-Stephanouplos, Chem. Eng. Sci. 49 (1995) 4871.

[115] A. Martínez-Arias, J. Soria, R. Cataluña, J.C. Conesa, V. Cortés Corberán, Stud. Surf. Sci. Cat. 116 (1998) 591. 
[116] A. Martínez-Arias, M. Fernández-García, J. Soria, J. C. Conesa, J. Catal. 182 (1999) 367.

[117] M. Machida, M. Uto, D. Kurogi and T. Kijima. Chem. Mater. 12 (2000) 3158

[118] G. Qi, R. T.Yang, R.Chang, Appl. Catal. B: Environ. 51,2, (2004) 93.

[119] B. Murugan, D. Srinivas, C.S. Gopinath, V. Ramaswamy, A.V. Ramaswamy, Chem. Mater. 17 (2005) 3983

[120] H. Chen, A. Sayari, A. adnot and F. Larachi., Appl. Catal. B 32 (2001) 195.

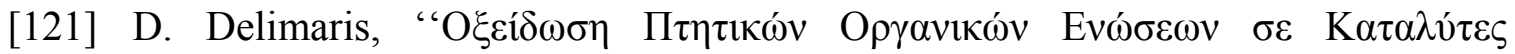

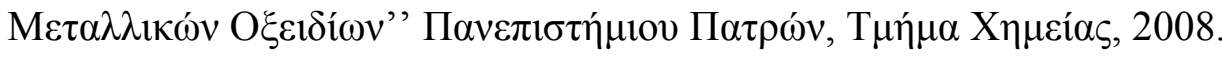

[122] S. Imamura, A. Dol, S. Ishido, Ind. Eng. Chem. Prod. Res. Dev. 24 (1985) 75.

[123] Z.Y. ding, L. Li, D. Wade, E.F. Gloyna, Ind. Eng. Chem. Res. 37 (1998) 1707.

[124] A. Wollner, F. Lange, Appl. Catal. 94 (1993) 181.

[125] Gong-Xin Qi, Xiao-Ming Zheng, Jin-Hua Fei, Zhao-Yin Hou, J.Molec. Catal. A: Chem. 176 (2001) 195.

[126] P. Porta. G. Moretti, M. Musicanti and A. Nardella, Catal. Today, 9 (1991) 211.

[127] J. Papavasiliou, G. Avgouropoulos, T.s Ioannides, J. Catal. 251 (2007) 7

[128] P. Porta. G. Moretti, M. Musicanti and A. Nardella, Sol. Sta. Ion. 63-65 (1993) 257

[129] Sukriti B. Kanungo, J. Catal. 58 (1978) 419

[130] V.S. Nunes-Halldorson, R.L. Steiner, G.B. Smith, Ecotox. and Envir. Safety, 57(2) 2004, 162-167.

[131] M.L. McWilliams, Guang-Di Chen, L.D. Fechter, Tox. and Appl. Pharmac. 167(1) 2000, 18-29.

[132] M. Murata, M.Tsujikawa, S. Kawanishi, Biochem. and Biophys. Res. Commun. 261(2), 1999. 


\section{KEФA $А A I O 3^{\circ}$}

\section{ПЕIPAMATIKO MEPOГ}

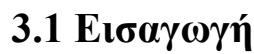

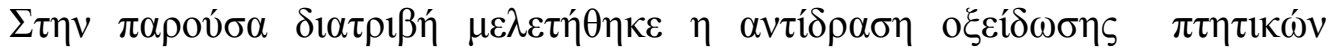

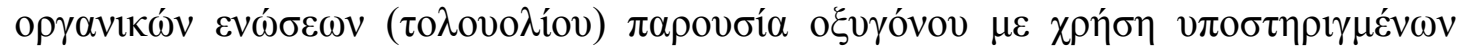

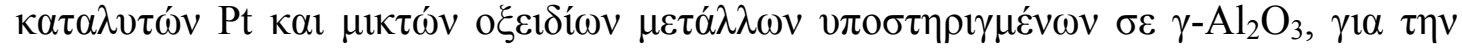

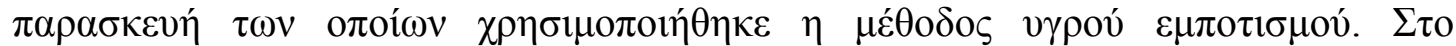

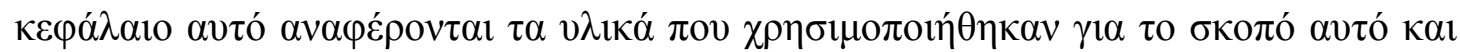

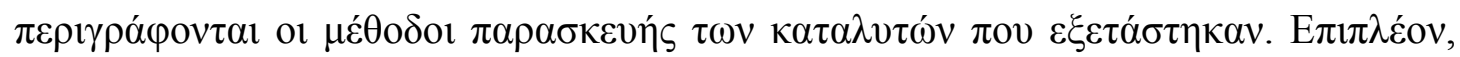

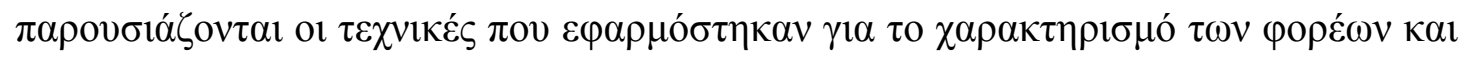

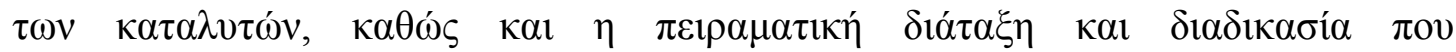

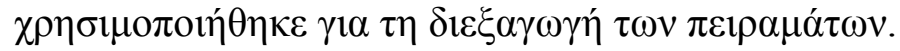




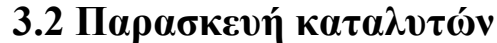

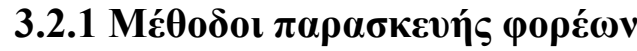

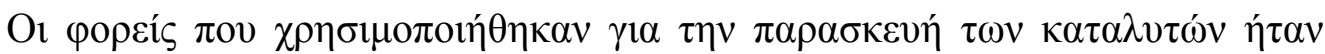

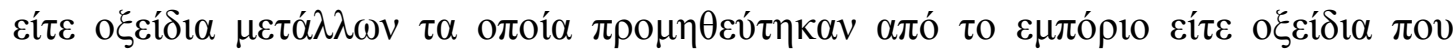

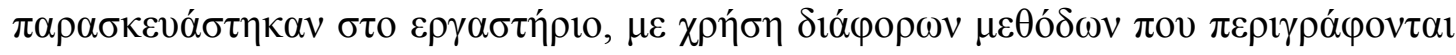

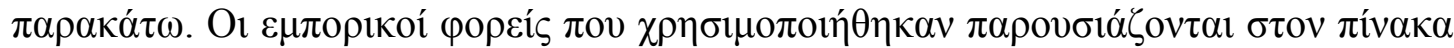
3.1

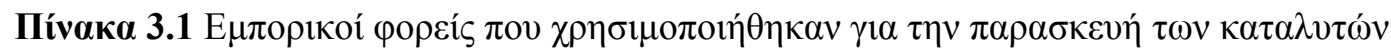

\begin{tabular}{|c|c|}
\hline 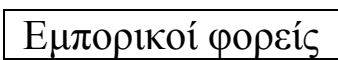 & 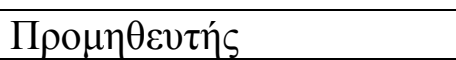 \\
\hline $\mathrm{CeO}_{2}$ & Alfa Products \\
\hline YSZ & Tosoh 8Y-SZ \\
\hline$\gamma-\mathrm{Al}_{2} \mathrm{O}_{3}$ & Alfa Products $\eta$ Engelhard \\
\hline $\mathrm{SiO}_{2}$ & Alfa Products \\
\hline $\mathrm{La}_{2} \mathrm{O}_{3}$ & Alfa Products \\
\hline $\mathrm{ZrO}_{2}$ & Alfa Products \\
\hline $\mathrm{MgO}$ & Alfa Products \\
\hline $\mathrm{MnO}$ & Alfa Products \\
\hline $\mathrm{TiO}_{2}$ & Degussa \\
\hline \multicolumn{2}{|c|}{$* \mathrm{Al}_{2} \mathrm{O}_{3}$ (Alfa Products) : $\mathrm{S}_{\mathrm{BET}}=90 \mathrm{~m}^{2} / \mathrm{g}$} \\
\hline \multicolumn{2}{|c|}{$* * \mathrm{Al}_{2} \mathrm{O}_{3}$ (Engelhard): $\mathrm{S}_{\mathrm{BET}}=190 \mathrm{~m}^{2} / \mathrm{g}$} \\
\hline
\end{tabular}

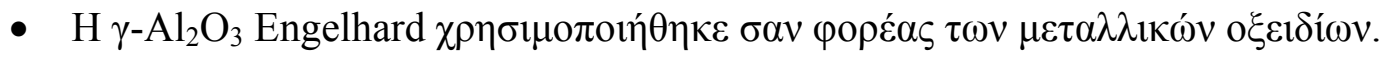

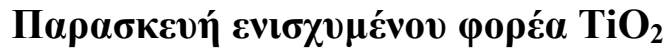

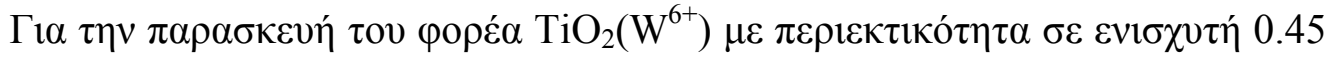

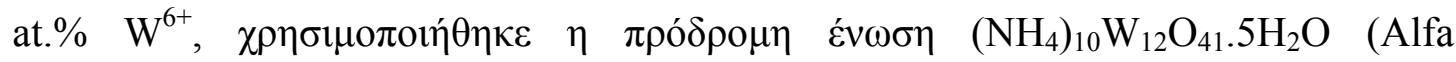

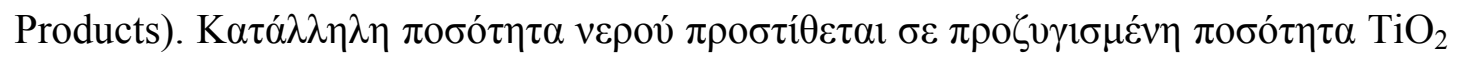

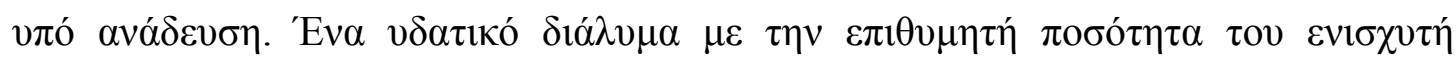

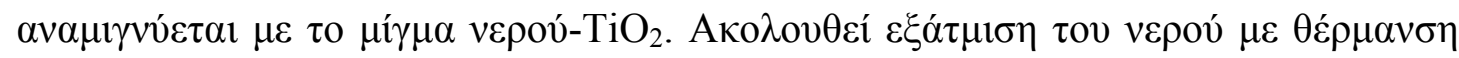

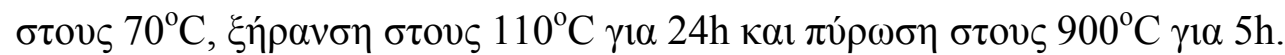

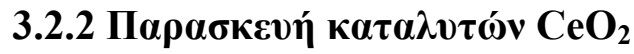

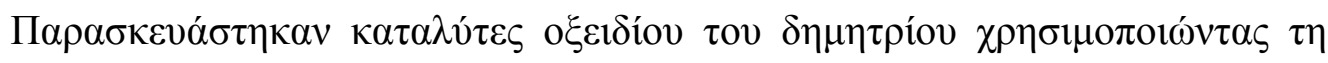

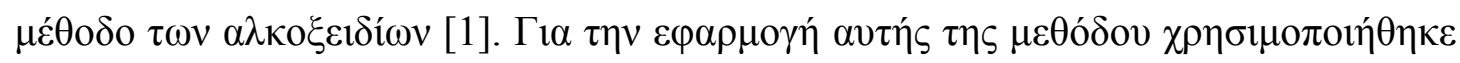




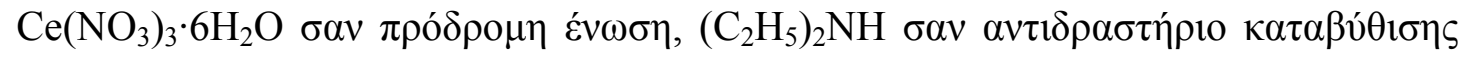

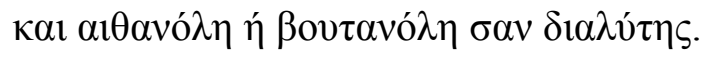

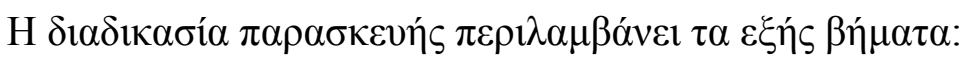

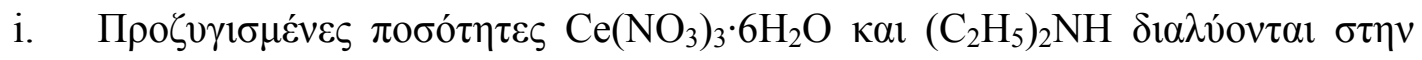

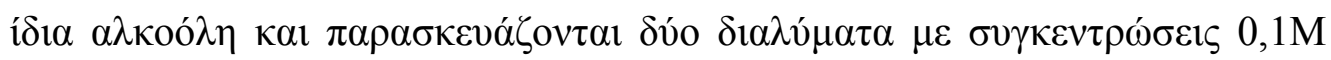

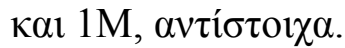

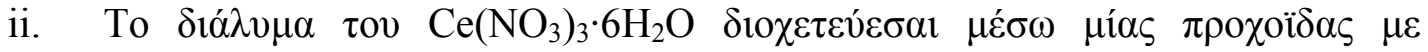

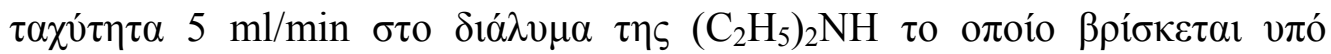
$\mu \varepsilon ́ \tau \rho i \alpha \alpha v \alpha ́ \delta \varepsilon v \sigma \eta$.

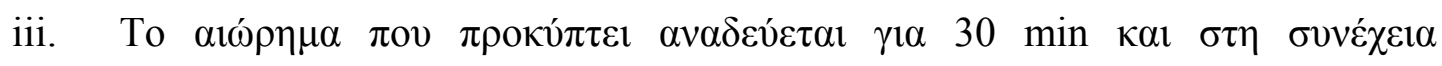
$\varphi \imath \lambda \tau \rho \alpha ́ \rho \varepsilon \tau \alpha \imath$

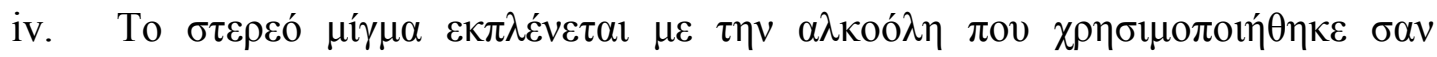

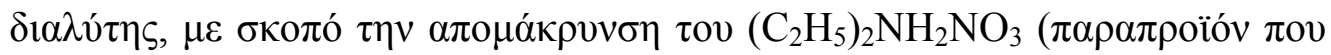

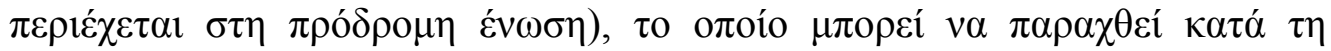

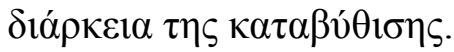

v. छท́

vi. $\pi u ́ \rho \omega \sigma \eta \sigma \tau o v \varsigma ~ 450^{\circ} \mathrm{C} \kappa \alpha 1600^{\circ} \mathrm{C} \gamma 1 \alpha 3 \mathrm{~h}$

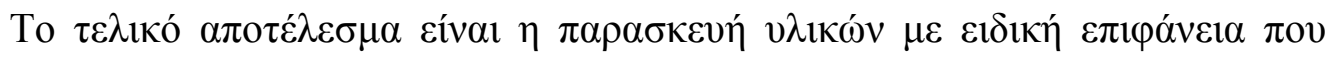

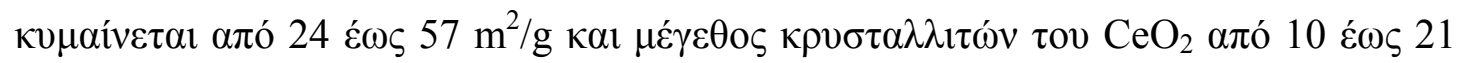
$\mathrm{nm}$.

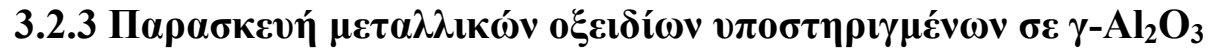

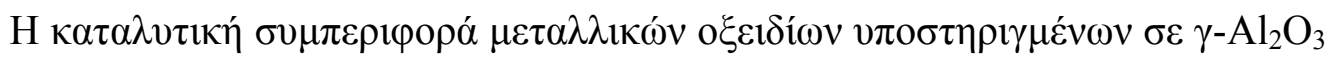

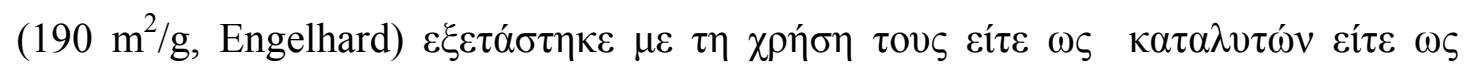

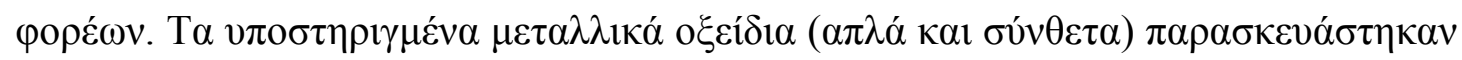

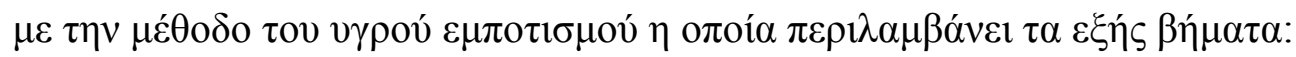

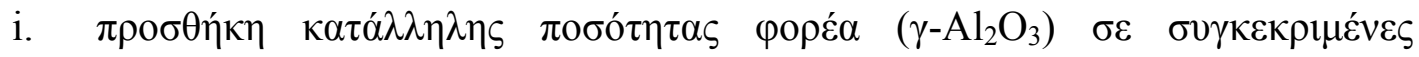

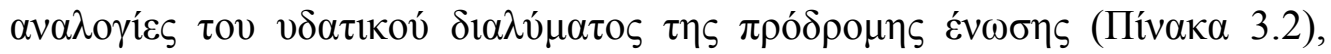

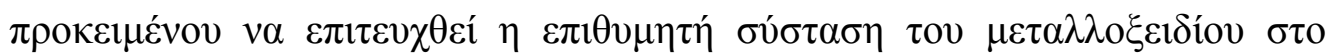

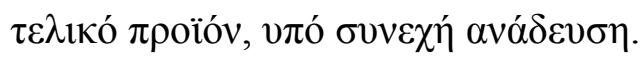

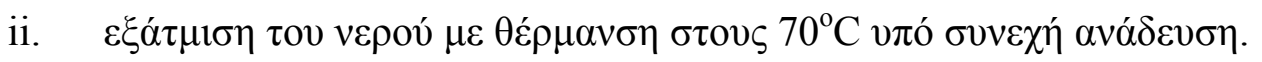

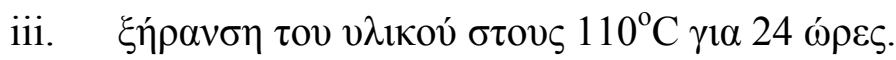

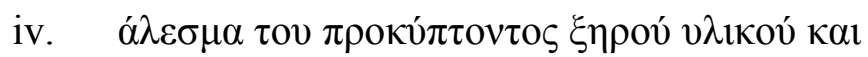

v. $\pi v ́ \rho \omega \sigma \eta \sigma \tau o v \varsigma 450^{\circ} \mathrm{C} \pi \alpha \rho o v \sigma i ́ \alpha \alpha \varepsilon \dot{\rho} \alpha$. 


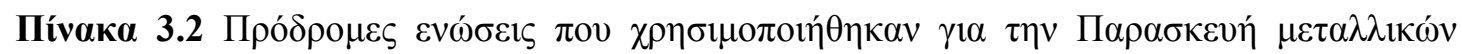
o $\xi \varepsilon 1 \delta i ́ \omega v$

\begin{tabular}{|c|c|c|c|}
\hline 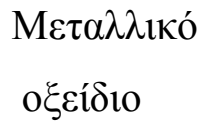 & 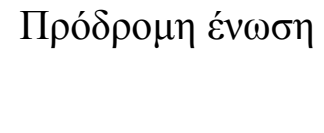 & 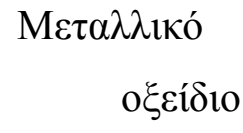 & 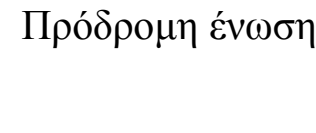 \\
\hline $\mathrm{CuO}$ & $\mathrm{Cu}\left(\mathrm{NO}_{3}\right)_{2} \cdot 3 \mathrm{H}_{2} \mathrm{O}$ & $\mathrm{Cr}_{2} \mathrm{O}_{3}$ & $\mathrm{Cr}\left(\mathrm{NO}_{3}\right)_{3} \cdot 9 \mathrm{H}_{2} \mathrm{O}$ \\
\hline $\mathrm{MnO}_{2}$ & $\mathrm{Mn}\left(\mathrm{NO}_{3}\right)_{2} \cdot \mathrm{xH}_{2} \mathrm{O}$ & $\mathrm{Nd}_{2} \mathrm{O}_{3}$ & $\mathrm{Nd}\left(\mathrm{NO}_{3}\right)_{3} \cdot 6 \mathrm{H}_{2} \mathrm{O}$ \\
\hline $\mathrm{CeO}_{2}$ & $\mathrm{Ce}\left(\mathrm{NO}_{3}\right)_{3} \cdot 6 \mathrm{H}_{2} \mathrm{O}$ & $\mathrm{ZrO}_{2}$ & $\mathrm{ZrO}\left(\mathrm{NO}_{3}\right)_{2} \cdot 6 \mathrm{H}_{2} \mathrm{O}$ \\
\hline $\mathrm{MgO}$ & $\mathrm{MgN}_{2} \mathrm{O}_{6} \cdot 6 \mathrm{H}_{2} \mathrm{O}$ & $\mathrm{CsO}$ & $\mathrm{CsNO}_{3}$ \\
\hline $\mathrm{V}_{2} \mathrm{O}_{5}$ & $\mathrm{NH}_{4} \mathrm{VO}_{3}$ & & \\
\hline
\end{tabular}

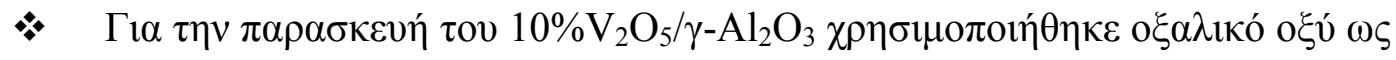

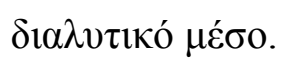

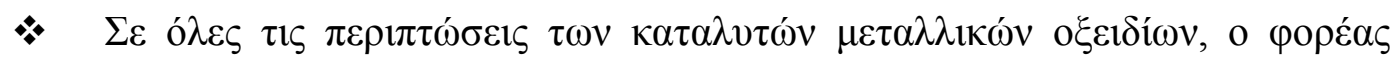
$\eta \dot{\tau} \alpha \nu \gamma-\mathrm{Al}_{2} \mathrm{O}_{3}$ (Engelhard).

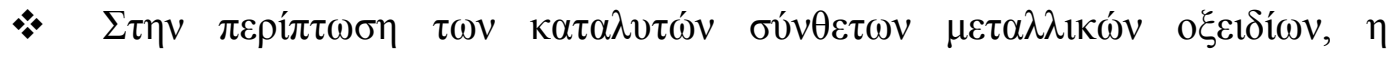

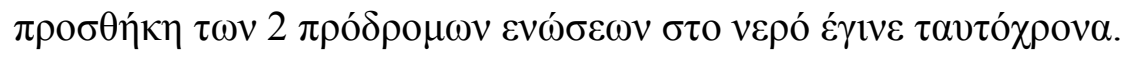

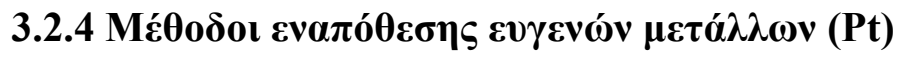

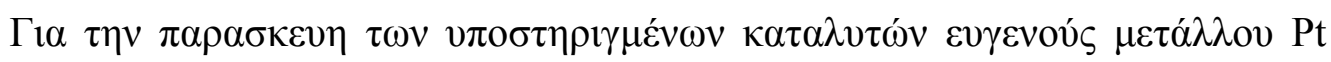

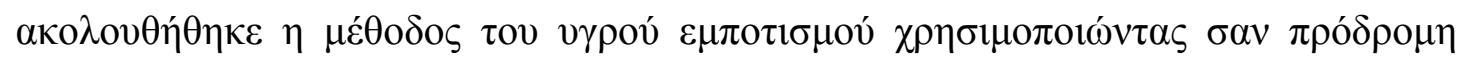

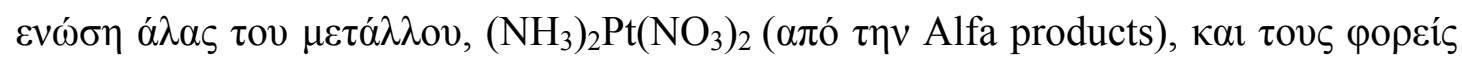

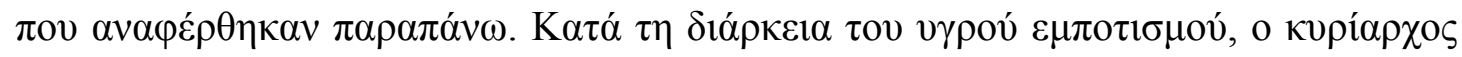

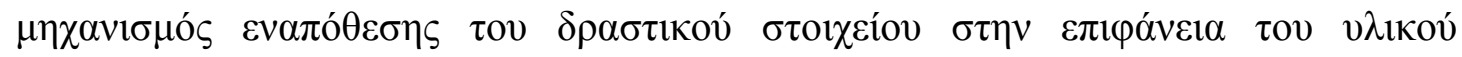

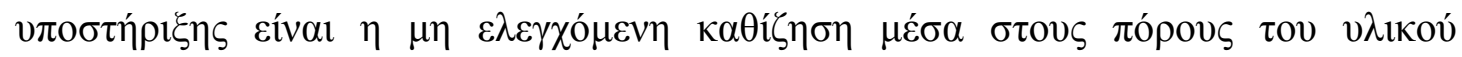

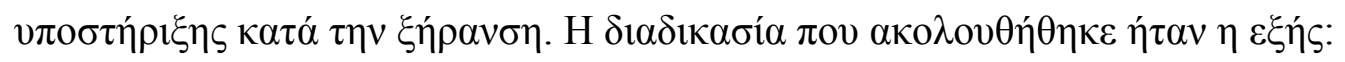

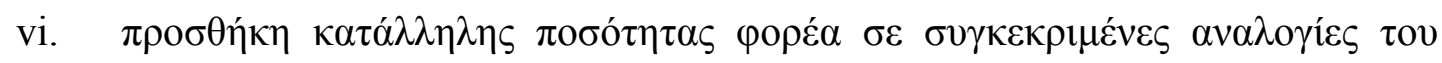

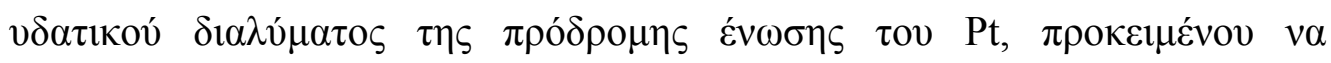

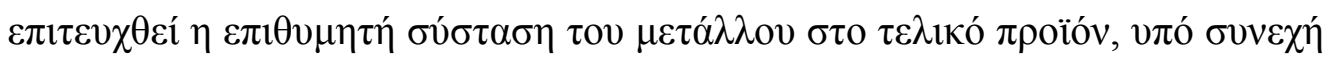
$\alpha v \alpha ́ \delta \varepsilon v \sigma \eta$.

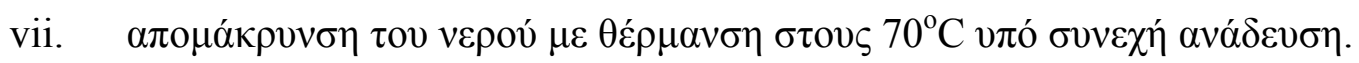

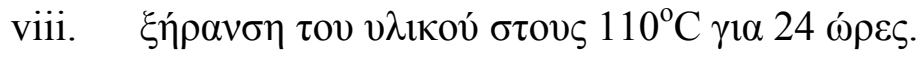

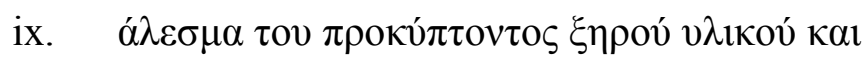

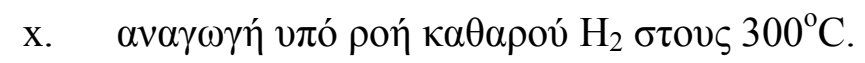




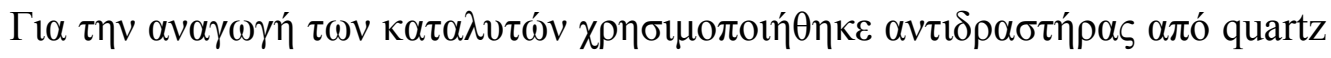

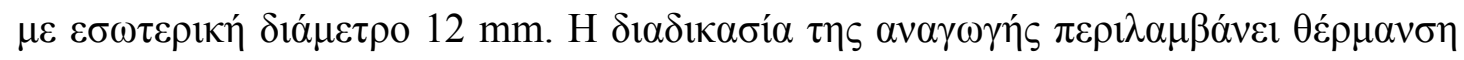

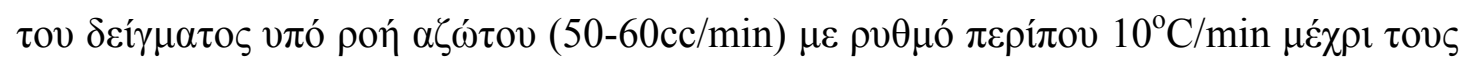

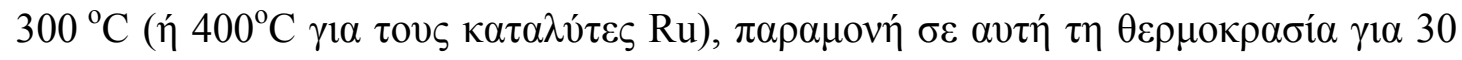

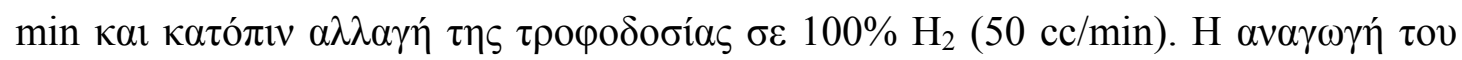

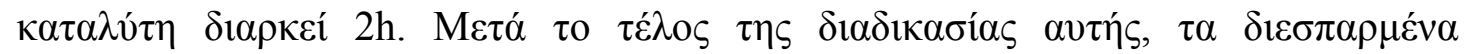

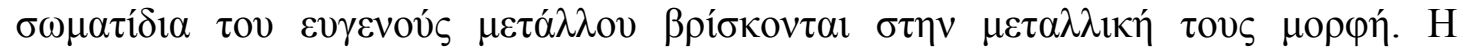

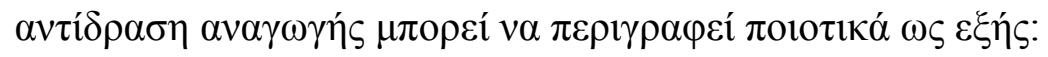

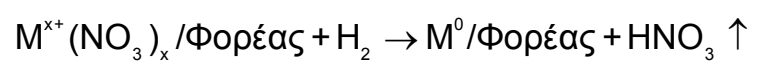

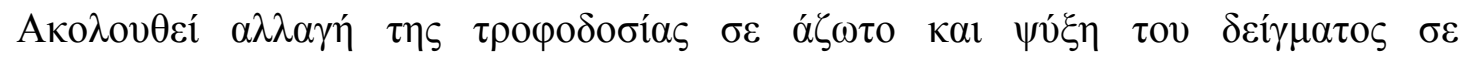
$\theta \varepsilon \rho \mu о \kappa \rho \alpha \sigma i ́ \alpha \delta \omega \mu \alpha \tau i ́ o v$

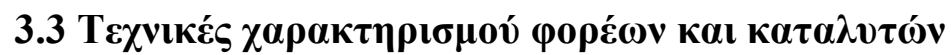

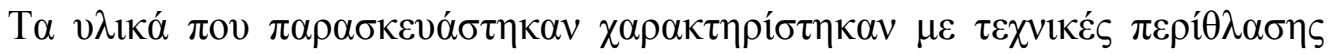

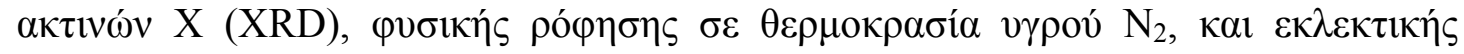

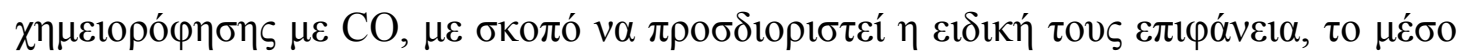

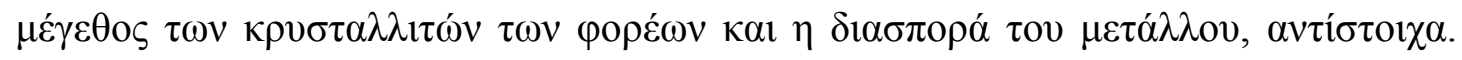

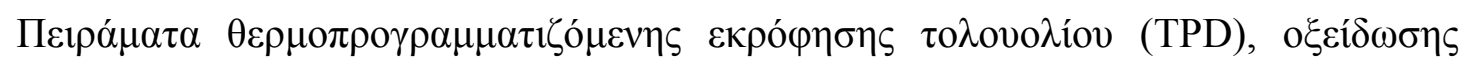

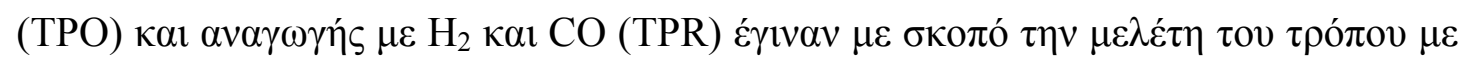

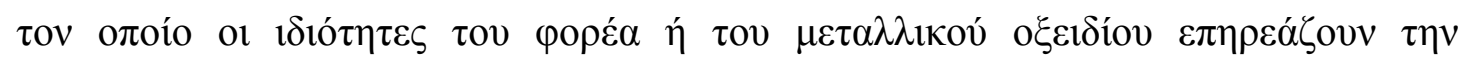
$\alpha v \tau i ́ \delta \rho \alpha \sigma \eta$.

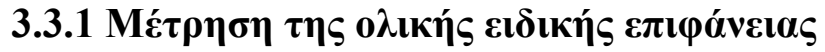

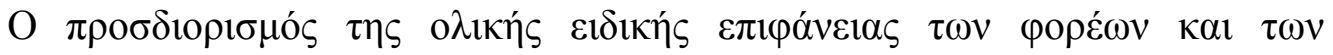

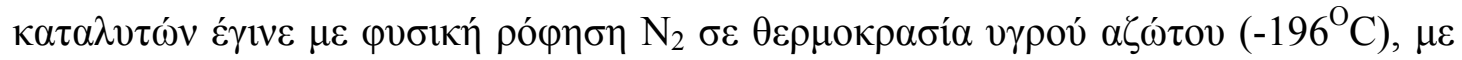

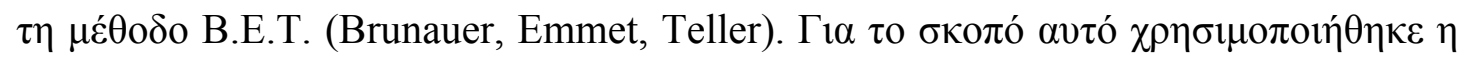

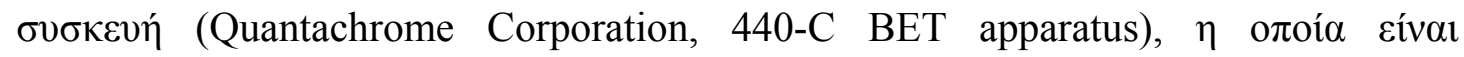

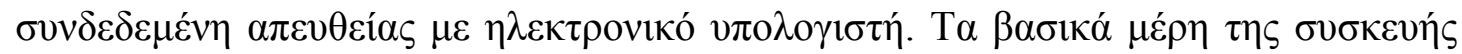

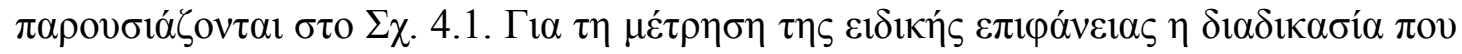

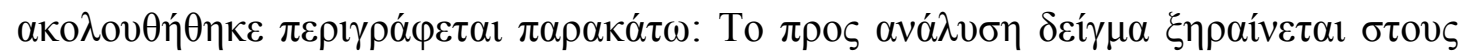

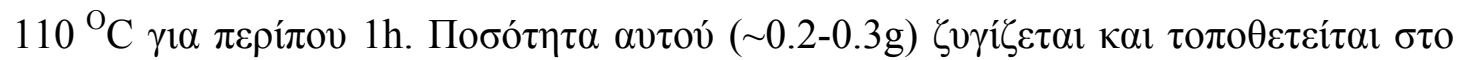

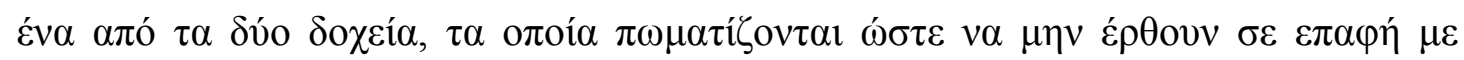

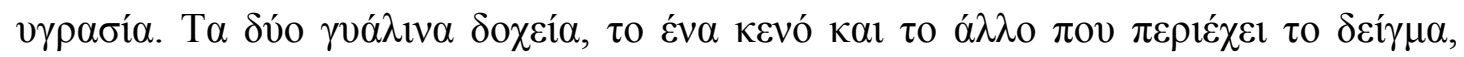




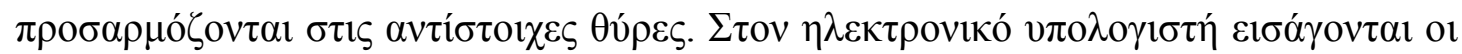

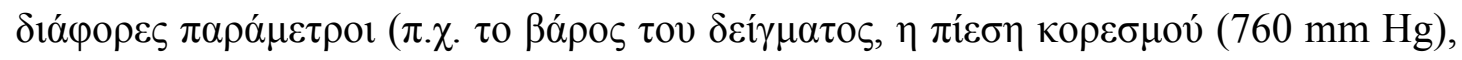

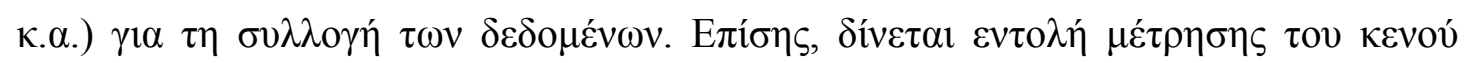

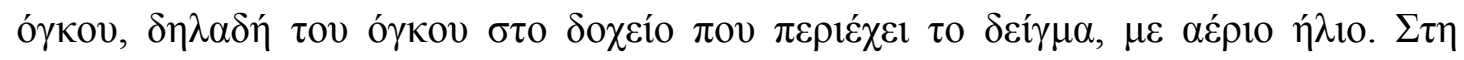

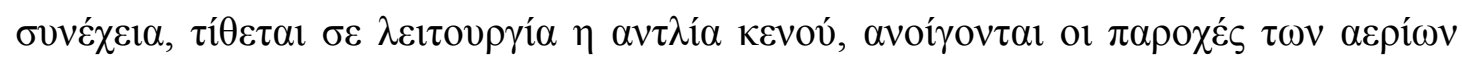

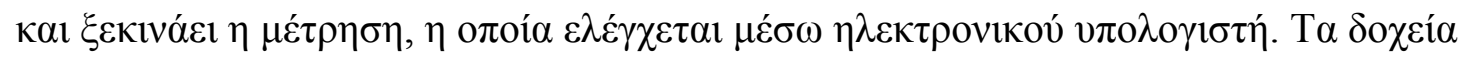

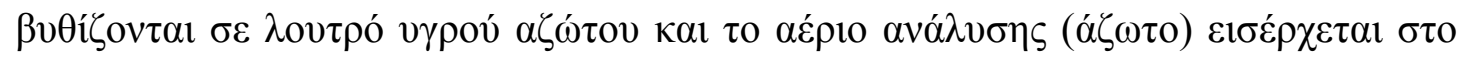

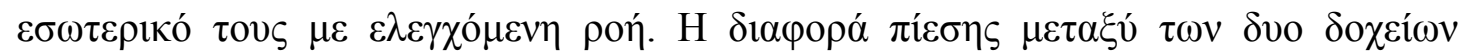

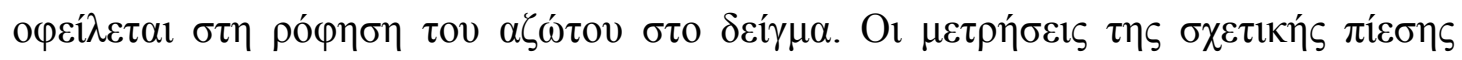

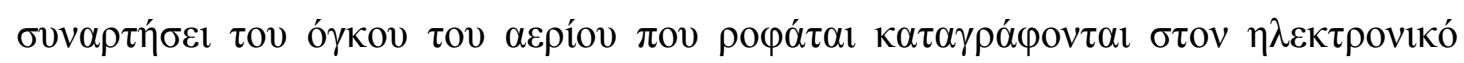

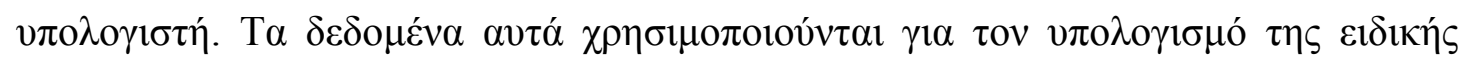

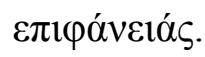

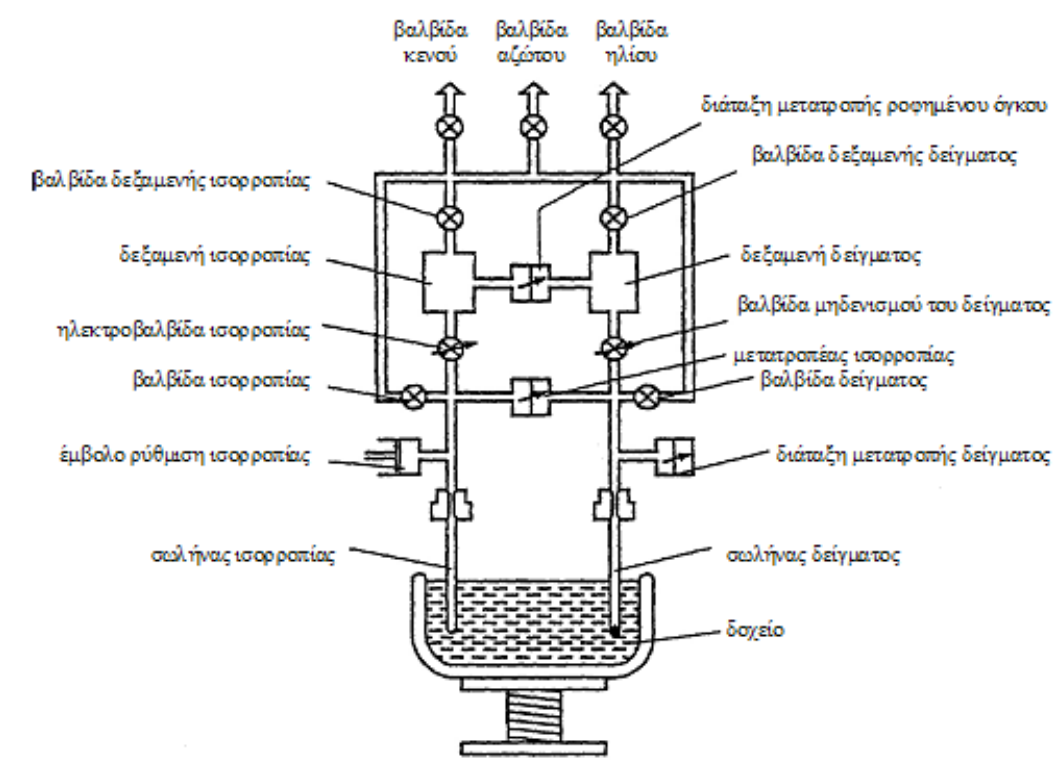

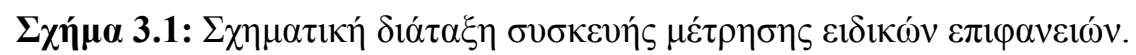

H $\varepsilon \pi \varepsilon \xi \varepsilon \rho \gamma \alpha \sigma i ́ \alpha$ $\tau \omega v$ a

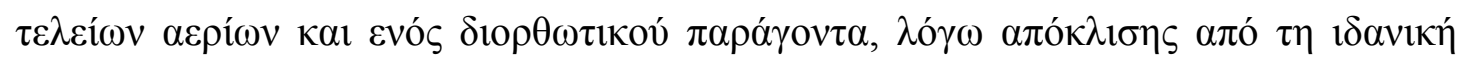

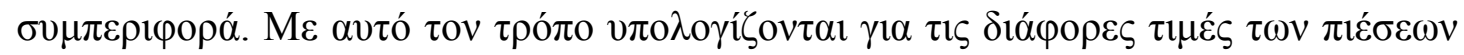

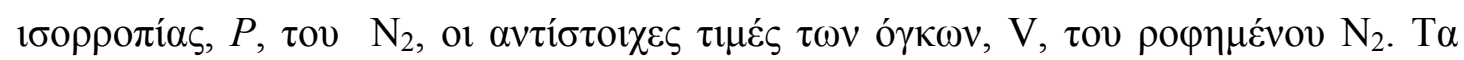

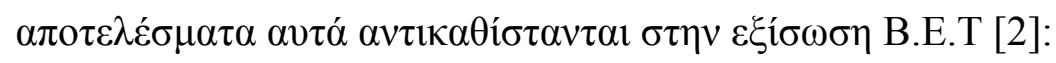




$$
\frac{P}{V \cdot\left(P_{0}-P\right)}=\frac{1}{V_{m} \cdot C}+\frac{(C-1) \cdot P}{V_{m} \cdot C \cdot P_{0}}
$$

ó

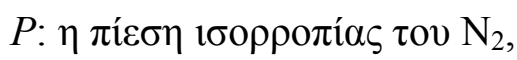

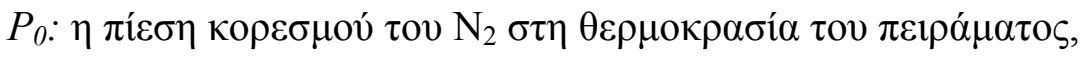

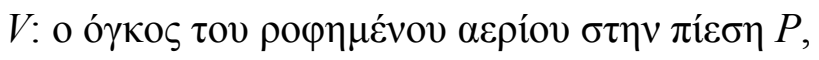

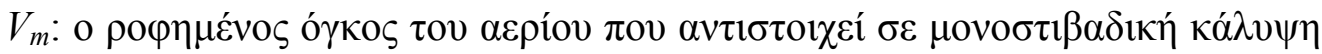

$\kappa \alpha 1$

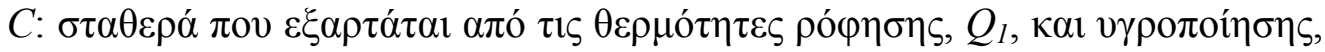

$Q_{2}$, tov acpíov $\left(C=\exp \left(Q_{1^{-}} Q_{2}\right) / R T\right)$.

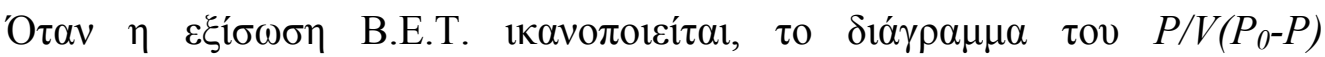

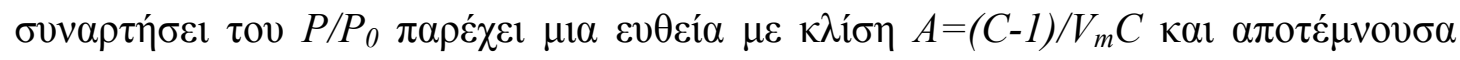

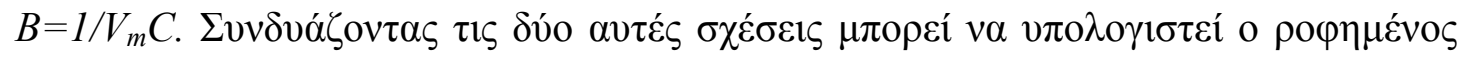
о́ $\gamma \kappa \kappa_{\mathrm{m}}$ :

$$
\mathrm{V}_{\mathrm{m}}=\frac{1}{\mathrm{~A}+\mathrm{B}}\left(\mathrm{cm}^{3} / \mathrm{g}\right)
$$

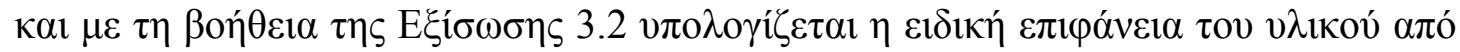
$\tau \eta \sigma \chi \varepsilon \dot{\varepsilon} \sigma:$

$$
\mathrm{S}_{\mathrm{g}}=4.36 \cdot 10^{4} \cdot \mathrm{V}_{\mathrm{m}}\left(\mathrm{cm}^{2} / \mathrm{g}\right)
$$

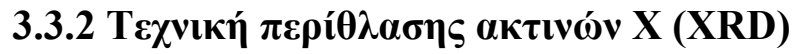

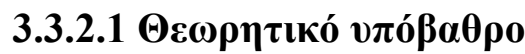

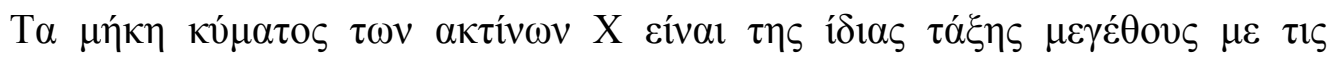

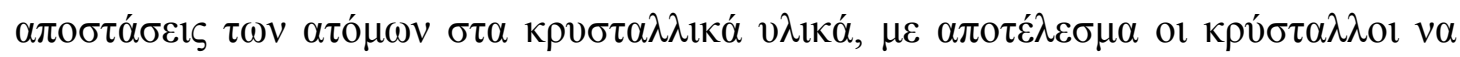

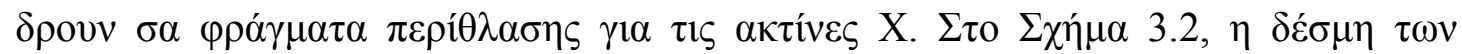

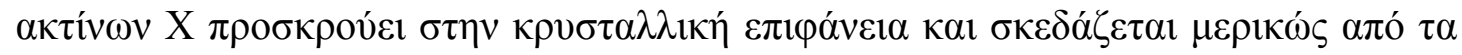

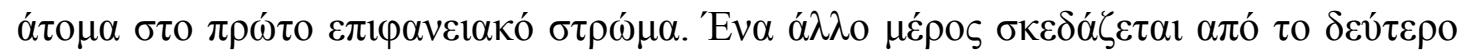

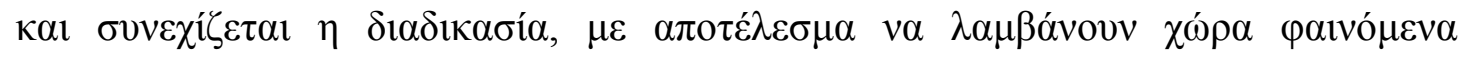

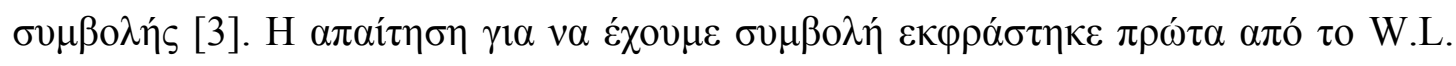
Bragg :

$$
\begin{aligned}
& \mathrm{A} \cdot \mathrm{P}+\mathrm{P} \cdot \mathrm{C}=\mathrm{n} \cdot \lambda \\
& \mathrm{A} \cdot \mathrm{P}=\mathrm{P} \cdot \mathrm{C}=\mathrm{d} \cdot \eta \mu \theta \\
& \mathrm{n} \cdot \lambda=2 \cdot \mathrm{d} \cdot \eta \mu \theta
\end{aligned}
$$




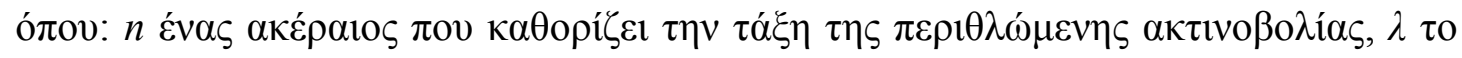

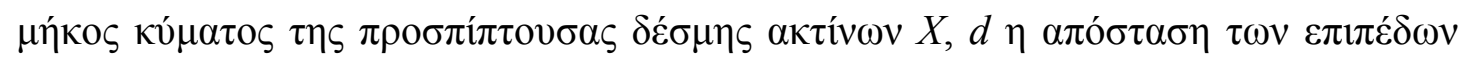

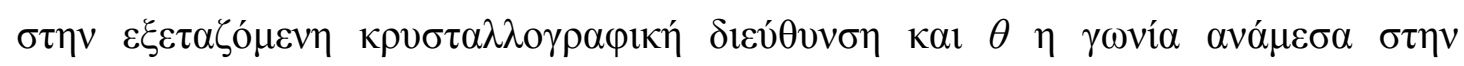

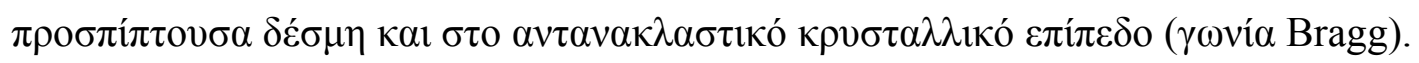

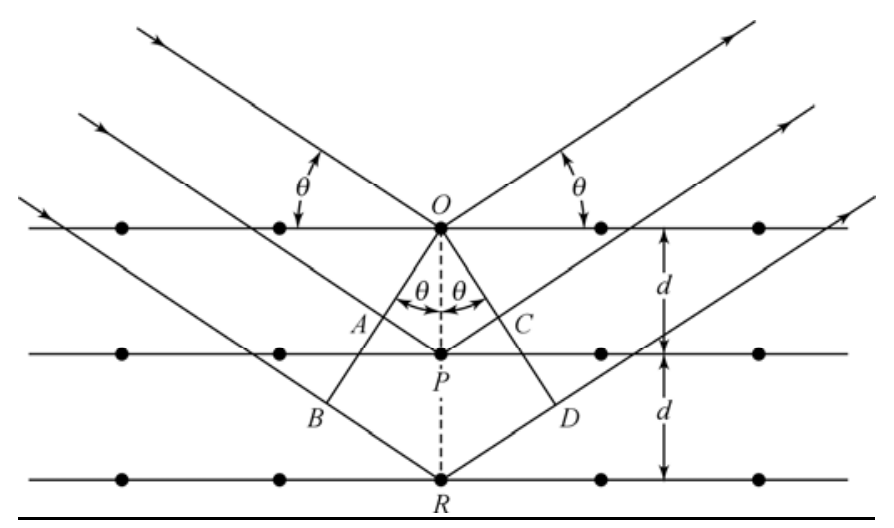

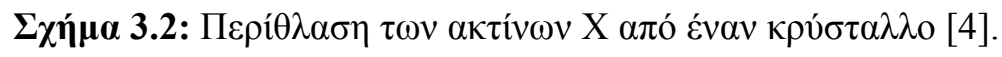

A

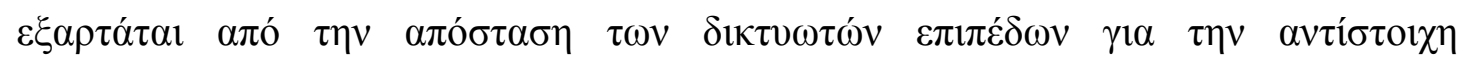

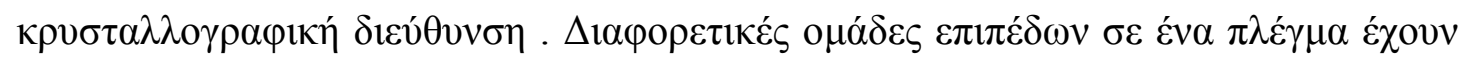

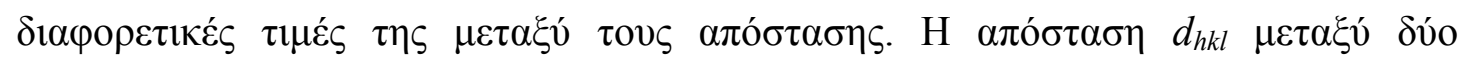

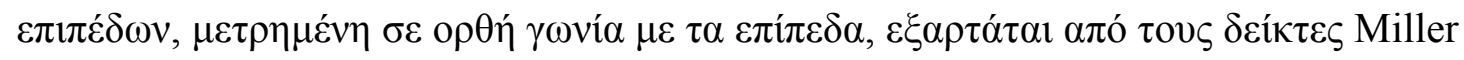

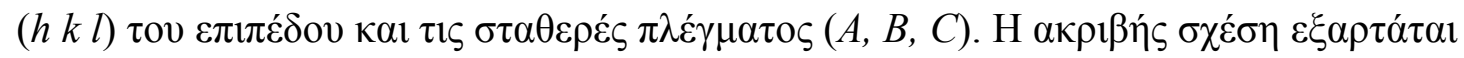

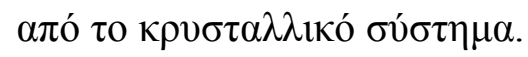

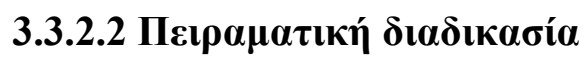

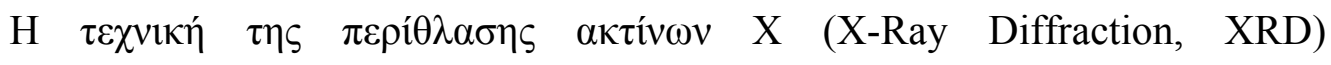

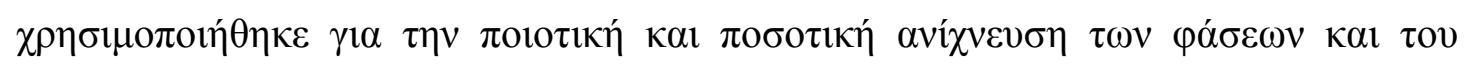

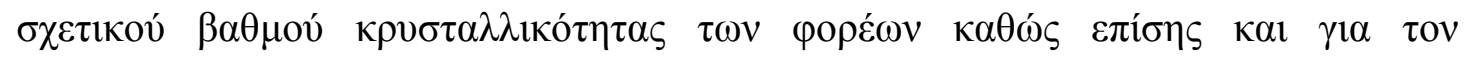

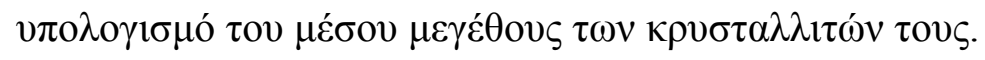

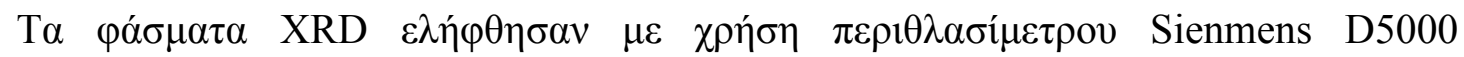

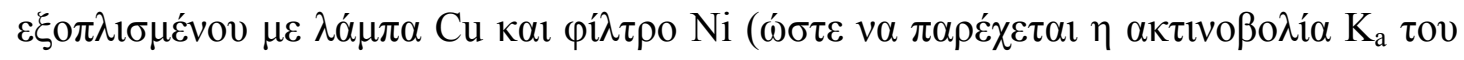

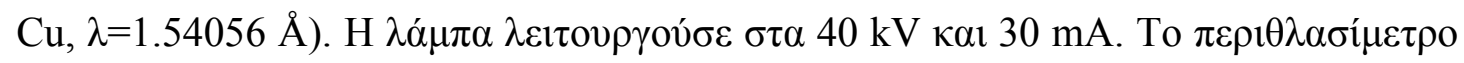

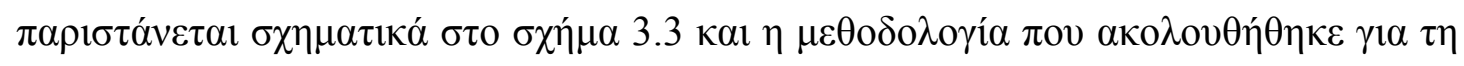

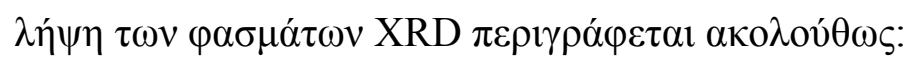




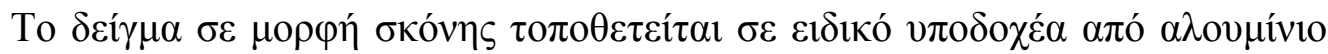

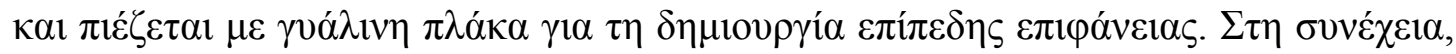

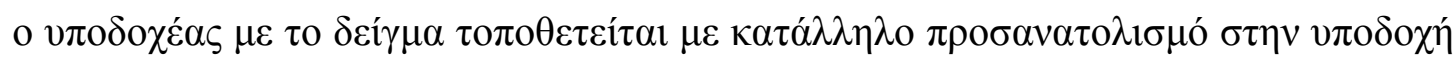

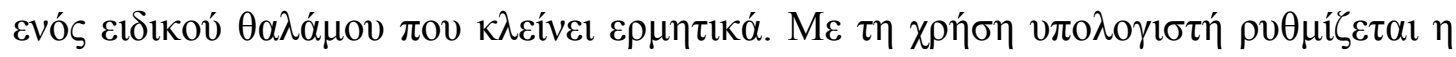

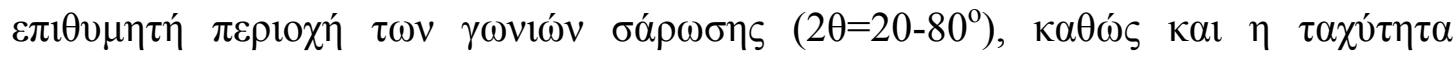

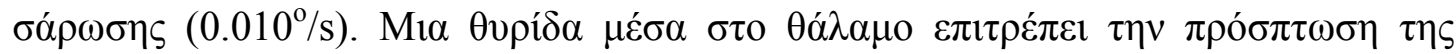

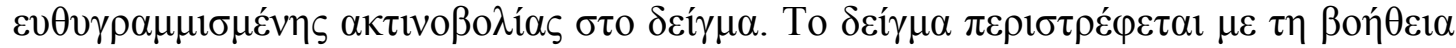

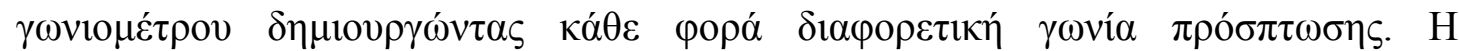

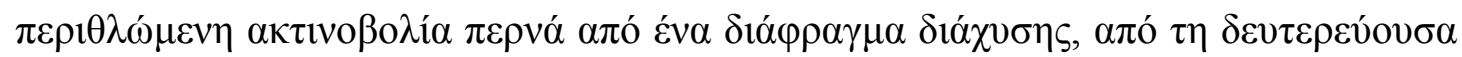

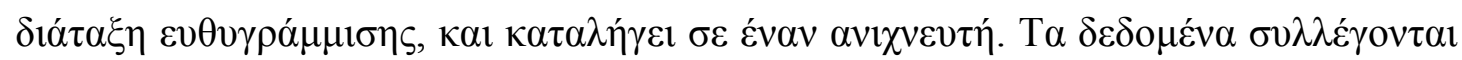

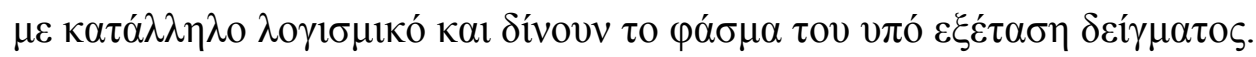

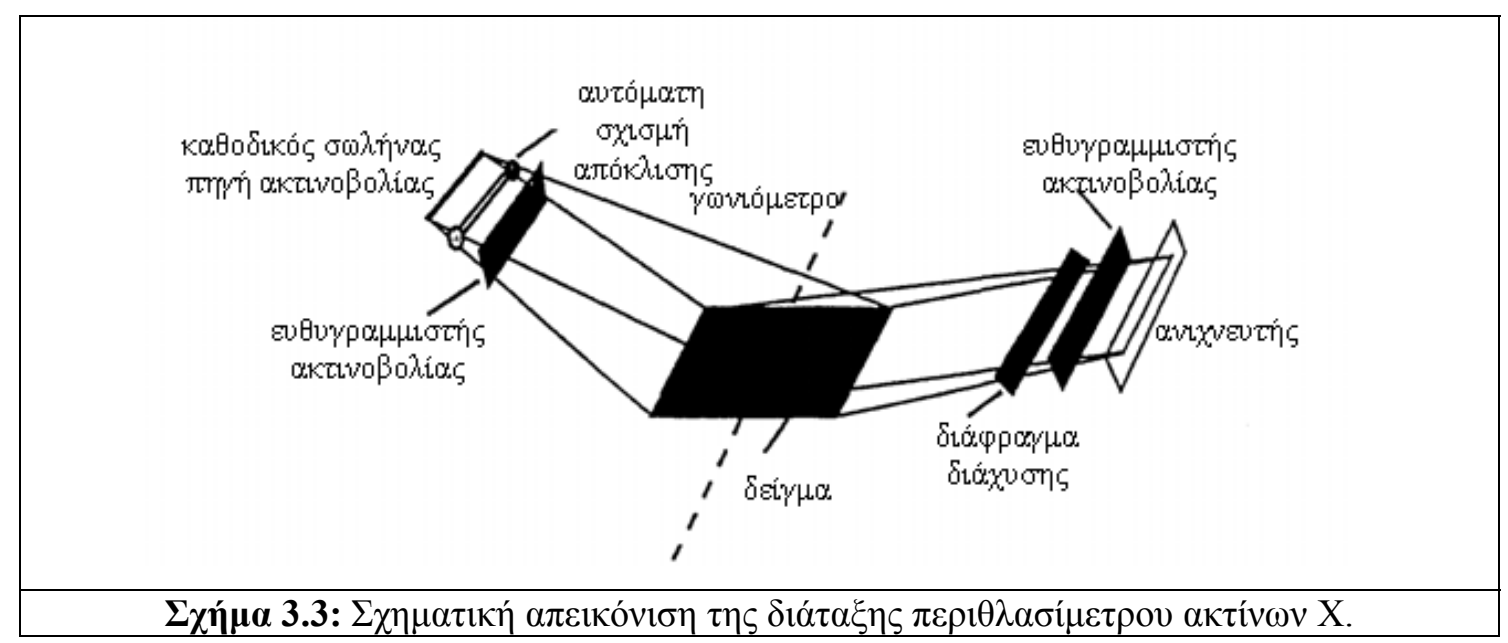

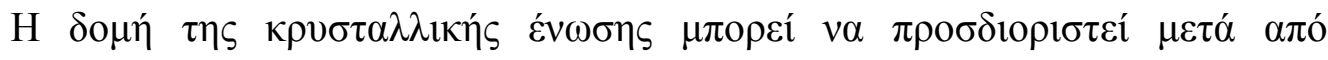

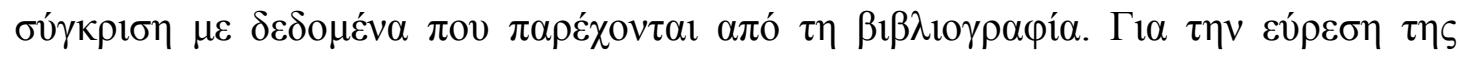

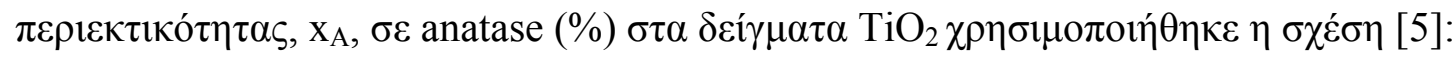

$$
\mathrm{x}_{\mathrm{A}}=\frac{1}{1+1.26 \cdot \frac{\mathrm{I}_{\mathrm{R}}}{\mathrm{I}_{\mathrm{A}}}} \cdot 100 \theta
$$

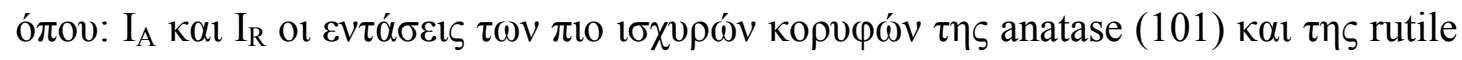

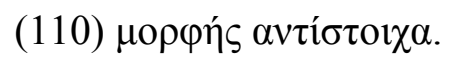




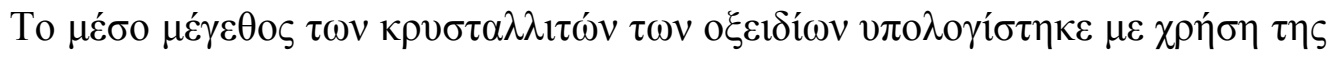
$\varepsilon \xi \hat{\imath} \sigma \omega \sigma \eta$ Scherrer [6-9]:

$$
\mathrm{d}=\frac{0.9 \cdot \lambda}{\mathrm{B} \cdot \cos \theta}
$$

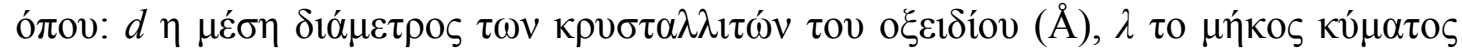

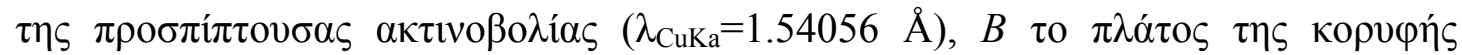

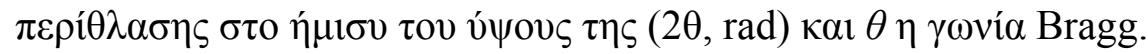

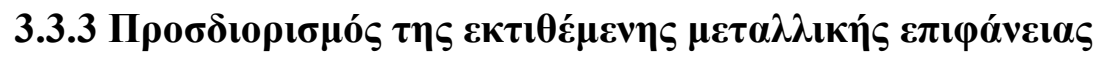

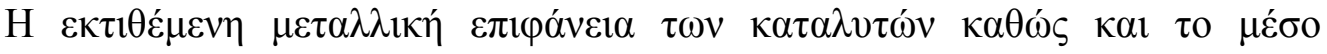

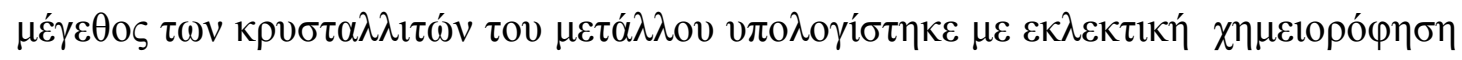

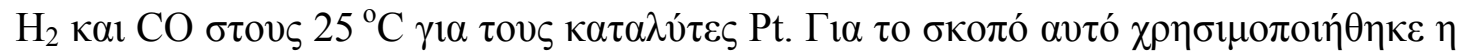

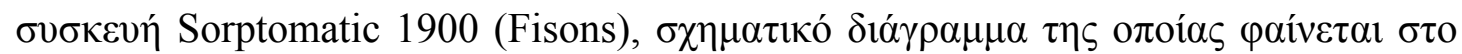

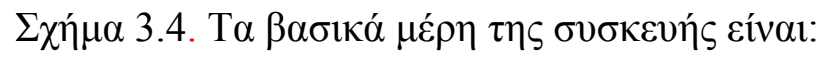

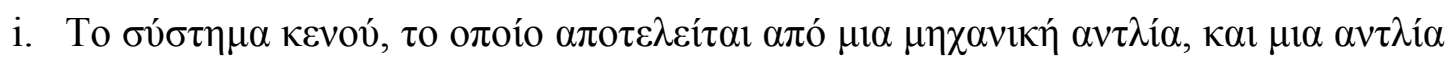

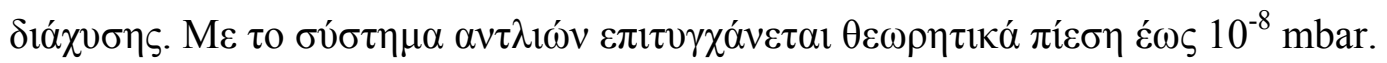

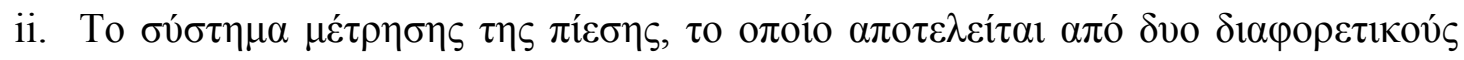

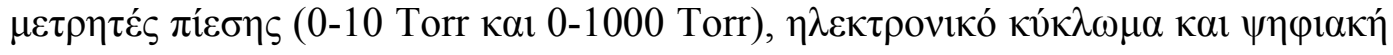
$\varepsilon \dot{v} \delta \varepsilon 1 \xi \eta$.

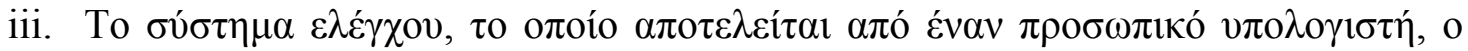

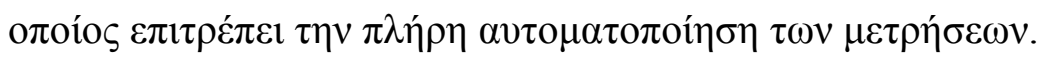

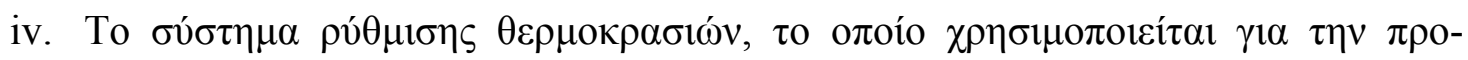
$\kappa \alpha \tau \varepsilon \rho \gamma \alpha \sigma i ́ \alpha \tau \omega \nu \delta \varepsilon 1 \gamma \mu \alpha \dot{\alpha} \omega \nu \sigma \varepsilon v \psi \eta \lambda \varepsilon \dot{\varsigma} \theta \varepsilon \rho \mu о \kappa \rho \alpha \sigma i ́ \varepsilon \varsigma\left(\varepsilon \dot{\varepsilon} \omega \varsigma 450^{\circ} \mathrm{C}\right)$.

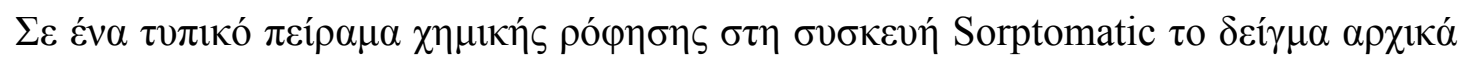

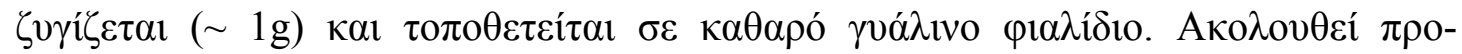

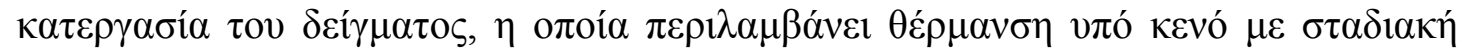

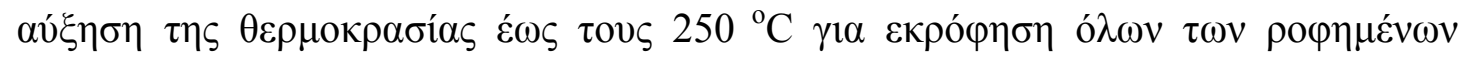

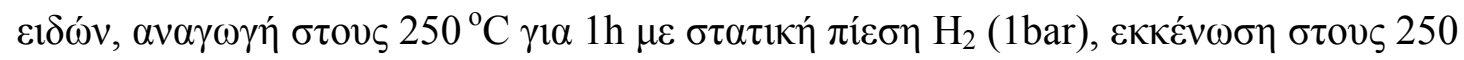

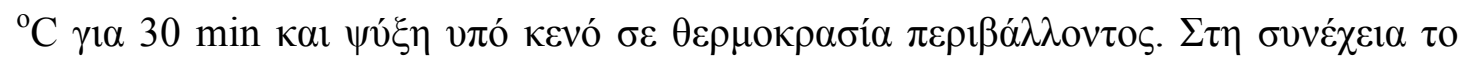

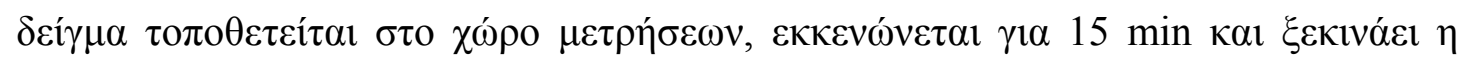

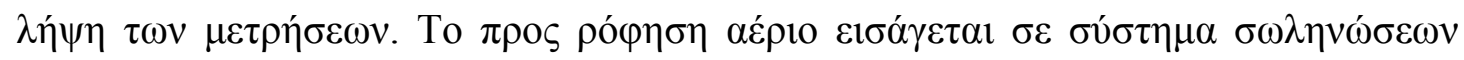

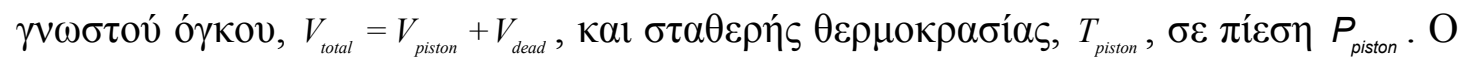




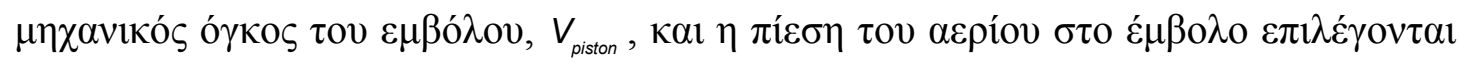

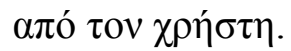

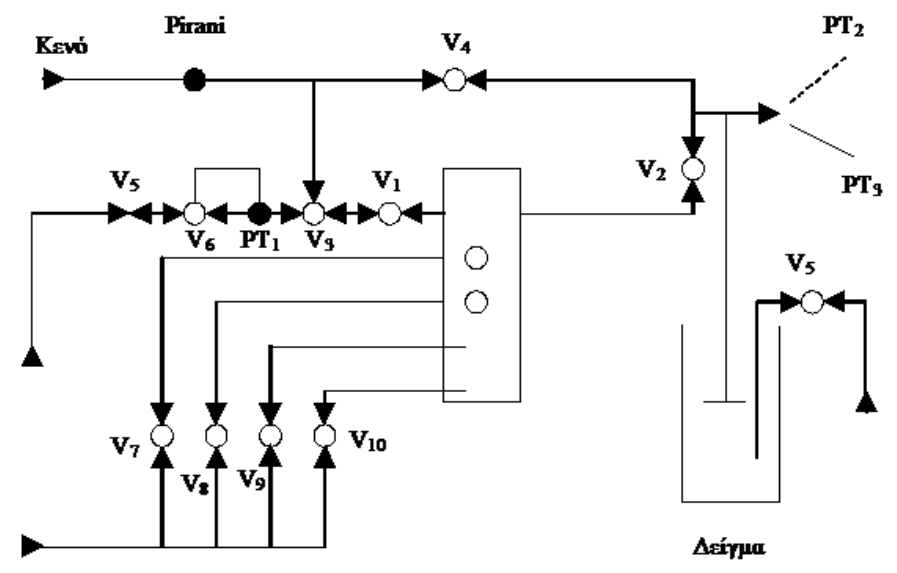

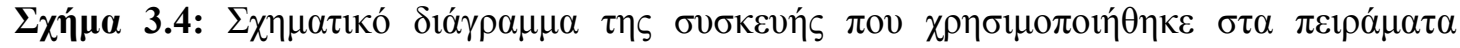

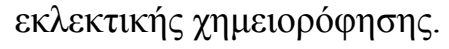

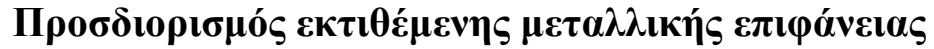

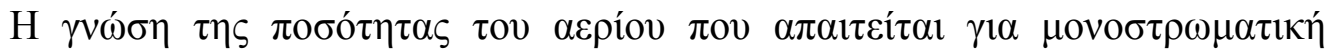

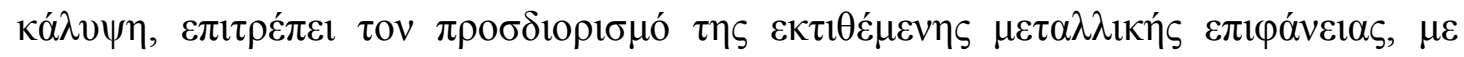

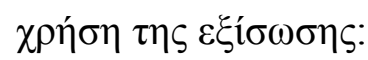

$$
\mathrm{S}_{\mathrm{sp}}=\frac{\mathrm{V}_{\mathrm{m}}}{\mathrm{V}_{\mathrm{mol}, \mathrm{STP}}} \cdot \mathrm{N}_{\mathrm{AV}} \cdot \mathrm{F}_{\mathrm{s}} \cdot \mathrm{S}_{\mathrm{t}} \cdot 10^{-20}
$$

о́

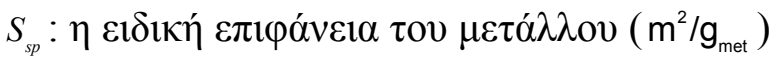

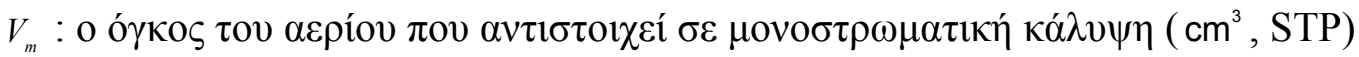

$N_{A V}$ : o $\alpha \rho \imath \theta \mu$ ós Avogadro $\left(6.023 \cdot 10^{23} \mu o ́ \rho l \alpha / m o l\right)$

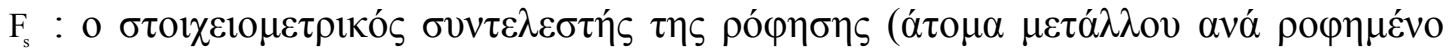

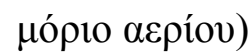

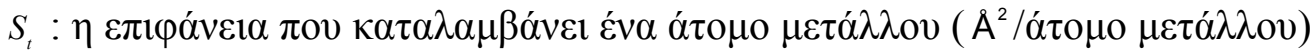

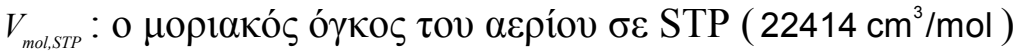




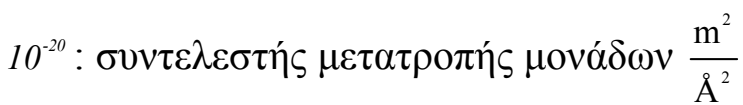

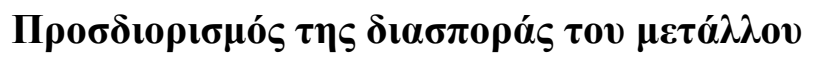

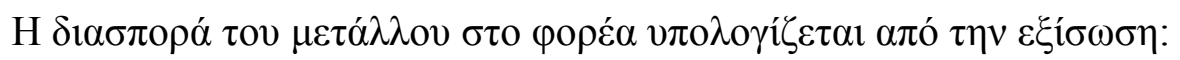

$$
\mathrm{D}=\frac{\text { surface atoms }}{\text { total atoms }}=\frac{\mathrm{S}_{\mathrm{sp}} \cdot \mathrm{AB}}{\mathrm{S}_{\mathrm{t}} \cdot \mathrm{N}_{\mathrm{AV}} \cdot 10^{-20}}
$$

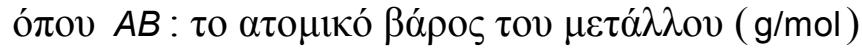

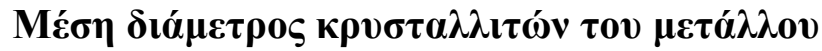

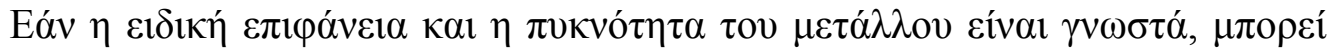

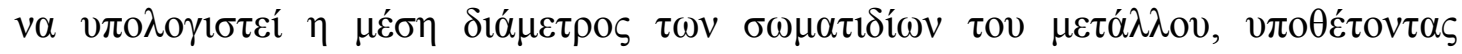

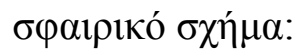

$$
\mathrm{d}=\frac{6}{\rho_{\text {met }} \cdot \mathrm{S}_{\mathrm{sp}}} \cdot 10^{4}
$$

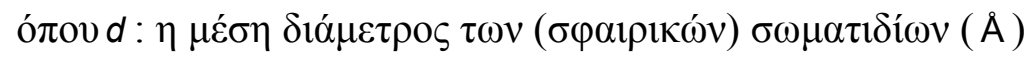

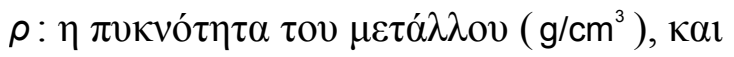

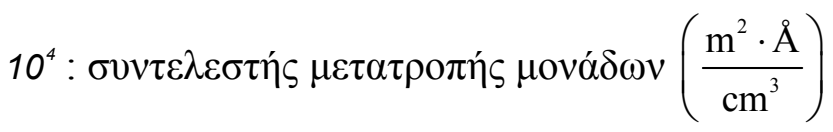

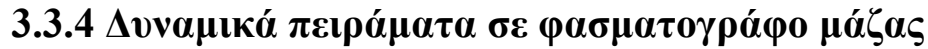

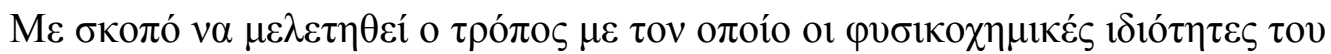


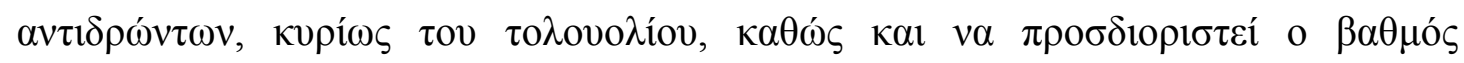

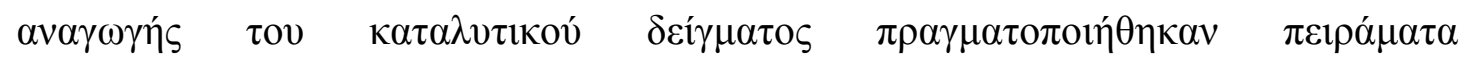

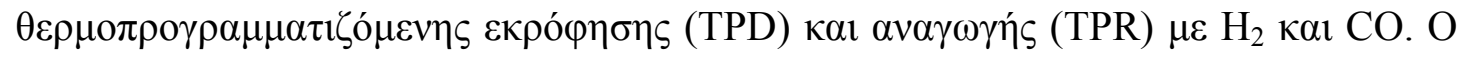

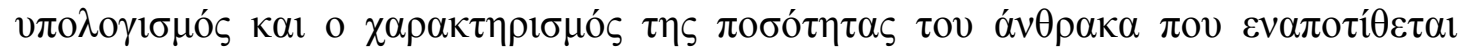

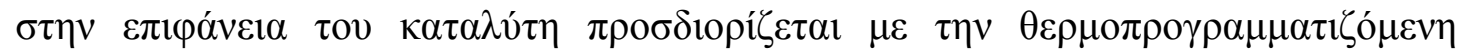

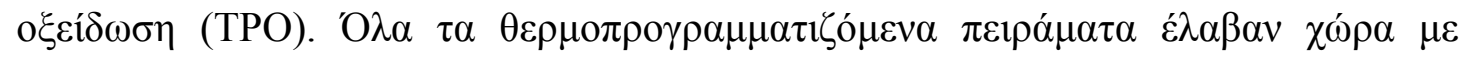

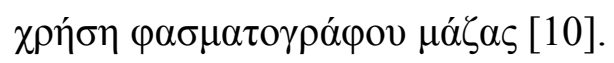

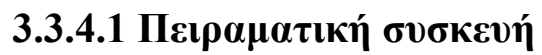




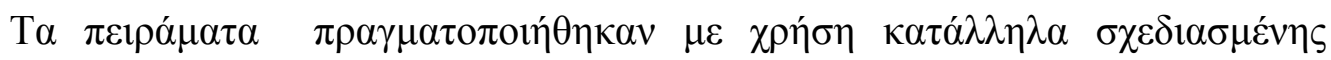

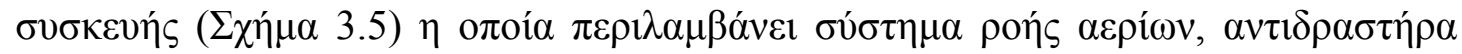

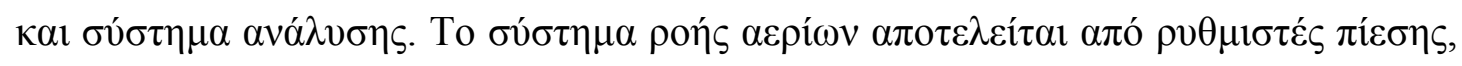

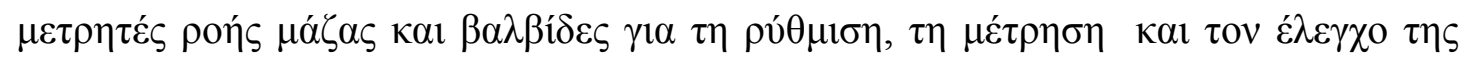

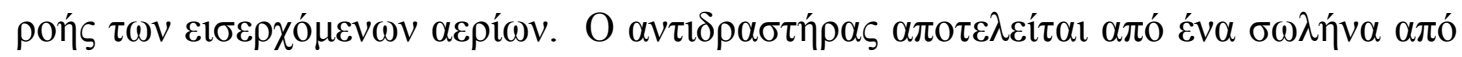

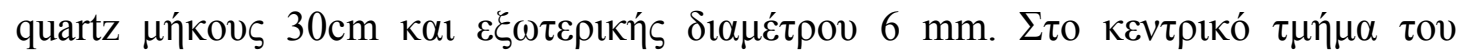

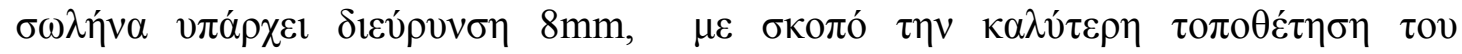

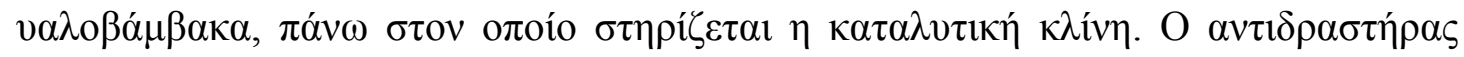

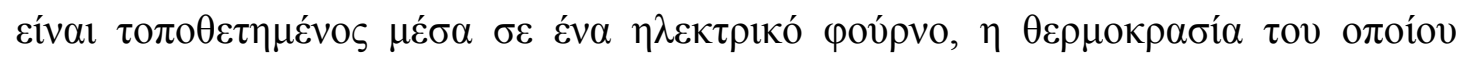

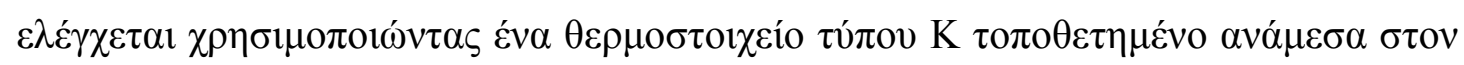

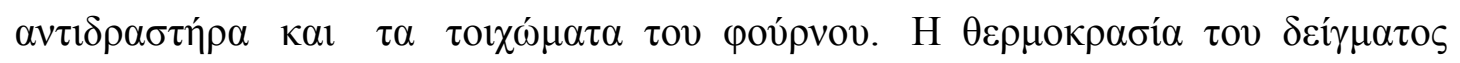

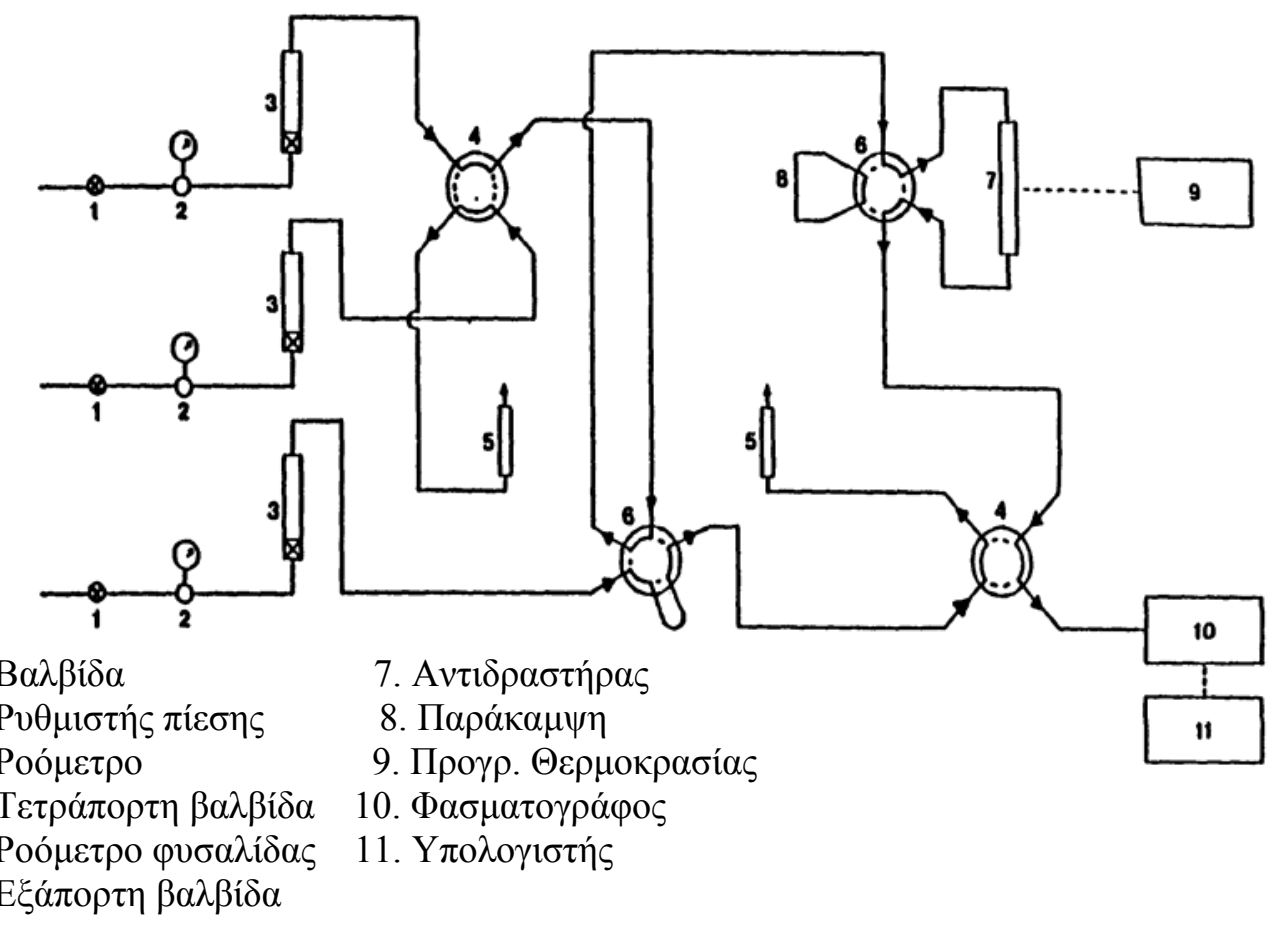

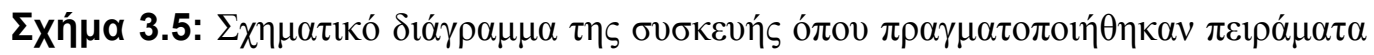

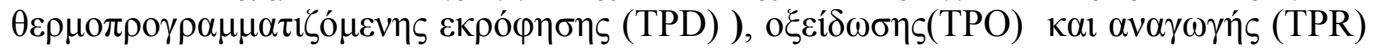
$\mu \varepsilon \mathrm{H}_{2} \kappa \alpha \mathrm{l} \mathrm{CO}$.

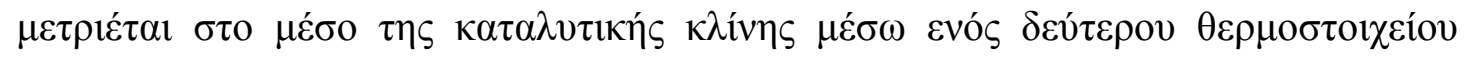

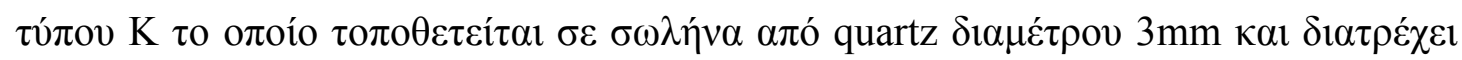

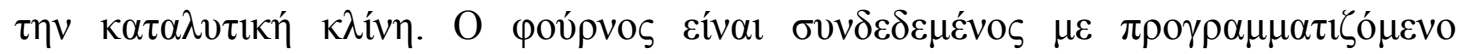




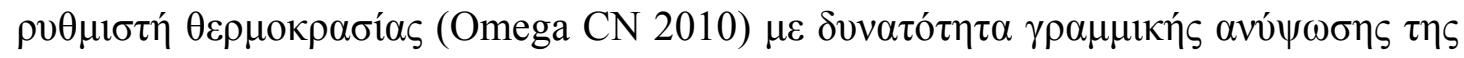

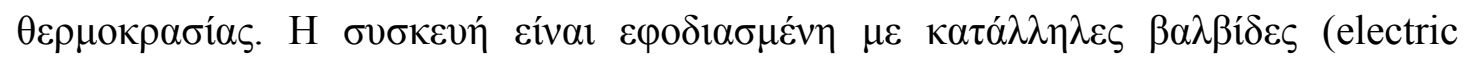

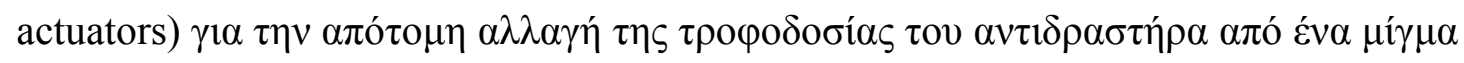

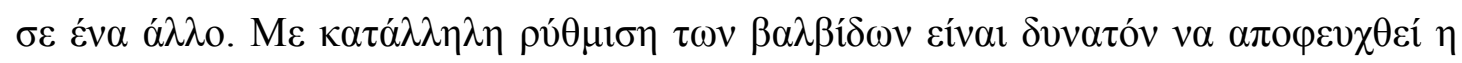

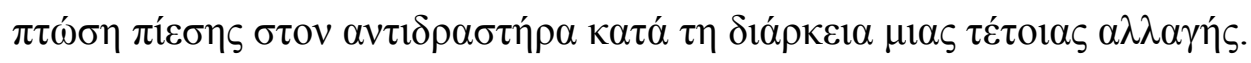

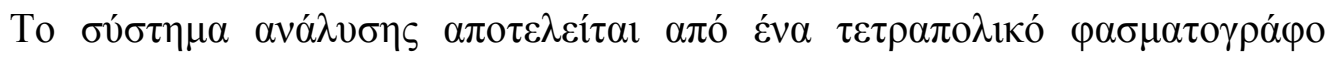

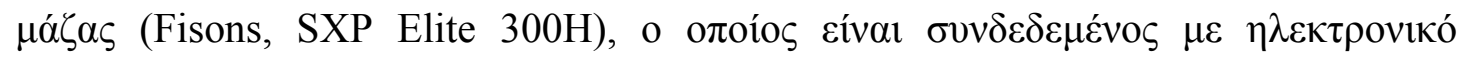

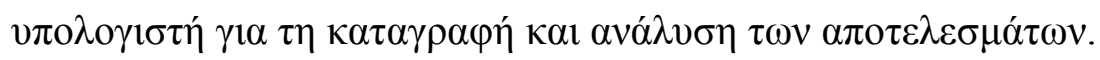

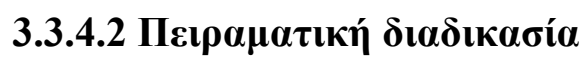

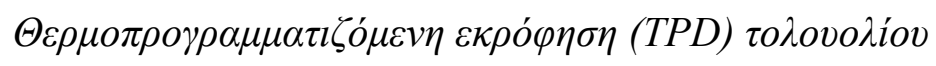

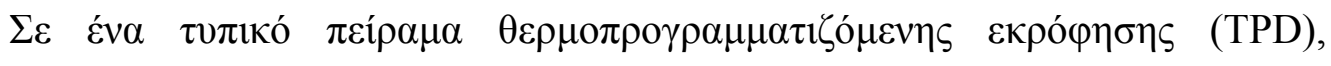

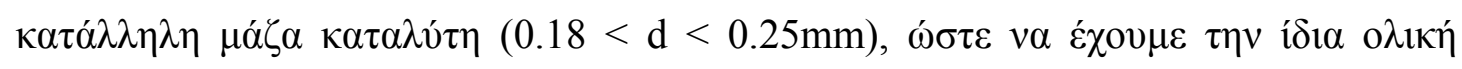

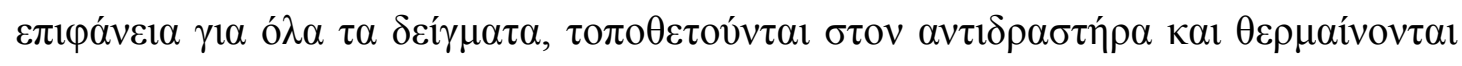

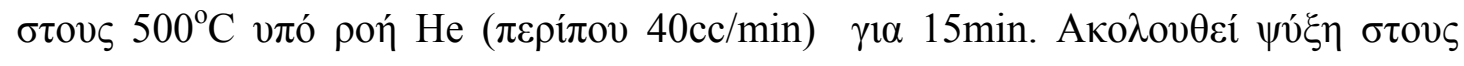

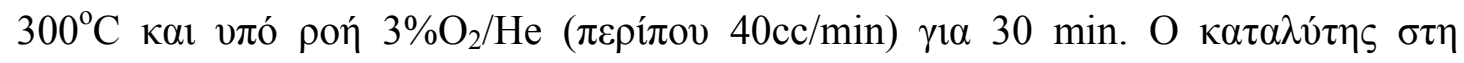

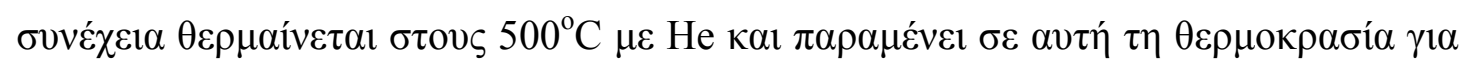

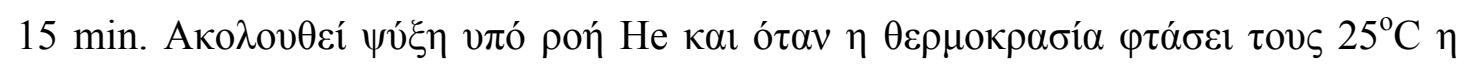

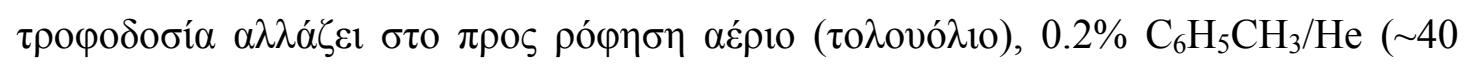

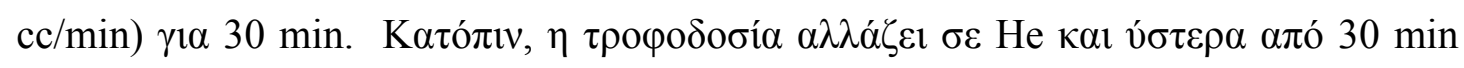

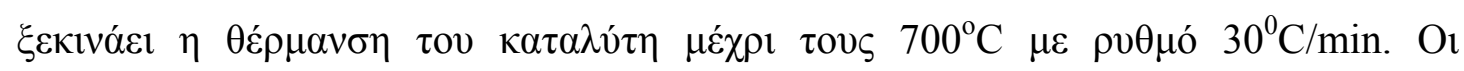

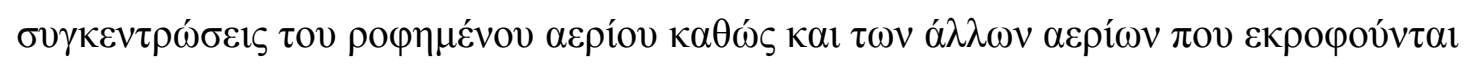

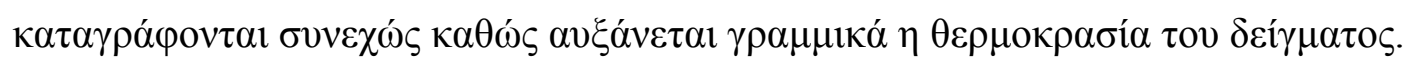

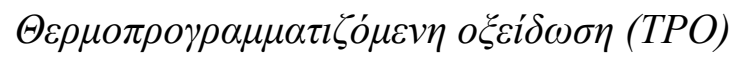

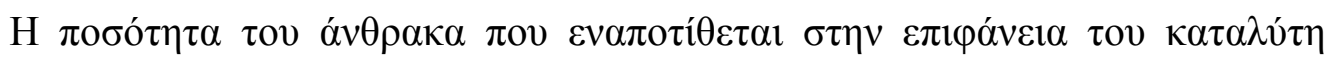

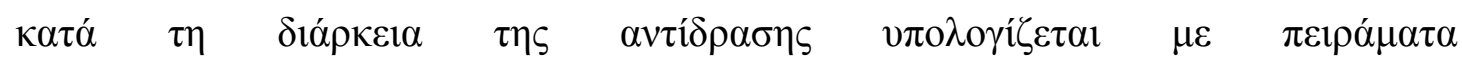

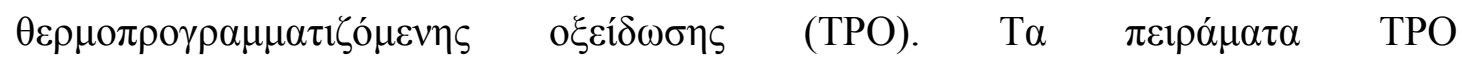

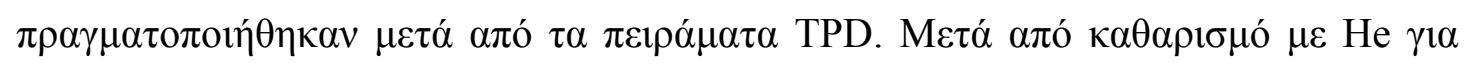

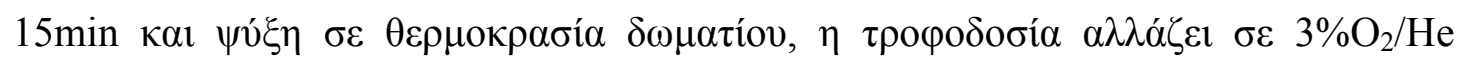

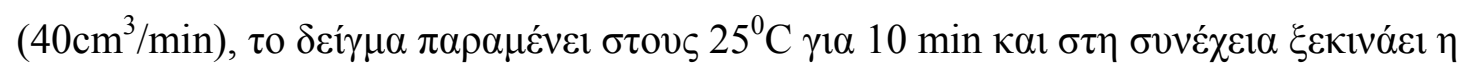

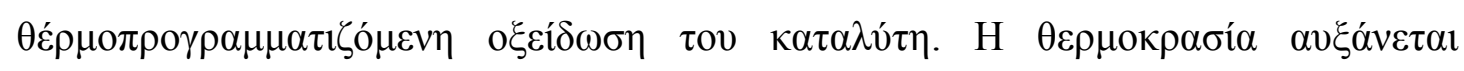

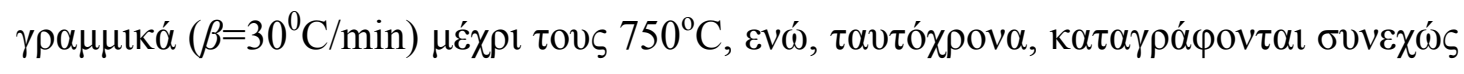

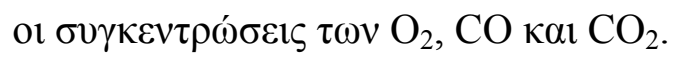


$\Theta \varepsilon \rho \mu о \pi \rho о \gamma \rho \alpha \mu \mu \alpha \tau \iota \zeta o ́ \mu \varepsilon v \eta \alpha \nu \alpha \gamma \omega \gamma \dot{\eta} \mu \varepsilon H_{2} \dot{\eta} C O(T P R)$

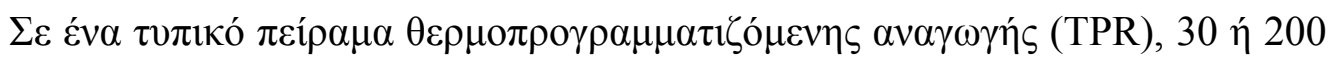

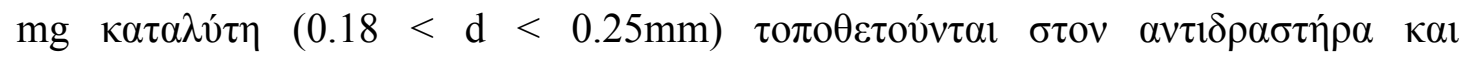

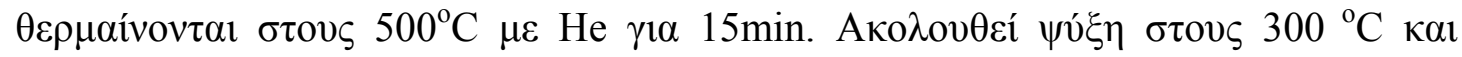

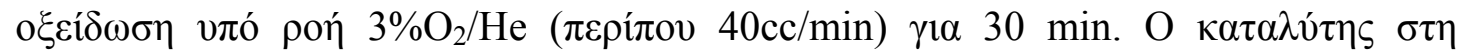

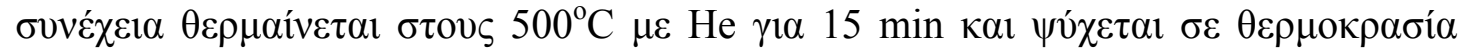

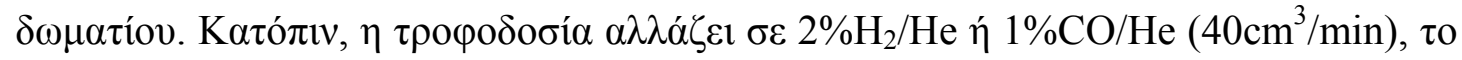

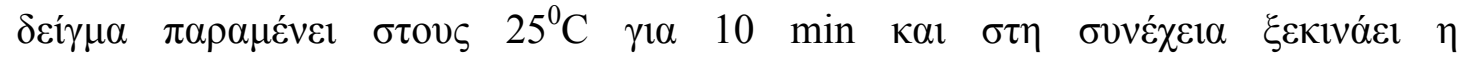

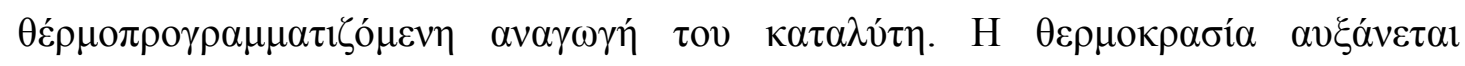

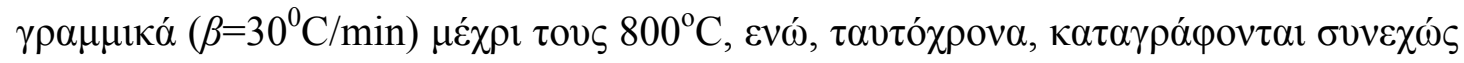

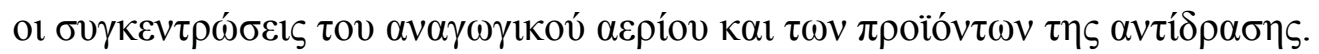

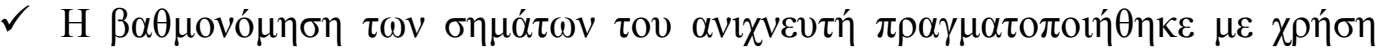

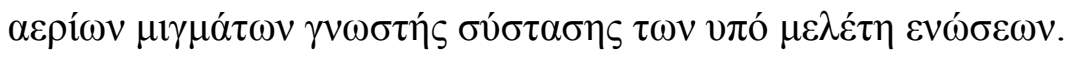

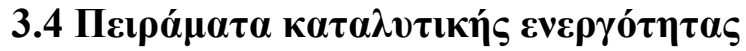

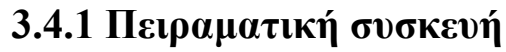

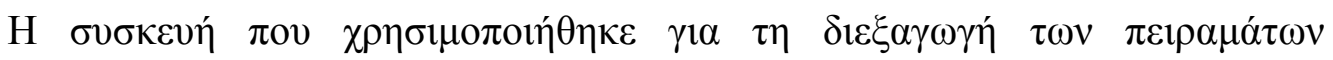

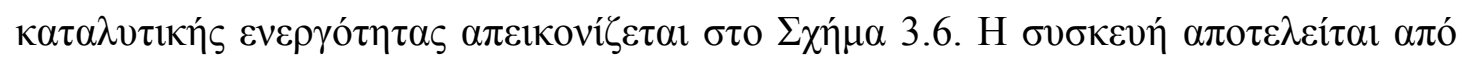

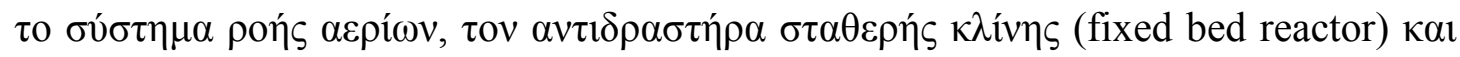

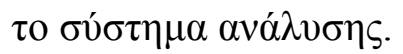

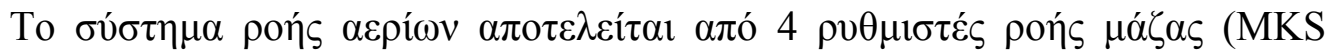

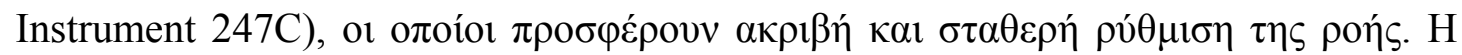

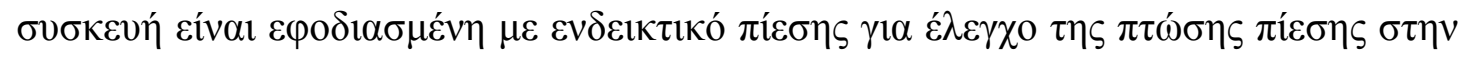

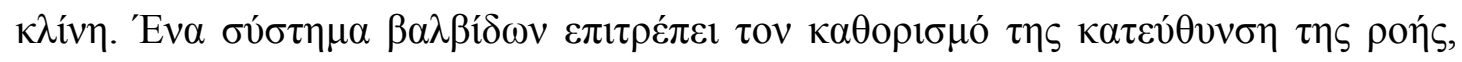

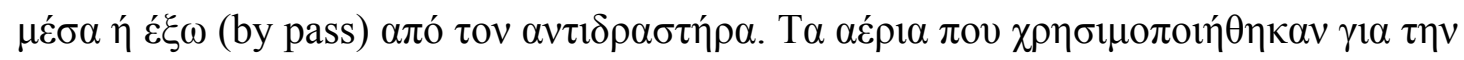

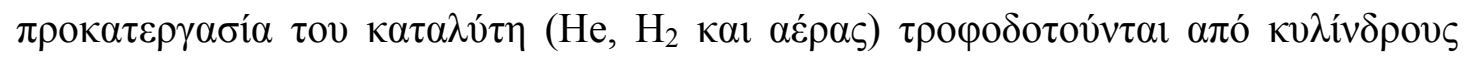

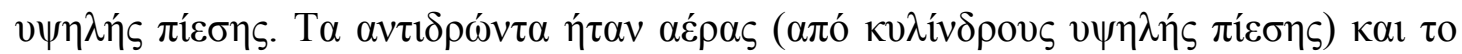

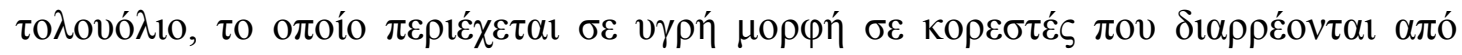

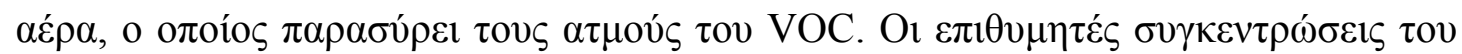

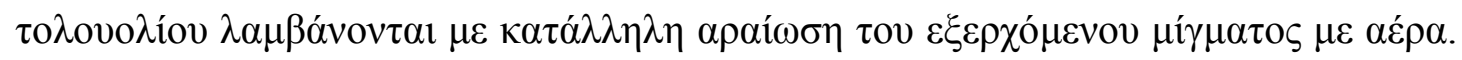

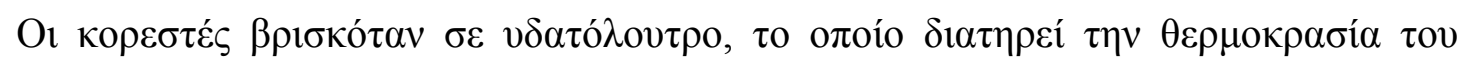




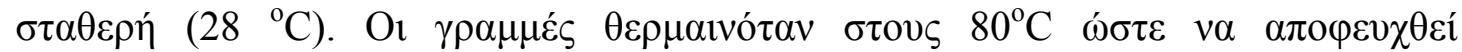

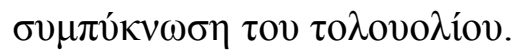

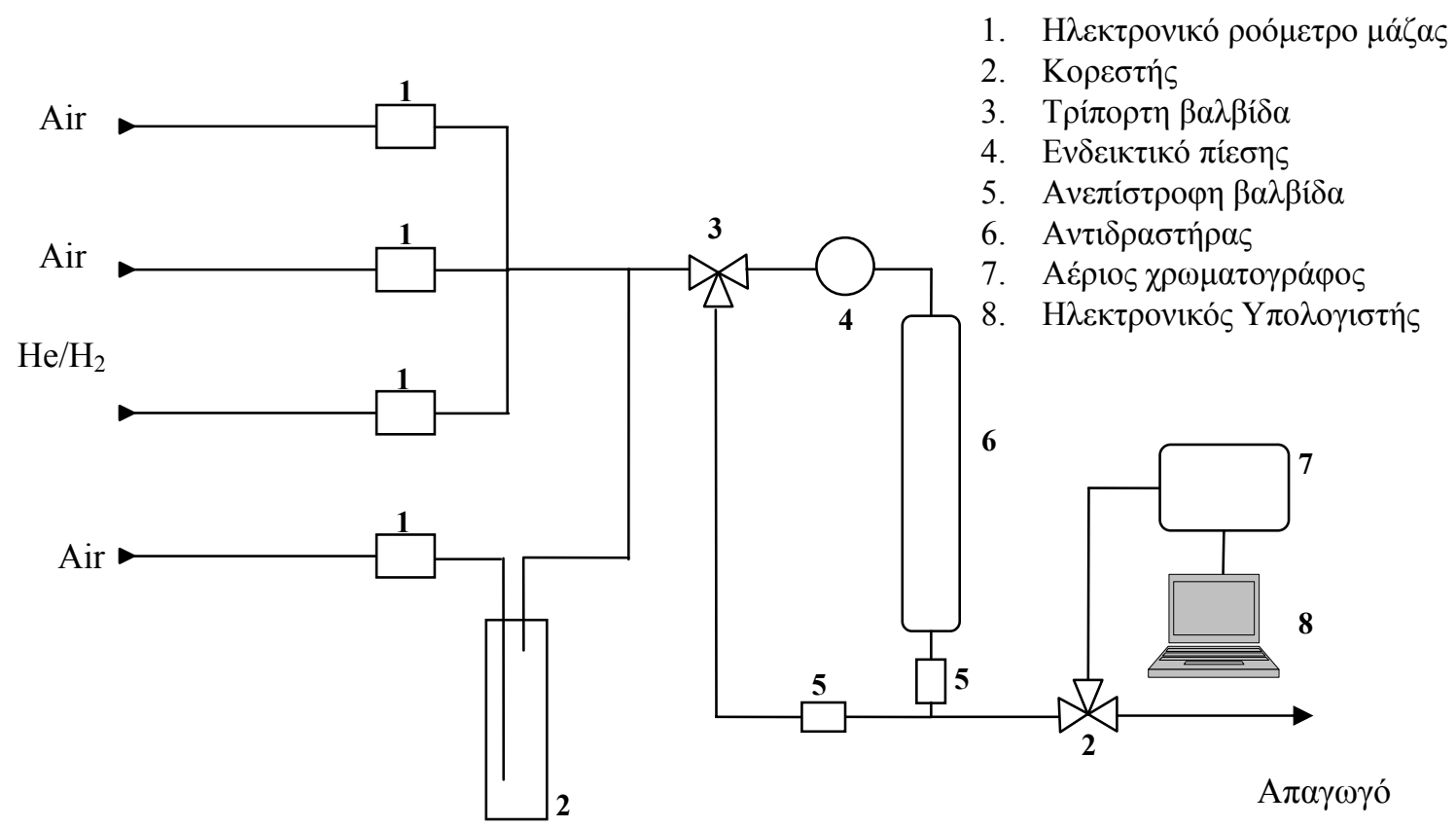

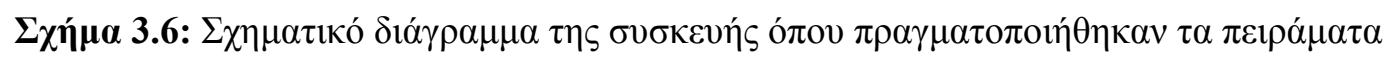

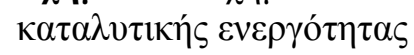

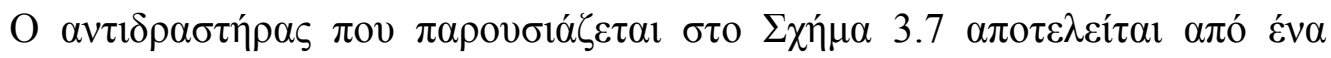

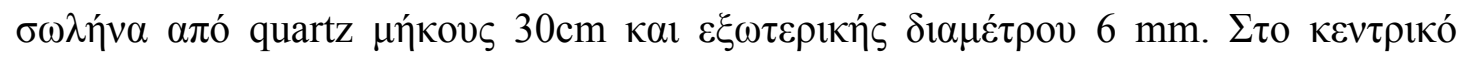

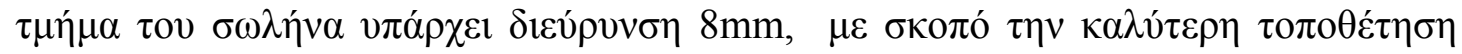

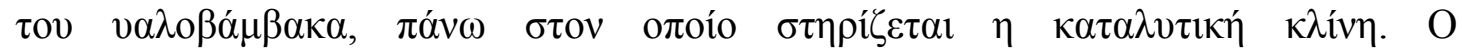

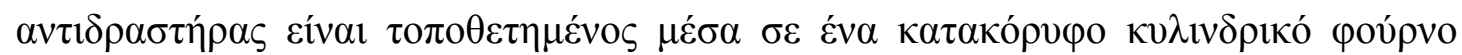

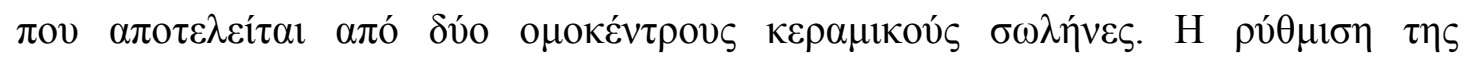

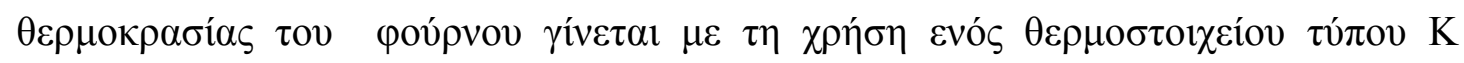

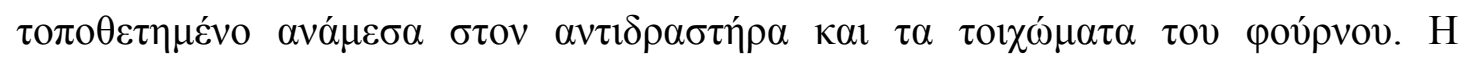

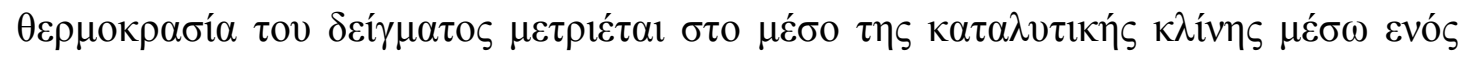

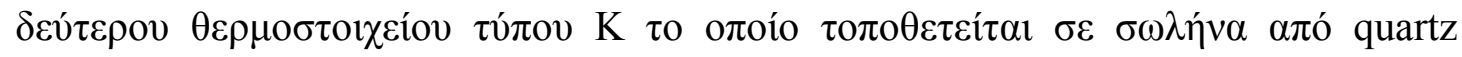

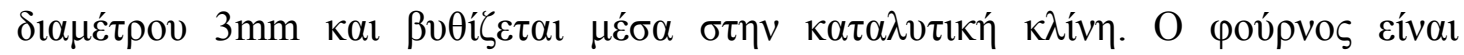

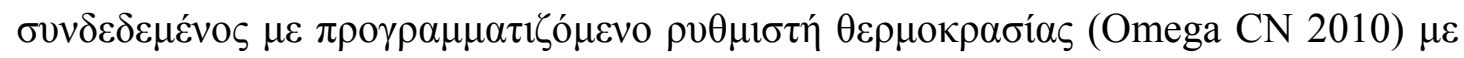

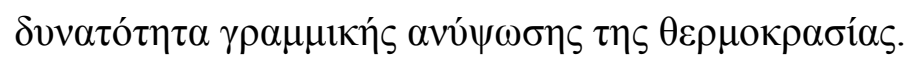




$$
\begin{aligned}
& \alpha: \beta \alpha ́ \sigma \eta ~ \theta \varepsilon \rho \mu о \sigma \tau о \chi \varepsilon i ́ o v \\
& \beta: \varphi о v ́ \rho v o \varsigma \\
& \gamma: \kappa \alpha \tau \alpha \lambda v \tau i \kappa \eta ́ ~ \kappa \lambda i ́ v \eta \\
& \delta: v \alpha \lambda o \beta \alpha ́ \mu \beta \alpha \kappa \alpha \varsigma \\
& \\
& \mathrm{d}_{1}=3 \mathrm{~mm} \\
& \mathrm{~d}_{2}=8 \mathrm{~mm} \\
& \mathrm{~d}_{3}=6 \mathrm{~mm} \\
& \mathrm{~h}=1 \mathrm{~mm}
\end{aligned}
$$

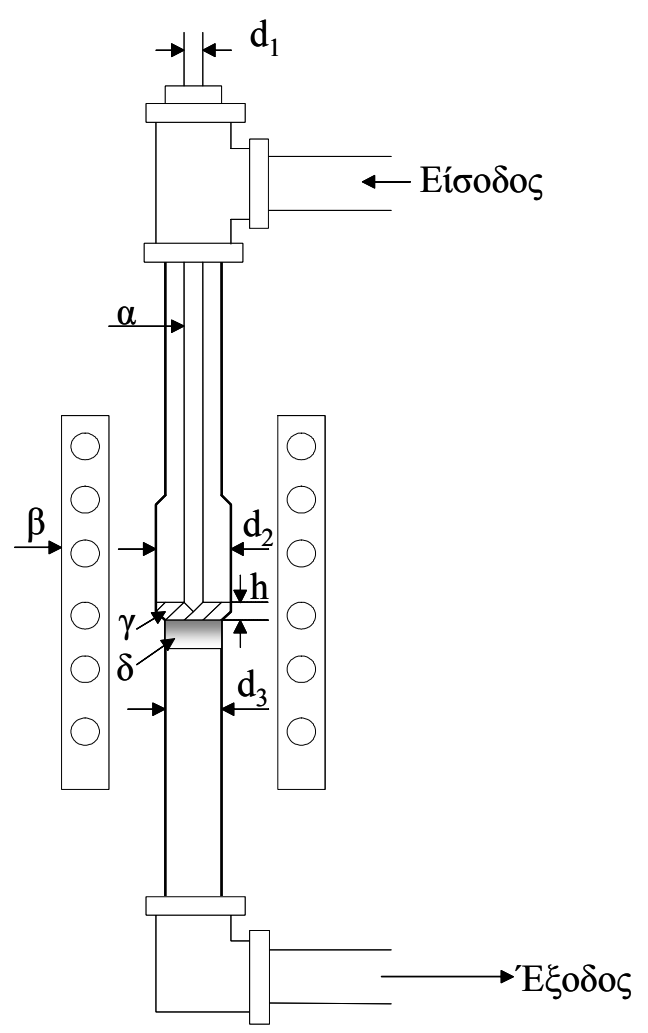

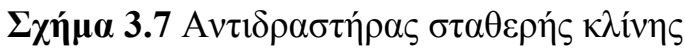

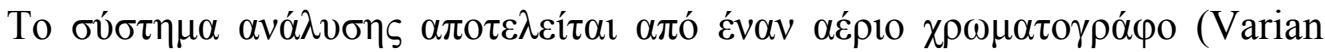

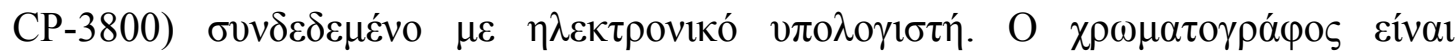

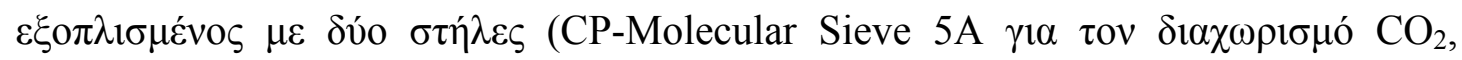

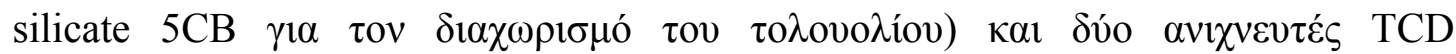

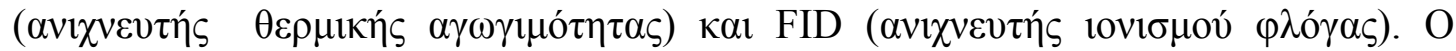

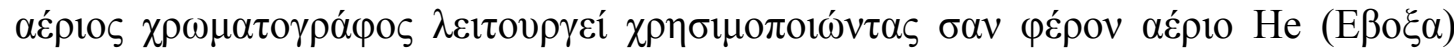

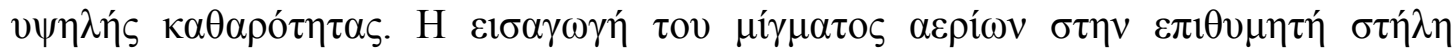

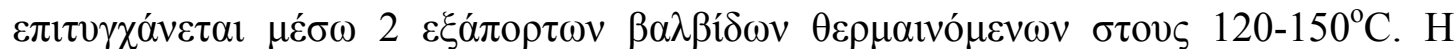

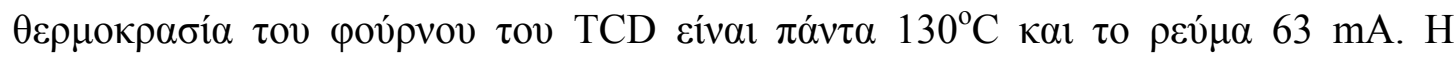

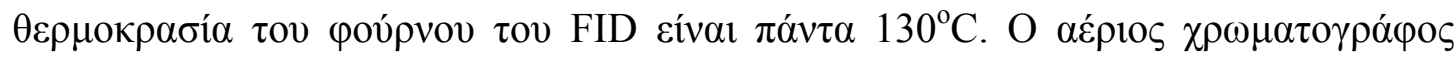

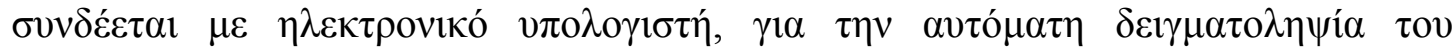

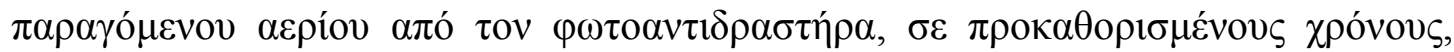

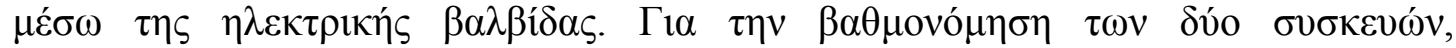

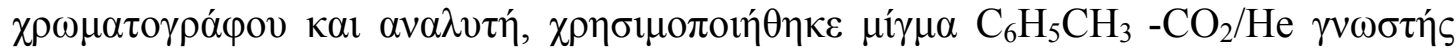

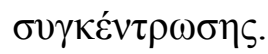




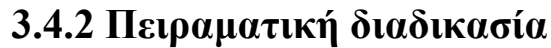

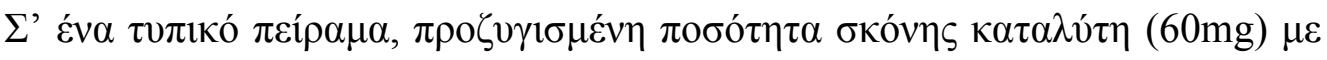

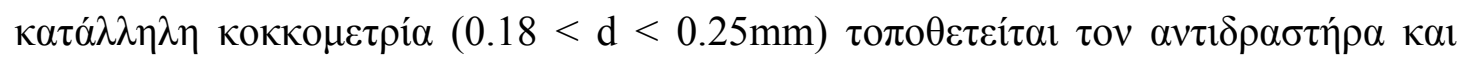

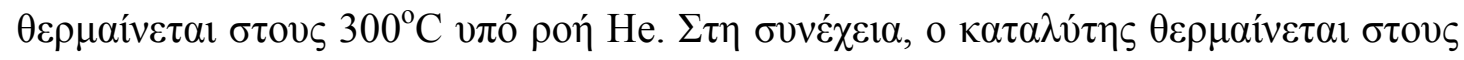

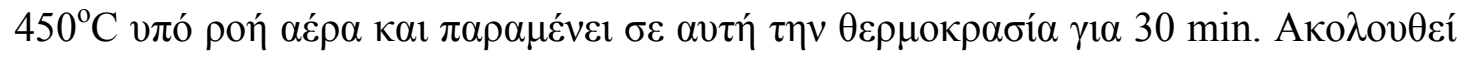

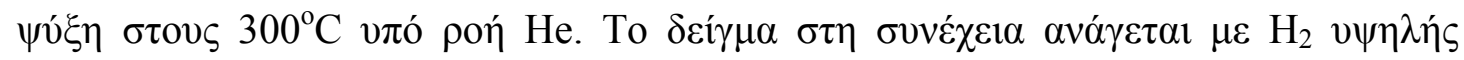

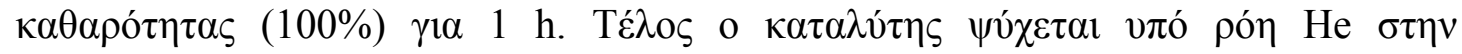

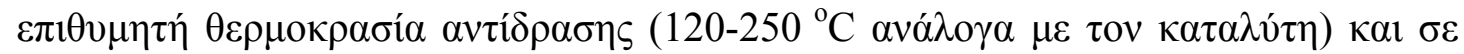

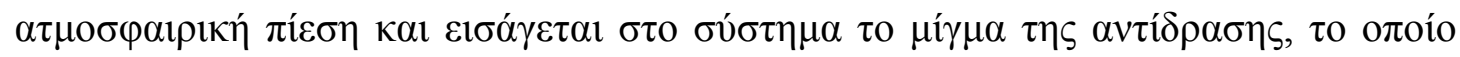

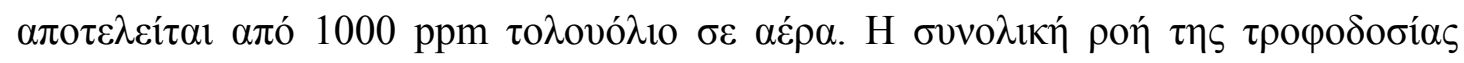

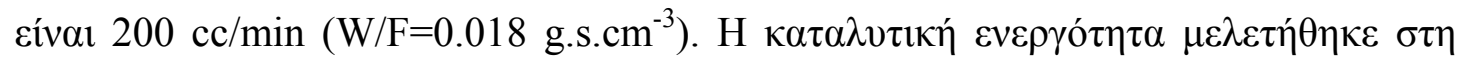

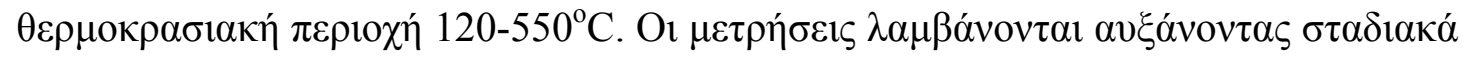

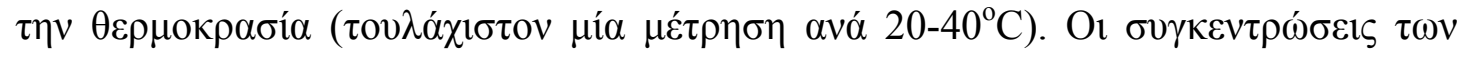

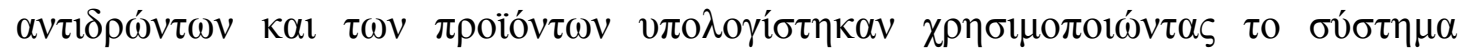

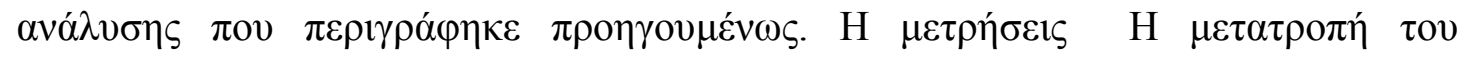

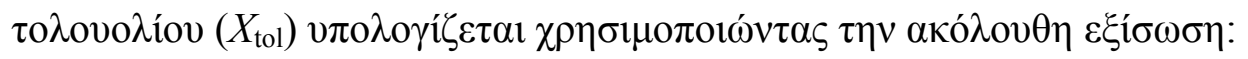

$$
X_{\mathrm{tol}}=\frac{C_{\mathrm{in}}-C_{\mathrm{out}}}{C_{\mathrm{in}}}
$$

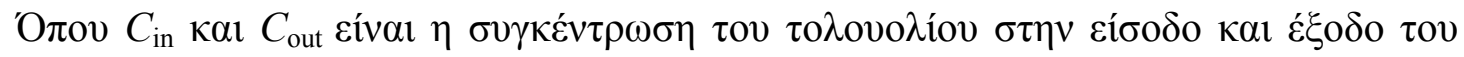

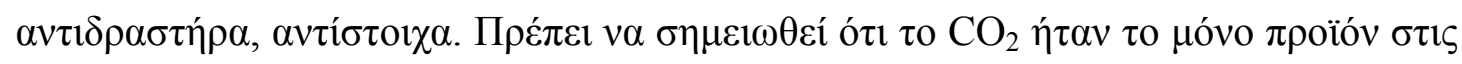

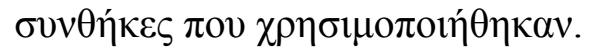

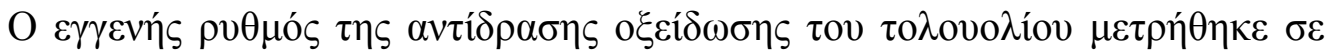

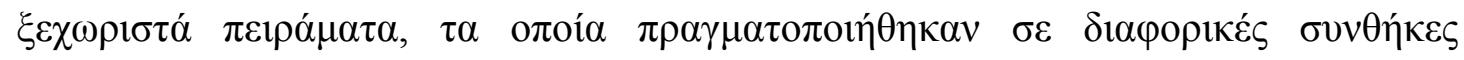

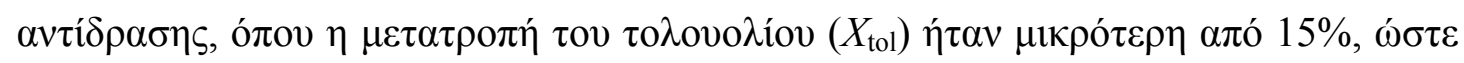

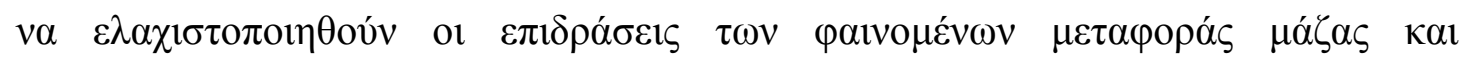

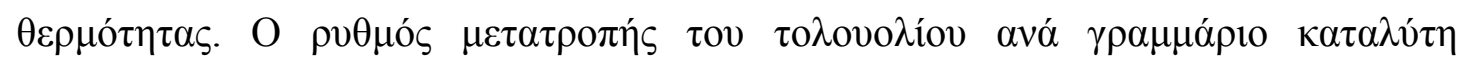

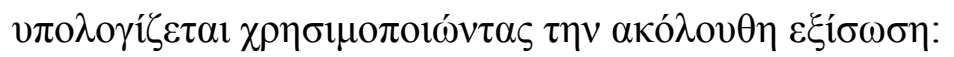

$$
r=\frac{\left(C_{\text {in }}-C_{\text {out }}\right) F}{W}
$$

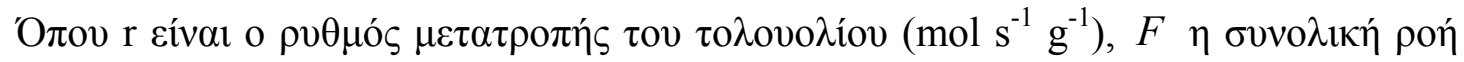

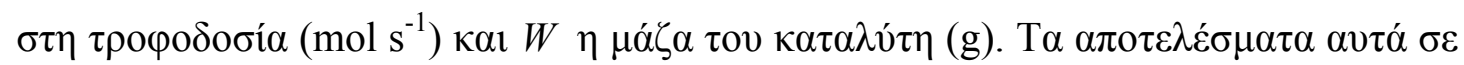

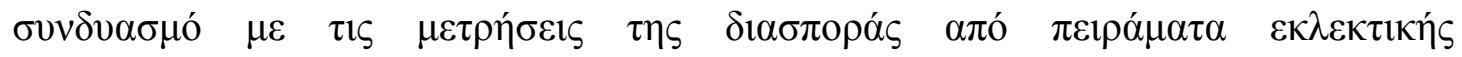

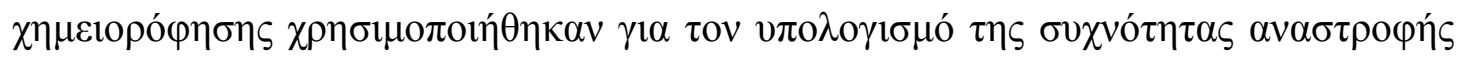




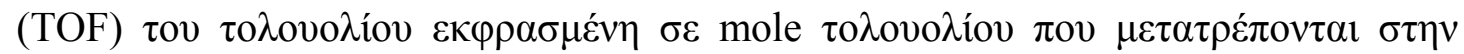

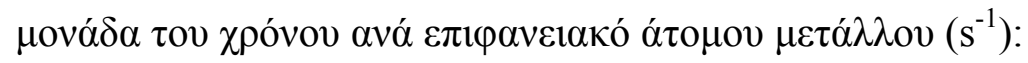

$$
T O F=\frac{r_{\text {tol. }} \cdot A B_{M}}{D \cdot X_{M}}
$$

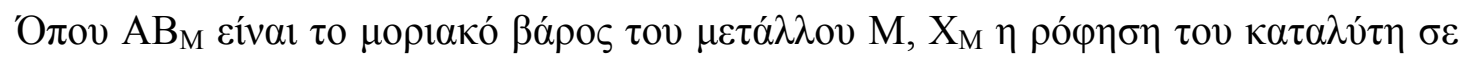
$\mu \varepsilon ́ \tau \alpha \lambda \lambda \mathrm{o}\left(\mathrm{g}_{\text {met. }} / \mathrm{g}_{\text {cat. }}\right) \kappa \alpha \mathrm{l} \mathrm{D} \eta \delta 1 \alpha \sigma \pi \mathrm{\rho} \alpha \dot{\text {. }}$

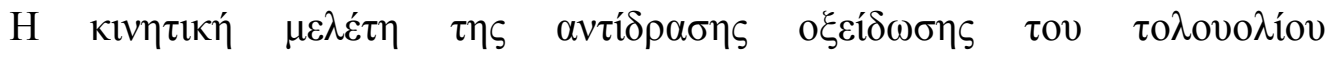

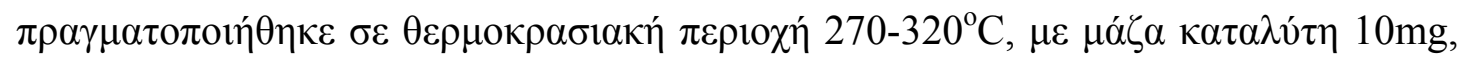

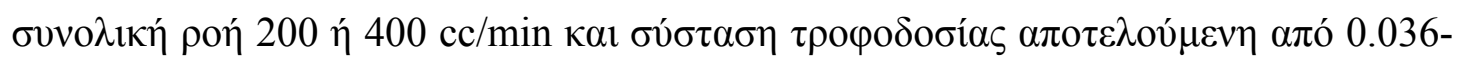

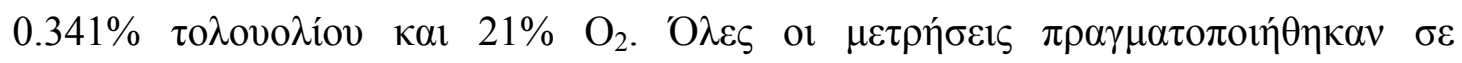

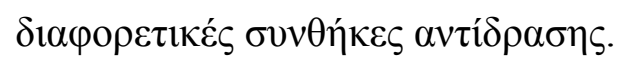




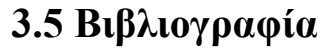

[1] J.-G, Li, T. Ikegami, J.-H, Lee, T. Mori, Acat mater. 49 (2001) 419.

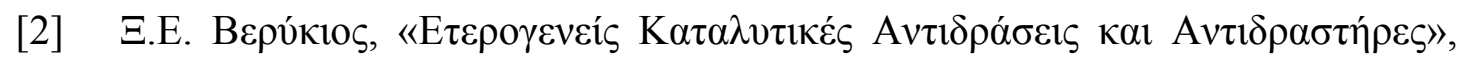

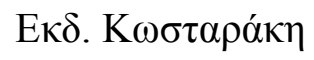

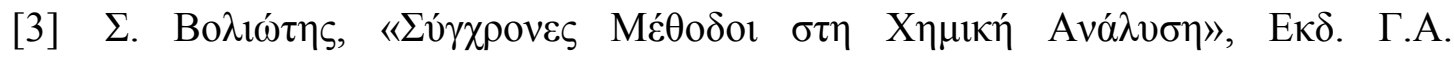

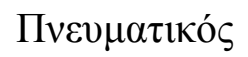

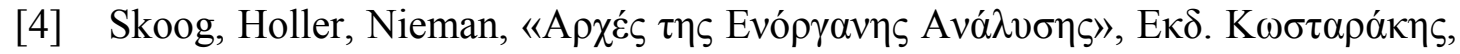
2005.

[5] R.A. Spurr, H. Myers, Anal. Chem. 59 (1957) 761.

[6] B.E. Warren, «X-Ray Diffraction», Addison-Wesley Publishing Company, Reading Massachusetts, 1969.

[7] A. Taylor, «X-Ray Metallography», John Willey \& Sons, New York, 1961

[8] B.D. Cullity, « Elements of X-Ray Diffraction», Addison- Wesley Publishing Company, Reading, MA,1978.

[9] D.M. Moore, R.C. Reynolds, Jr. «X-Ray Diffraction and the Identification and Analysis of Clay Minerals», $2^{\text {nd }}$ Edition, Oxford University Press. N. York, 1997

[10] Efstathiou and X.E. Verykios, Appl. Catal. A: Gen. 151 (1997) 109. 


\section{KEФAイAIO $4^{o}$}

\section{H ANTI $\triangle \mathrm{PA} \Sigma \mathrm{H}$ OEEI $\Delta \Omega \Sigma \mathrm{H} \Sigma$ TOY TO $\Lambda O Y O \Lambda I O Y \quad \Sigma \mathrm{E}$

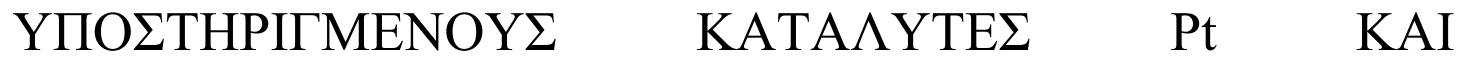 META $\Lambda \Lambda I K \Omega N$ OEEI $\Delta \mathrm{I} \Omega \mathrm{N}$}

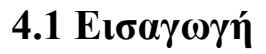

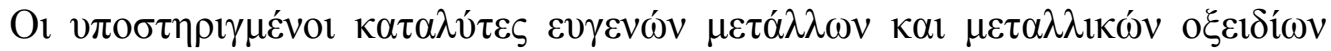

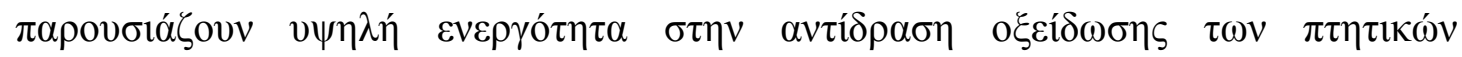

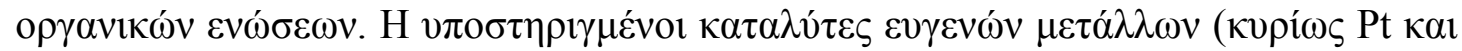

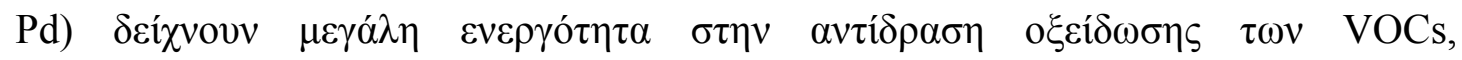

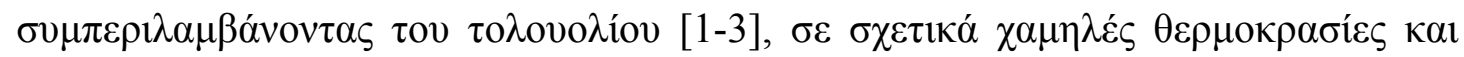

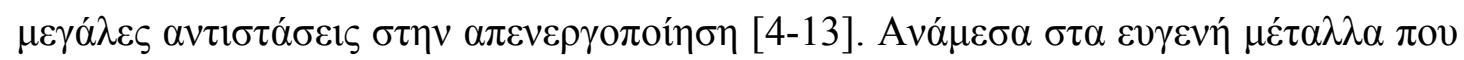

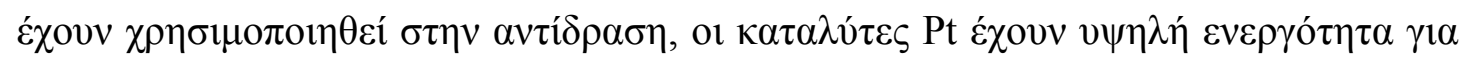

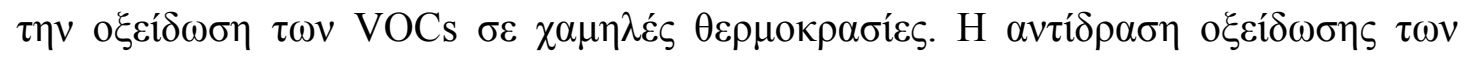

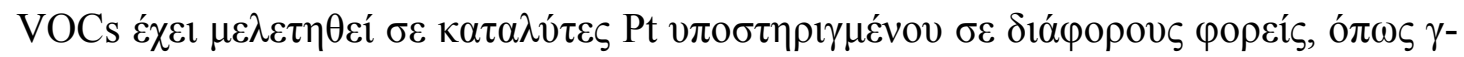


$\mathrm{Al}_{2} \mathrm{O}_{3}[2,6,10,11], \mathrm{TiO}_{2}\left(\mathrm{~W}^{6+}\right)[4,6], \mathrm{SiO}_{2}[6], \mathrm{TiO}_{2}[6,11,13] \kappa \alpha \iota \varepsilon \pi i ́ \sigma \eta \varsigma \sigma \varepsilon \kappa \alpha \tau \alpha \lambda u ́ \tau \varepsilon \varsigma$

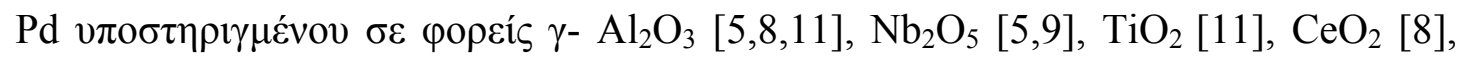

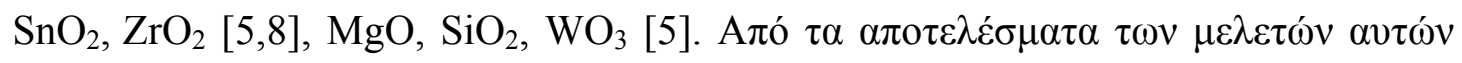

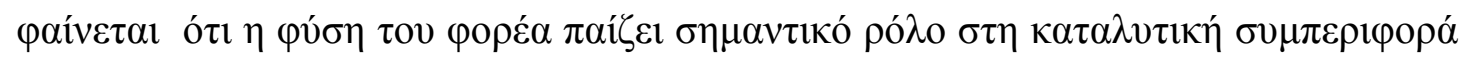

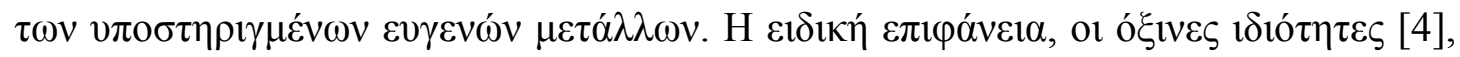

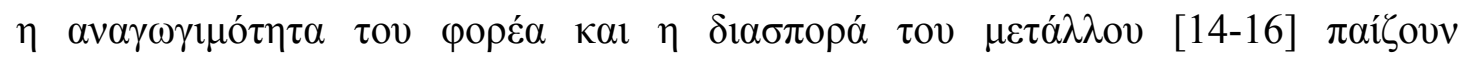

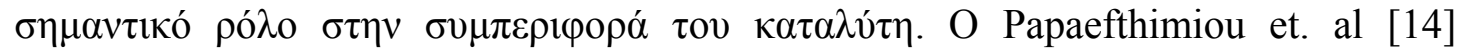

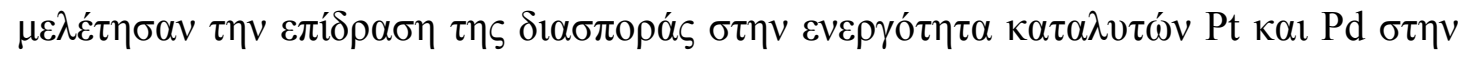

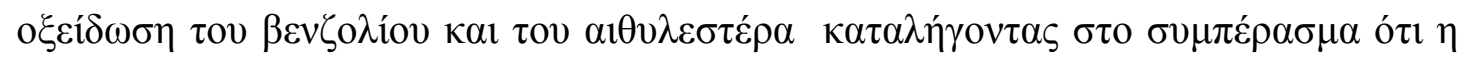

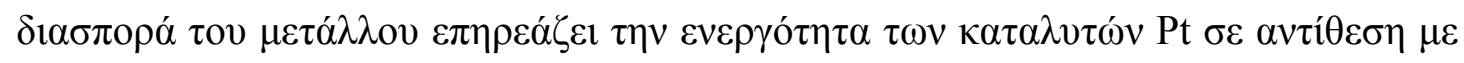

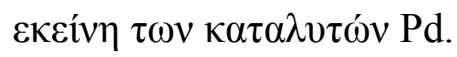

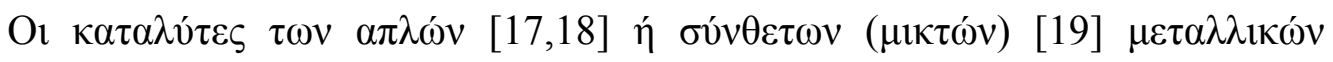

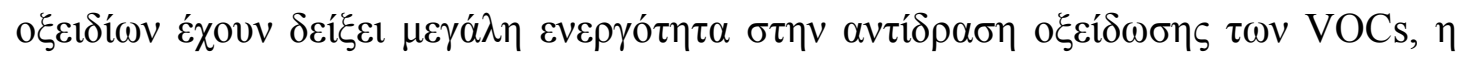

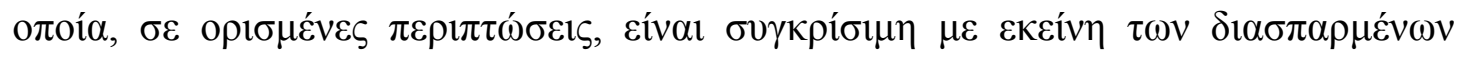

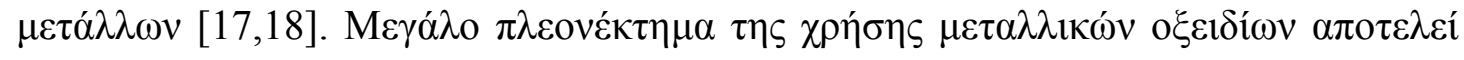

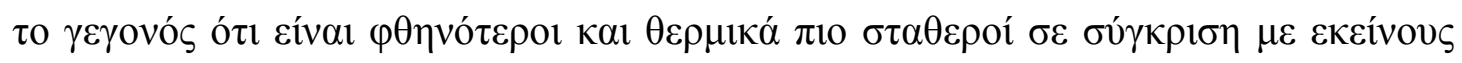
$\tau \omega v \varepsilon v \gamma \varepsilon v \omega ́ v ~ \mu \varepsilon \tau \alpha \dot{\alpha} \lambda \lambda \omega v$.

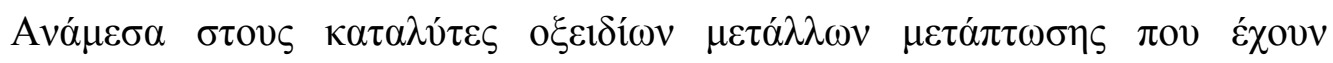

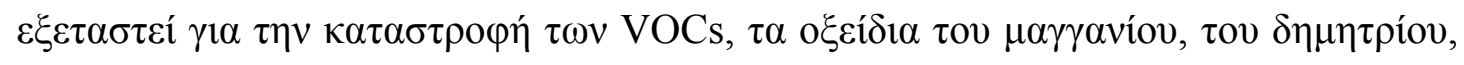

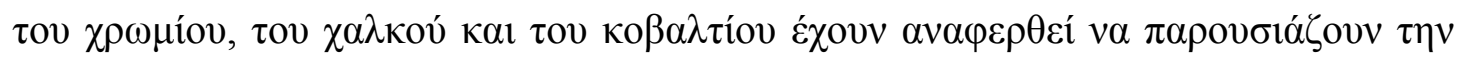

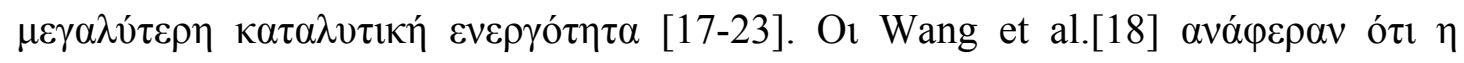

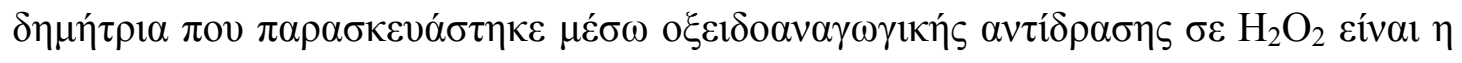

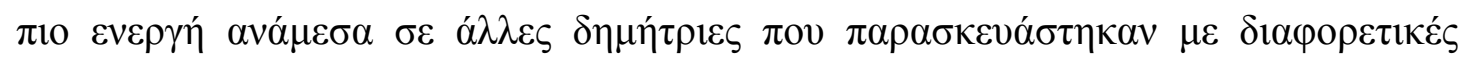
$\mu \varepsilon \theta$ ó

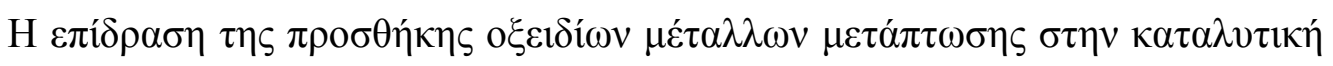

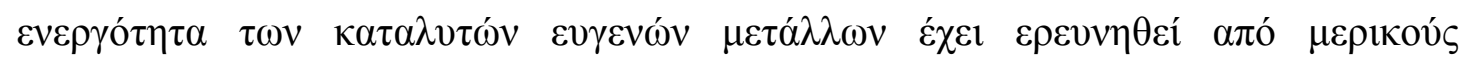

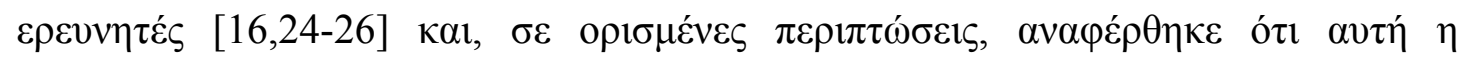

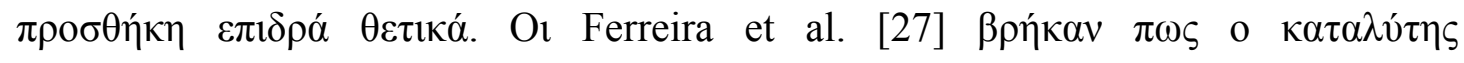

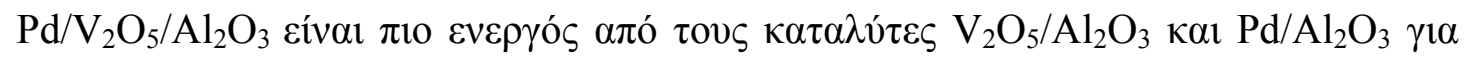

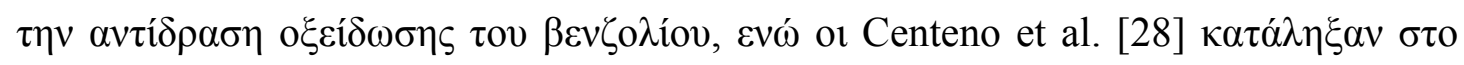

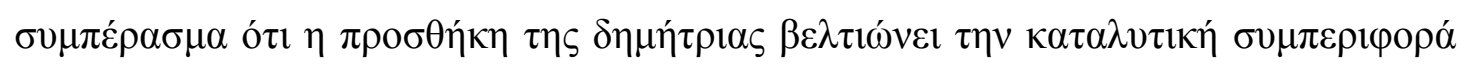

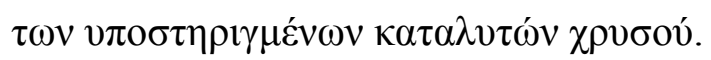

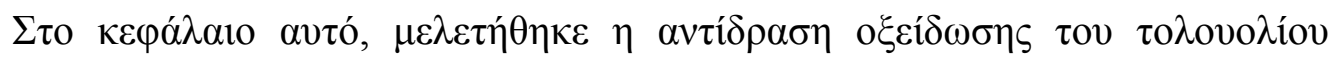

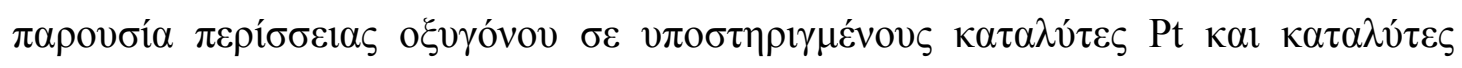




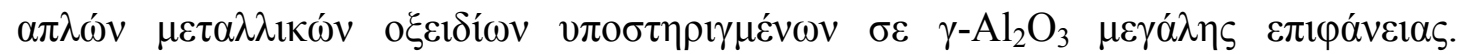

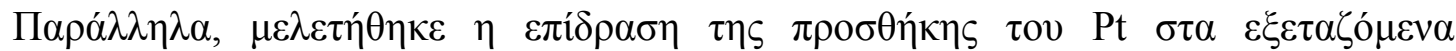

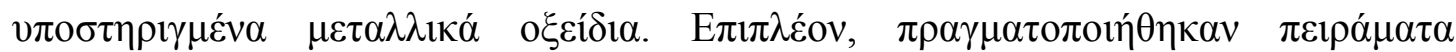

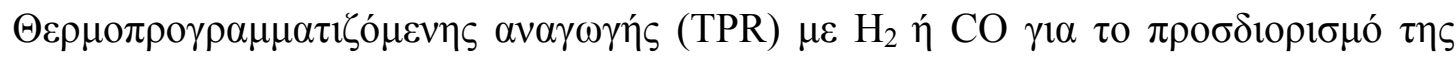

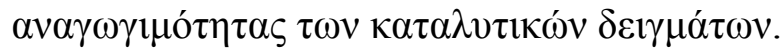

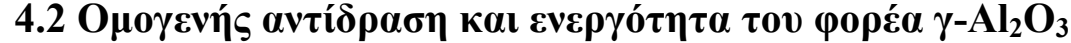

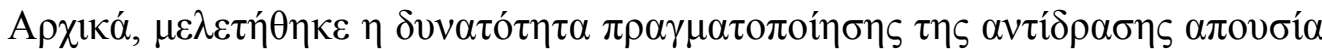

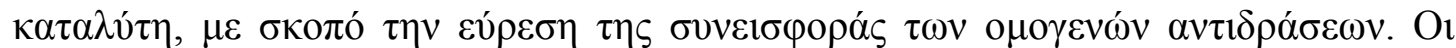
o

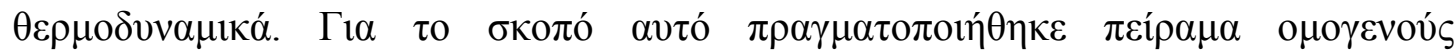

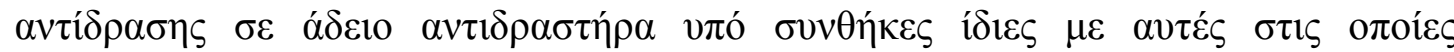

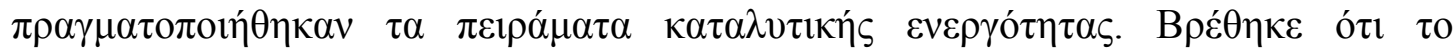

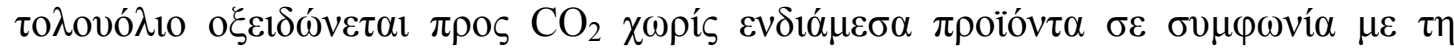

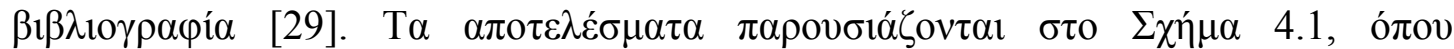

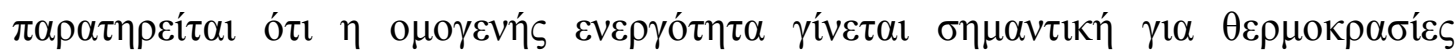
$\mu \varepsilon \gamma \alpha \lambda \hat{\tau} \varepsilon \rho \varepsilon \varsigma \alpha \pi$ ó $300^{\circ} \mathrm{C}$.

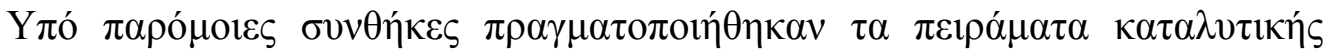

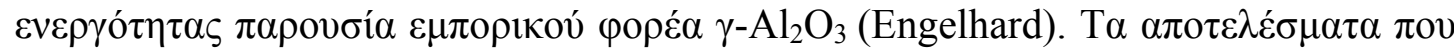

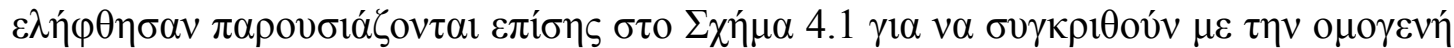

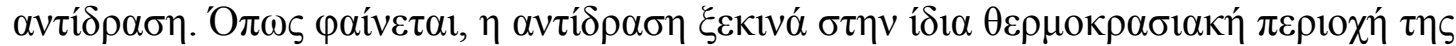

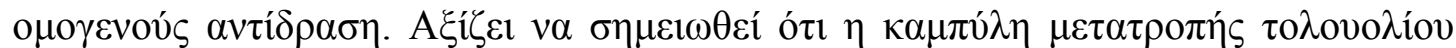

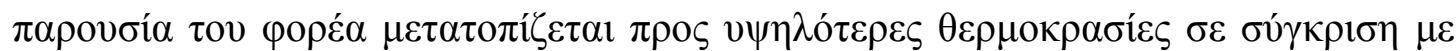

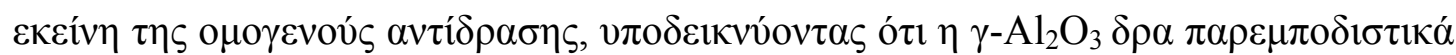

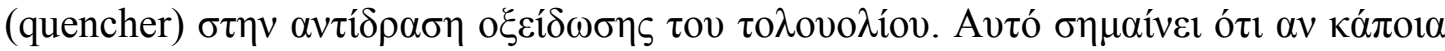

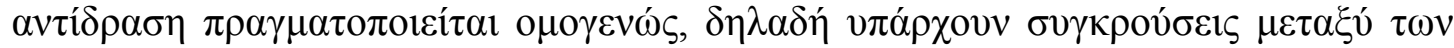

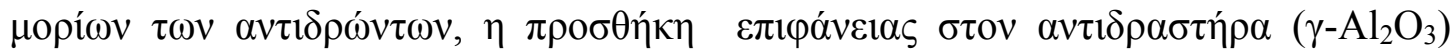

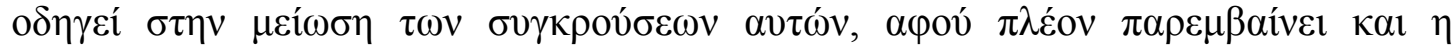

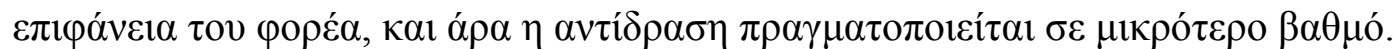




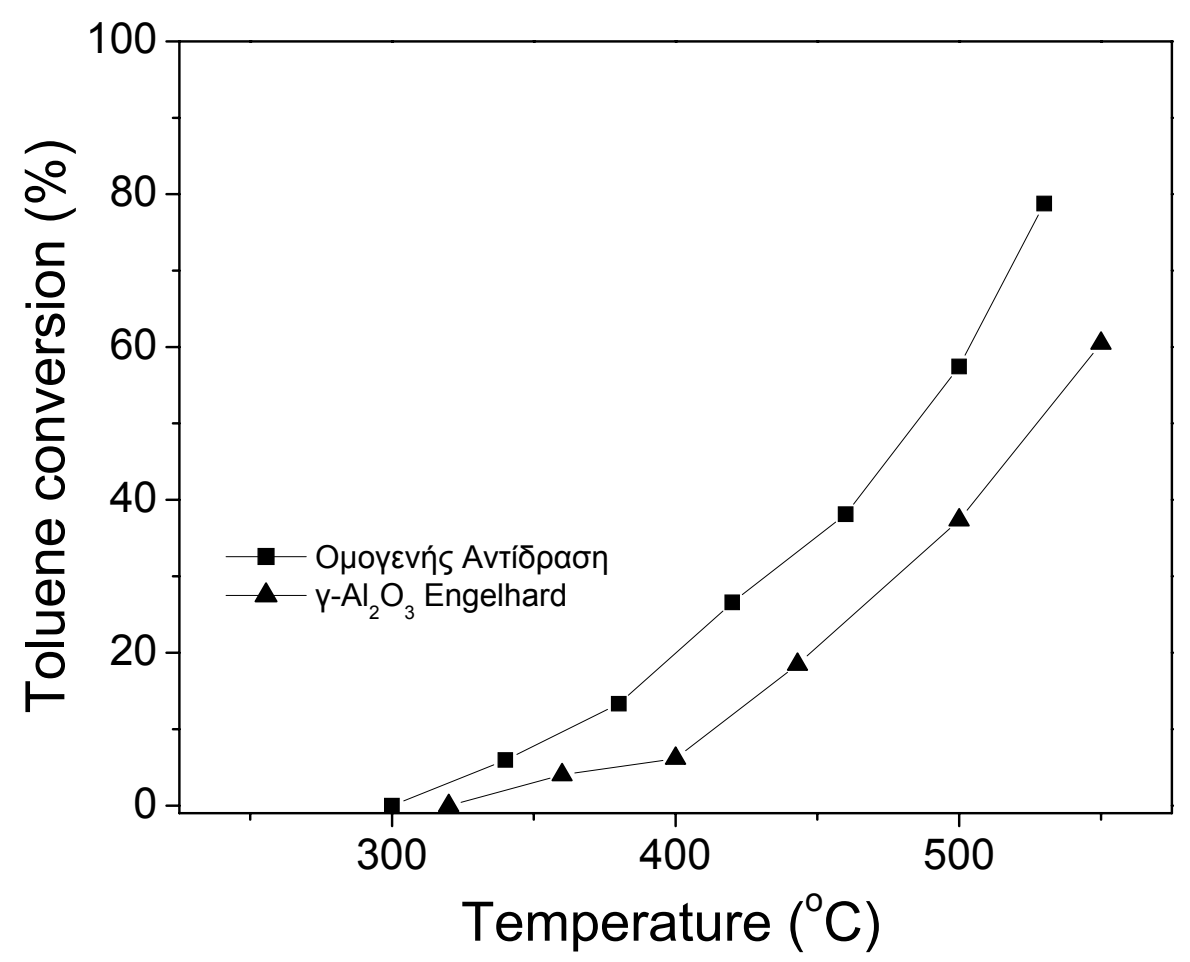

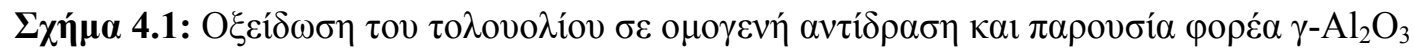

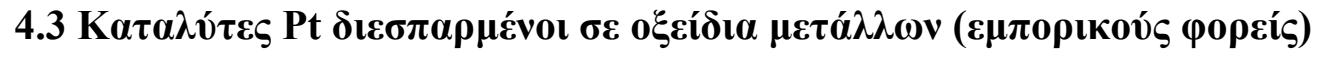

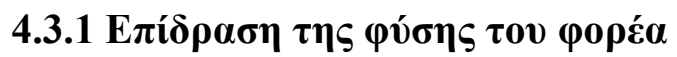

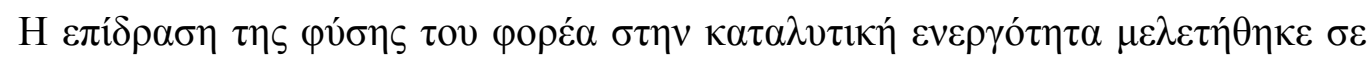

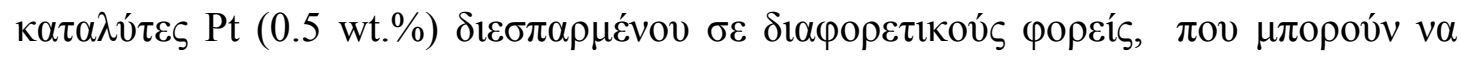

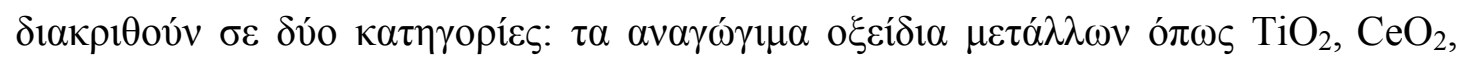

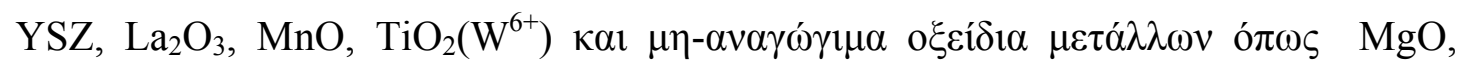

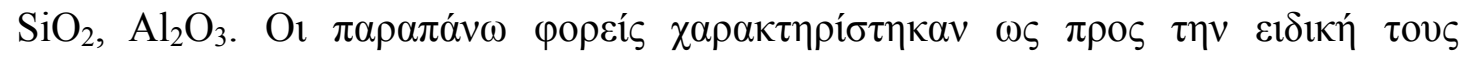

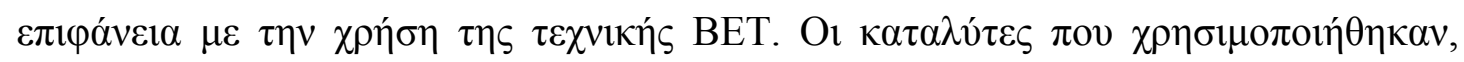

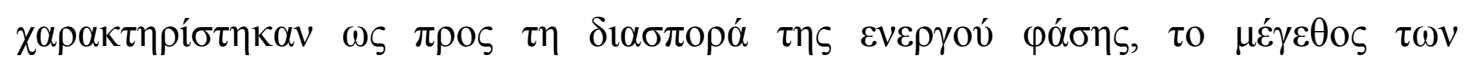

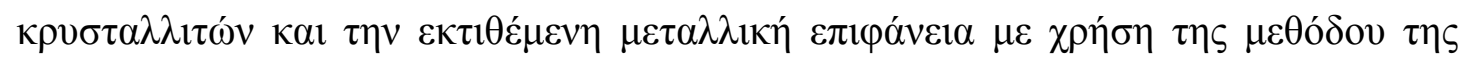

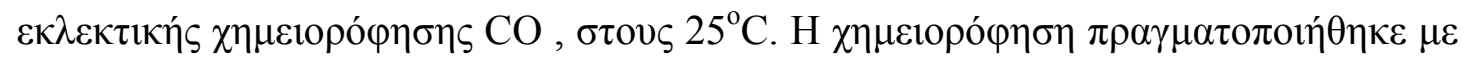

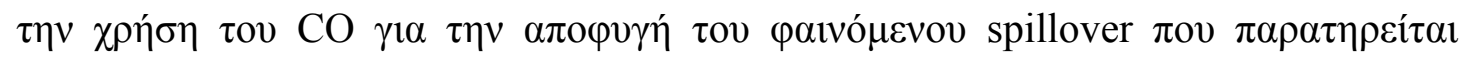

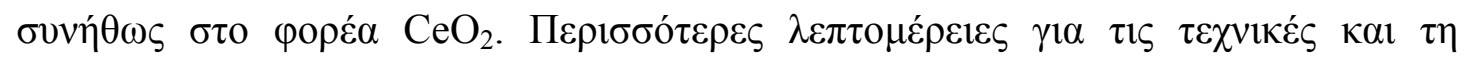

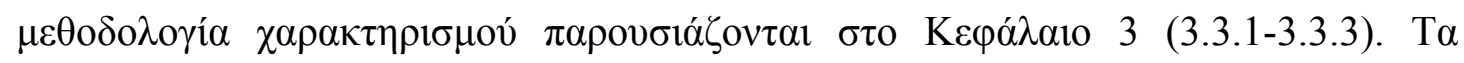

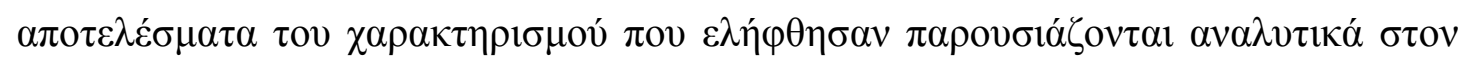

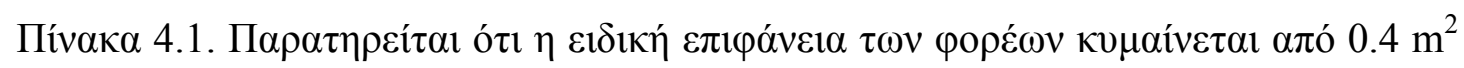




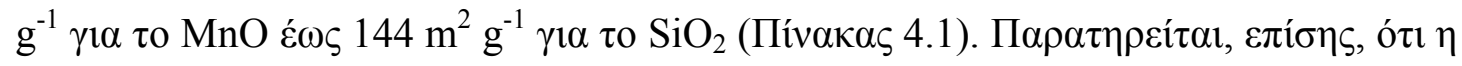

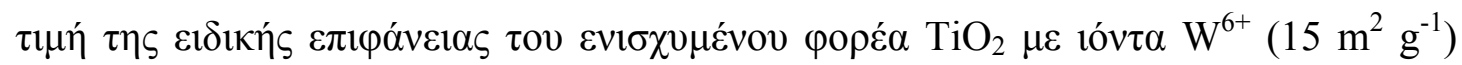

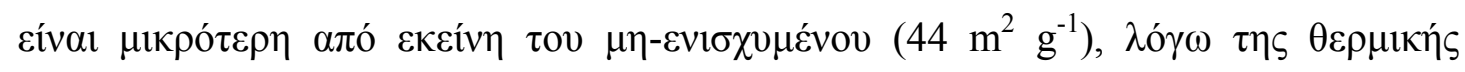

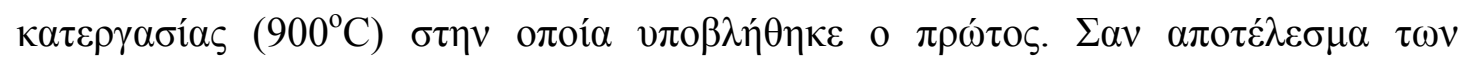

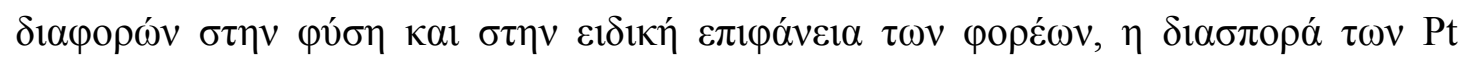

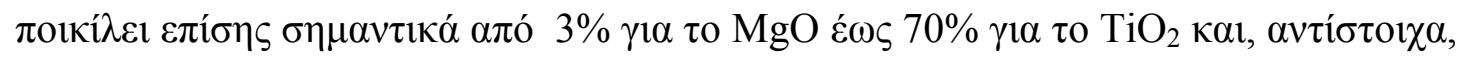

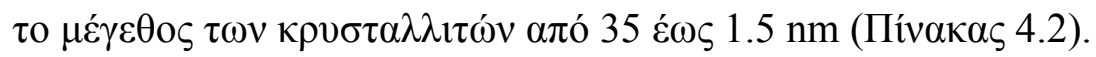

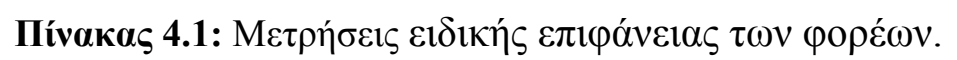

\begin{tabular}{|c|c|c|c|}
\hline Форе́ $\alpha \varsigma$ & 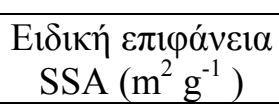 & Форе́ $\alpha \varsigma$ & 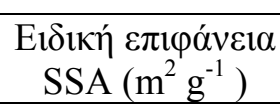 \\
\hline $\mathrm{SiO}_{2}$ & 144 & YSZ & 12 \\
\hline $\mathrm{Al}_{2} \mathrm{O}_{3}$ & 83 & $\mathrm{La}_{2} \mathrm{O}_{3}$ & 7 \\
\hline $\mathrm{TiO}_{2}$ & 44 & $\mathrm{ZrO}_{2}$ & 4 \\
\hline $\mathrm{MgO}$ & 22 & $\mathrm{CeO}_{2}$ & 3 \\
\hline $\mathrm{TiO}_{2}\left(\mathrm{~W}^{6+}\right)$ & 15 & $\mathrm{MnO}$ & 0.4 \\
\hline
\end{tabular}

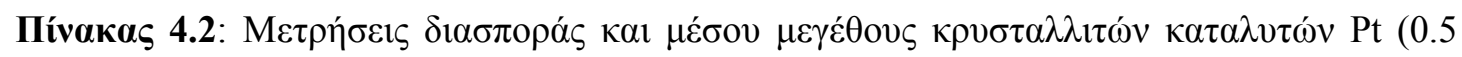

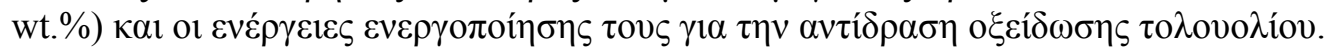

\begin{tabular}{|c|c|c|c|}
\hline 0.5 wt. $\%$ Pt/M $\mathrm{M}_{x} \mathrm{O}_{y}$ & $\begin{array}{c}\Delta \mathrm{t} \alpha \sigma \pi \mathrm{\rho} \rho \alpha ́ \\
\text { (D) }(\%)\end{array}$ & 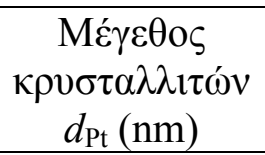 & 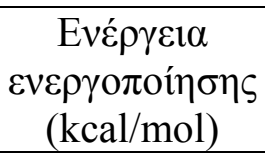 \\
\hline $\mathrm{SiO}_{2}$ & 53 & 1.9 & 53.1 \\
\hline $\mathrm{Al}_{2} \mathrm{O}_{3}$ & 62 & 1.7 & 85.2 \\
\hline $\mathrm{TiO}_{2}$ & 70 & 1.5 & 28.9 \\
\hline $\mathrm{MgO}$ & 3 & 35 & 52 \\
\hline $\mathrm{La}_{2} \mathrm{O}_{3}$ & 11 & 8.9 & 27.4 \\
\hline $\mathrm{ZrO}_{2}$ & 30 & 3.3 & 51.6 \\
\hline $\mathrm{CeO}_{2}$ & 41 & 2.5 & 34.3 \\
\hline $\mathrm{MnO}$ & 9 & 11.5 & 42.6 \\
\hline $\mathrm{TiO}_{2}\left(\mathrm{~W}^{6+}\right)$ & 25.7 & 4 & 43.3 \\
\hline YSZ & 22.9 & 4.4 & 138.5 \\
\hline
\end{tabular}




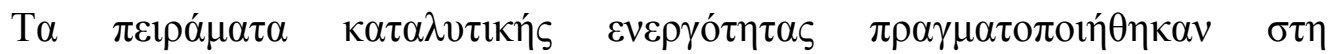

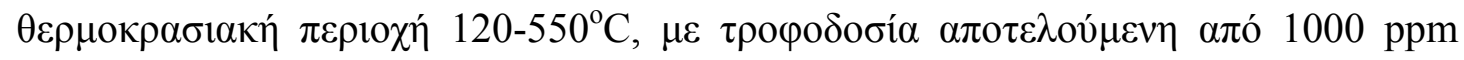

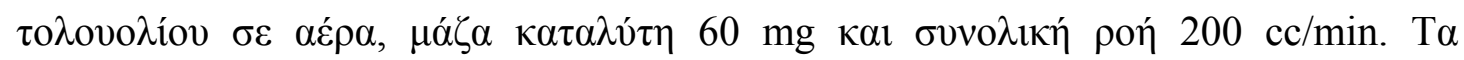

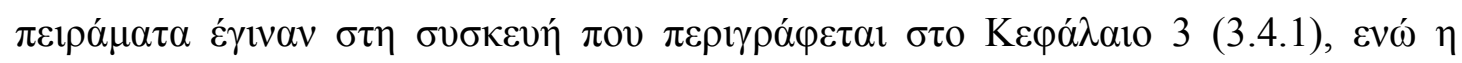

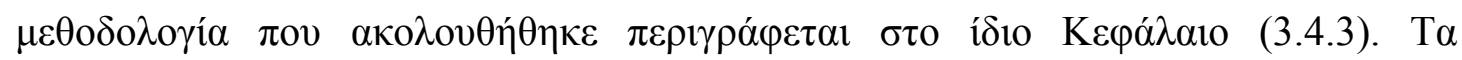

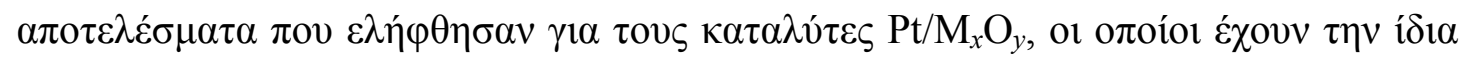

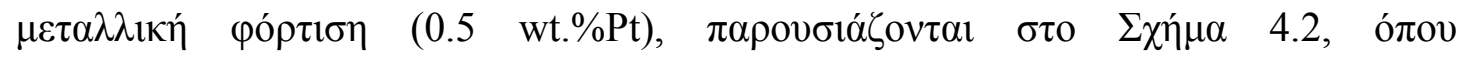

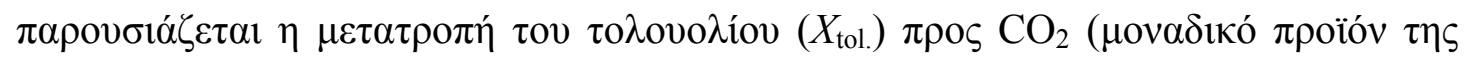

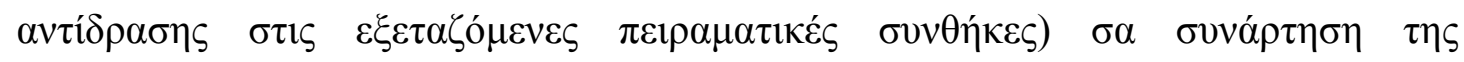

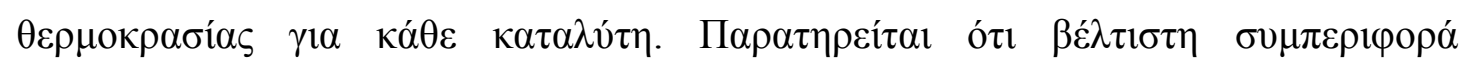

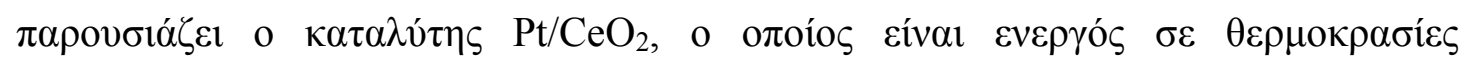

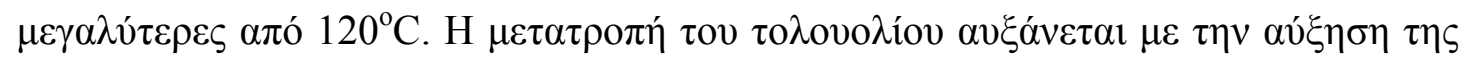

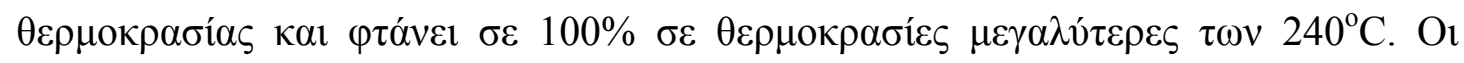

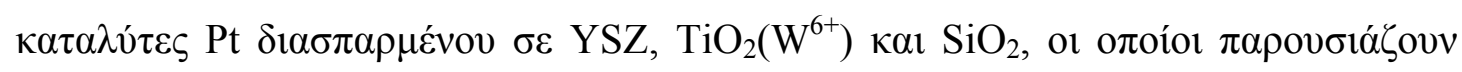

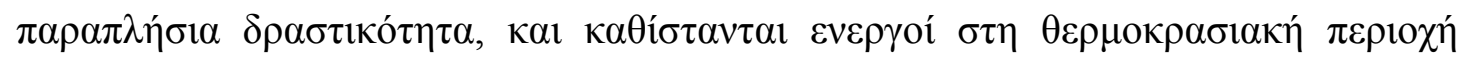

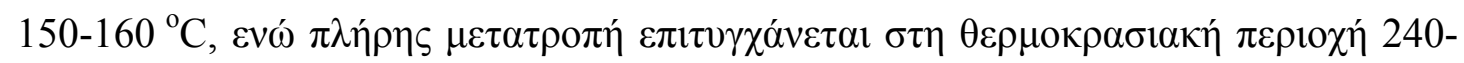

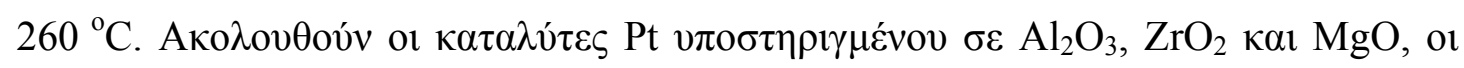

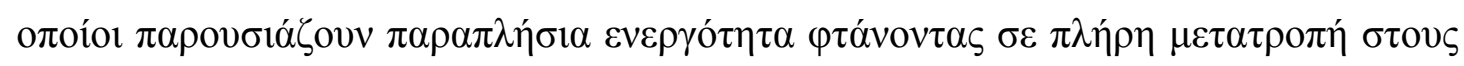

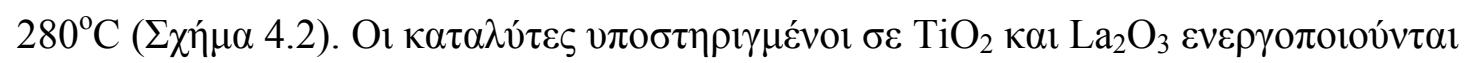

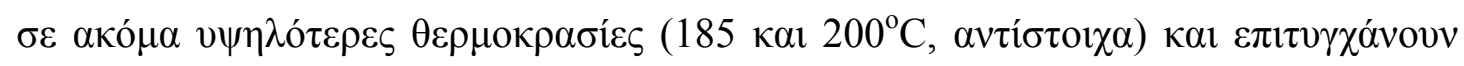

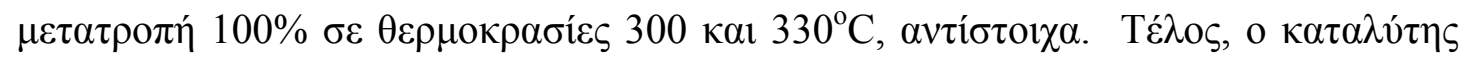

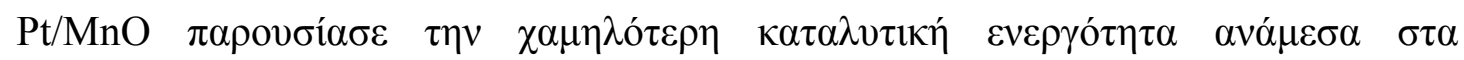

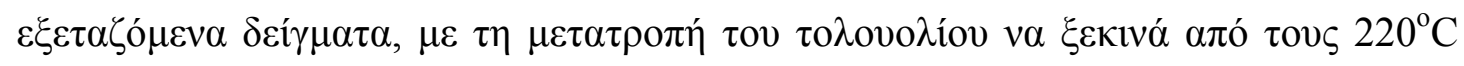

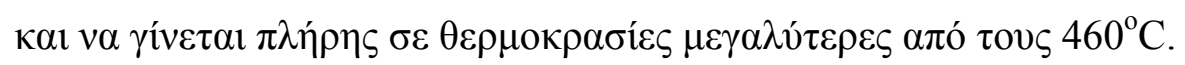

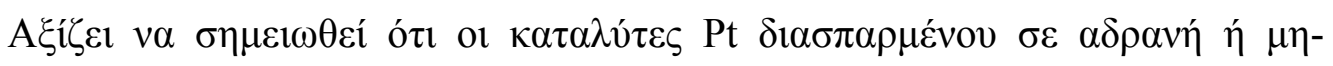

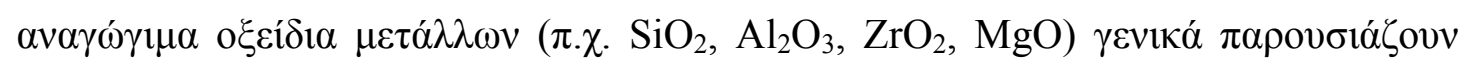

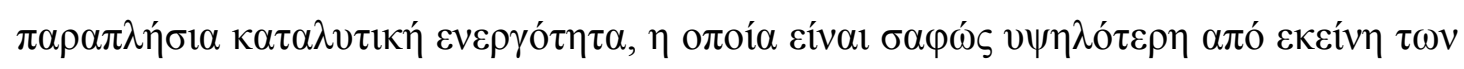

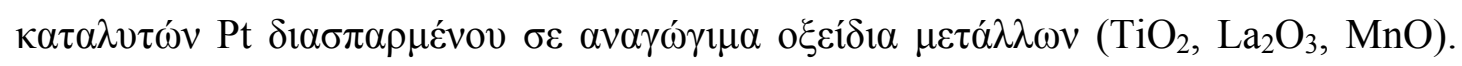

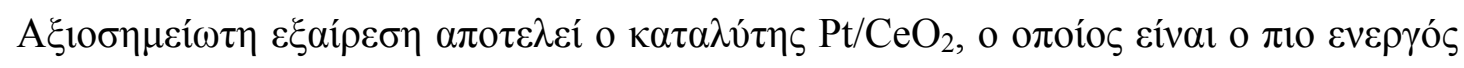

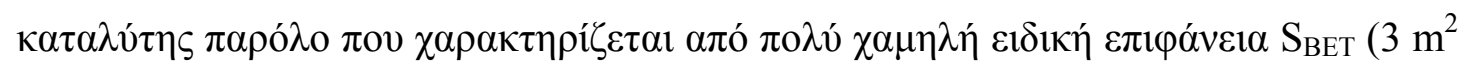
$\left.\mathrm{g}^{-1}\right)$. 




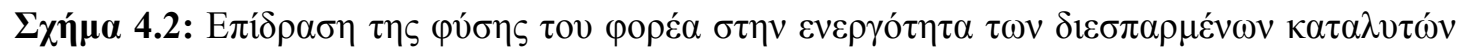
$\operatorname{Pt}(0.5$ wt.\%).

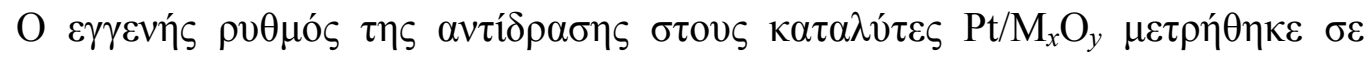

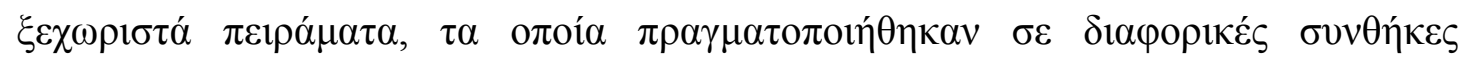

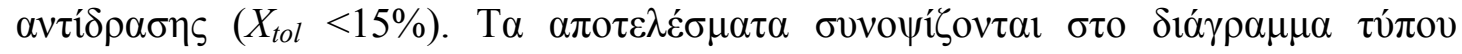

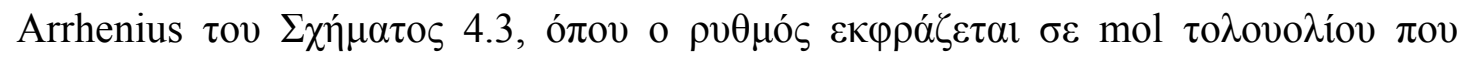

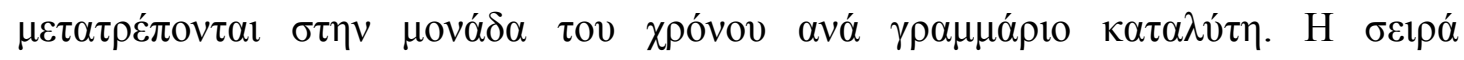

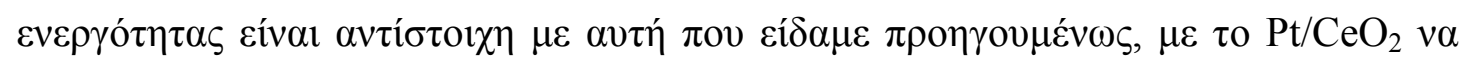

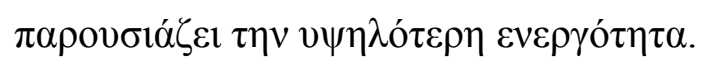

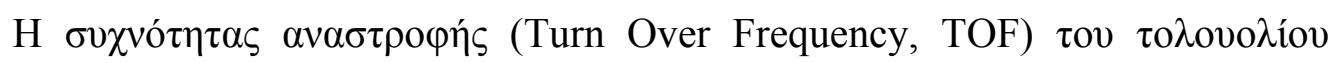

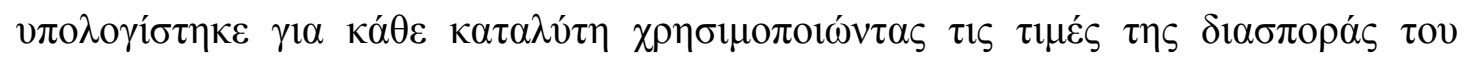

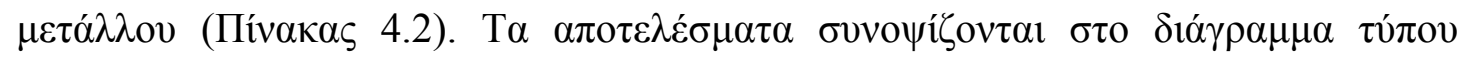

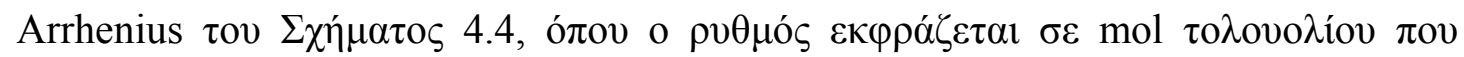

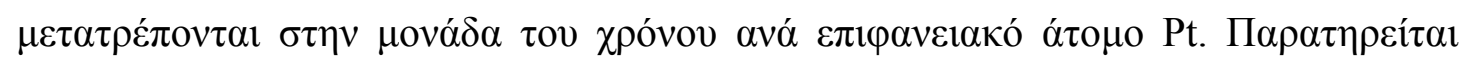

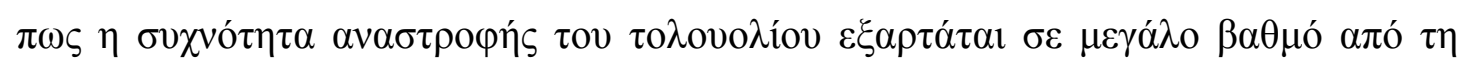

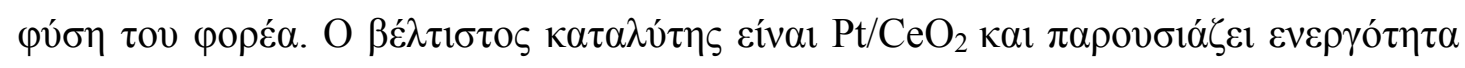

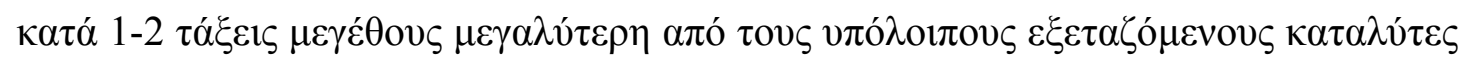

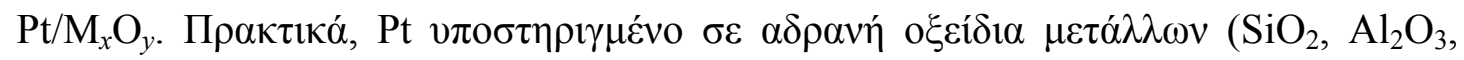

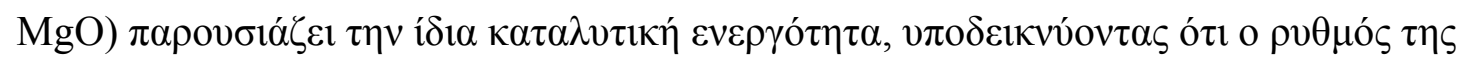




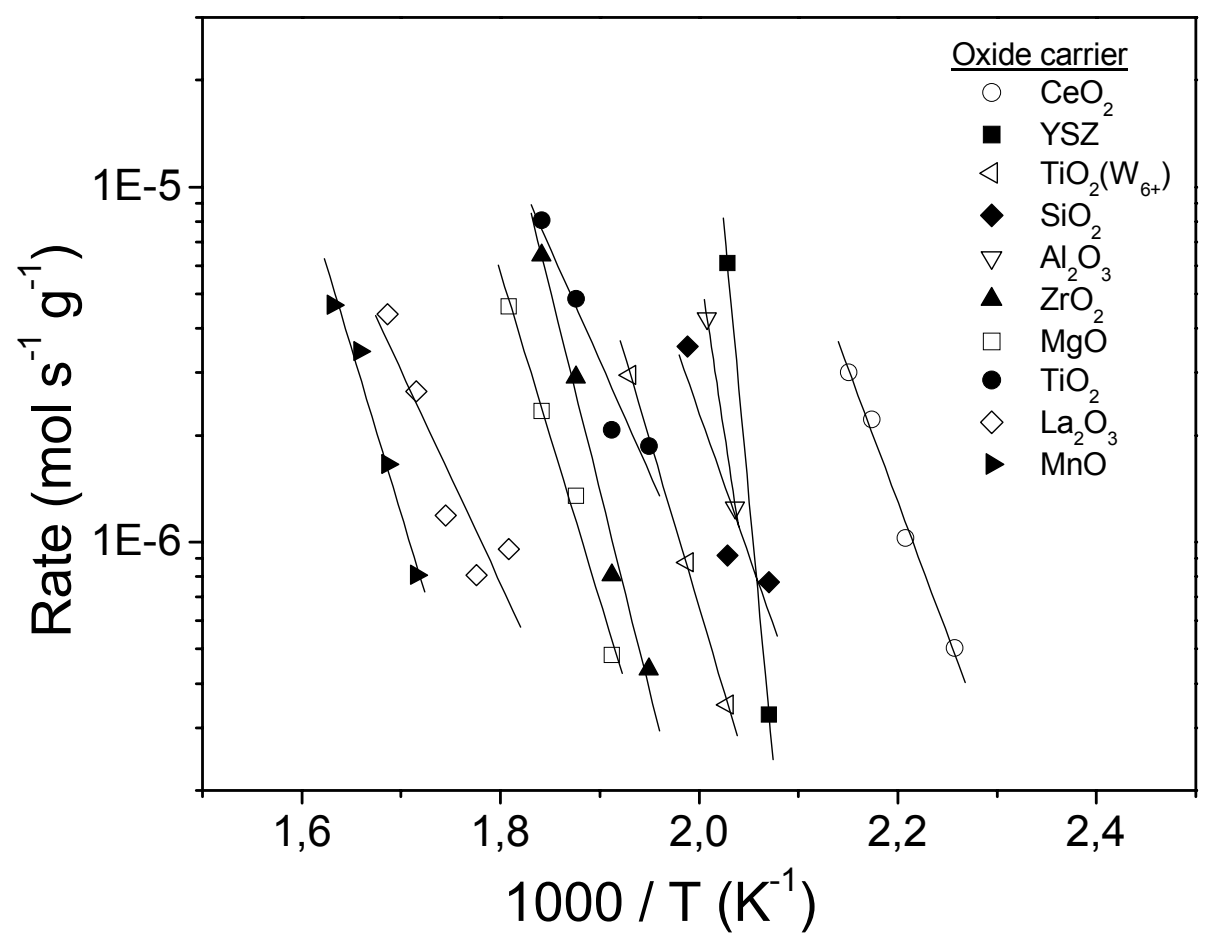

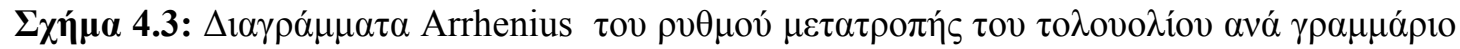
$\kappa \alpha \tau \alpha \lambda \dot{\tau} \tau \eta \sigma \varepsilon \kappa \alpha \tau \alpha \lambda v ́ \tau \varepsilon \varsigma 0.5 \% \mathrm{Pt} / \mathrm{M}_{x} \mathrm{O}_{y}$

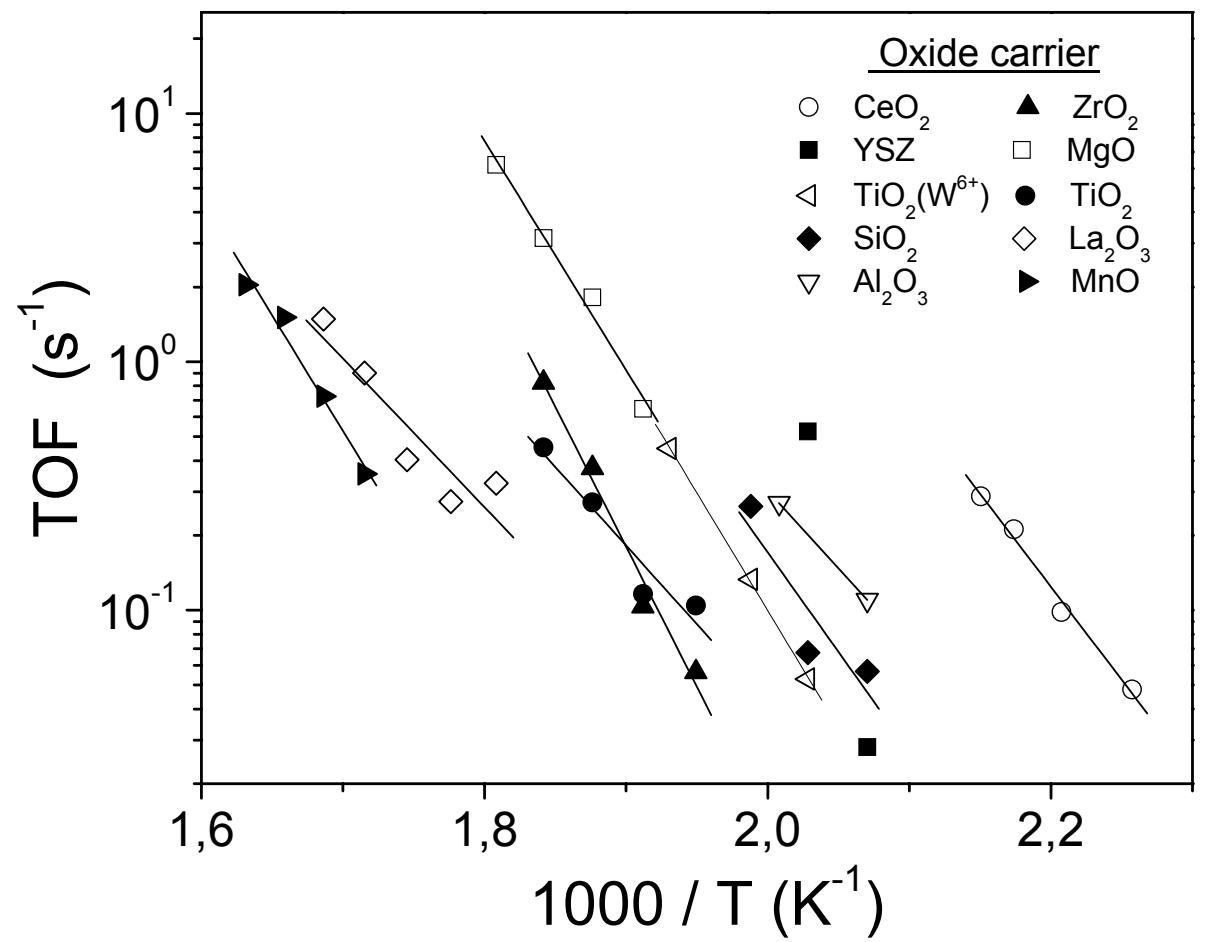

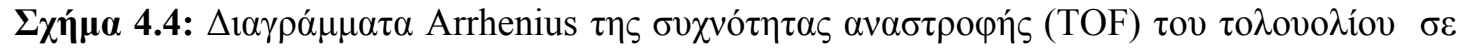

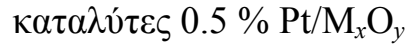




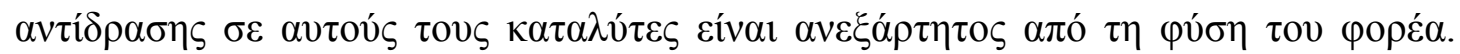

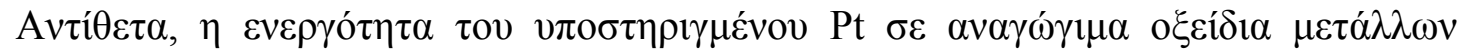

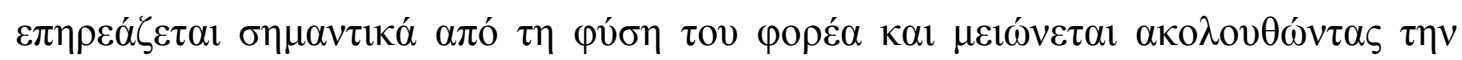

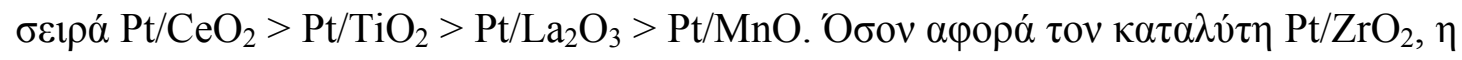

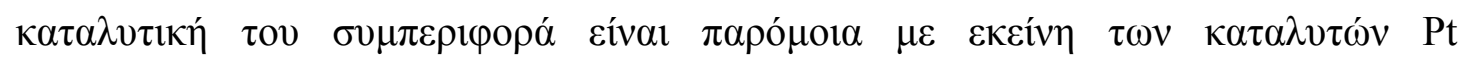

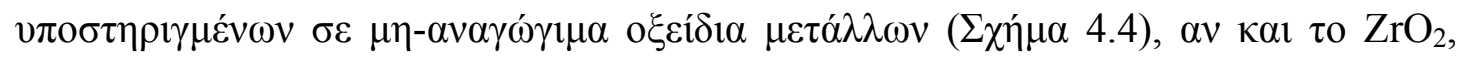

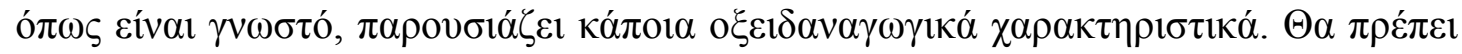

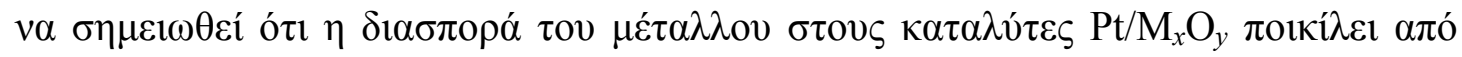

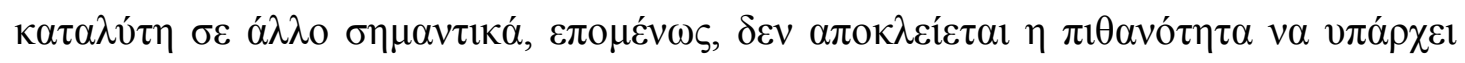

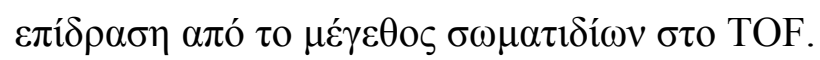

A

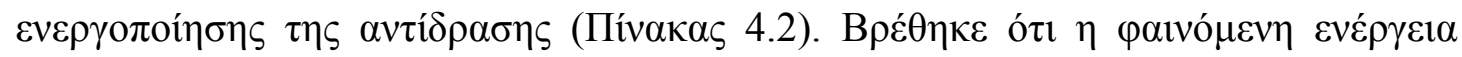

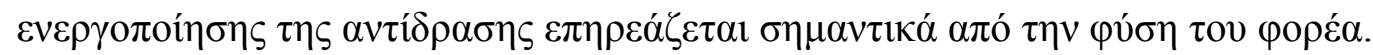

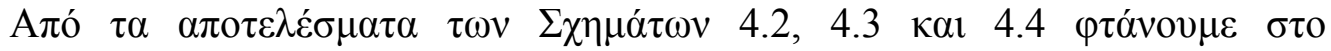

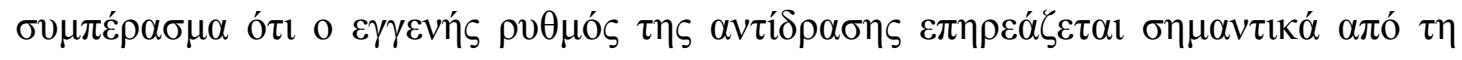

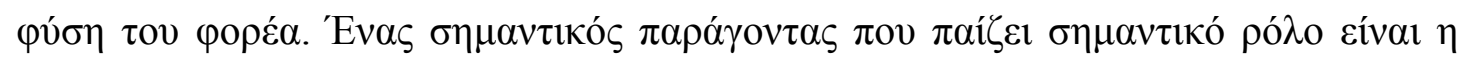

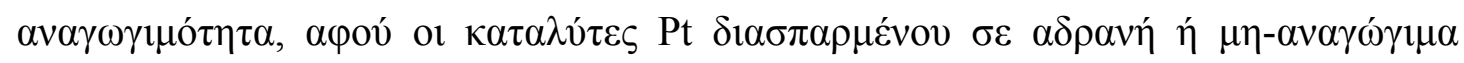

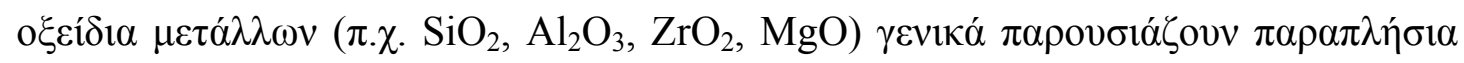

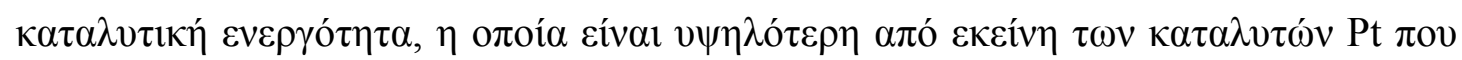

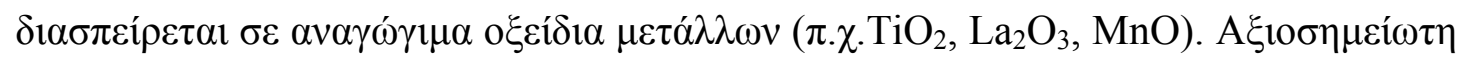

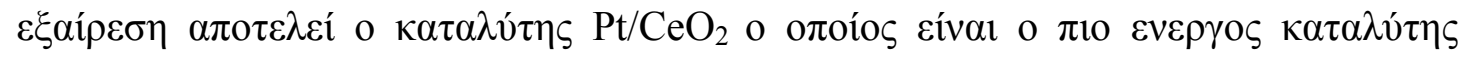

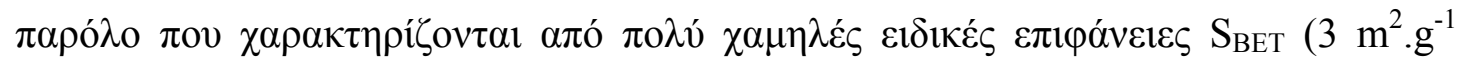

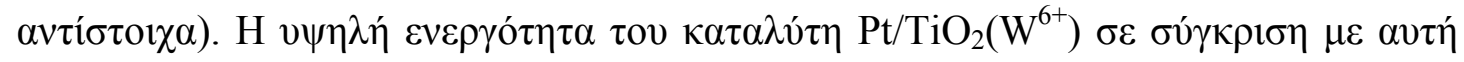

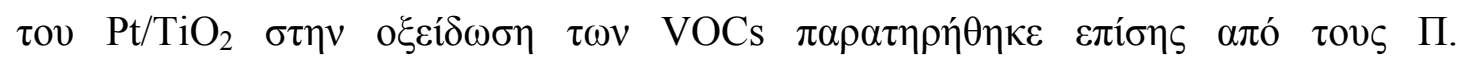

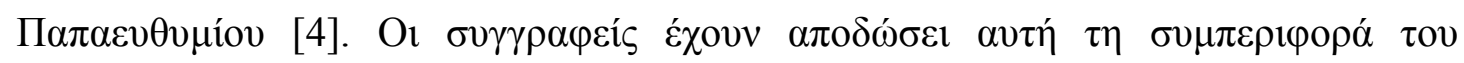

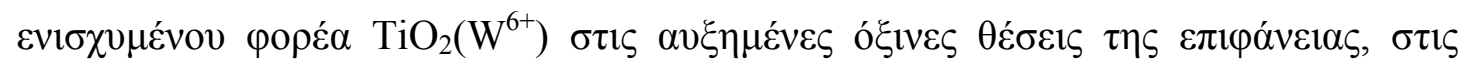

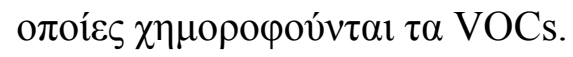

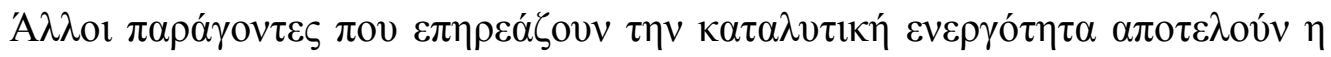
$\varepsilon \imath \delta 1 \kappa \eta ́ ~ \varepsilon \pi \imath \varphi \alpha ́ v \varepsilon ı \alpha \mathrm{S}_{\mathrm{BET}} \kappa \alpha \imath \eta \eta$

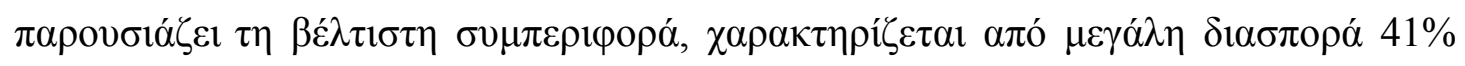

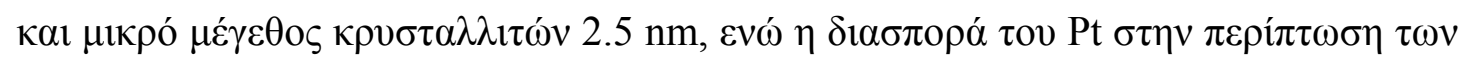

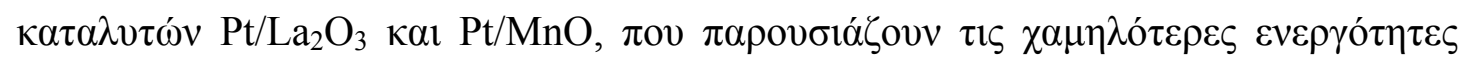

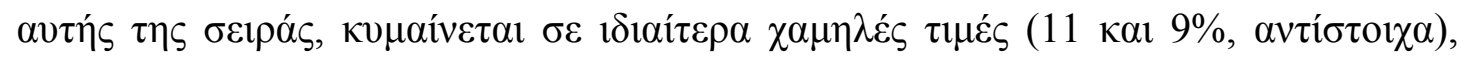

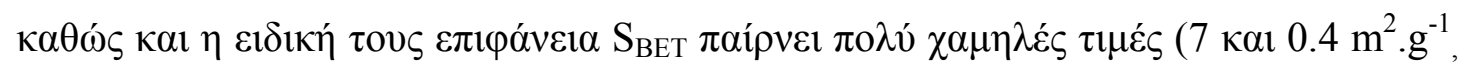




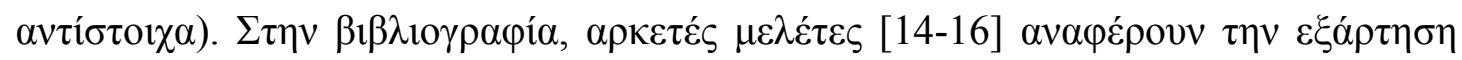

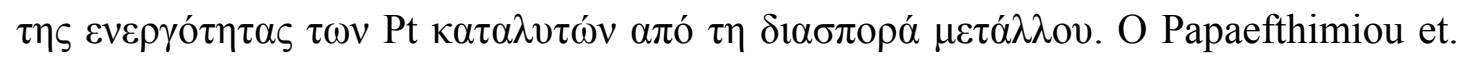

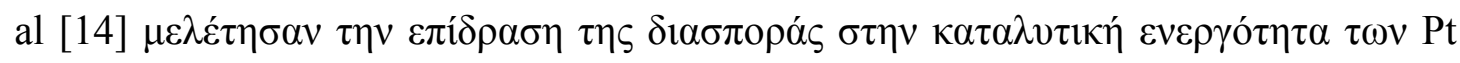

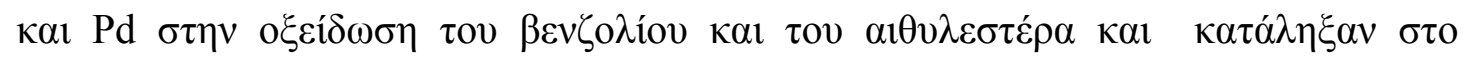

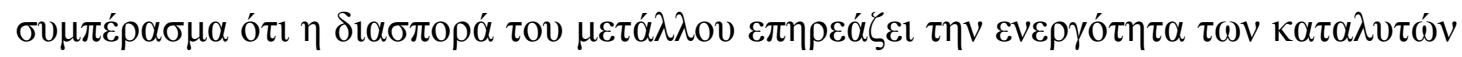

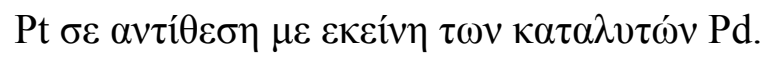

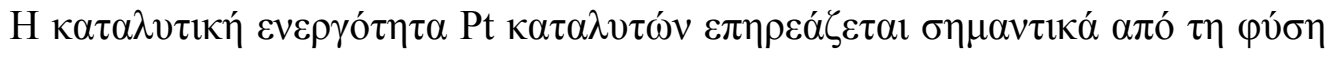

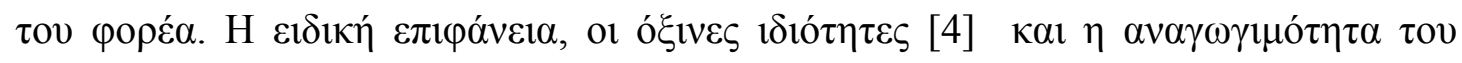

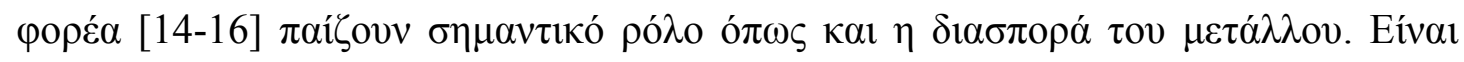

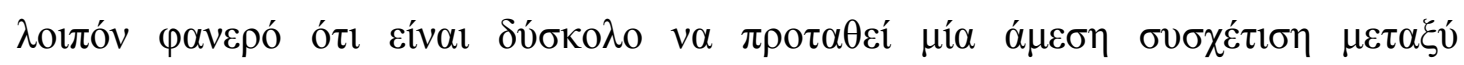

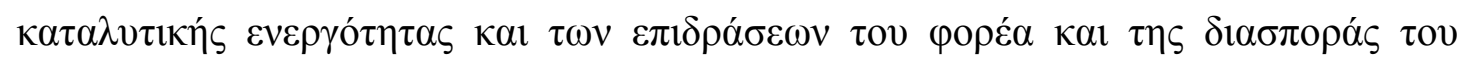
$\mu \varepsilon \tau \dot{\alpha} \lambda \lambda \mathrm{ov}$.

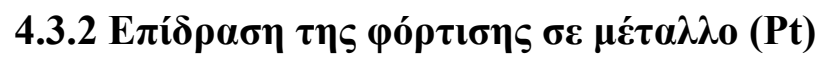

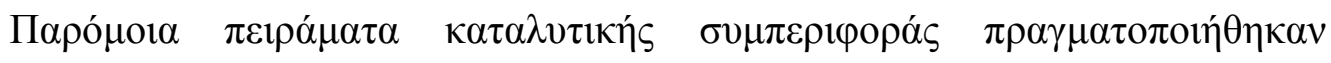

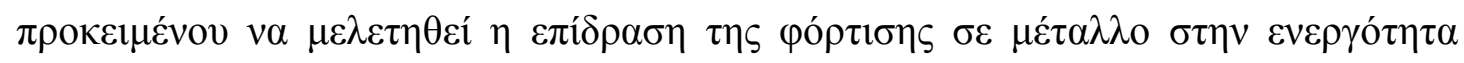

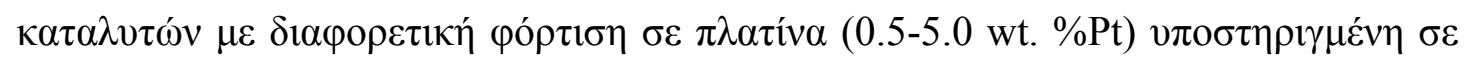

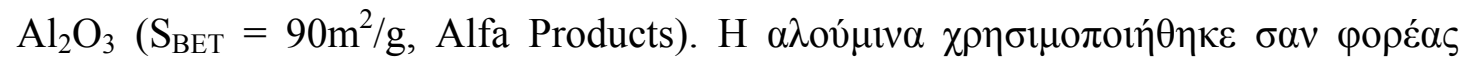

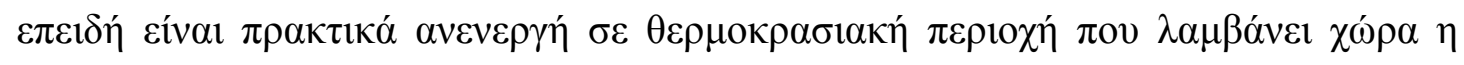
$\alpha v \tau i ́ \delta \rho \alpha \eta \eta$.

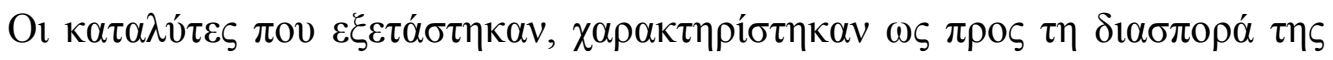

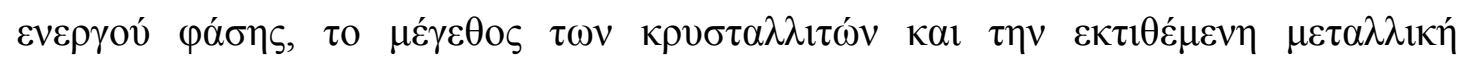

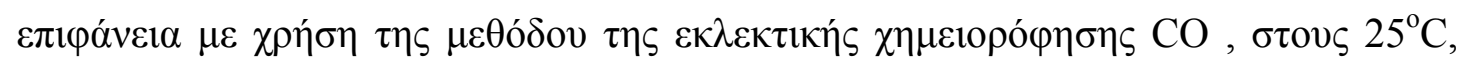

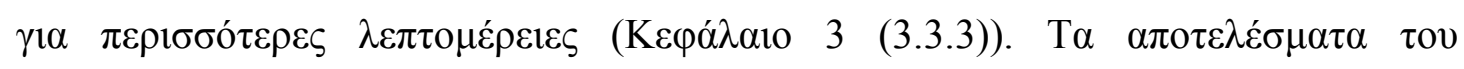

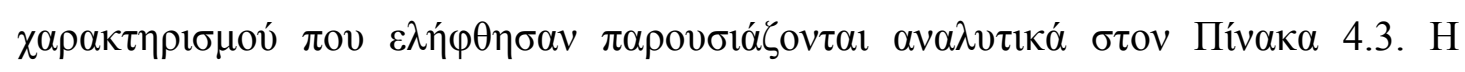

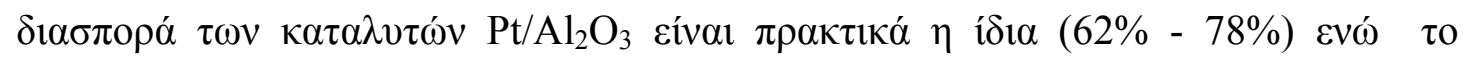

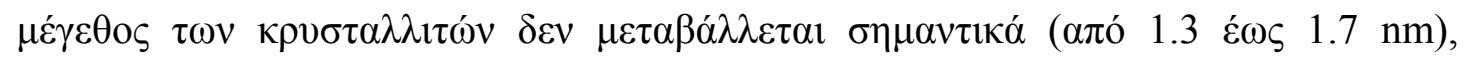
(Пívakas 4.3). 


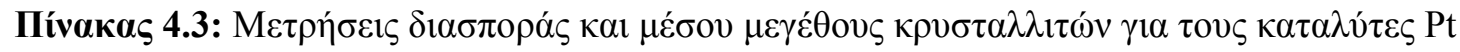
$(0.5-5$ wt. $\%) / \mathrm{Al}_{2} \mathrm{O}_{3}$.

\begin{tabular}{|c|c|c|}
\hline wt.\% Pt/ $\mathrm{Al}_{2} \mathrm{O}_{3}$ & $\begin{array}{c}\Delta 1 \alpha \sigma \pi \mathrm{o} \rho \dot{\alpha} \\
\text { (D) (\%) }\end{array}$ & 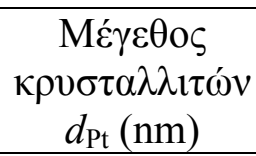 \\
\hline 0.5 & 62 & 1.7 \\
\hline 1 & 74 & 1.4 \\
\hline 2 & 78 & 1.3 \\
\hline 5 & 75 & 1.4 \\
\hline
\end{tabular}

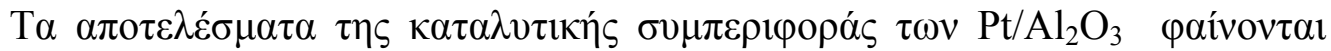

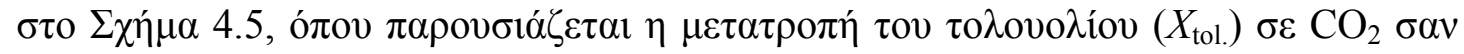

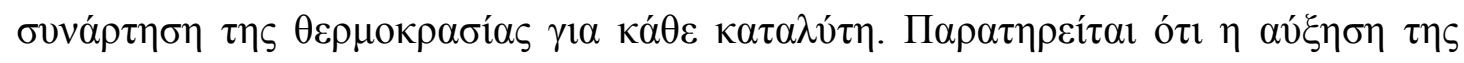

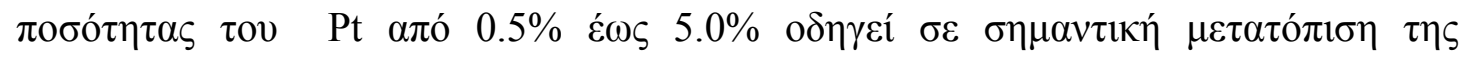

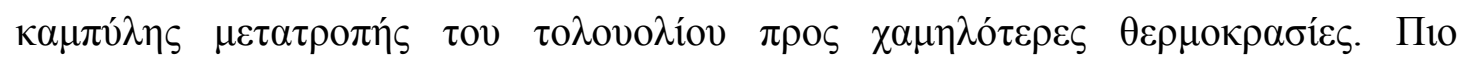

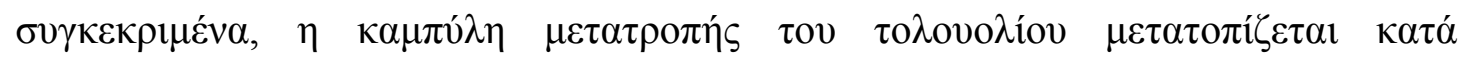

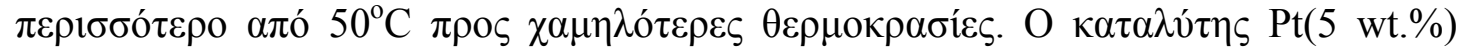

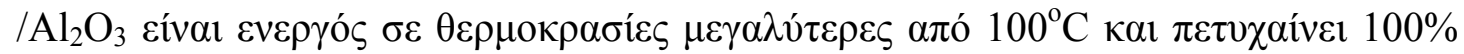

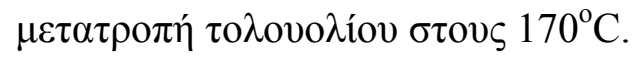

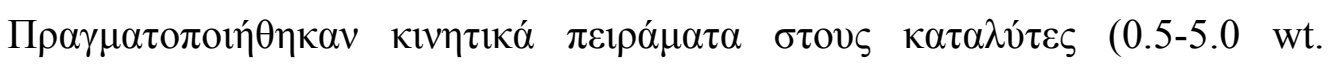

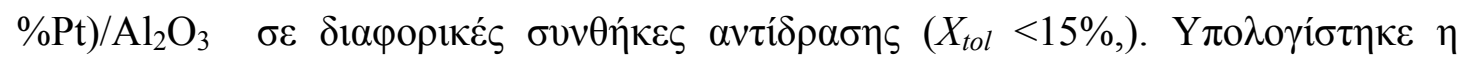

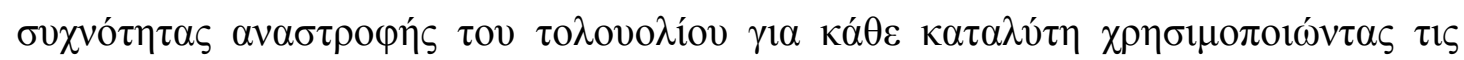

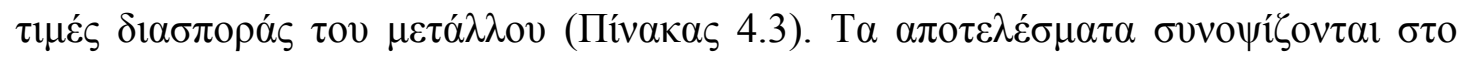

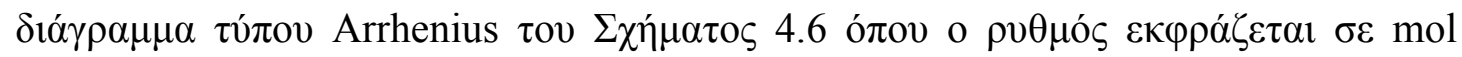

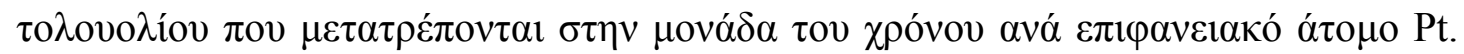

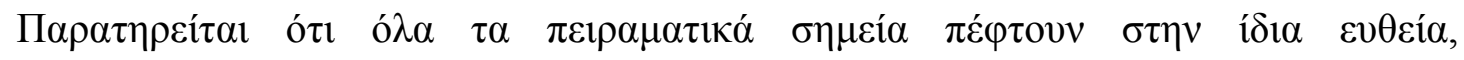

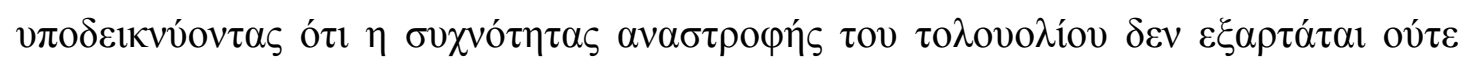

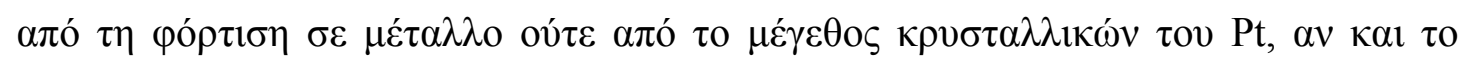

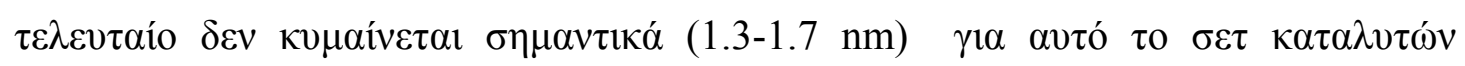

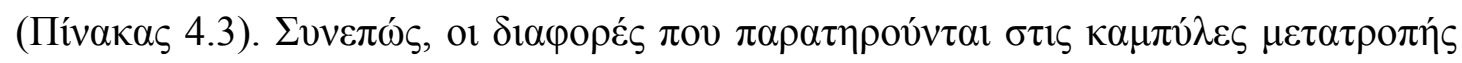

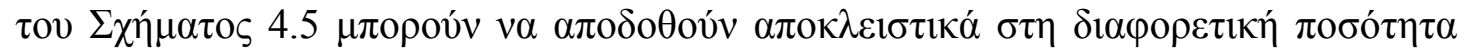

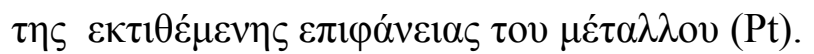




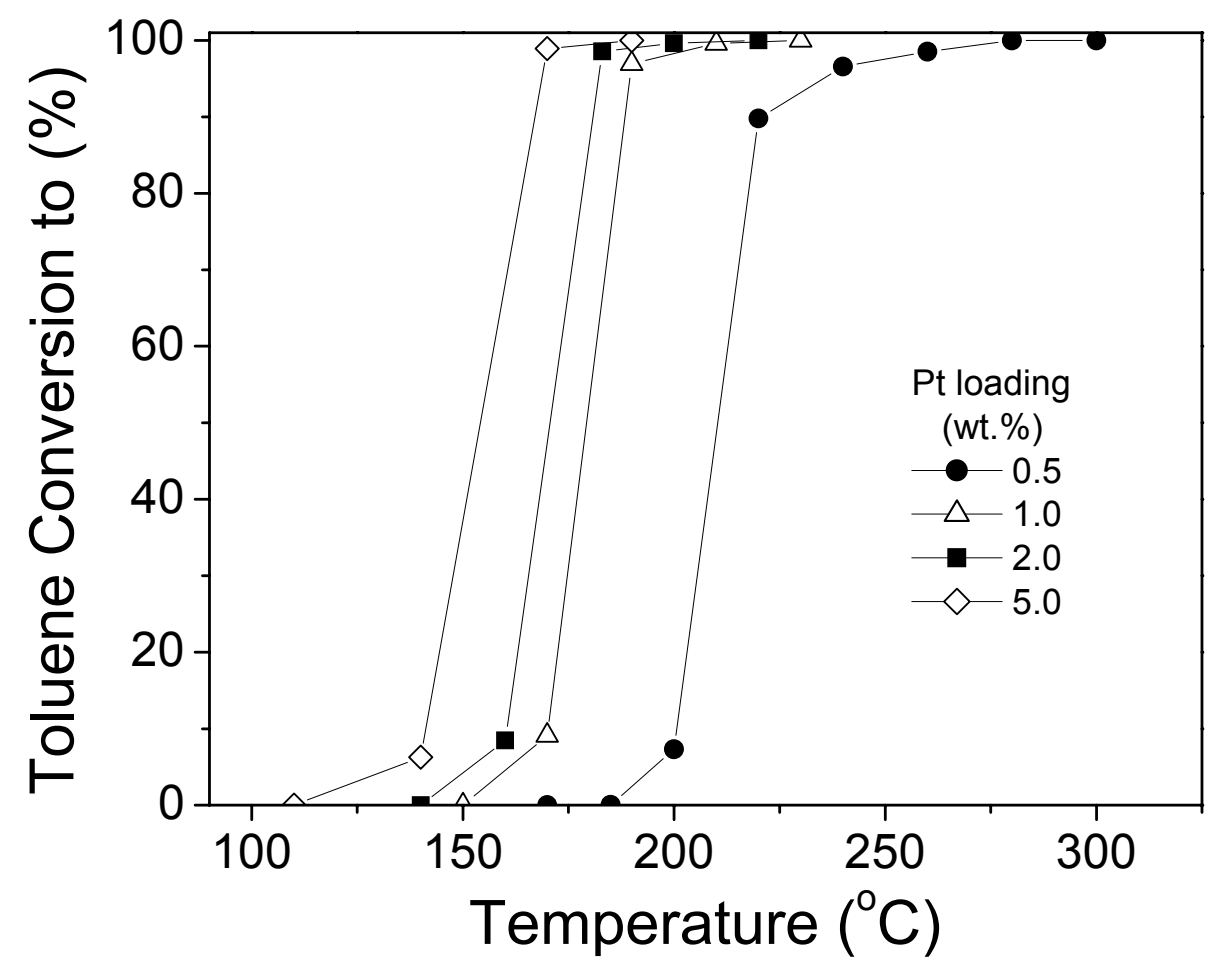

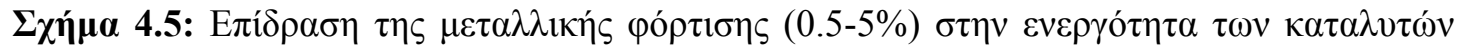
$\mathrm{Pt} / \mathrm{Al}_{2} \mathrm{O}_{3}$.

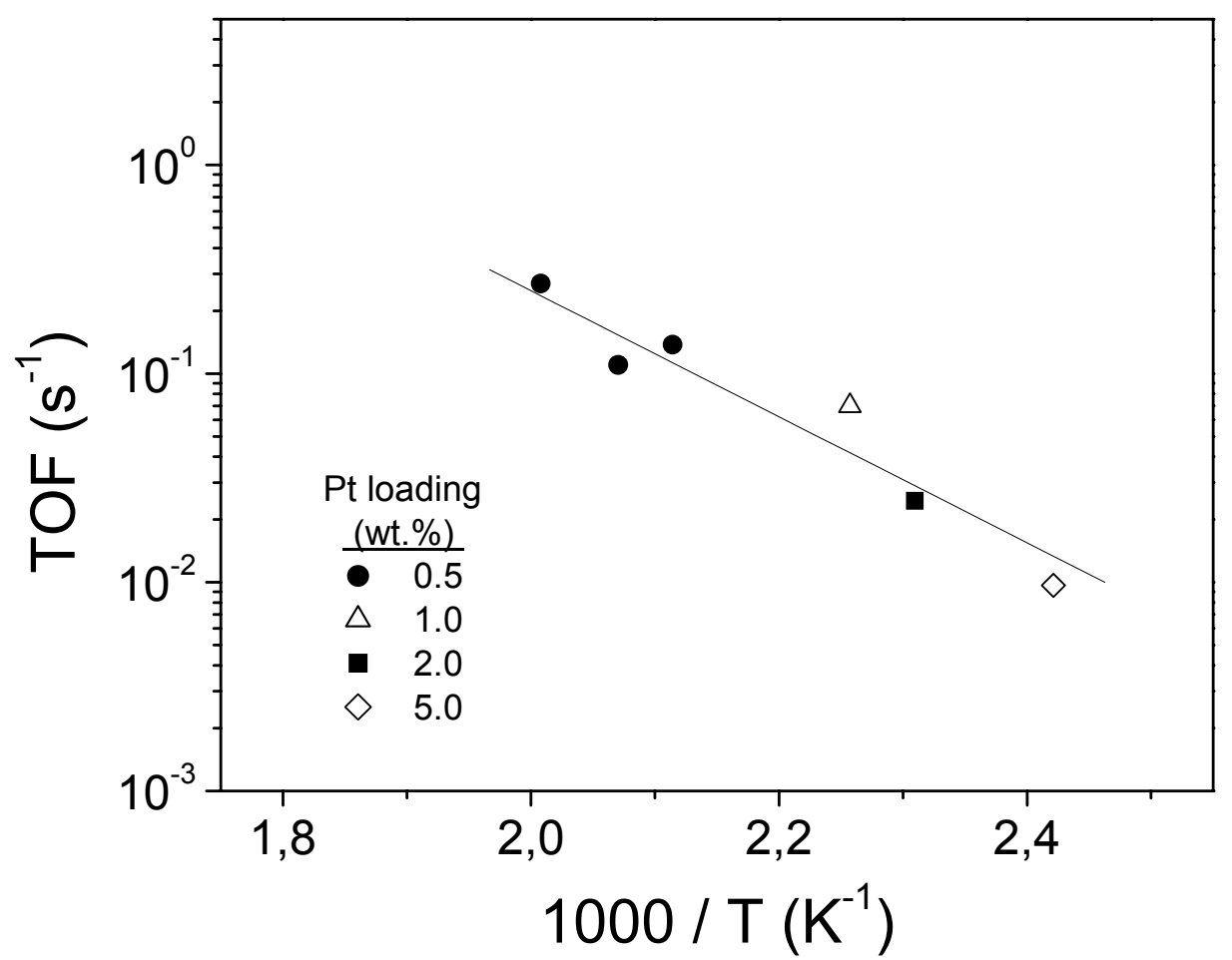

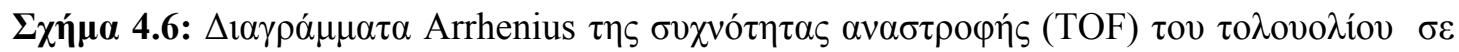

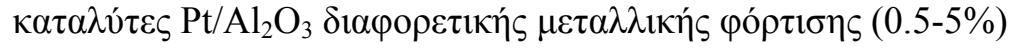




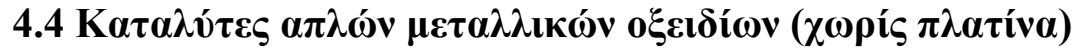

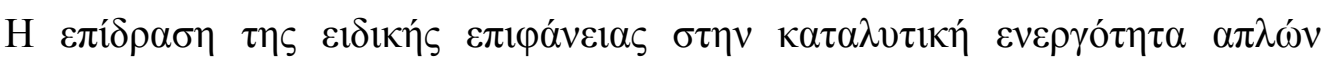

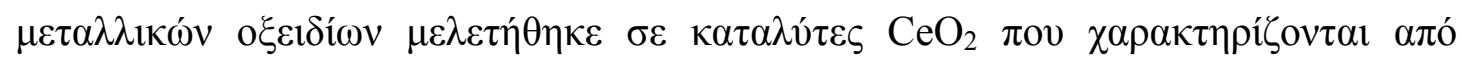

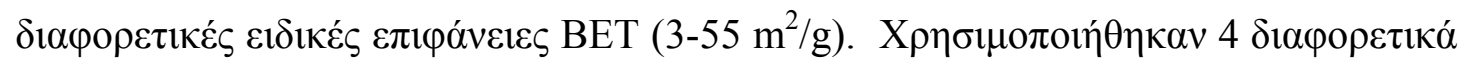

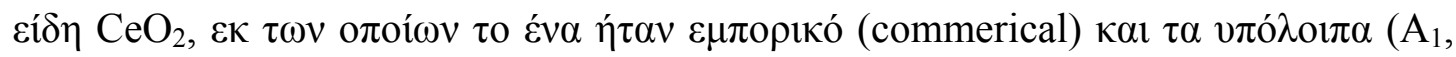

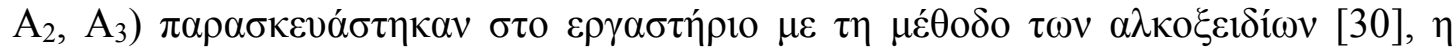

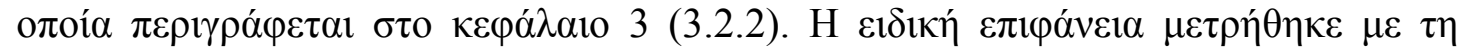

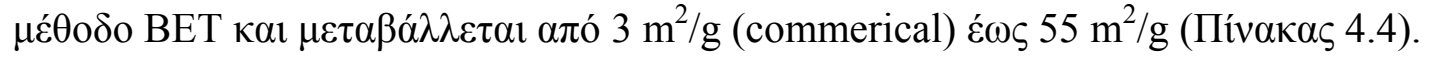

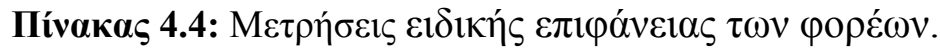

\begin{tabular}{|c|c|}
\hline Форе́as & 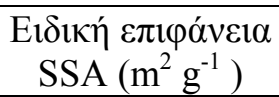 \\
\hline commercial & 3 \\
\hline $\mathrm{A}_{1}$ & 28 \\
\hline $\mathrm{A}_{2}$ & 32 \\
\hline $\mathrm{A}_{3}$ & 55 \\
\hline
\end{tabular}

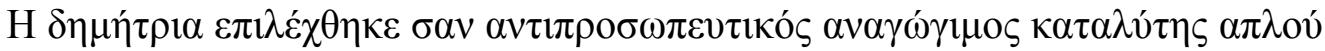

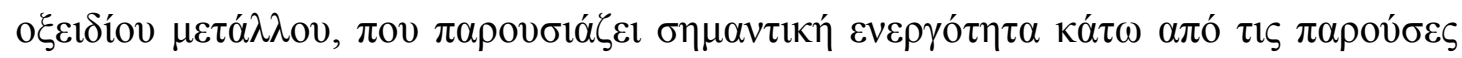

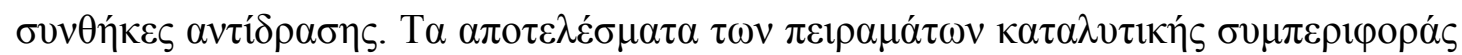

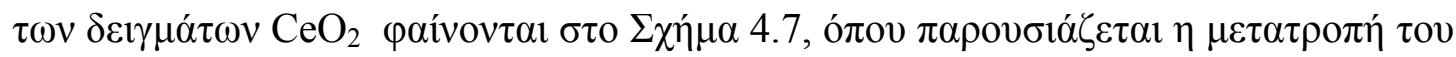

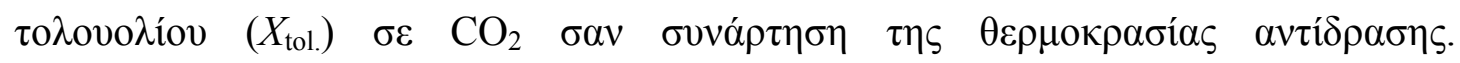

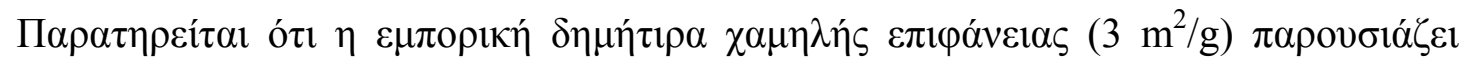

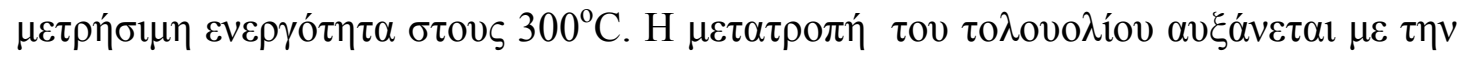

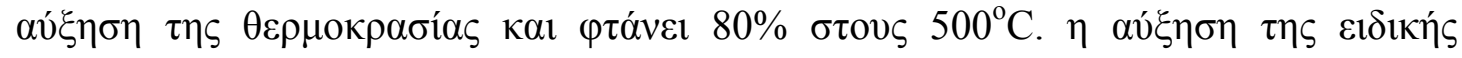

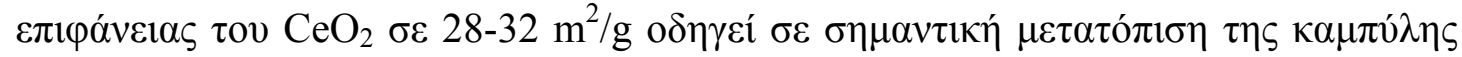

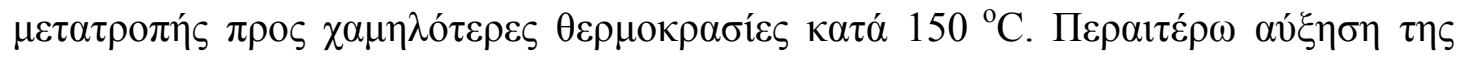

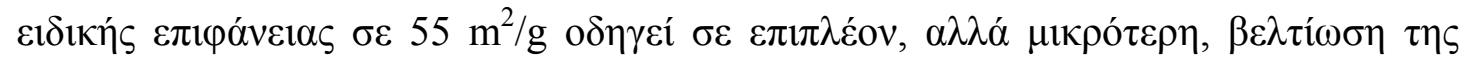

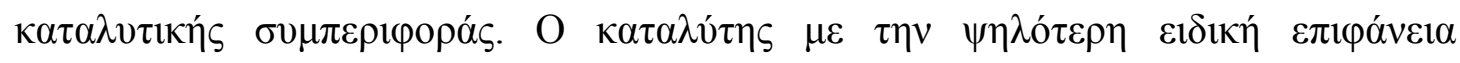

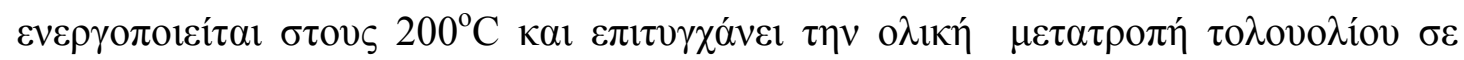

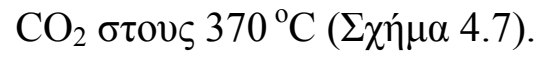




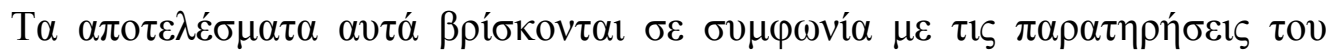

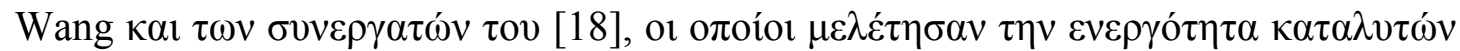

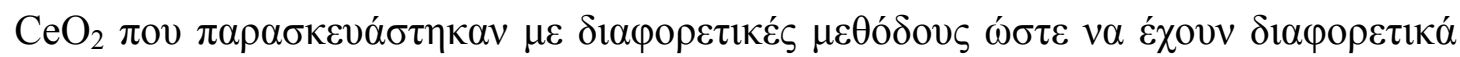

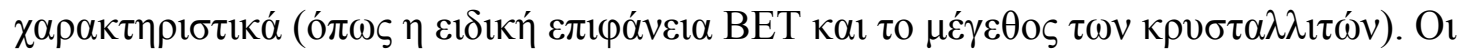

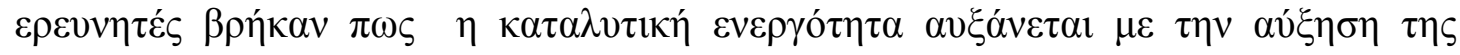

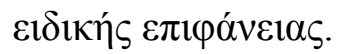

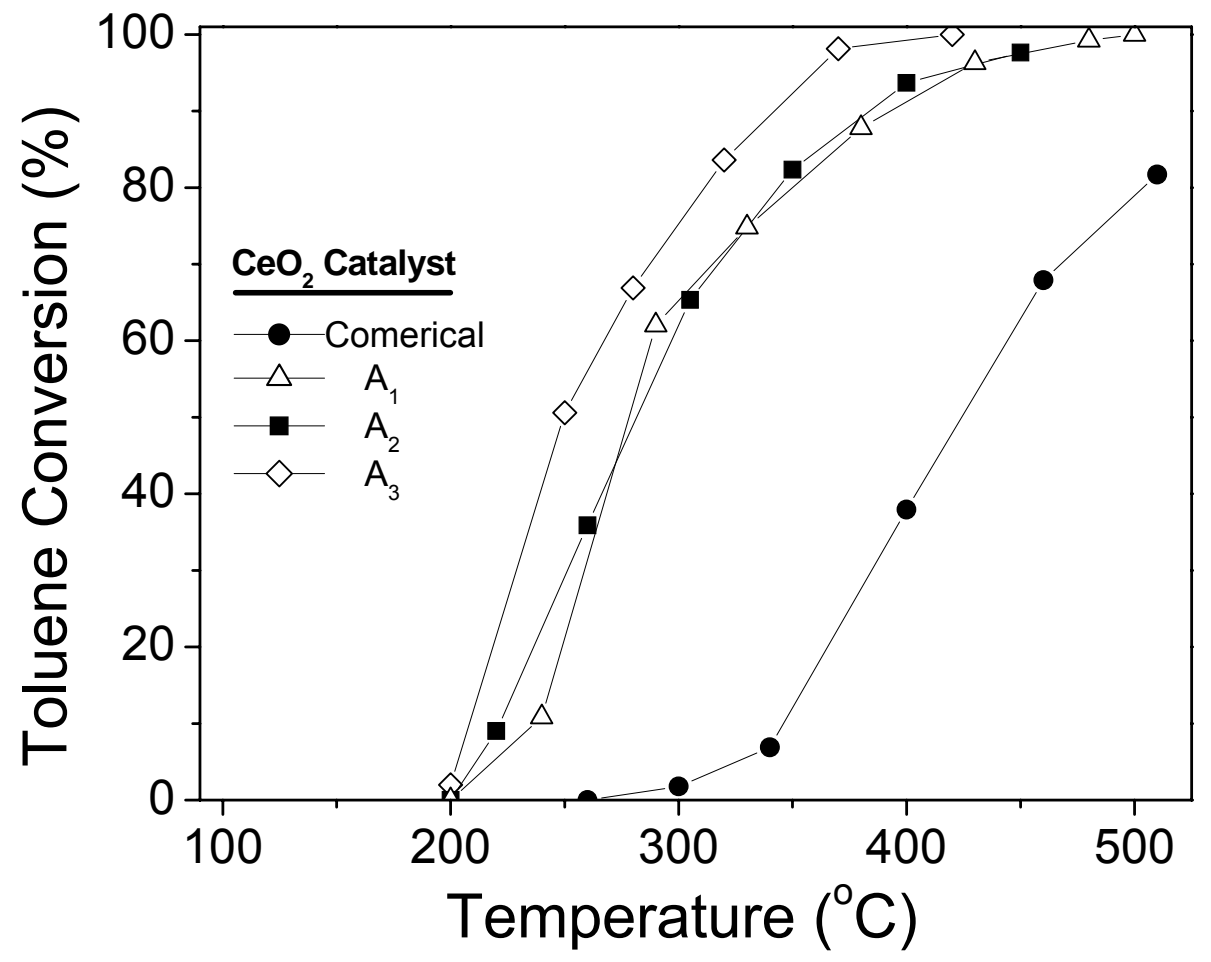

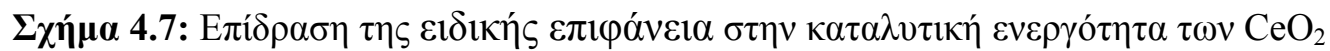

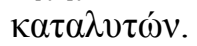

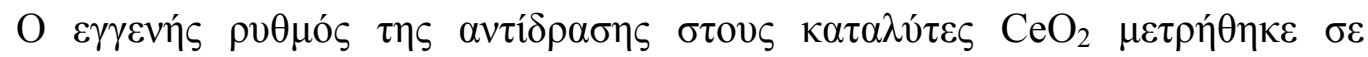

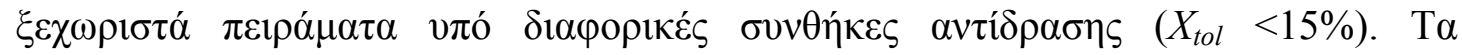

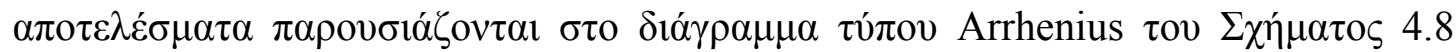

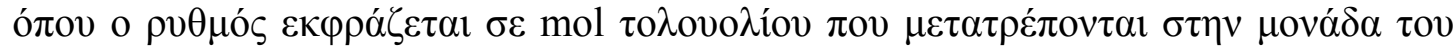

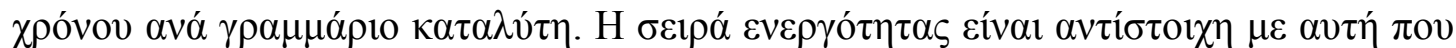

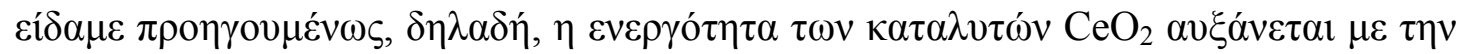

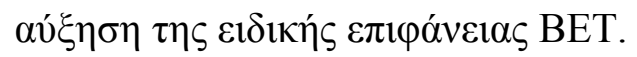




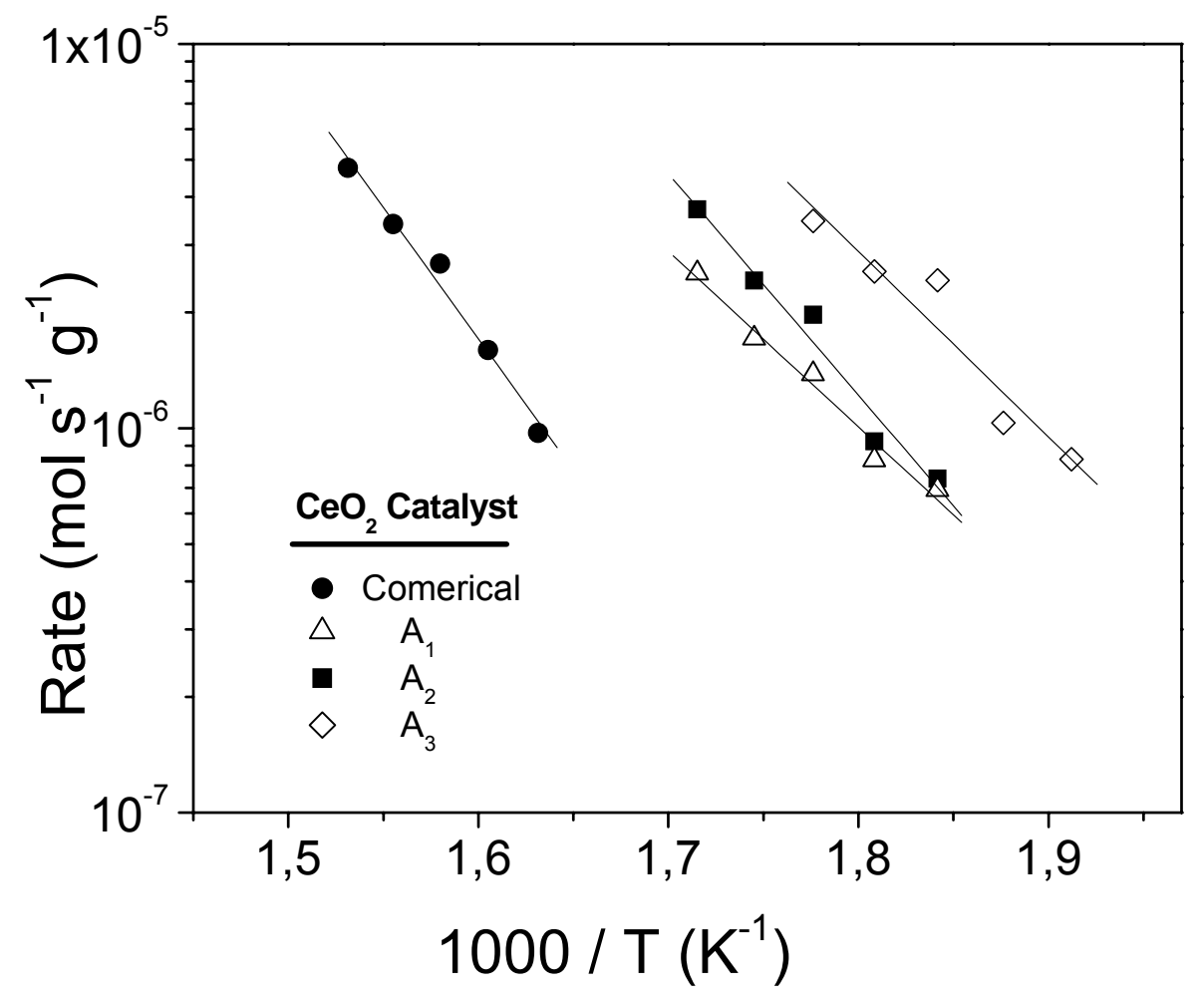

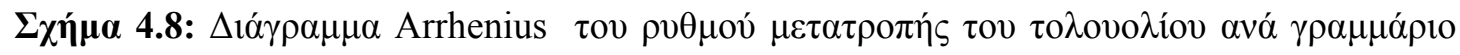

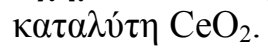

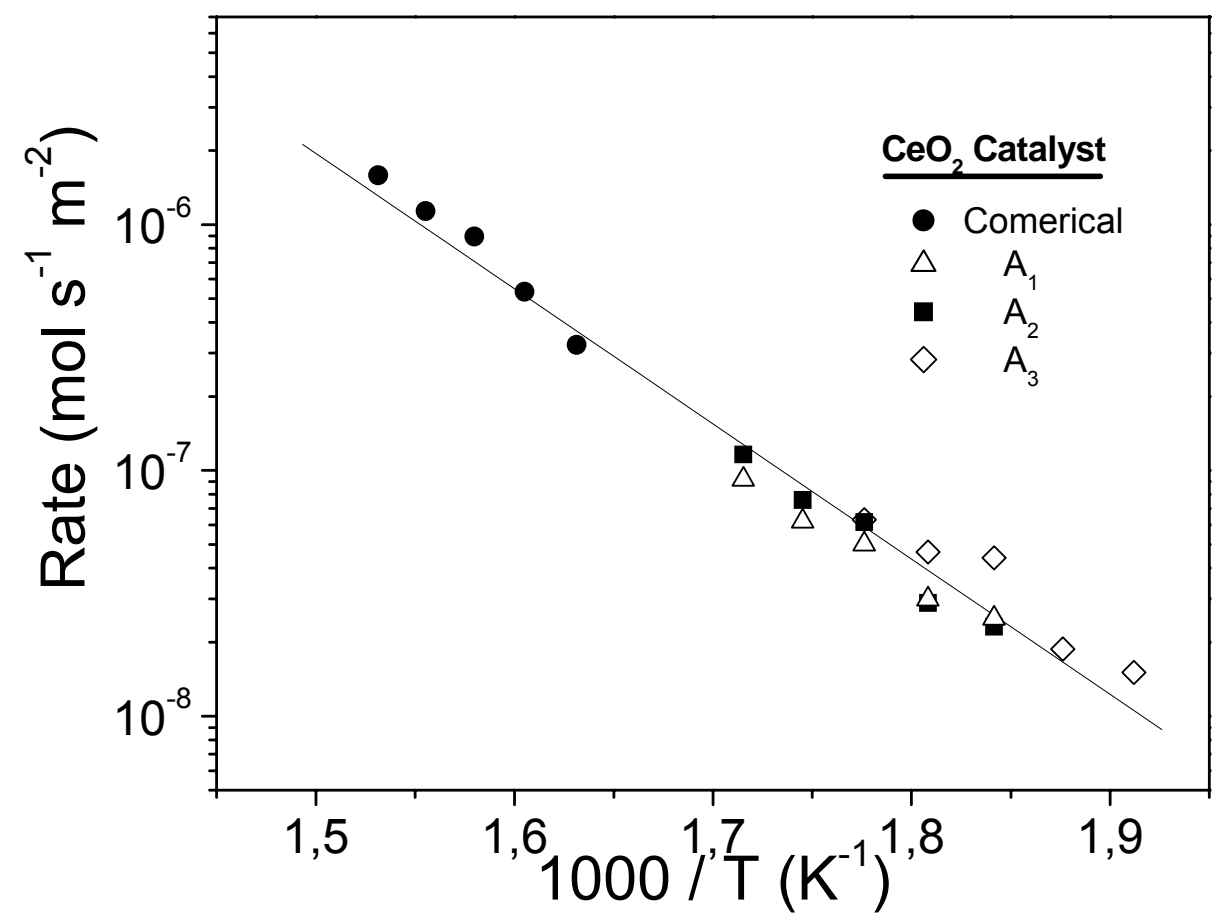

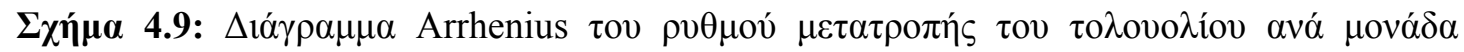
$\varepsilon \pi \iota \varphi \alpha ́ v \varepsilon 1 \alpha \varsigma$ $\kappa \alpha \tau \alpha \lambda u ́ \tau \eta \mathrm{CeO}_{2}$. 


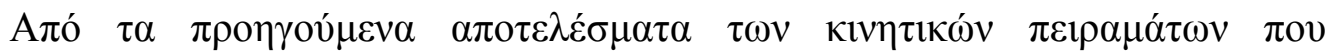

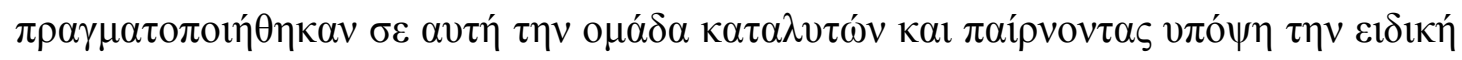

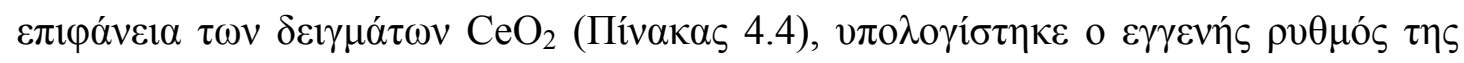

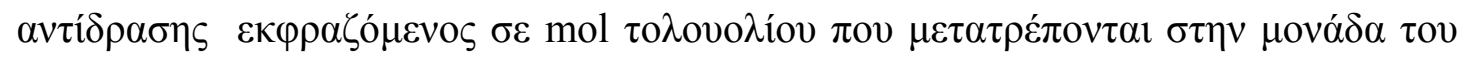

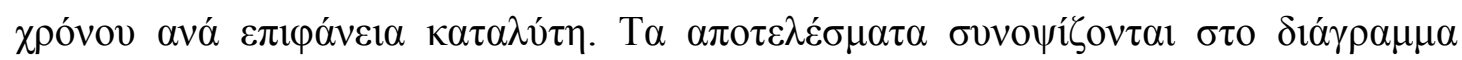

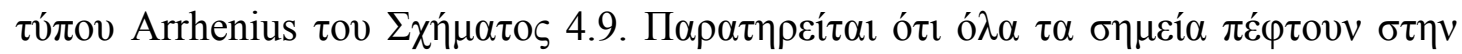

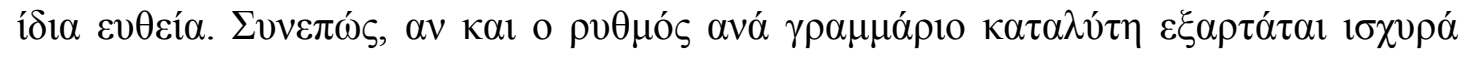

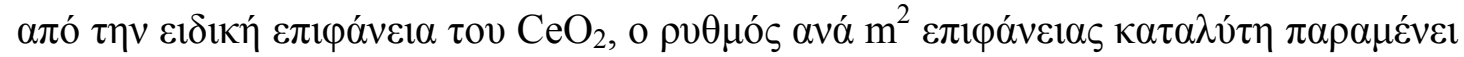

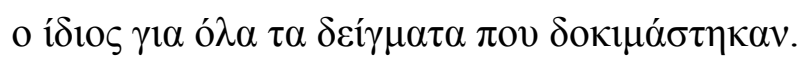

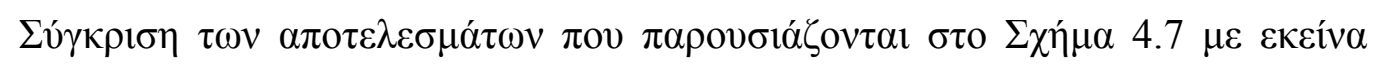

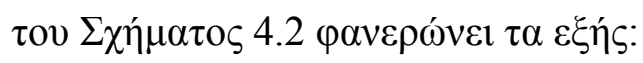

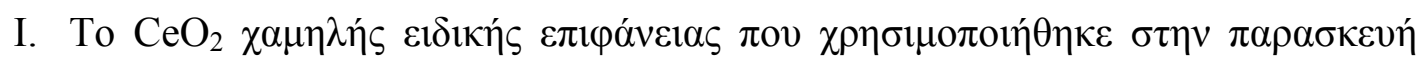

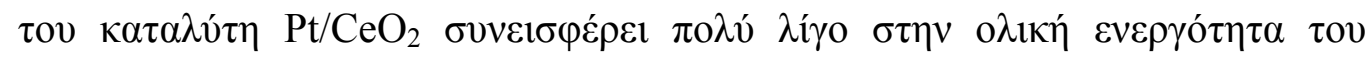

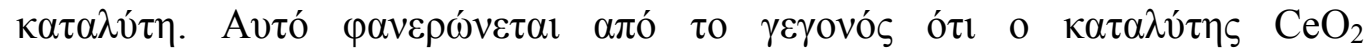

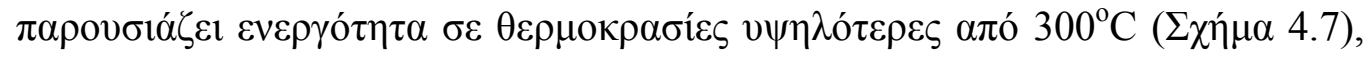

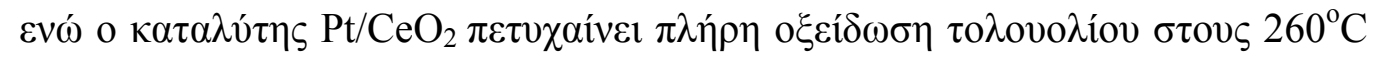

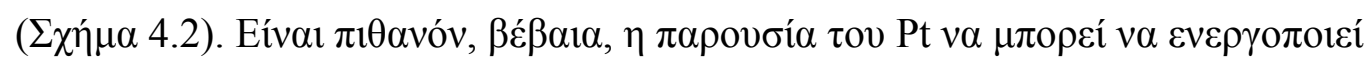

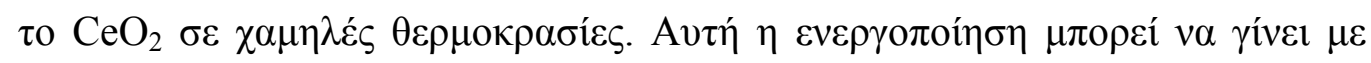

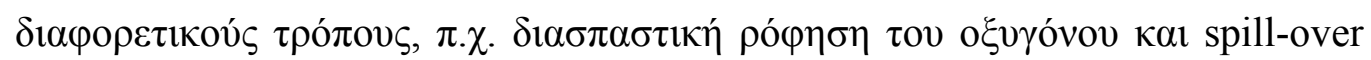

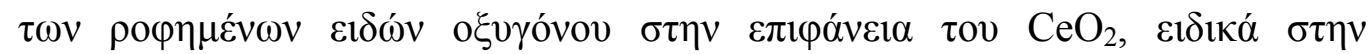

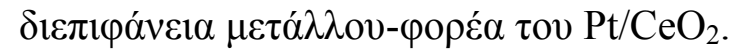

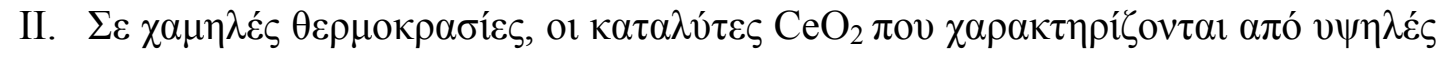

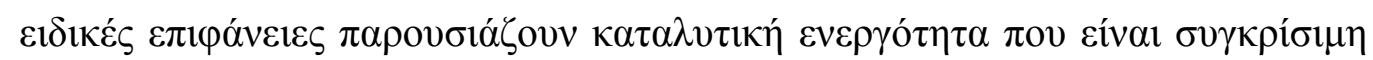

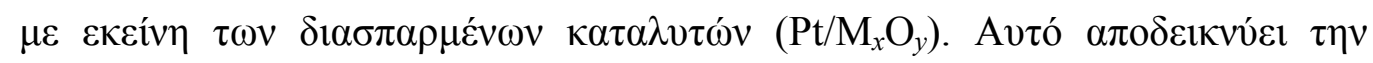

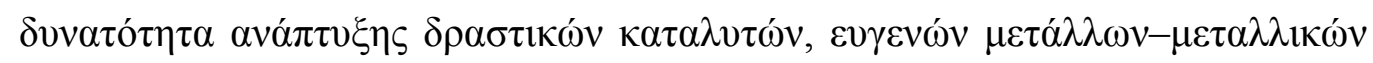

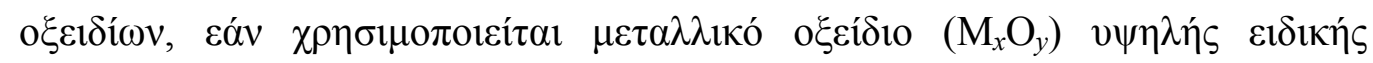
$\varepsilon \pi 1 \varphi \alpha ́ v \varepsilon i a s$.

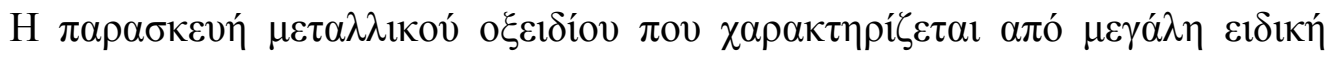

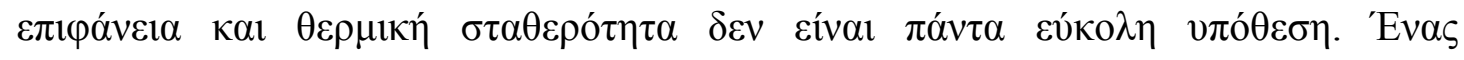

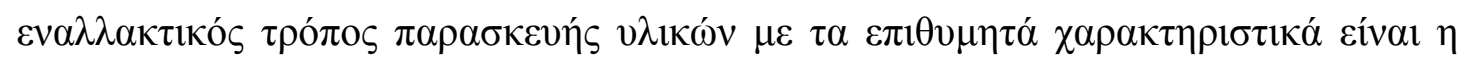

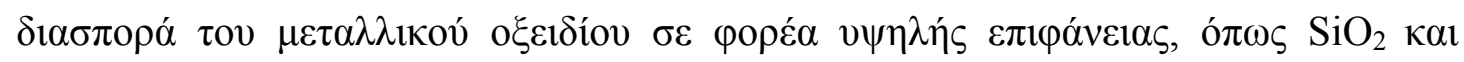

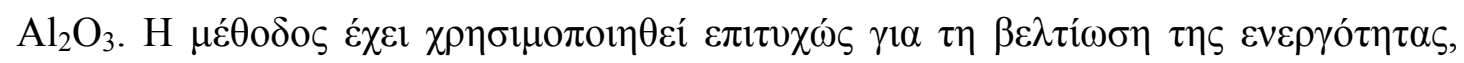

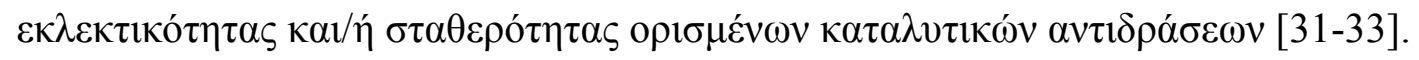




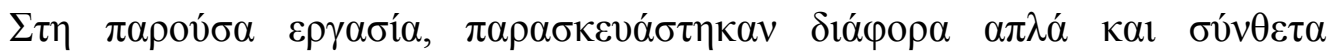

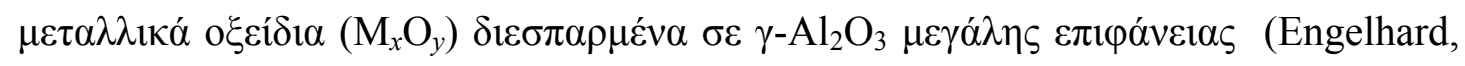

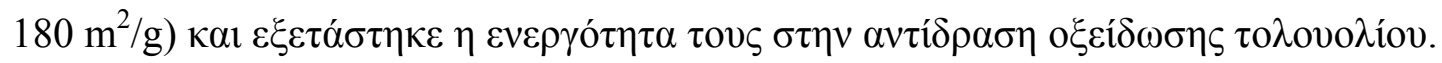

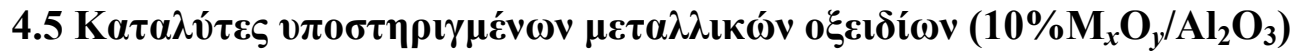

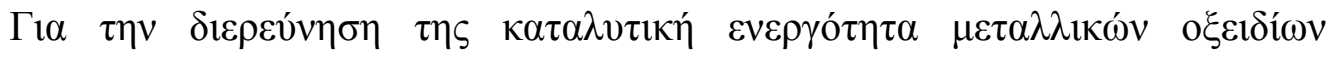

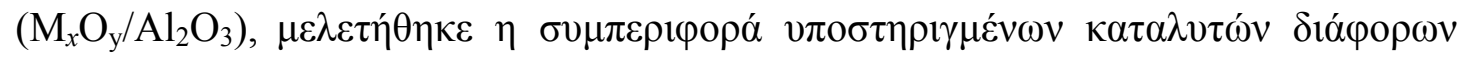

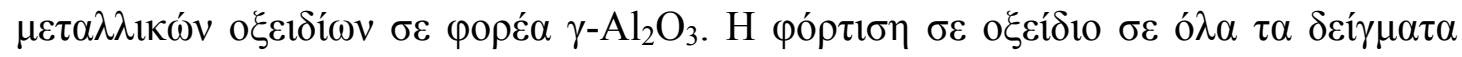

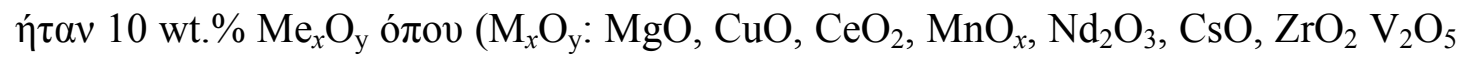

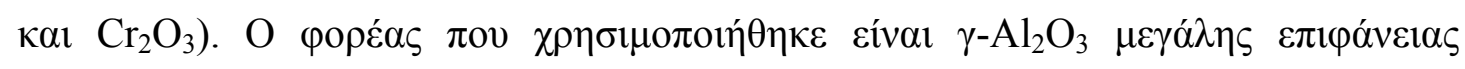

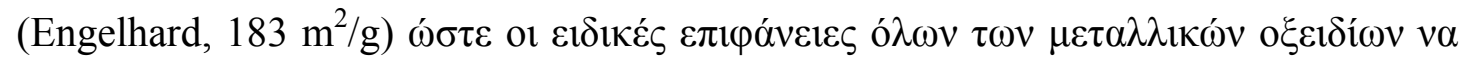

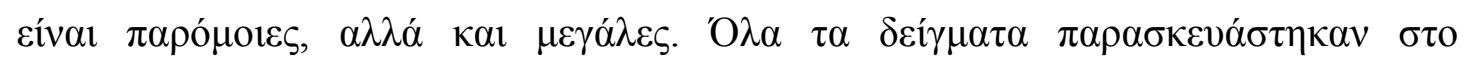

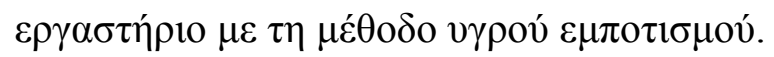

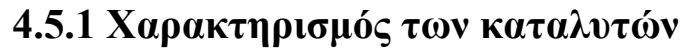

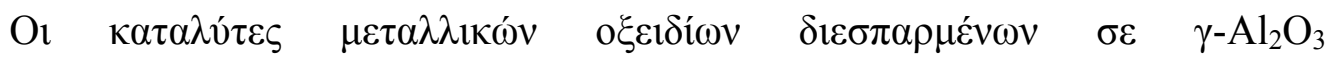

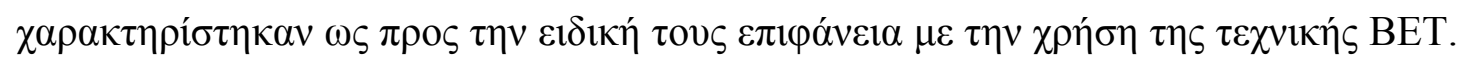

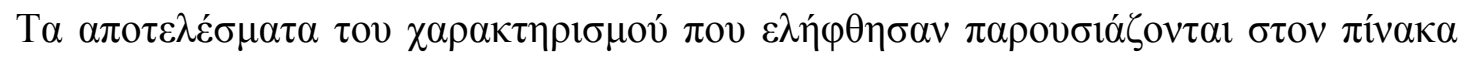

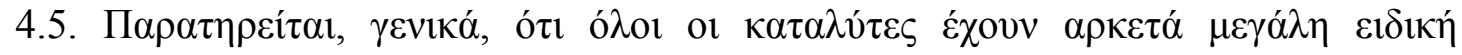

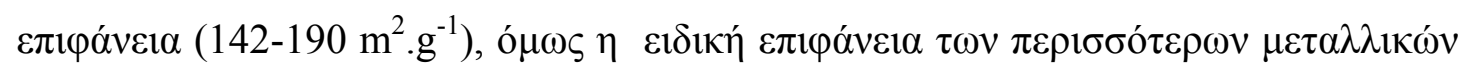

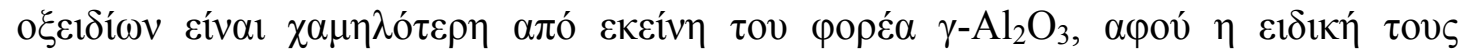

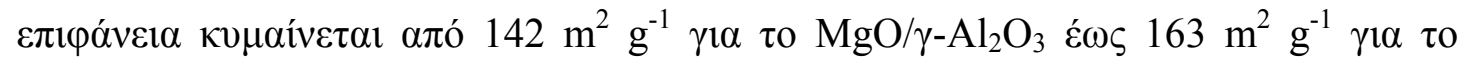

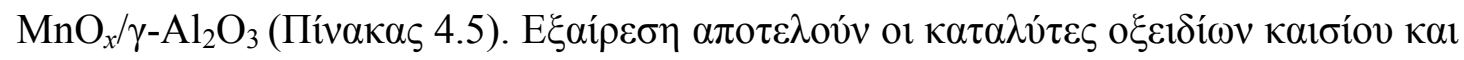

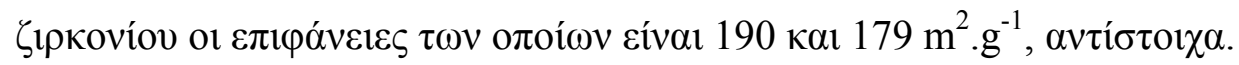

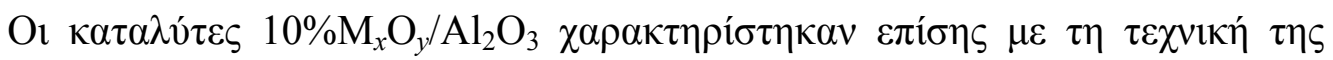

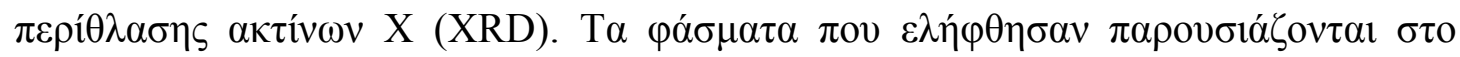

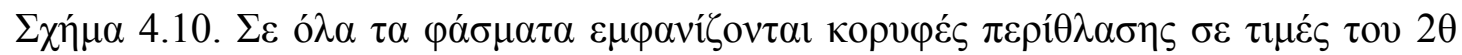

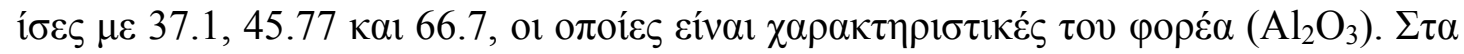

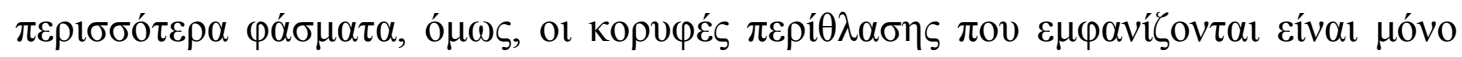

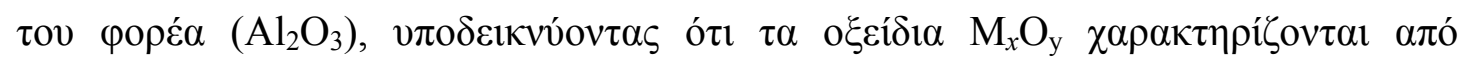

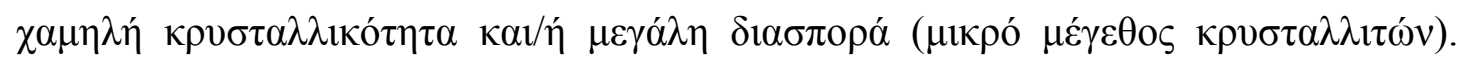

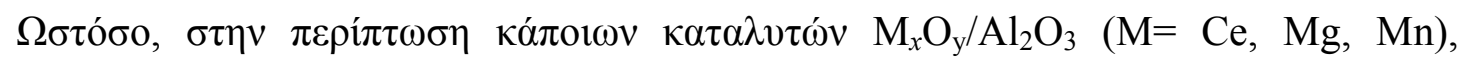

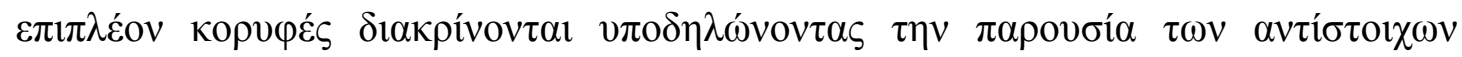

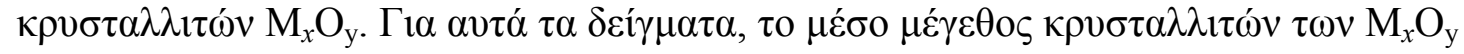




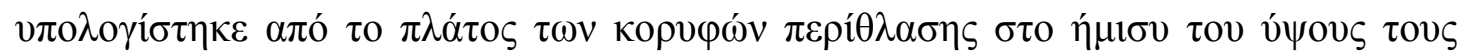

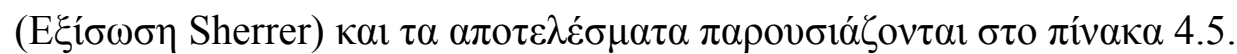

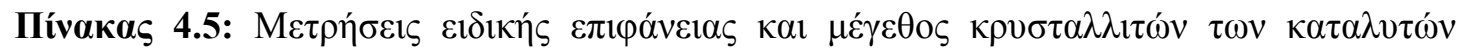
$10 \% \mathrm{M}_{x} \mathrm{O}_{\mathrm{y}} / \mathrm{Al}_{2} \mathrm{O}_{3}(\mathrm{M}=\mathrm{V}, \mathrm{Cu}, \mathrm{Cr}, \mathrm{Cs}, \mathrm{Nd}, \mathrm{Zr}, \mathrm{Ce}, \mathrm{Mg}, \mathrm{Mn})$.

\begin{tabular}{|c|c|c|}
\hline $\begin{array}{c}10 \% \mathrm{M}_{x} \mathrm{O}_{y} / \\
\mathrm{Al}_{2} \mathrm{O}_{3}\end{array}$ & 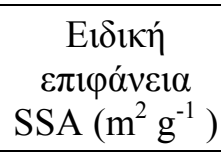 & 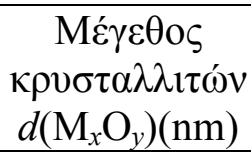 \\
\hline None $\left(\mathrm{Al}_{2} \mathrm{O}_{3}\right)$ & 183 & - \\
\hline $\mathrm{CsO}$ & 190 & n.d. \\
\hline $\mathrm{ZrO}_{2}$ & 179 & n.d. \\
\hline $\mathrm{MnO}_{x}$ & 163 & 8 \\
\hline $\mathrm{CeO}_{2}$ & 161 & 6 \\
\hline $\mathrm{Nd}_{2} \mathrm{O}_{3}$ & 153 & n.d. \\
\hline $\mathrm{Cr}_{2} \mathrm{O}_{3}$ & 152 & n.d. \\
\hline $\mathrm{CuO}$ & 152 & n.d. \\
\hline $\mathrm{V}_{2} \mathrm{O}_{5}$ & 151 & n.d. \\
\hline $\mathrm{MgO}$ & 142 & n.d. \\
\hline
\end{tabular}

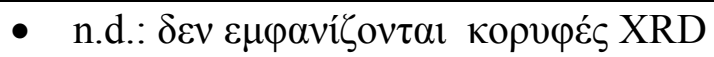

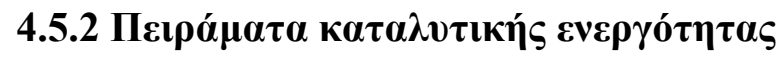

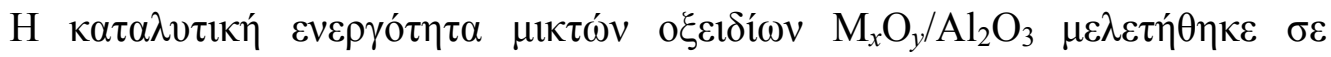

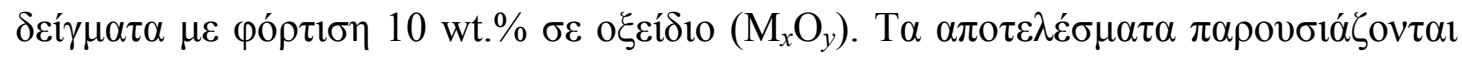

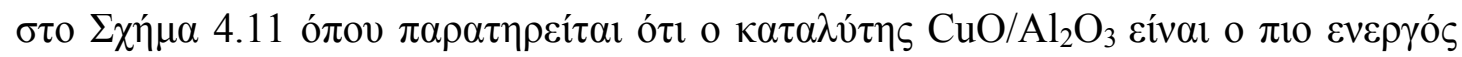

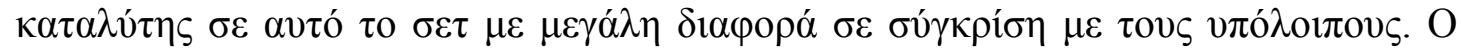

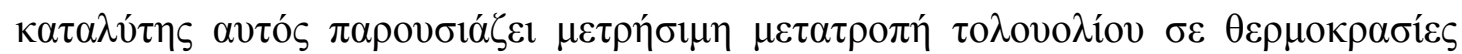

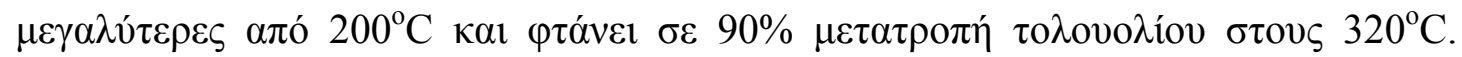

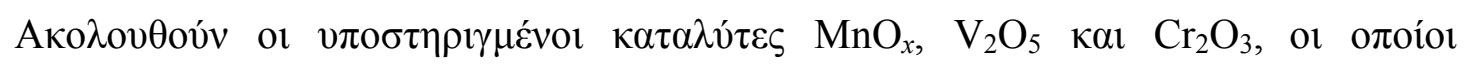

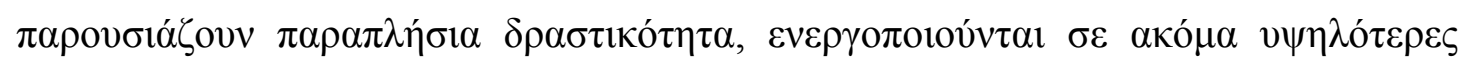

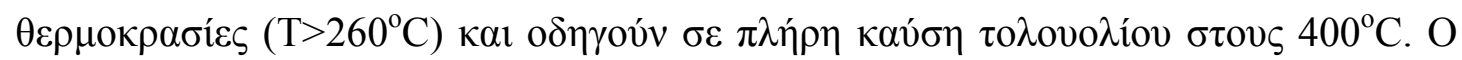

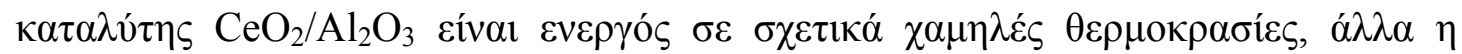

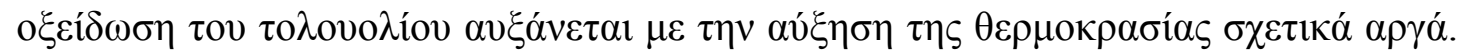




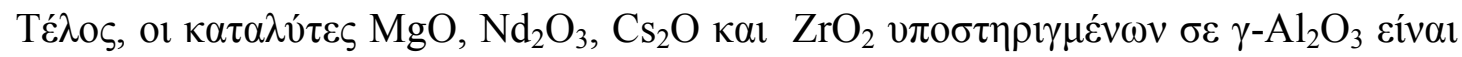

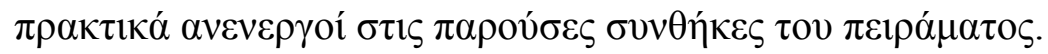

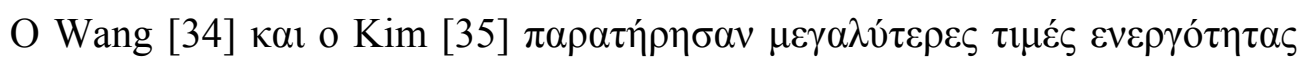

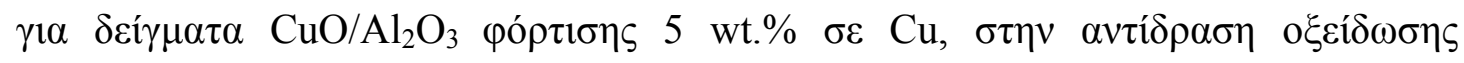

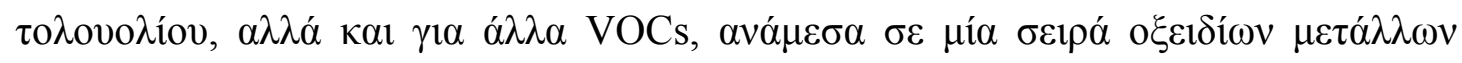

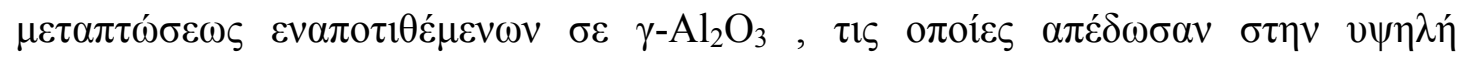

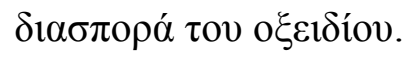

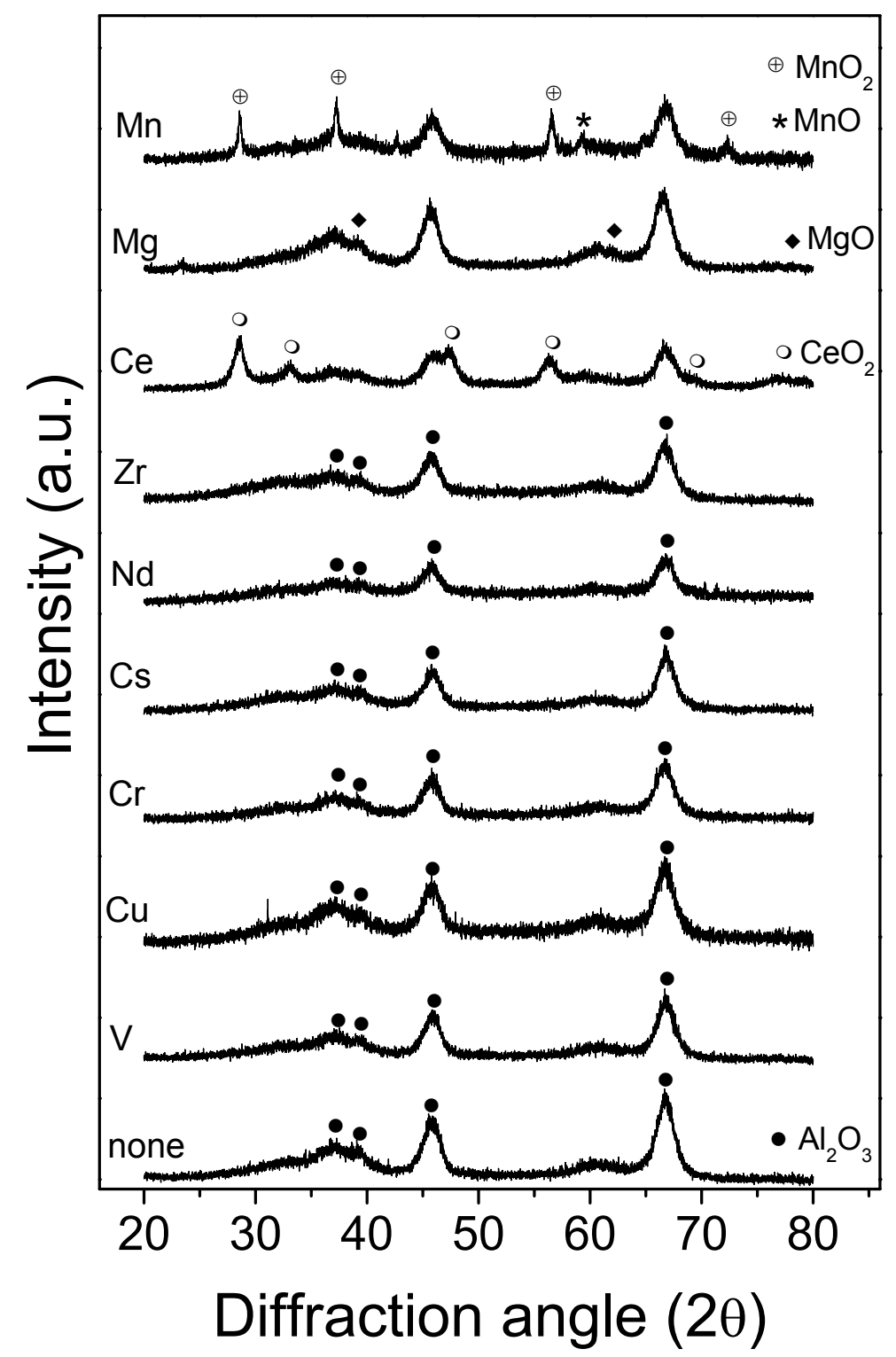

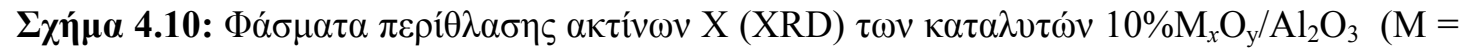
$\mathrm{V}, \mathrm{Cu}, \mathrm{Cr}, \mathrm{Cs}, \mathrm{Nd}, \mathrm{Zr}, \mathrm{Ce}, \mathrm{Mg}, \mathrm{Mn})$. 


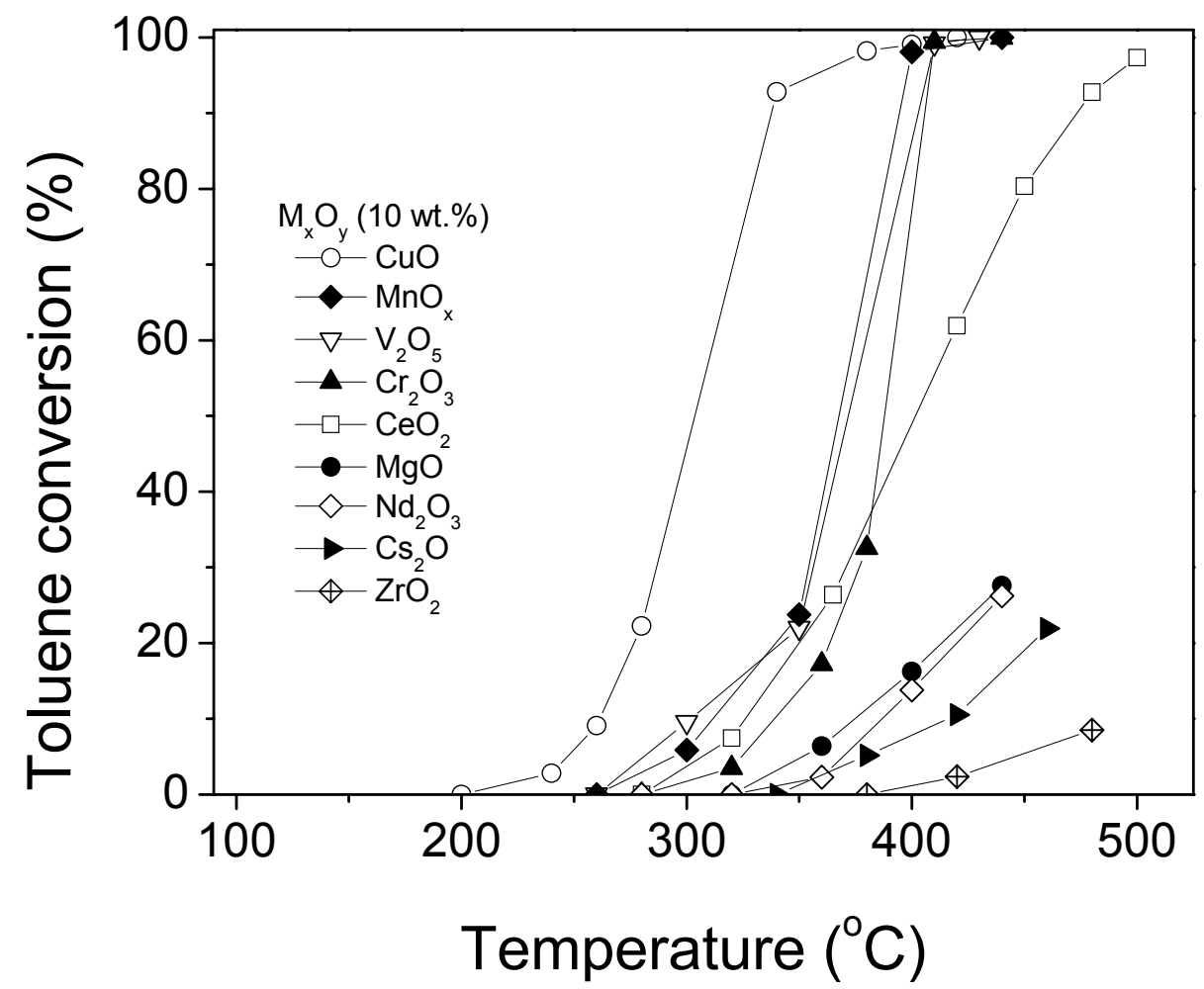

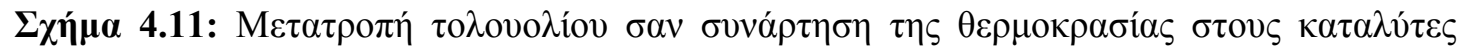
$10 \% \mathrm{M}_{x} \mathrm{O}_{\mathrm{y}} / \mathrm{Al}_{2} \mathrm{O}_{3}(\mathrm{M}=\mathrm{V}, \mathrm{Cu}, \mathrm{Cr}, \mathrm{Cs}, \mathrm{Nd}, \mathrm{Zr}, \mathrm{Ce}, \mathrm{Mg}, \mathrm{Mn})$.

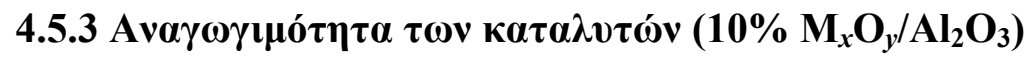

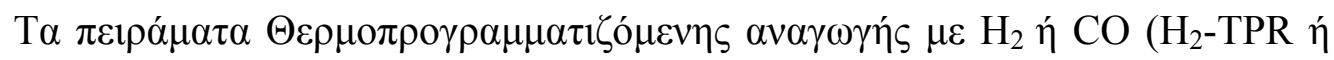

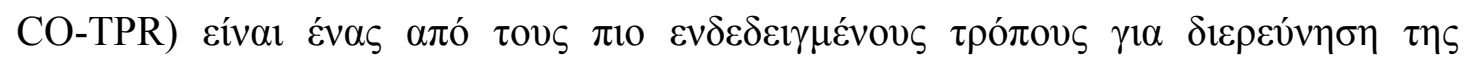

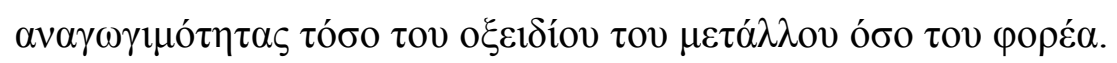

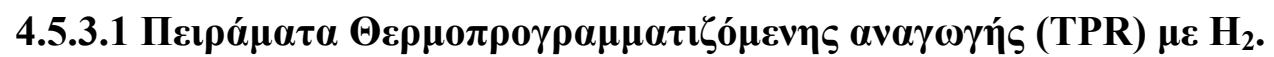

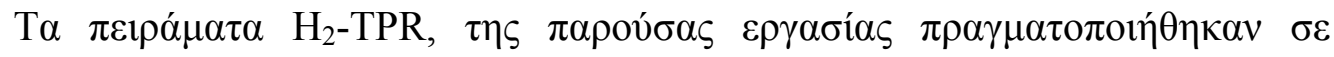

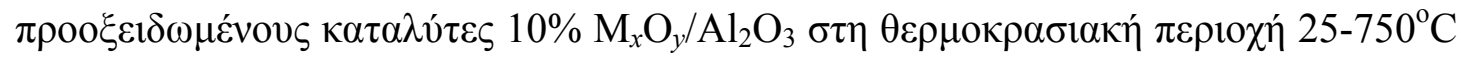
$\left(\beta=30^{\circ} \mathrm{C} / \mathrm{min}\right) \mu \varepsilon \quad 0.5 \% \mathrm{H}_{2} / \mathrm{He}\left(40 \mathrm{~cm}^{3} / \mathrm{min}\right), \chi \rho \eta \sigma \iota \mu$ о

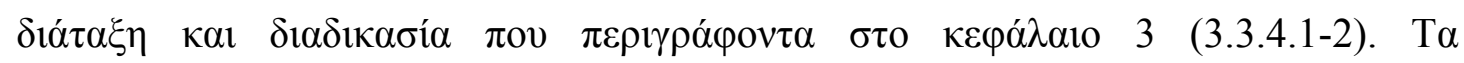

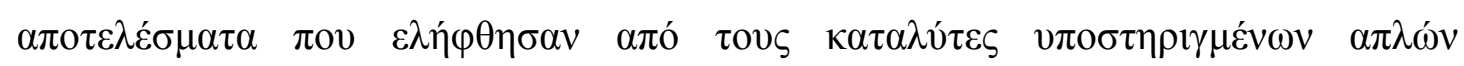

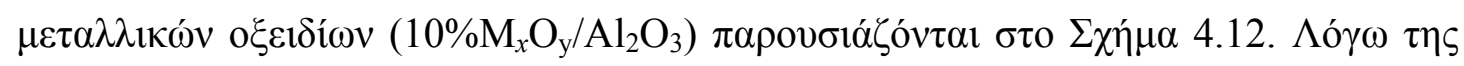

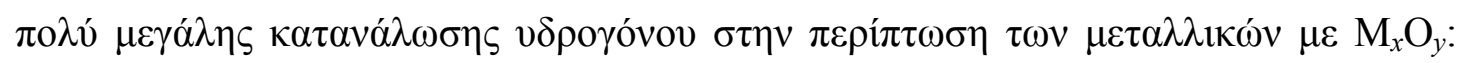

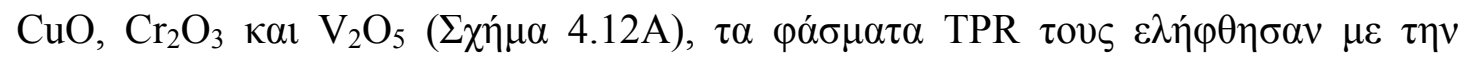

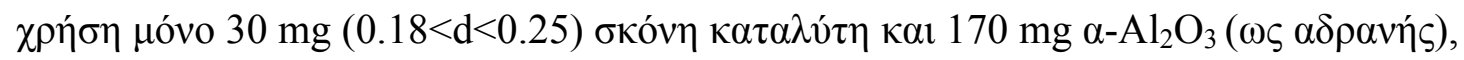




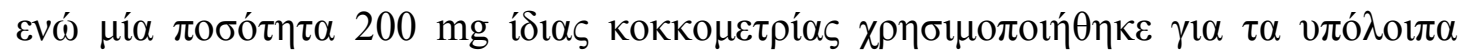

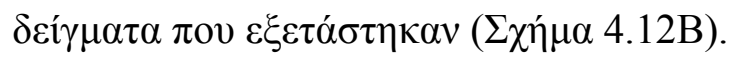

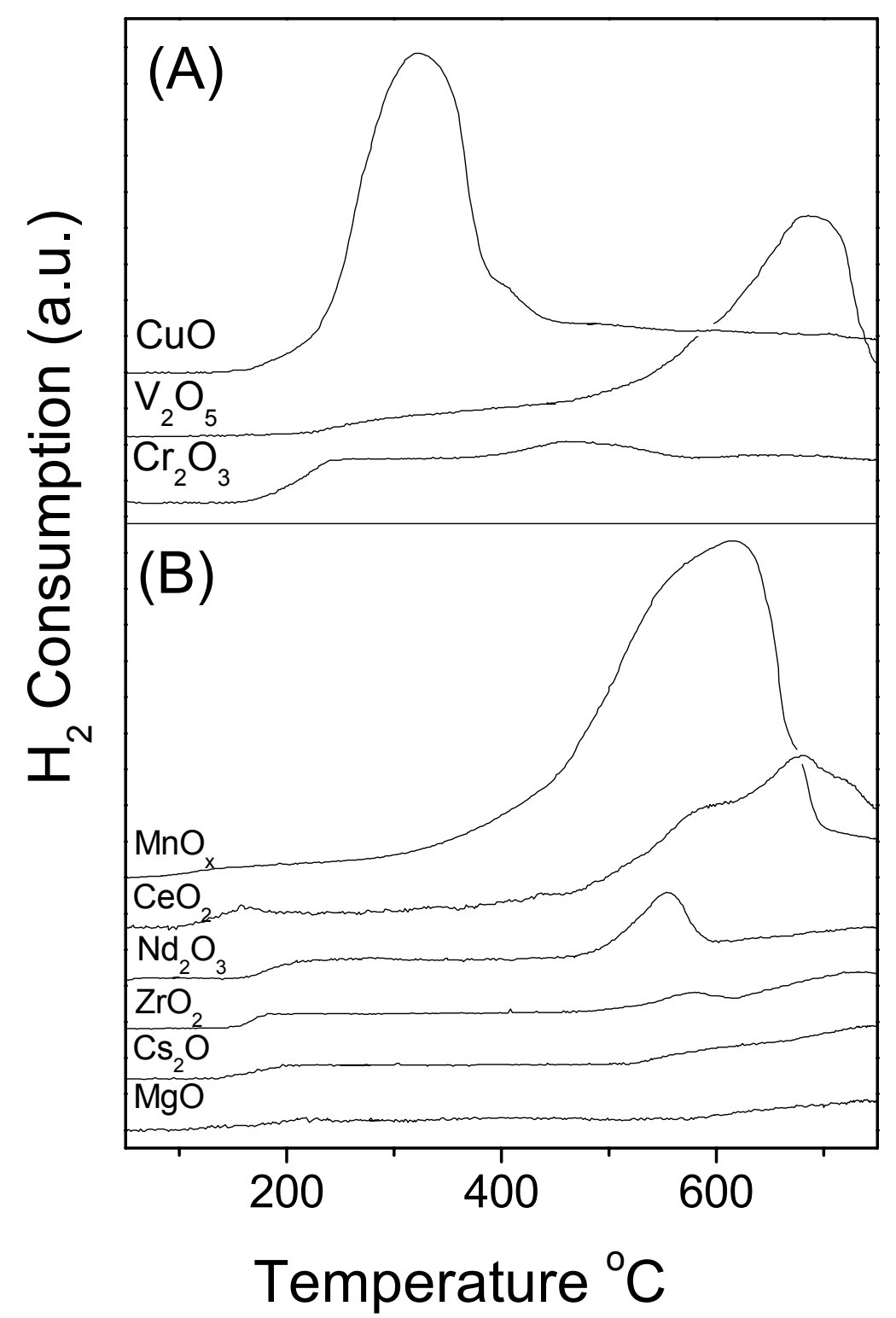

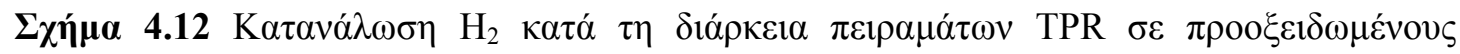
$\kappa \alpha \tau \alpha \lambda \nu$ $\varepsilon \varsigma, 10 \% \mathrm{M}_{x} \mathrm{O}_{\mathrm{y}} / \mathrm{Al}_{2} \mathrm{O}_{3}$.

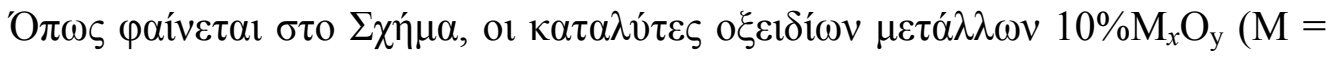

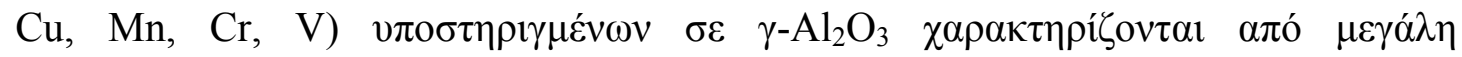

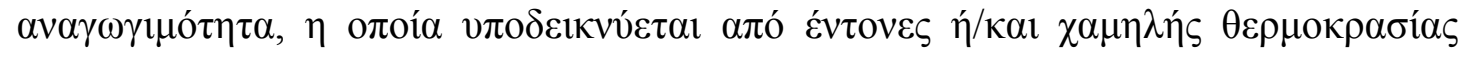

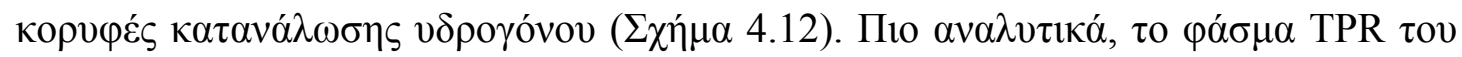

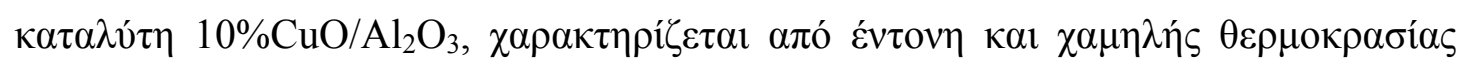




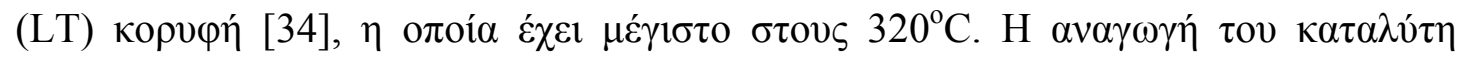

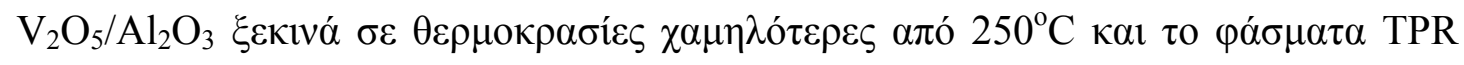

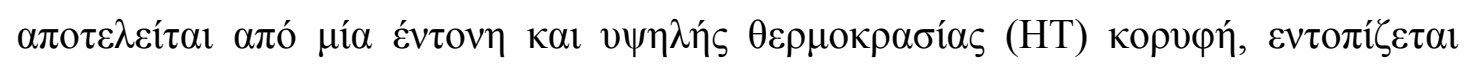

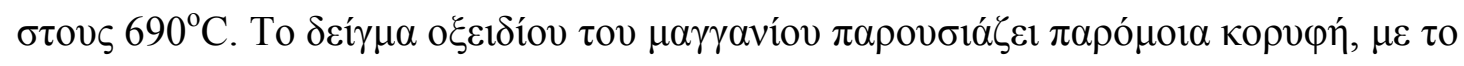

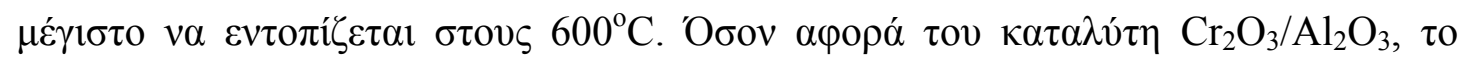

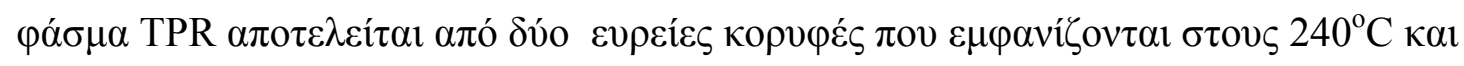

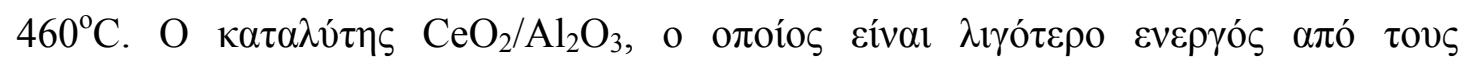

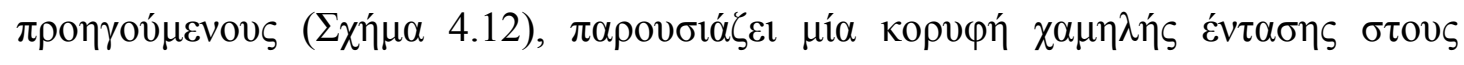

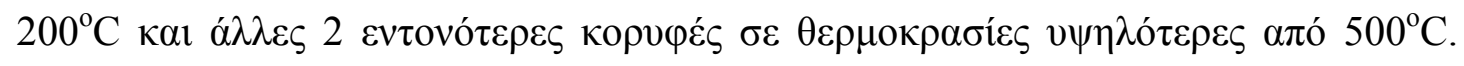

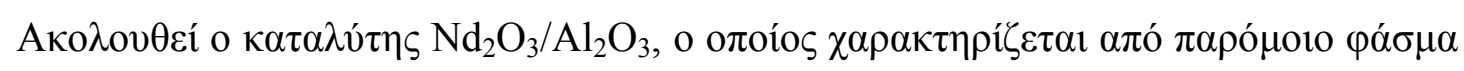

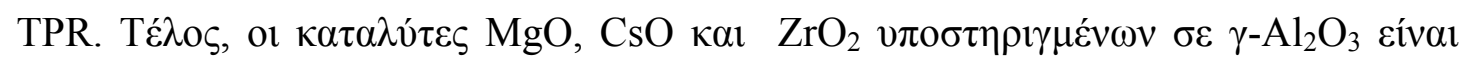

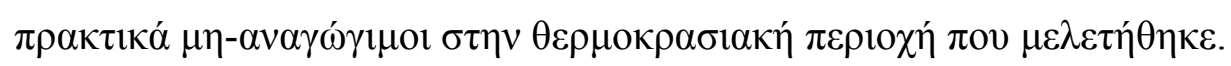

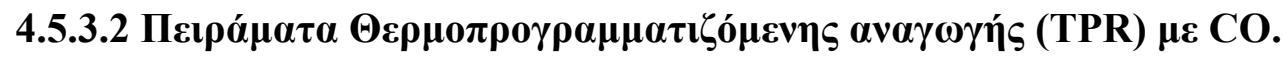

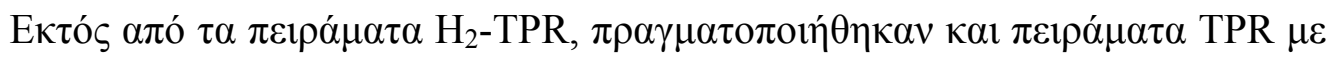

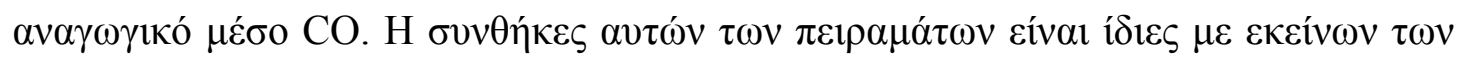

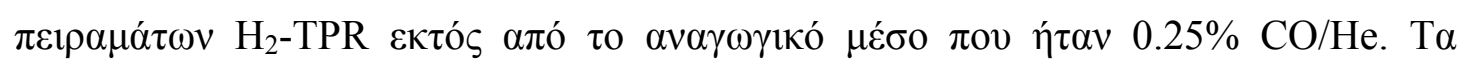

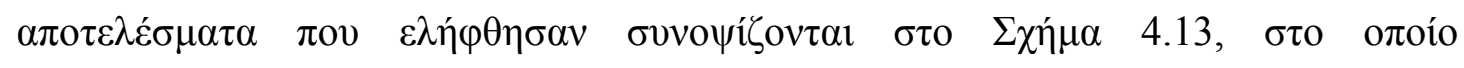

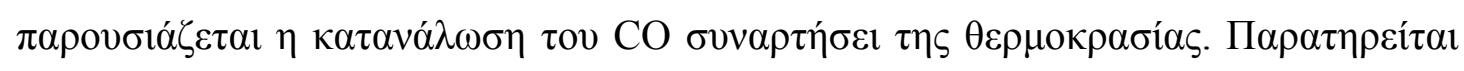

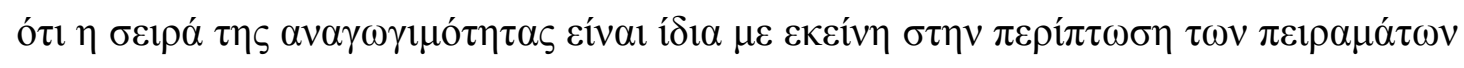

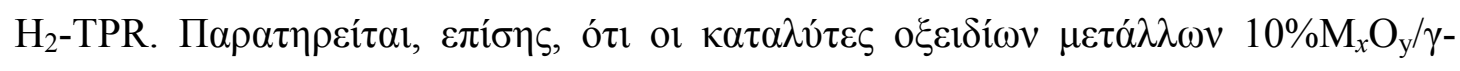

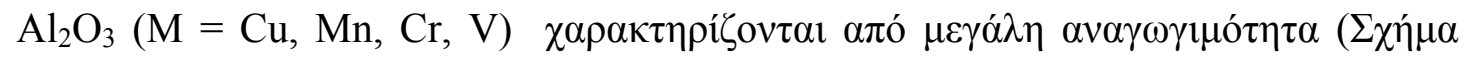

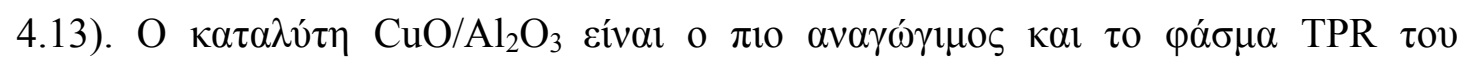

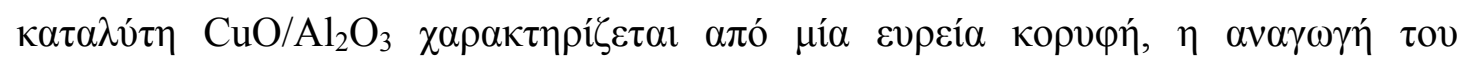

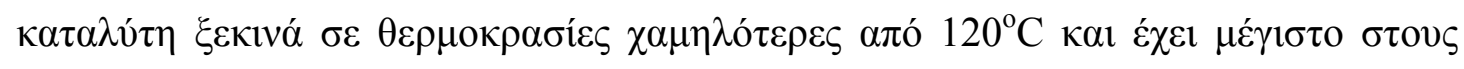

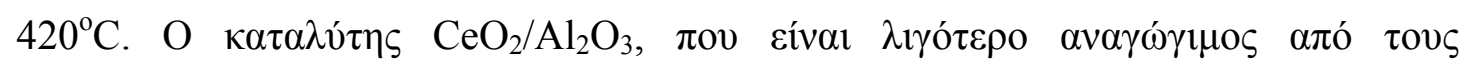

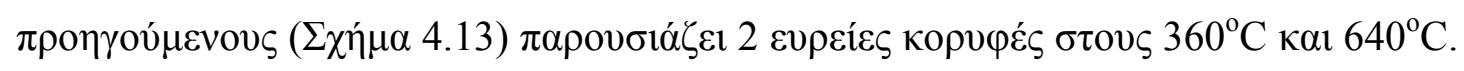

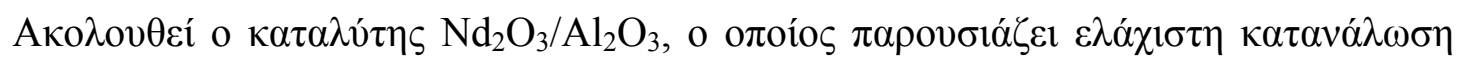

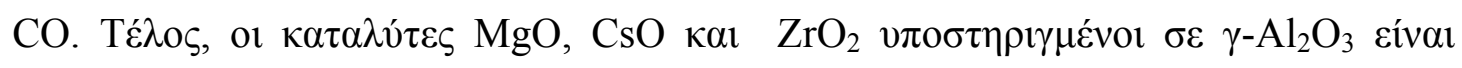

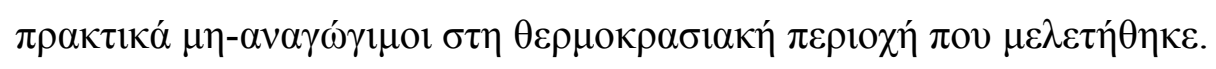




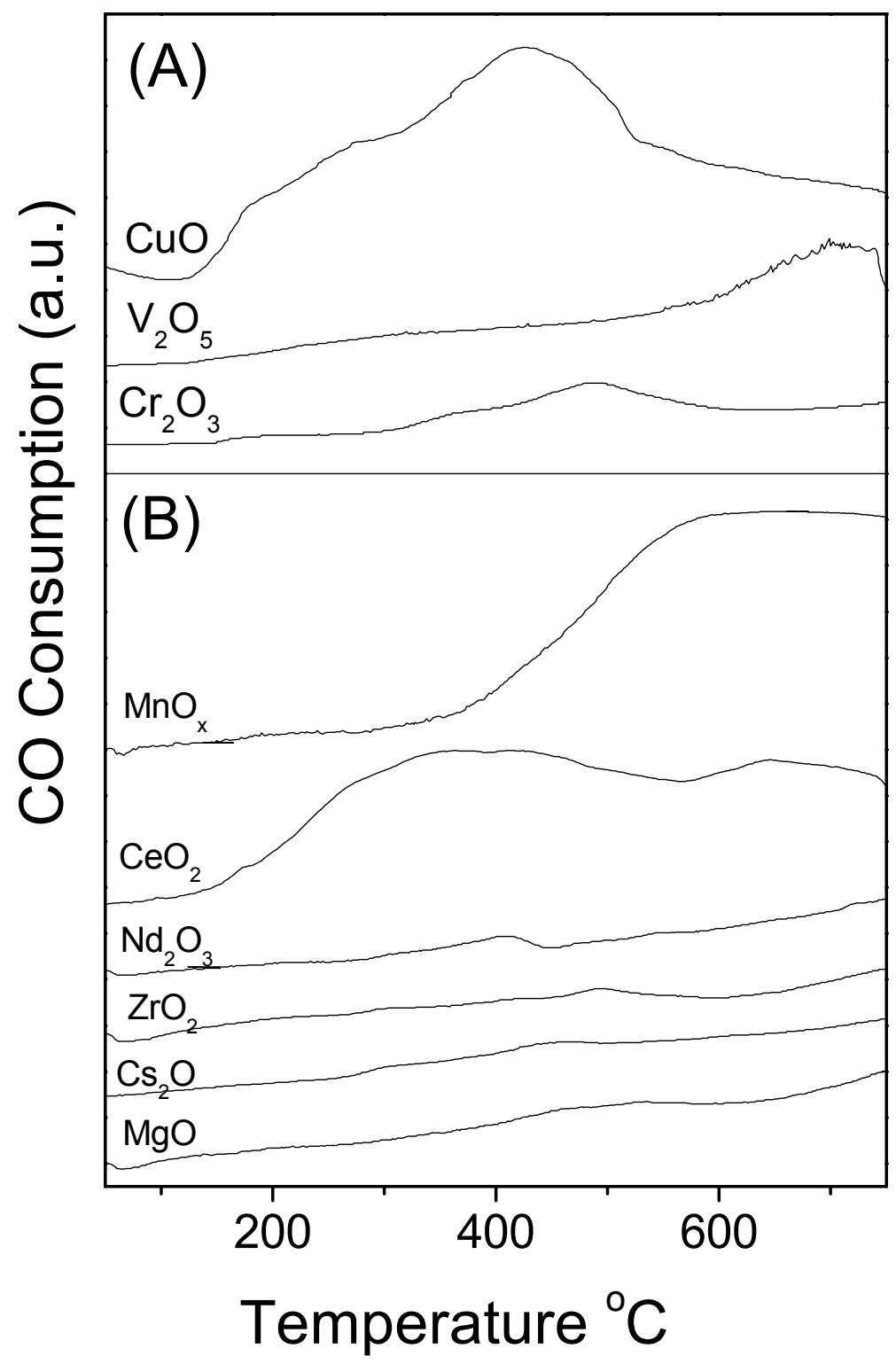

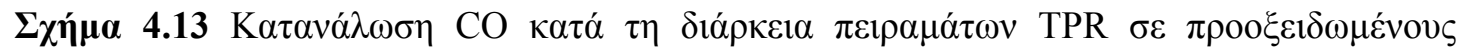
$\kappa \alpha \tau \alpha \lambda v ́ \tau \varepsilon \varsigma 10 \% \mathrm{M}_{x} \mathrm{O}_{\mathrm{y}} / \mathrm{Al}_{2} \mathrm{O}_{3}$.

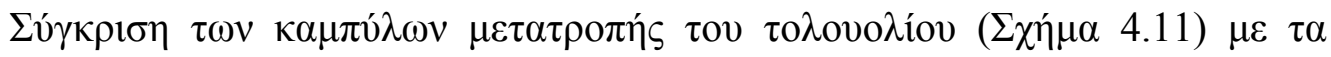

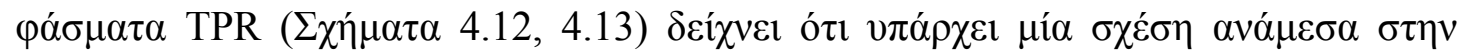

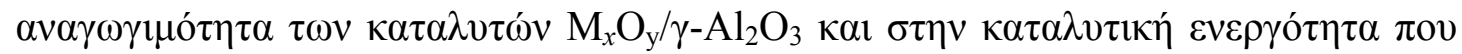

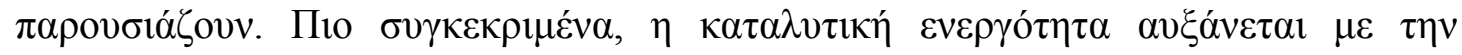

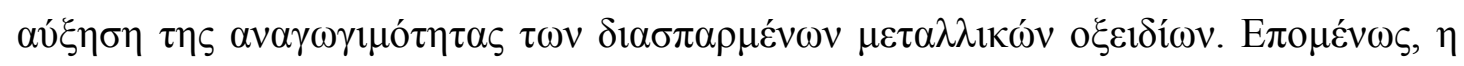

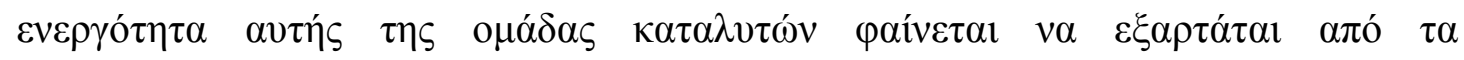

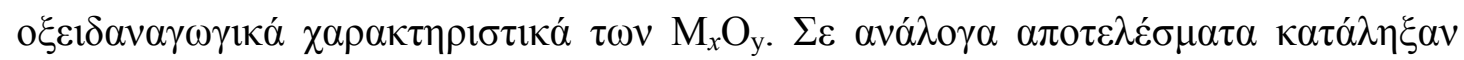

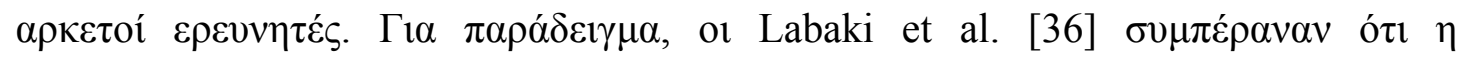

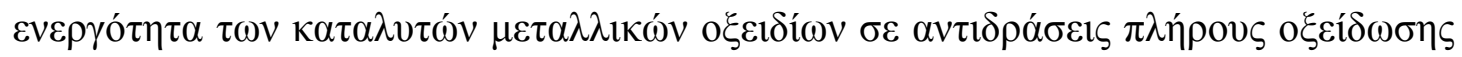




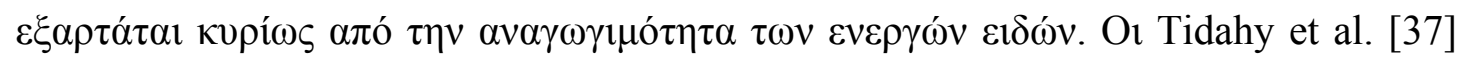

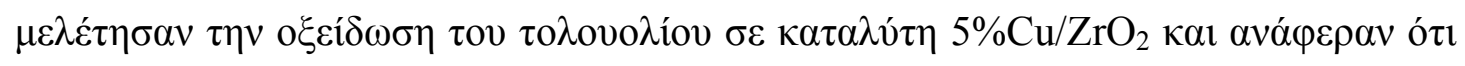

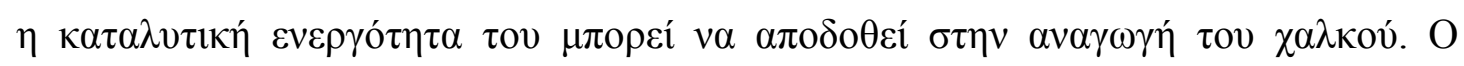

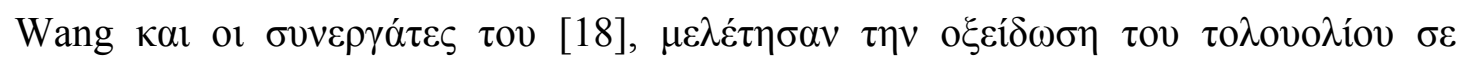

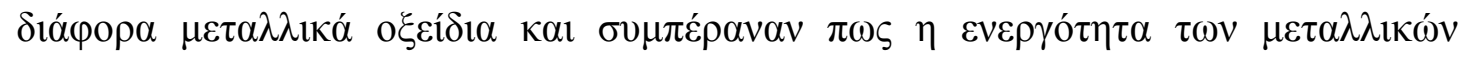

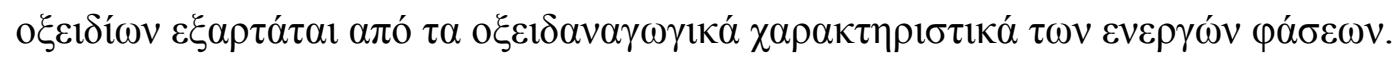

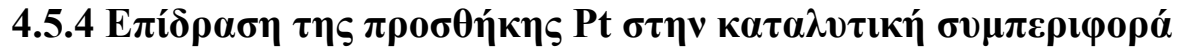

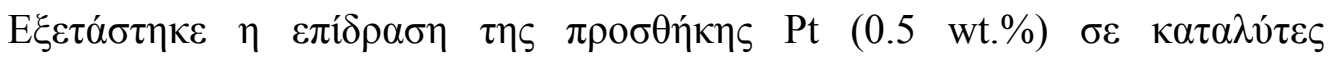

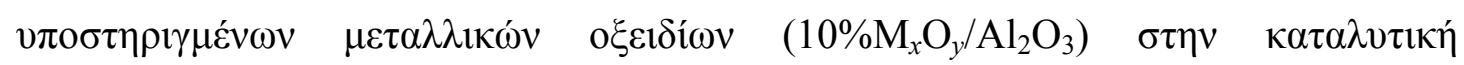

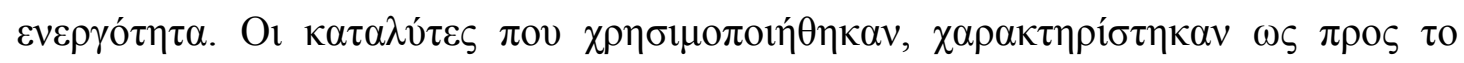

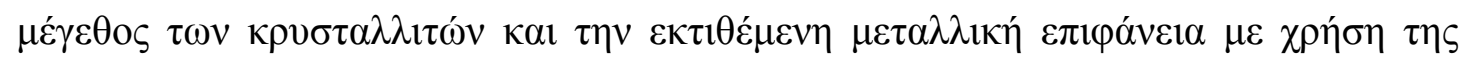

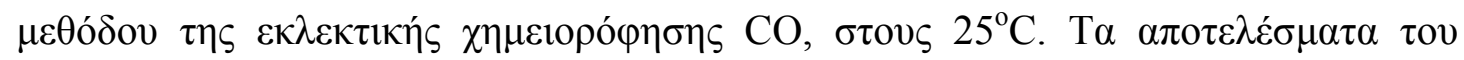

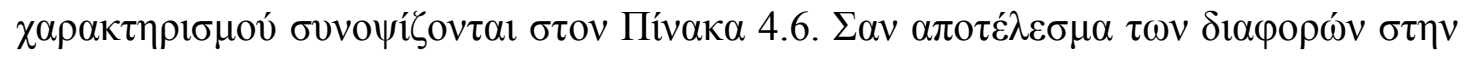

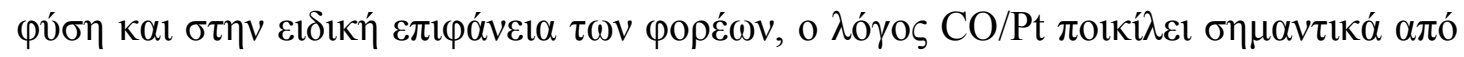

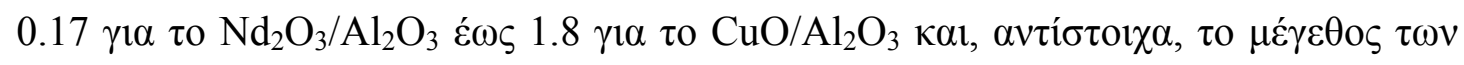

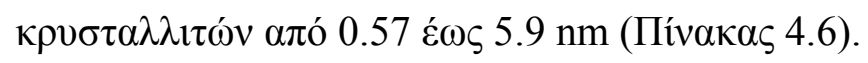

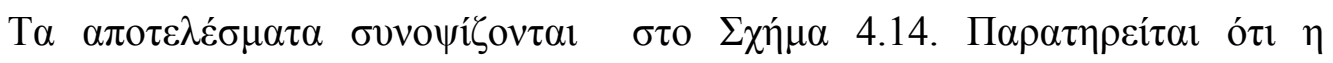

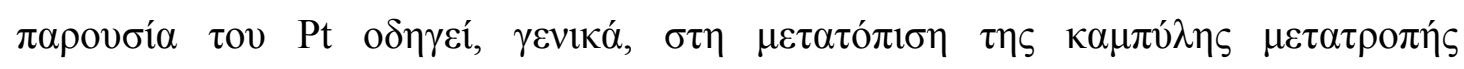

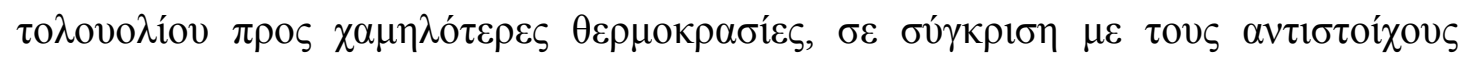

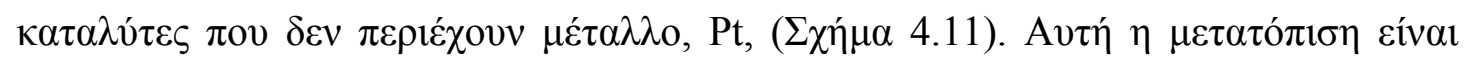

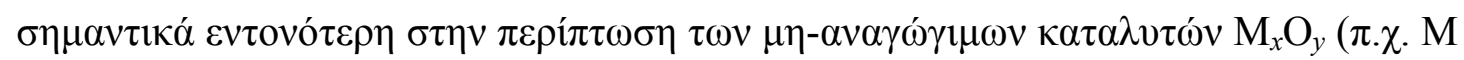

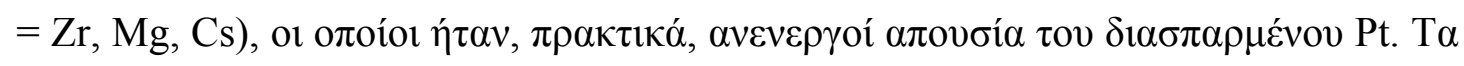

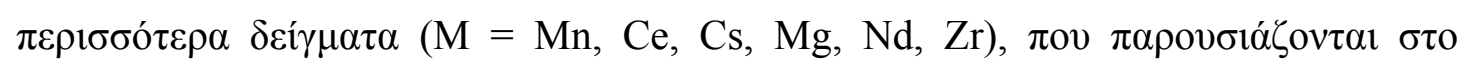

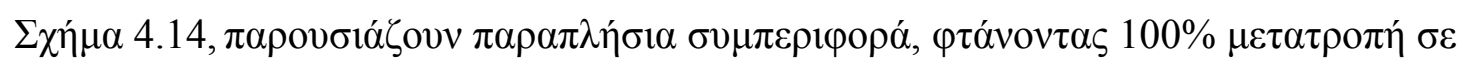

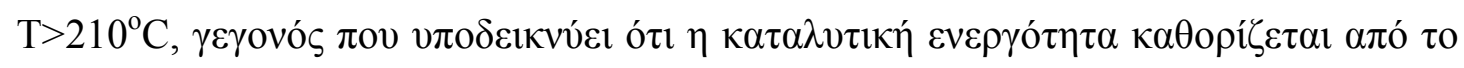

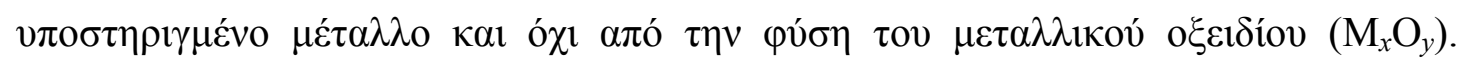

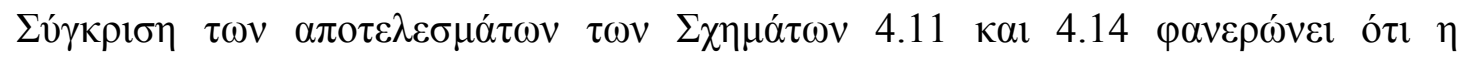

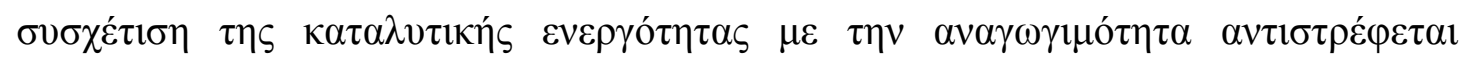

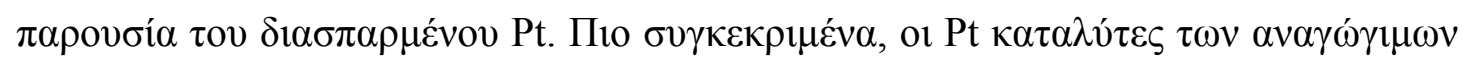

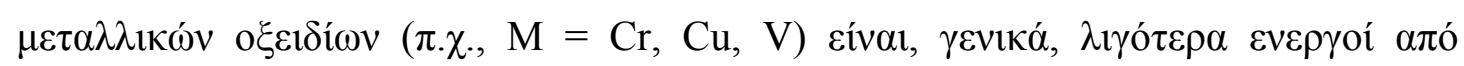

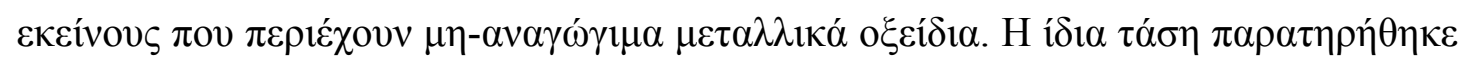

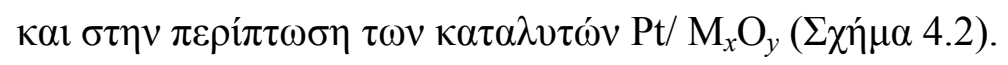




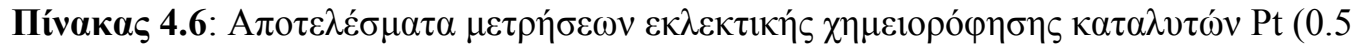

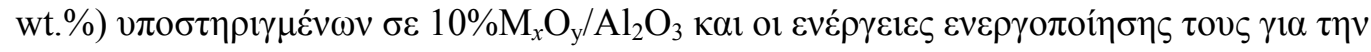

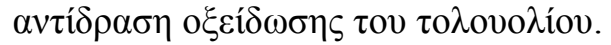

\begin{tabular}{|c|c|c|c|}
\hline $\mathrm{M}_{x} \mathrm{O}_{y}$ & $\mathrm{CO} / \mathrm{Pt}$ & 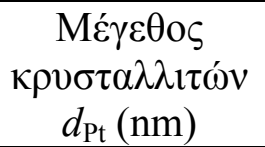 & 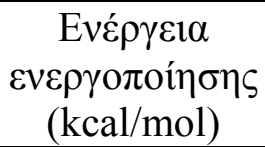 \\
\hline $\mathrm{CuO}$ & 1.8 & 0.57 & 24 \\
\hline $\mathrm{CeO}_{2}$ & 1.04 & 1.0 & 63.2 \\
\hline $\mathrm{ZrO}_{2}$ & 0.64 & 1.6 & 13.6 \\
\hline $\mathrm{Cr}_{2} \mathrm{O}_{3}$ & 0.58 & 1.8 & 18.1 \\
\hline $\mathrm{MgO}$ & 0.52 & 2.0 & 104.7 \\
\hline $\mathrm{MnO}_{x}$ & 0.21 & 4.9 & 30.4 \\
\hline $\mathrm{CsO}$ & 0.38 & 2.8 & 41.9 \\
\hline $\mathrm{V}_{2} \mathrm{O}_{5}$ & 0.18 & 5.5 & - \\
\hline $\mathrm{Nd}_{2} \mathrm{O}_{3}$ & 0.17 & 5.9 & 20.1 \\
\hline
\end{tabular}

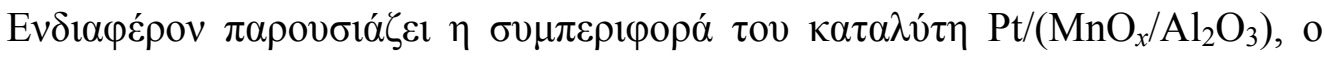

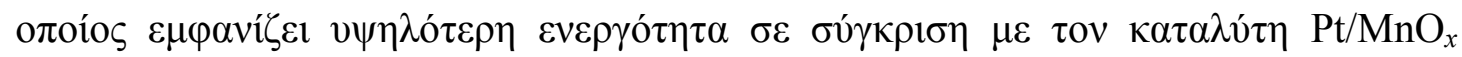

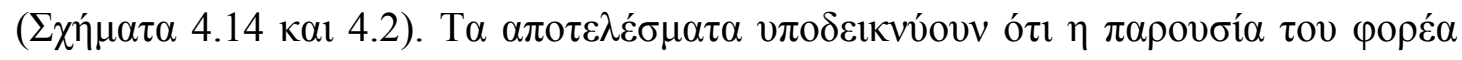

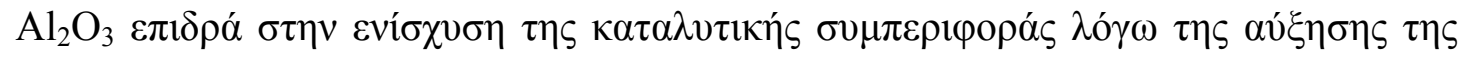

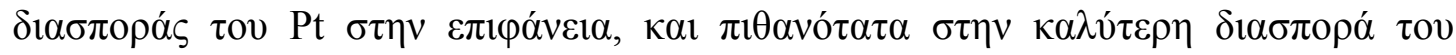

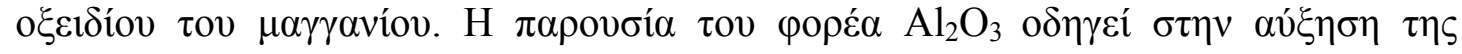

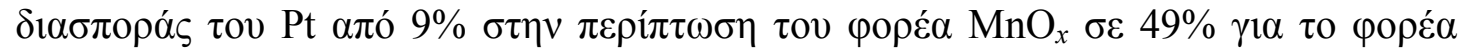

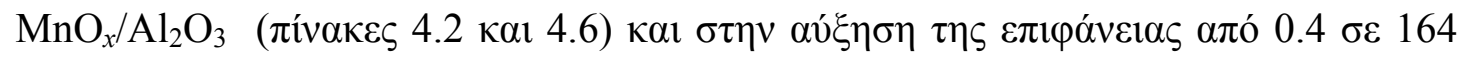

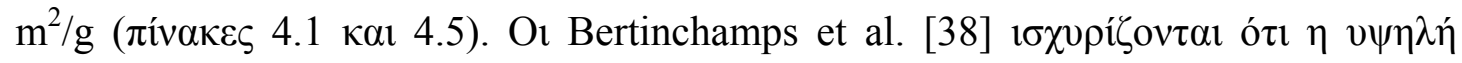

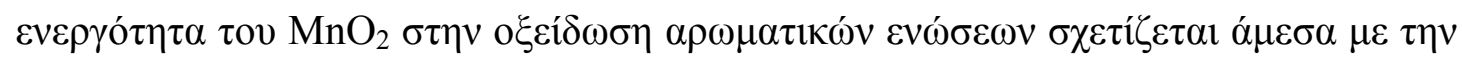
$\varepsilon \imath \delta \imath \kappa \eta ́ ~ \varepsilon \pi ı \varphi \alpha ́ v \varepsilon ı \alpha$.

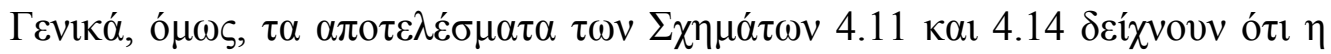

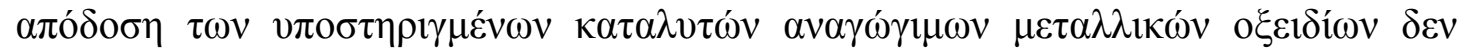

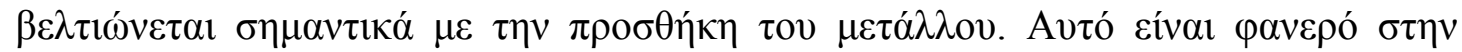

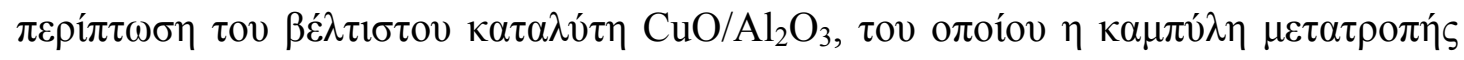

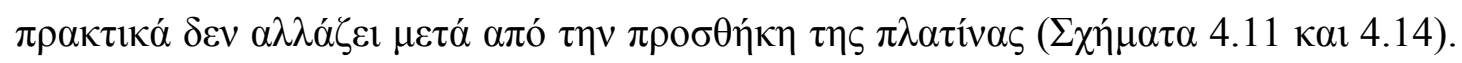

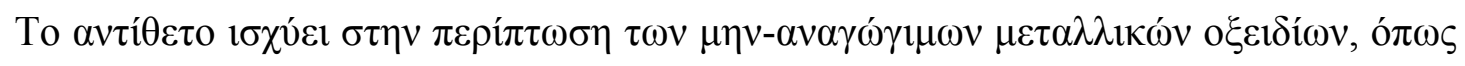

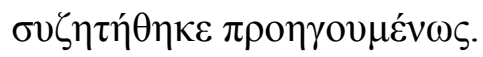




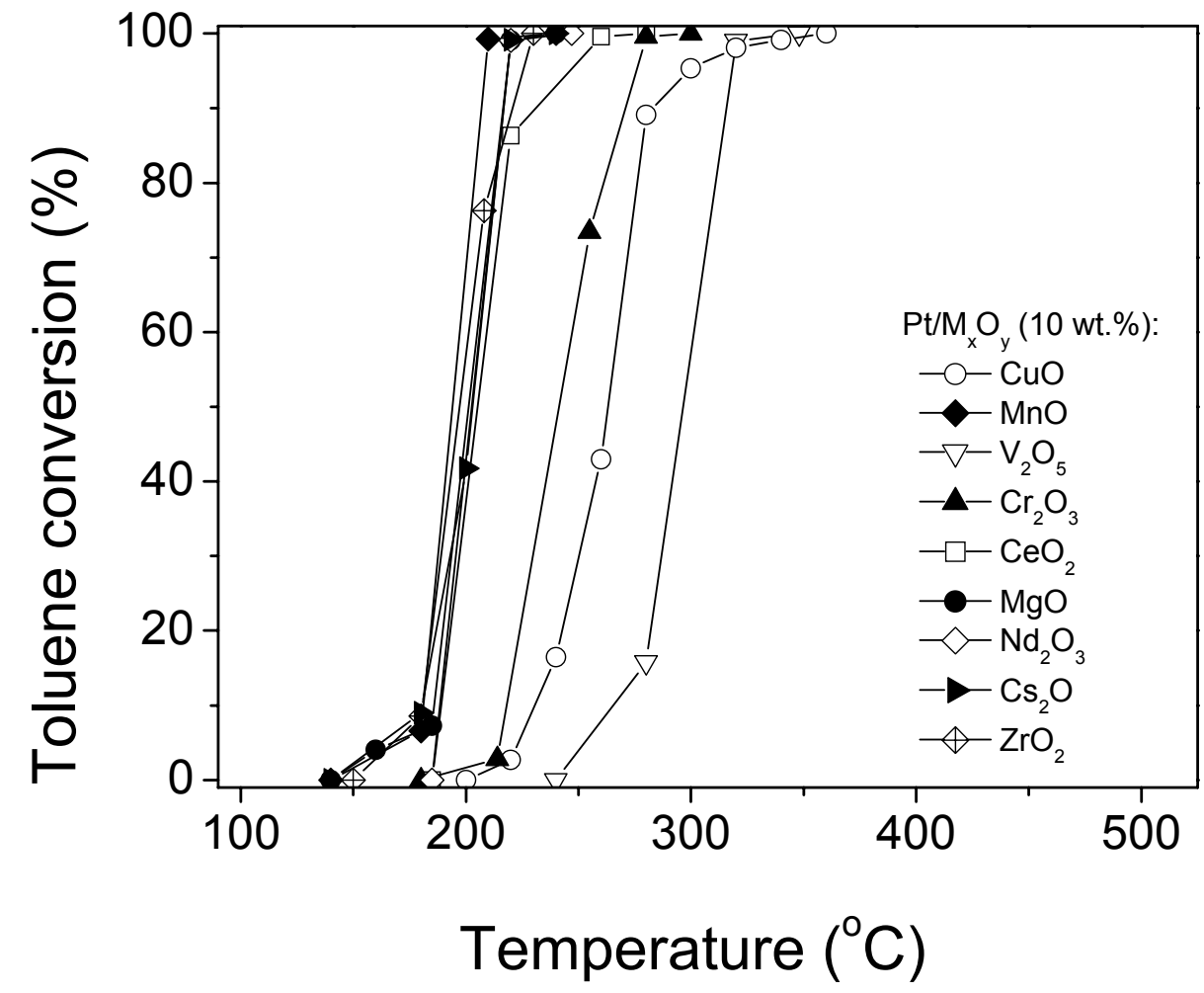

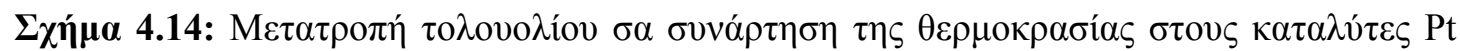
$(0.5$ wt. $\%) / 10 \%\left(\mathrm{M}_{x} \mathrm{O}_{\mathrm{y}} / \mathrm{Al}_{2} \mathrm{O}_{3}\right)(\mathrm{M}=\mathrm{V}, \mathrm{Cu}, \mathrm{Cr}, \mathrm{Cs}, \mathrm{Nd}, \mathrm{Zr}, \mathrm{Ce}, \mathrm{Mg}, \mathrm{Mn})$.

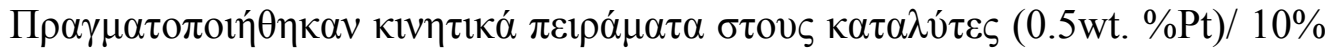

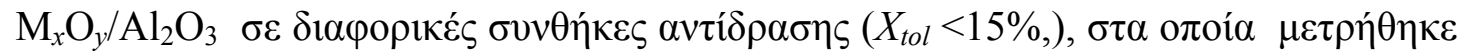

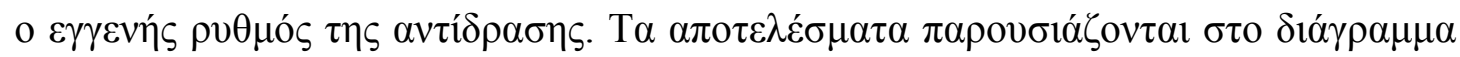

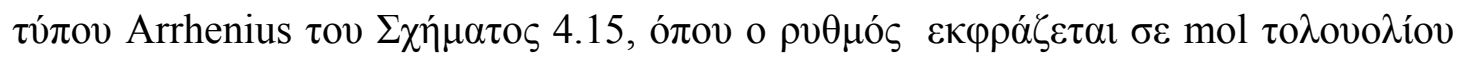

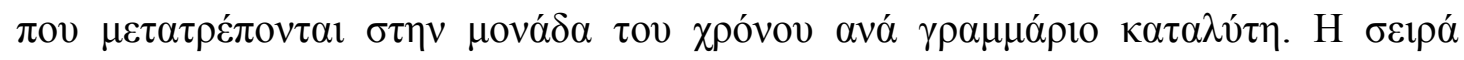

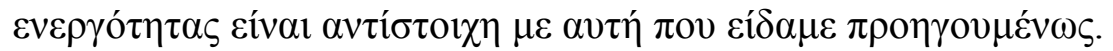




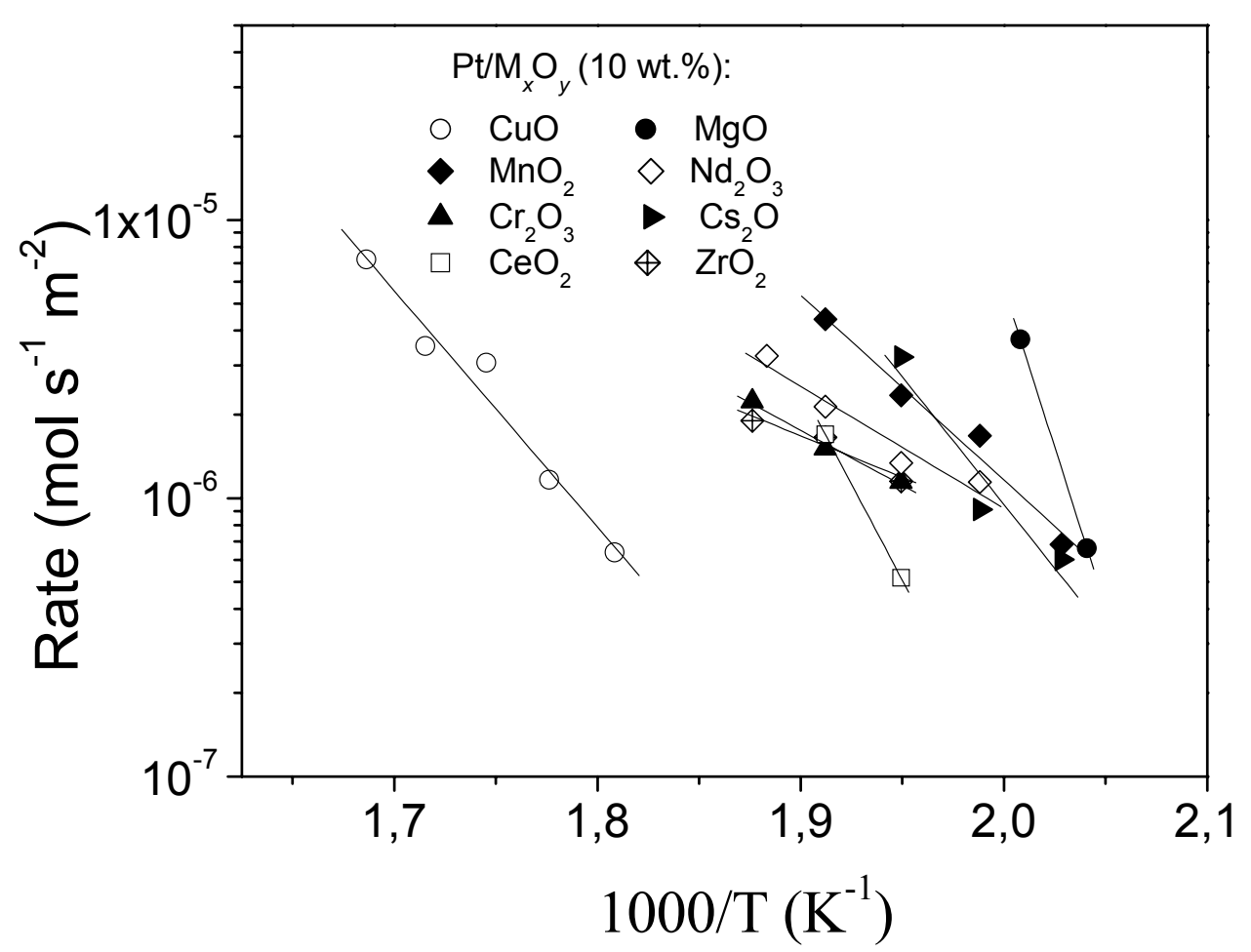

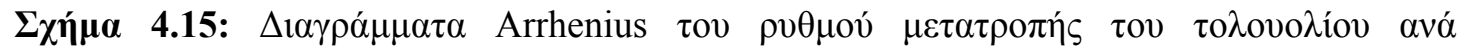

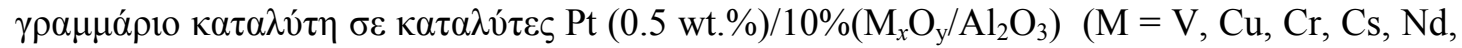
$\mathrm{Zr}, \mathrm{Ce}, \mathrm{Mg}, \mathrm{Mn})$.

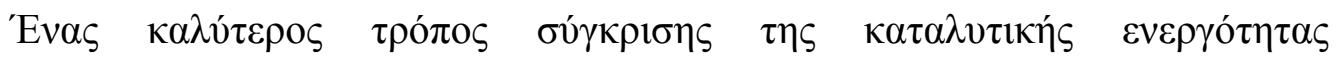

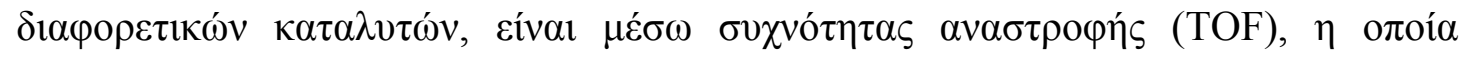

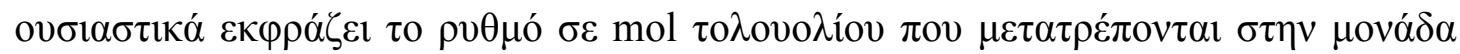

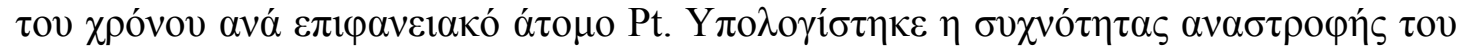

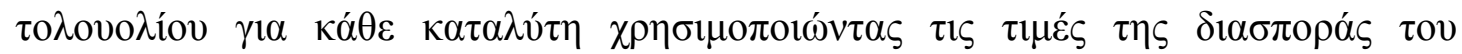

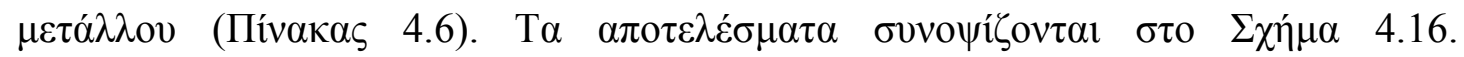

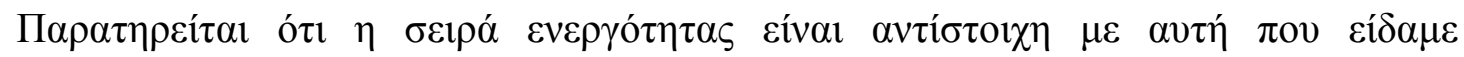

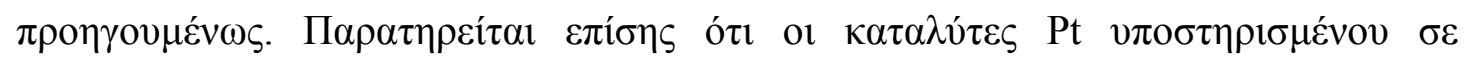

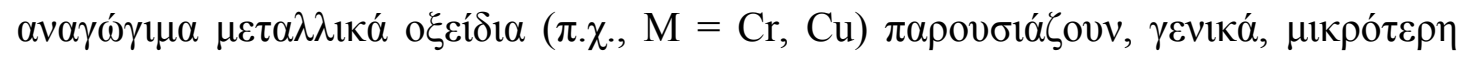

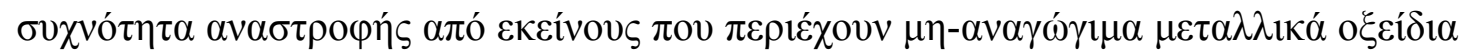

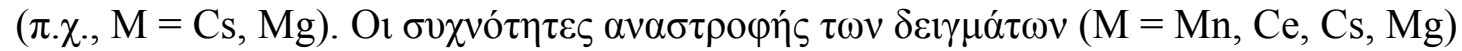

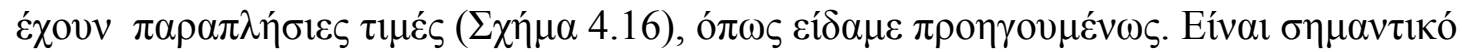

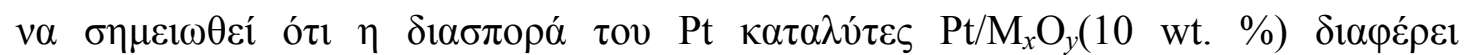

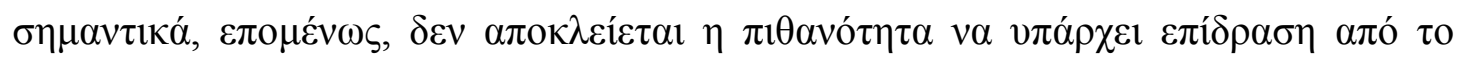
$\mu \varepsilon \dot{\gamma \varepsilon \theta \text { o } \sigma \omega \mu \alpha \tau \imath \delta i ́ \omega v ~ \sigma \tau о ~ T O F . ~}$ 
A

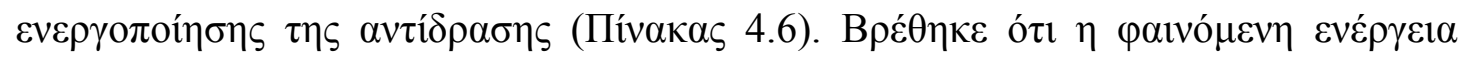

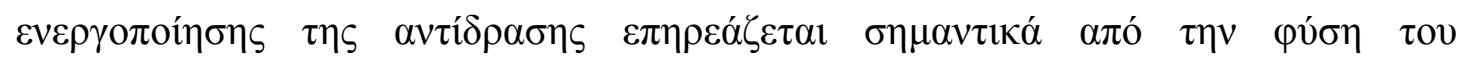

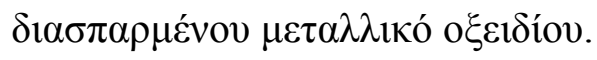

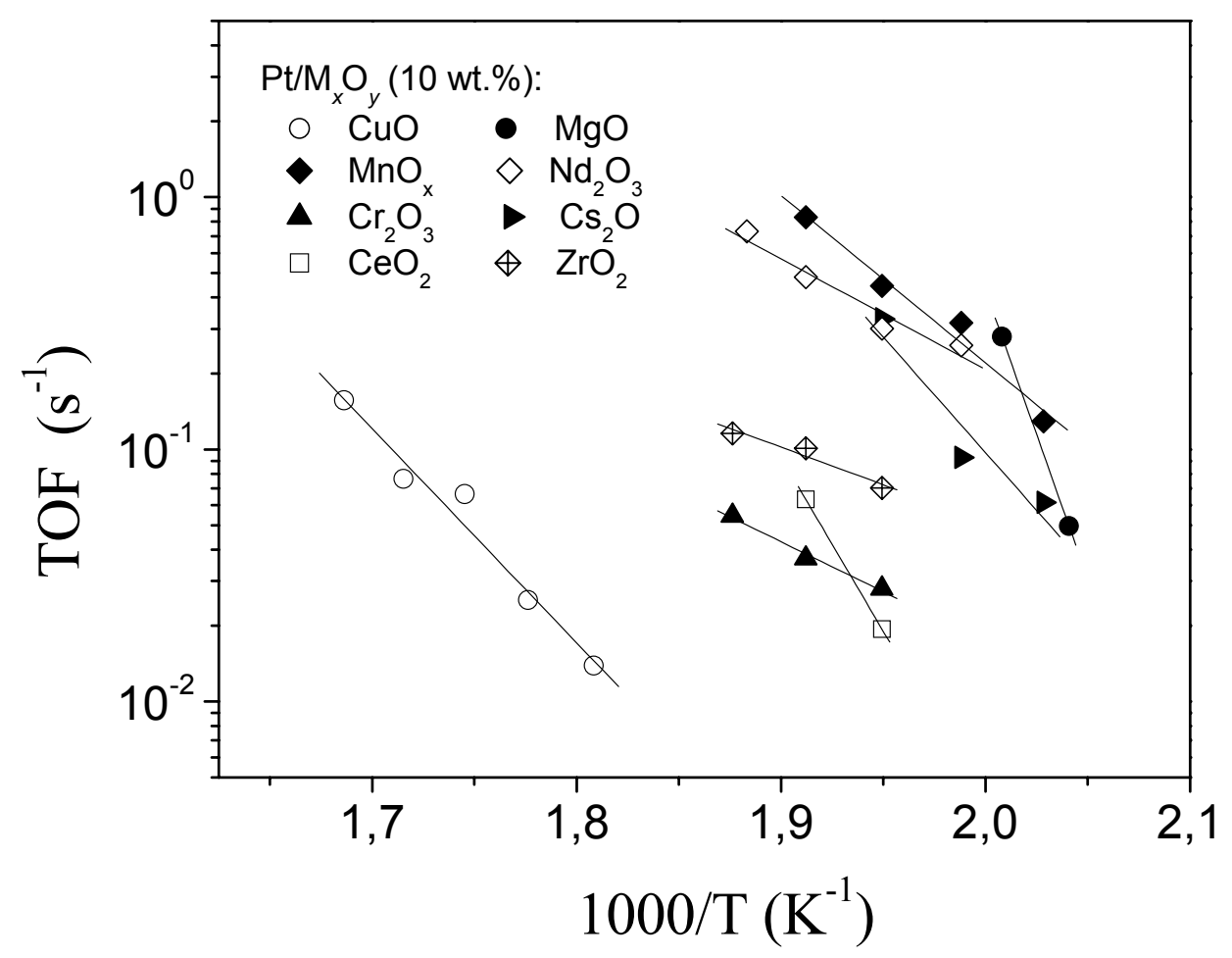

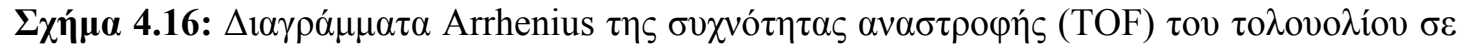

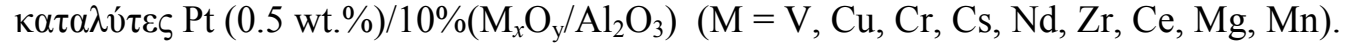

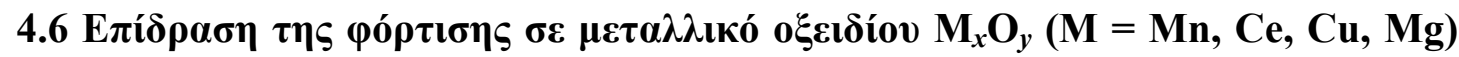

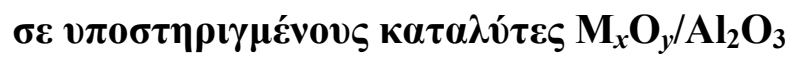

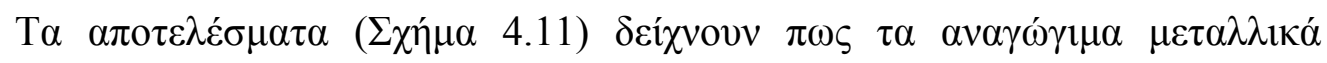

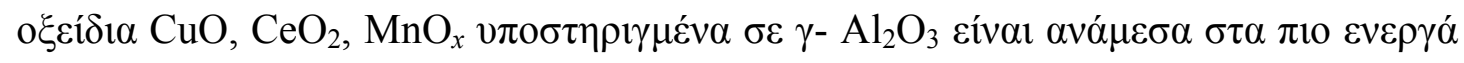

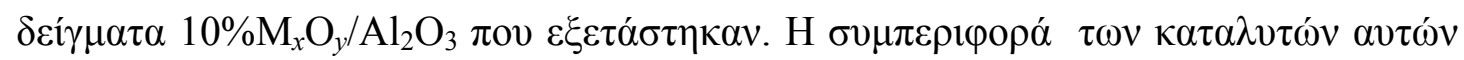

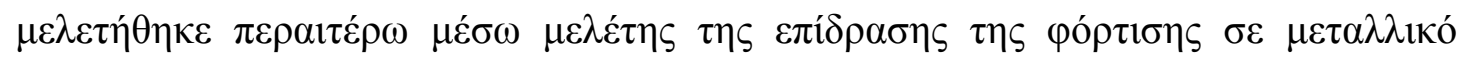

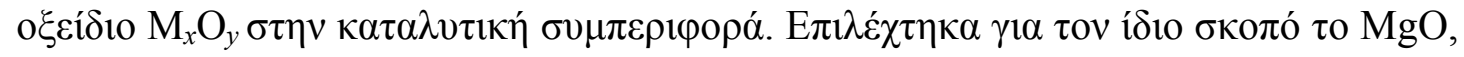

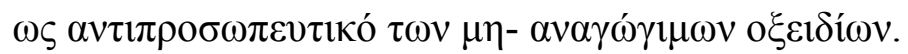




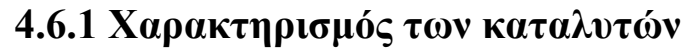

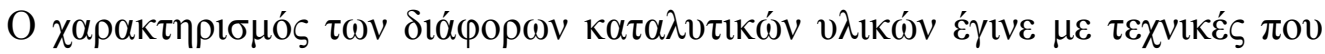

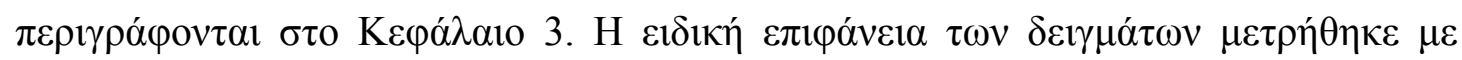

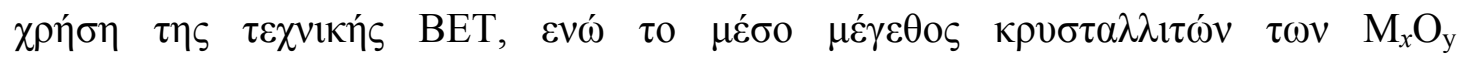

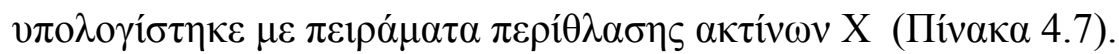

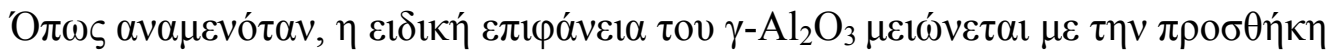

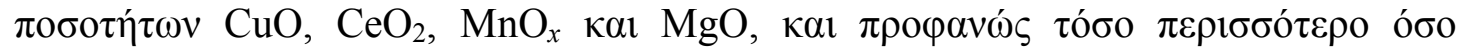

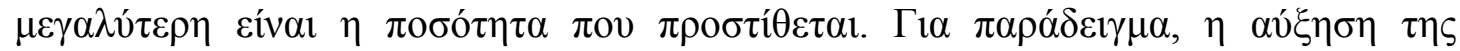

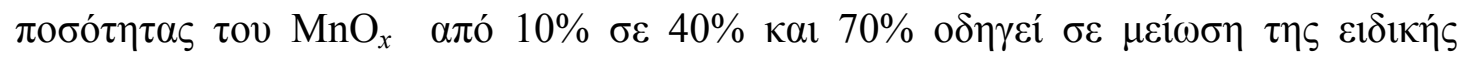

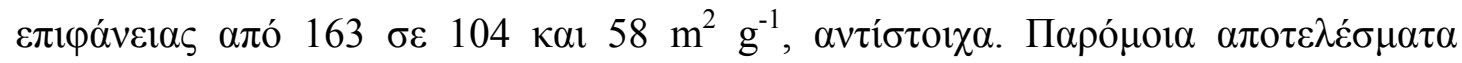

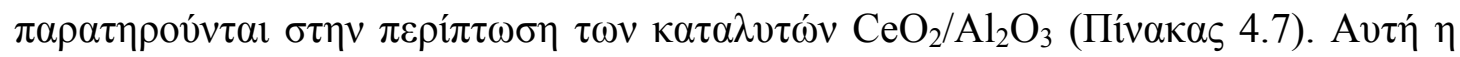

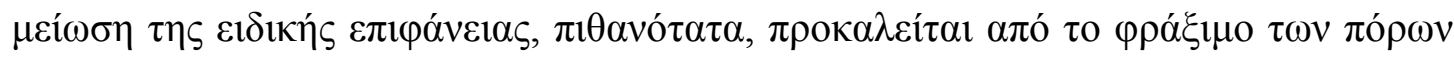

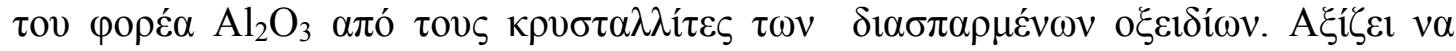

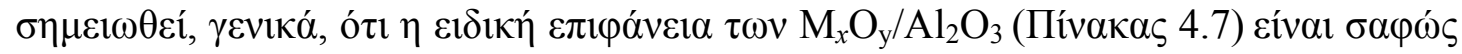

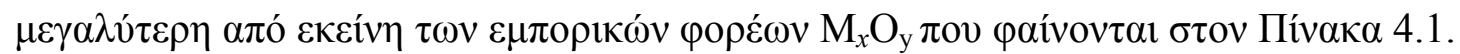

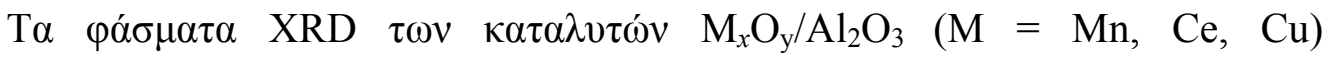

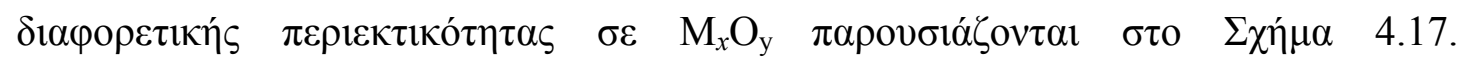

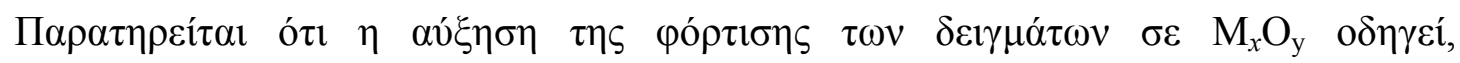

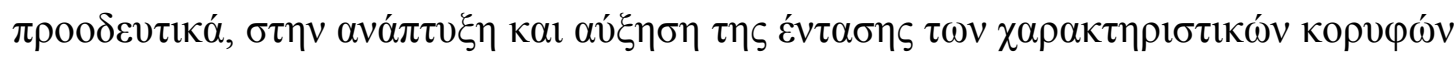

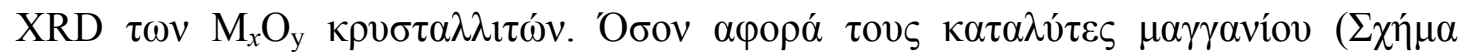

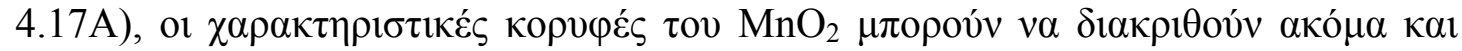

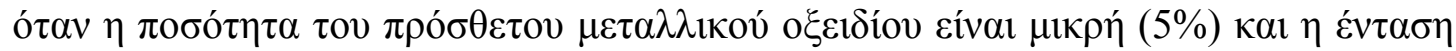

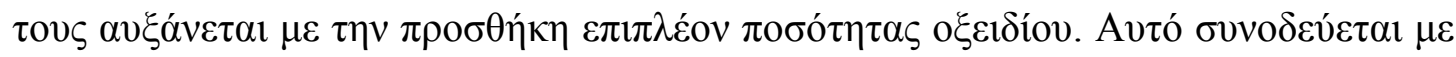

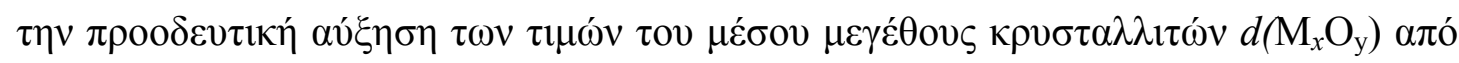

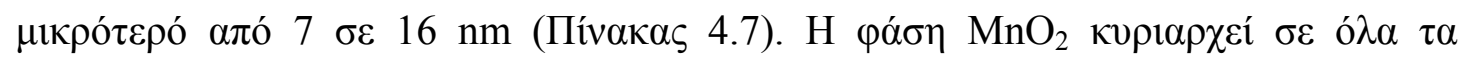

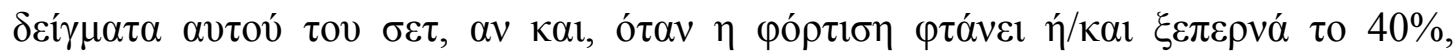

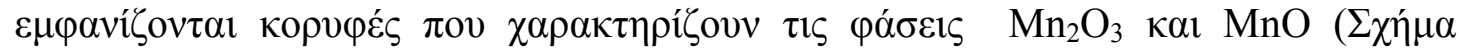

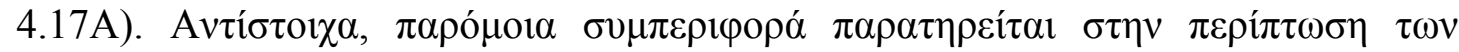

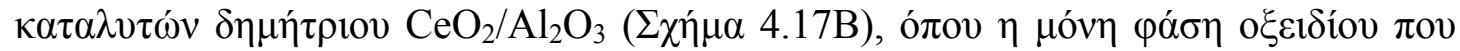

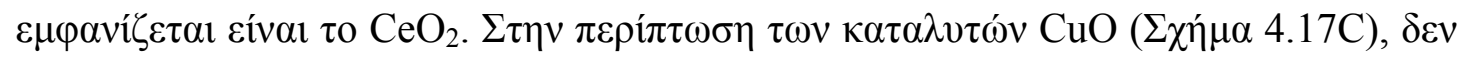

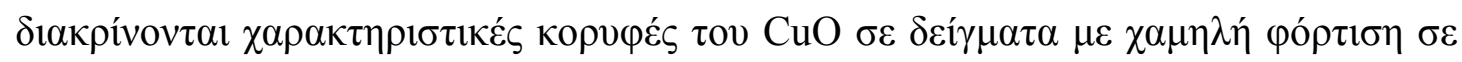

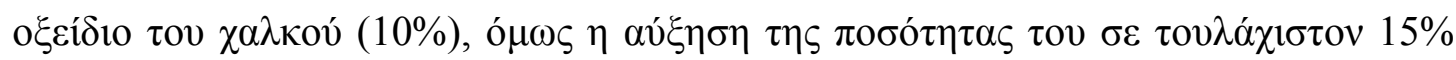

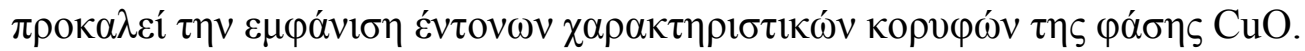




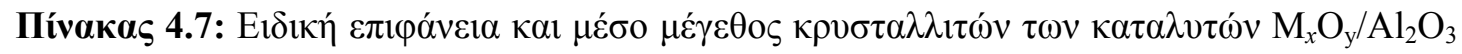
$(\mathrm{M}=\mathrm{Cu}, \mathrm{Mn}, \mathrm{Ce}, \mathrm{Mg})$.

\begin{tabular}{|c|c|c|c|}
\hline \multicolumn{2}{|c|}{$\mathrm{M}_{x} \mathrm{O}_{y}$} & \multirow{2}{*}{$\begin{array}{c}\text { Еıљıк'́ } \\
\varepsilon \pi ı \alpha^{\prime} v \varepsilon ı \alpha \text { SSA } \\
\left(\mathrm{m}^{2} \mathrm{~g}^{-1}\right)\end{array}$} & \multirow{2}{*}{ 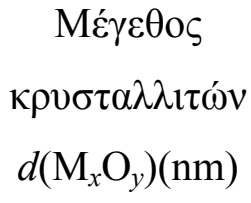 } \\
\hline $\begin{array}{c}\text { Méc } \alpha \lambda \lambda \mathrm{o} \\
\text { (M) }\end{array}$ & 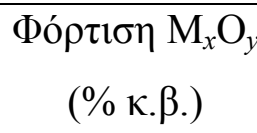 & & \\
\hline None $\left(\mathrm{Al}_{2} \mathrm{O}_{3}\right)$ & - & 183 & - \\
\hline \multirow[t]{4}{*}{$\mathrm{Cu}$} & 5 & - & n.d. \\
\hline & 10 & 152 & n.d \\
\hline & 15 & 151 & 33 \\
\hline & 20 & - & 31 \\
\hline \multirow[t]{6}{*}{$\mathrm{Mn}$} & 5 & - & $<7$ \\
\hline & 10 & 163 & 8 \\
\hline & 20 & - & 10 \\
\hline & 30 & 122 & 9 \\
\hline & 40 & 104 & 14 \\
\hline & 70 & 58 & 16 \\
\hline \multirow[t]{7}{*}{$\mathrm{Ce}$} & 5 & - & 6 \\
\hline & 10 & 161 & 7 \\
\hline & 20 & - & 9 \\
\hline & 30 & - & 8 \\
\hline & 50 & 104 & 8 \\
\hline & 70 & 82 & 9 \\
\hline & 90 & - & 10 \\
\hline $\mathrm{Mg}$ & 10 & 142 & n.d. \\
\hline
\end{tabular}



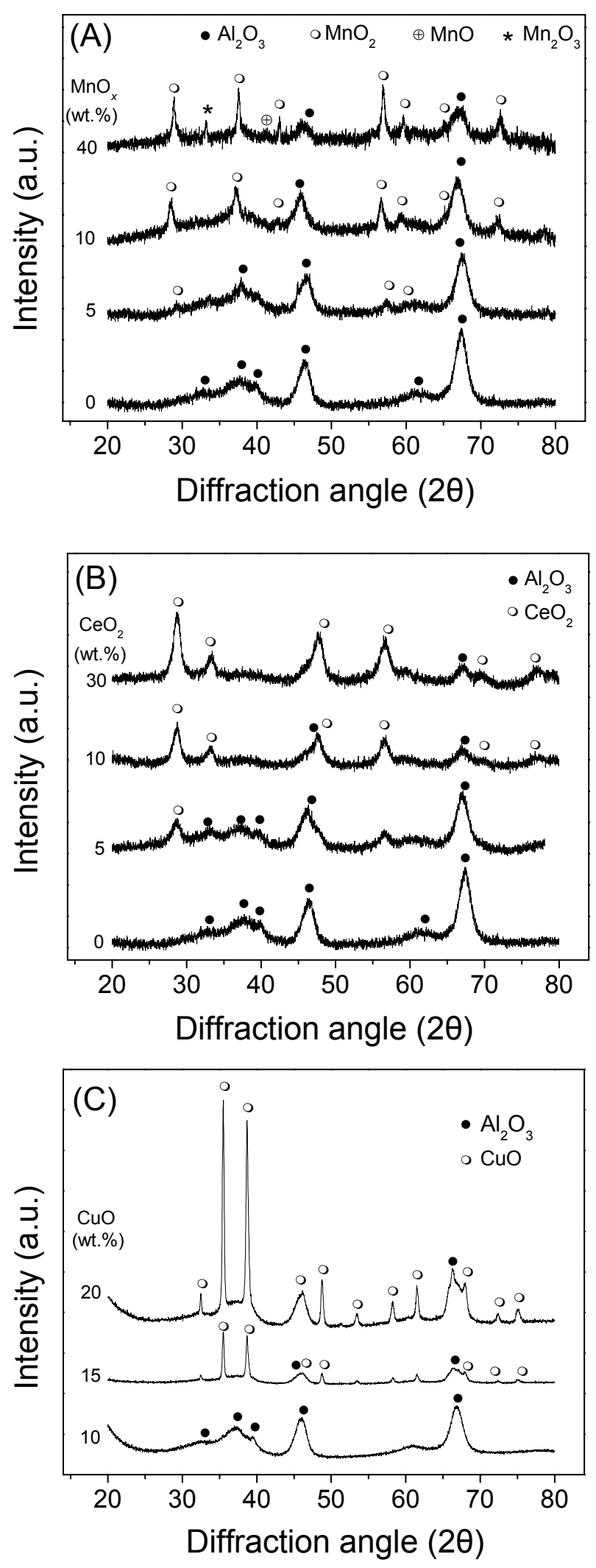

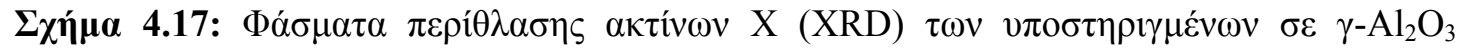

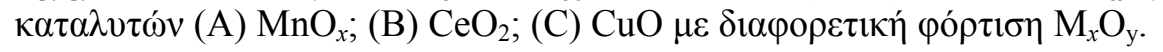




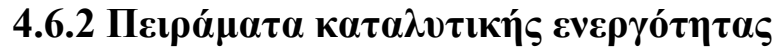

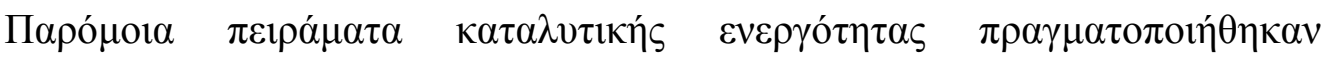

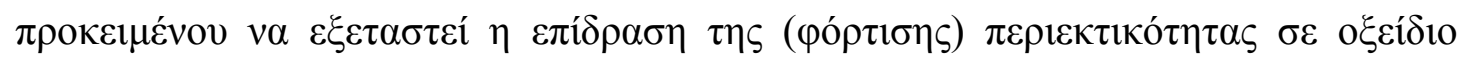

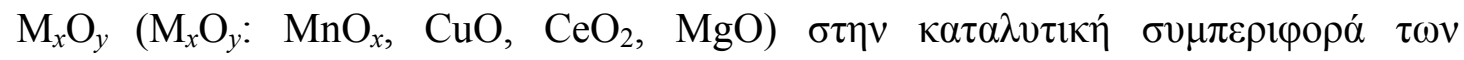

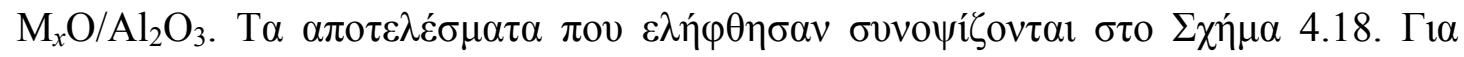

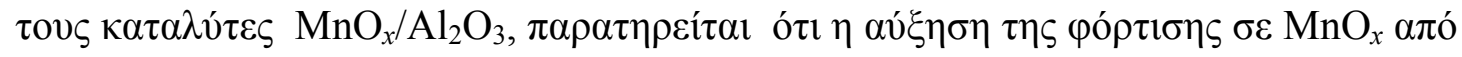

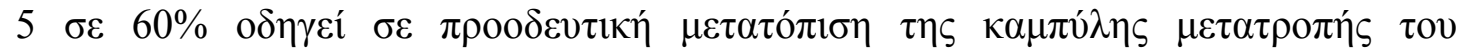

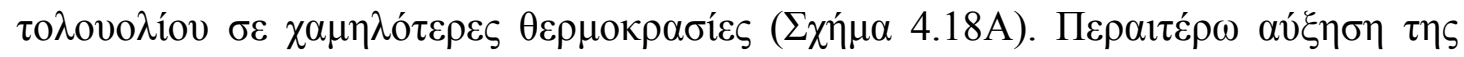

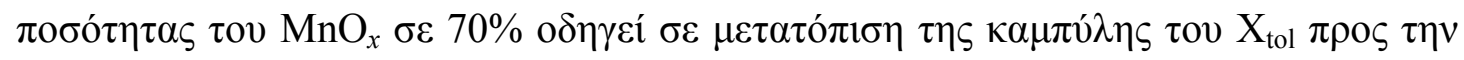
$\alpha v \tau i ́ \theta \varepsilon \tau \eta ~ \kappa \alpha \tau \varepsilon v ́ \theta v v \sigma \eta$.

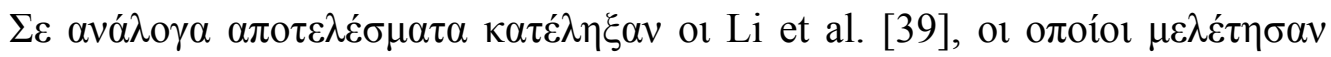

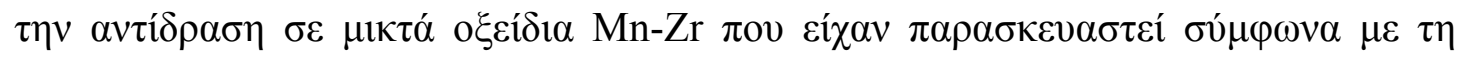

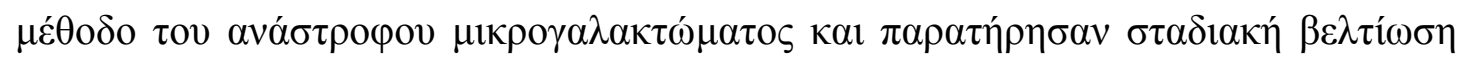

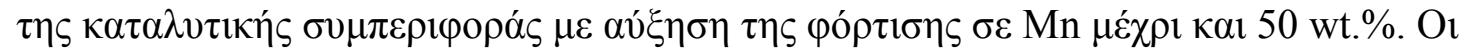

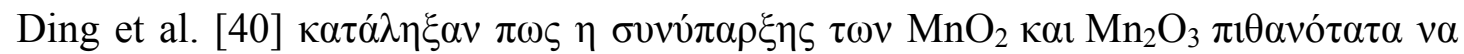

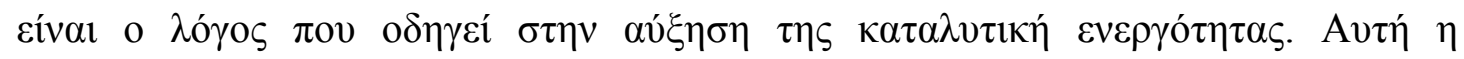

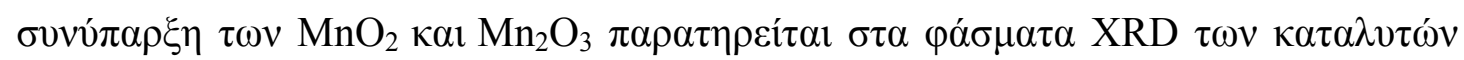

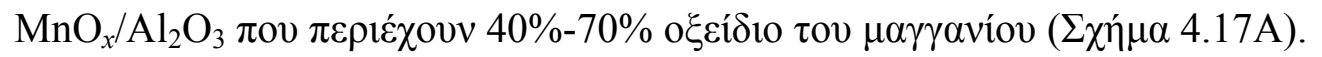

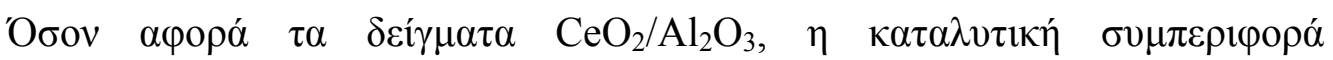

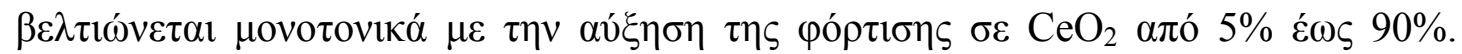

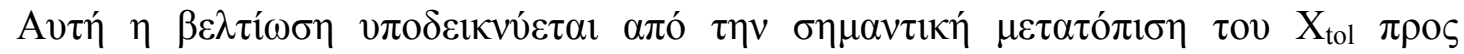

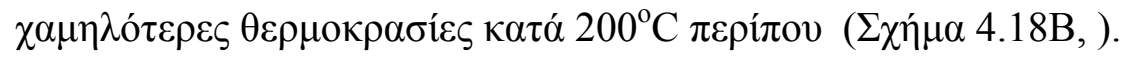

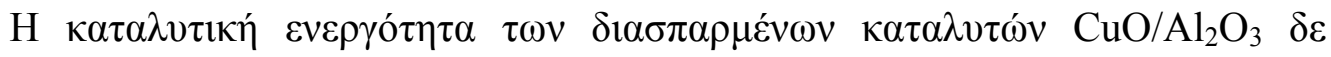

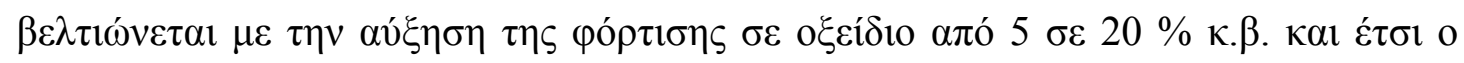

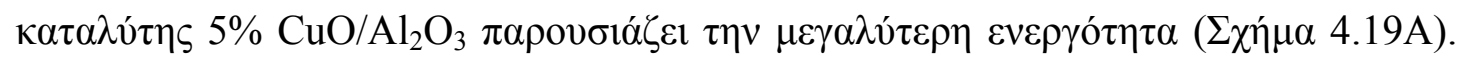

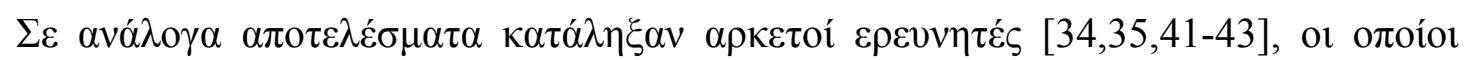

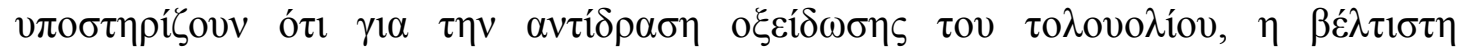

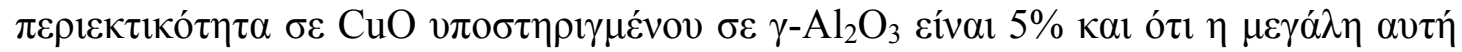

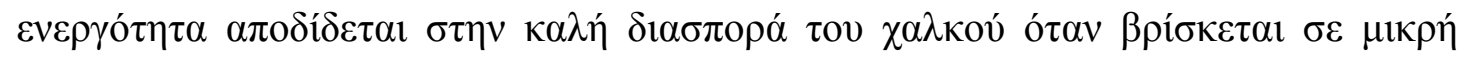
$\pi$

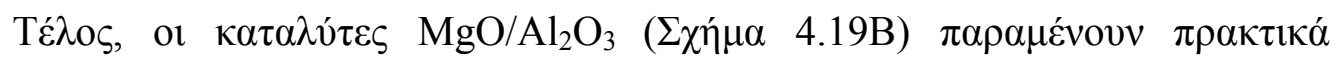

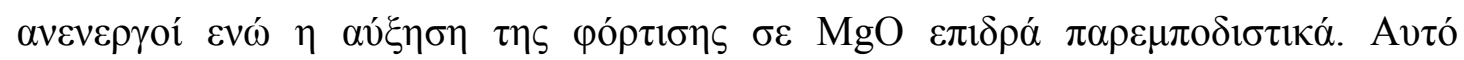

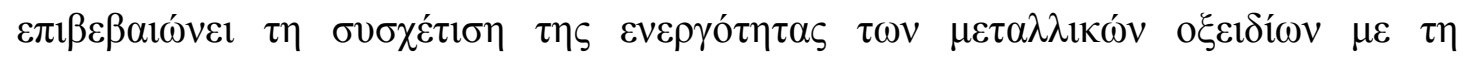

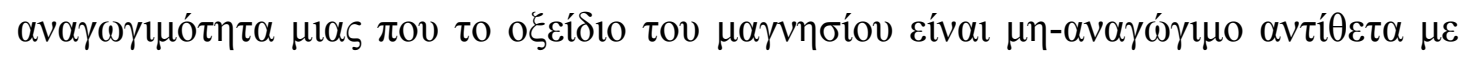




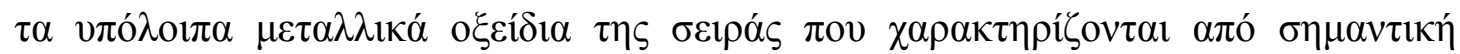
$\alpha v \alpha \gamma \omega \gamma \mu \operatorname{có}_{\tau \eta \tau \alpha .}$
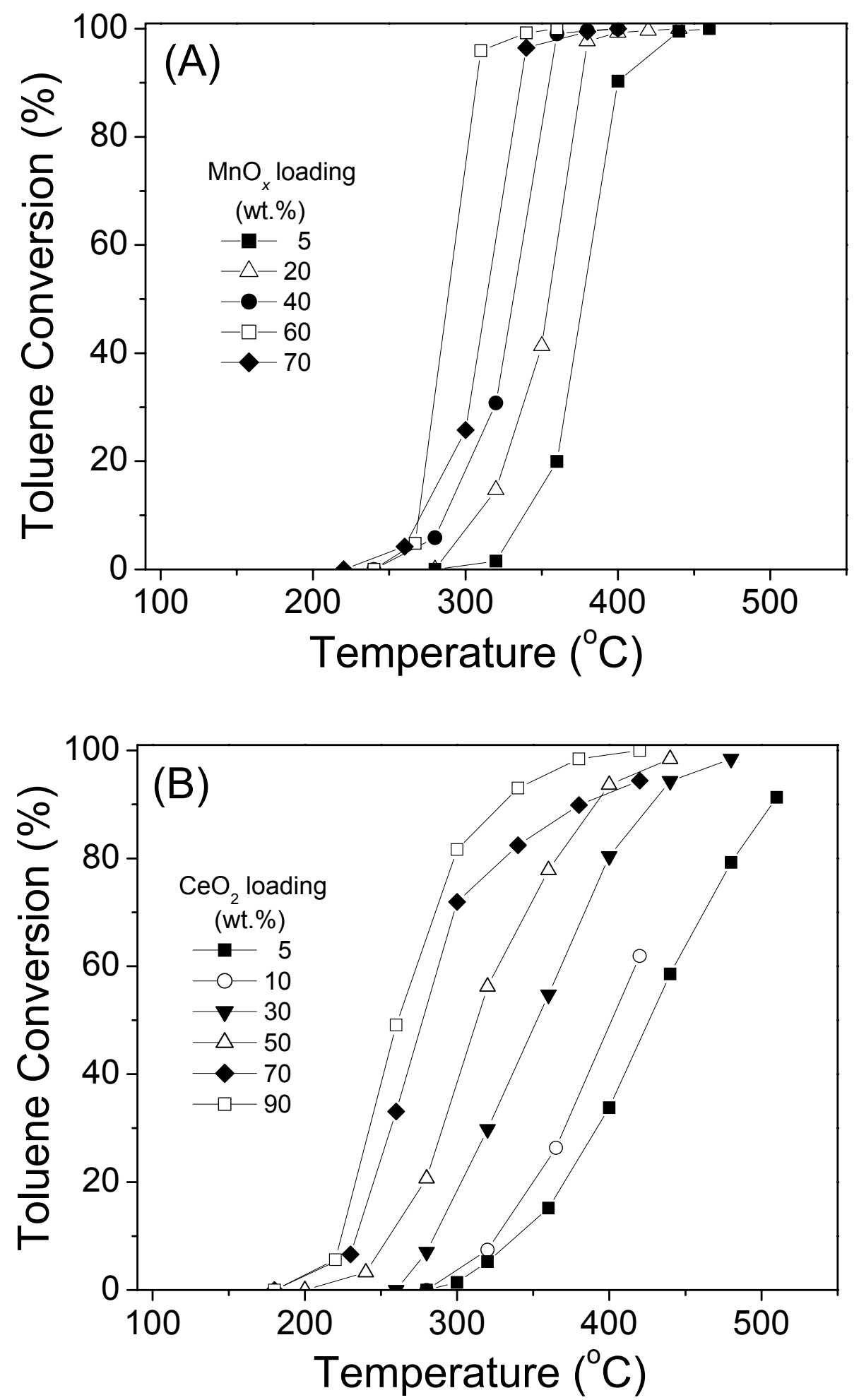

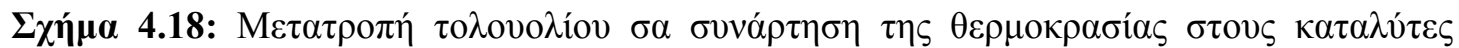

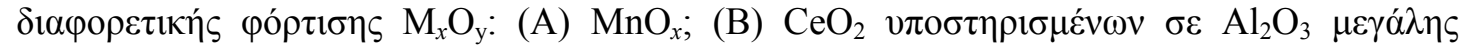
$\varepsilon \pi ı \alpha_{v} \varepsilon 1 \alpha \varsigma$. 

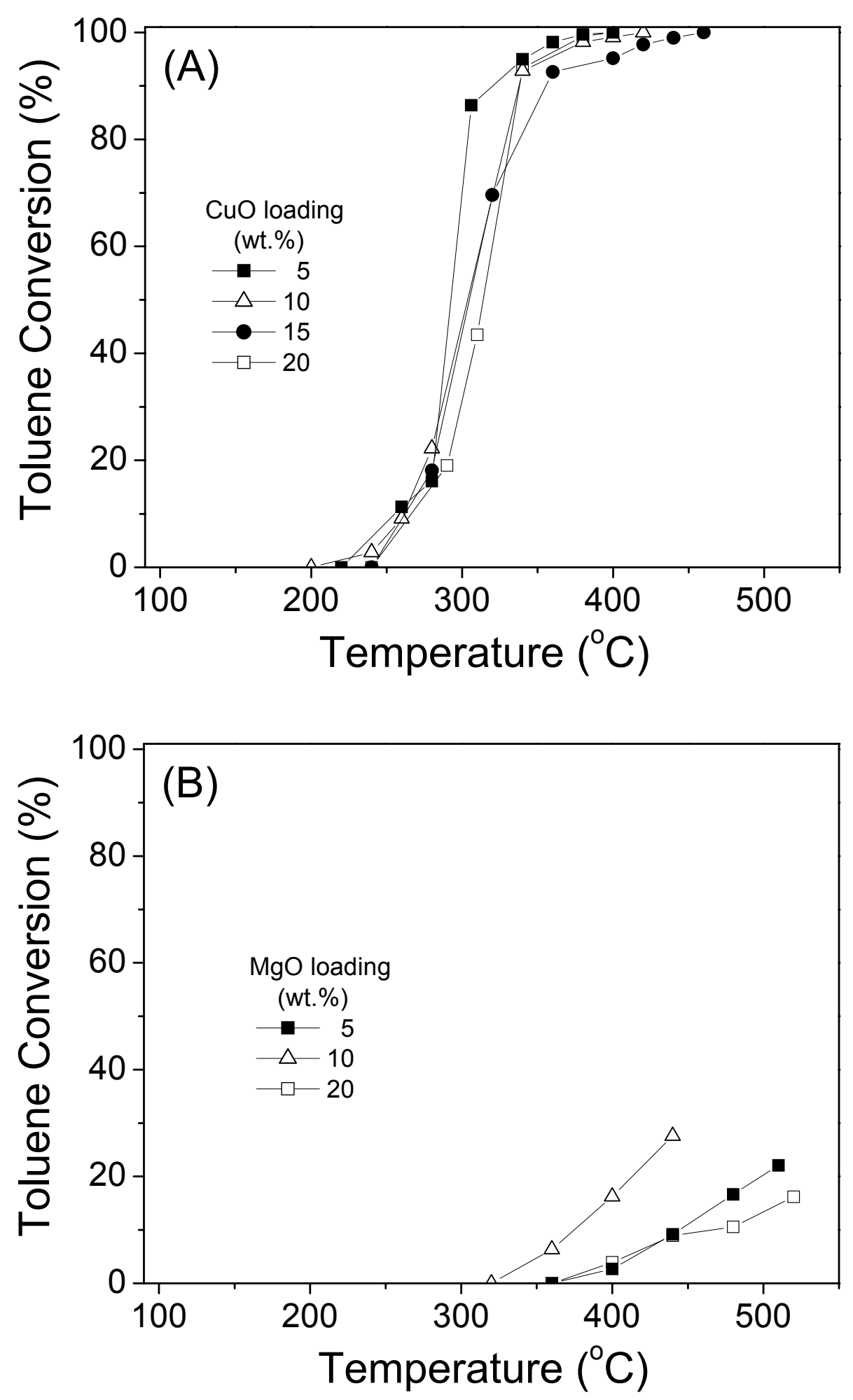

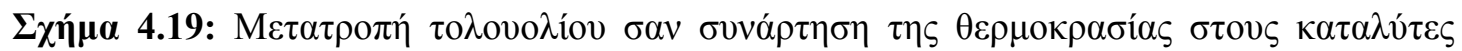

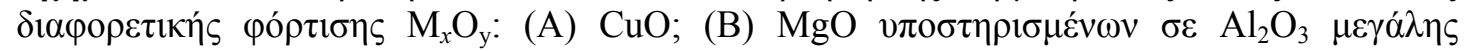

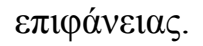




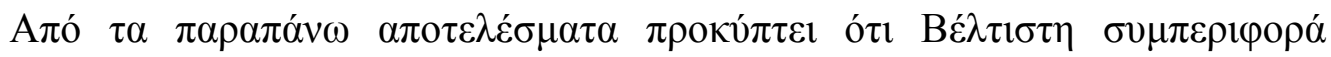

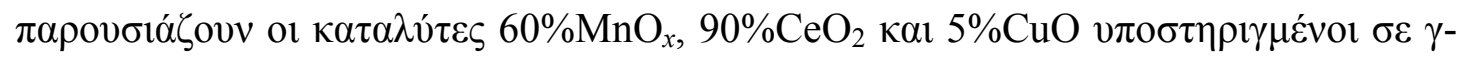

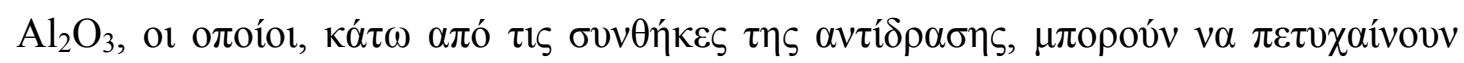

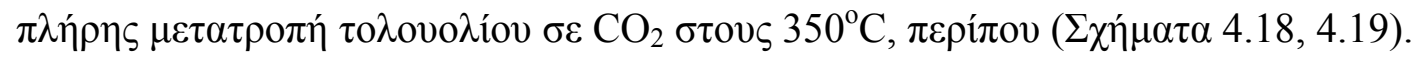

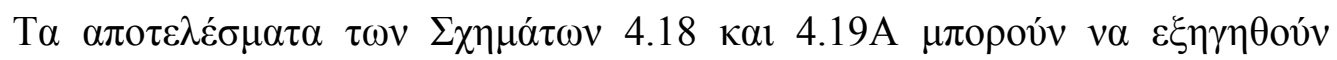

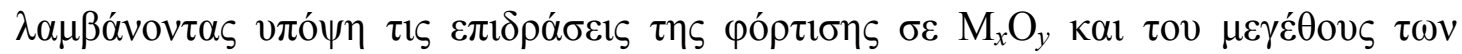

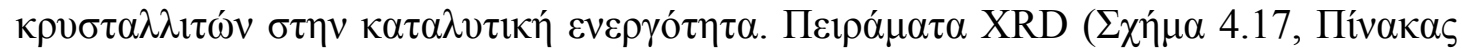

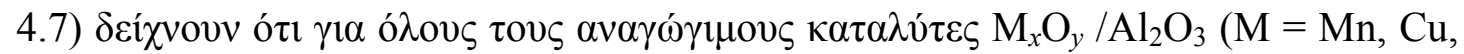

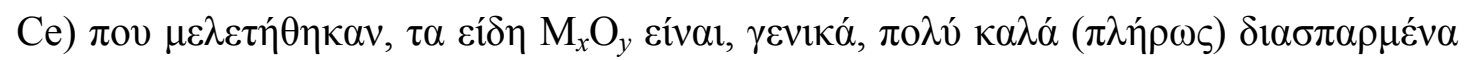

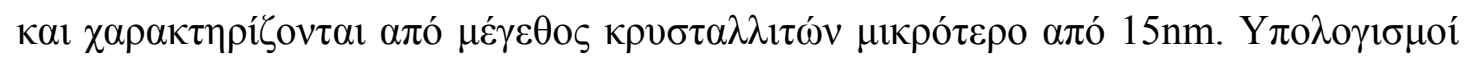

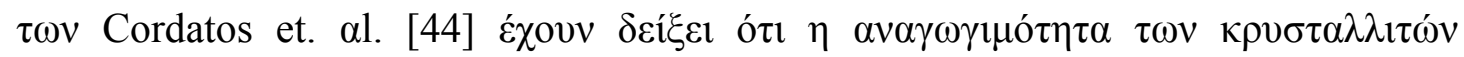

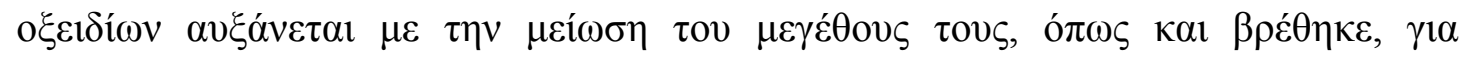

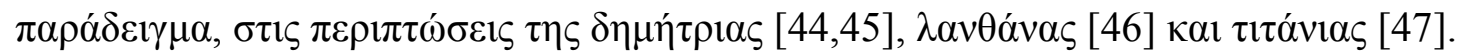

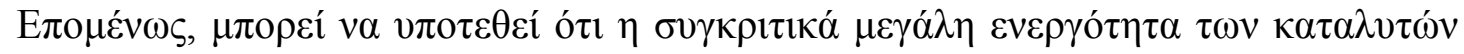

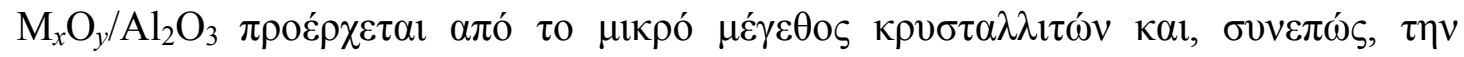

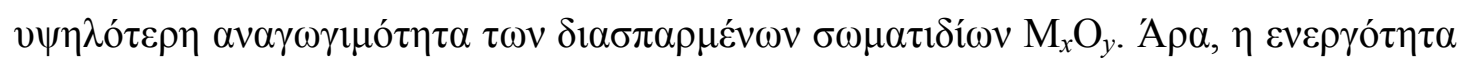

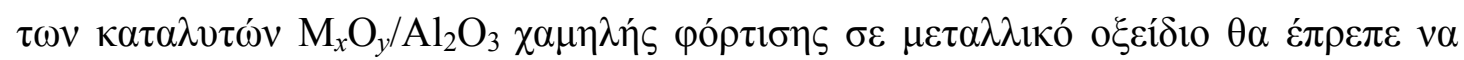

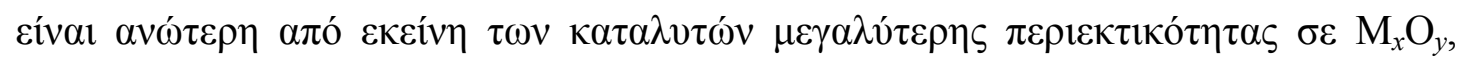

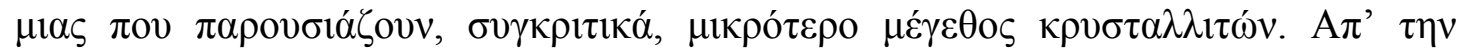

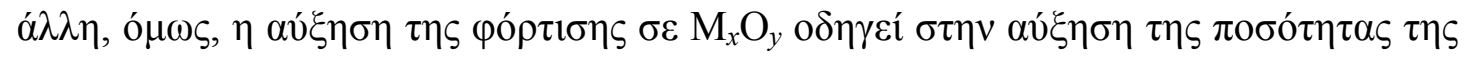

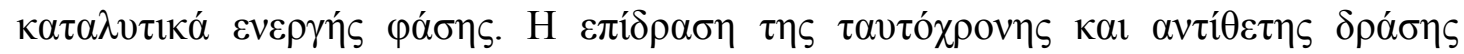

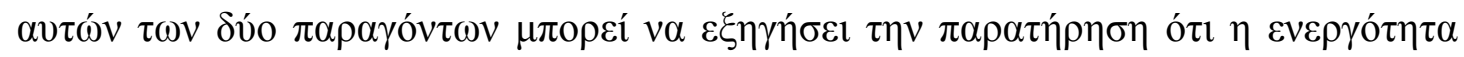

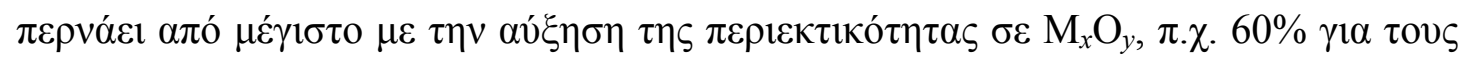

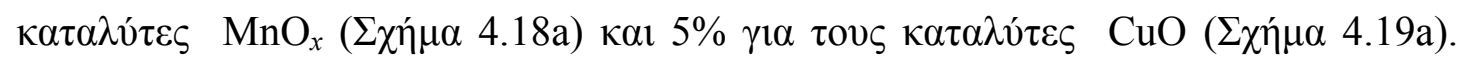

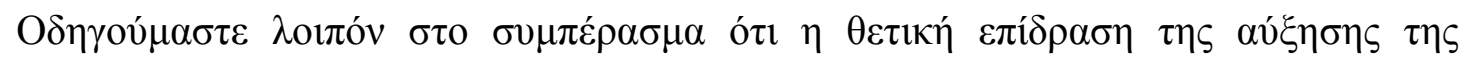

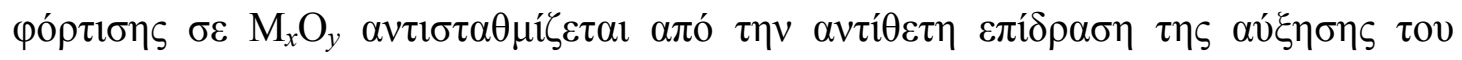

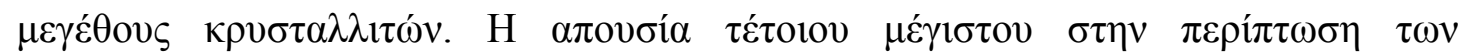

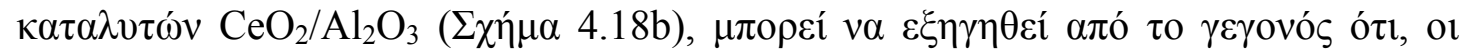

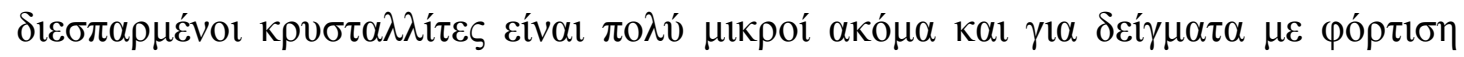

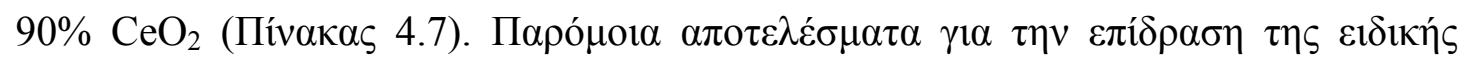

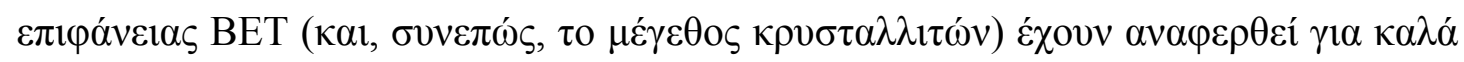

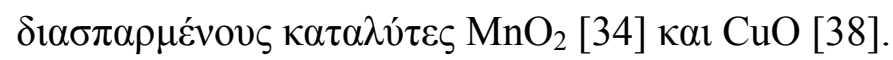




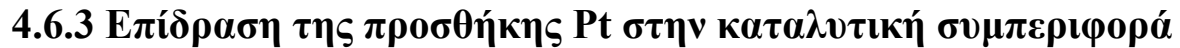

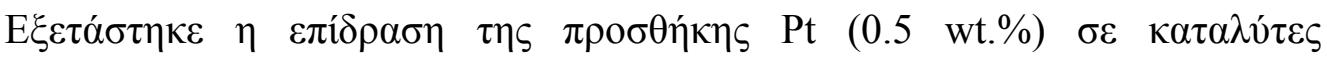

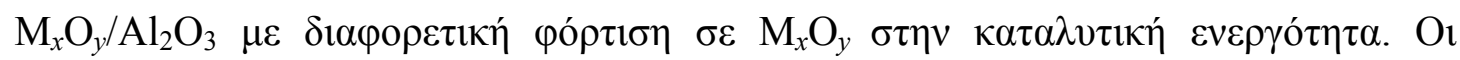

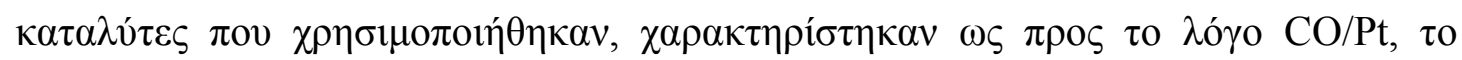

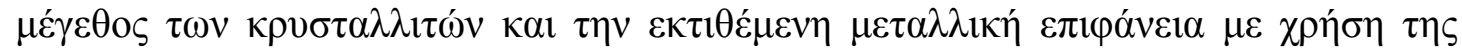

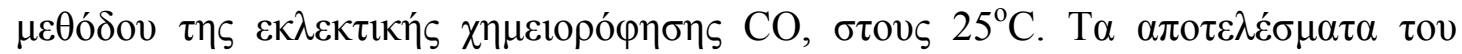

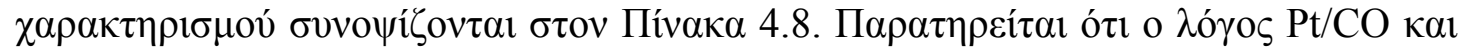

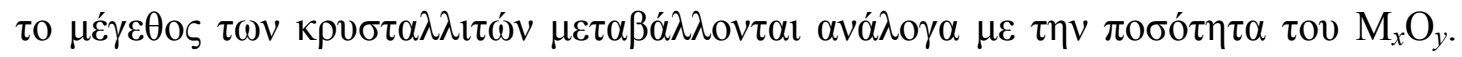

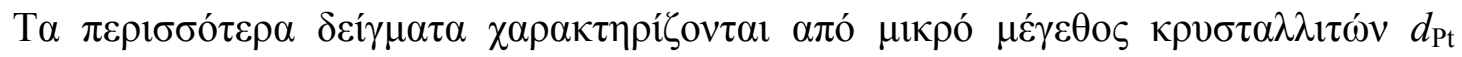

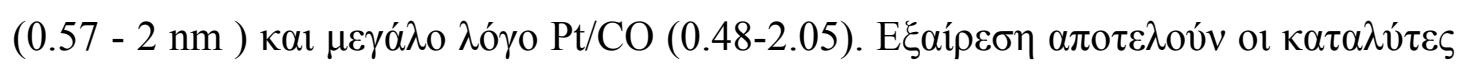

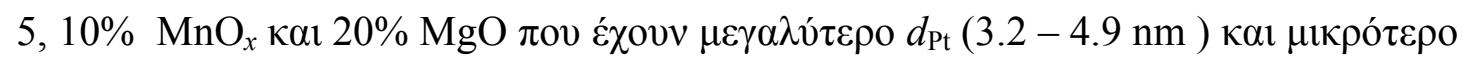
$\lambda$ ó yo Pt/CO (0.21-0.32).

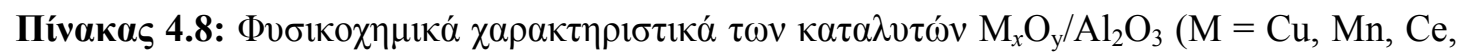
$\mathrm{Mg})$.

\begin{tabular}{|c|c|c|c|}
\hline \multicolumn{2}{|c|}{$\mathrm{M}_{x} \mathrm{O}_{y}$} & \multirow[t]{2}{*}{$\mathrm{CO} / \mathrm{Pt}$ ratio } & \multirow{2}{*}{ 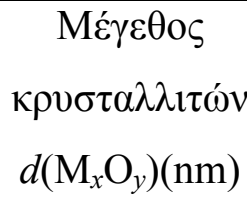 } \\
\hline $\begin{array}{c}\text { Mźc } \alpha \lambda \lambda \mathrm{o} \\
\text { (M) }\end{array}$ & 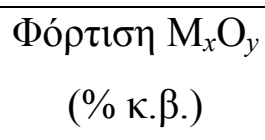 & & \\
\hline \multirow[t]{4}{*}{$\mathrm{Mn}$} & 5 & 0.32 & 3.2 \\
\hline & 10 & 0.21 & 4.9 \\
\hline & 20 & 0.50 & 2 \\
\hline & 30 & 0.48 & 2.1 \\
\hline \multirow[t]{4}{*}{$\mathrm{Ce}$} & 5 & 2.05 & 0.5 \\
\hline & 10 & 1.04 & 1.0 \\
\hline & 20 & 0.73 & 1.4 \\
\hline & 30 & 0.99 & 1.3 \\
\hline \multirow[t]{3}{*}{$\mathrm{Cu}$} & 5 & 1.68 & 0.61 \\
\hline & 10 & 1.8 & 0.57 \\
\hline & 20 & 1.59 & 0.64 \\
\hline \multirow[t]{3}{*}{$\mathrm{Mg}$} & 5 & 1.01 & 1.0 \\
\hline & 10 & 0.52 & 2.0 \\
\hline & 20 & 0.31 & 3.3 \\
\hline
\end{tabular}




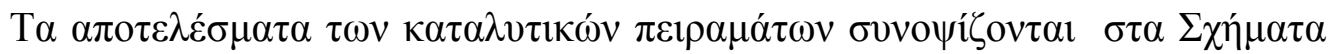

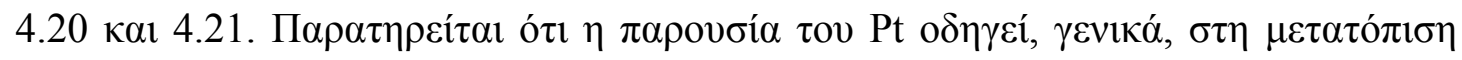

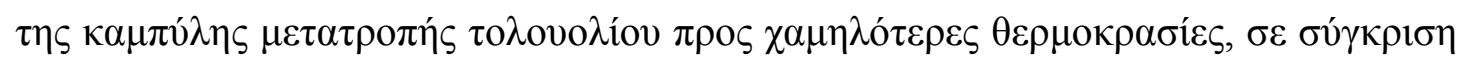

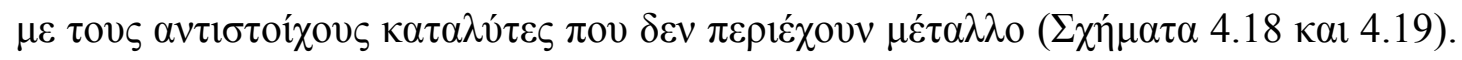

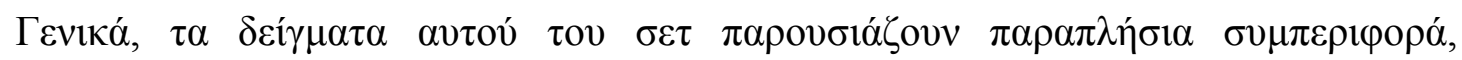

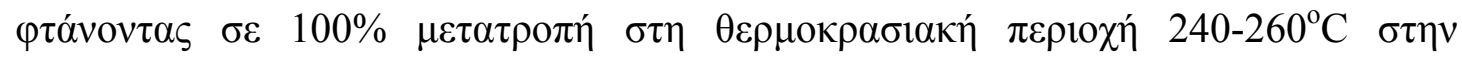

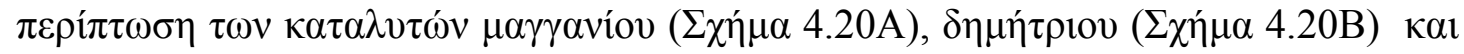

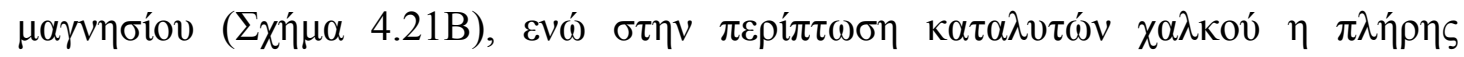

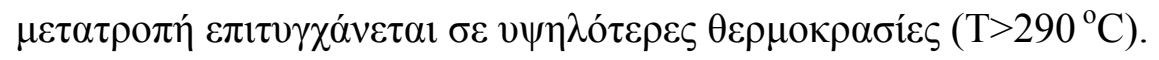

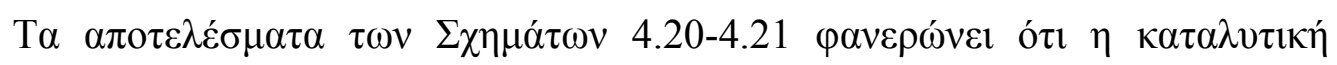

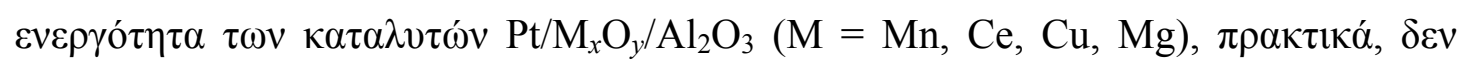

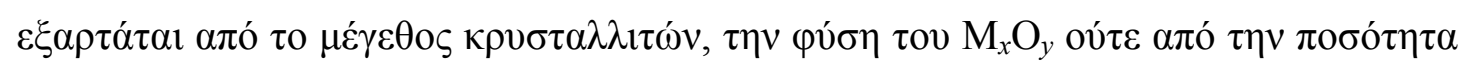

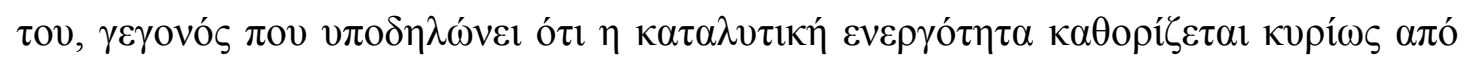
$\tau$ o $\delta 1 \alpha \sigma \pi \alpha \rho \mu \varepsilon ́ v o ~ \mu \varepsilon ́ \tau \alpha \lambda \lambda \mathrm{o}, \mathrm{Pt}$.

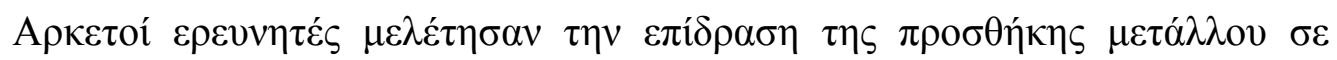

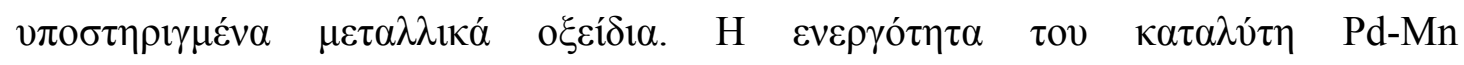

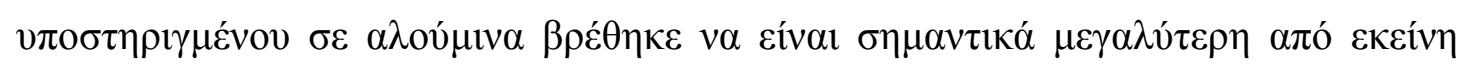

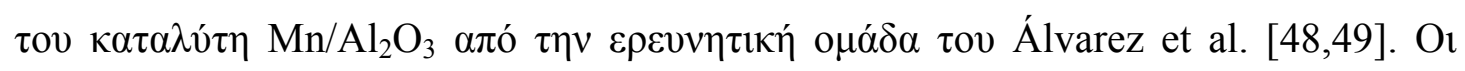

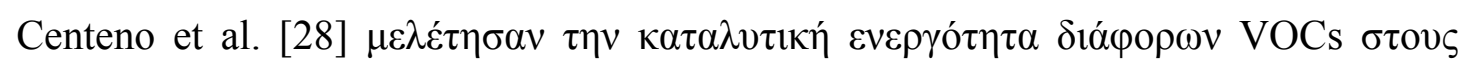

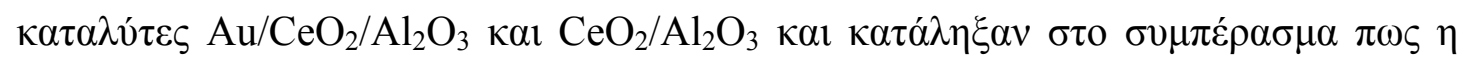

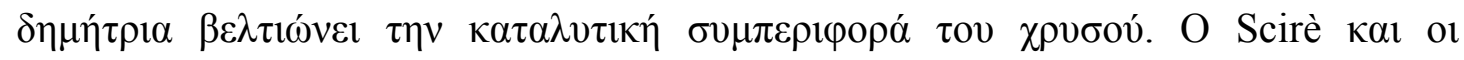

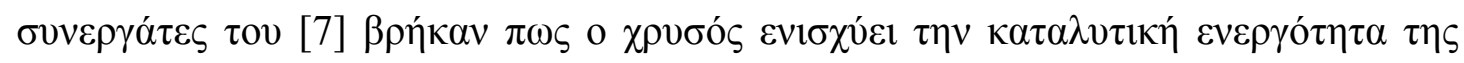

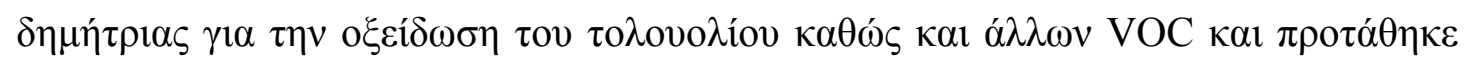

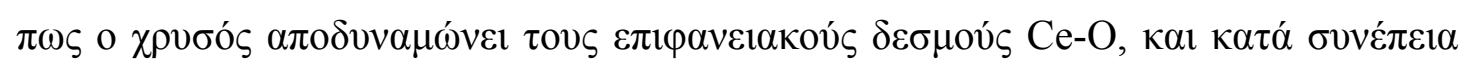

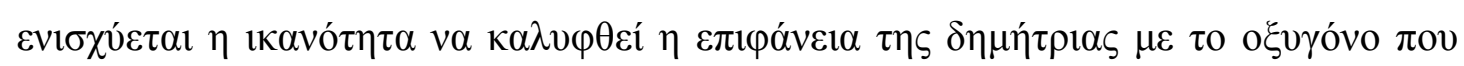

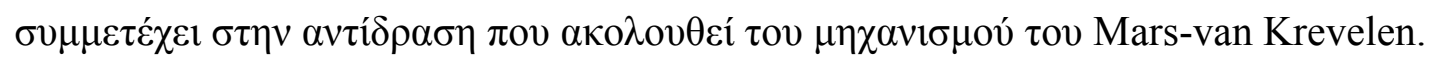



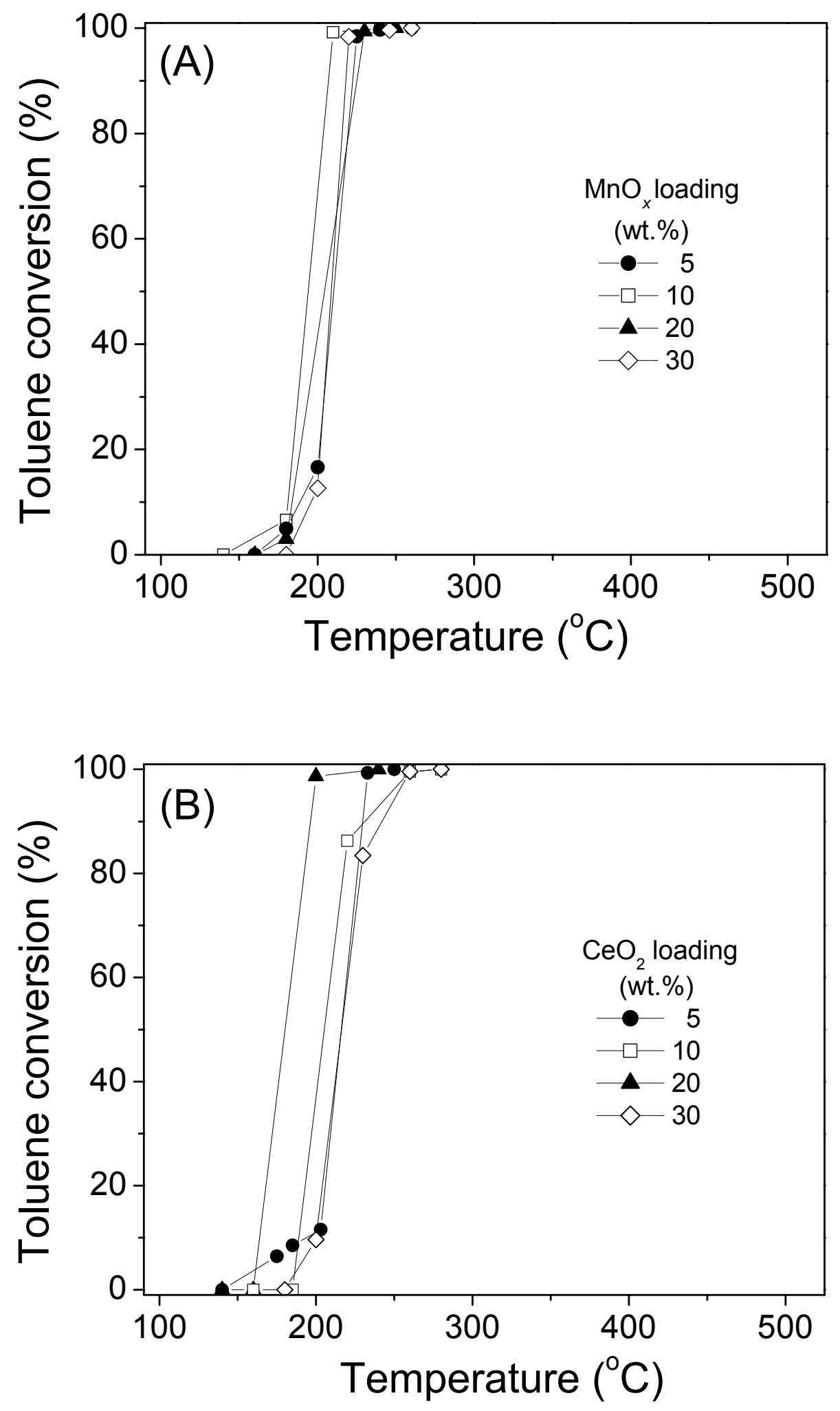

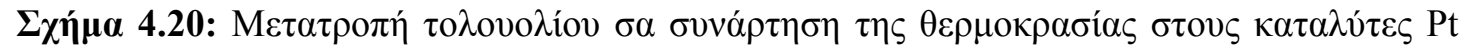

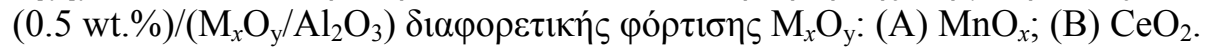



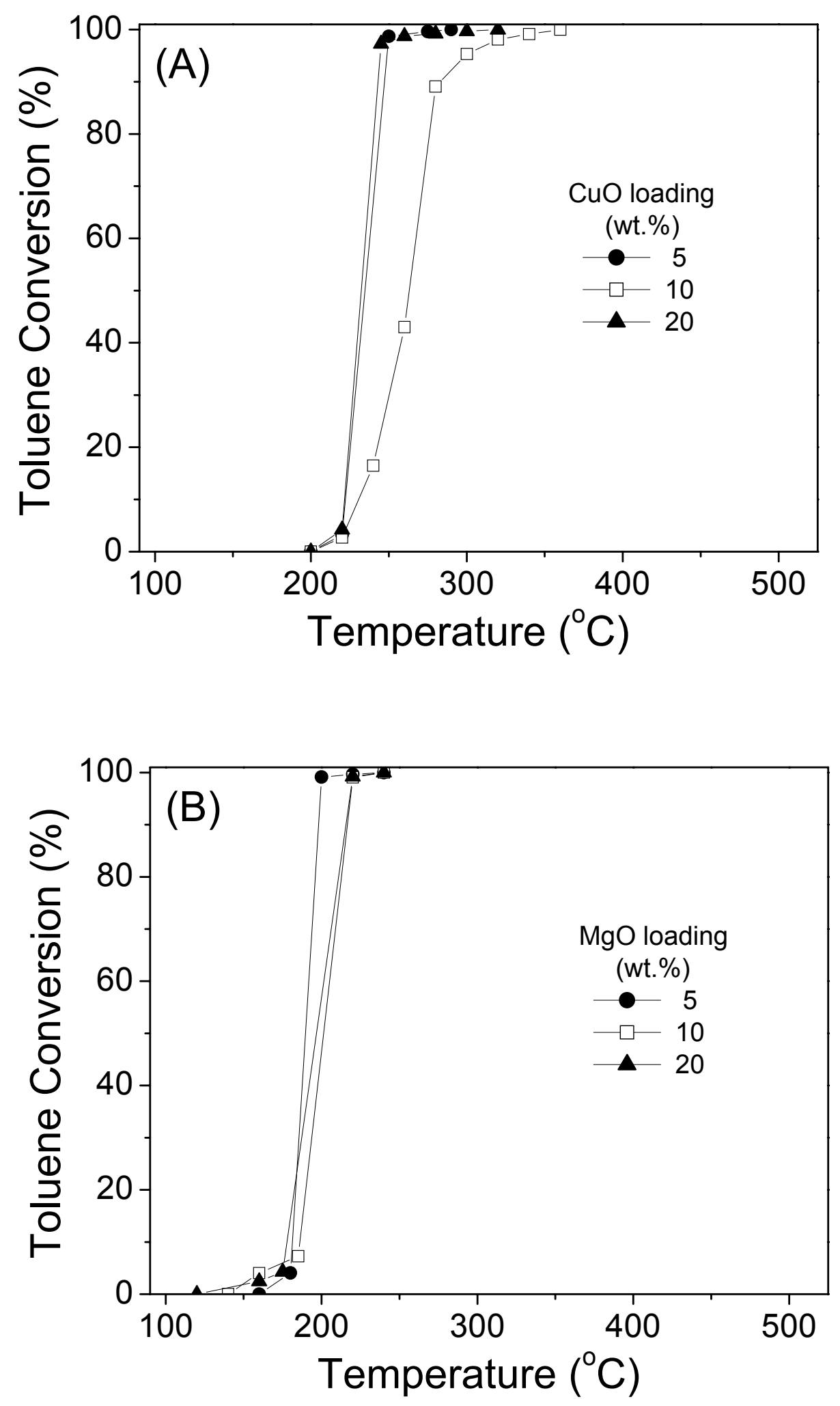

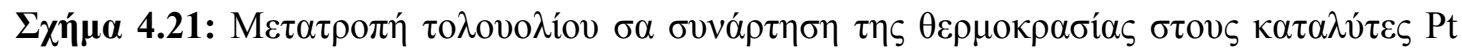

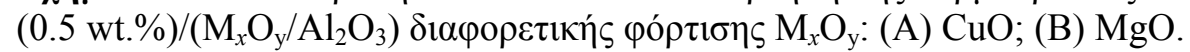




\section{$4.7 \Sigma v \mu \pi \varepsilon \rho \alpha ́ \sigma \mu \alpha \tau \alpha$}

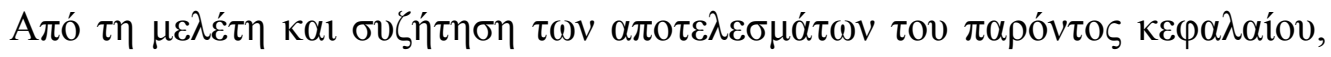

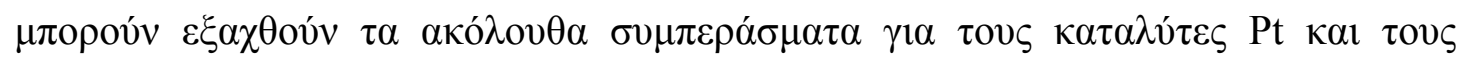

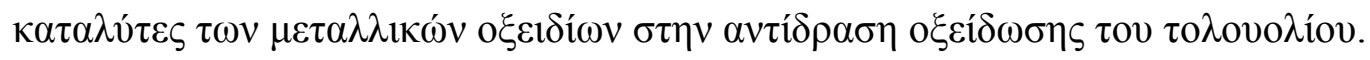

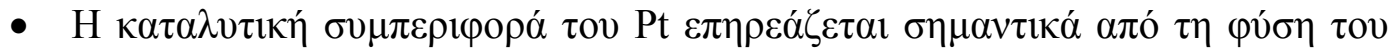

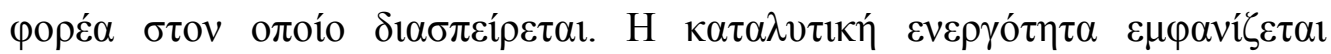

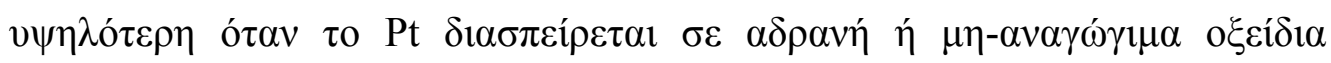
$\mu \varepsilon \tau \alpha \dot{\lambda} \lambda \lambda \omega v, \pi \cdot \chi . \mathrm{SiO}_{2}, \mathrm{Al}_{2} \mathrm{O}_{3}, \mathrm{ZrO}_{2}, \mathrm{MgO}, \sigma \varepsilon \sigma \chi \varepsilon \dot{\sigma} \eta \mu \varepsilon \tau \eta \nu \pi \varepsilon \rho i ́ \pi \tau \omega \sigma \eta \pi$

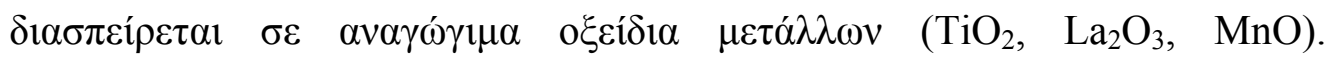

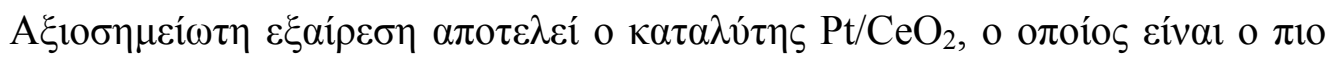

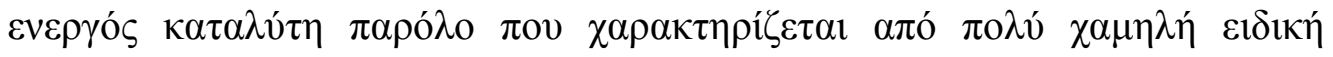
$\varepsilon \pi \imath \varphi \alpha ́ v \varepsilon \imath \alpha \mathrm{S}_{\mathrm{BET}}\left(3 \mathrm{~m}^{2} \mathrm{~g}^{-1}\right)$.

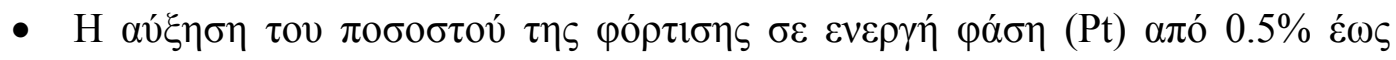

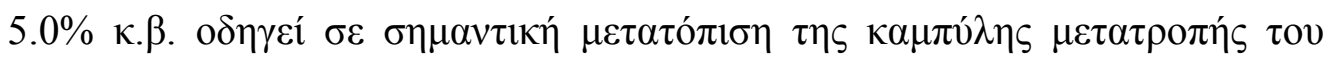

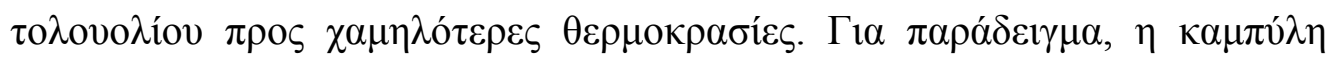

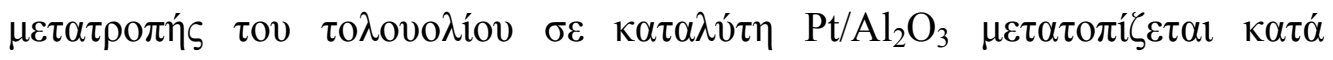

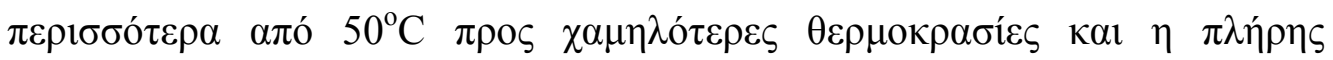

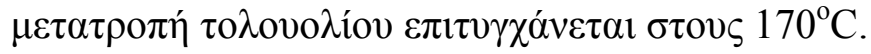

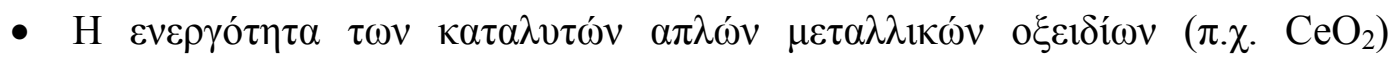

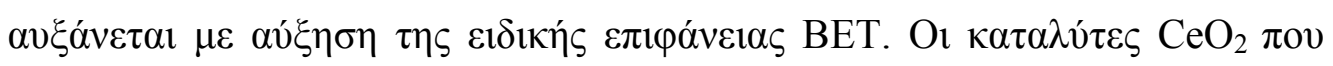

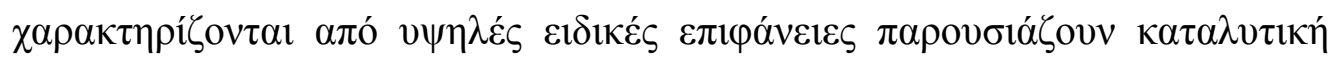

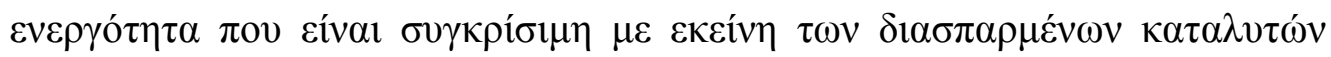

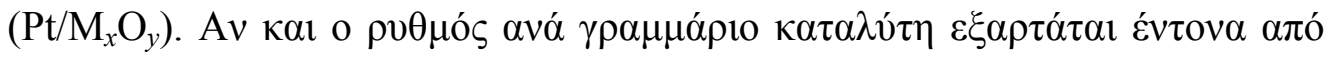

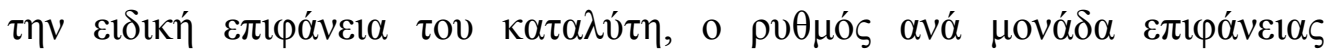

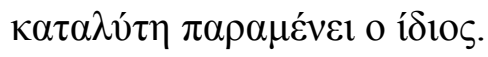

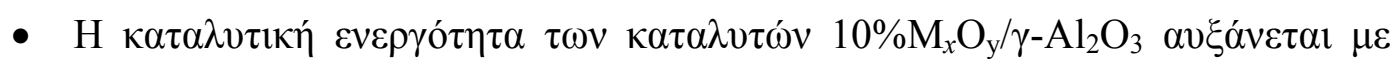

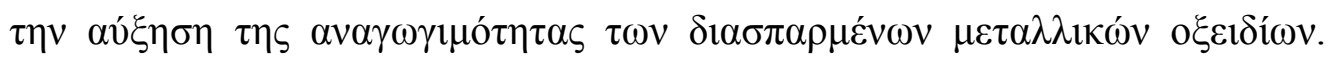

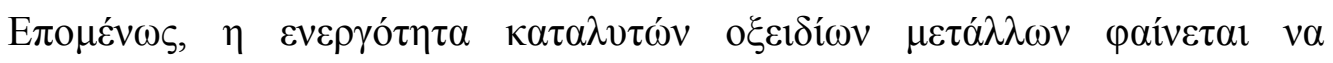

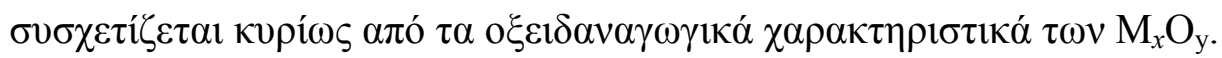

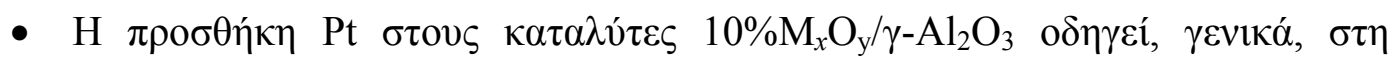

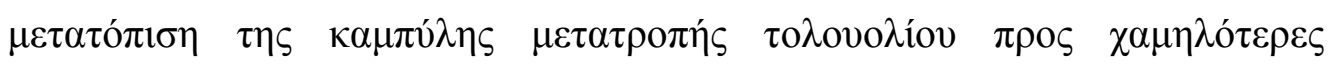

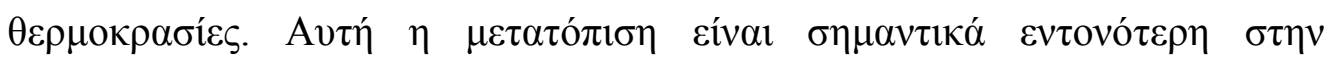


$\pi \varepsilon \rho i ́ \pi \tau \omega \sigma \eta \tau \omega \nu \mu \eta-\alpha v \alpha \gamma \omega ́ \gamma \gamma \mu \omega \nu \mathrm{M}_{x} \mathrm{O}_{y} \kappa \alpha \tau \alpha \lambda v \tau \omega ́ v(\pi . \chi . \mathrm{M}=\mathrm{Zr}, \mathrm{Mg}, \mathrm{Cs}$ ), ol

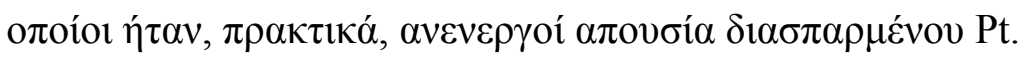

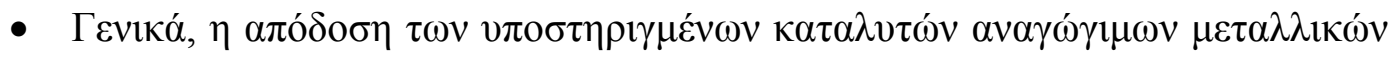

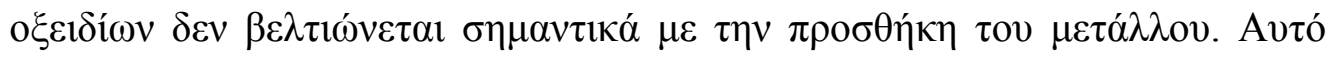

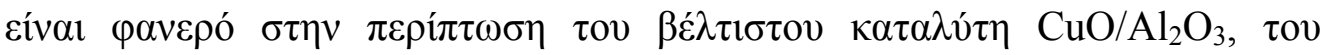

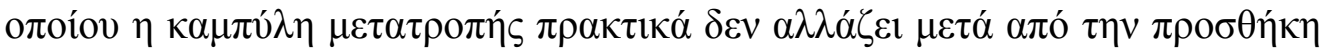
$\tau \eta \varsigma \pi \lambda \alpha \tau i ́ v \alpha \varsigma$.

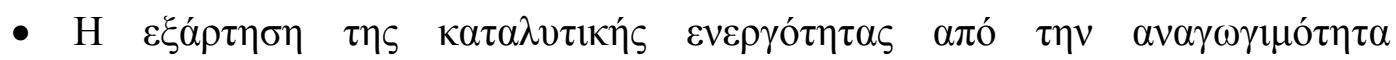

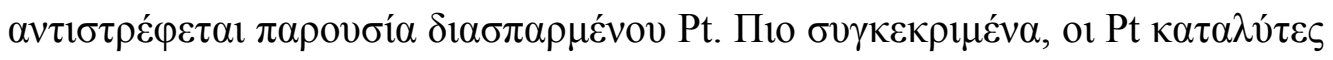

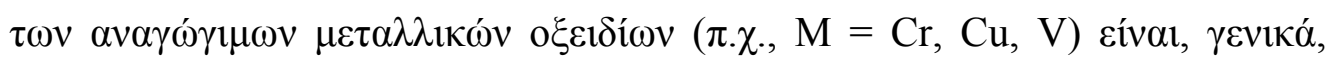

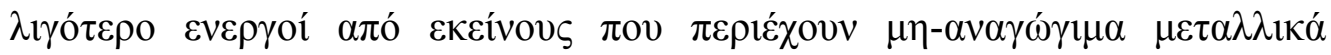

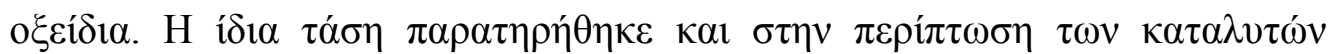
$\mathrm{Pt} / \mathrm{M}_{x} \mathrm{O}_{y}$.

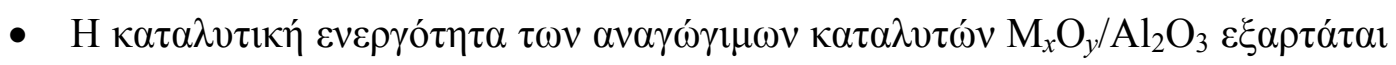

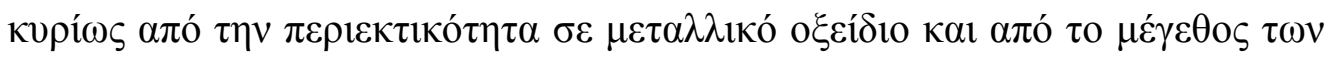

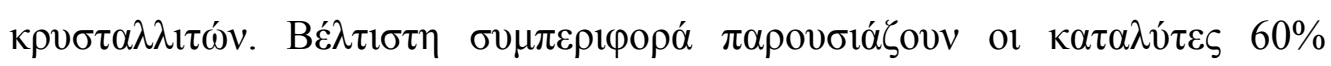

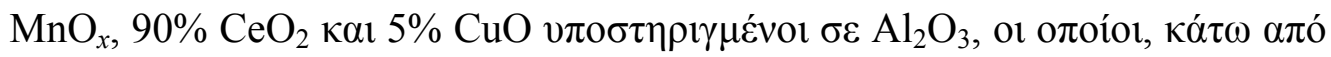

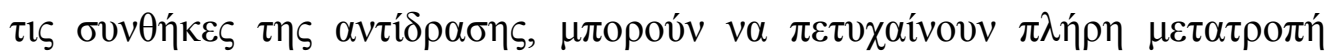

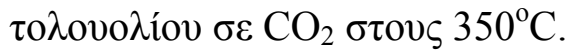

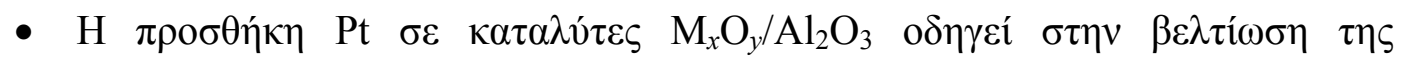

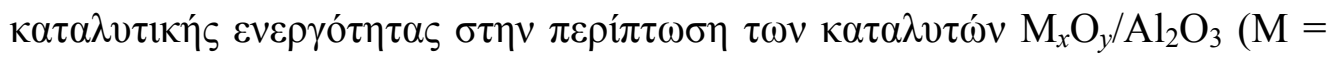

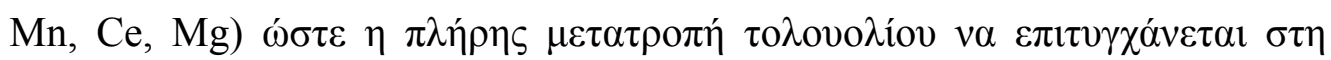

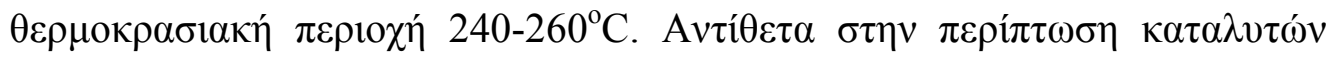

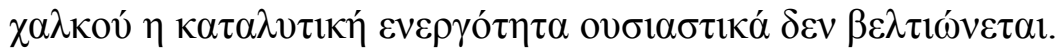

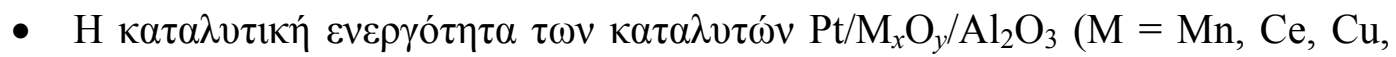

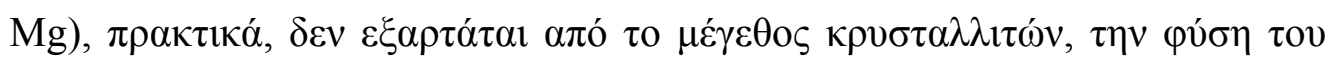

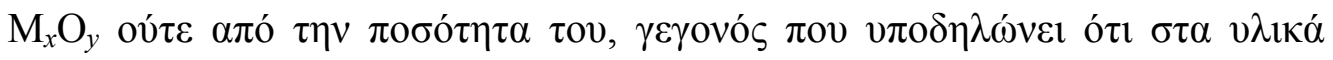

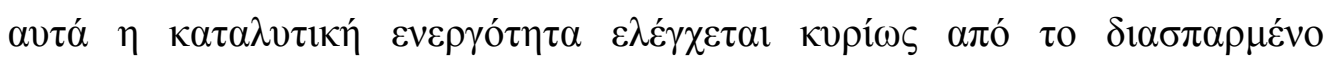
$\mu \varepsilon ́ \tau \alpha \lambda \lambda \mathrm{o}, \mathrm{Pt}$. 


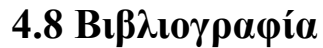

[1] J. Carpentier, J.F. Lamonier, S. Siffert, E.A. Zhilinskaya, A. Aboukaïs, Appl. Catal. A 234 (2002) 91.

[2] M. Paulis, H. Peyrard, M. Montes, J. Catal. 199 (2001) 30.

[3] J.C.S. Wu, T.-Y. Chang, Catal. Today 44 (1998) 111.

[4] P. Papaefthimiou, T. Ioannides, X.E. Verykios, Appl. Catal. B 15 (1998) 75.

[5] K. Okumura, T. Kobayashi, H. Tanaka, M. Niwa, Appl. Catal. B 44 (2003) 325.

[6] P. Papaefthimiou, T. Ioannides, X.E. Verykios, Catal. Today 54 (1999) 81.

[7] S. Scirè, S. Minicò, C. Crisafulli, C. Satriano, A. Pistone, Appl. Catal. B 40 (2003) 43.

[8] T. Takeguchi, S. Aoyama, J. Ueda, R. Kikuchi, K. Eguchi, Top. Catal. 23 (2003) 159.

[9] R. Brayner, D. dos Santos Cunha, F. Bozon-Verduraz, Catal. Today 78 (2003) 419.

[10] T.-K. Tseng, H. Chu, T.-H. Ko, L.-K. Chung, J. Hazard. Mater. 122 (2005) 155.

[11] A. Janbey, W. Clark, E. Noordally, S. Grimes, S. Tahir, Chemosphere 52 (2003) 1041.

[12] J. Tsou, L. Pinard, P. Magnoux, J.L. Figueiredo, M. Guisnet, Appl. Catal. B 46 (2003) 371 .

[13] C. Zhang, H. He, K. Tanaka, Catal. Commun. 6 (2005) 211.

[14] P. Papaefthimiou, T. Ioannides, X. E. Verykios, Appl. Catal. B: Env. 13 (1997) 175

[15] H. S. Kim, T. W. Kim, H. L. Koh, S. Ho Lee, B. R. Min, Appl. Catal A: General $280(2005) 125$.

[16] P. Chantaravitoon, S. Chavadej, J. Schwank, Chem. Eng. J. 97 (2004) 161.

[17] C. Lahousse, A. Bernier, P. Grange, B. Delmon, P. Papaefthimiou, T. Ioannides, X. Verykios, J. Catal. 178 (1998) 214.

[18] Ching-Huei Wang, Shiow-Shyung Lin, Appl. Catal. A: Gen. 268 (2004) 227.

[19] D. Delimaris, T. Ioannides, Appl. Catal. B: Environ 84 (2008) 303.

[20] H. Rajesh, U.S. Ozkan, Ind. Eng. Chem. Res. 32 (1993) 1622.

[21] T. García, B. Solsona, S.H. Taylor, Appl. Catal. B 66 (2006) 92.

[22] C. Hettige, K.R.R. Mahanama, D.P. Dissanayake, Chemosphere 43 (2001) 1079.

[23] S.-C. Kim, J. Hazard. Mater. 91 (2002) 285. 
[24] G. Pecchi, P. Reyes, T. López, R. Gómez, J. Non-Cryst. Solids 345\&346 (2004) 624.

[25] V.A. de la Peña O’Shea, M.C. Álvarez-Galván, J.L.G. Fierro, P.L. Arias, Appl. Catal. B 57 (2005) 191.

[26] B. Grbic, N. Radic, B. Markovic, P. Stefanov, D. Stoychev, Ts. Marinova, Appl. Catal. B 64 (2006) 51.

[27] R.S.G. Ferreira, P.G.P. de Oliveira, F.B. Noronha, Appl. Catal. B 50 (2004) 243.

[28] M.A. Centeno, M. Paulis, M. Montes, J.A. Odriozola, Appl. Catal. A 234 (2002) 65 .

[29] A. Zieba, T. Banaszak, R. Miller, Appl.Catal. A: General 124 ( 1995$) 47$.

[30] J.-G, Li, T. Ikegami, J.-H, Lee, T. Mori, Acat mater. 49 (2001) 419.

[31] P. Panagiotopoulou, D.I. Kondarides, Catal. Today 127 (2007) 319.

[32] R.J.H. Grisel, C.J. Weststrate, A. Goossens, M.W.J. Crajé, A.M. van der Kraan, B.E. Nieuwenhuys, Catal. Today 72 (2002) 123.

[33] R.J.H. Grisel, B.E. Nieuwenhuys, Catal. Today 64 (2001) 69.

[34] C.H. Wang, Chemosphere 55 (2004) 11.

[35] S.-C. Kim, J. Hazard. Mater. B91 (2002) 285.

[36] M. Labaki, J-F. Lamonier, S. Siffert, A. Aboukaïs, Thermochem. Acta 427 (2005) 193.

[37] H.L. Tidahy, S. Siffert, F. Wyrwalski, J-F. Lamonier, A. Aboukaïs, Catal. Today 119 (2007) 317.

[38] F. Bertinchamps, C. Grégoire, E.M. Gaigneaux, Appl. Catal. B 66 (2004) 10.

[39] W.B. Li, W.B. Chu, M. Zhuang, J. Hua, Catal. Today 93-95 (2004) 205.

[40] Z.Y. ding, L. Li, D. Wade, E.F. Gloyna, Ind. Eng. Chem. Res. 37 (1998) 1707.

[41] F. Severino, J. Brito, O. Carias, J. Laine, J. Catal. 102 (1986) 172.

[42] M.C. Marion, E. Garbowski, M. Primet, J. Chem. Soc., Faraday Trans. 86 (1990) 3027.

[43] M.C. Marion, E. Garbowski, M. Primet, J. Chem. Soc., Faraday Trans. 87 (1991) 1795.

[44] H. Cordatos, D. Ford, R.J. Gorte, J. Phys. Chem. 100 (1996) 18128.

[45] H. Cordatos, T. Bunluesin, J. Stubenrauch, J.M. Vohs, R.J. Gorte, J. Phys. Chem. 100 (1996) 785.

[46] E.S. Putna, B. Sherek, R.J. Gorte, Appl. Catal. B 17 (1998) 101. 
[47] P. Panagiotopoulou, A. Christodoulakis, D.I. Kondarides, S. Boghosian, J. Catal. 240 (2006) 114.

[48] M.C. Álvarez-Galván, V.A. de la Peña O’Shea, J.L.G. Fierro, P.L. Arias, Catal. Commun. 4 (2003) 223.

[49] M.C. Álvarez-Galván, B. Pawalec, V.A. de la Peña O’Shea, J.L.G. Fierro, P.L. Arias, Appl. Catal. B 51 (2004) 83. 


\section{KEФA $А A I O 5^{\circ}$}

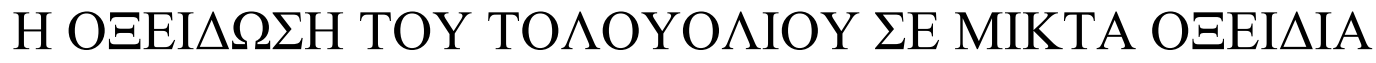

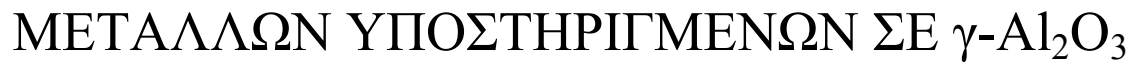

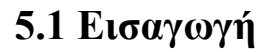

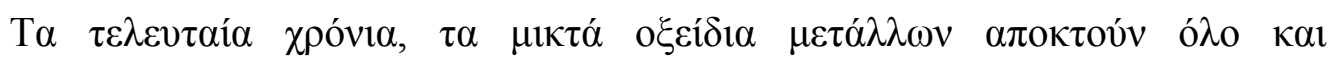

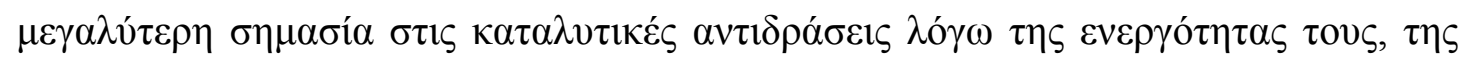

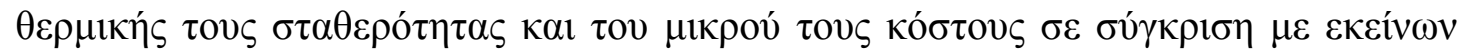

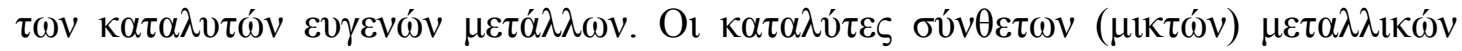

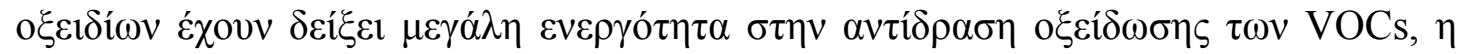

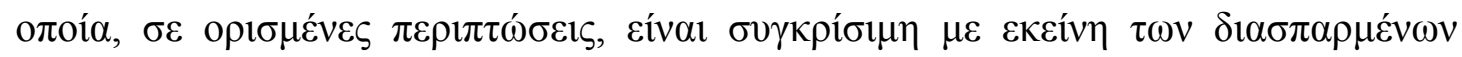
$\mu \varepsilon \tau \alpha \dot{\lambda} \lambda \lambda \omega \nu[1-3]$.

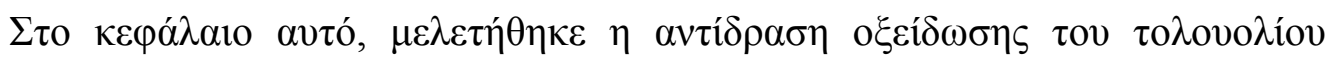

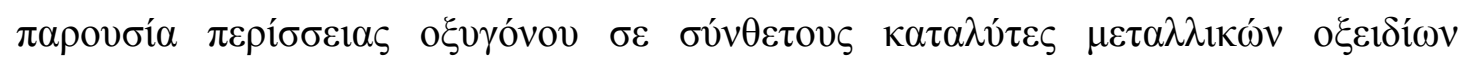

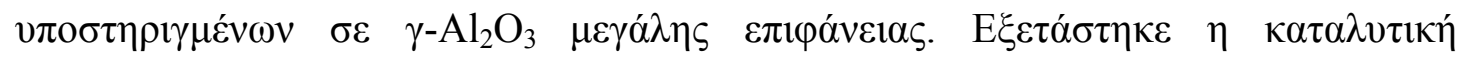

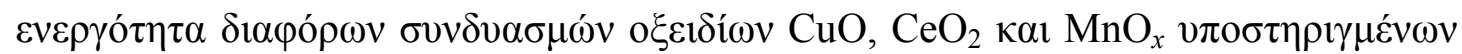




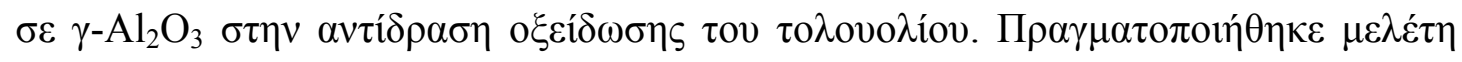

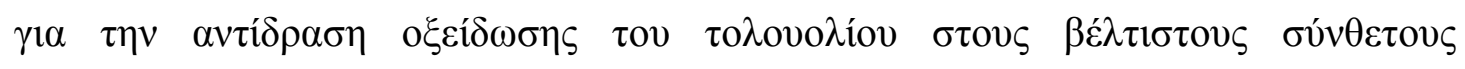

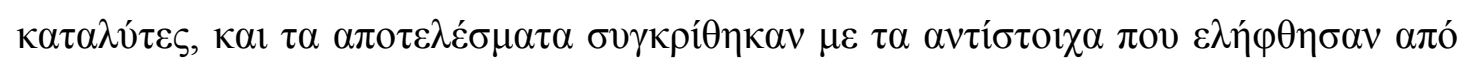

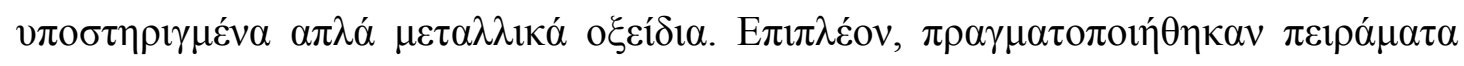

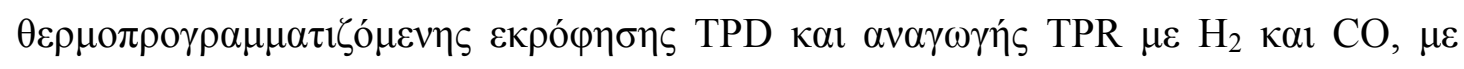

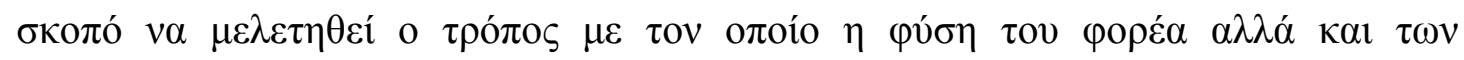

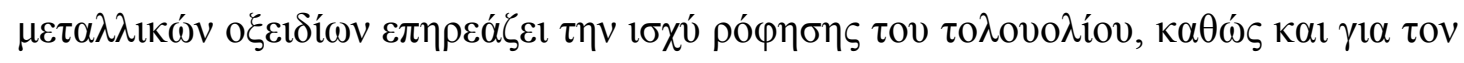

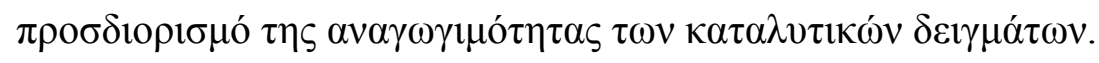

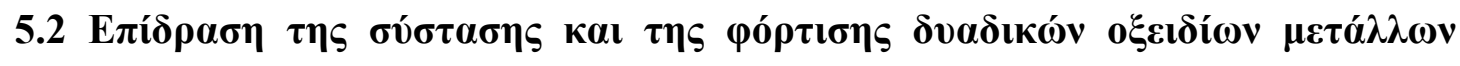

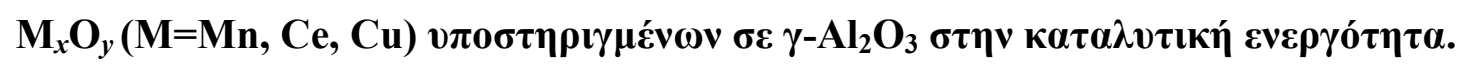

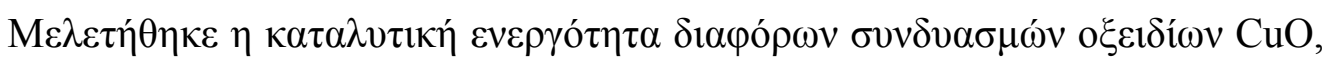

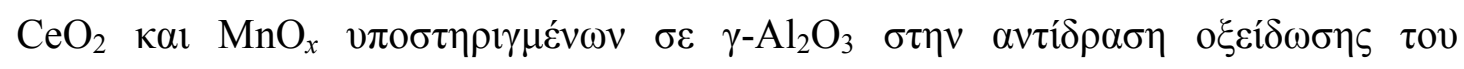

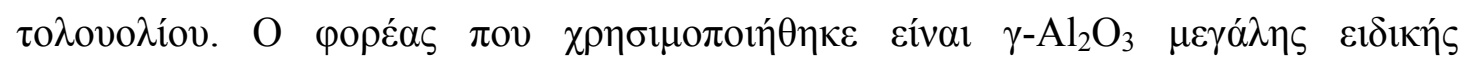

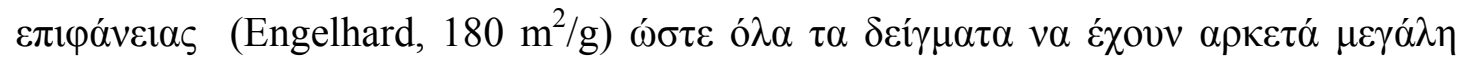

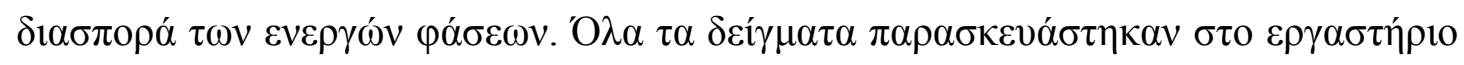

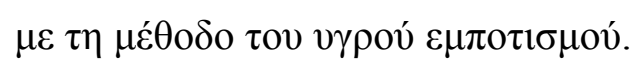

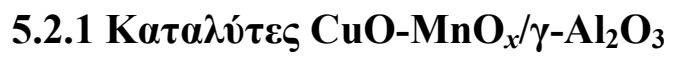

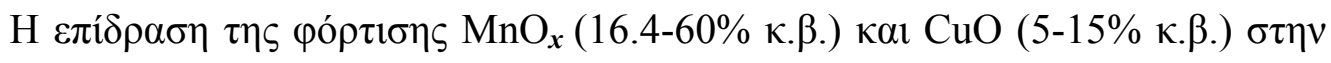

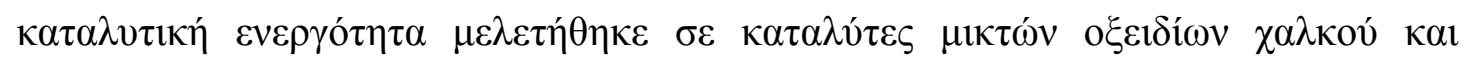

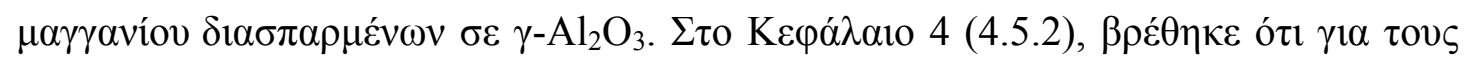

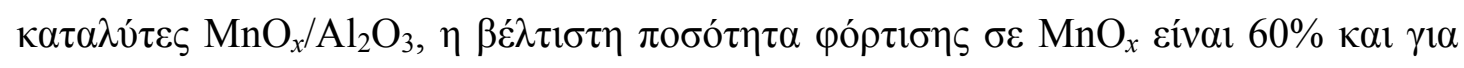

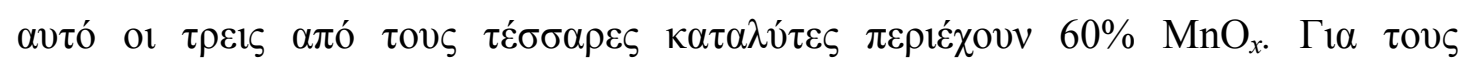

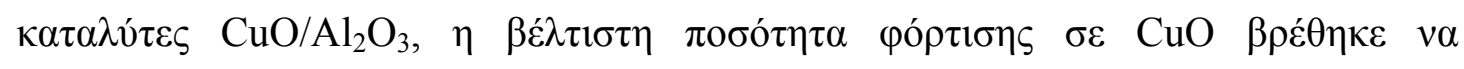

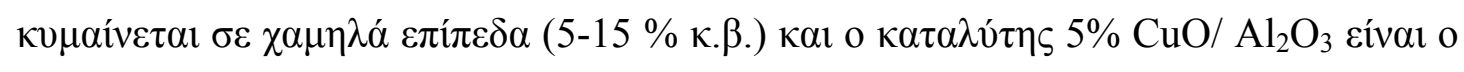

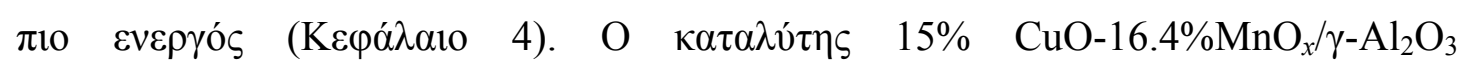

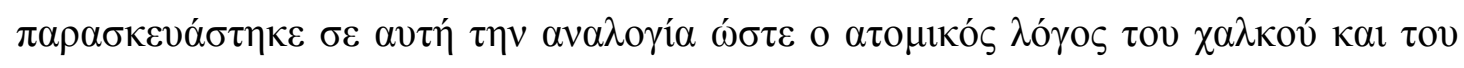

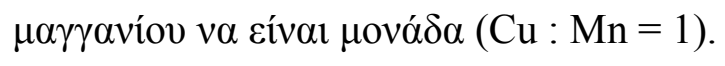




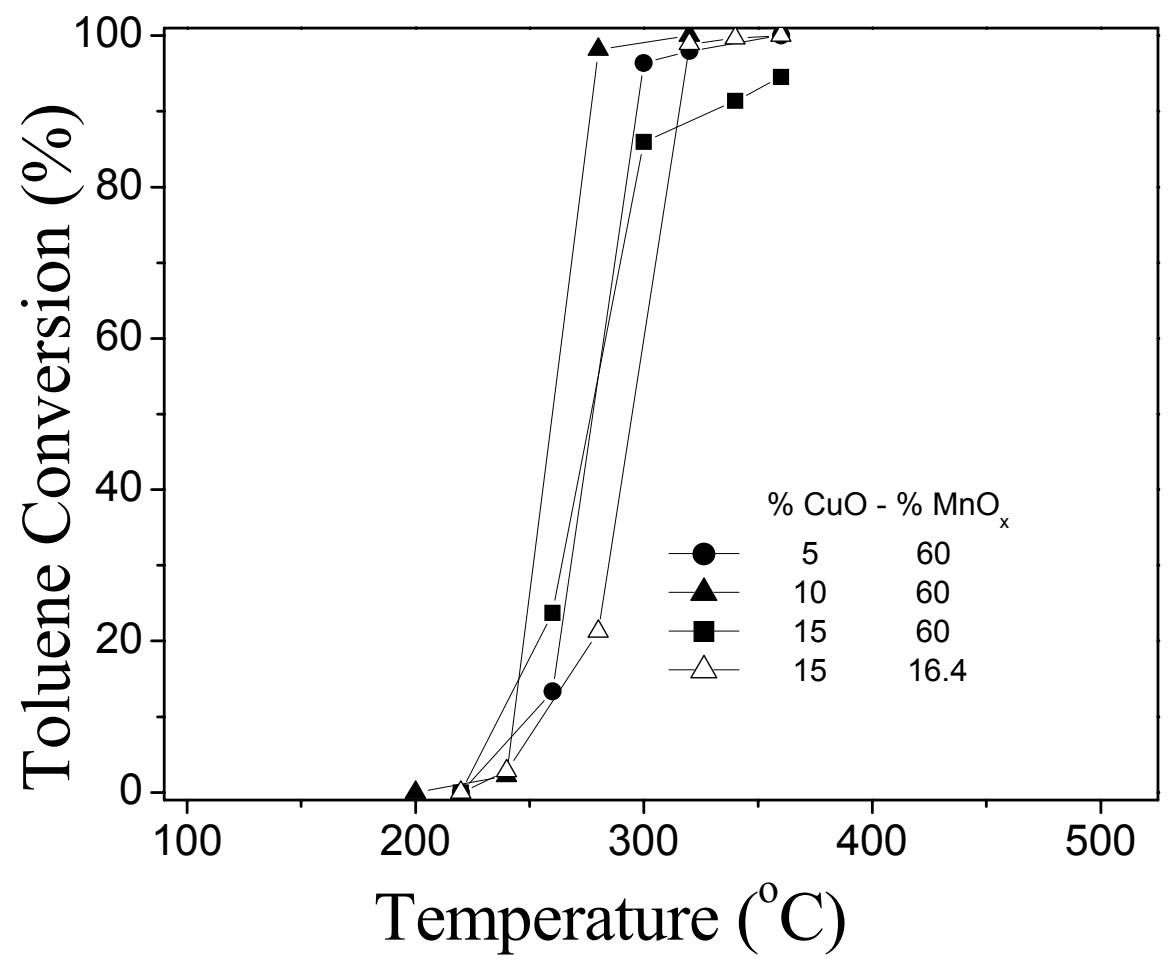

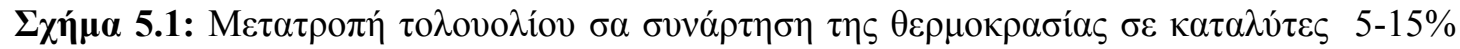

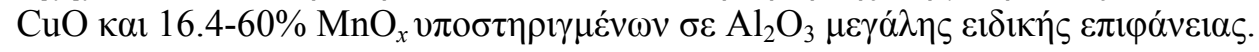

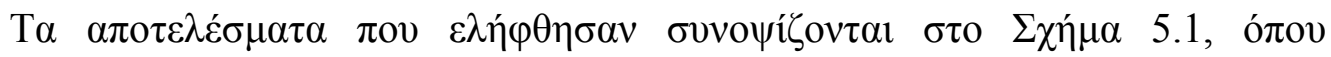

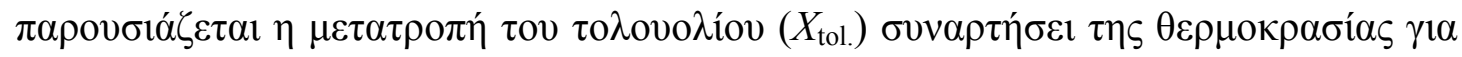

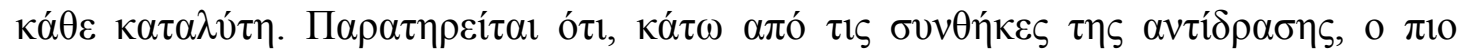

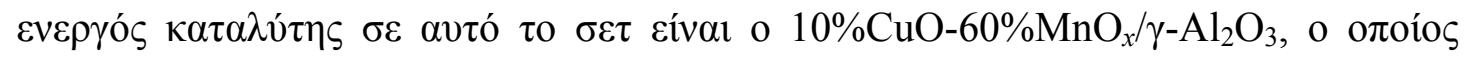

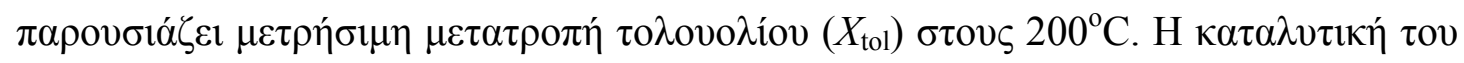

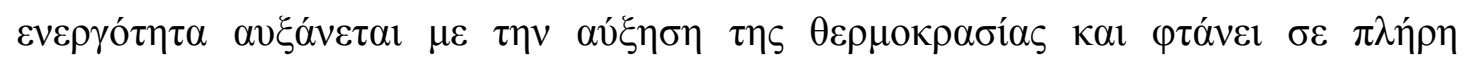

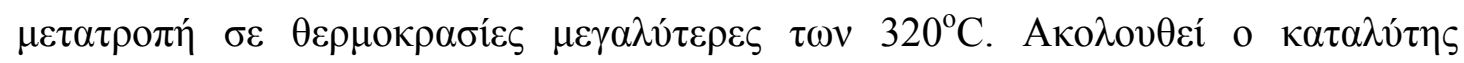

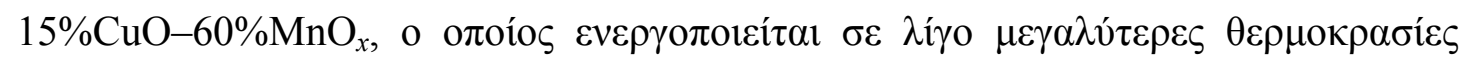

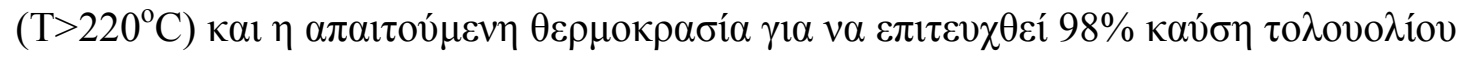

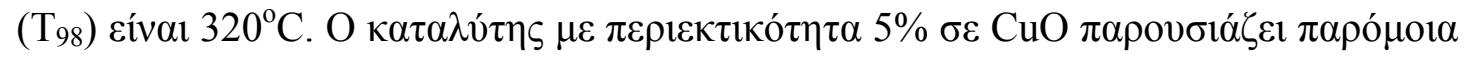

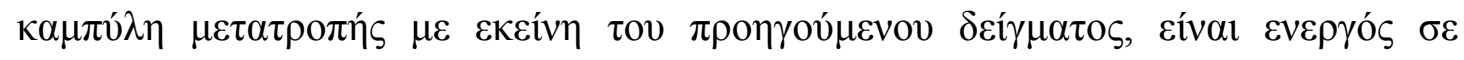

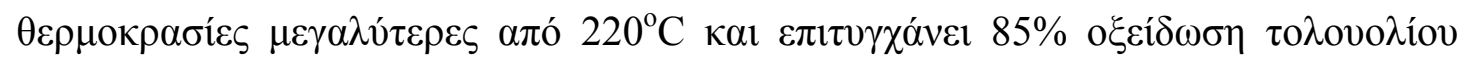

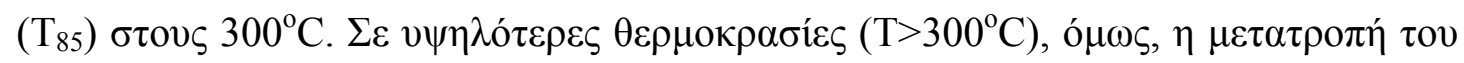

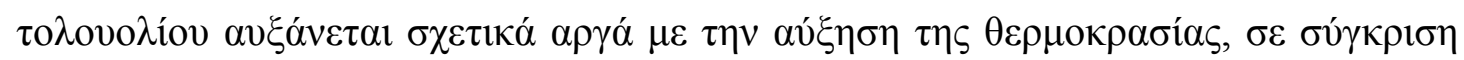

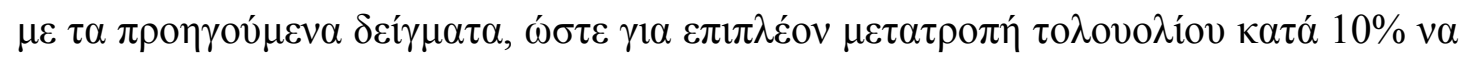

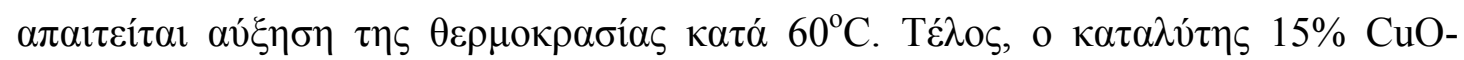




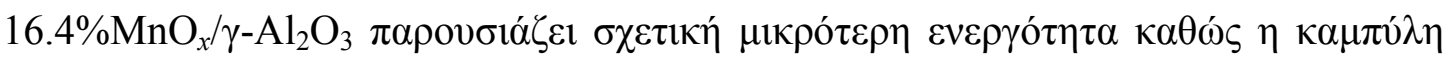

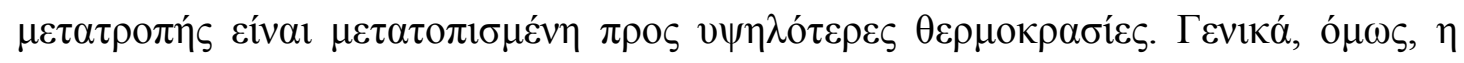

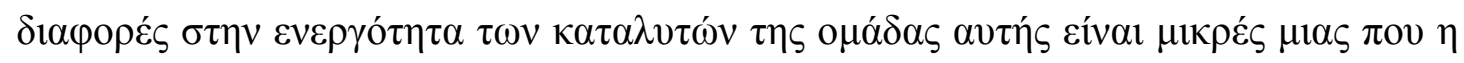

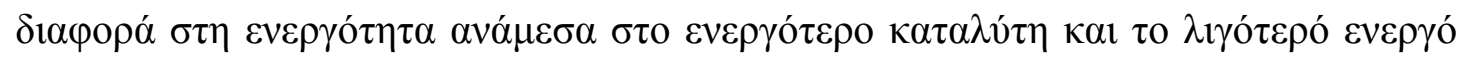

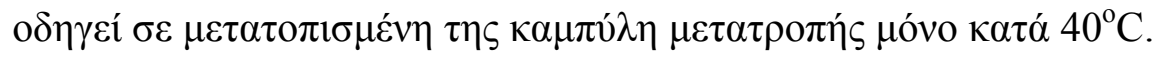

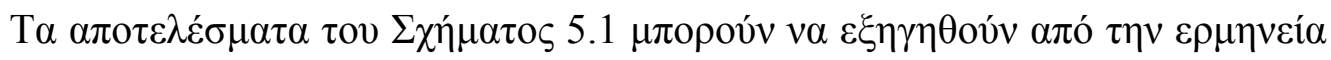

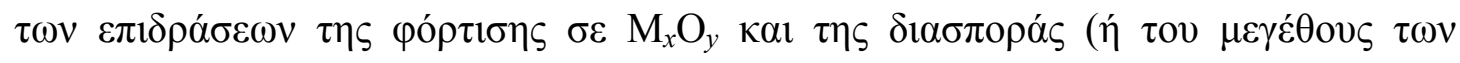

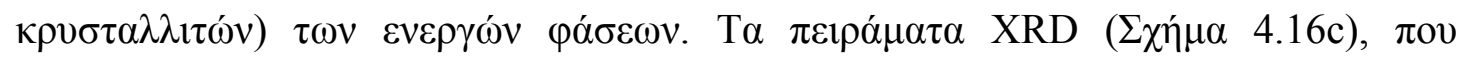

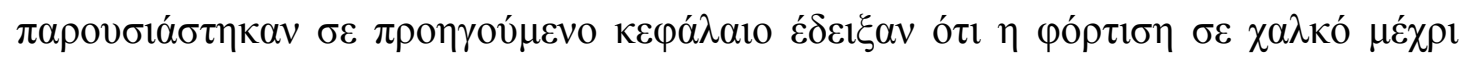

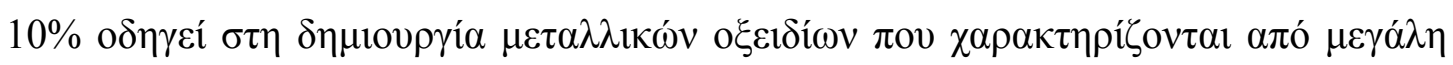

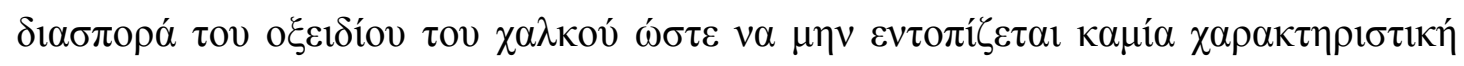

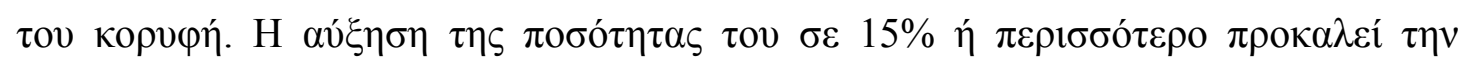

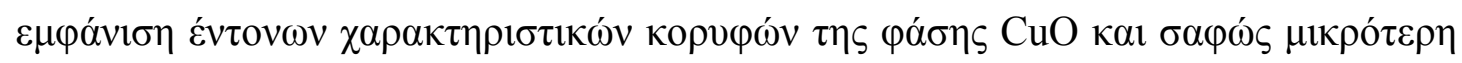

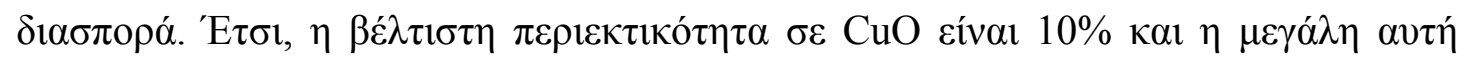

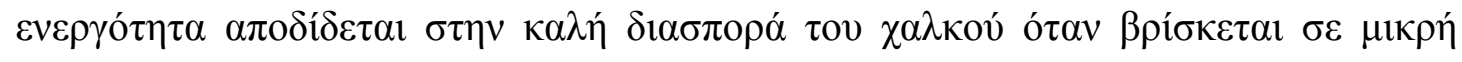

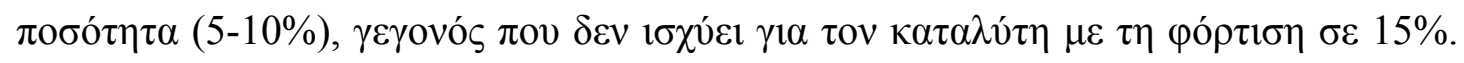

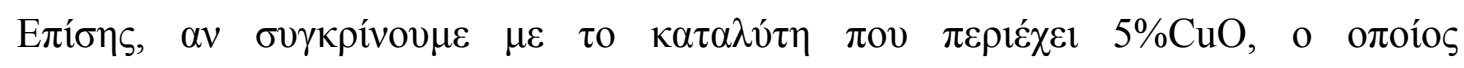

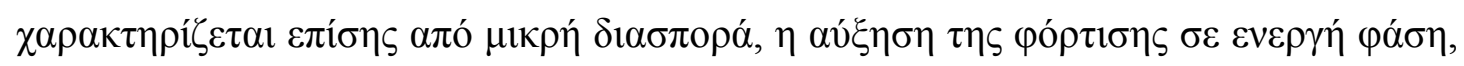

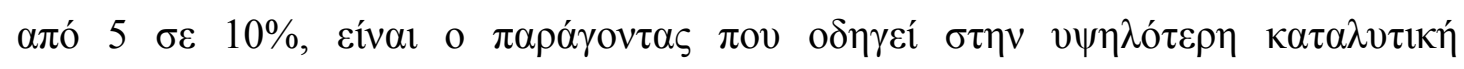

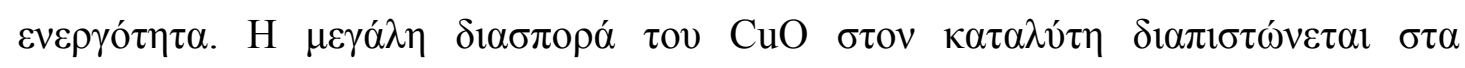

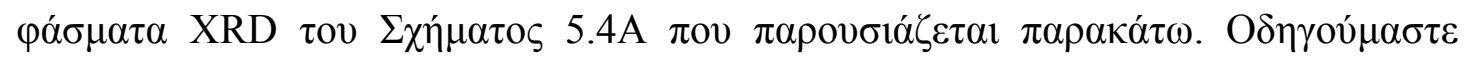

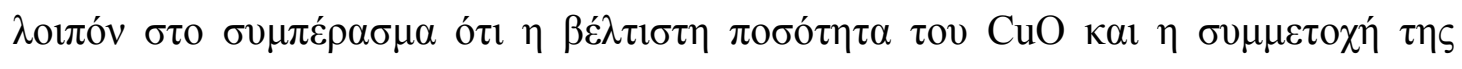

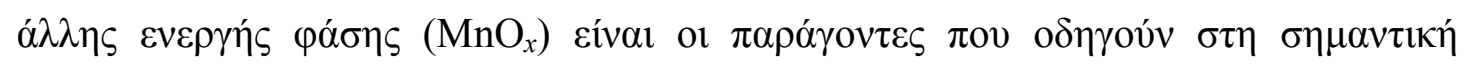

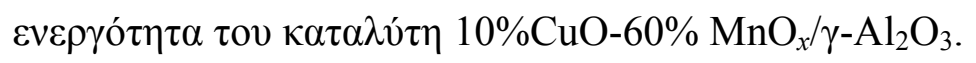

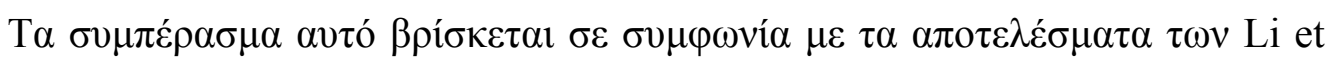

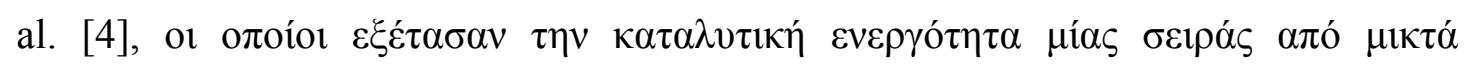

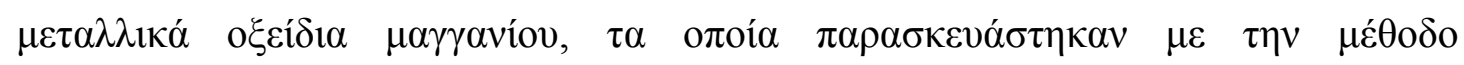

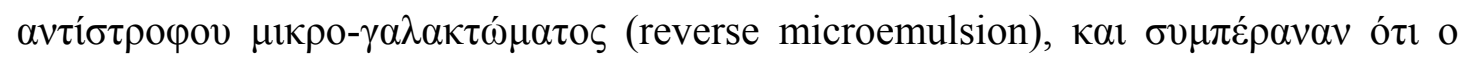

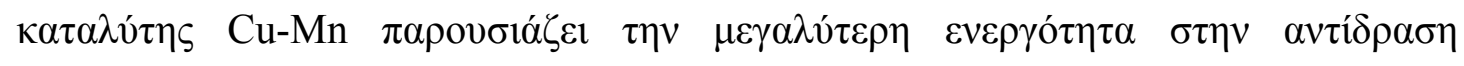

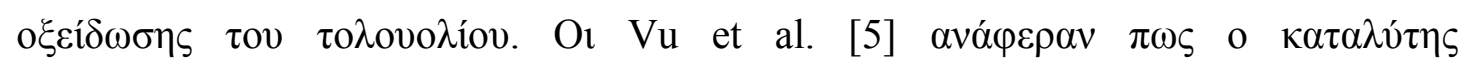

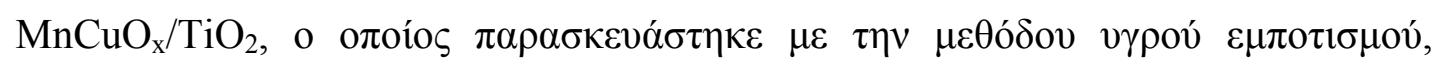

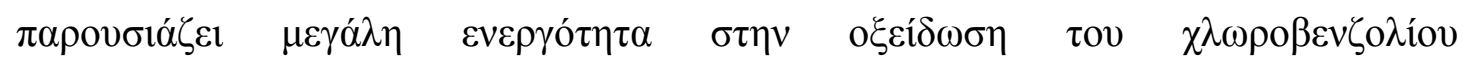

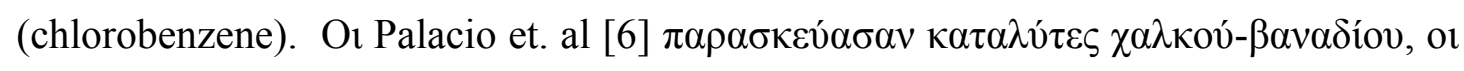

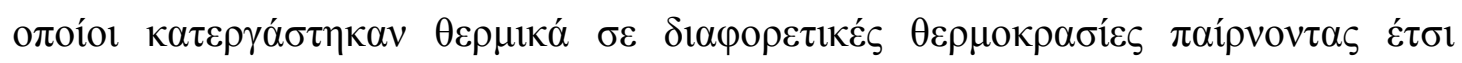




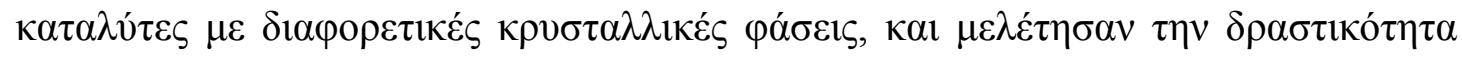

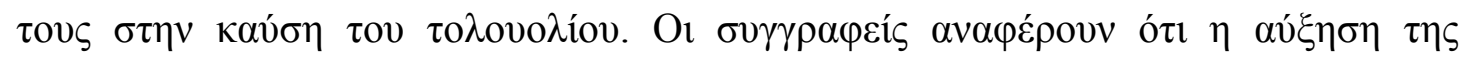

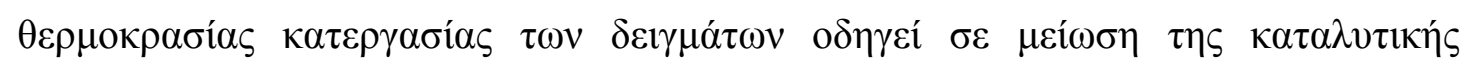

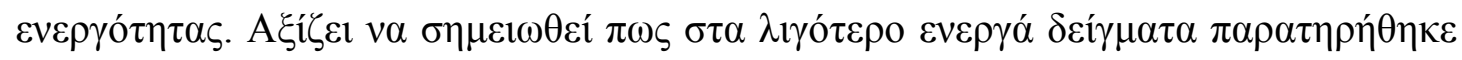

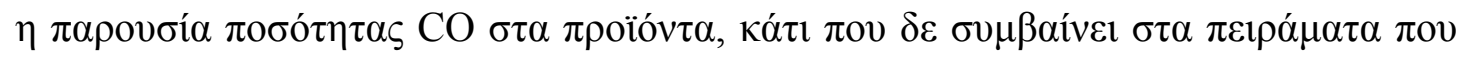

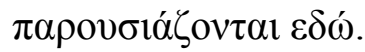

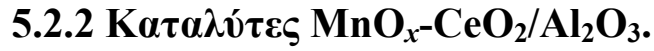

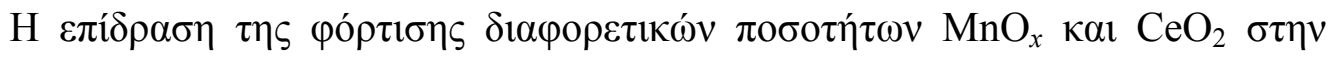

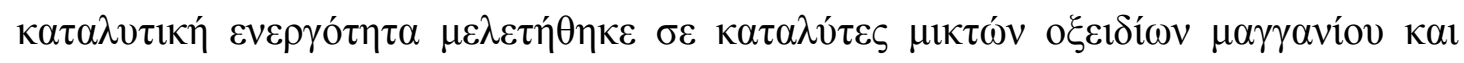

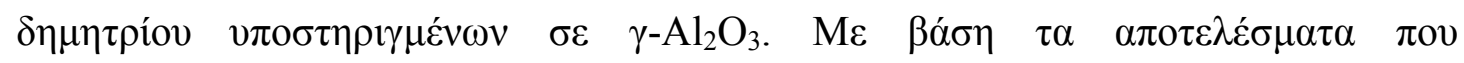

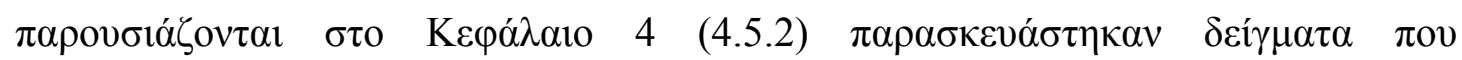

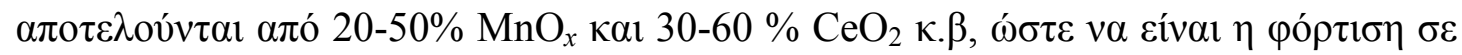

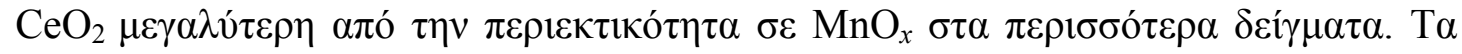

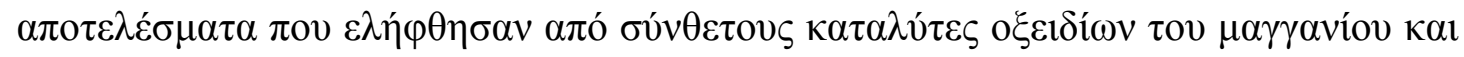

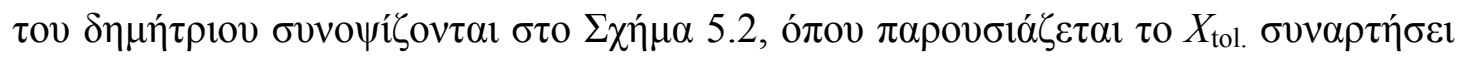

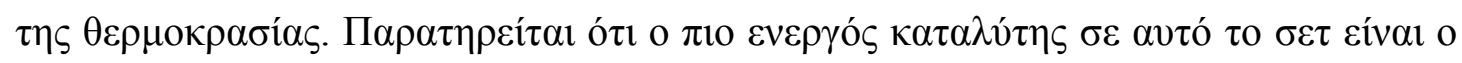

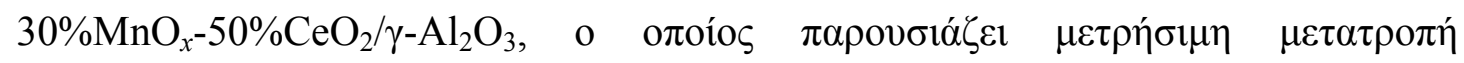

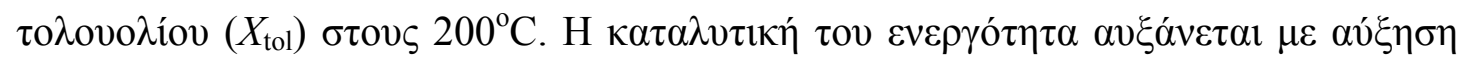

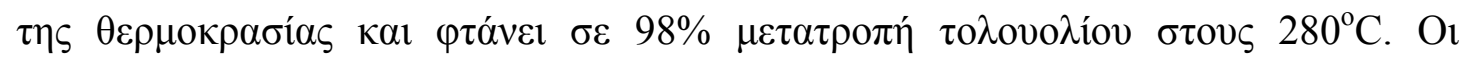

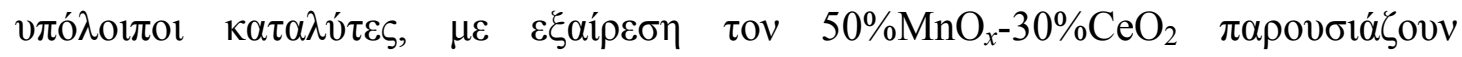

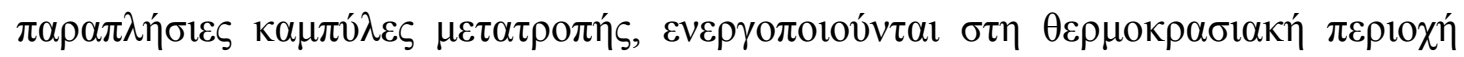

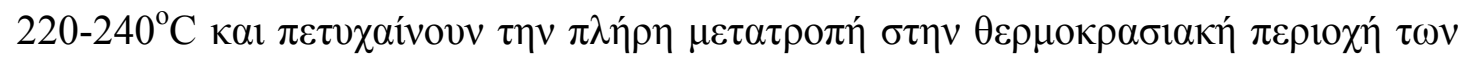

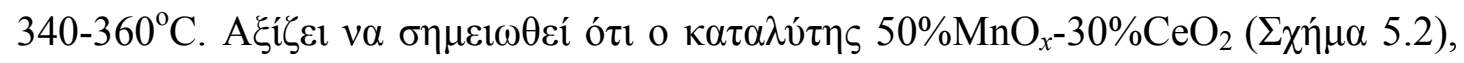

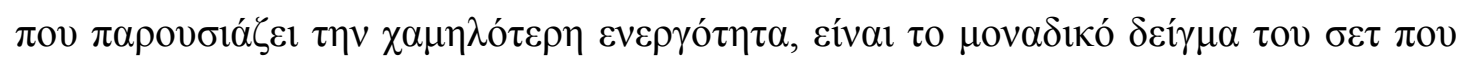

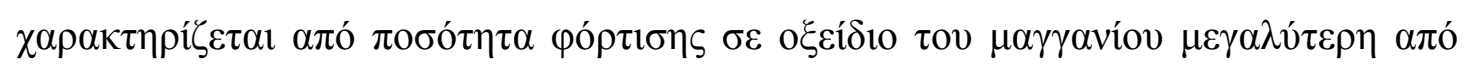

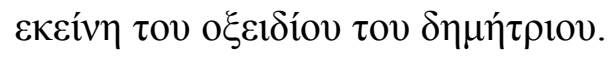

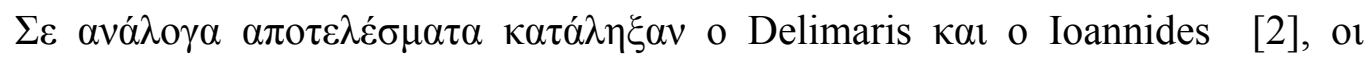

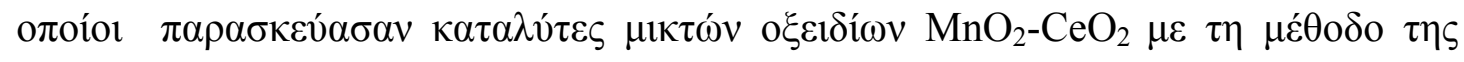

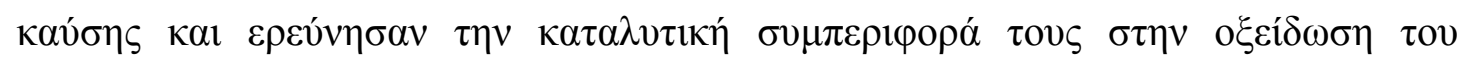

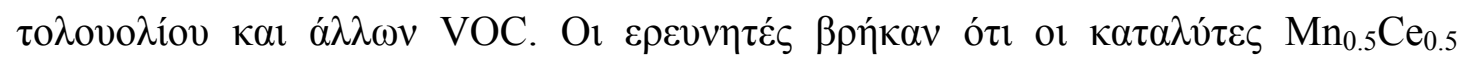

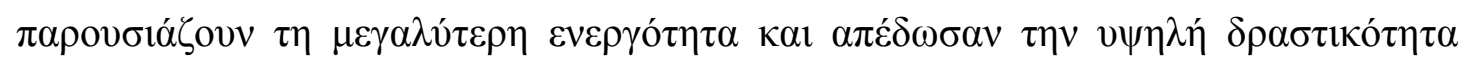

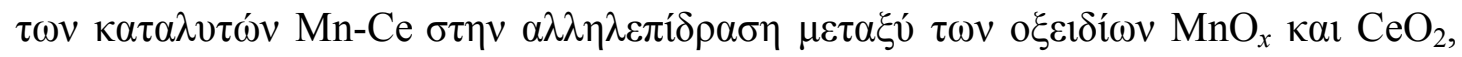

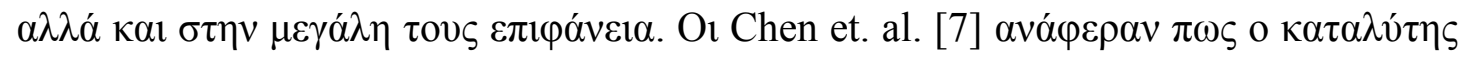




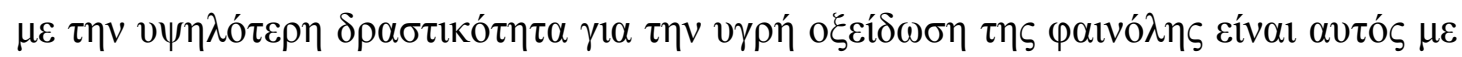

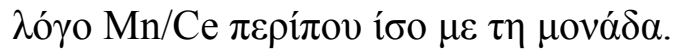

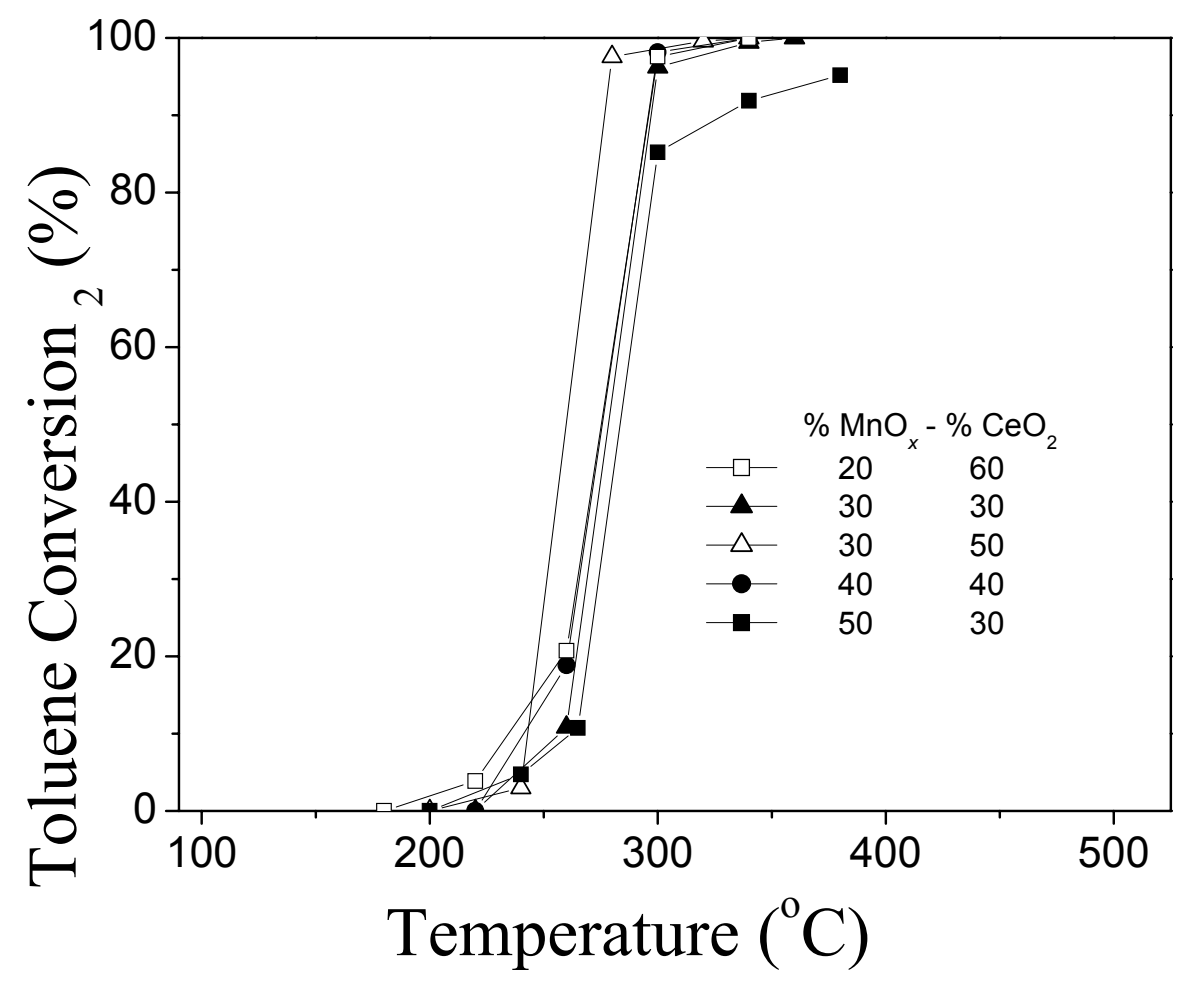

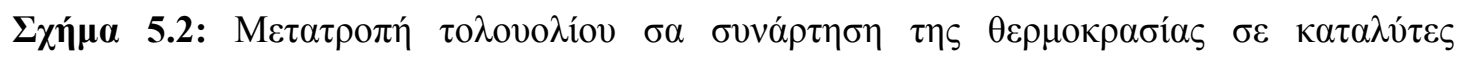

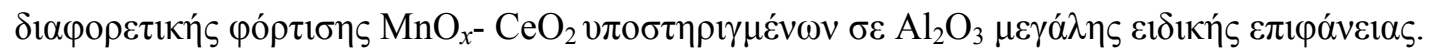

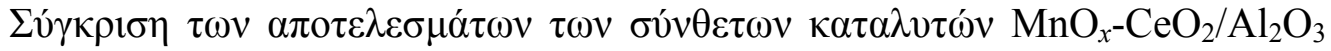

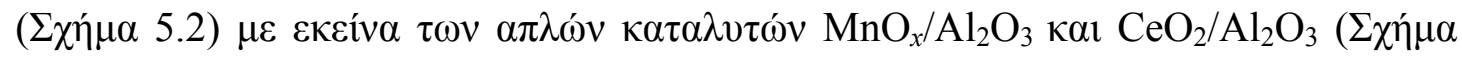

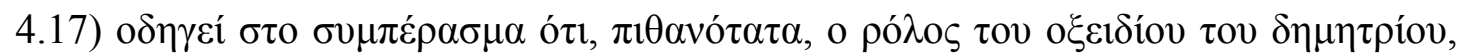

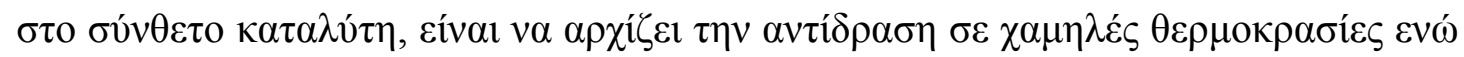

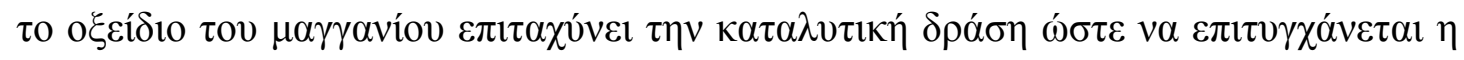

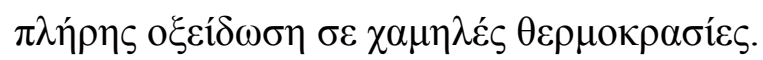

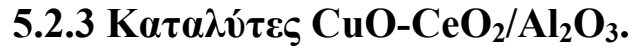

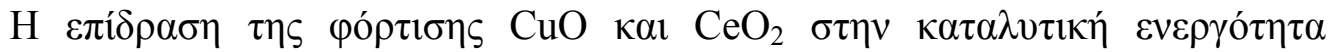

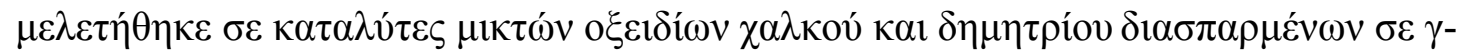

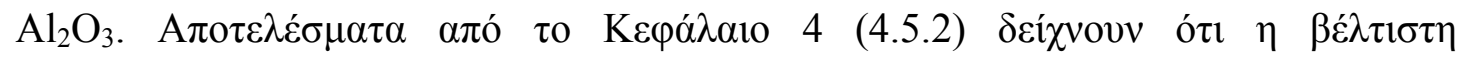

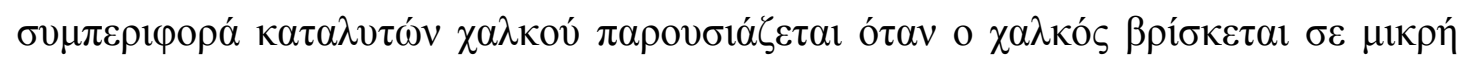




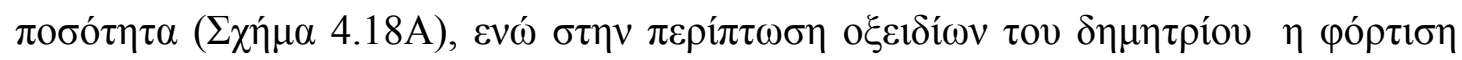

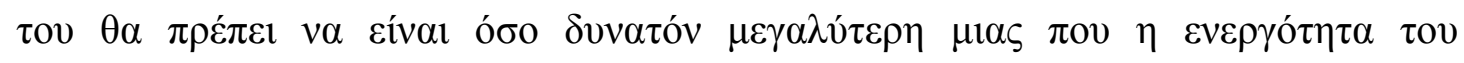

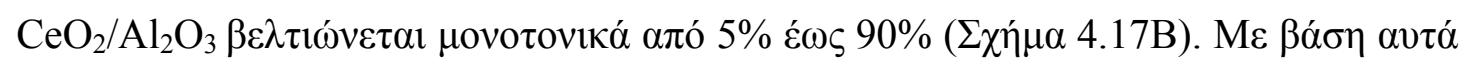

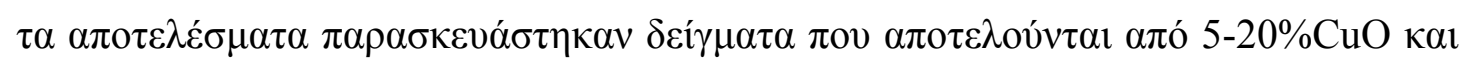

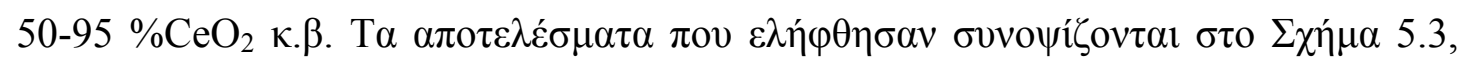

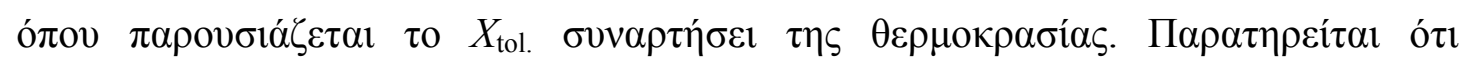

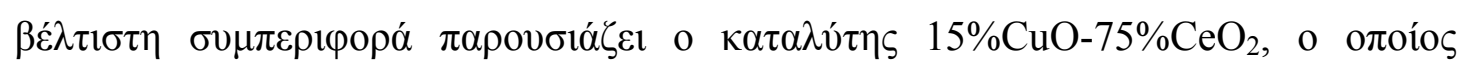

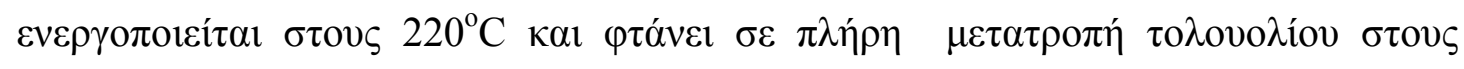

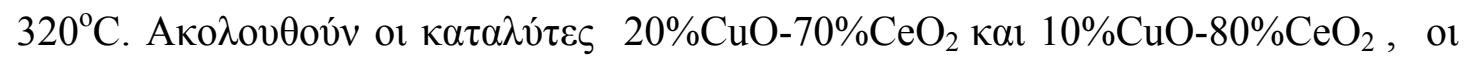

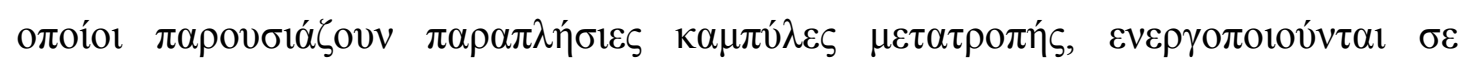

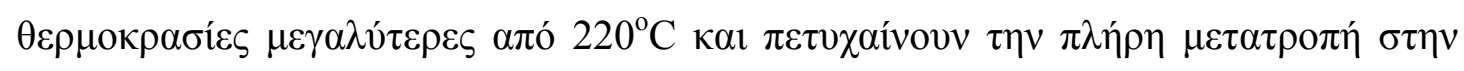

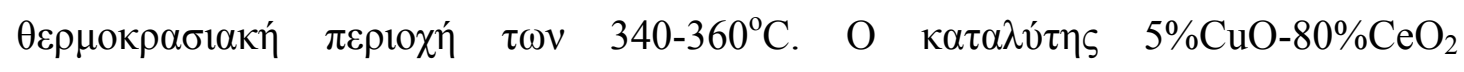

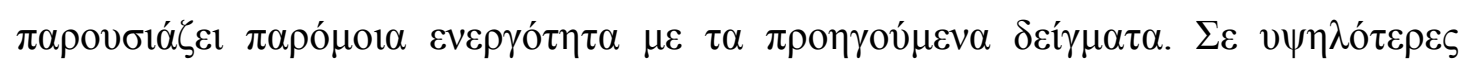

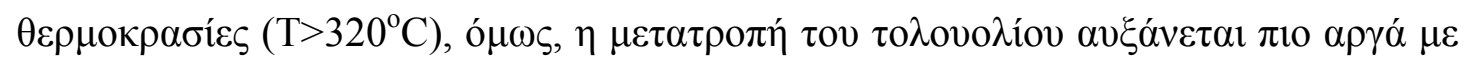

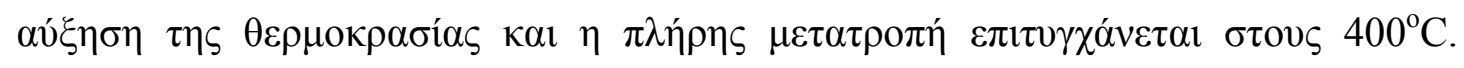

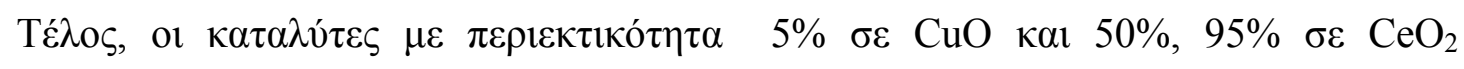

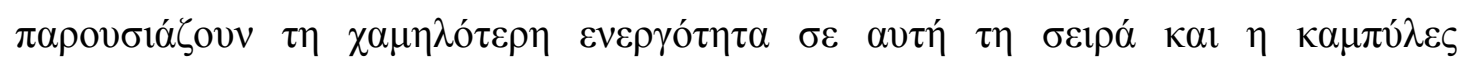

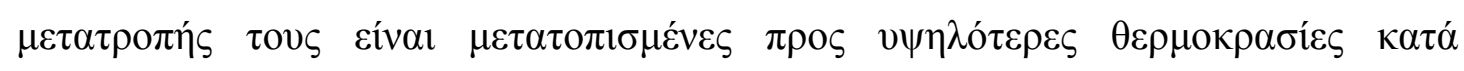

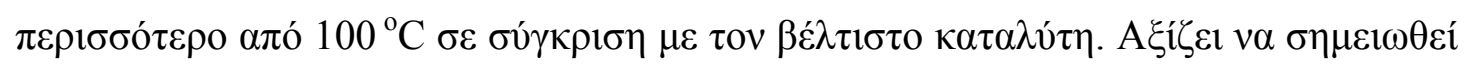

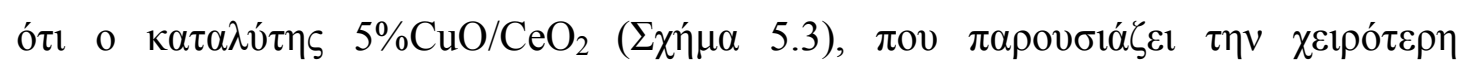

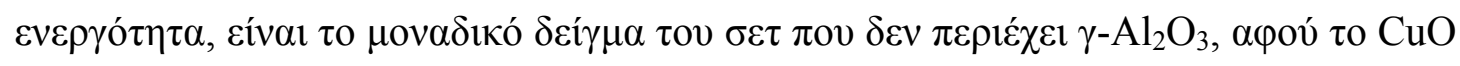

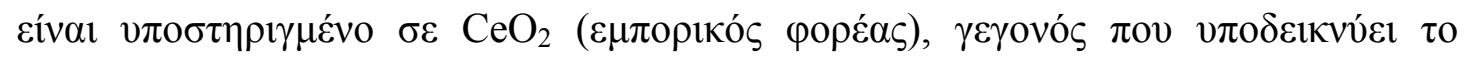

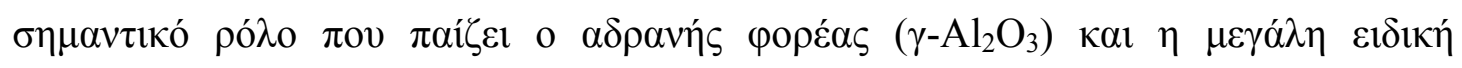

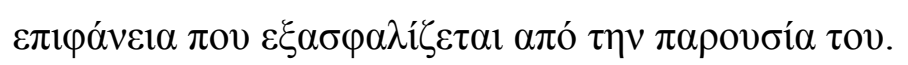

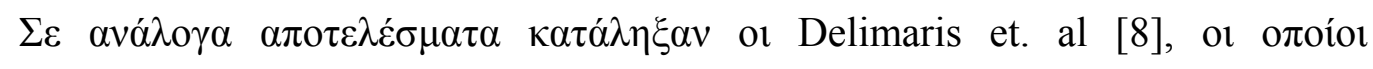

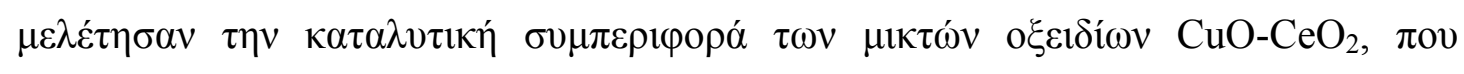

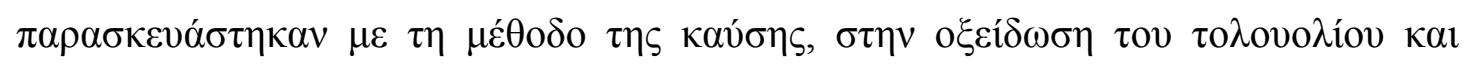

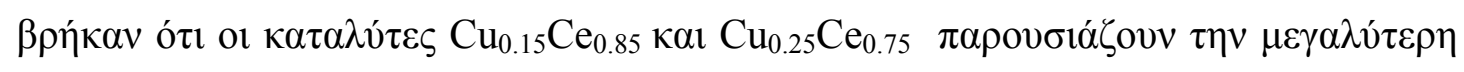

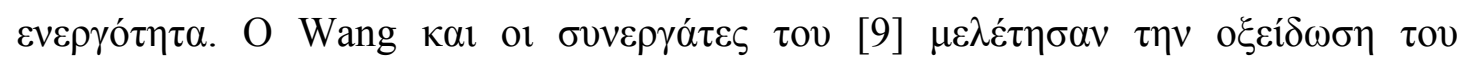

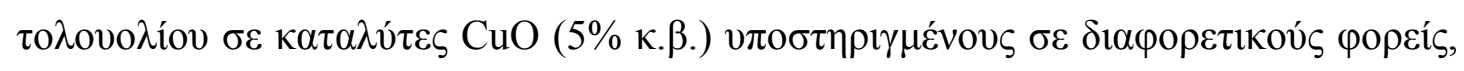

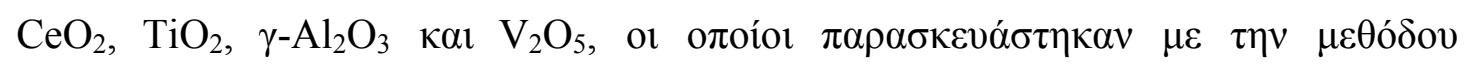

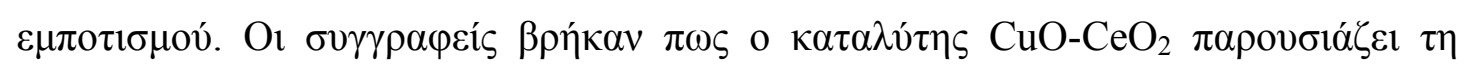

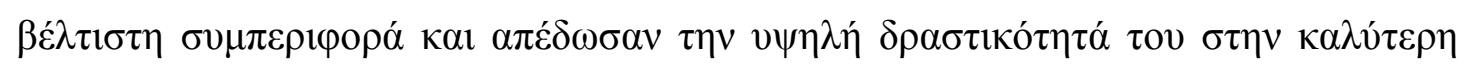

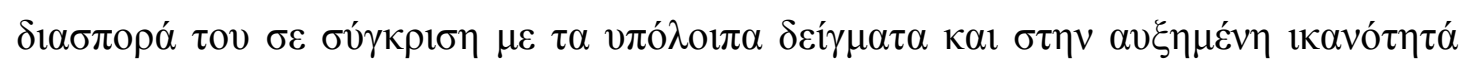




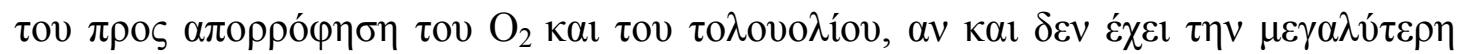
$\varepsilon 1 \delta เ \kappa \eta ́ ~ \varepsilon \pi \imath \varphi \alpha ́ v \varepsilon \imath \alpha$.

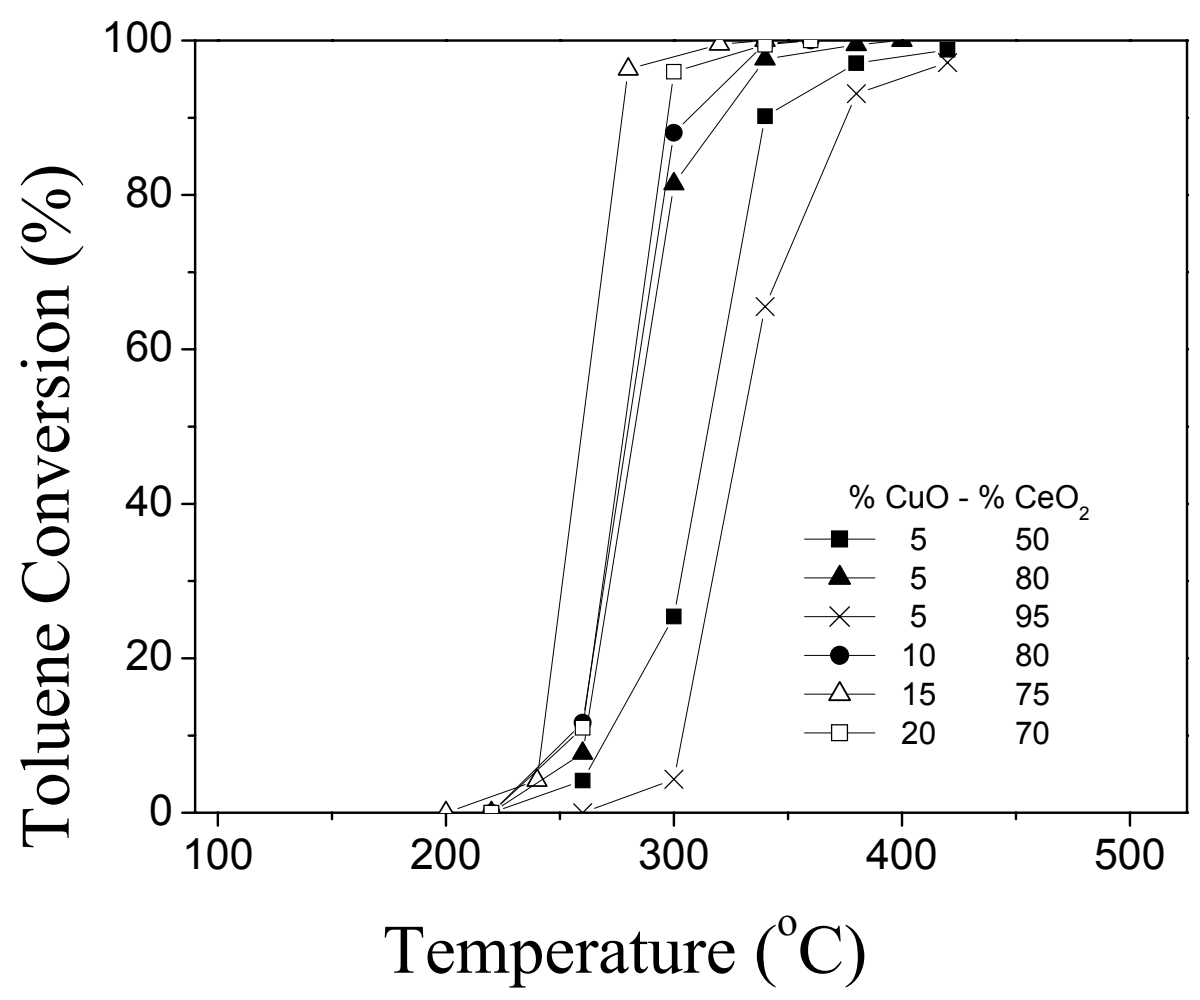

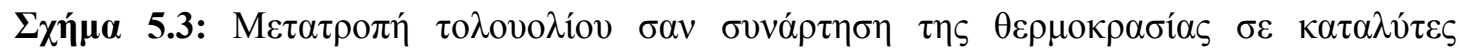

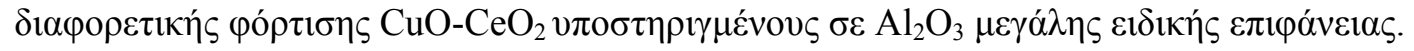

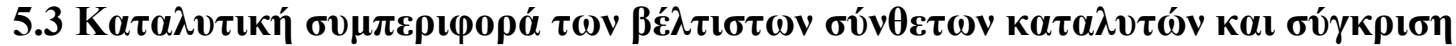

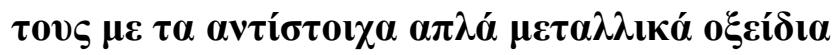

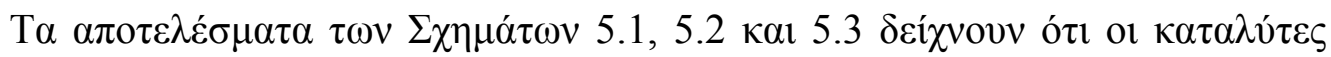

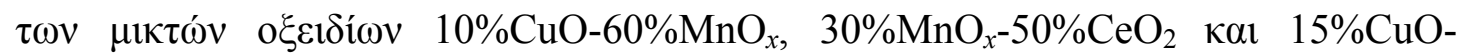

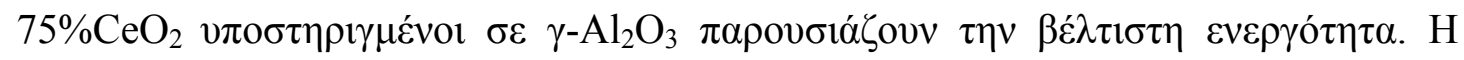

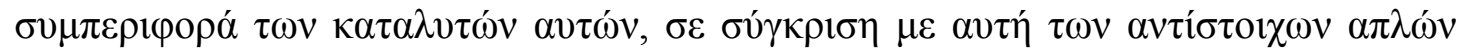

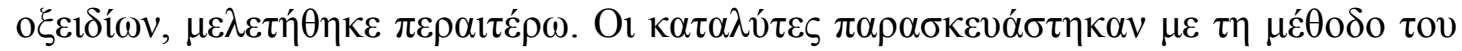

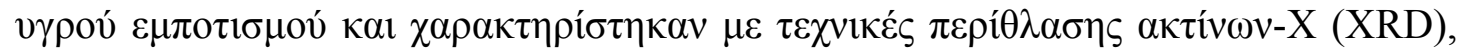

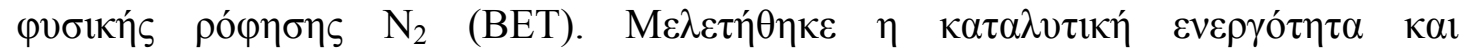

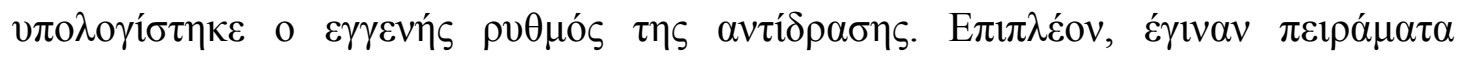

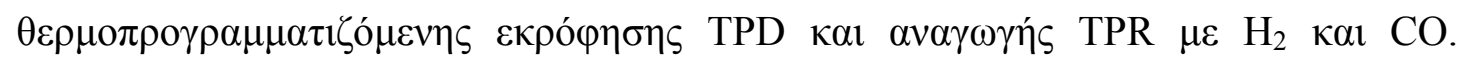




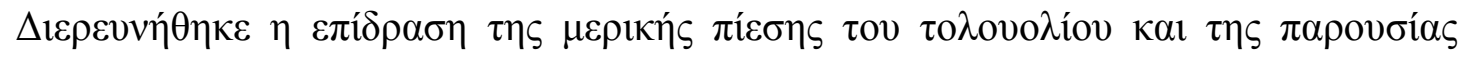

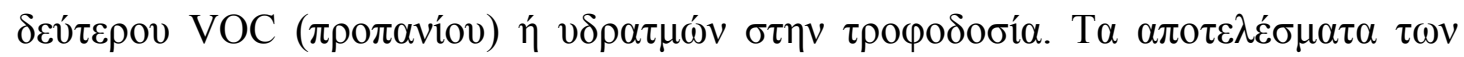

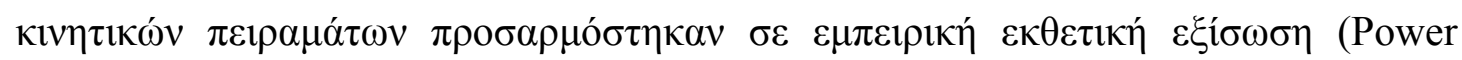

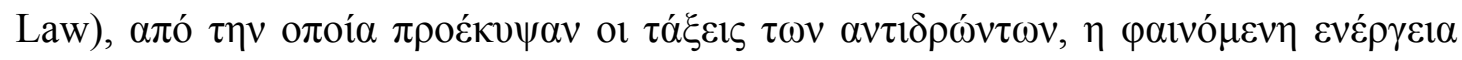

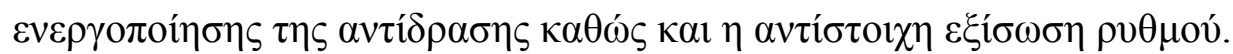

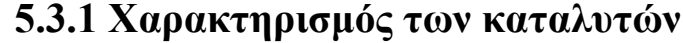

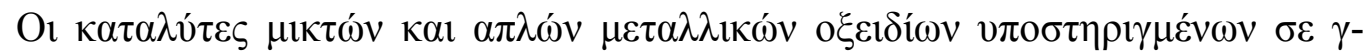

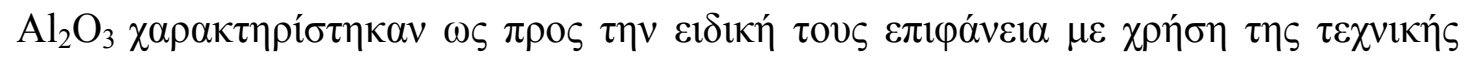
BET. Т $\alpha$ a

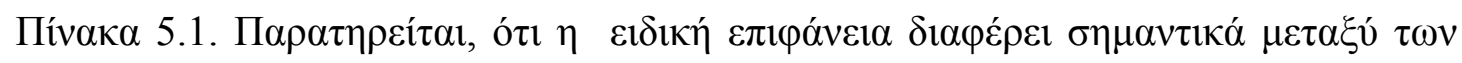

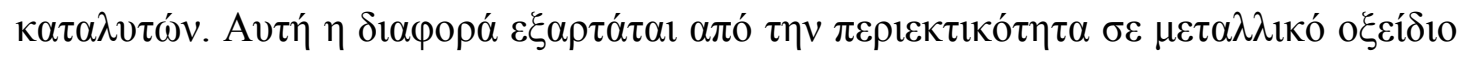

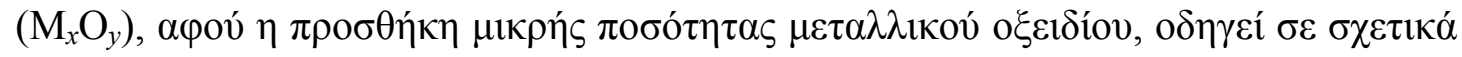

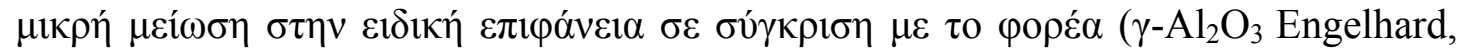

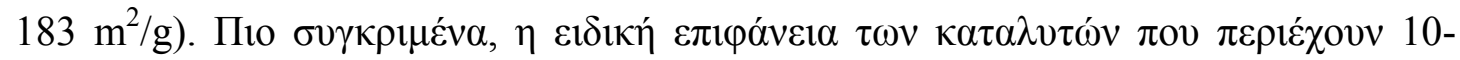

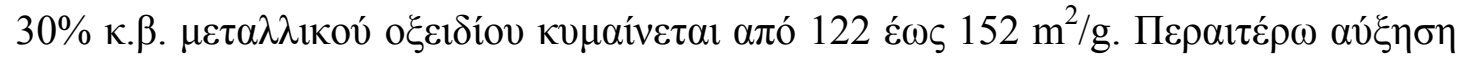

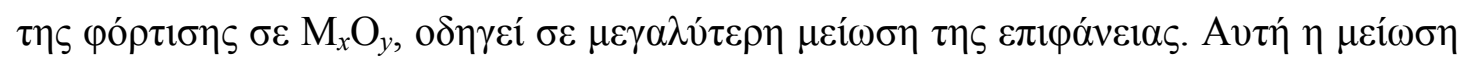

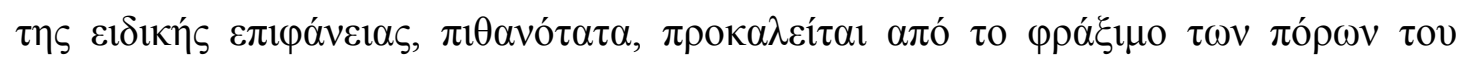

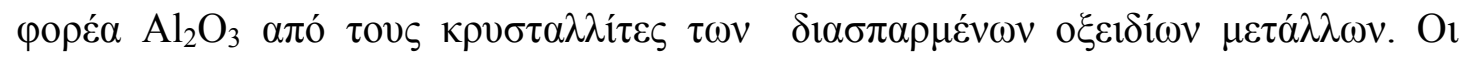

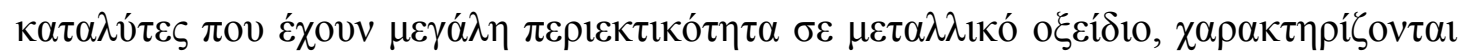

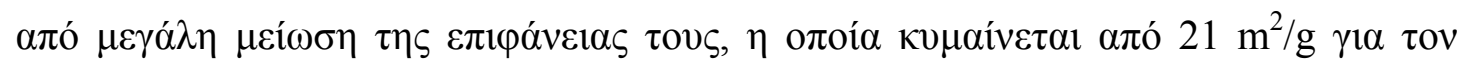

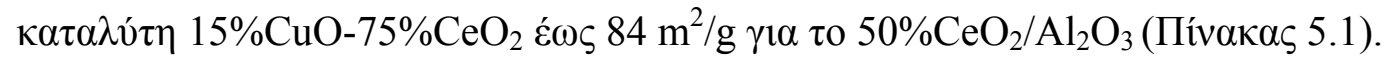

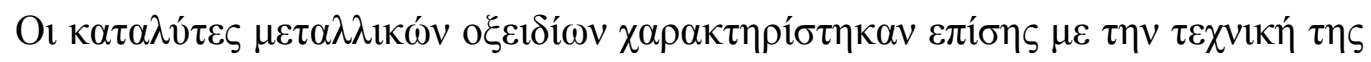

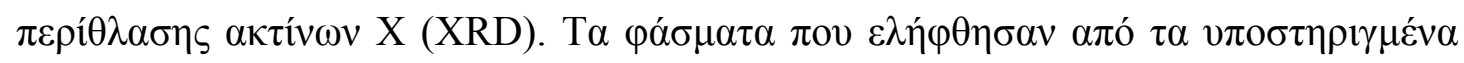

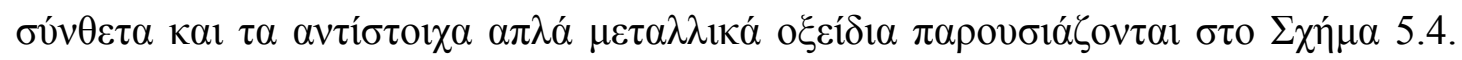

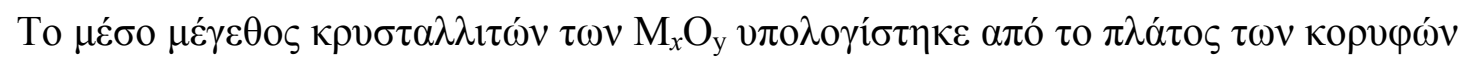

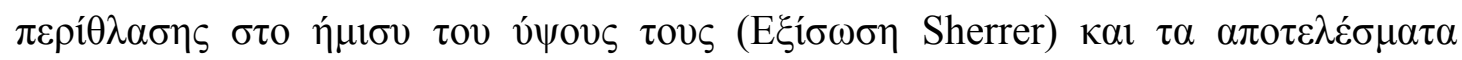

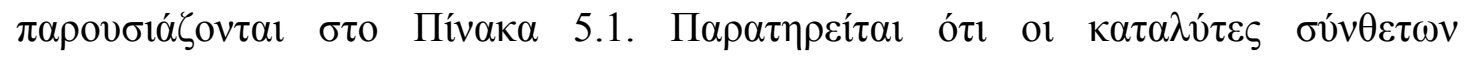

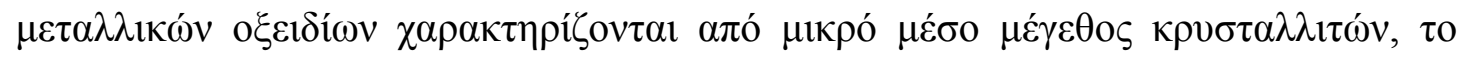

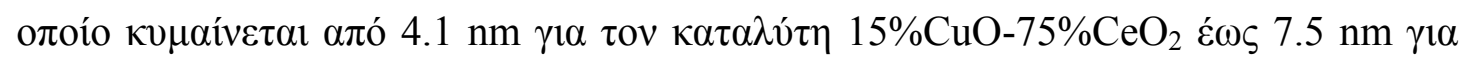

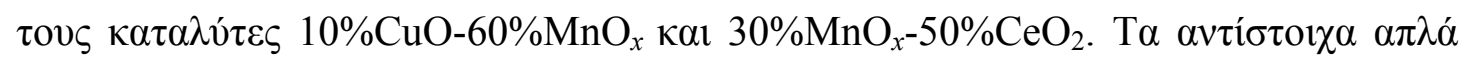

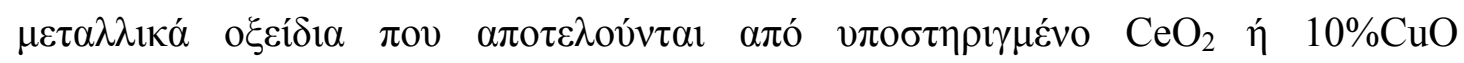

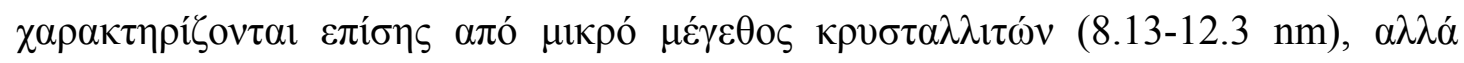

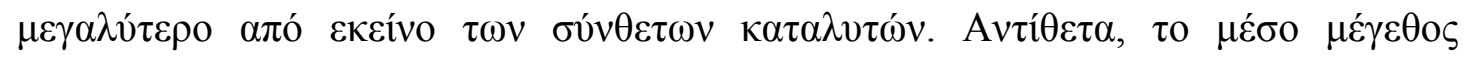




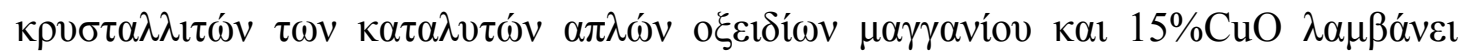

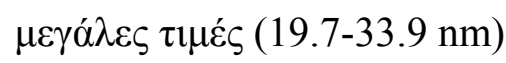

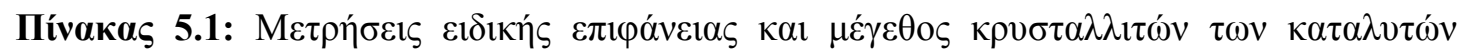

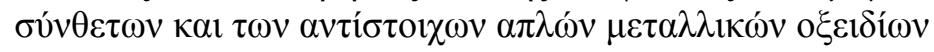

\begin{tabular}{|c|c|c|c|}
\hline 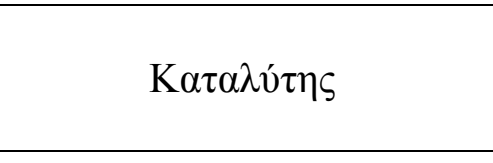 & 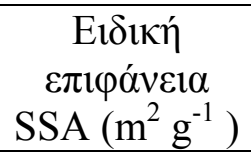 & 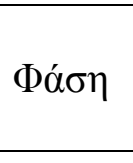 & 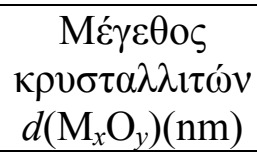 \\
\hline$\gamma-\mathrm{Al}_{2} \mathrm{O}_{3}$ (Engelhard) & 183 & $\mathrm{Al}_{2} \mathrm{O}_{3}$ & 5.1 \\
\hline $10 \% \mathrm{CuO}-60 \% \mathrm{MnO}_{x} / \mathrm{Al}_{2} \mathrm{O}_{3}$ & 49.5 & $\mathrm{MnO}_{x}$ & 7.5 \\
\hline $10 \% \mathrm{CuO} / \mathrm{Al}_{2} \mathrm{O}_{3}$ & 152 & $\mathrm{Al}_{2} \mathrm{O}_{3}$ & 12.3 \\
\hline $60 \% \mathrm{MnO}_{x} / \mathrm{Al}_{2} \mathrm{O}_{3}$ & 53 & $\mathrm{MnO}_{x}$ & 19.7 \\
\hline $15 \% \mathrm{CuO}-75 \% \mathrm{CeO}_{2} / \mathrm{Al}_{2} \mathrm{O}_{3}$ & 21 & $\mathrm{CeO}_{2}$ & 4.1 \\
\hline $15 \% \mathrm{CuO} / \mathrm{Al}_{2} \mathrm{O}_{3}$ & 151 & $\mathrm{CuO}$ & 33.9 \\
\hline $75 \% \mathrm{CeO}_{2} / \mathrm{Al}_{2} \mathrm{O}_{3}$ & 89.6 & $\mathrm{CeO}_{2}$ & 8.5 \\
\hline $30 \% \mathrm{MnO}_{x}-50 \% \mathrm{CeO}_{2} / \mathrm{Al}_{2} \mathrm{O}_{3}$ & 79 & $\mathrm{CeO}_{2}$ & 7.5 \\
\hline $30 \% \mathrm{MnO}_{x} / \mathrm{Al}_{2} \mathrm{O}_{3}$ & 122 & $\mathrm{MnO}_{x}$ & 33.7 \\
\hline $50 \% \mathrm{CeO}_{2} / \mathrm{Al}_{2} \mathrm{O}_{3}$ & 83.6 & $\mathrm{CeO}_{2}$ & 8.3 \\
\hline
\end{tabular}

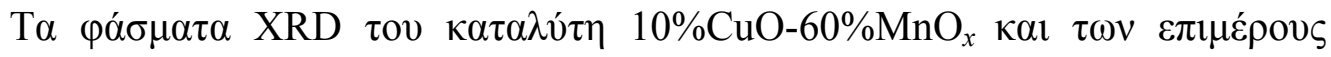

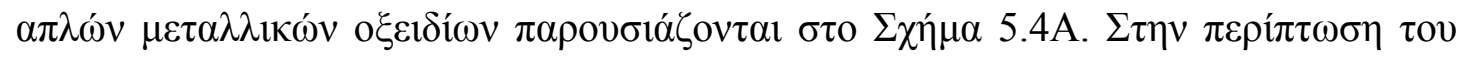

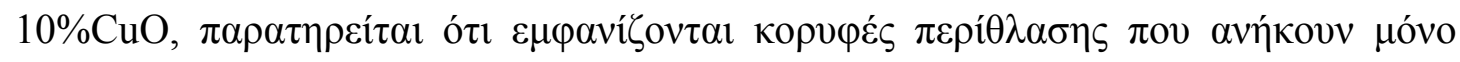

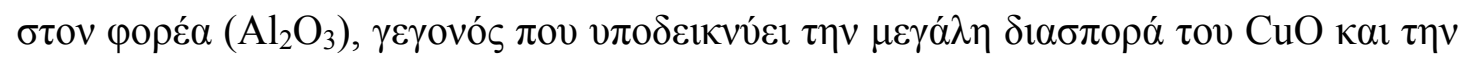

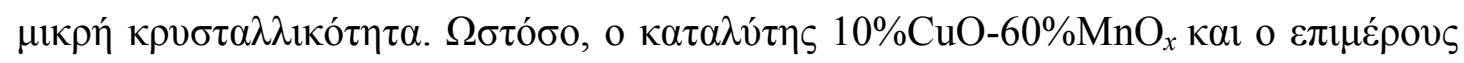

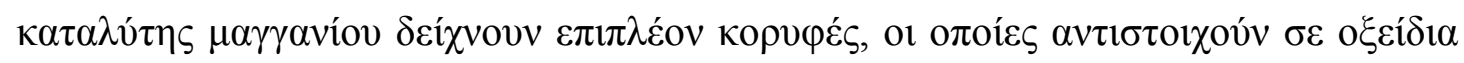

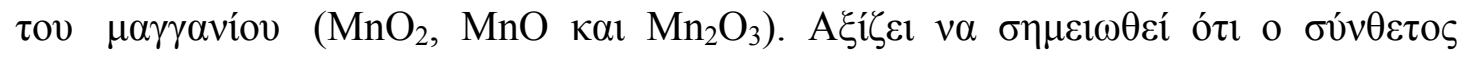

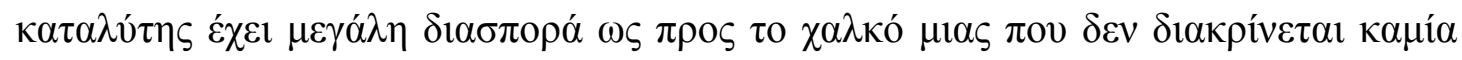

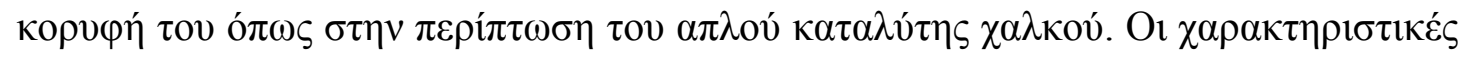

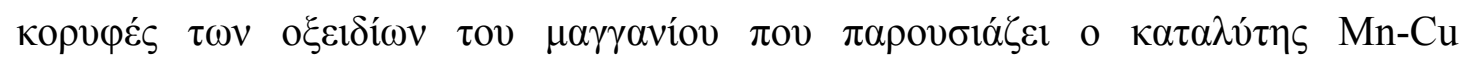

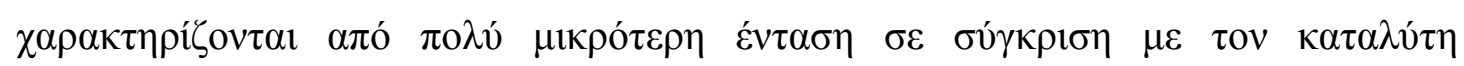

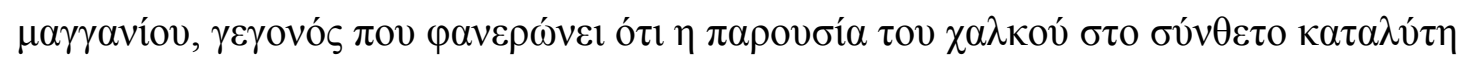

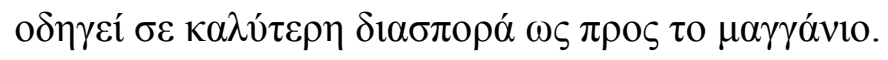



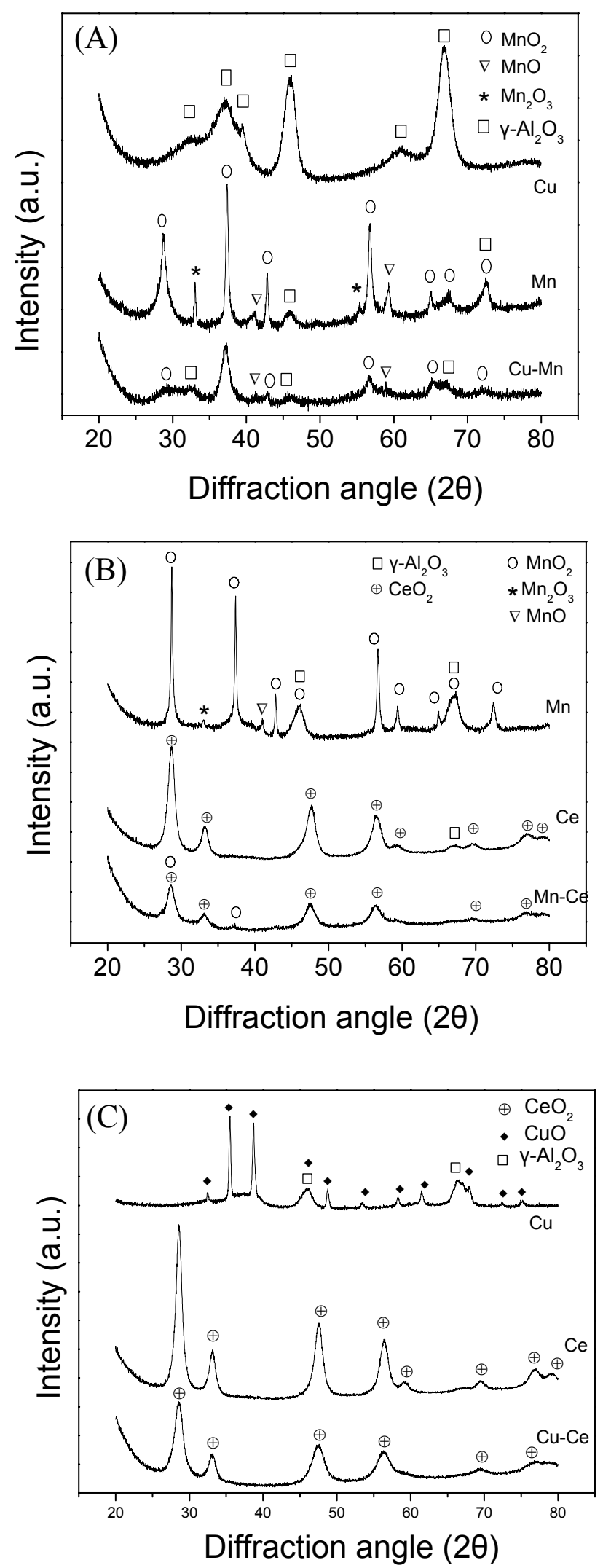

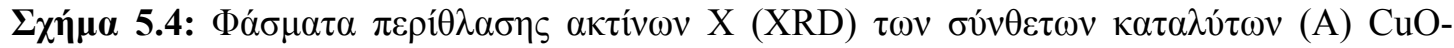

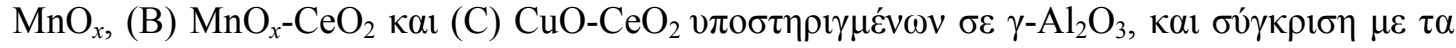

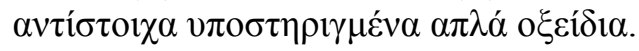




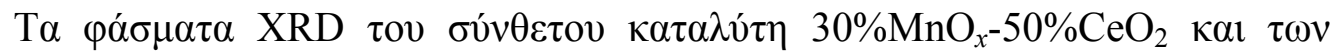

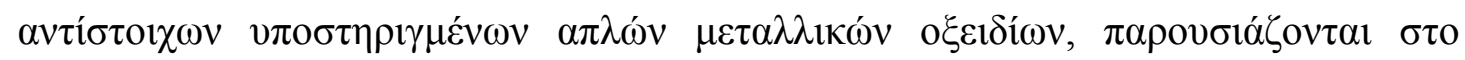

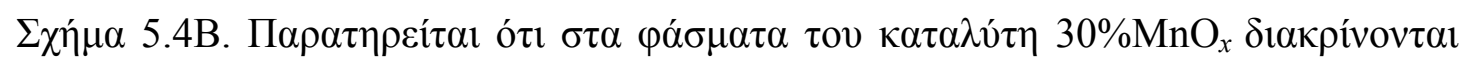

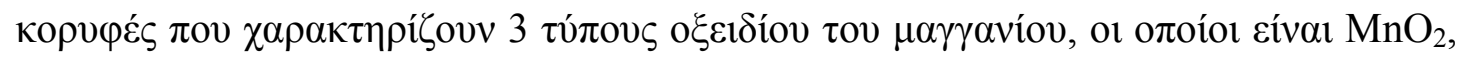

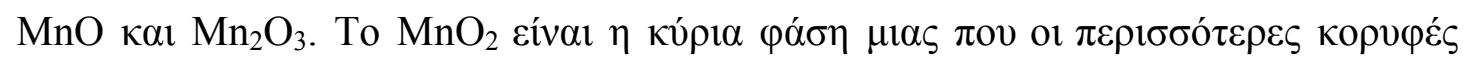

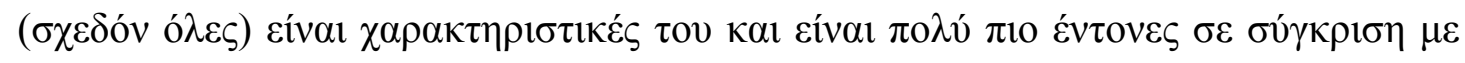

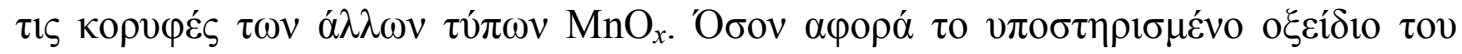

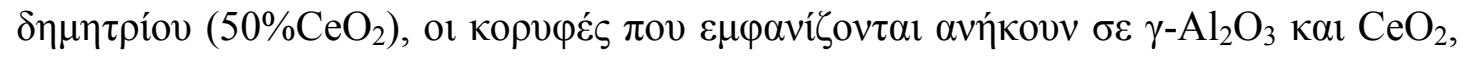

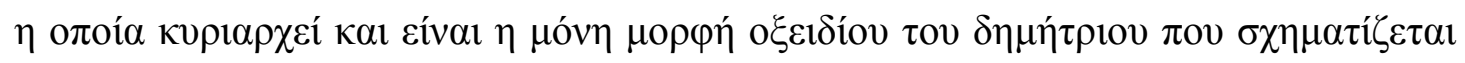

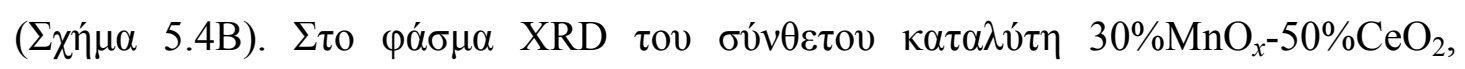

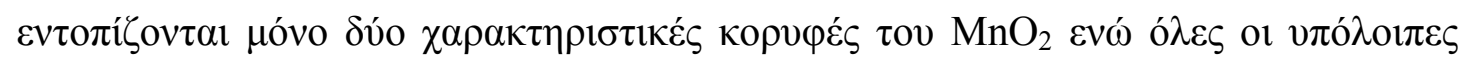

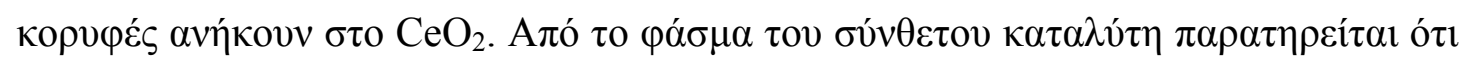

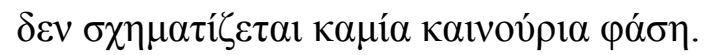

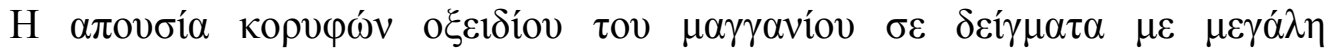

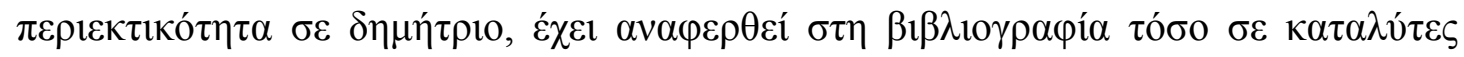

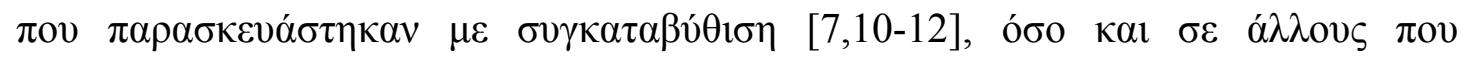

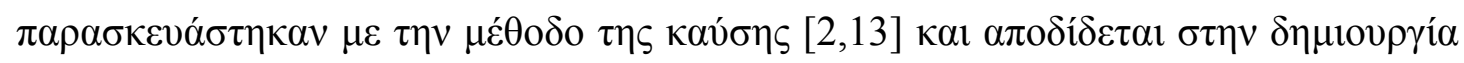

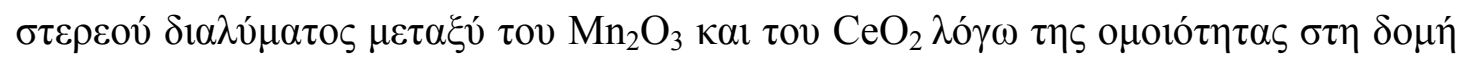

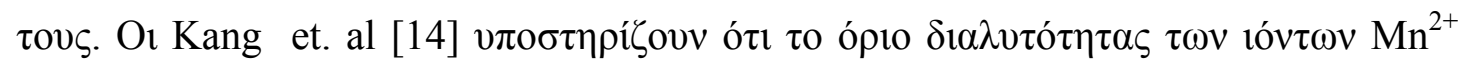

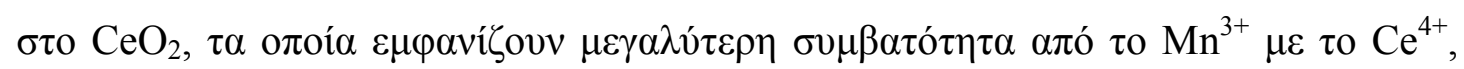

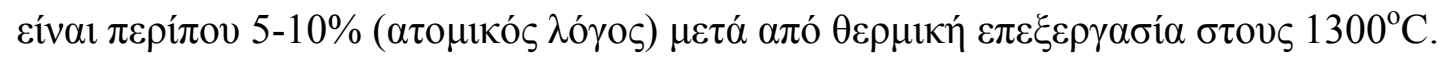

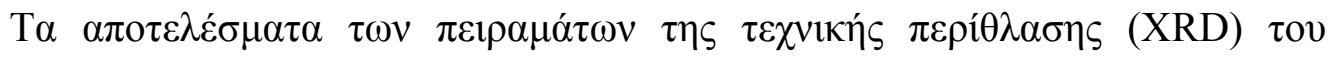

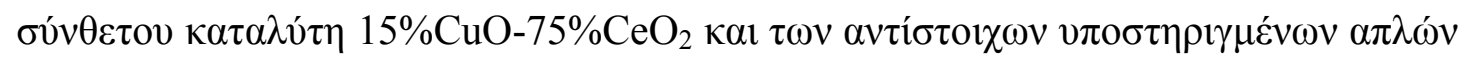

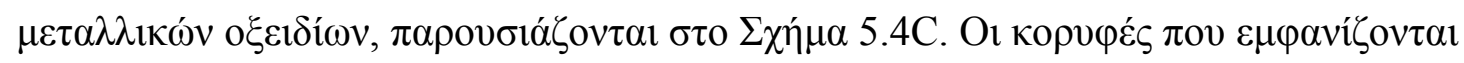

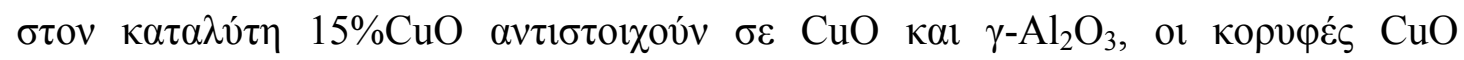

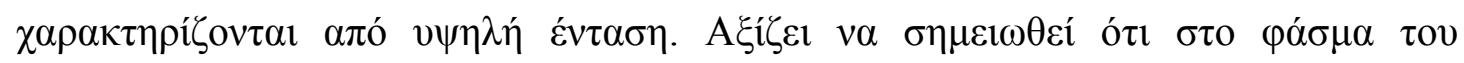

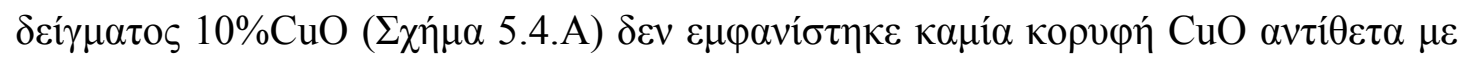

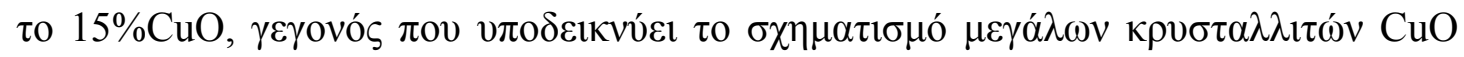

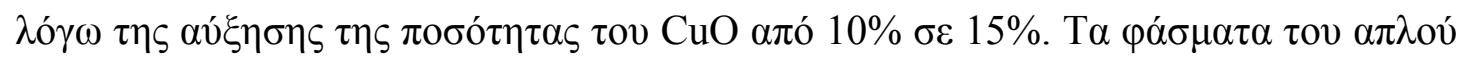

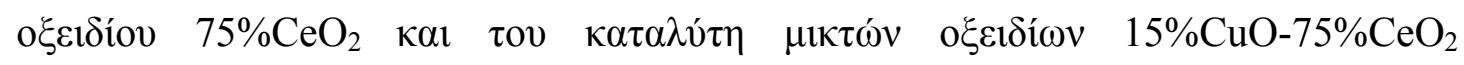

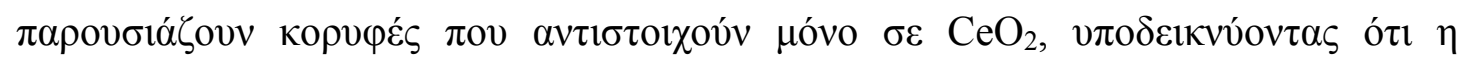
$\pi \alpha \rho o v \sigma i ́ \alpha$

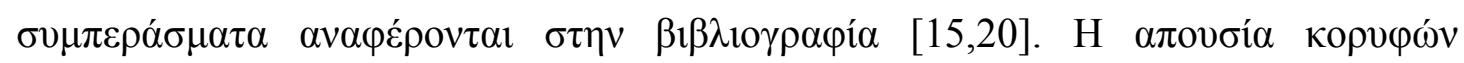

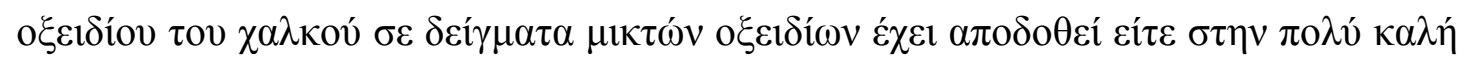




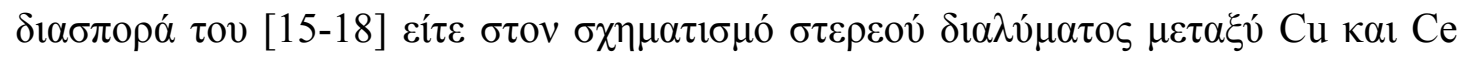
$[19,20]$.

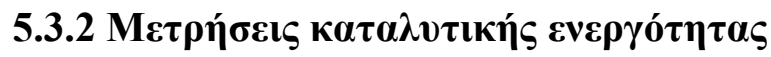

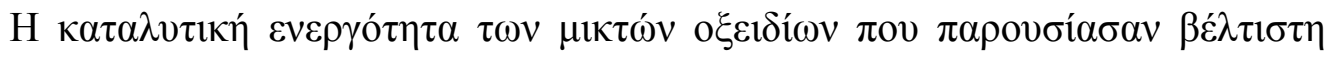

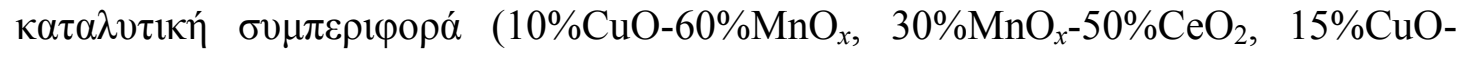

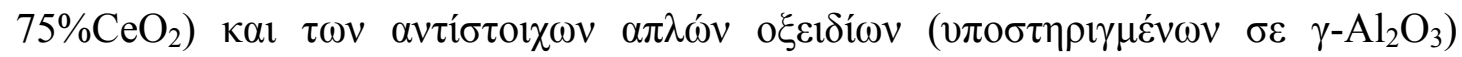

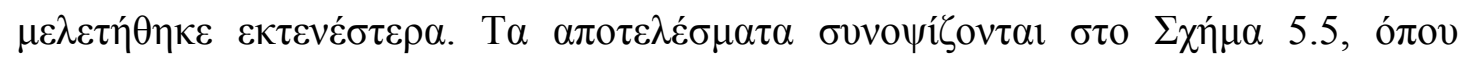

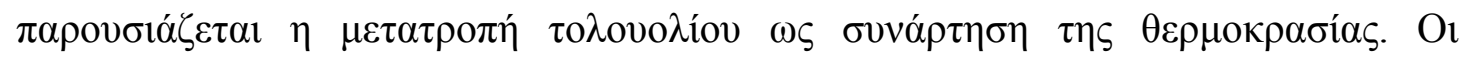

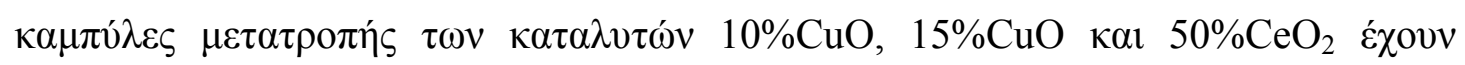

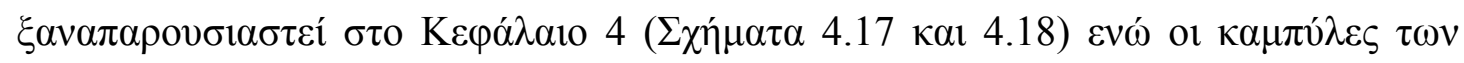

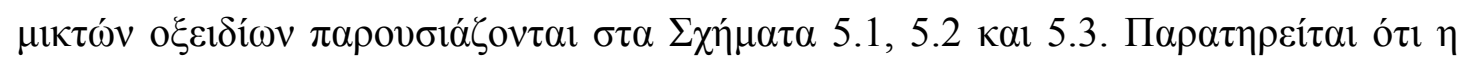

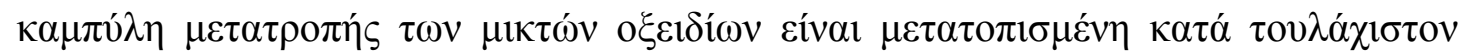

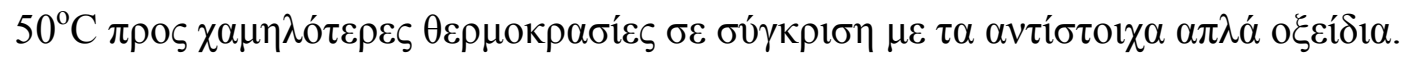



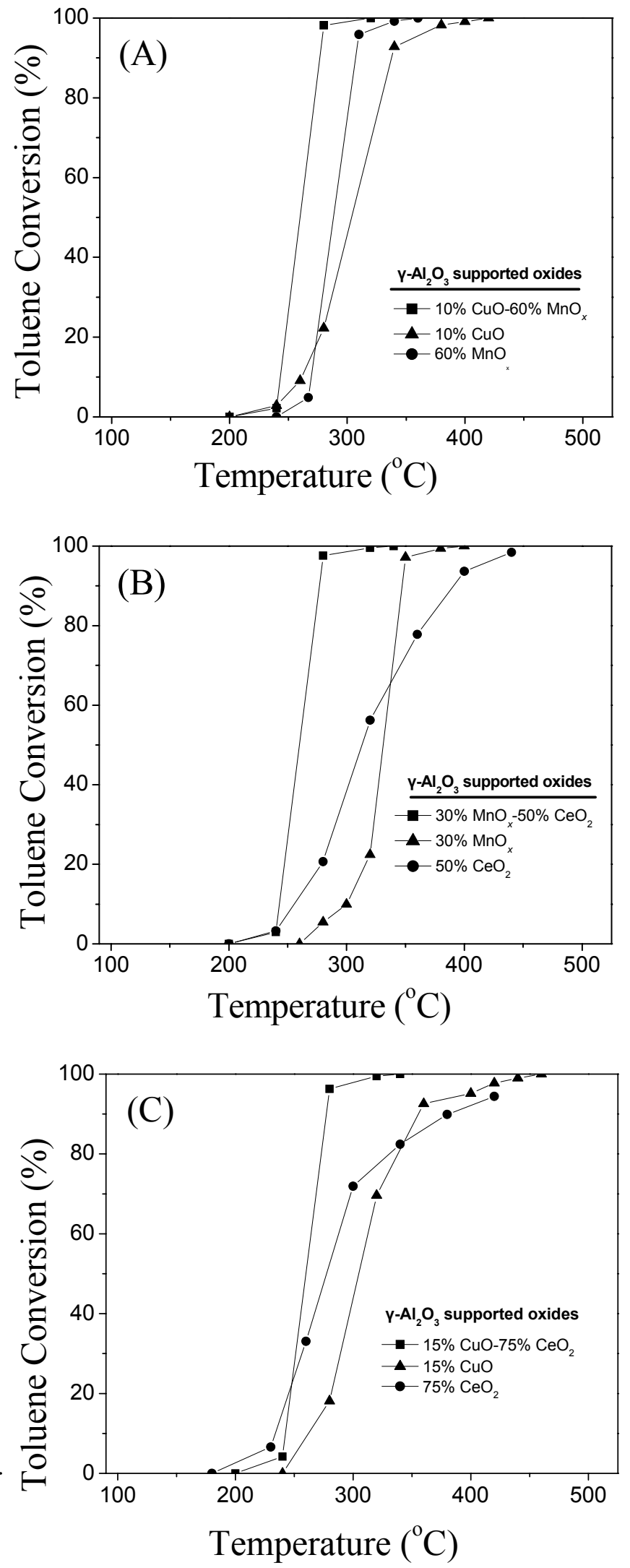

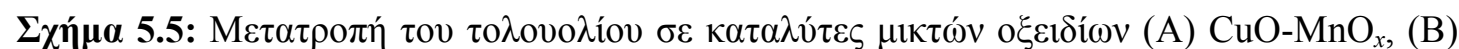

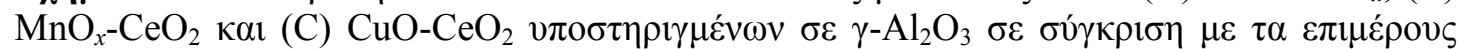

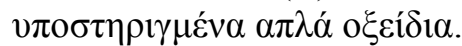




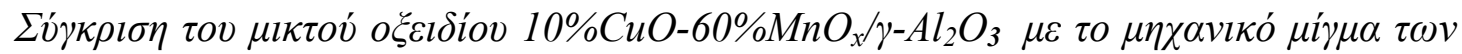

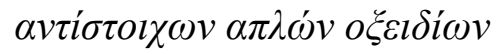

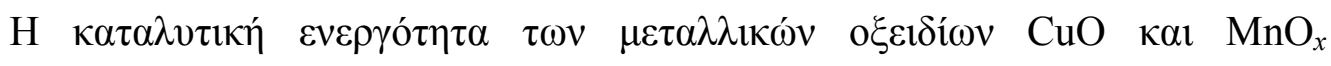

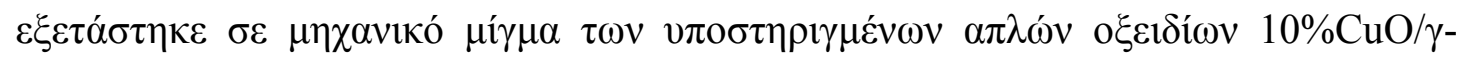

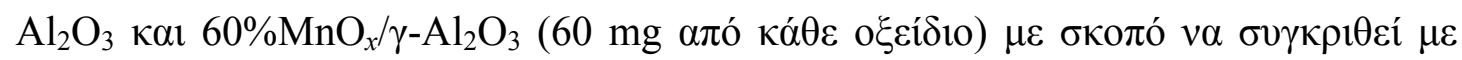

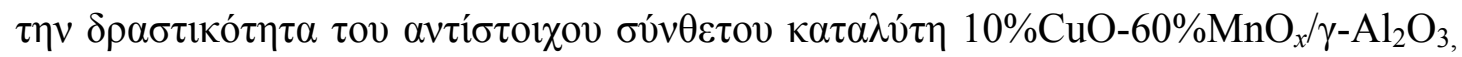

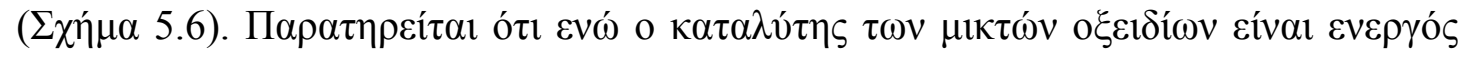

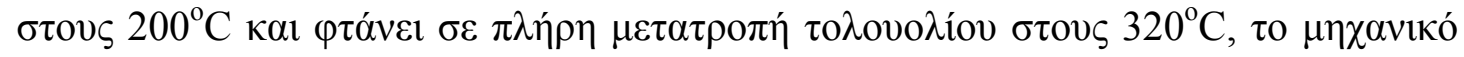

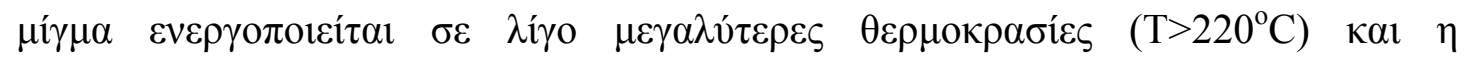

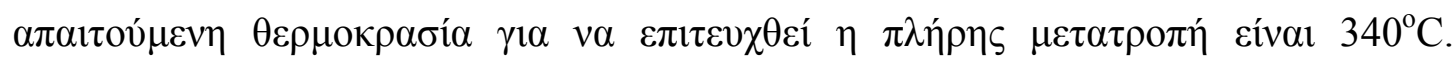

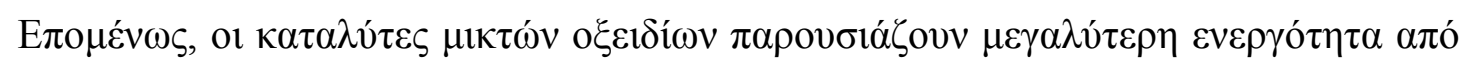

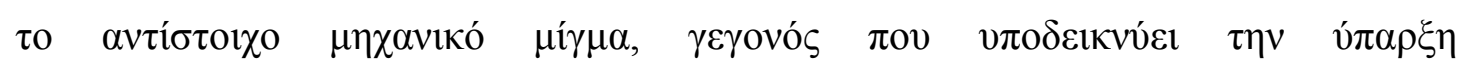

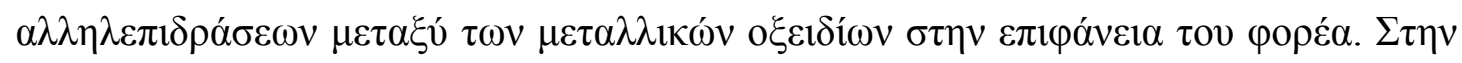

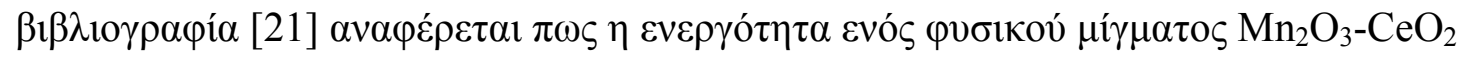

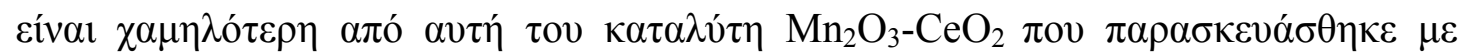

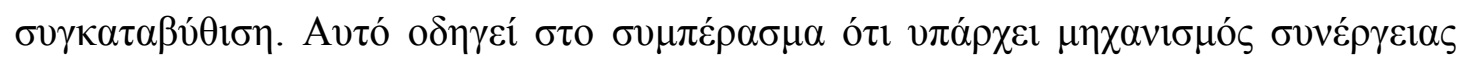

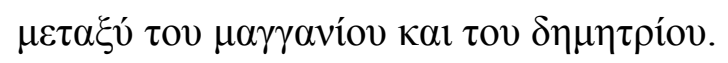

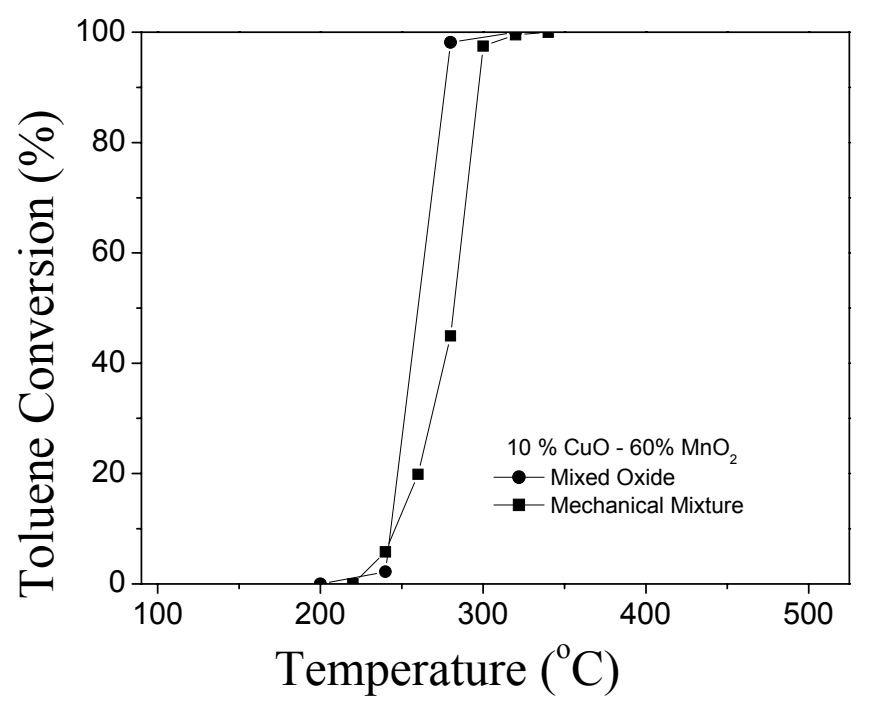

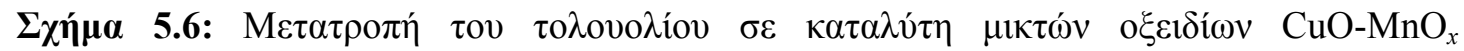

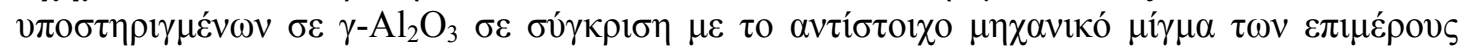
$\alpha \pi \lambda \omega ́ v 0 \xi \varepsilon 1 \delta i ́ \omega v$. 


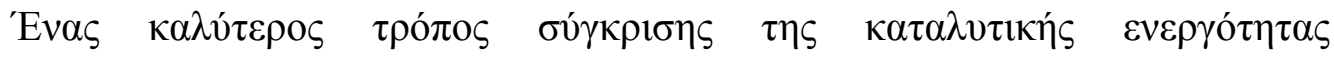

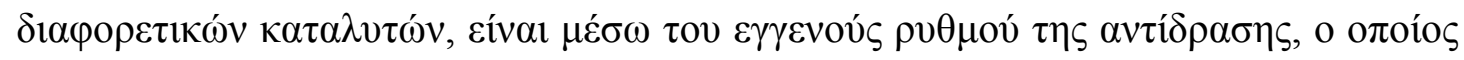

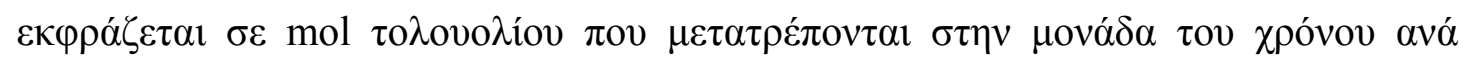

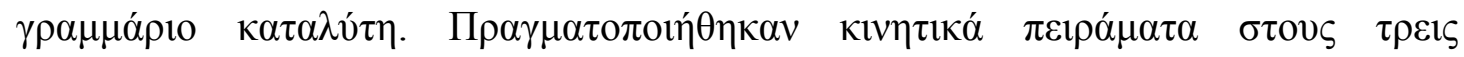

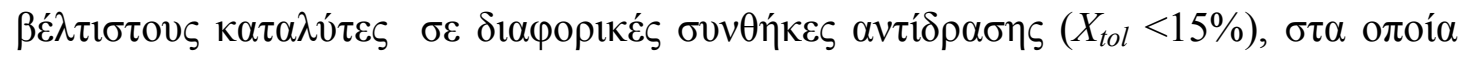

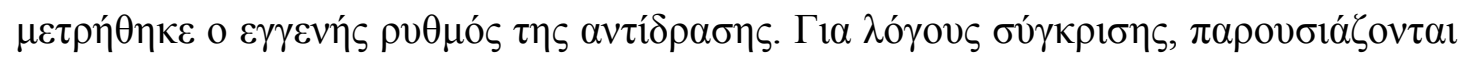

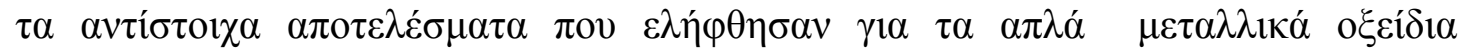

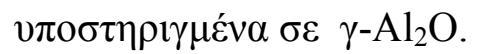

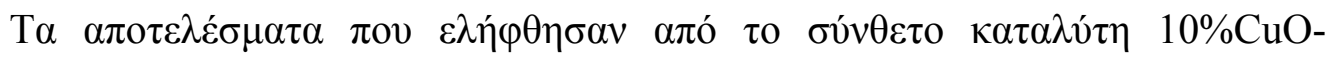

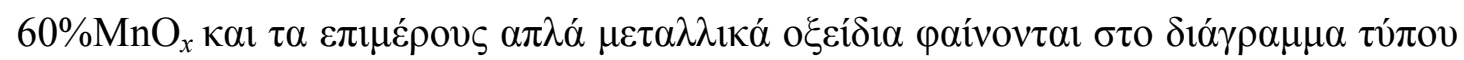

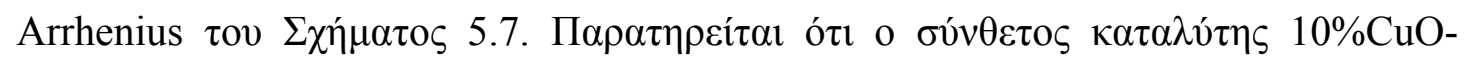

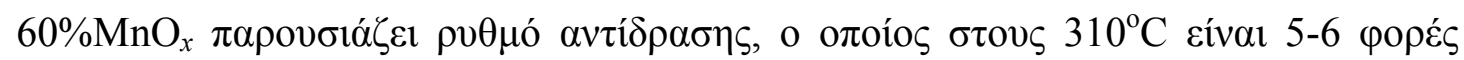

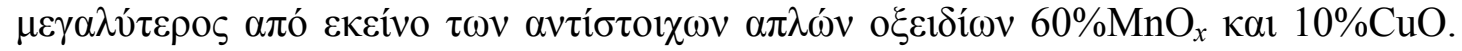

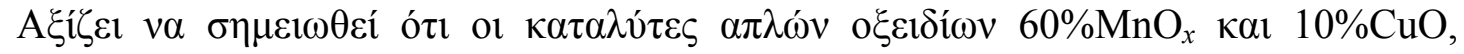

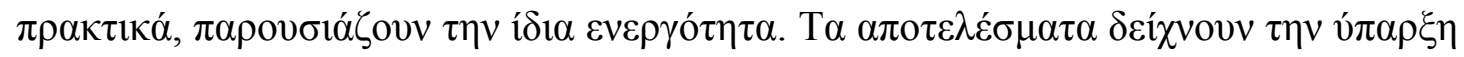

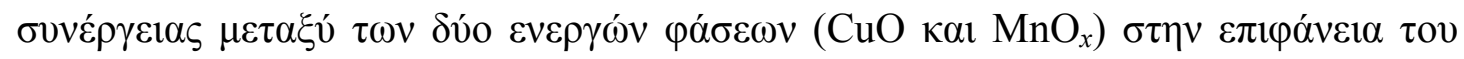

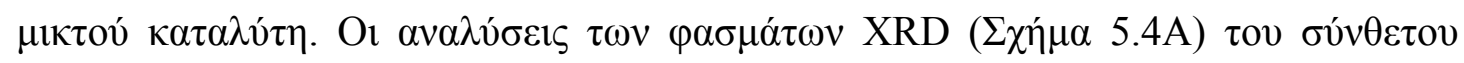

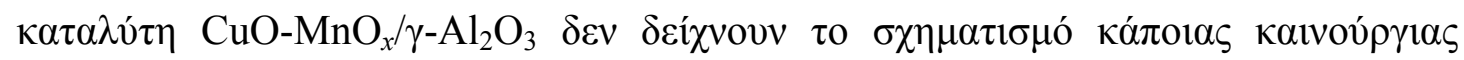

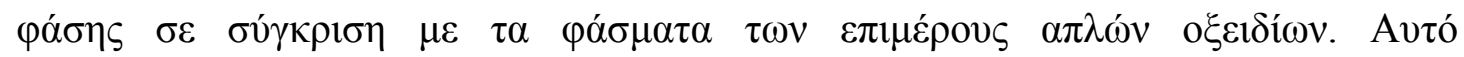

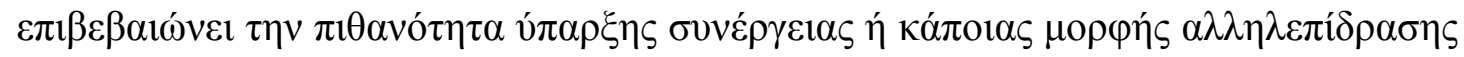

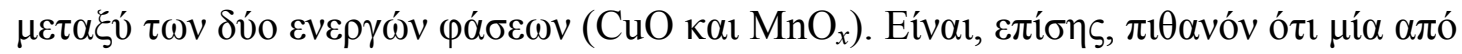

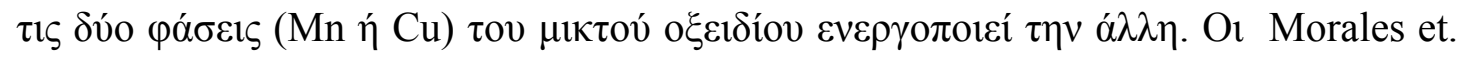

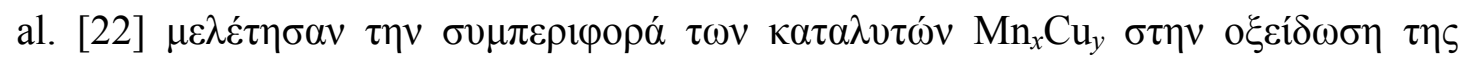

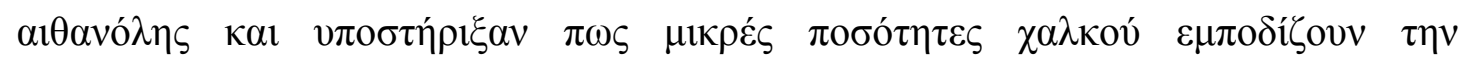

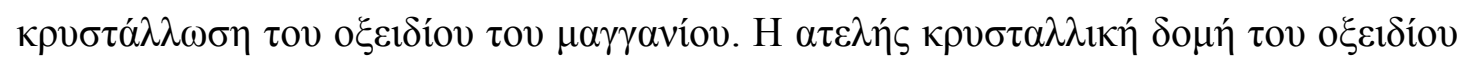

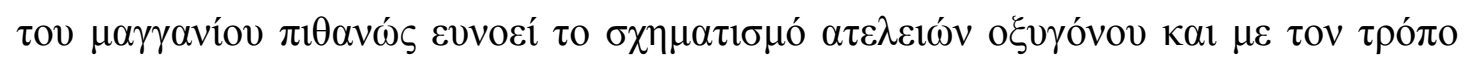

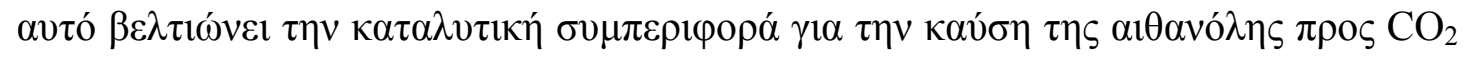
$[22]$.

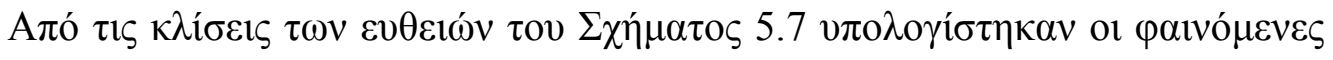

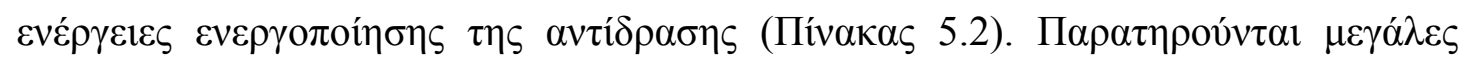

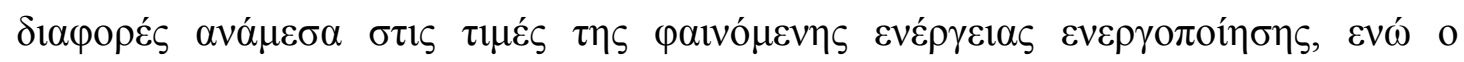

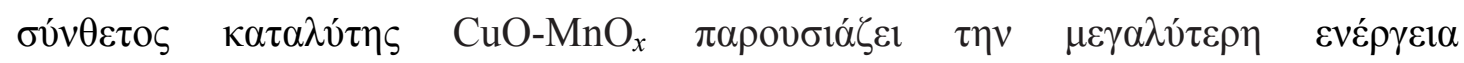

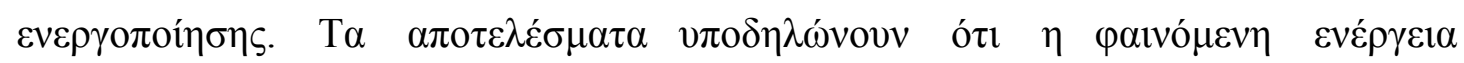




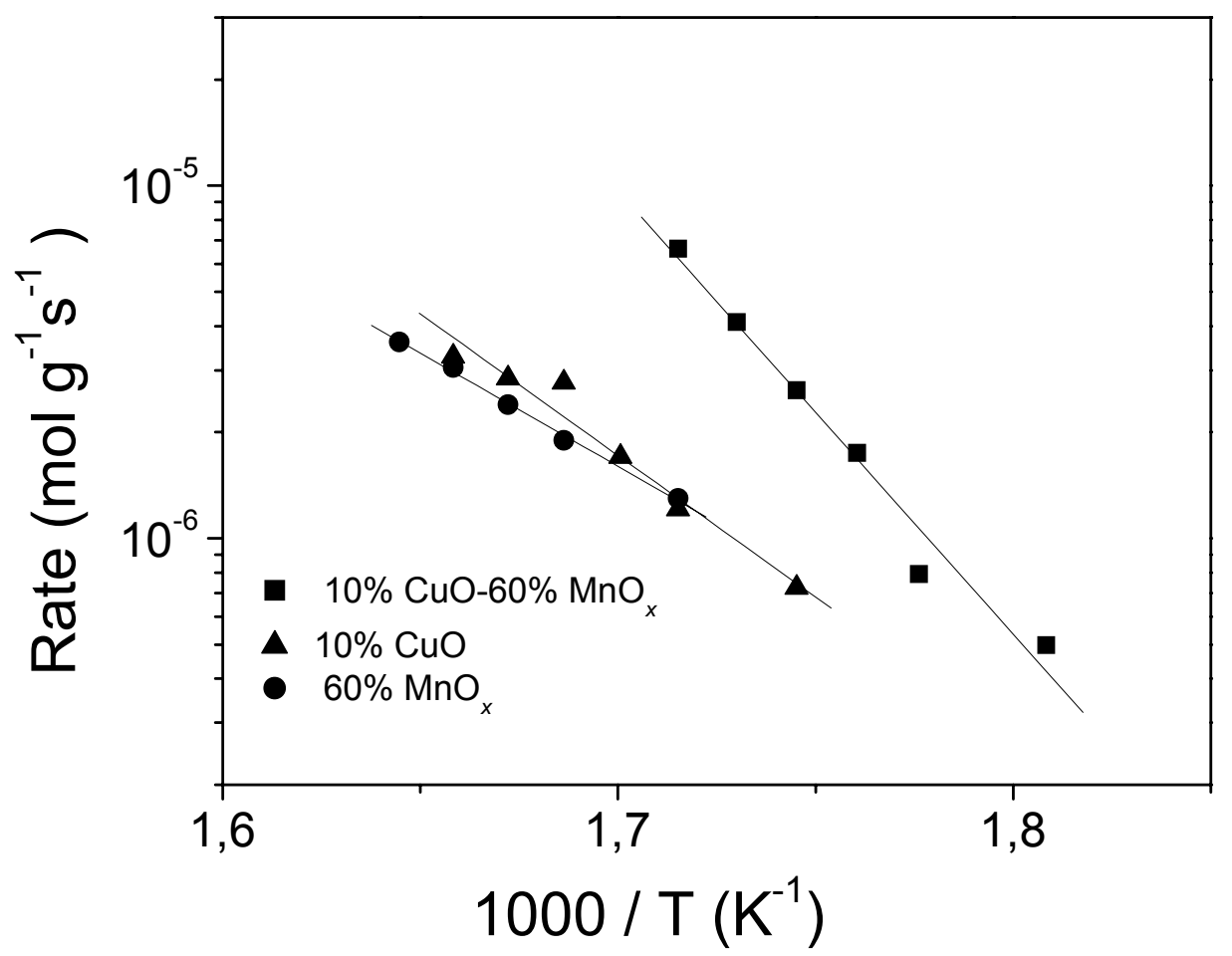

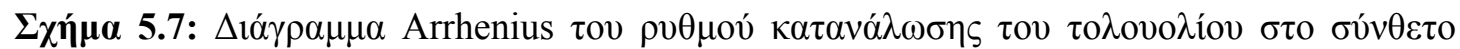

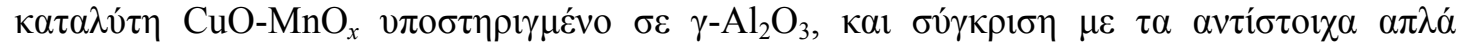
o $\xi \varepsilon i \delta 1 \alpha$.

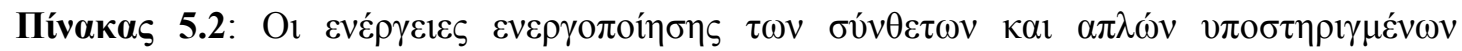

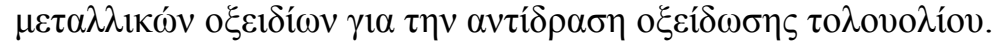

\begin{tabular}{|c|c|}
\hline 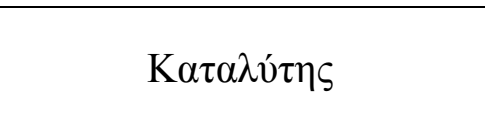 & 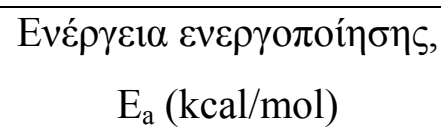 \\
\hline $10 \% \mathrm{CuO}-60 \% \mathrm{MnO}_{x} / \mathrm{Al}_{2} \mathrm{O}_{3}$ & 57.5 \\
\hline $60 \% \mathrm{MnO}_{x} / \mathrm{Al}_{2} \mathrm{O}_{3}$ & 42.3 \\
\hline $10 \% \mathrm{CuO} / \mathrm{Al}_{2} \mathrm{O}_{3}$ & 36.7 \\
\hline $30 \% \mathrm{MnO}_{x}-50 \% \mathrm{CeO}_{2} / \mathrm{Al}_{2} \mathrm{O}_{3}$ & 37.1 \\
\hline $50 \% \mathrm{CeO}_{2} / \mathrm{Al}_{2} \mathrm{O}_{3}$ & 18.3 \\
\hline $30 \% \mathrm{MnO}_{x} / \mathrm{Al}_{2} \mathrm{O}_{3}$ & 37.6 \\
\hline $15 \% \mathrm{CuO}-75 \% \mathrm{CeO}_{2} / \mathrm{Al}_{2} \mathrm{O}_{3}$ & 41 \\
\hline $15 \% \mathrm{CuO} / \mathrm{Al}_{2} \mathrm{O}_{3}$ & 23.5 \\
\hline $75 \% \mathrm{CeO}_{2} / \mathrm{Al}_{2} \mathrm{O}_{3}$ & 26.6 \\
\hline
\end{tabular}




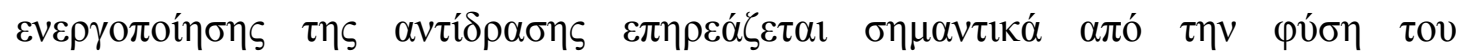

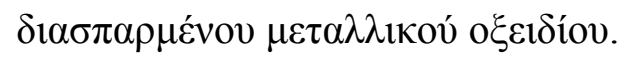

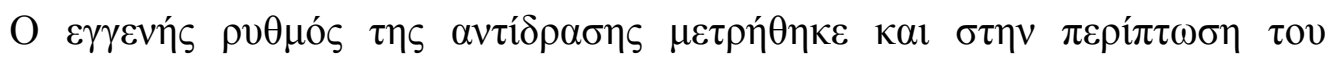

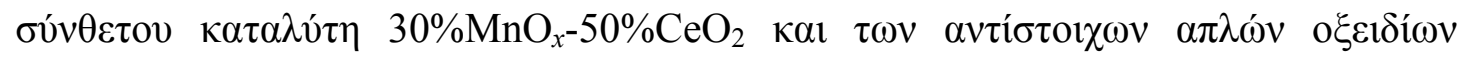

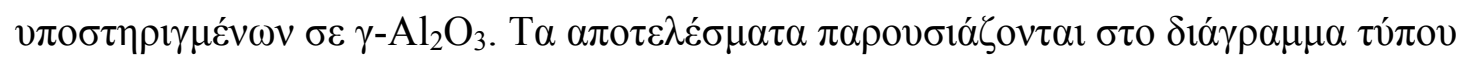

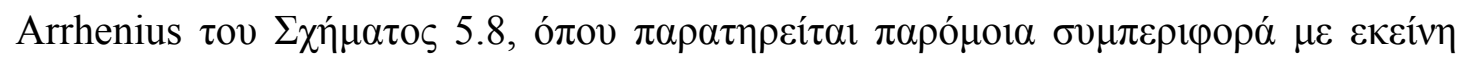

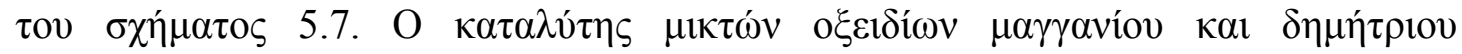

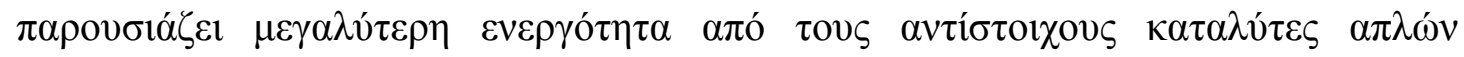

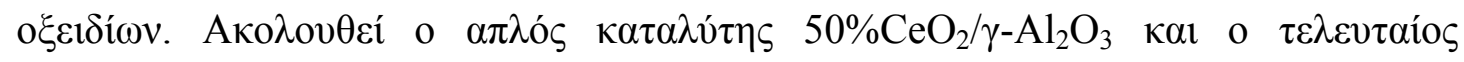

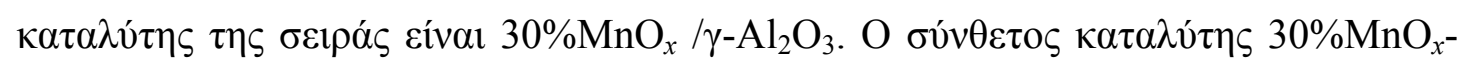

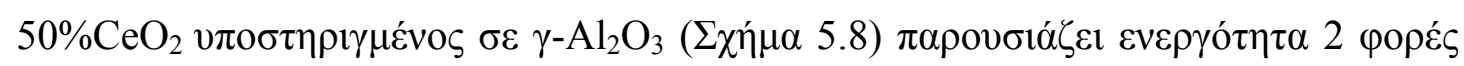

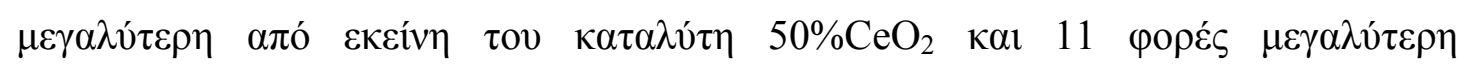

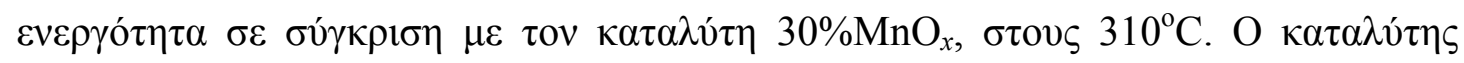

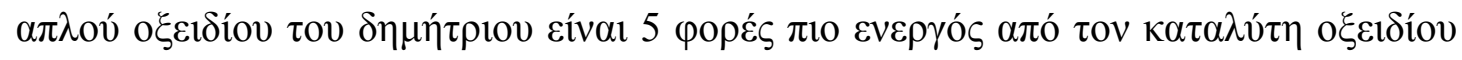

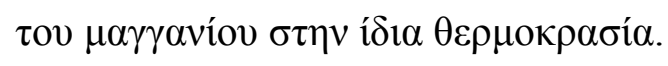

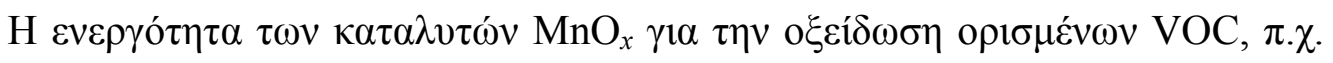

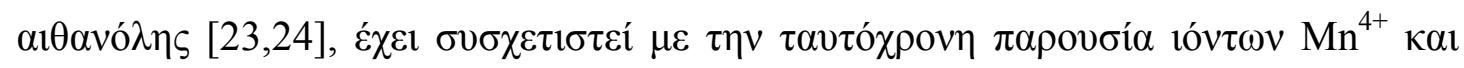

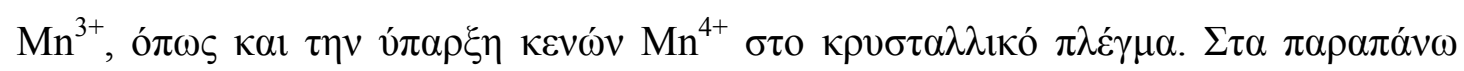

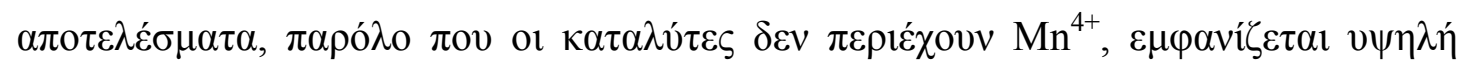

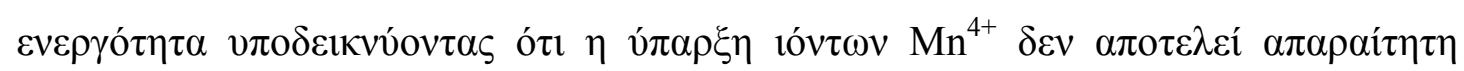

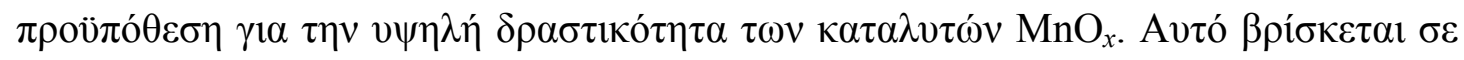

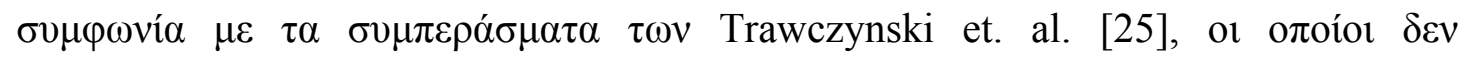

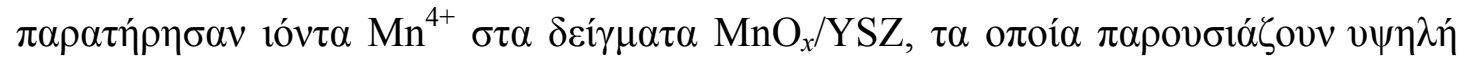
$\kappa \alpha \tau \alpha \lambda v \tau 1 \kappa \eta ́ ~ \varepsilon v \varepsilon \rho \gamma o ́ \tau \eta \tau \alpha$.

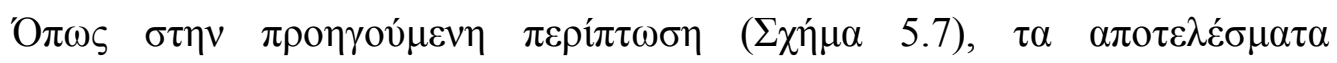

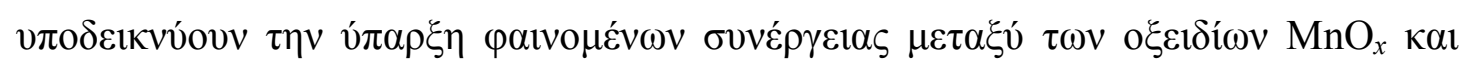

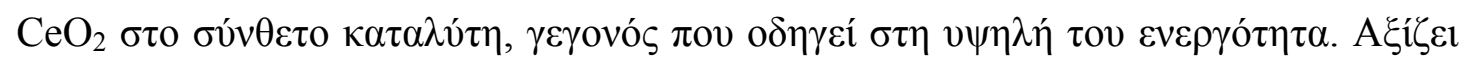

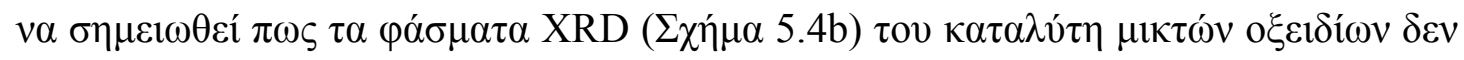

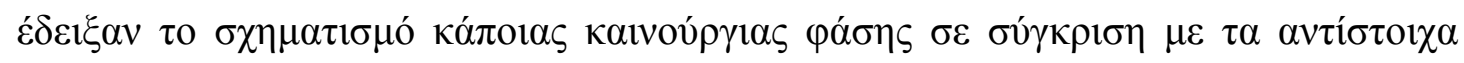
$\alpha \pi \lambda \alpha \dot{o}$ o $\varepsilon i \delta 1 \alpha$.

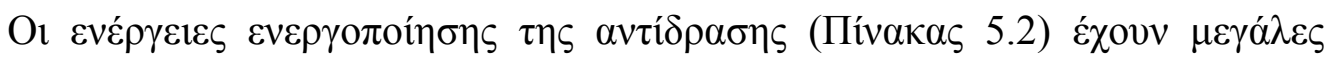

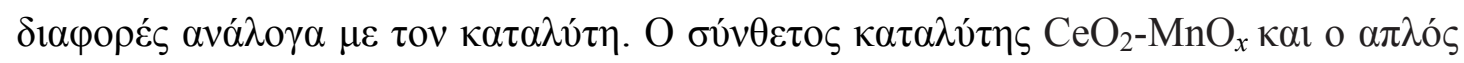

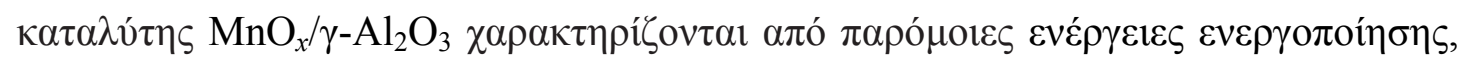

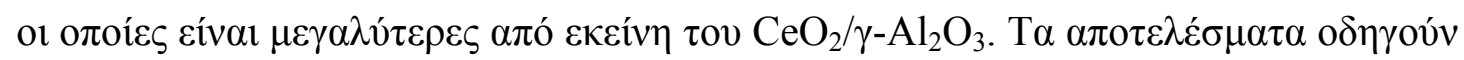




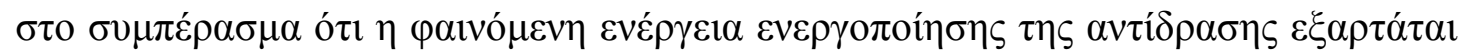

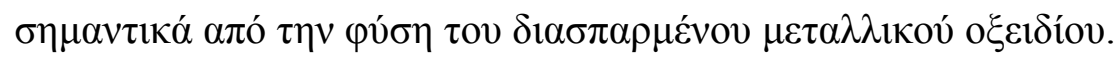

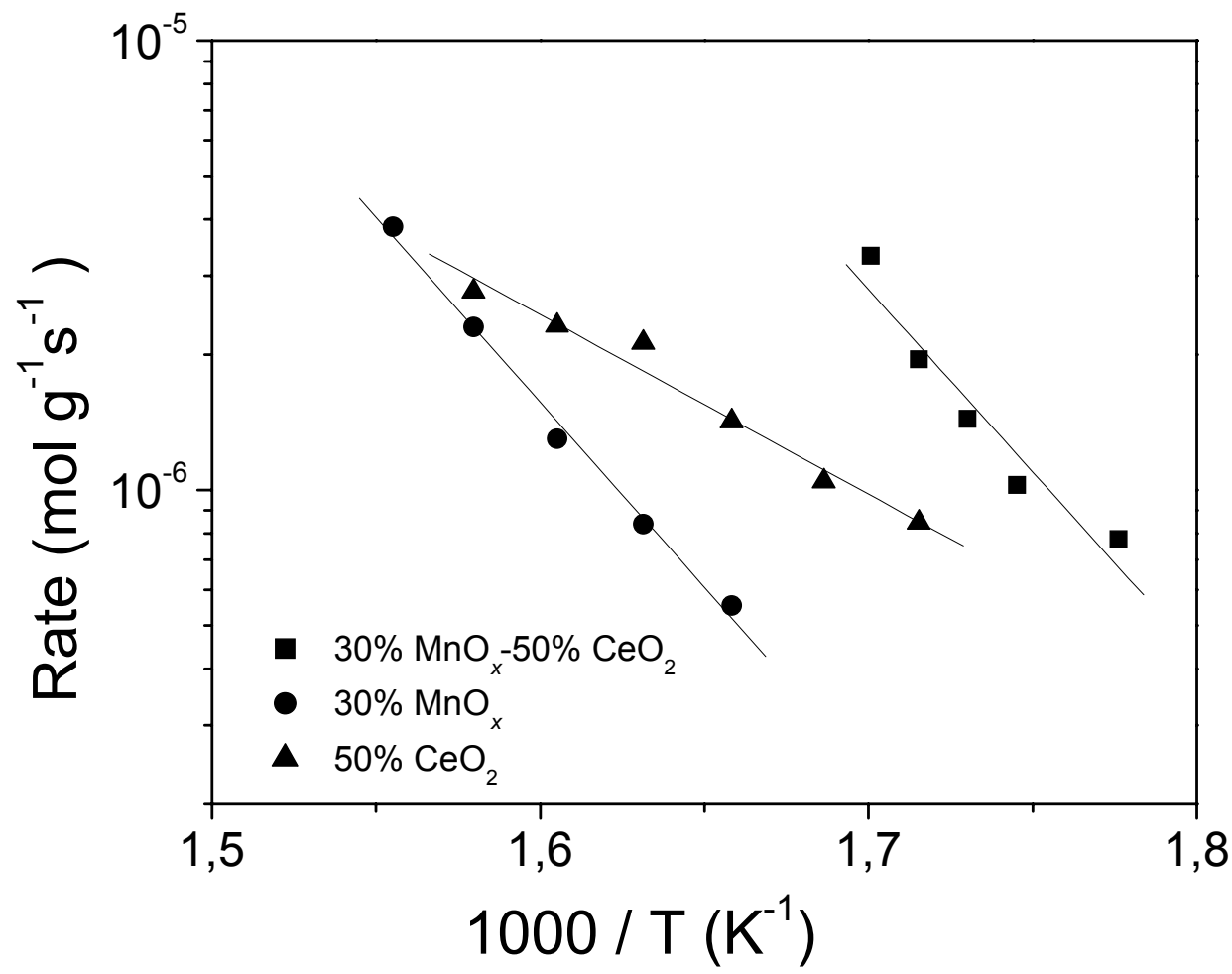

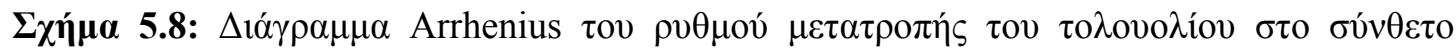

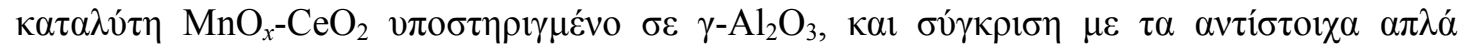
o $\xi \varepsilon i \delta \delta 1 \alpha$.

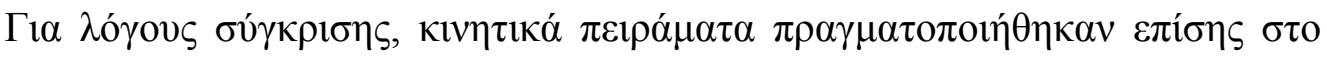

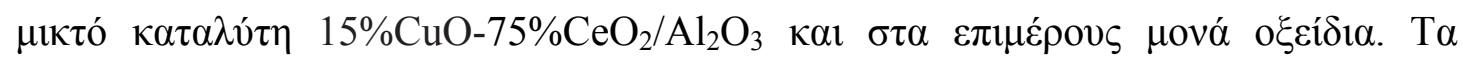

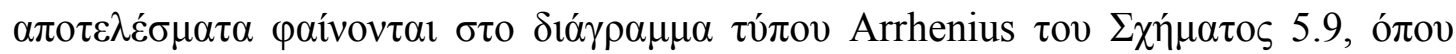

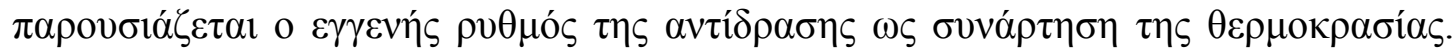

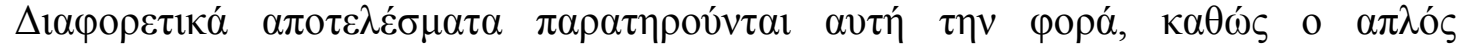

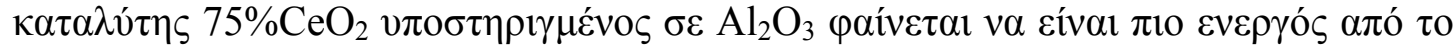

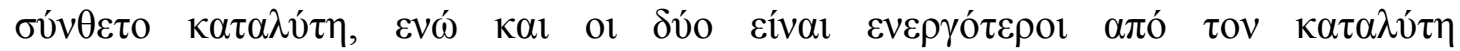

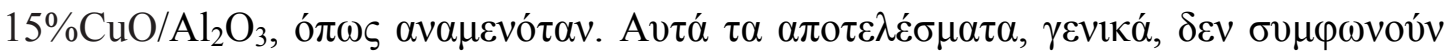

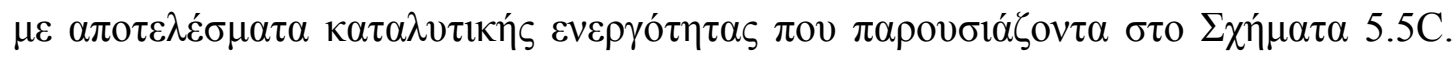

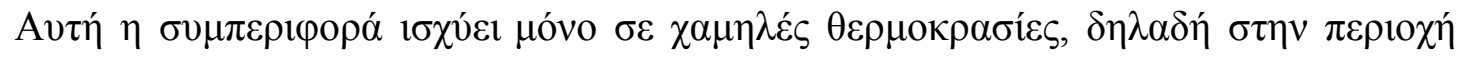

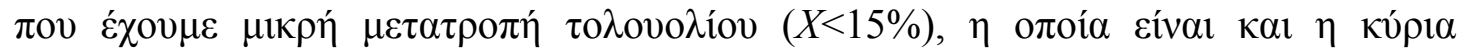

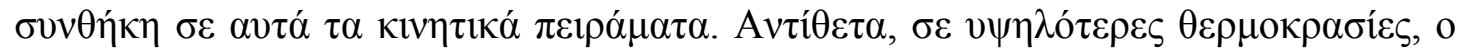




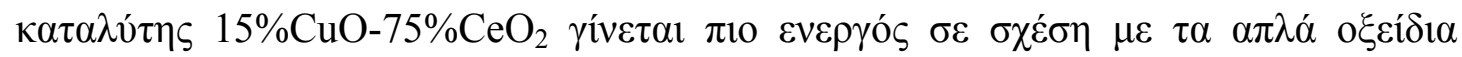

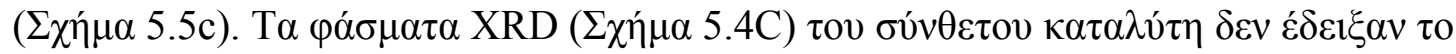

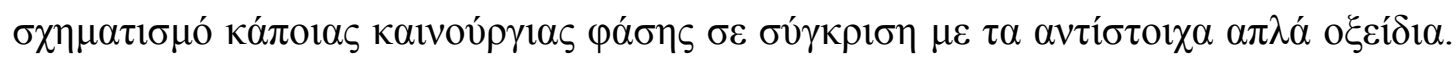

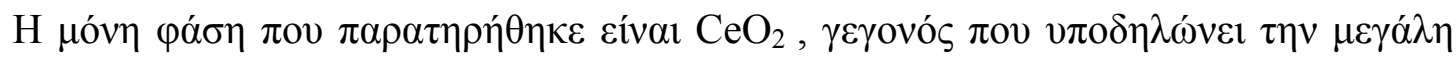

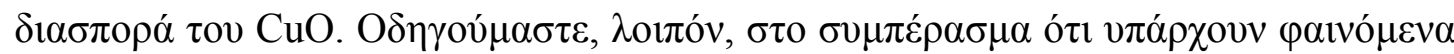

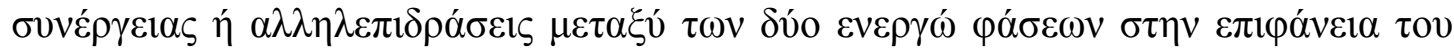
$\mu 1 \kappa \tau \circ \operatorname{v́}_{\kappa} \alpha \tau \alpha \lambda v ́ \tau \eta$.

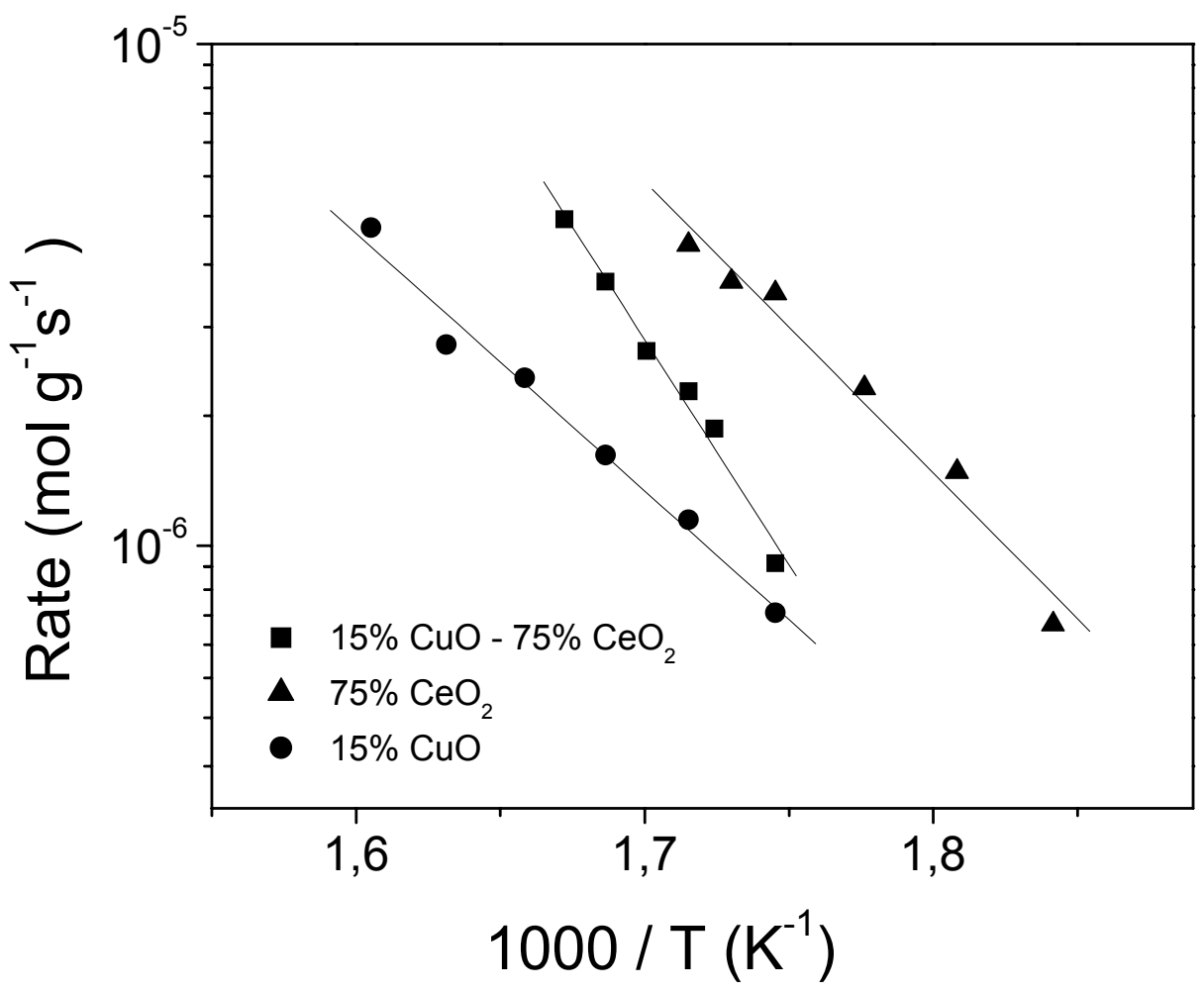

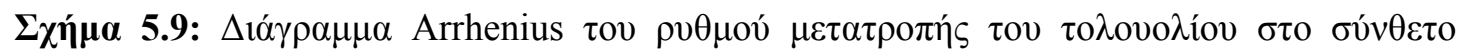

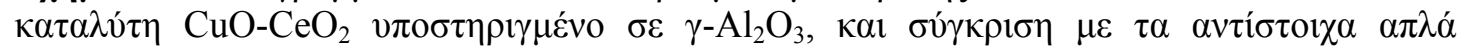
o $\xi \varepsilon i \delta \delta$.

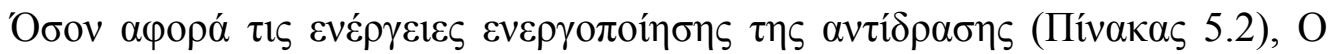

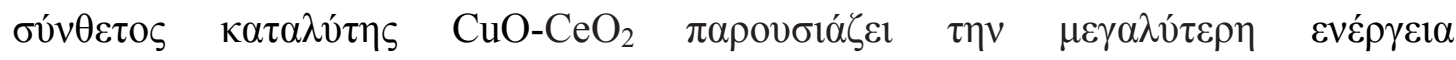

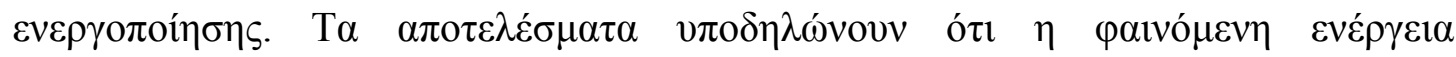

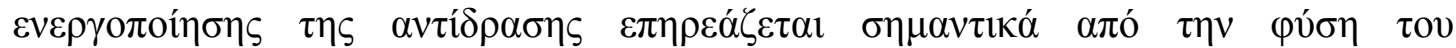

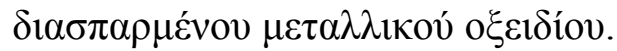




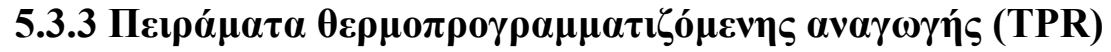

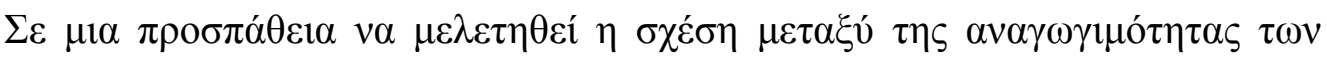

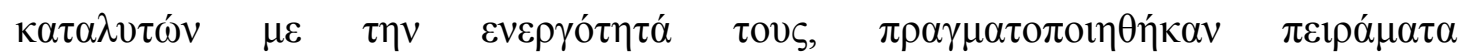

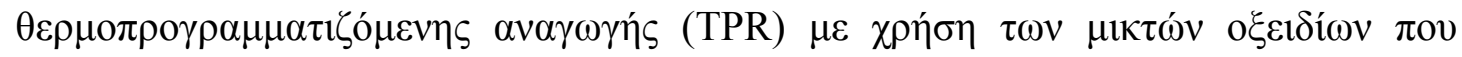

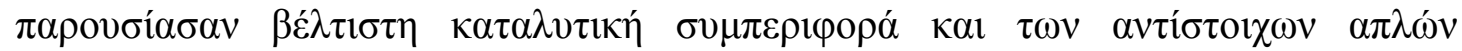

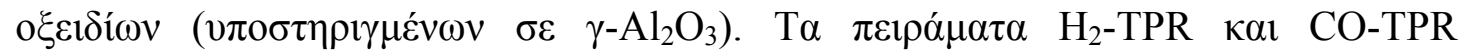

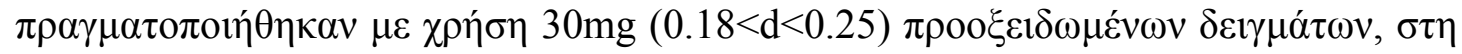

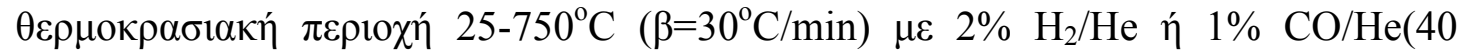

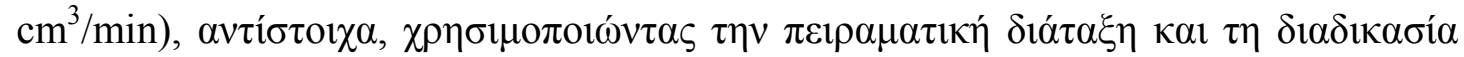

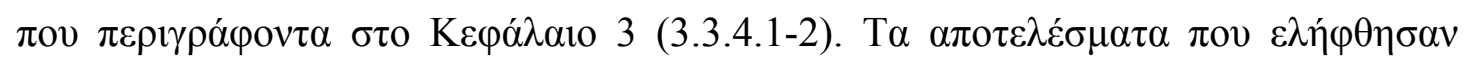

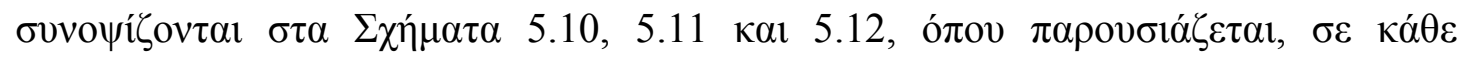

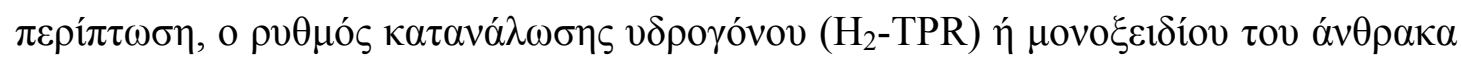

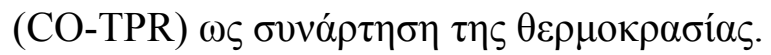

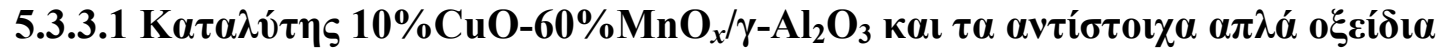

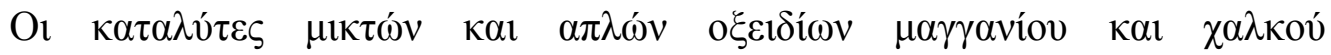

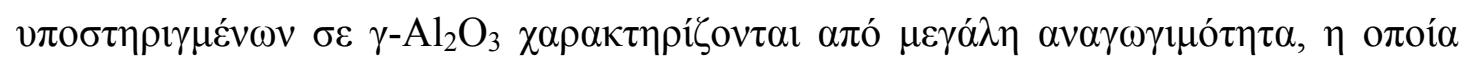

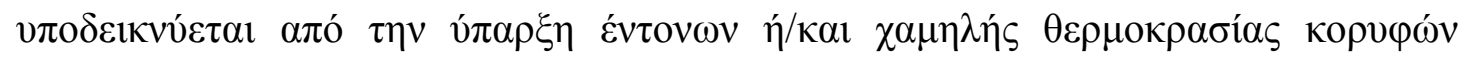

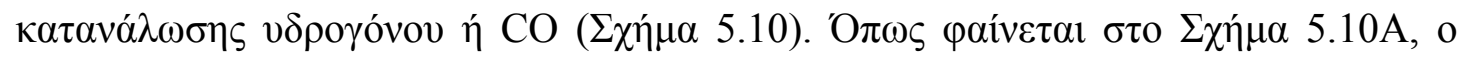

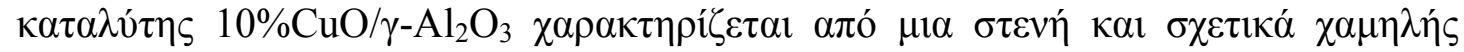

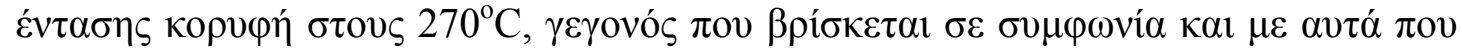

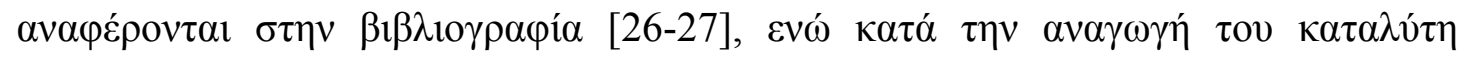

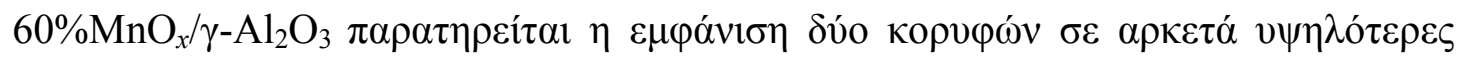

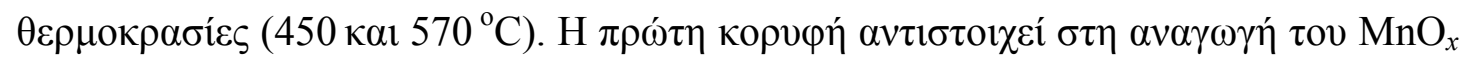

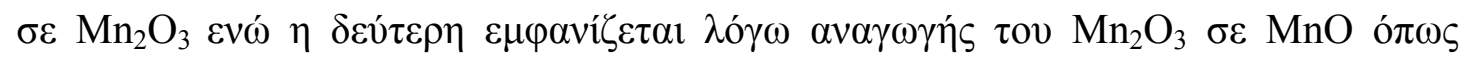

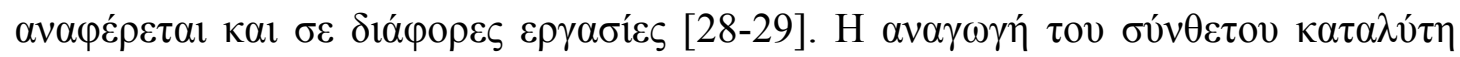

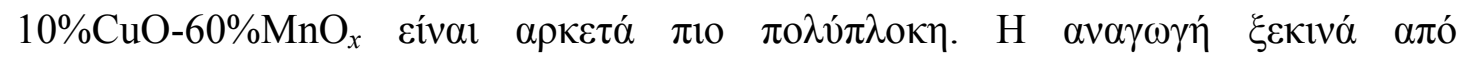

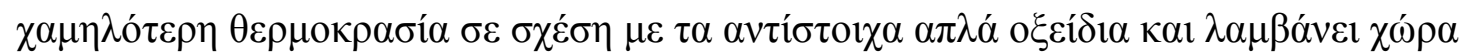

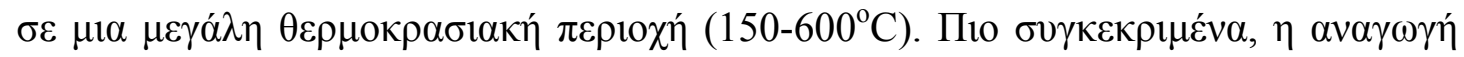

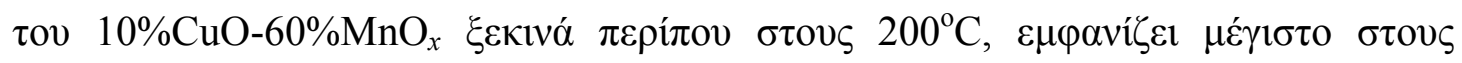

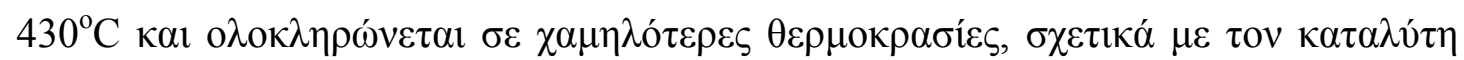
$\left.60 \% \mathrm{MnO}_{x} / \mathrm{Al}_{2} \mathrm{O}_{3}\left(\mathrm{~T}>600^{\circ} \mathrm{C}\right)\right)$. 

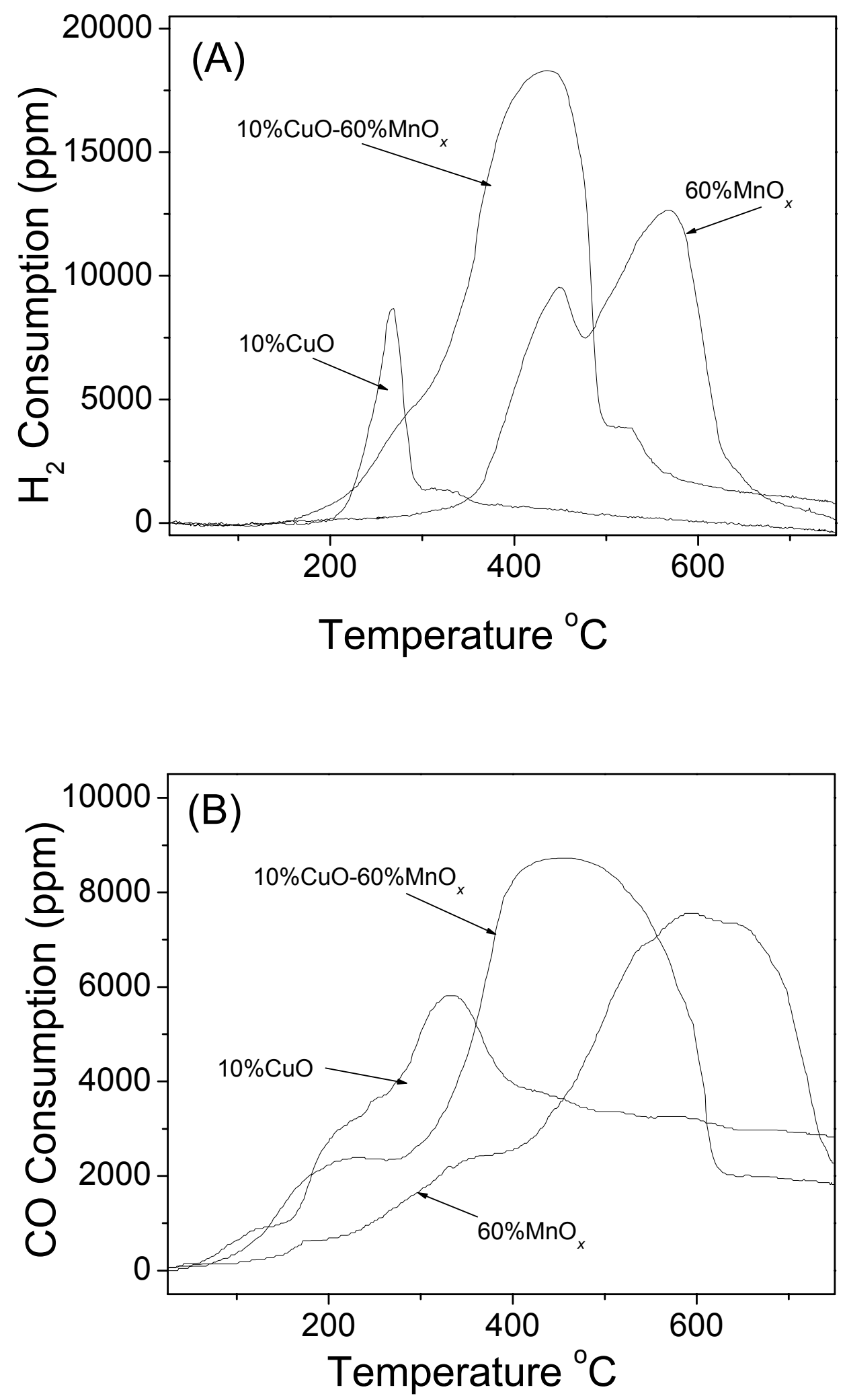

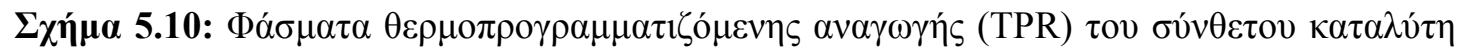

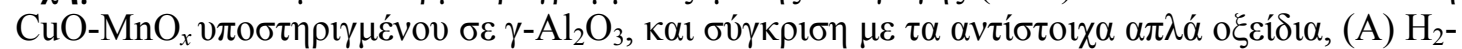
TPR (B) CO-TPR. 


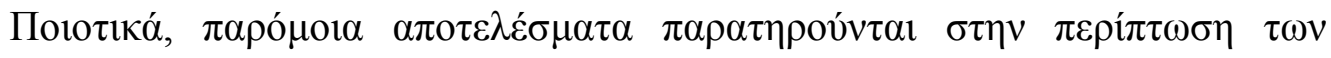

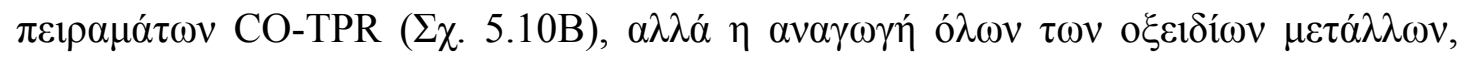

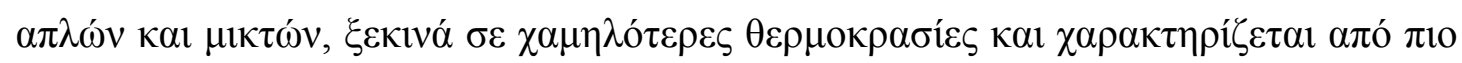

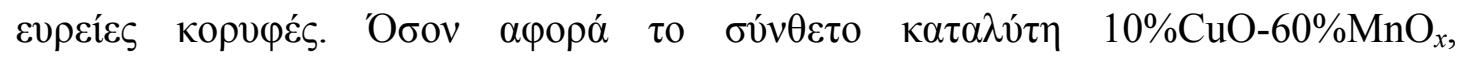

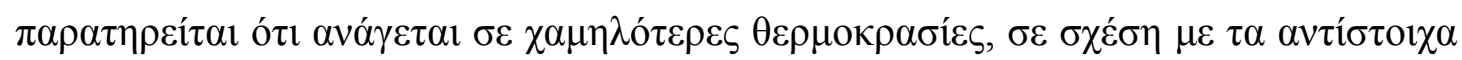

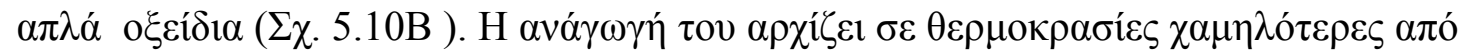

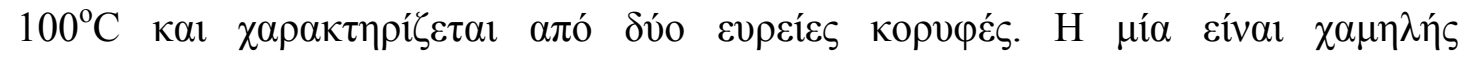

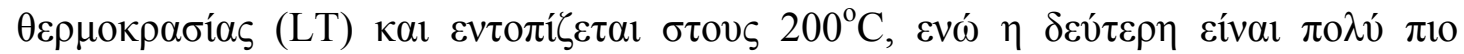

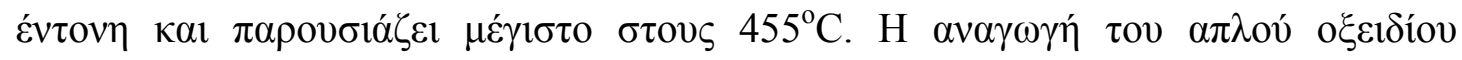

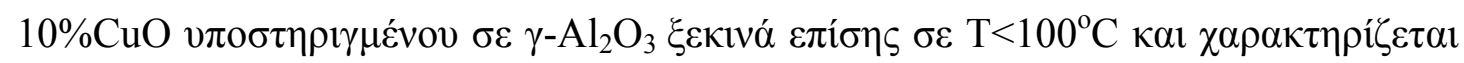

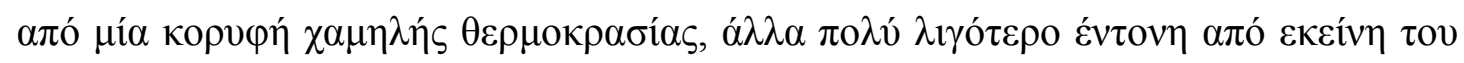

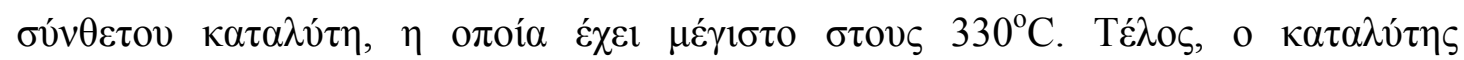

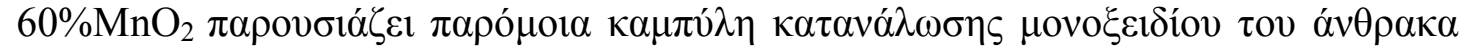

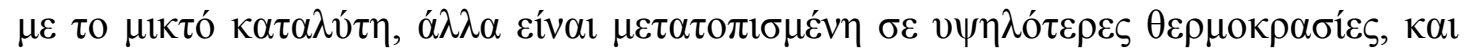

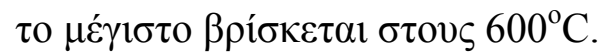

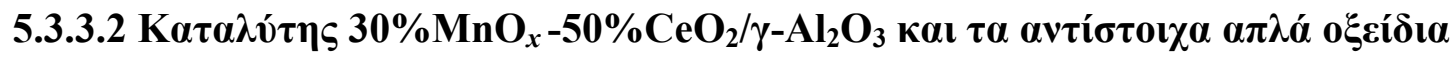

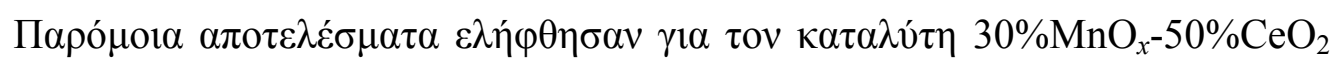

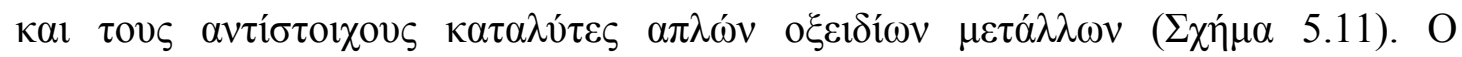

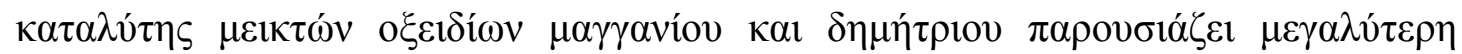

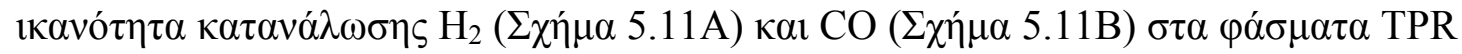

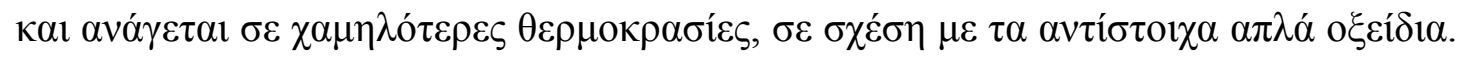

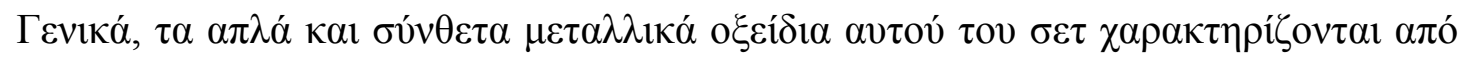

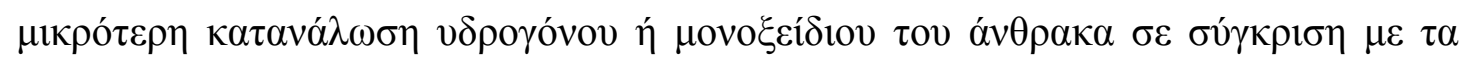

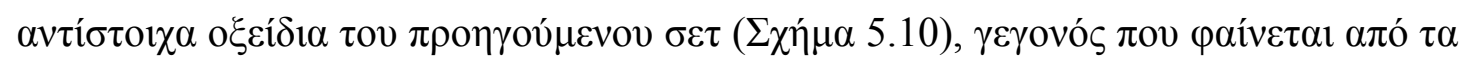

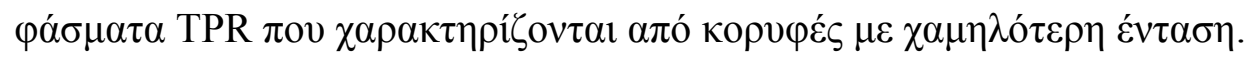

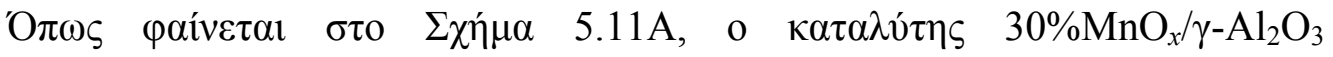

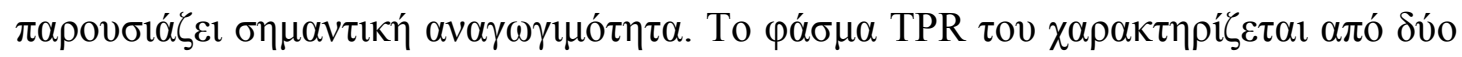

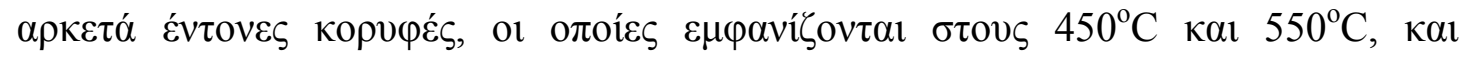

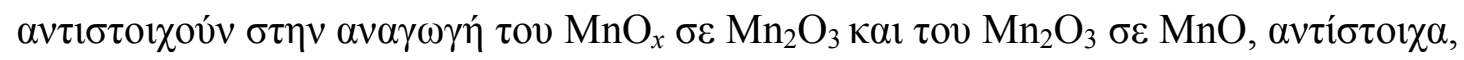

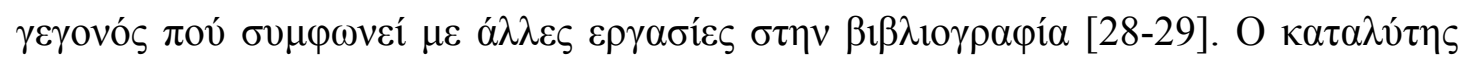
$50 \% \mathrm{CeO}_{2} / \gamma-\mathrm{Al}_{2} \mathrm{O}_{3}$, о олоíos $\pi \alpha \rho o v \sigma i \alpha ́ \zeta \varepsilon 1 ~ \tau \eta ~ \chi \alpha \mu \eta \lambda o ́ \tau \varepsilon \rho \eta ~ \kappa \alpha \tau \alpha \lambda v \tau 1 \kappa \eta ́ ~ \varepsilon v \varepsilon \rho \gamma o ́ \tau \eta \tau \alpha ~ \sigma \varepsilon$

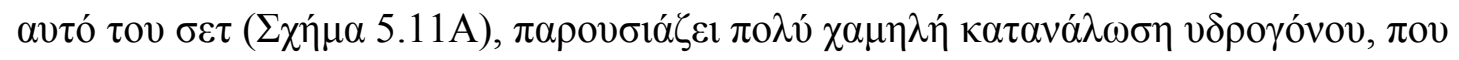



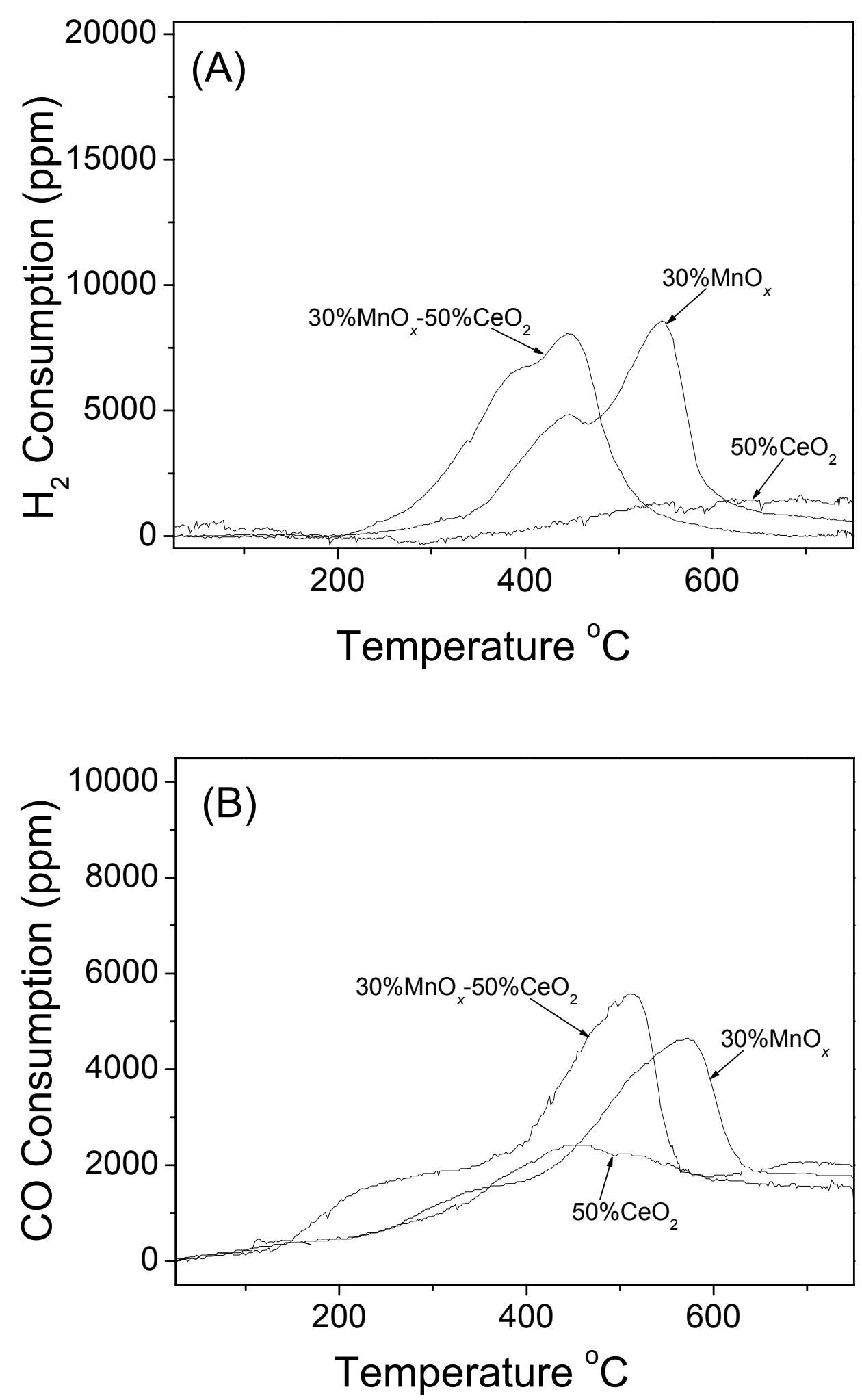

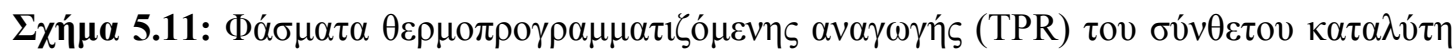

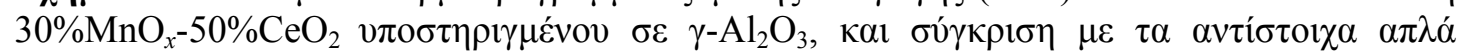
o $\xi \varepsilon i \delta 1 \alpha,(\mathrm{A}) \mathrm{H}_{2}$-TPR (B) CO-TPR. 


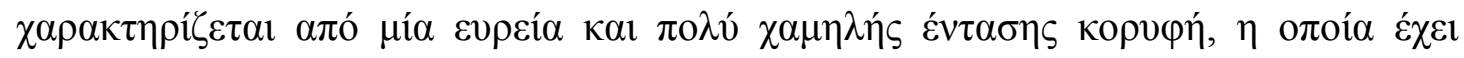

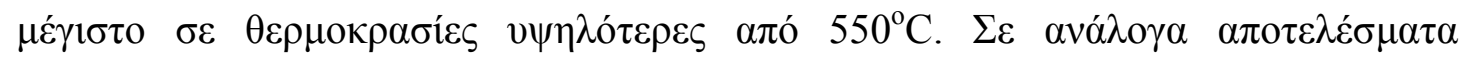

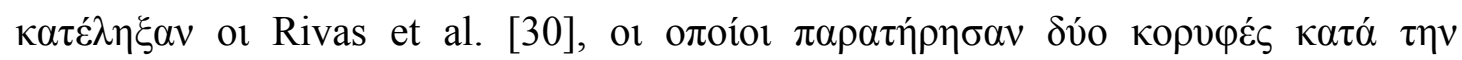

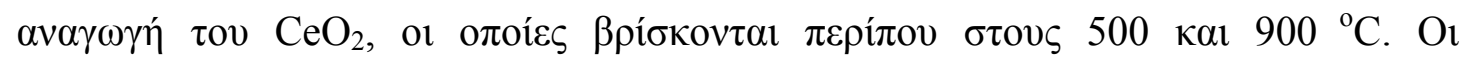

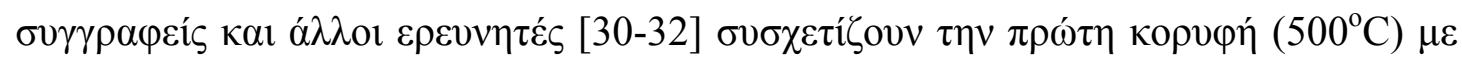

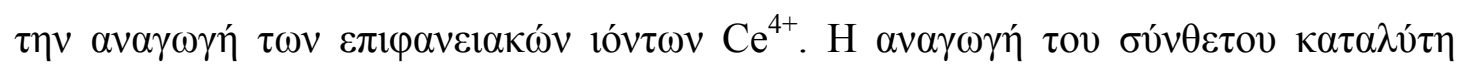

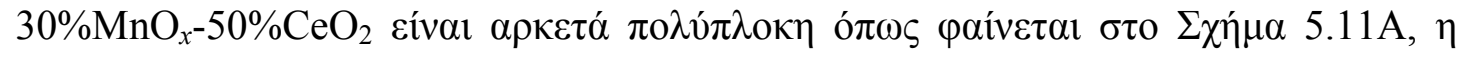

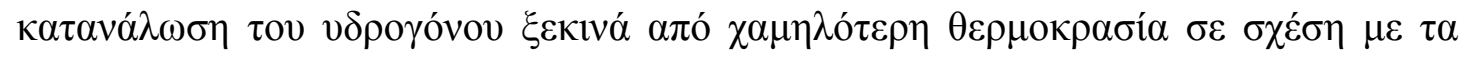

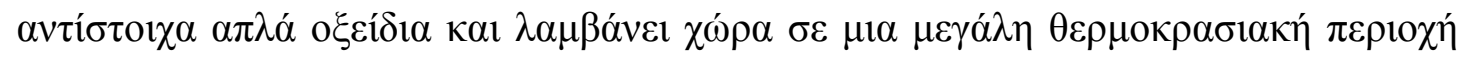

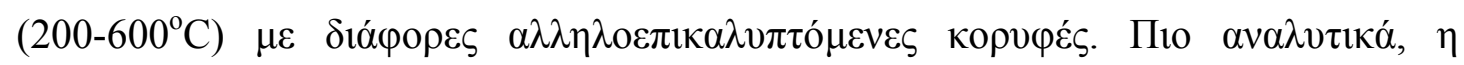

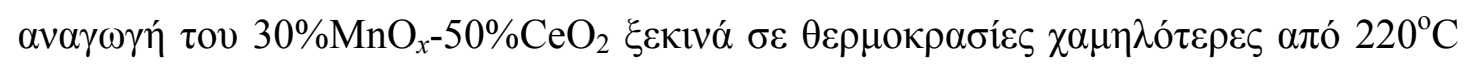

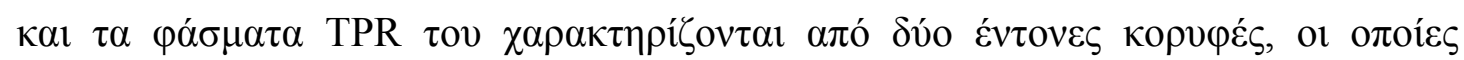

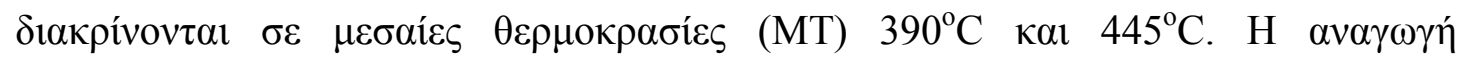

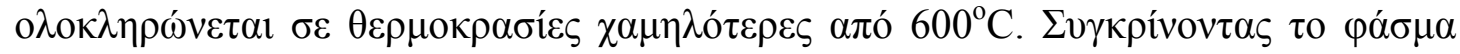

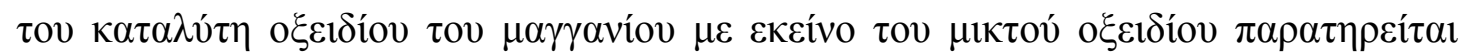

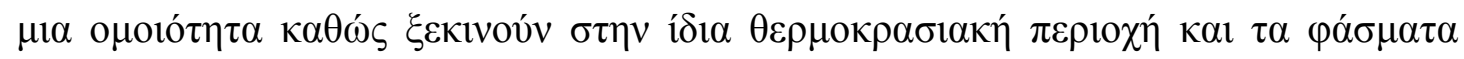

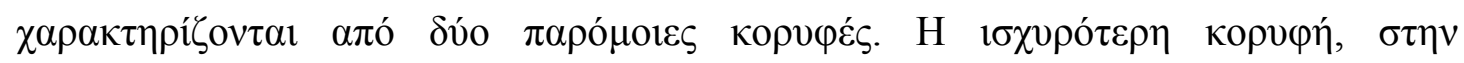

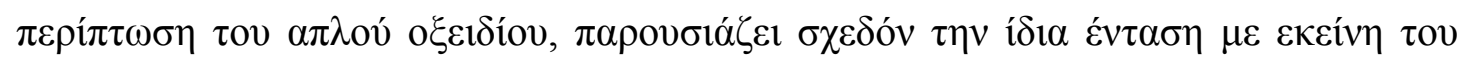

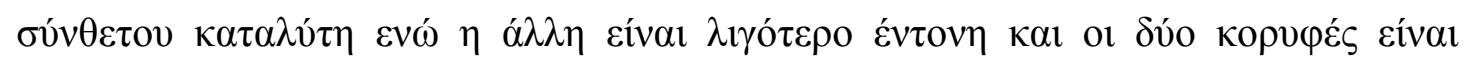

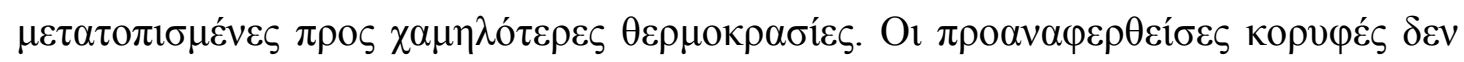

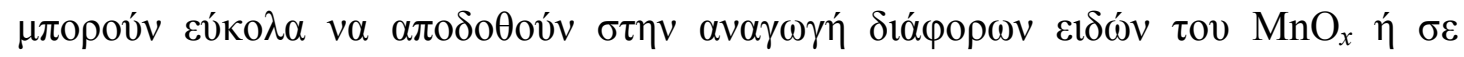

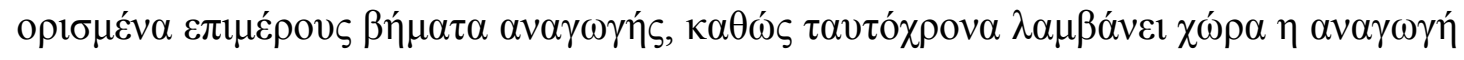

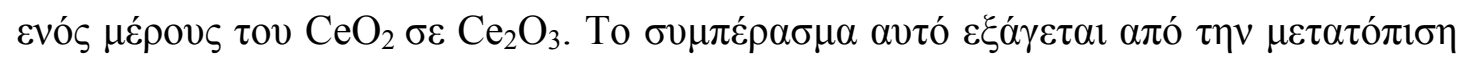

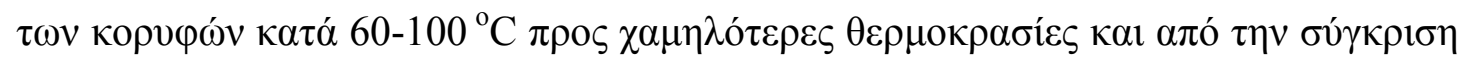

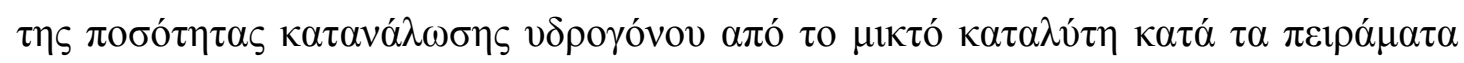

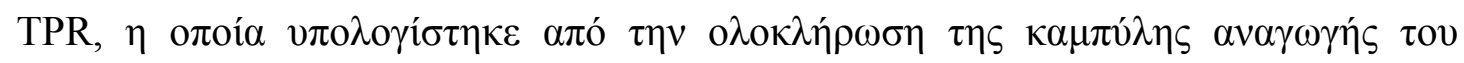

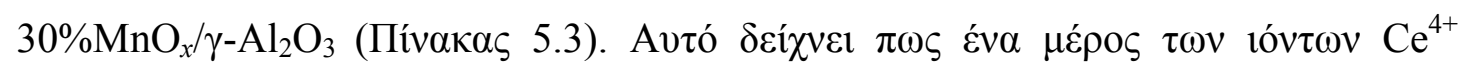

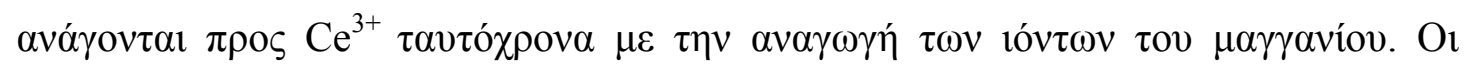

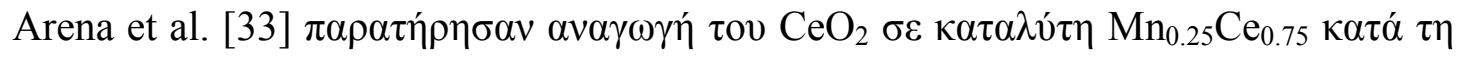

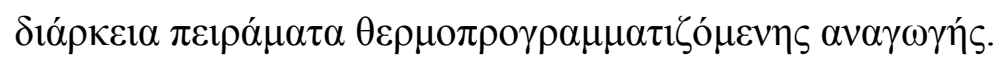




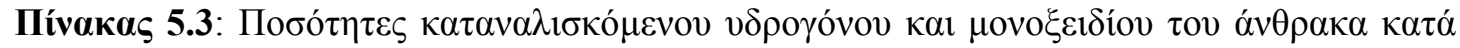

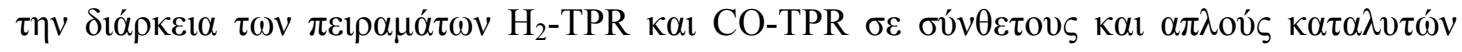

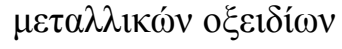

\begin{tabular}{|c|c|c|}
\hline $\mathrm{K} \alpha \tau \alpha \lambda v ́ \tau\rceil \varsigma$ & mmol $\mathrm{H}_{2} / \mathrm{g}$ cat. & mmol CO/g cat. \\
\hline $10 \% \mathrm{CuO}-60 \% \mathrm{MnO}_{x} / \mathrm{Al}_{2} \mathrm{O}_{3}$ & 7.1 & 6.3 \\
\hline $60 \% \mathrm{MnO}_{x} / \mathrm{Al}_{2} \mathrm{O}_{3}$ & 5 & 5.9 \\
\hline $10 \% \mathrm{CuO} / \mathrm{Al}_{2} \mathrm{O}_{3}$ & 1.2 & 4.9 \\
\hline $30 \% \mathrm{MnO}_{x}-50 \% \mathrm{CeO}_{2} / \mathrm{Al}_{2} \mathrm{O}_{3}$ & 3.2 & 3.4 \\
\hline $30 \% \mathrm{MnO}_{x} / \mathrm{Al}_{2} \mathrm{O}_{3}$ & 2.8 & 3 \\
\hline $50 \% \mathrm{CeO}_{2} / \mathrm{Al}_{2} \mathrm{O}_{3}$ & 0.9 & 2.4 \\
\hline $15 \% \mathrm{CuO}-75 \% \mathrm{CeO}_{2} / \mathrm{Al}_{2} \mathrm{O}_{3}$ & 3.6 & 3.7 \\
\hline $15 \% \mathrm{CuO} / \mathrm{Al}_{2} \mathrm{O}_{3}$ & 3 & 2.6 \\
\hline $75 \% \mathrm{CeO}_{2} / \mathrm{Al}_{2} \mathrm{O}_{3}$ & 0.8 & 1.9 \\
\hline
\end{tabular}

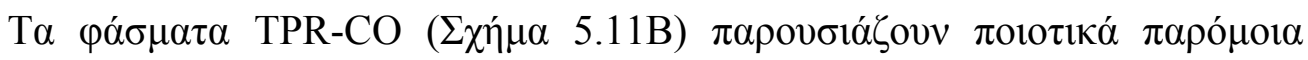

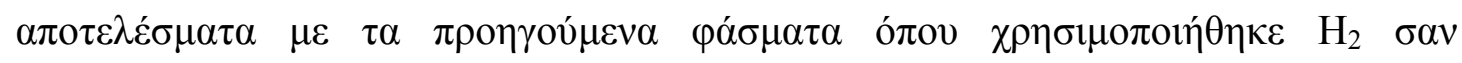

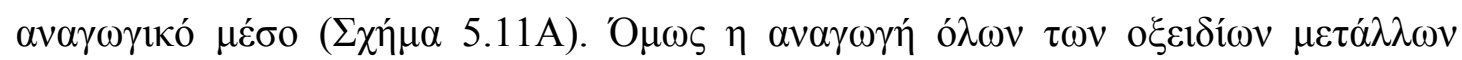

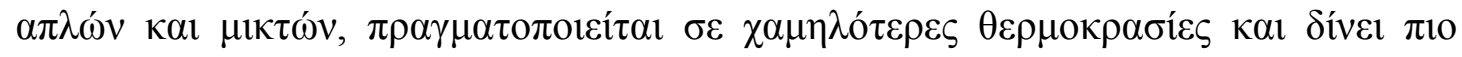

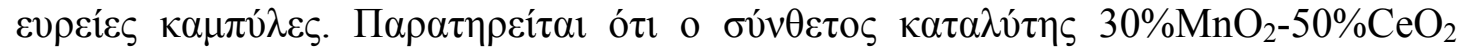

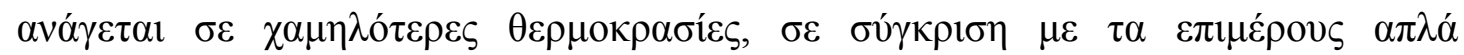

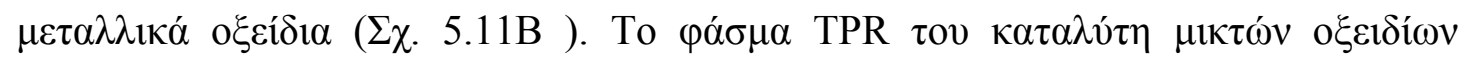

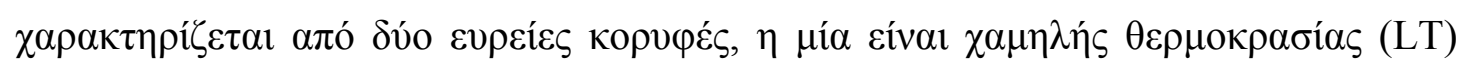

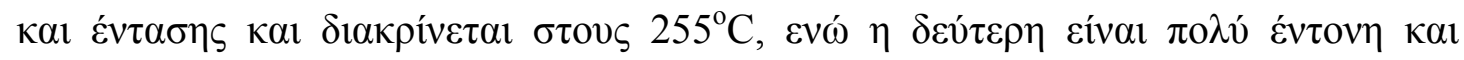

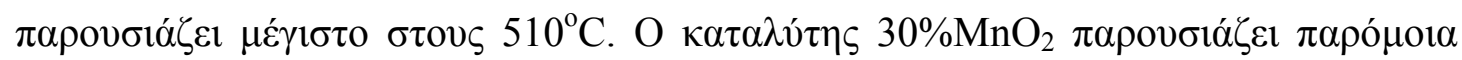

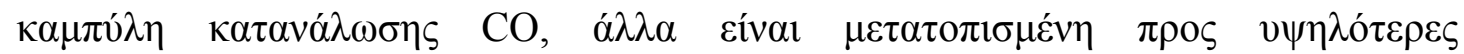

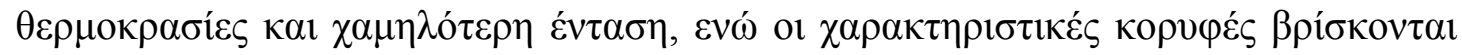

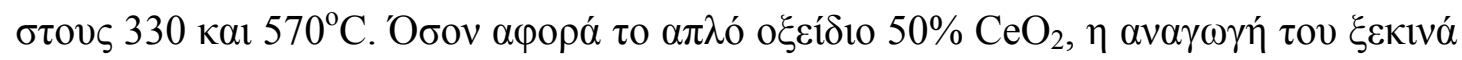

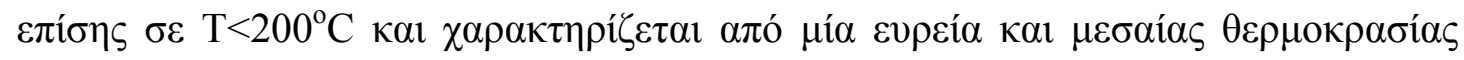

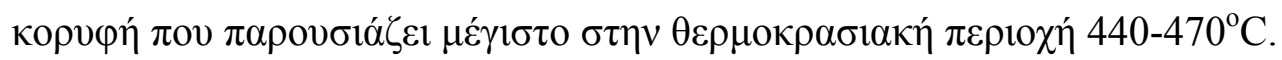

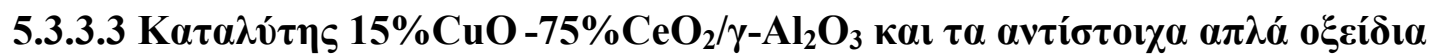

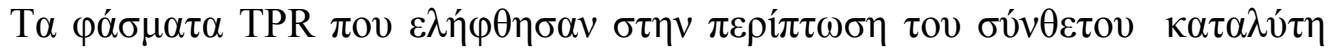

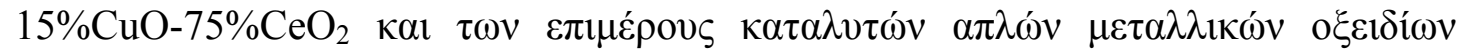




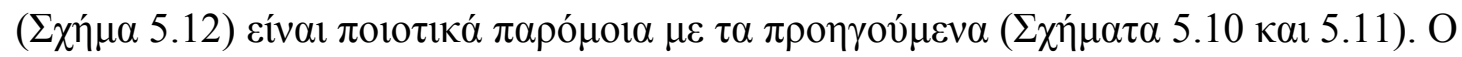

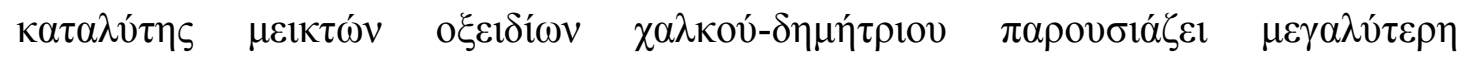

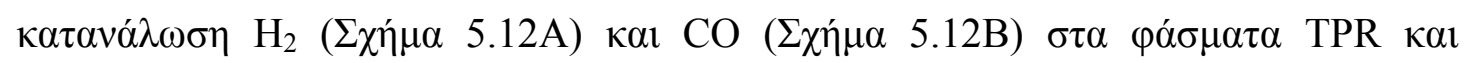

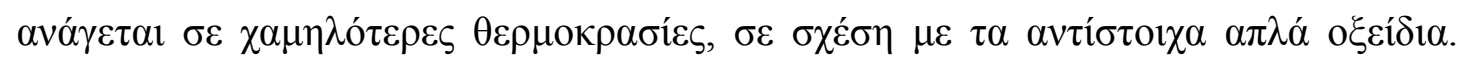

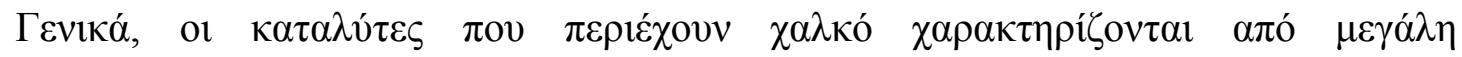

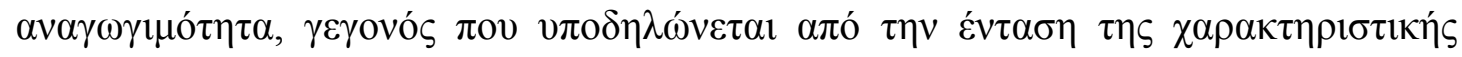

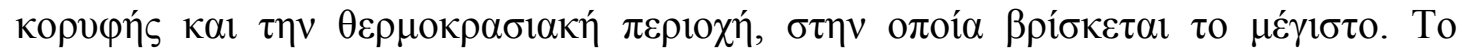

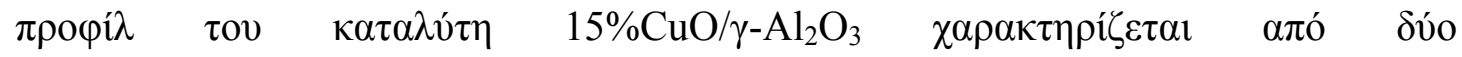
$\alpha \lambda \lambda \eta \lambda$

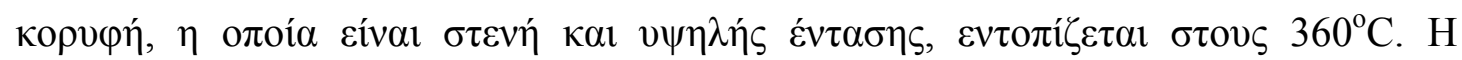

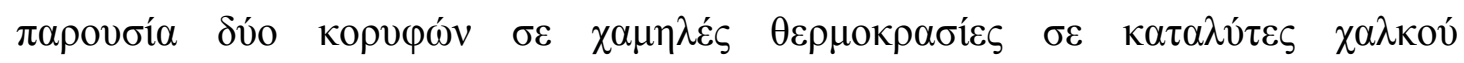

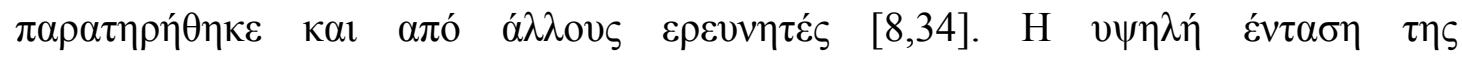

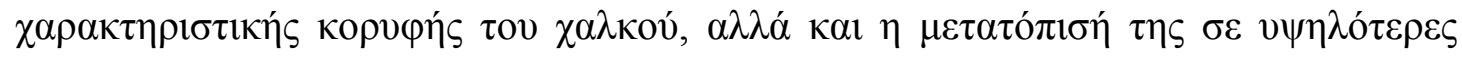

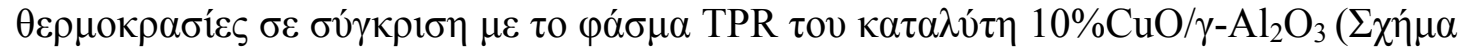

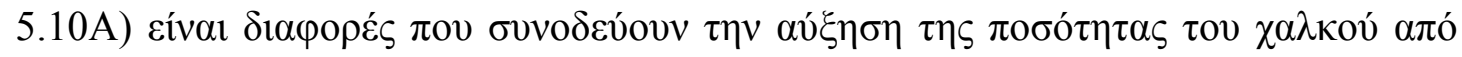

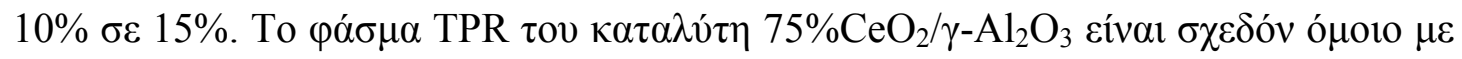

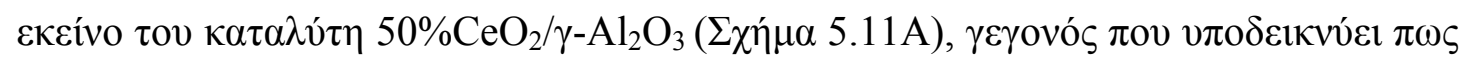

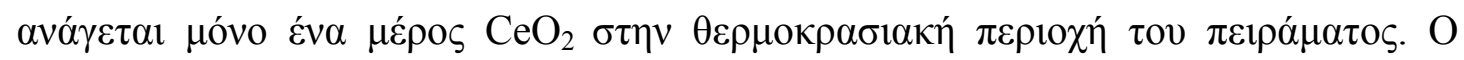

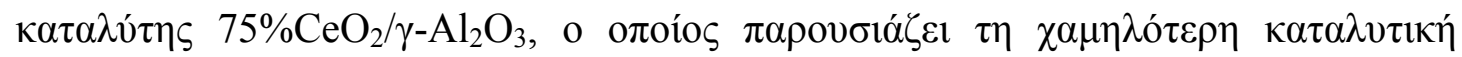

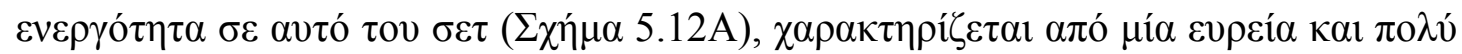

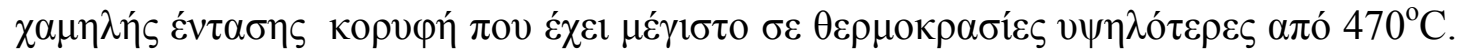

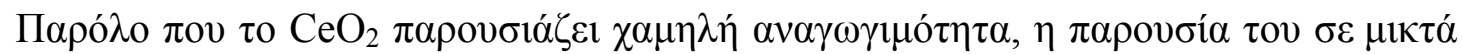

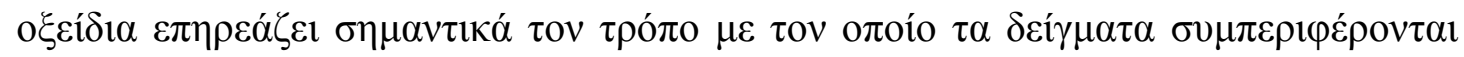

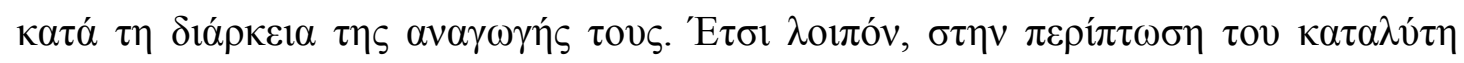

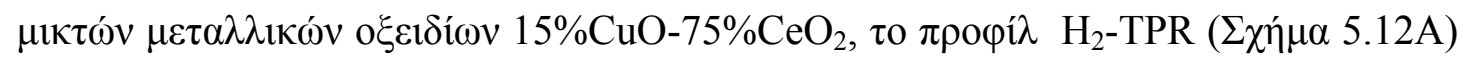

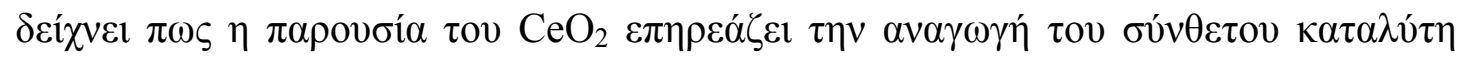

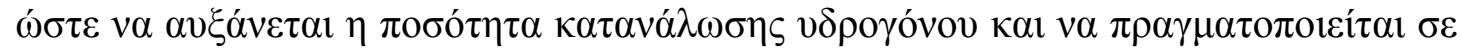

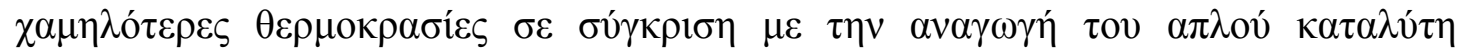

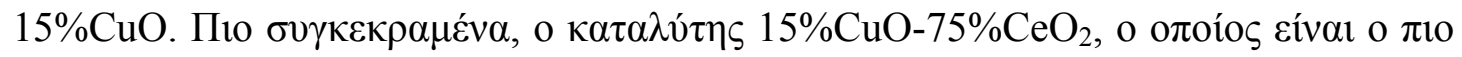

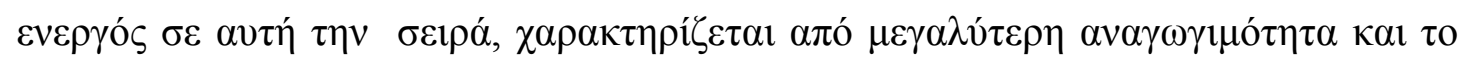

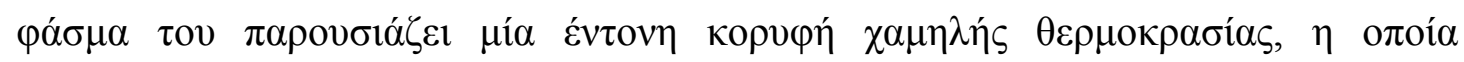

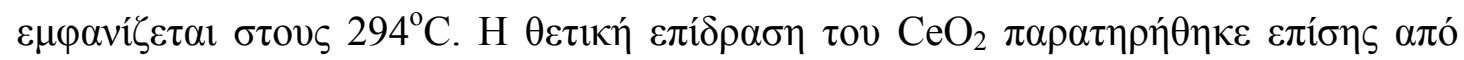

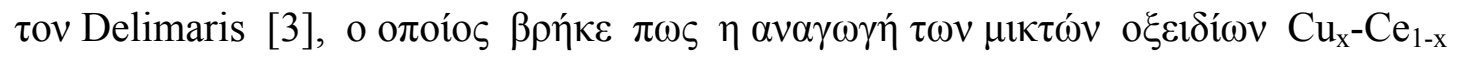



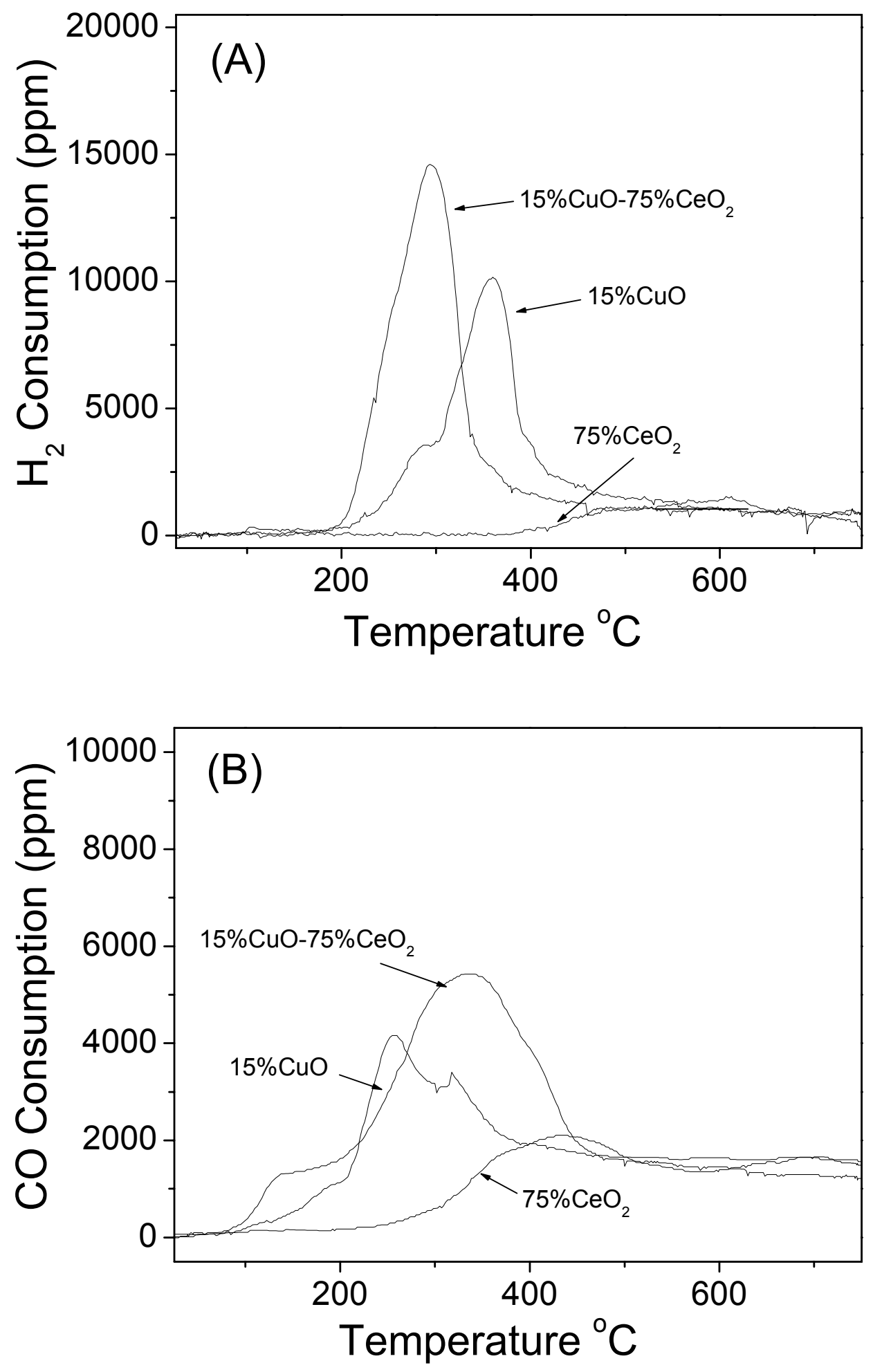

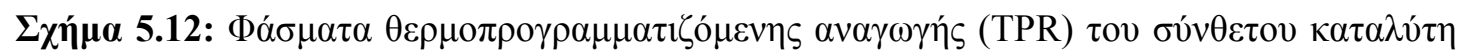

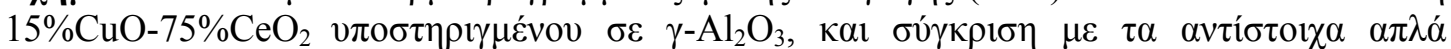
o $\xi \varepsilon i \delta i \alpha$, (A) $\mathrm{H}_{2}$-TPR (B) CO-TPR. 


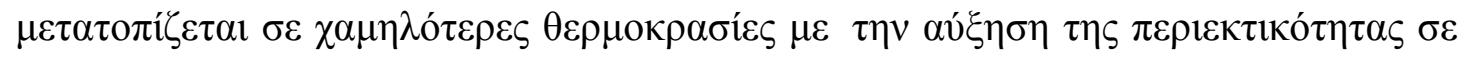
Ce.

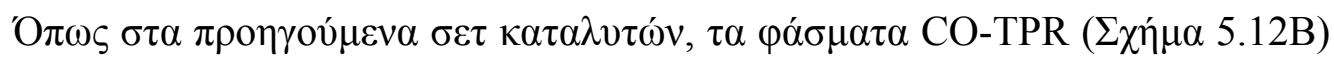

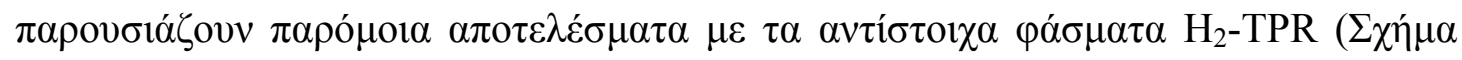

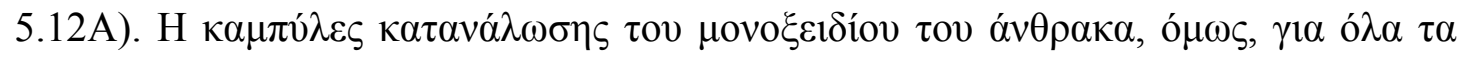

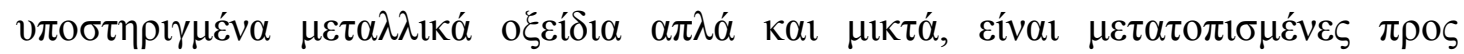

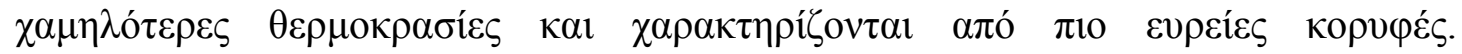

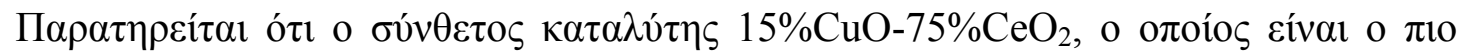

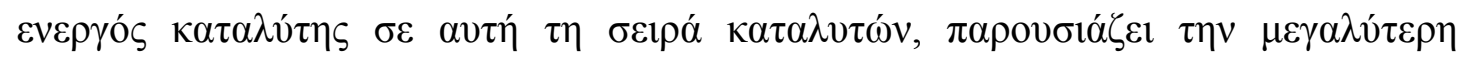

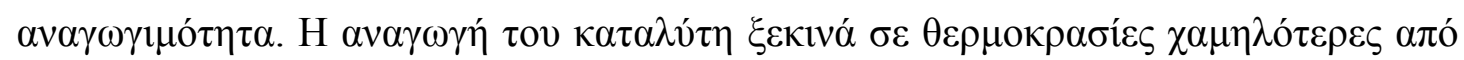

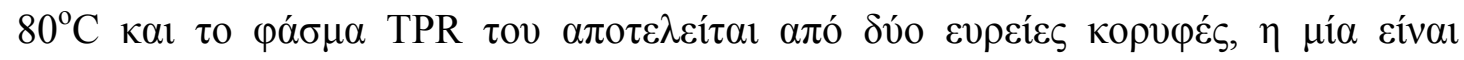

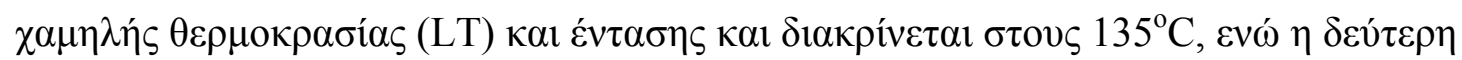

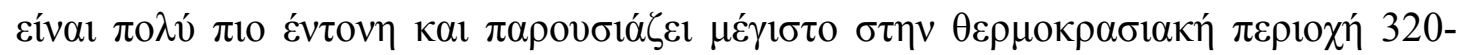

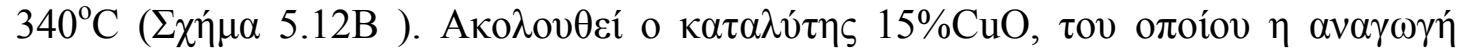

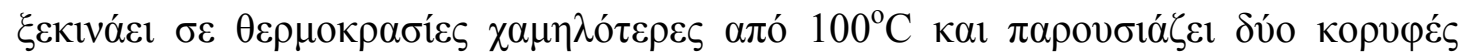

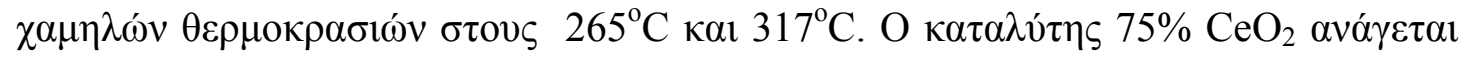

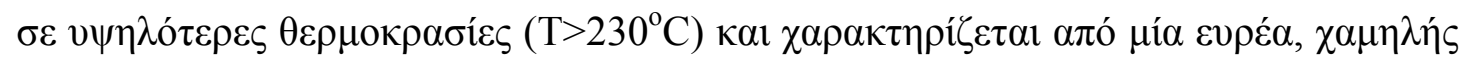

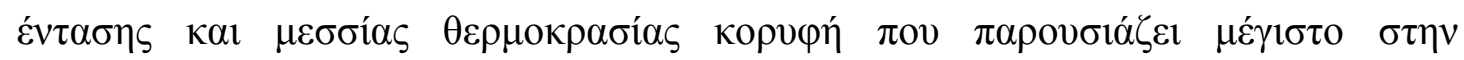

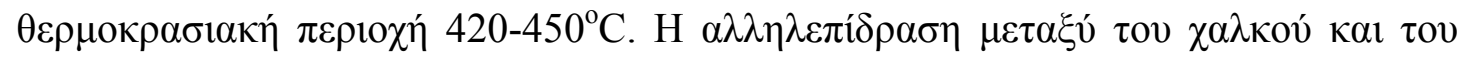

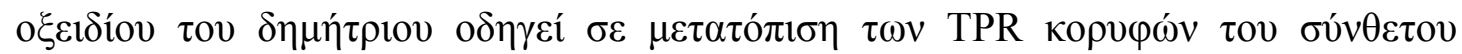

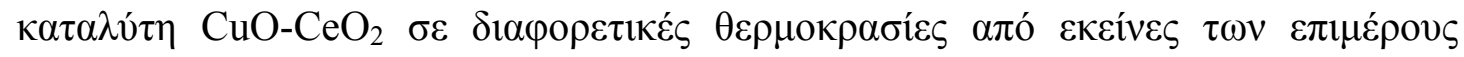

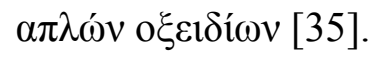

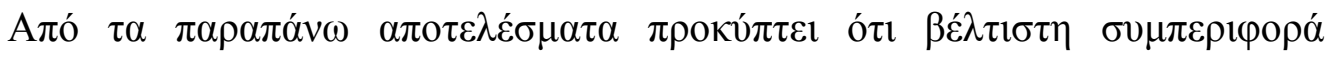

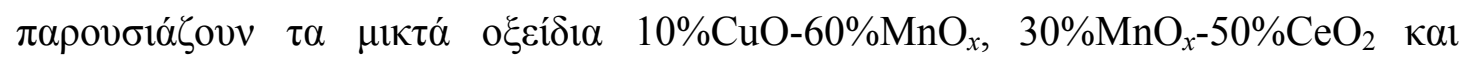

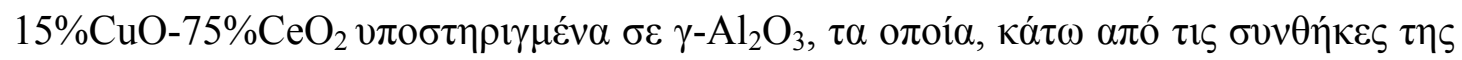

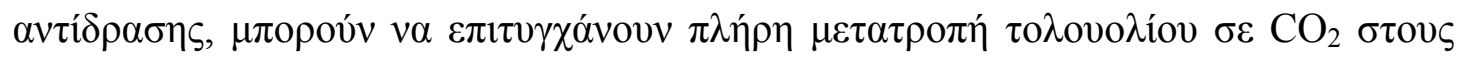

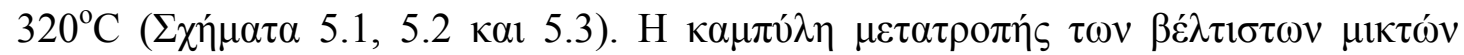

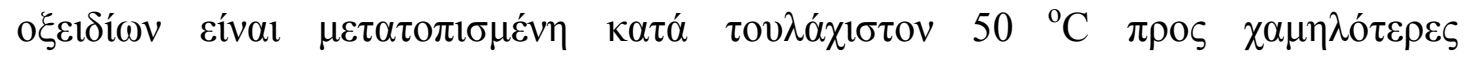

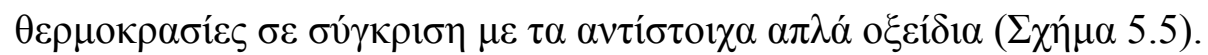

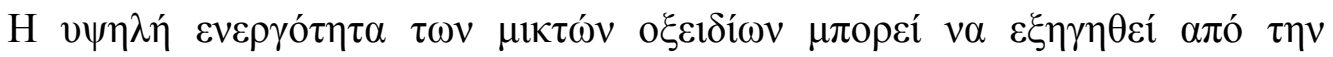

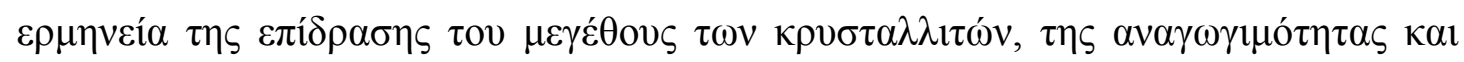

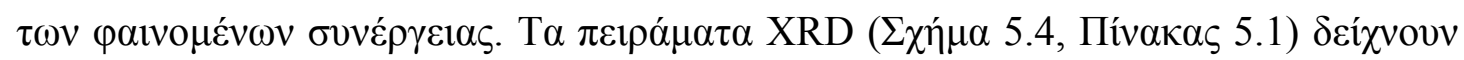

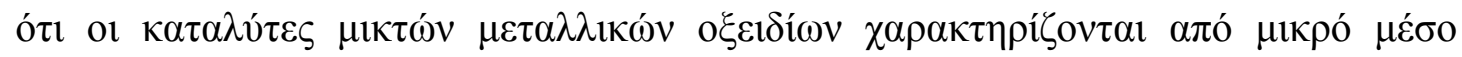

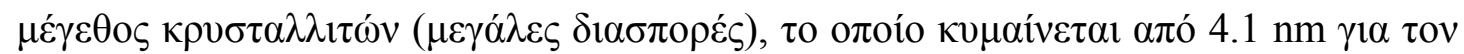




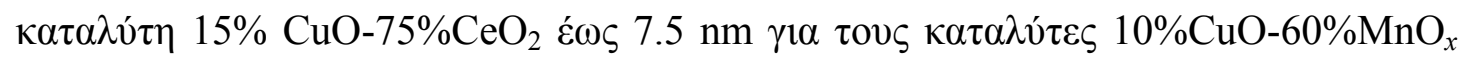

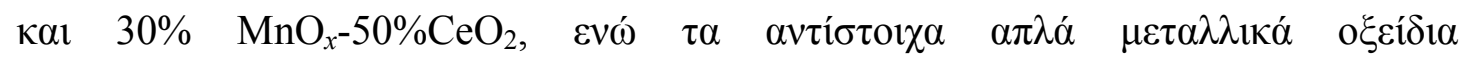

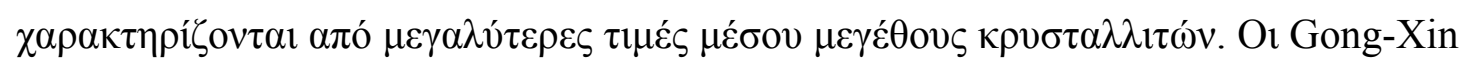

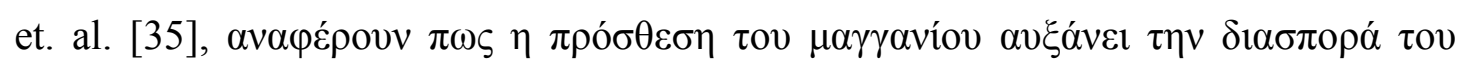

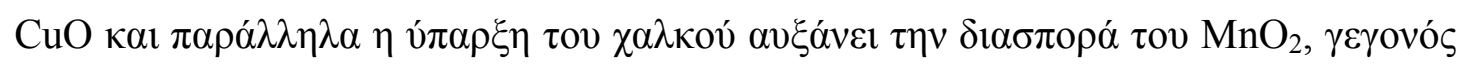

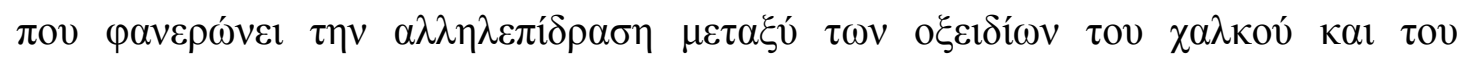

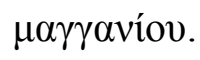

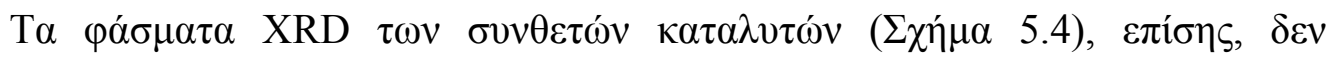

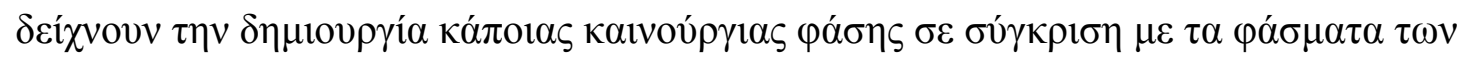

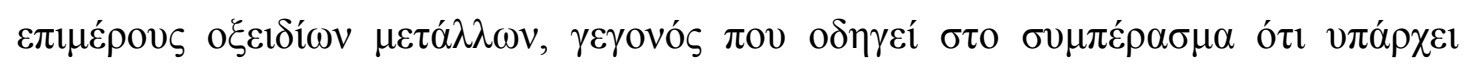

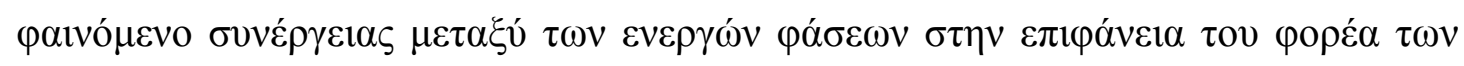

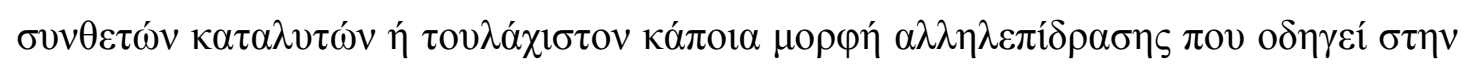

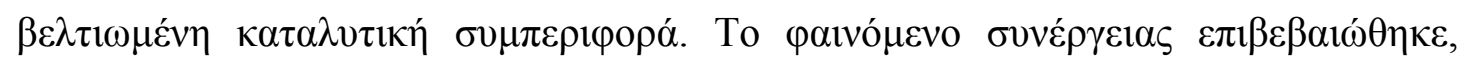

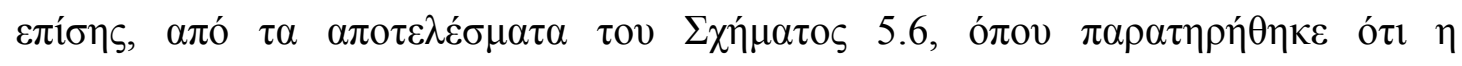

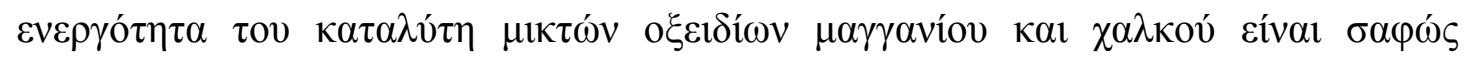

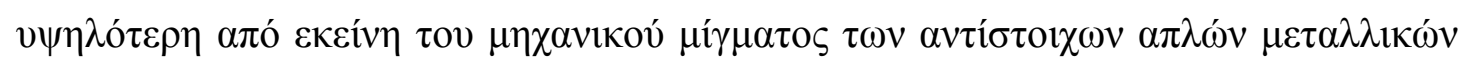

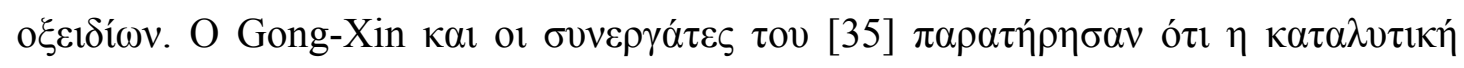

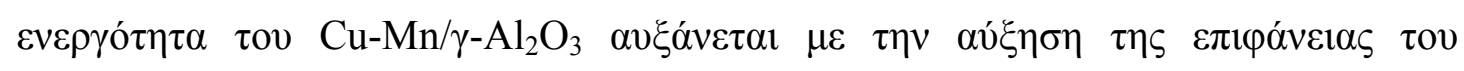

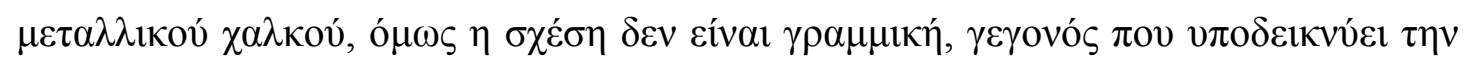

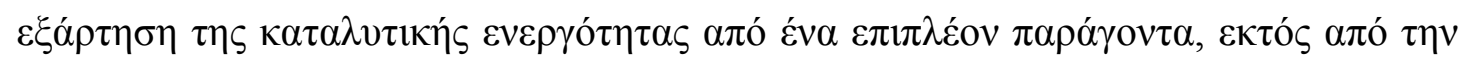

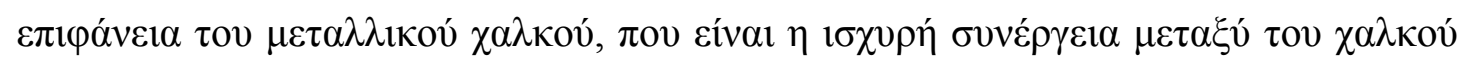

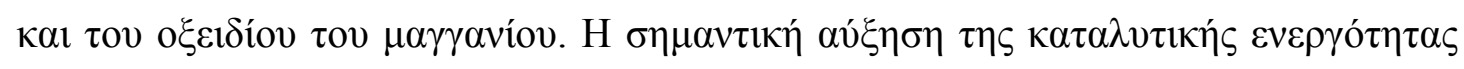

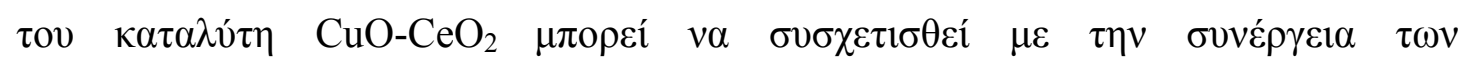

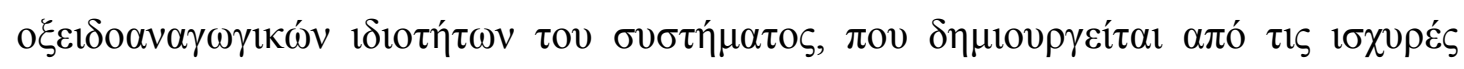

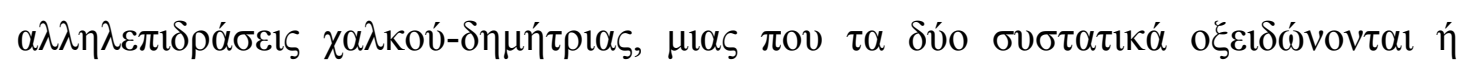

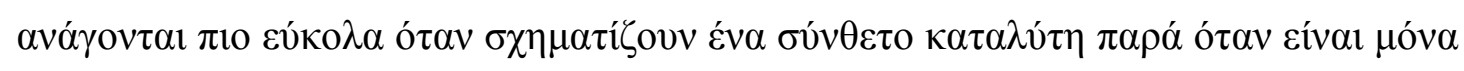

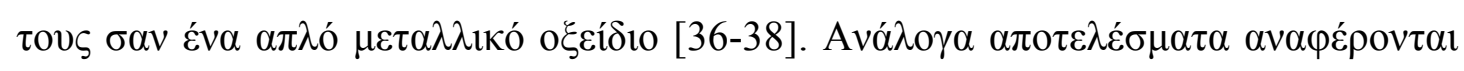

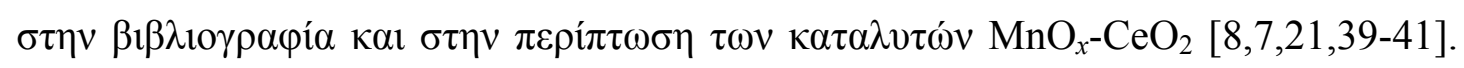

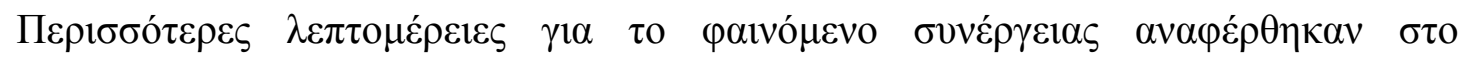

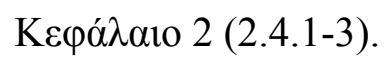

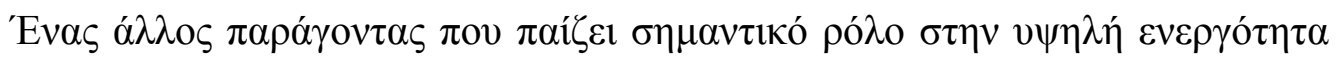

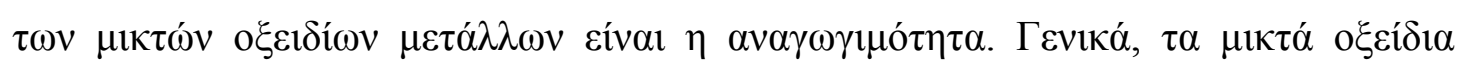

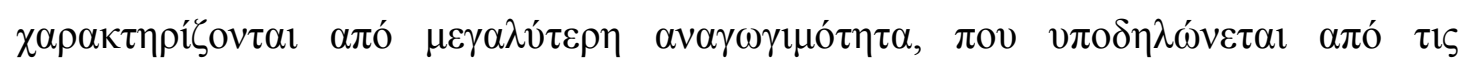

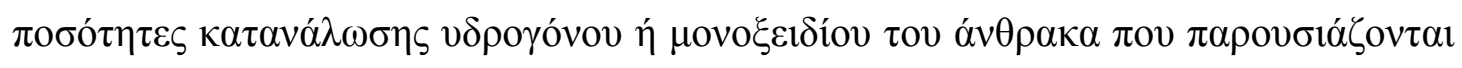




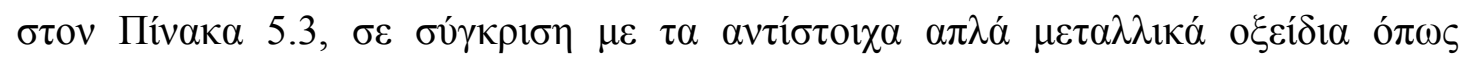

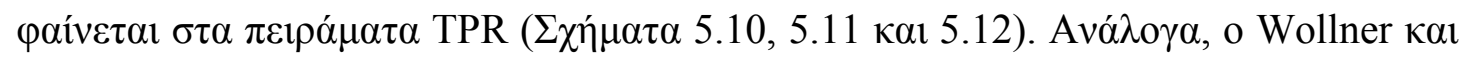

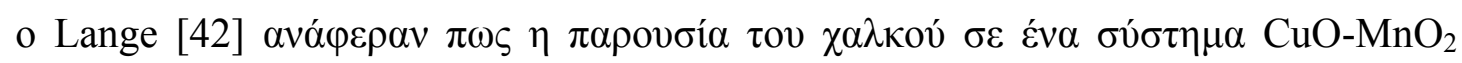

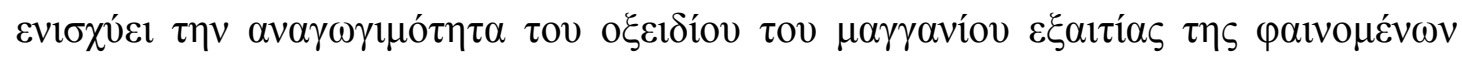

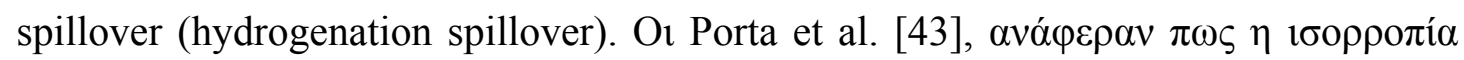

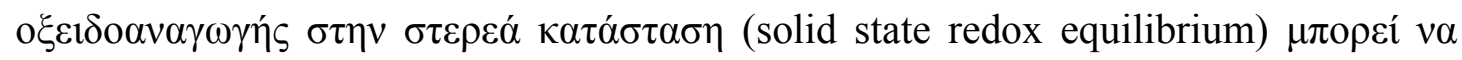

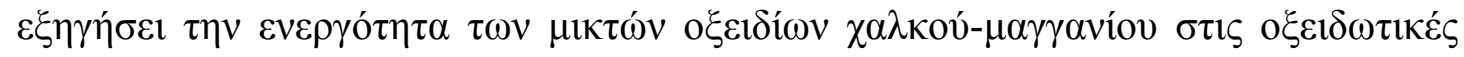

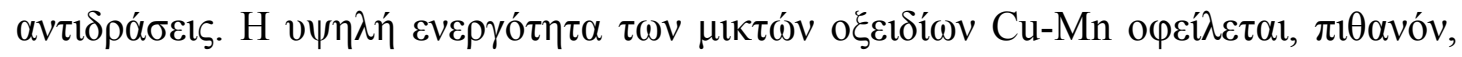

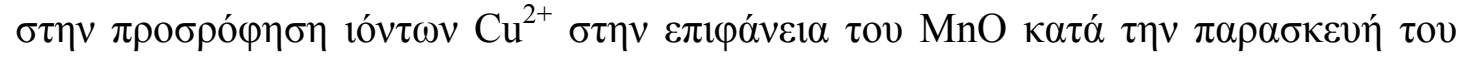

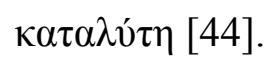

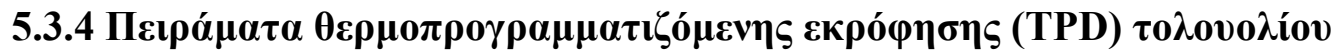

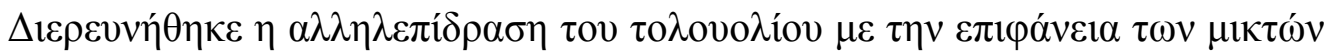

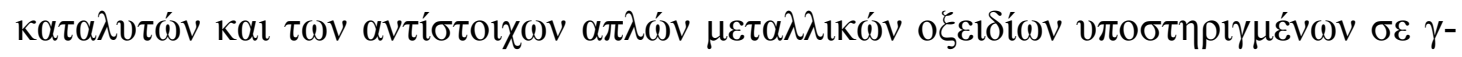

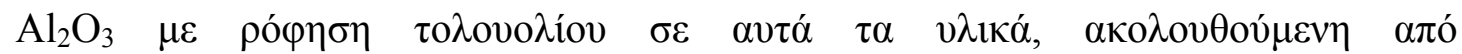

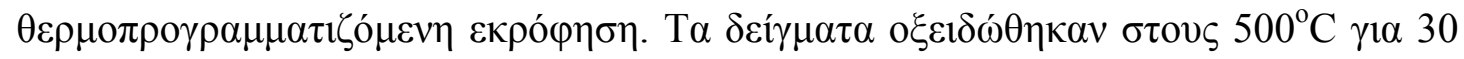

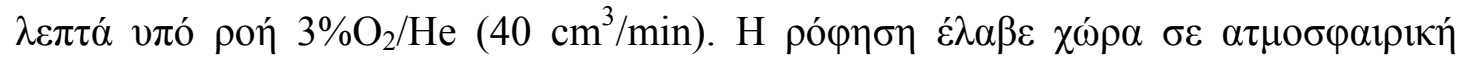

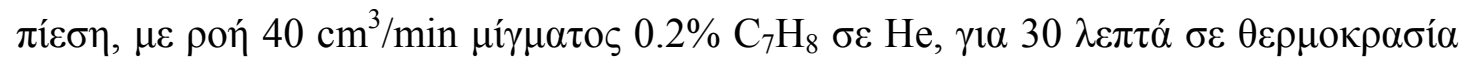

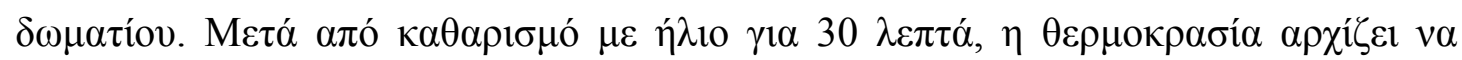

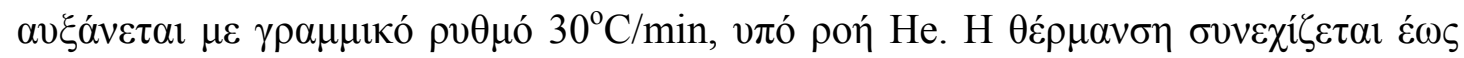

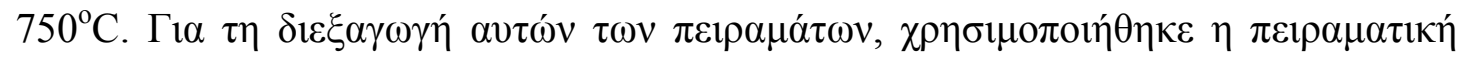

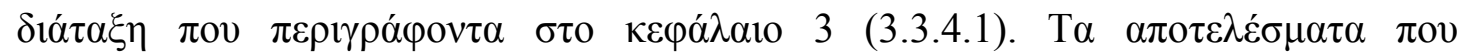

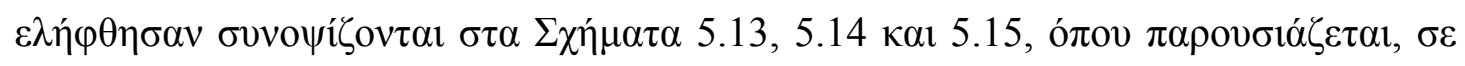

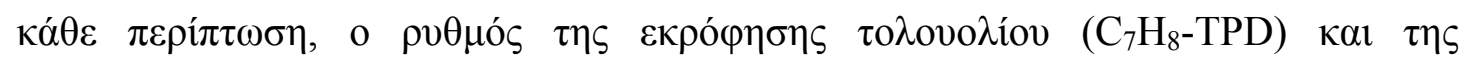

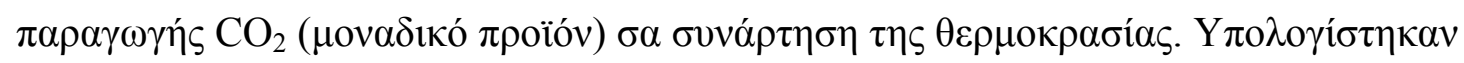

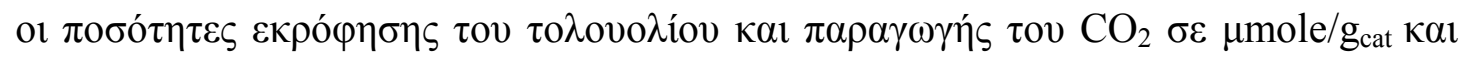

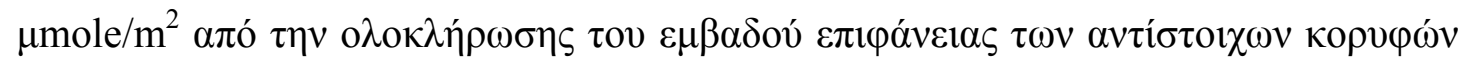

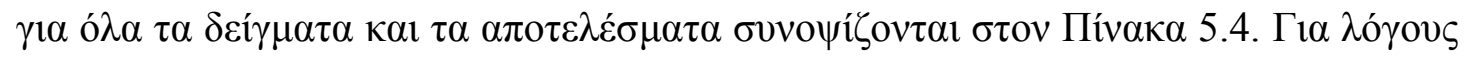

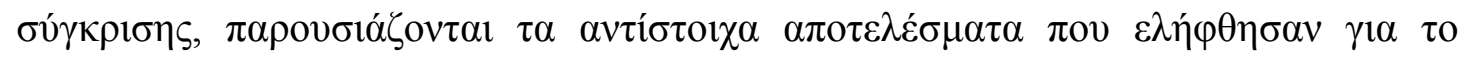
форе́ $\alpha \gamma-\mathrm{Al}_{2} \mathrm{O}_{3}$. 


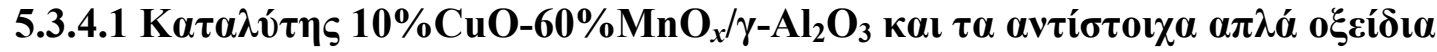

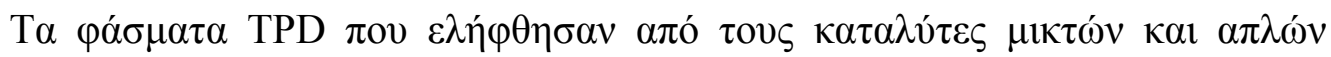

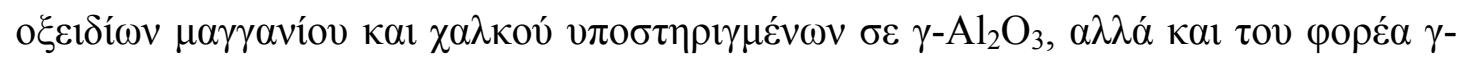

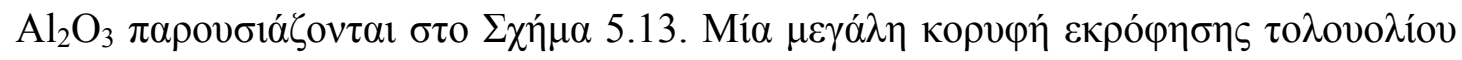

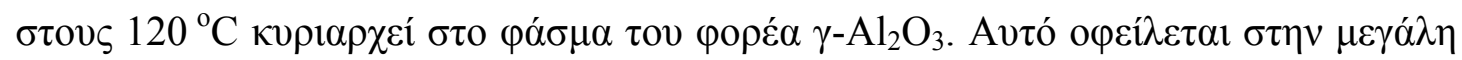

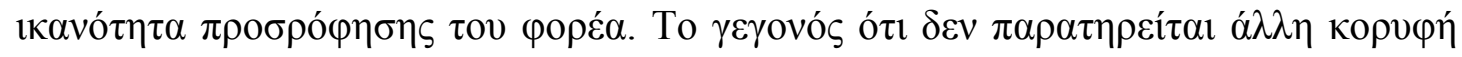

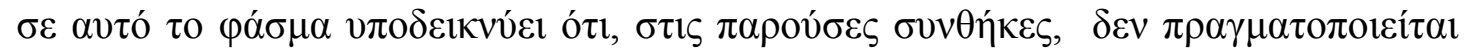

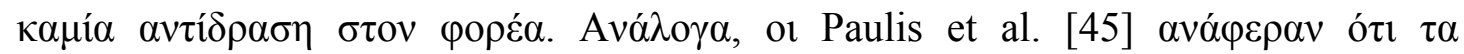

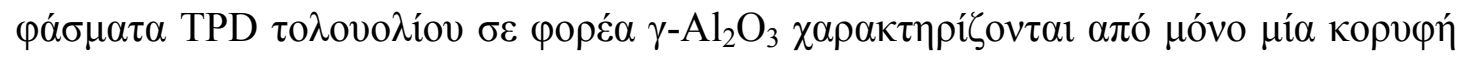

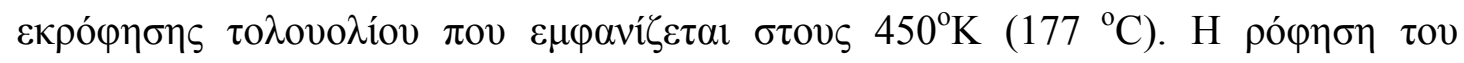

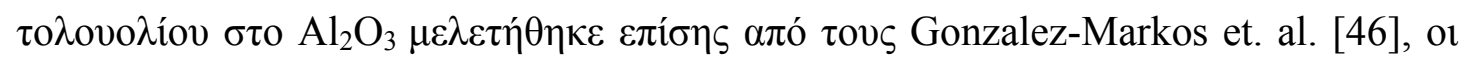

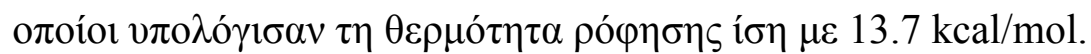

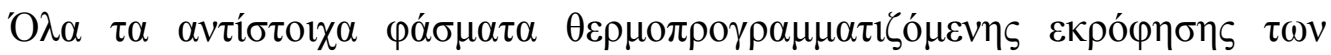

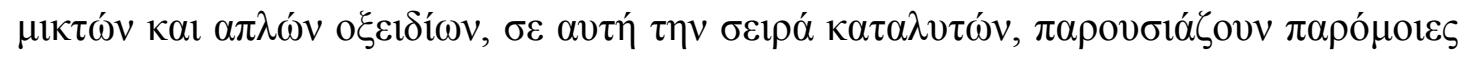

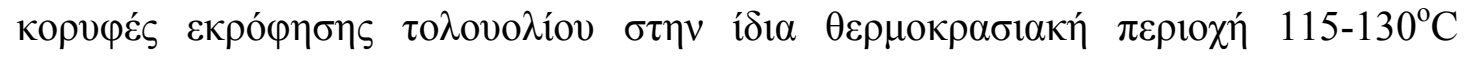

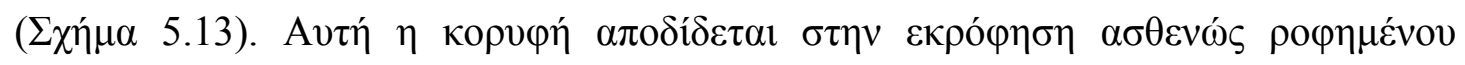

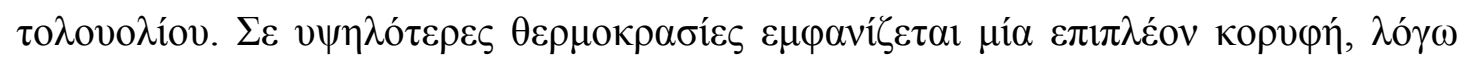

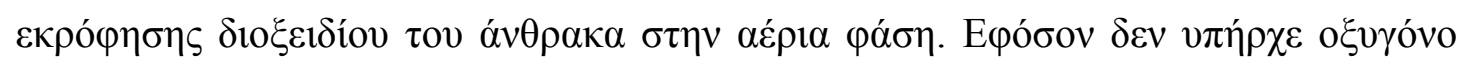

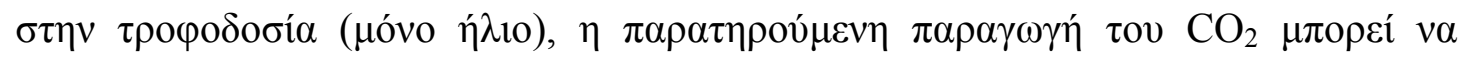

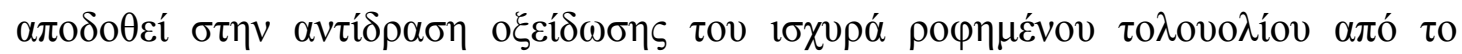

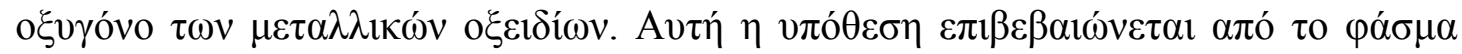

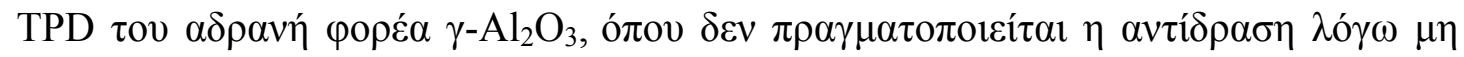

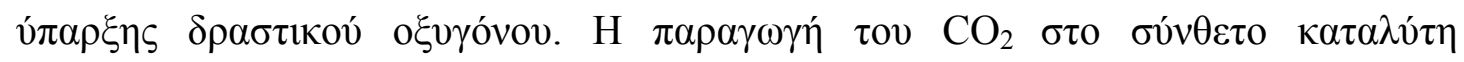

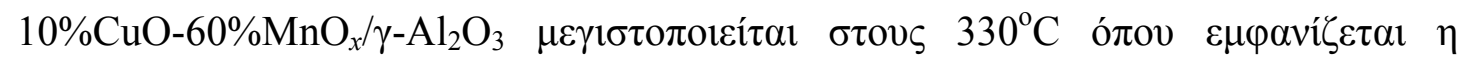

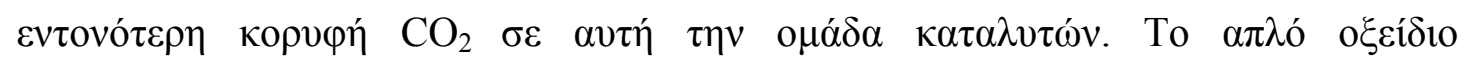

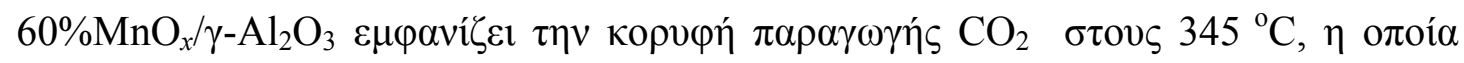

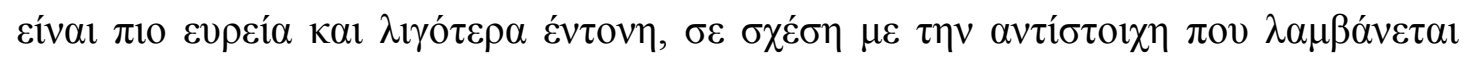

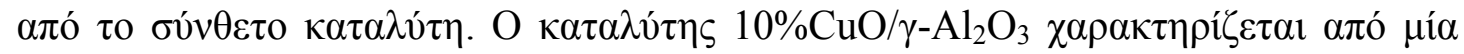

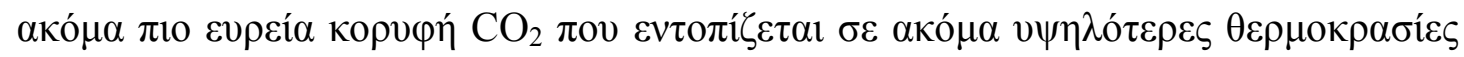

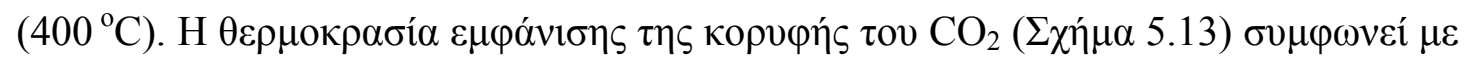

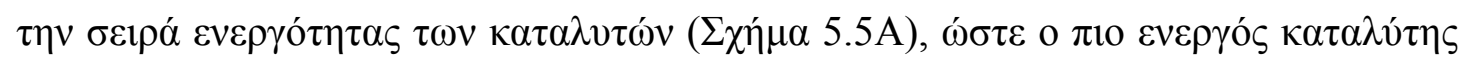

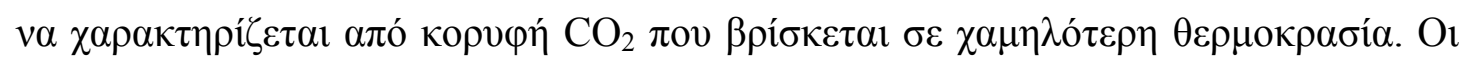

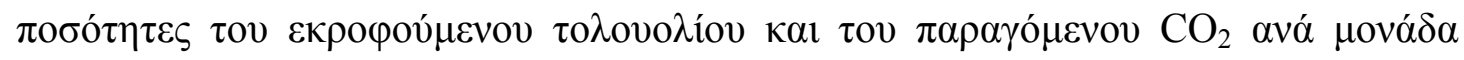

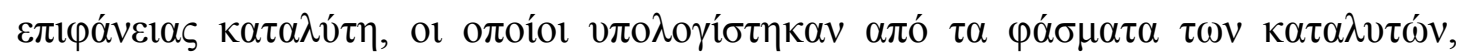




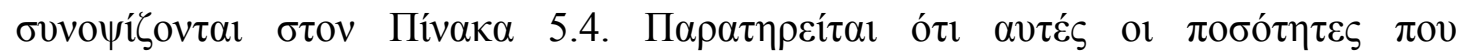

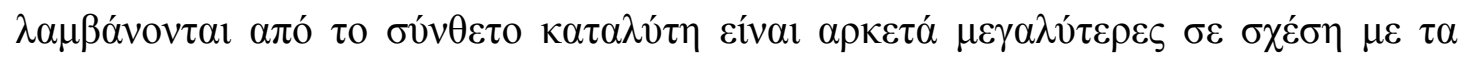

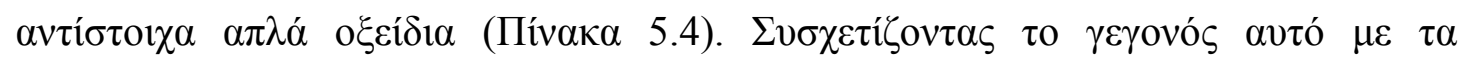

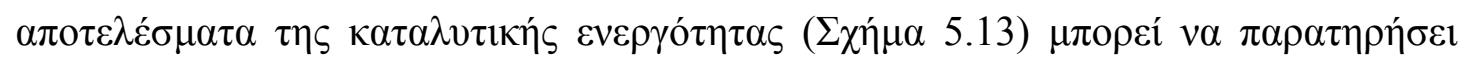

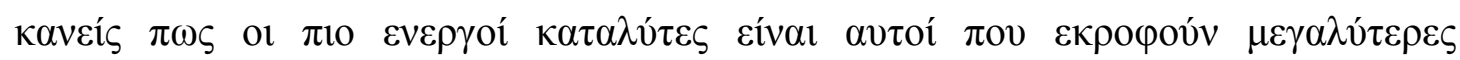

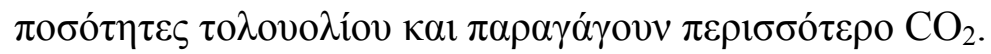

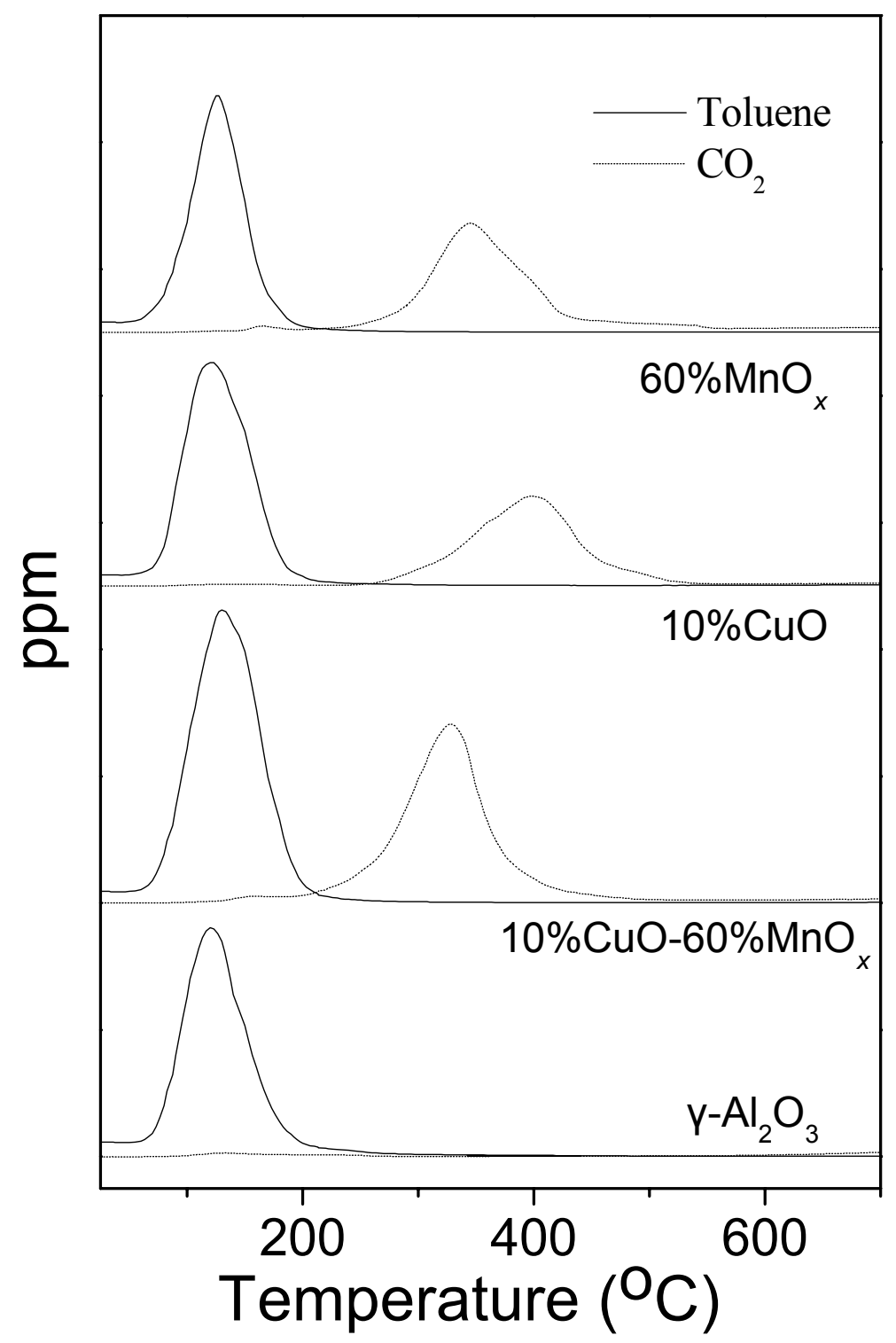

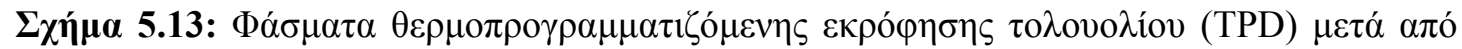

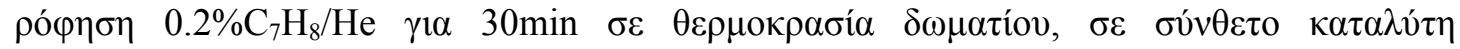

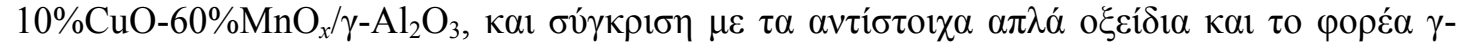
$\mathrm{Al}_{2} \mathrm{O}_{3}$. 


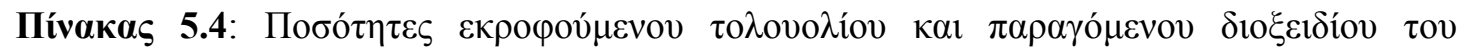

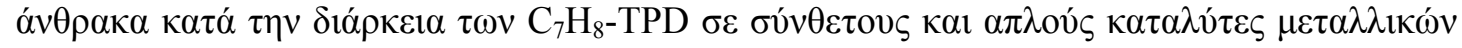
o $\xi \varepsilon 1 \delta i ́ \omega v$

\begin{tabular}{|c|c|c|c|c|}
\hline \multirow{2}{*}{ 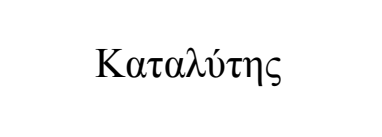 } & \multicolumn{2}{|c|}{ Toluene } & \multicolumn{2}{|c|}{$\mathrm{CO}_{2}$} \\
\hline & $\mu \mathrm{mol} / \mathrm{g}_{\mathrm{cat}}$ & $\mu \mathrm{mol} / \mathrm{m}^{2}$ & $\mu \mathrm{mol} / \mathrm{g}_{\mathrm{cat}}$ & $\mu \mathrm{mol} / \mathrm{m}^{2}$ \\
\hline$\gamma-\mathrm{Al}_{2} \mathrm{O}_{3}$ & 100.4 & 0.55 & 0 & 0 \\
\hline $10 \% \mathrm{CuO}-60 \% \mathrm{MnO}_{x}$ & 39.4 & 0.80 & 35.1 & 0.71 \\
\hline $10 \% \mathrm{CuO}$ & 88.18 & 0.58 & 63.4 & 0.42 \\
\hline $60 \% \mathrm{MnO}_{x}$ & 28 & 0.53 & 26.93 & 0.51 \\
\hline $30 \% \mathrm{MnO}_{x}-50 \% \mathrm{CeO}_{2}$ & 109.7 & 1.39 & 77.7 & 0.98 \\
\hline $30 \% \mathrm{MnO}_{x}$ & 40 & 0.33 & 16.8 & 0.14 \\
\hline $50 \% \mathrm{CeO}_{2}$ & 141.4 & 1.69 & 42.5 & 0.51 \\
\hline $15 \% \mathrm{CuO}-75 \% \mathrm{CeO}_{2}$ & 64.01 & 3.05 & 49.1 & 2.34 \\
\hline $15 \% \mathrm{CuO}$ & 60.7 & 0.40 & 48.0 & 0.3 \\
\hline $75 \% \mathrm{CeO}_{2}$ & 137.1 & 1.71 & 26.7 & 0.33 \\
\hline
\end{tabular}

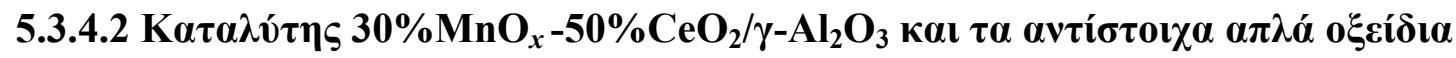

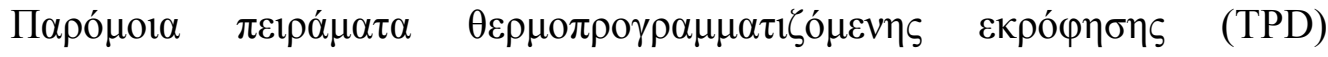

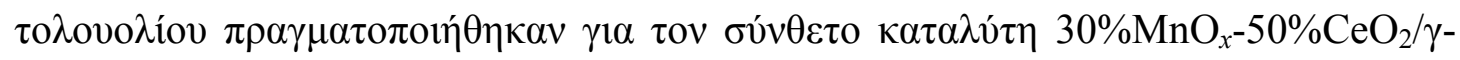

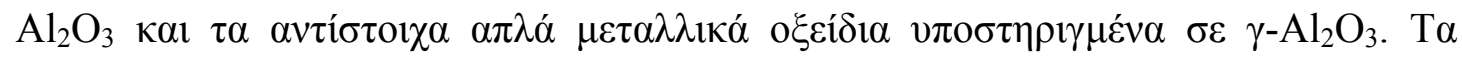

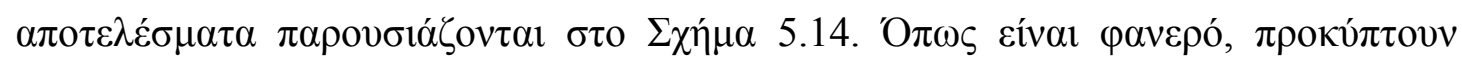

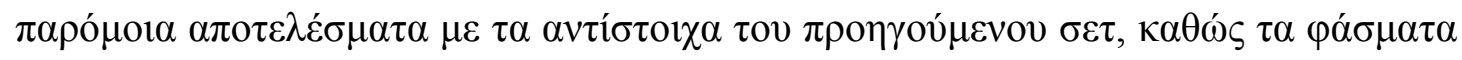

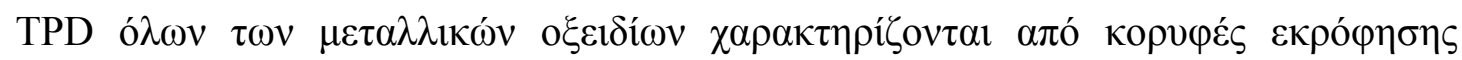

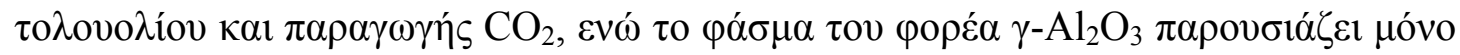

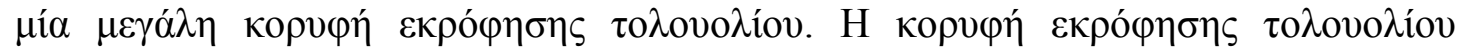

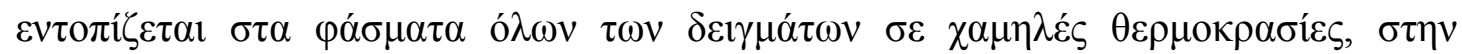

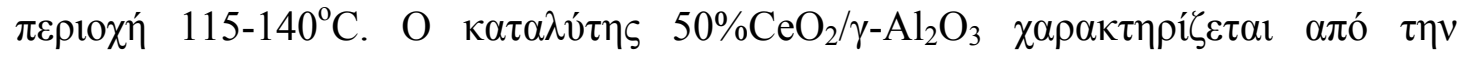

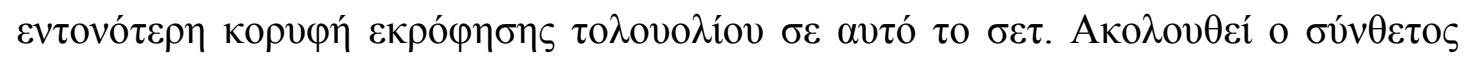

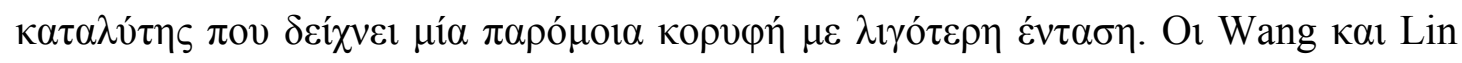

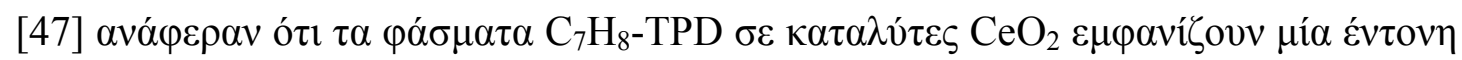

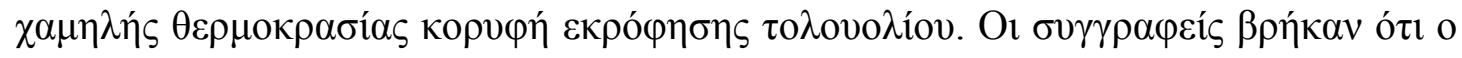




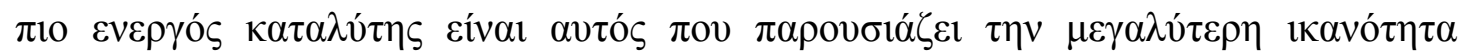

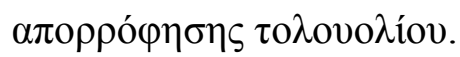

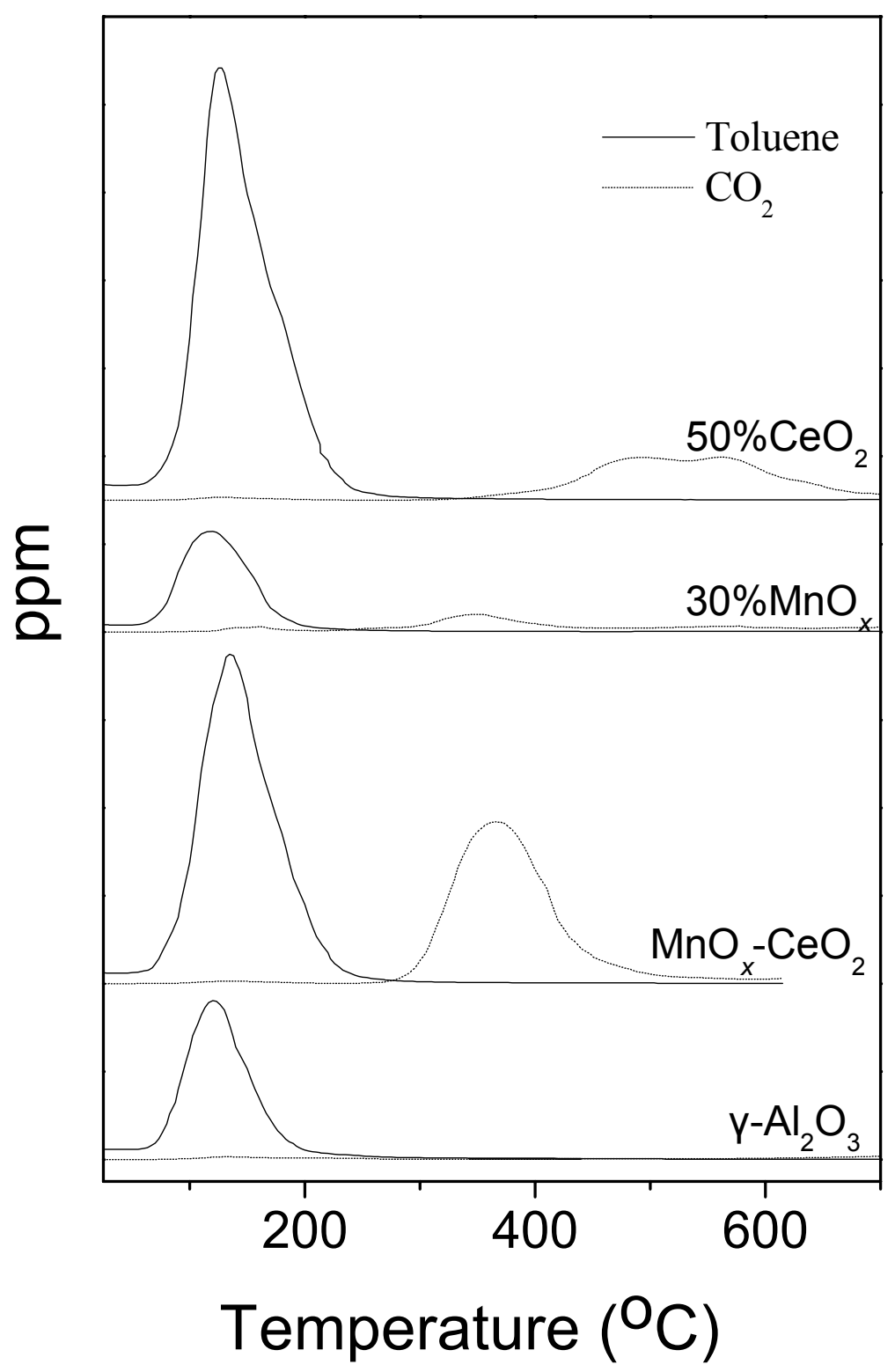

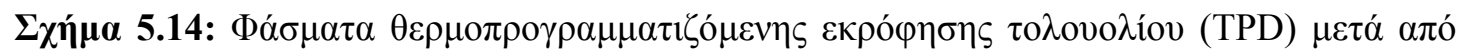

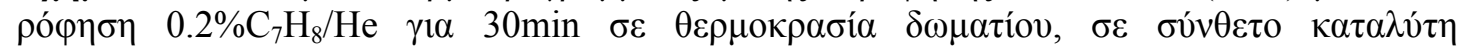

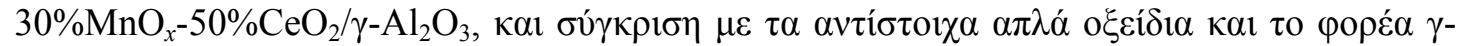
$\mathrm{Al}_{2} \mathrm{O}_{3}$.

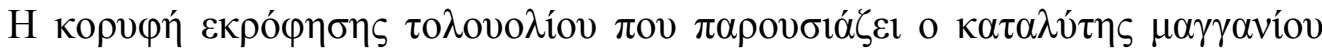

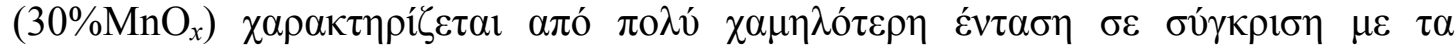

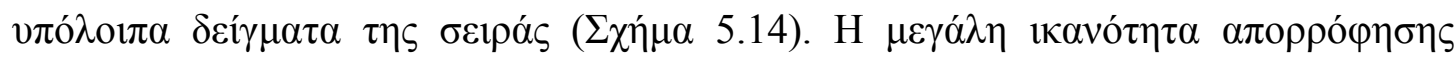




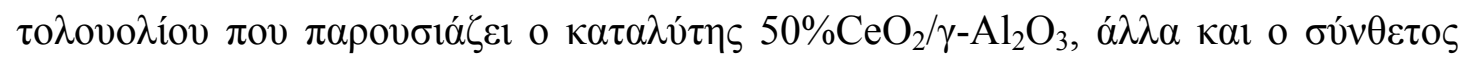

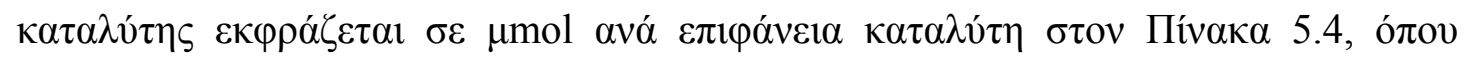

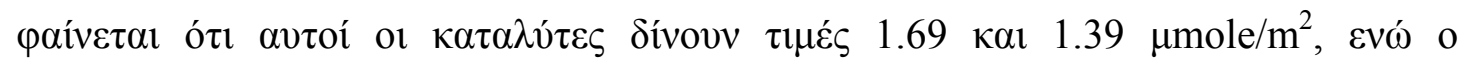

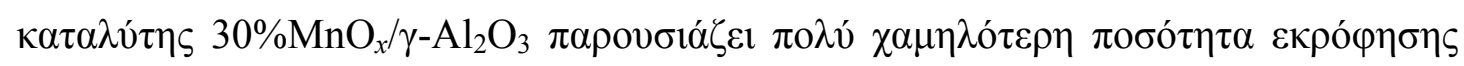

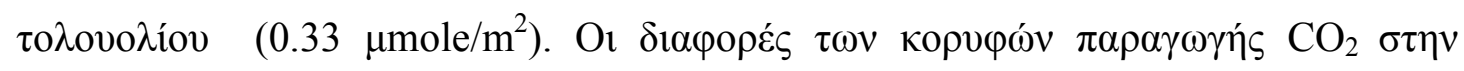

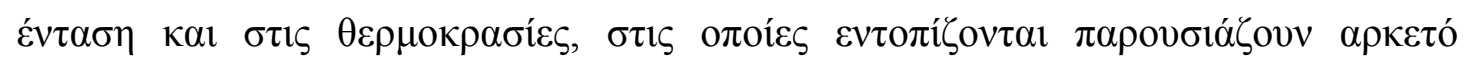

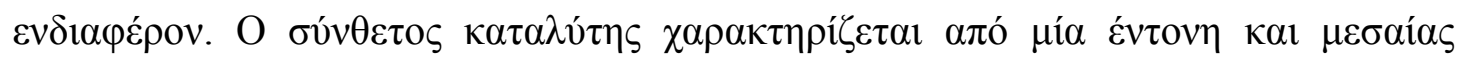

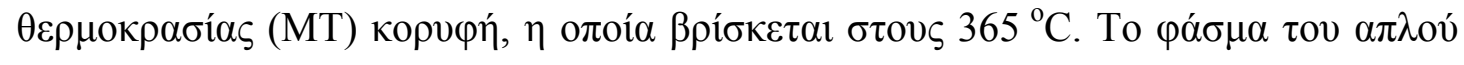

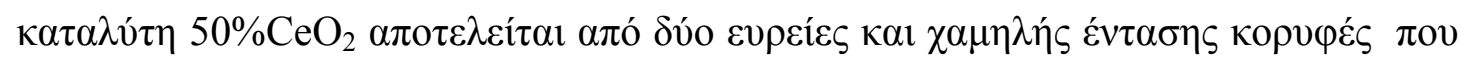

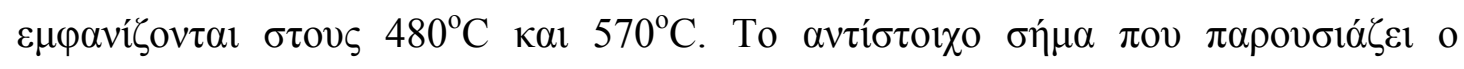

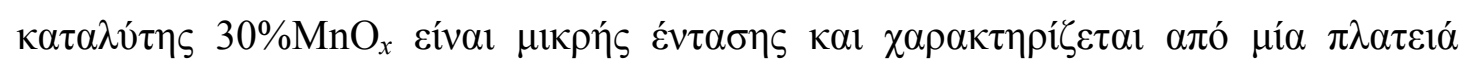

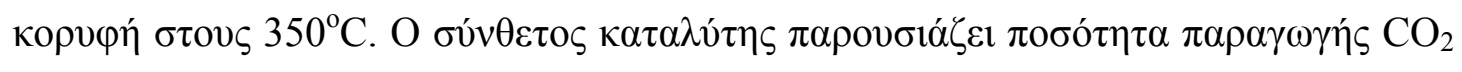

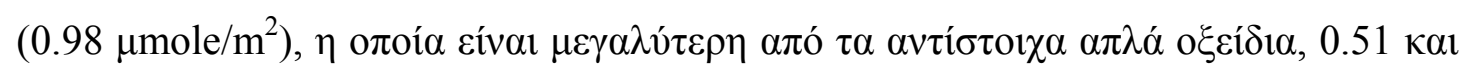

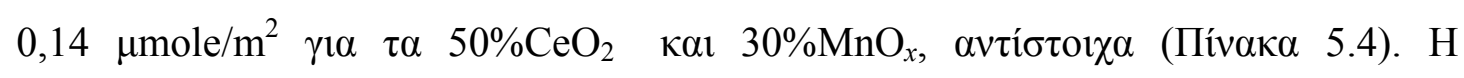

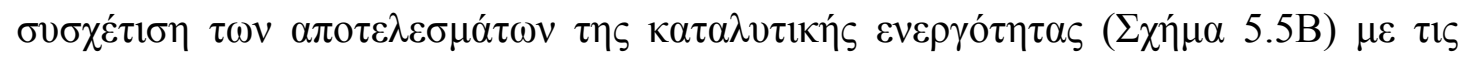

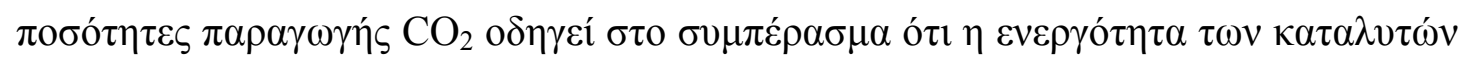

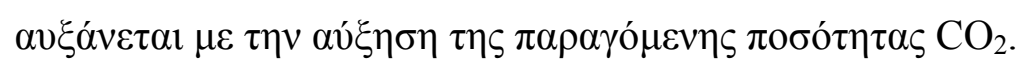

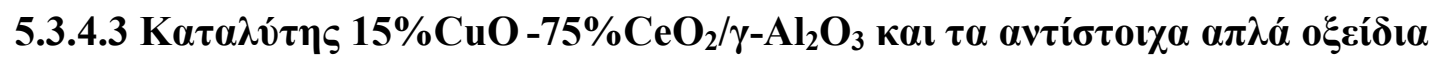

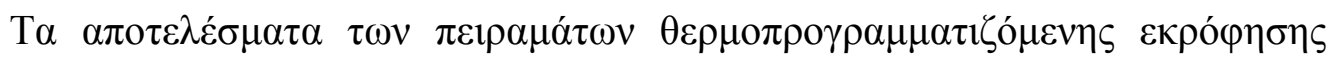

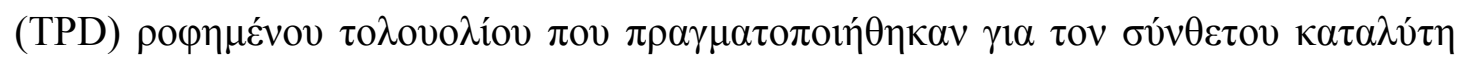

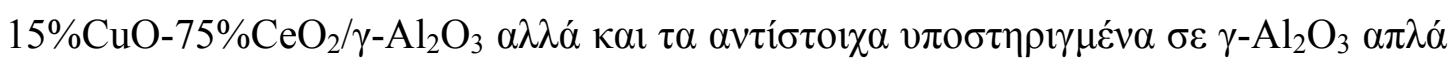

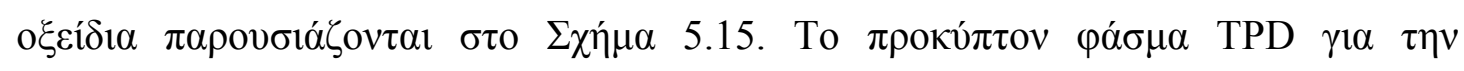

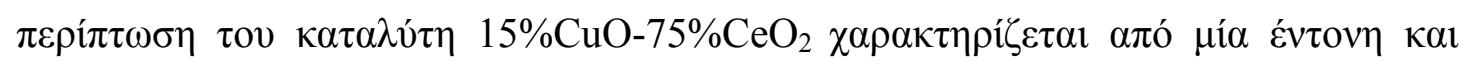

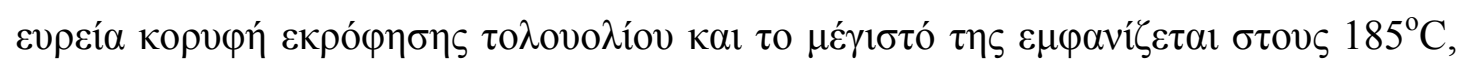

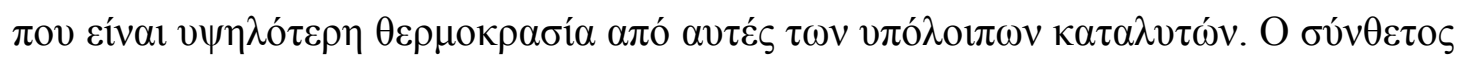

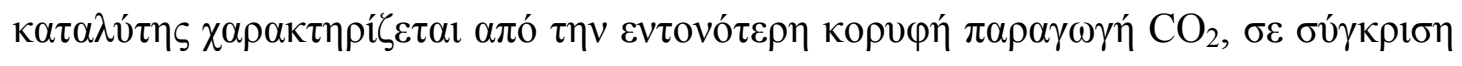

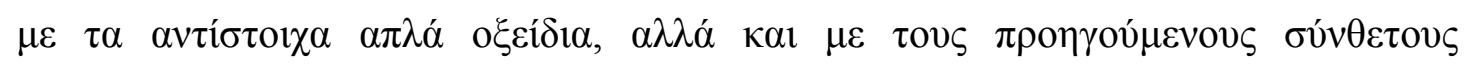

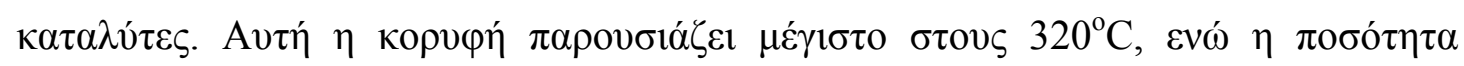

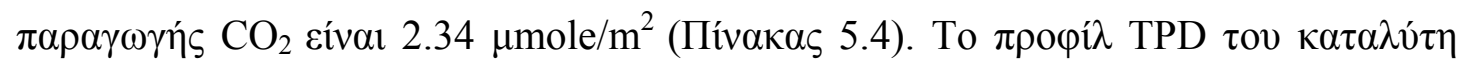

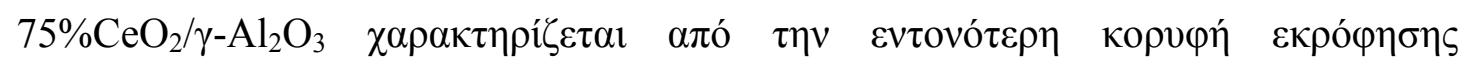

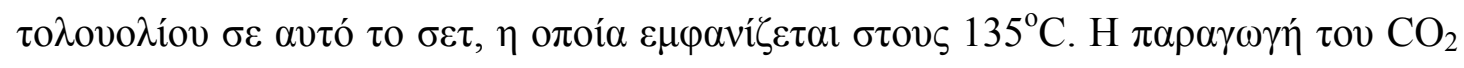

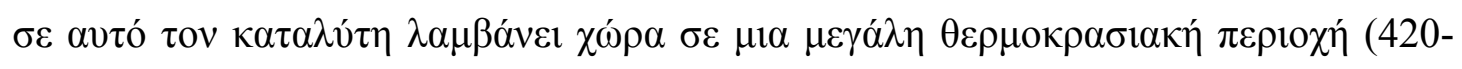




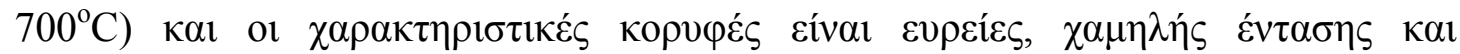

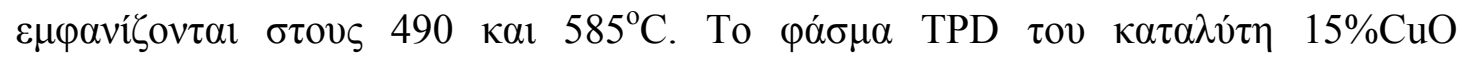

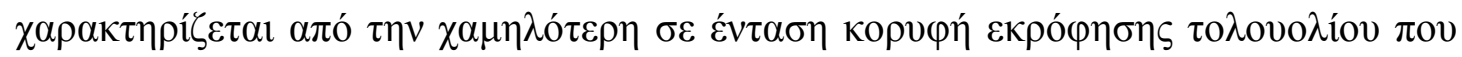

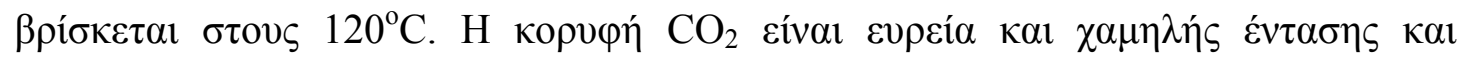

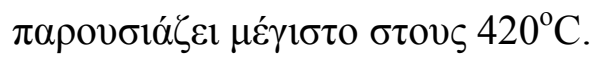

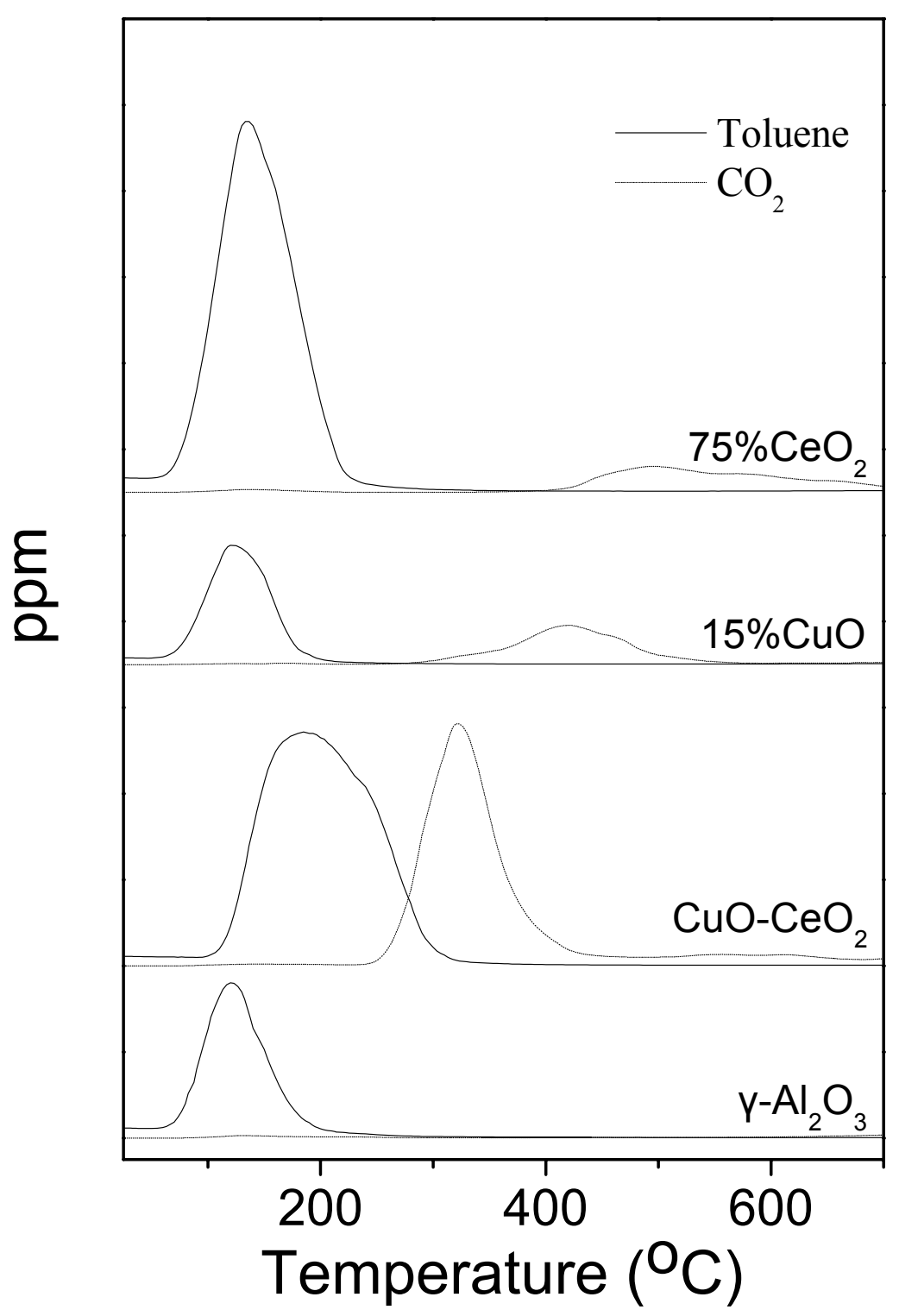

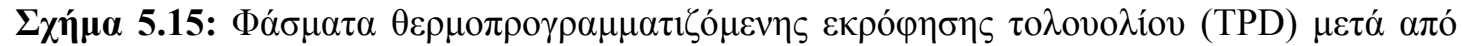

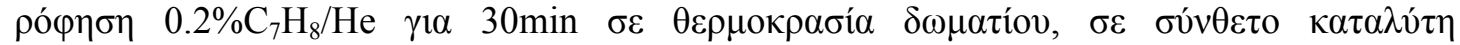

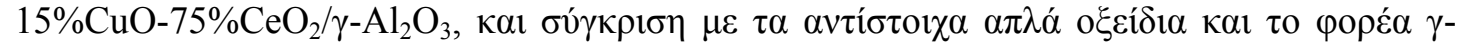
$\mathrm{Al}_{2} \mathrm{O}_{3}$. 


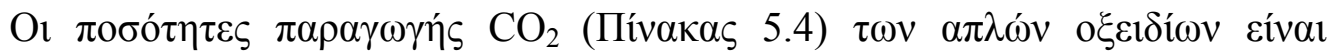

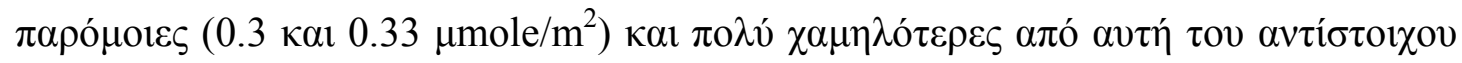

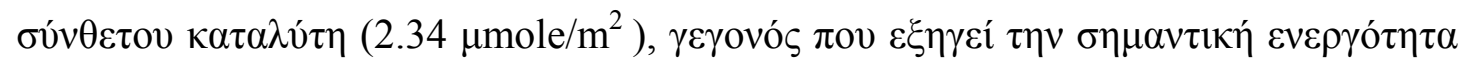

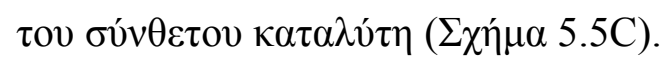

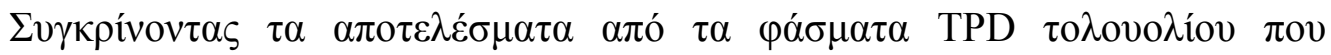

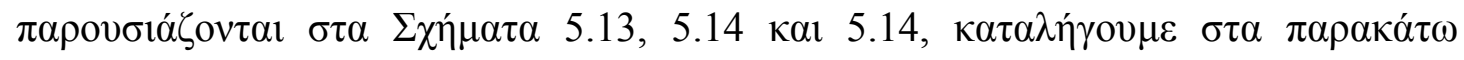
$\sigma v \mu \pi \varepsilon \rho \alpha ́ \sigma \mu \alpha \tau \alpha:$

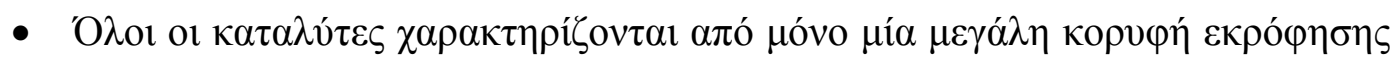

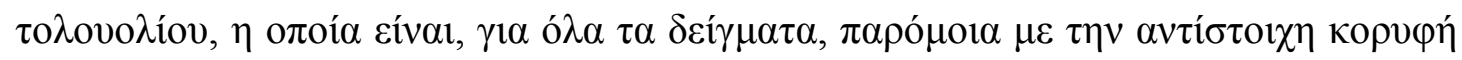

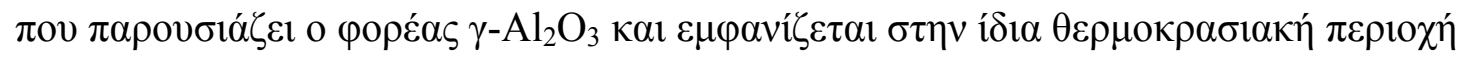

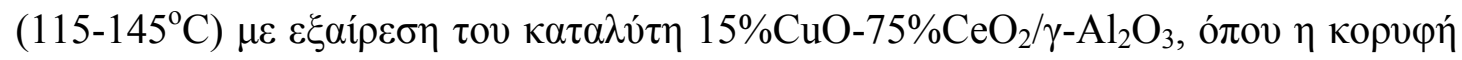

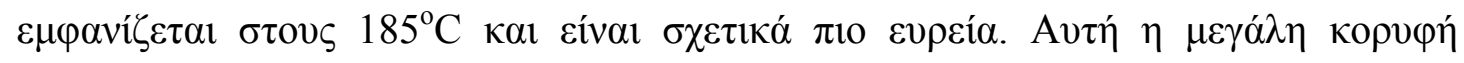

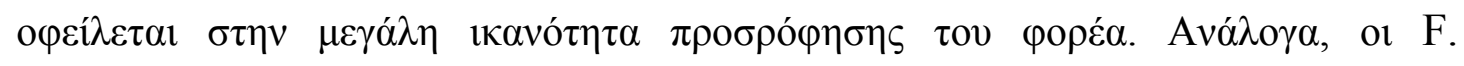

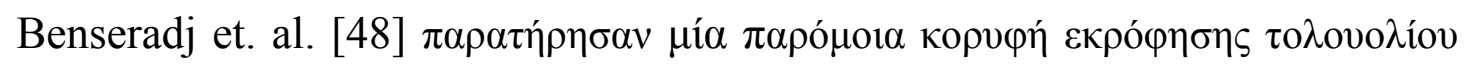

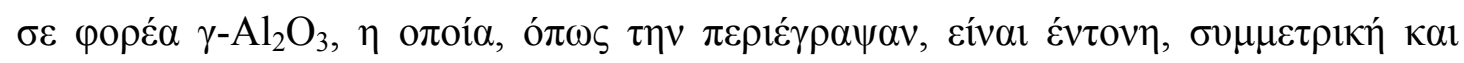
$\chi \alpha \mu \eta \lambda \eta ́ \varsigma, \theta \varepsilon \rho \mu о \kappa \rho \alpha \alpha^{\alpha} \alpha \varsigma\left(180^{\circ} \mathrm{C}\right)$.

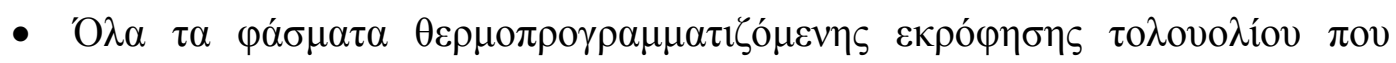

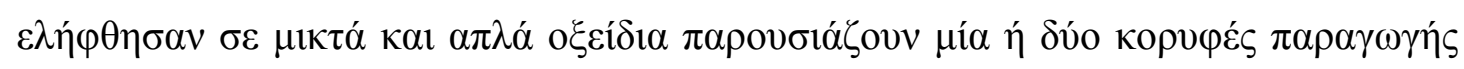

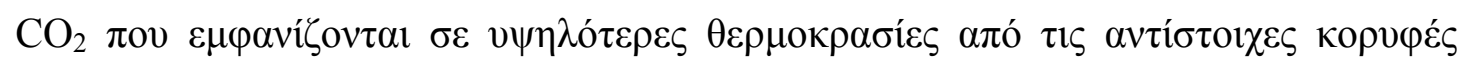

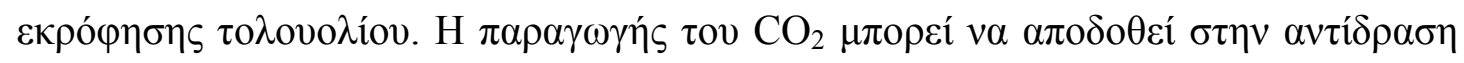

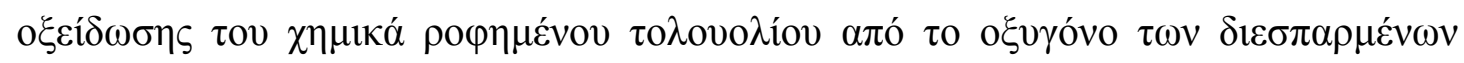

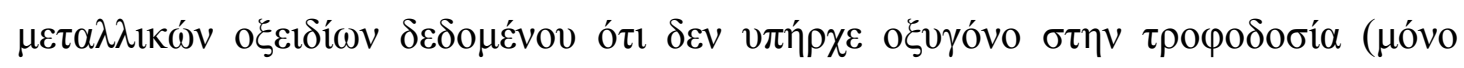

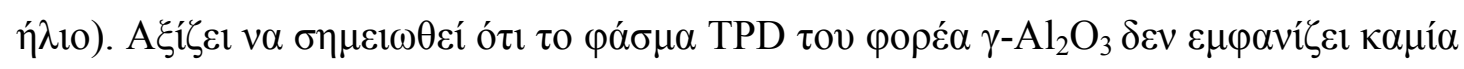

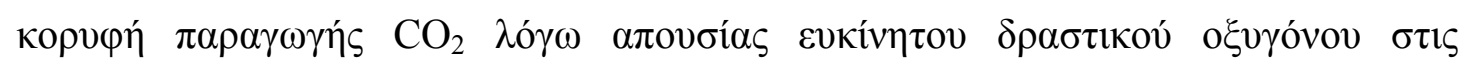

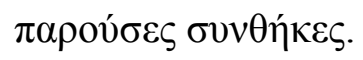

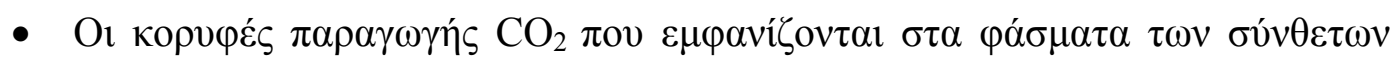

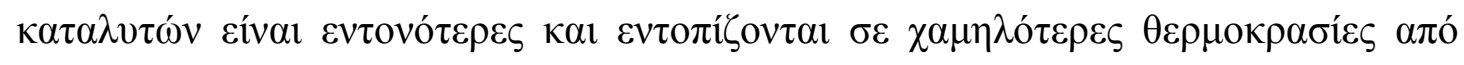

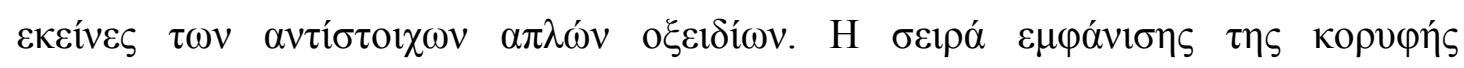

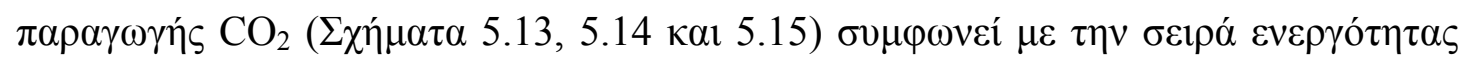

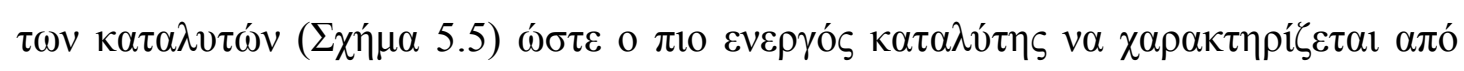

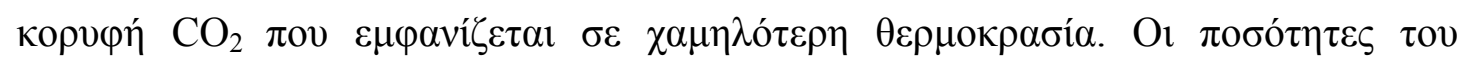

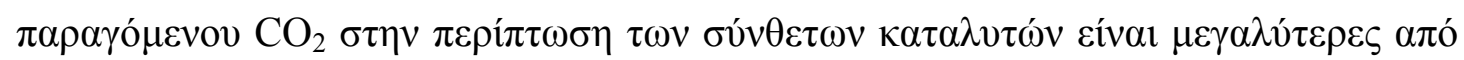




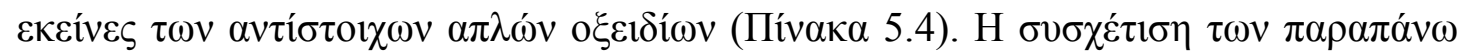

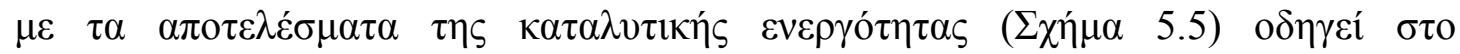

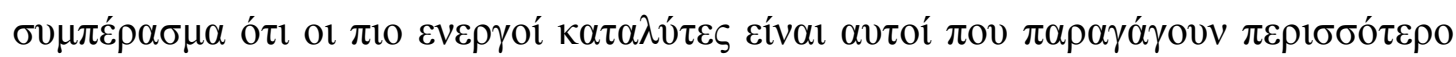

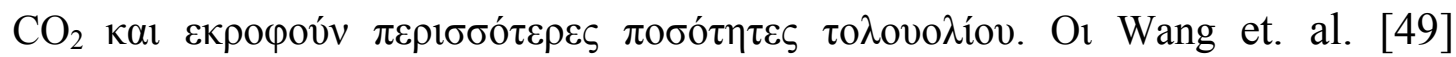

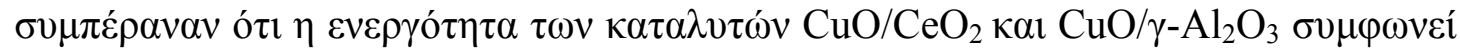

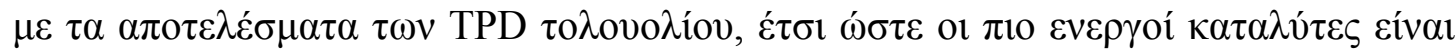

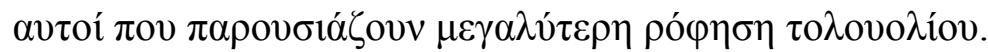

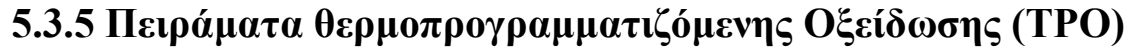

To

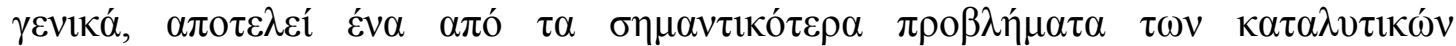

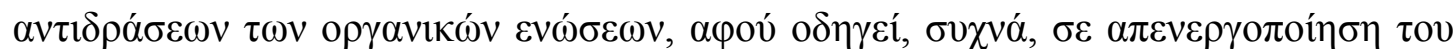

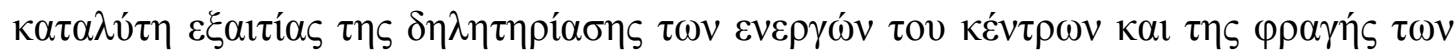

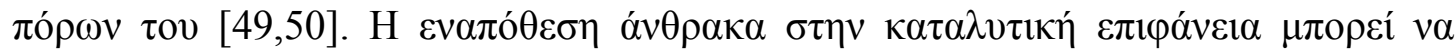

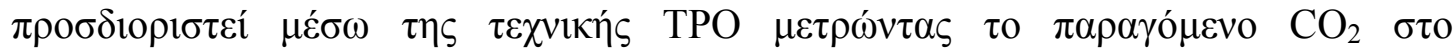

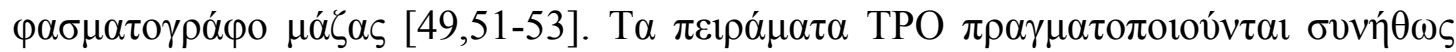

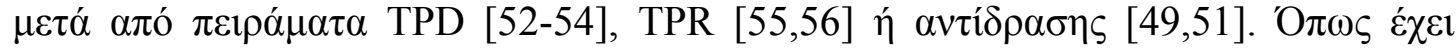

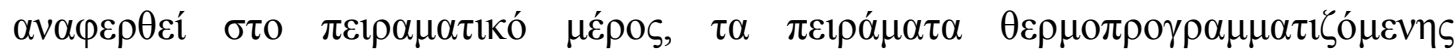

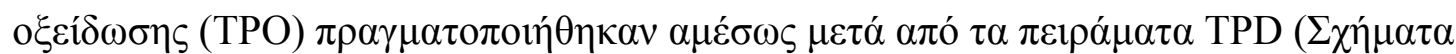

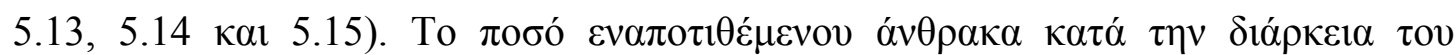

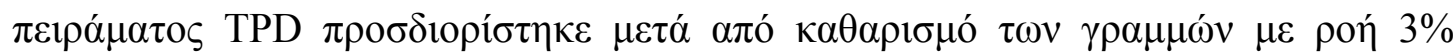

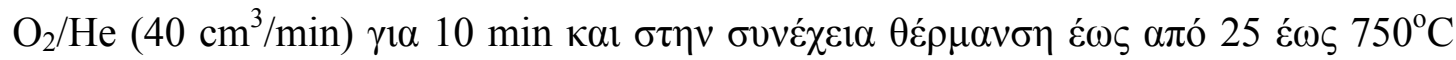

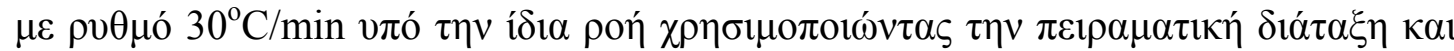

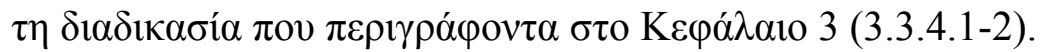

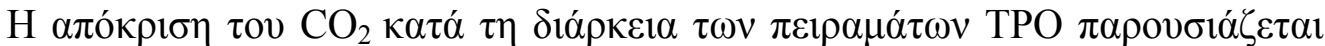

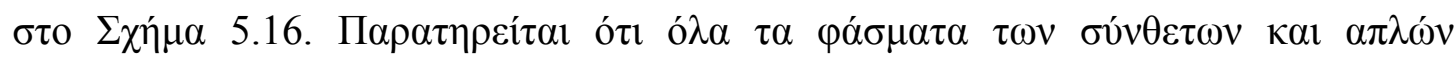

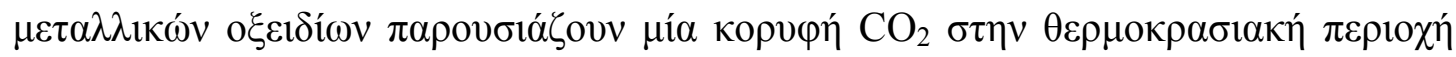

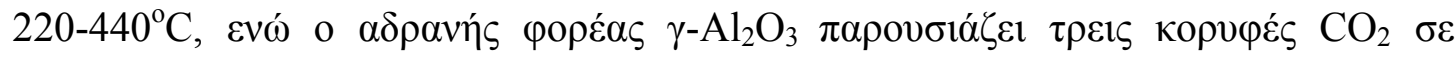

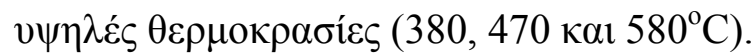

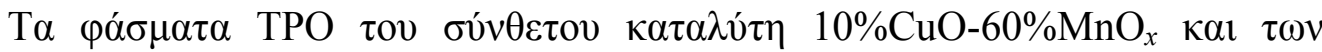

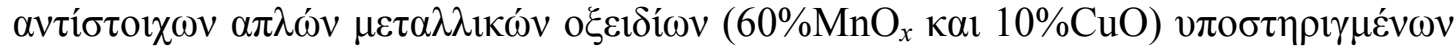

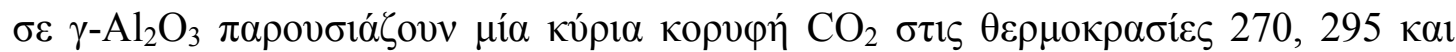

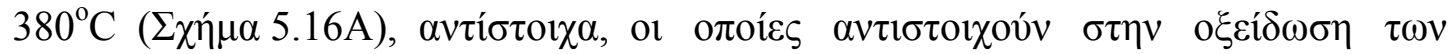



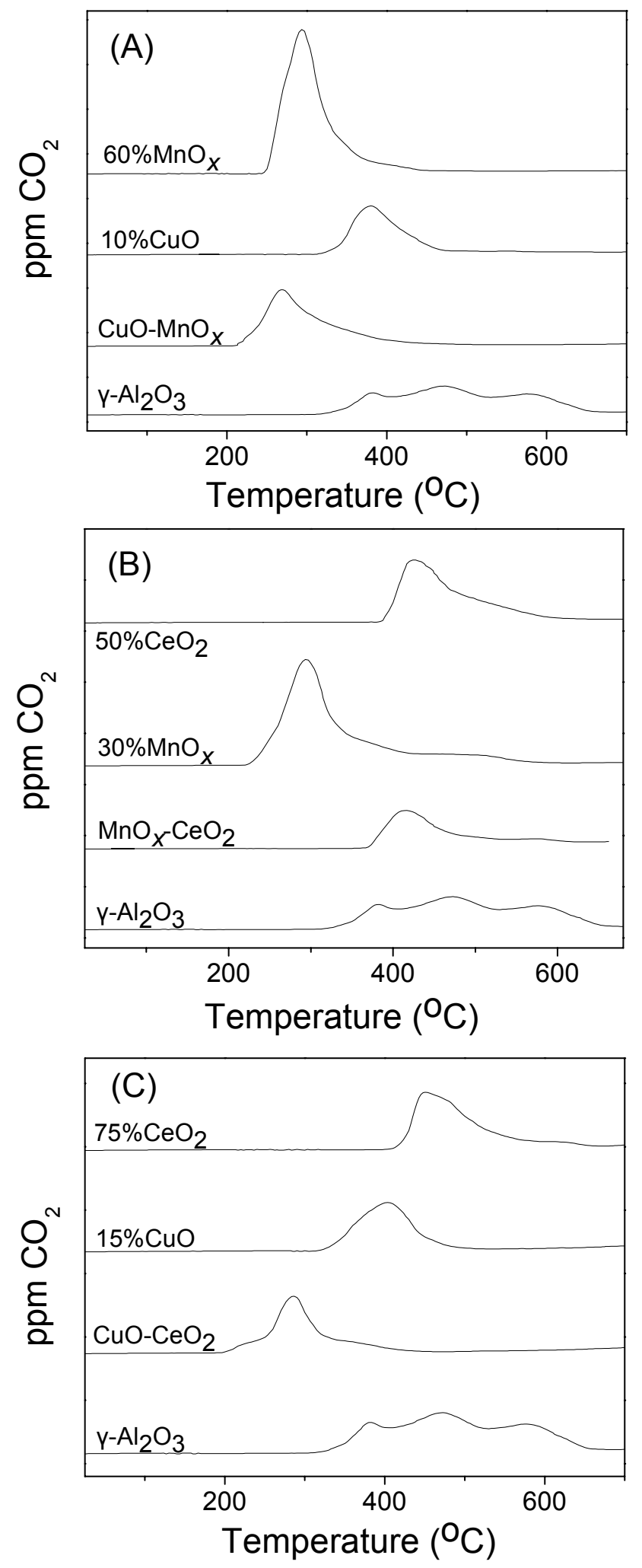

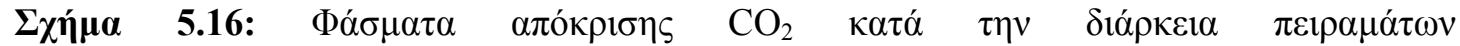

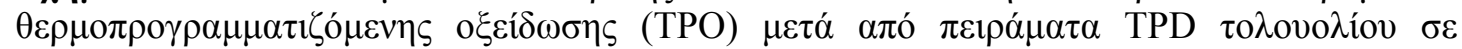

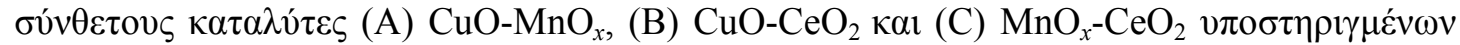

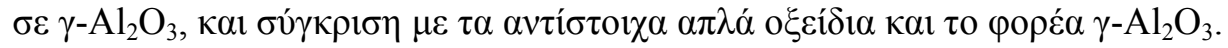




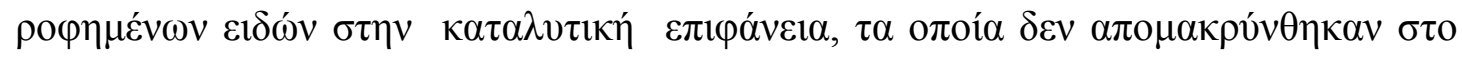

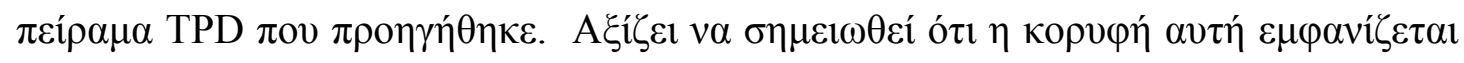

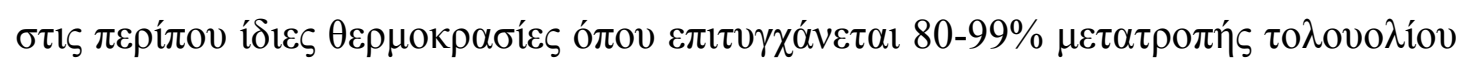
$(\Sigma \chi \eta \dot{\eta} \mu \alpha .5 \mathrm{~A})$.

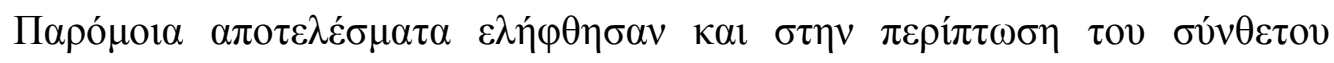

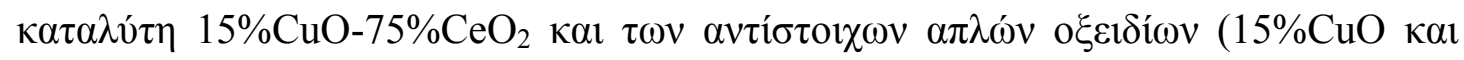

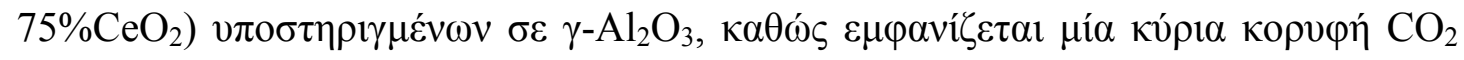

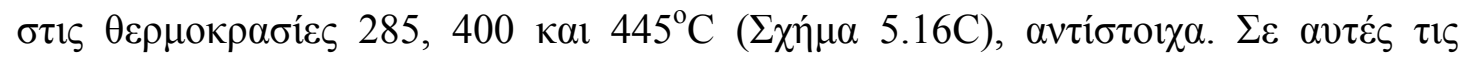

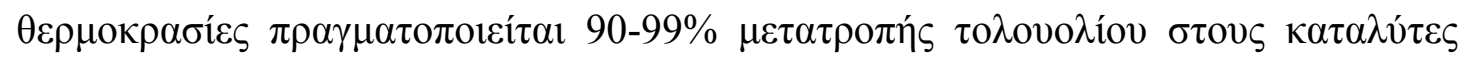

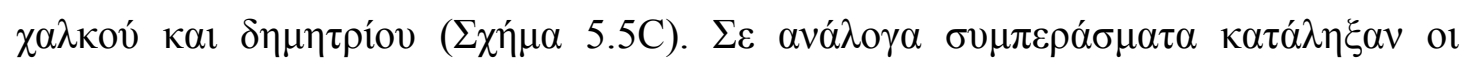

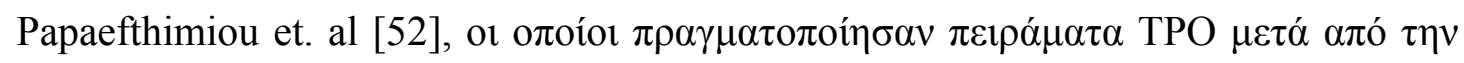

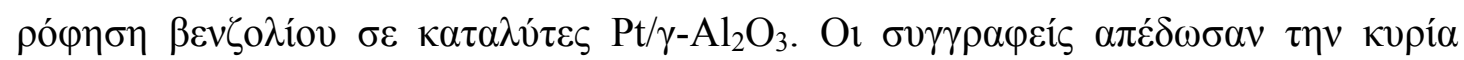

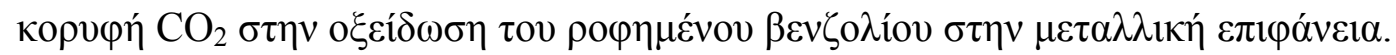

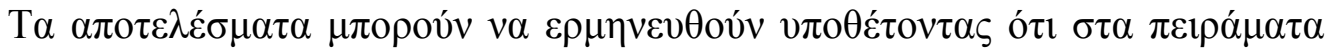

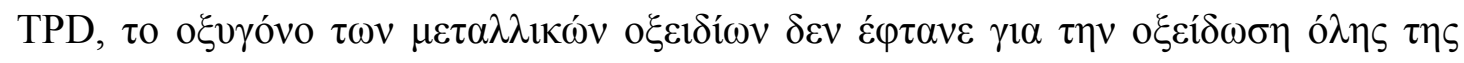

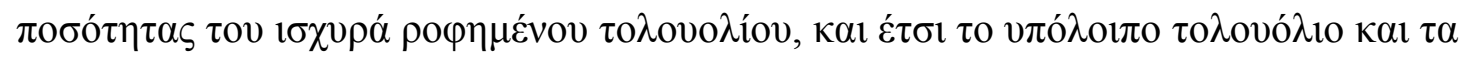

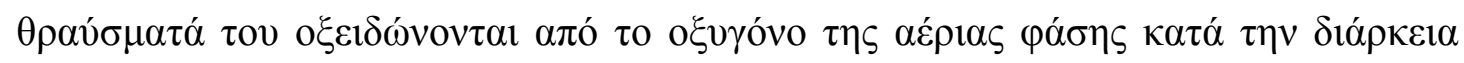

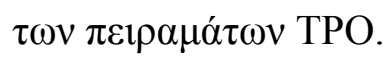

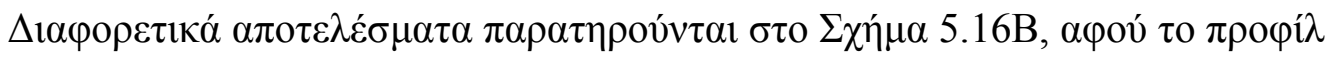

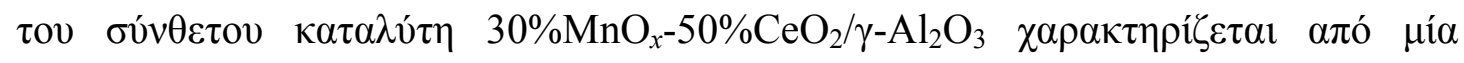

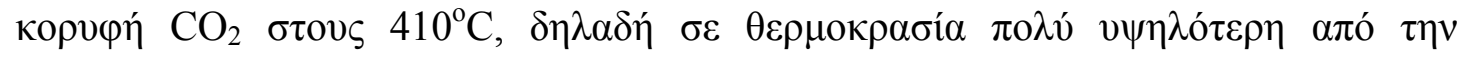

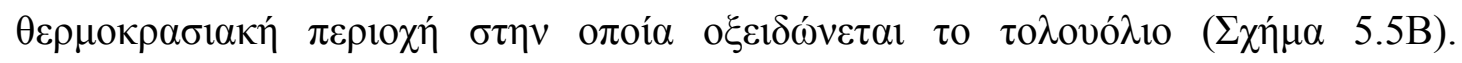

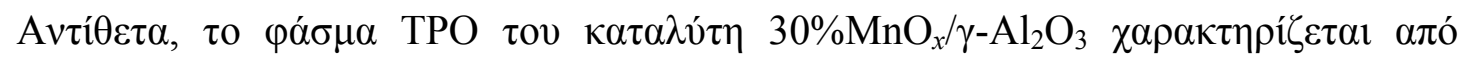

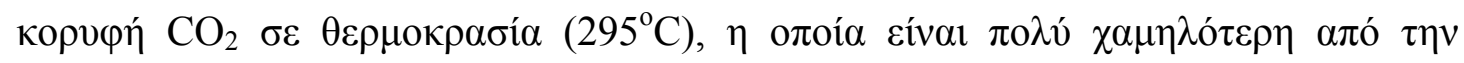

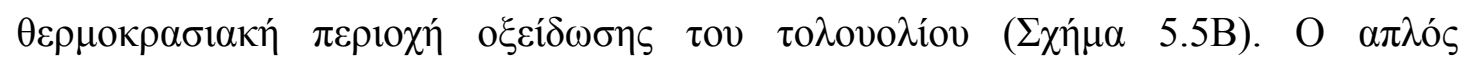

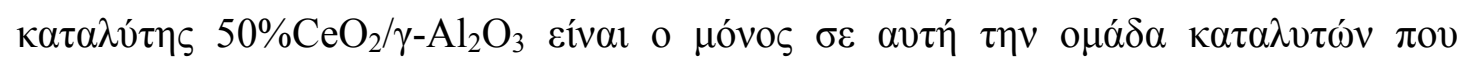

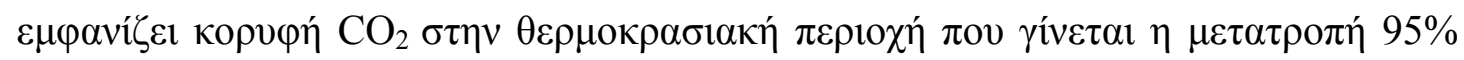

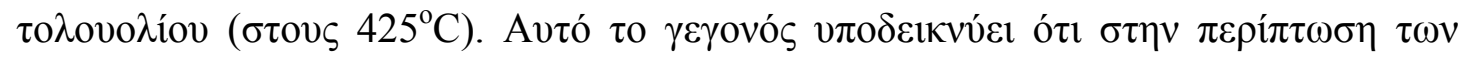

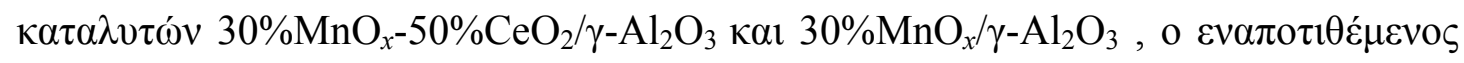

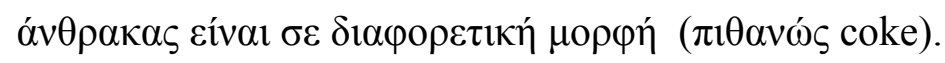

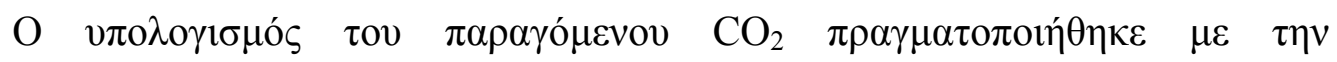

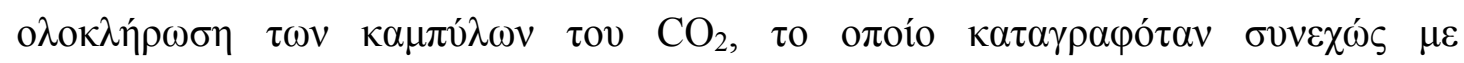

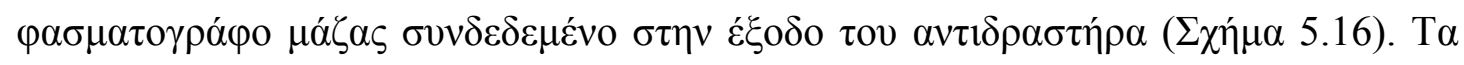

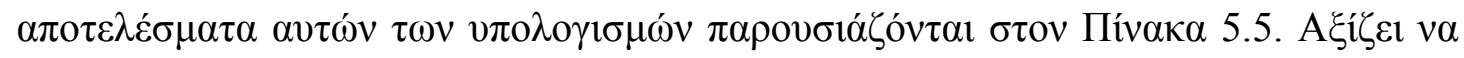




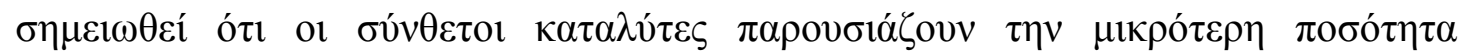

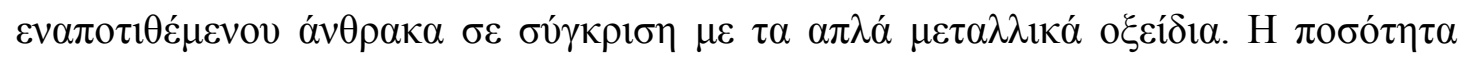

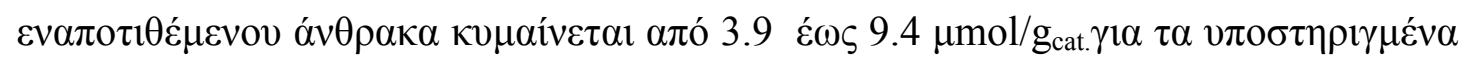

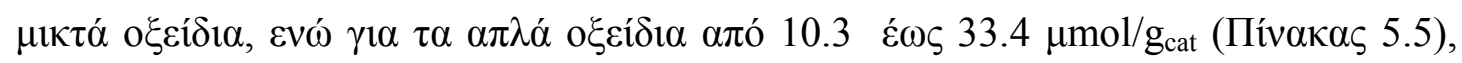

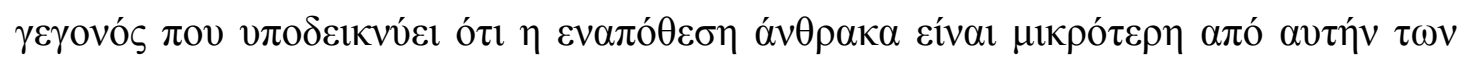

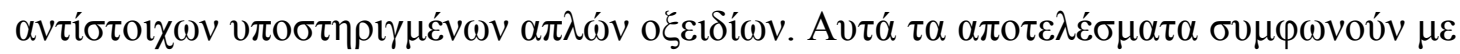

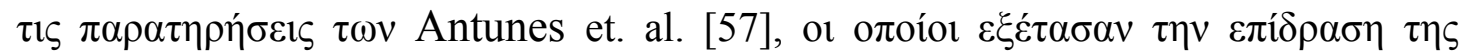

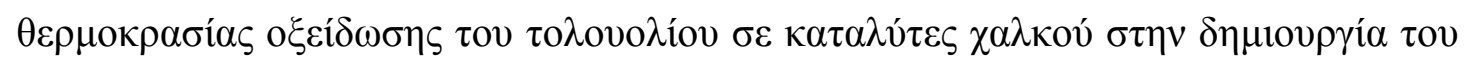

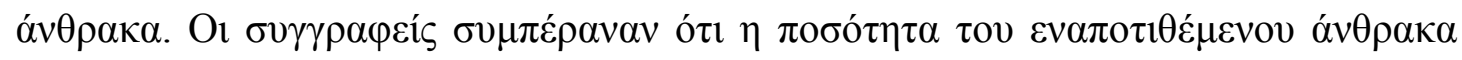

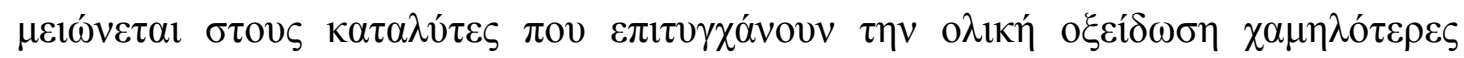

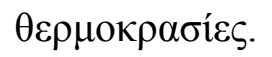

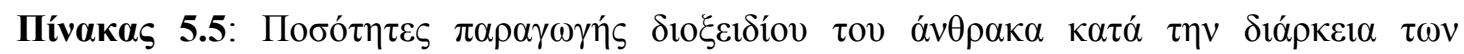

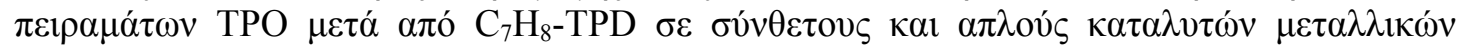
o $\xi \varepsilon 1 \delta i ́ \omega v$

\begin{tabular}{|c|c|c|}
\hline \multirow{2}{*}{ 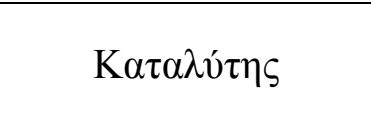 } & \multicolumn{2}{|c|}{ Побо́ $\tau \eta \varepsilon \varsigma \varsigma \alpha \rho \alpha \gamma o ́ \mu \varepsilon v o v \mathrm{CO}_{2}$} \\
\hline & $\mu \mathrm{mol} / \mathrm{g}_{\mathrm{cat}}$ & $\mu \mathrm{mol} / \mathrm{m}^{2}$ \\
\hline $100 \% \mathrm{Al}_{2} \mathrm{O}_{3}$ & 33.3 & 0.18 \\
\hline $10 \% \mathrm{CuO}-60 \% \mathrm{MnO}_{x}$ & 8.6 & 0.17 \\
\hline $10 \% \mathrm{CuO}$ & 18.3 & 0.12 \\
\hline $60 \% \mathrm{MnO}_{x}$ & 14 & 0.26 \\
\hline $30 \% \mathrm{MnO}_{x}-50 \% \mathrm{CeO}_{2}$ & 9.4 & 0.12 \\
\hline $30 \% \mathrm{MnO}_{x}$ & 30.9 & 0.25 \\
\hline $50 \% \mathrm{CeO}_{2}$ & 13.2 & 0.16 \\
\hline $15 \% \mathrm{CuO}-75 \% \mathrm{CeO}_{2}$ & 3.9 & 0.18 \\
\hline $15 \% \mathrm{CuO}$ & 14 & 0.09 \\
\hline $75 \% \mathrm{CeO}_{2}$ & 10.3 & 0.13 \\
\hline
\end{tabular}

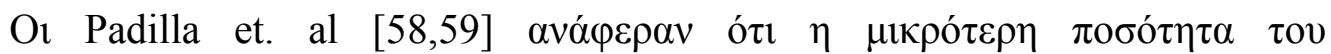

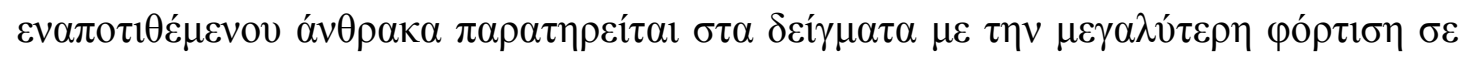

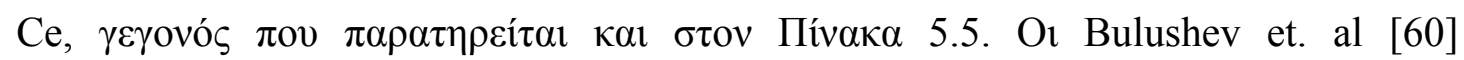

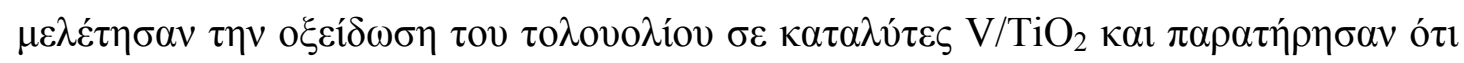




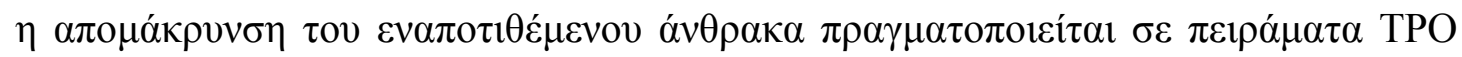

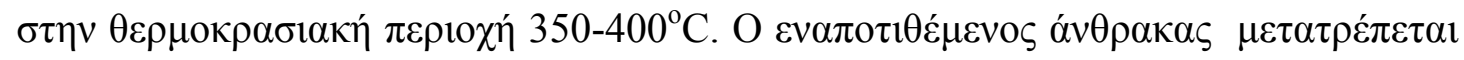

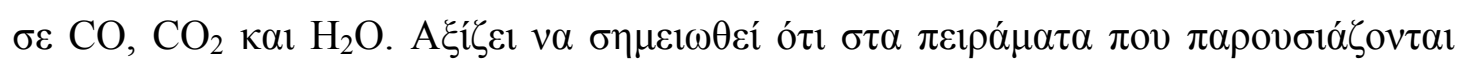

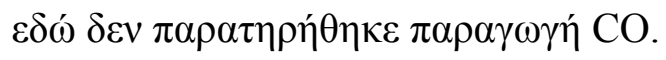

\section{$5.4 \Sigma v \mu \pi \varepsilon \rho \alpha ́ \sigma \mu \alpha \tau \alpha$}

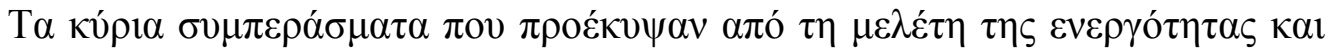

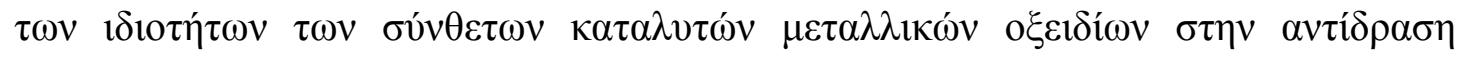

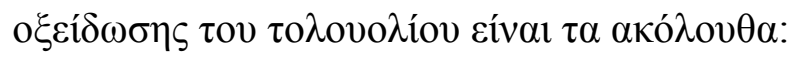

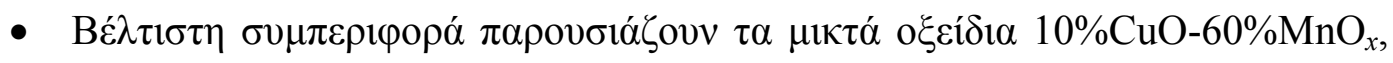

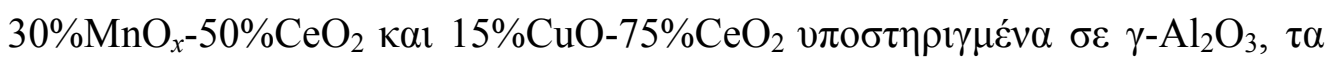

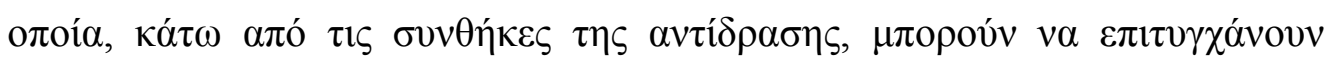

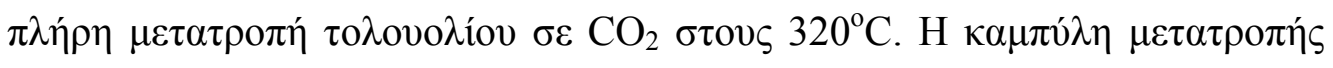

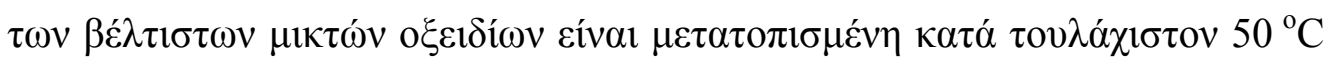

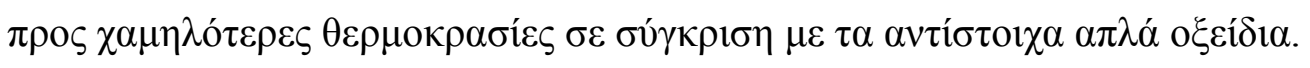

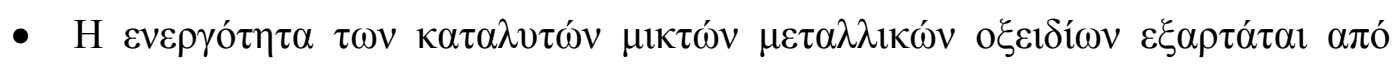

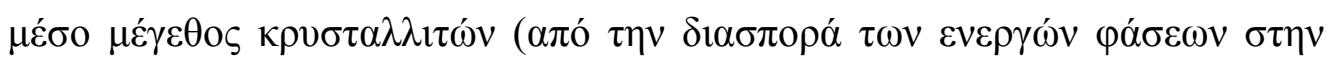

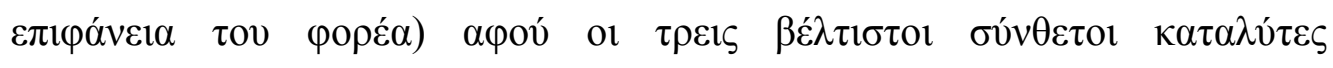

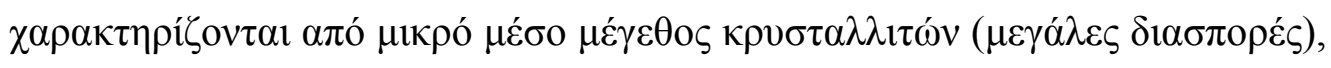

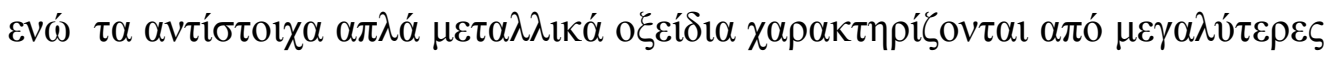

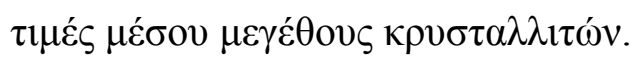

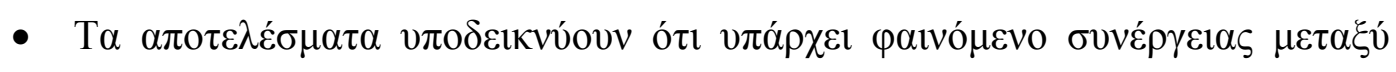

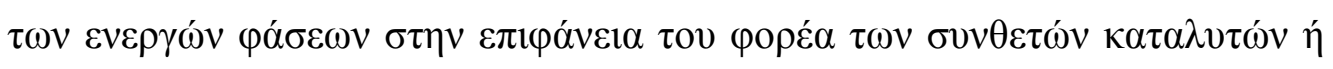

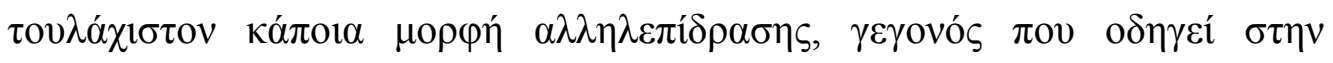

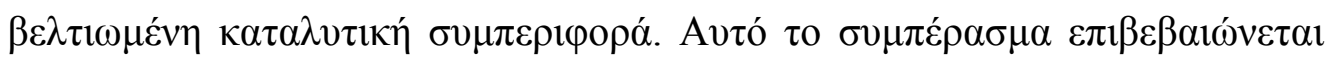

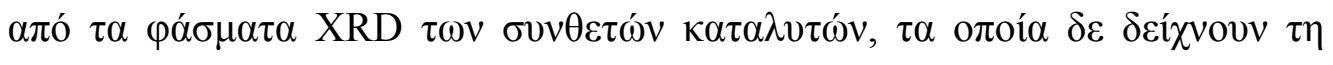

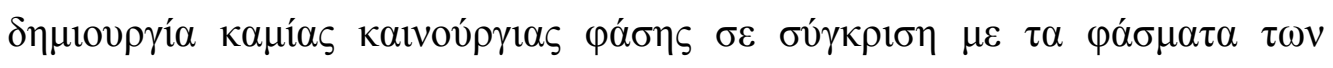

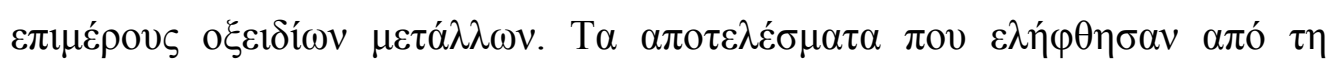

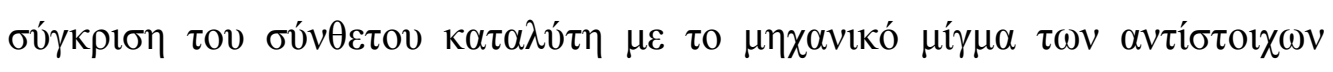

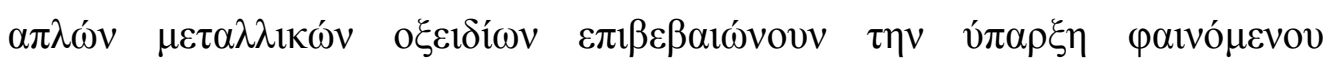

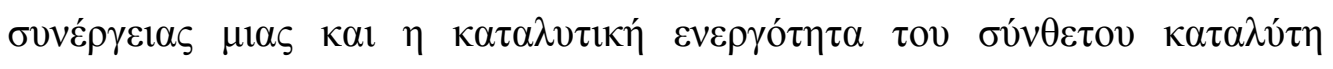

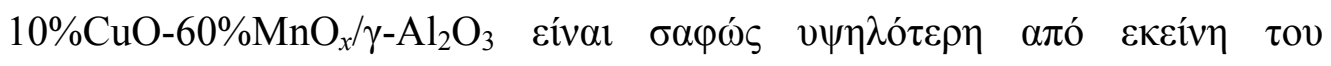

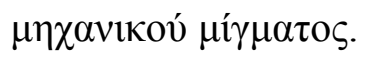




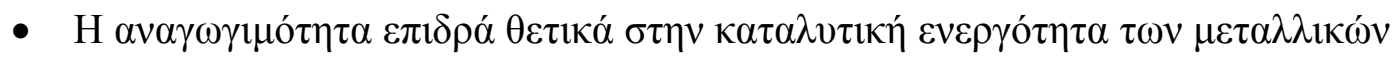

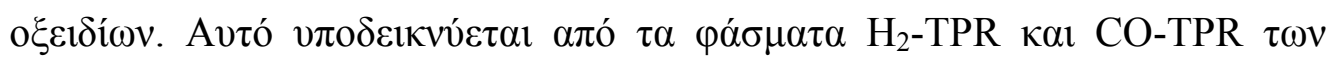

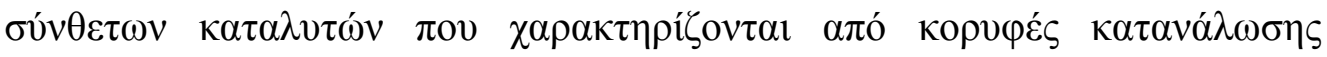

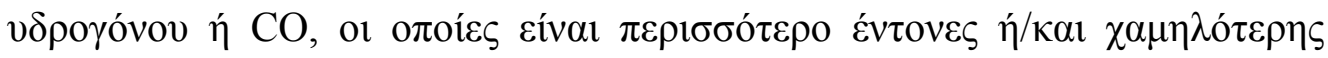

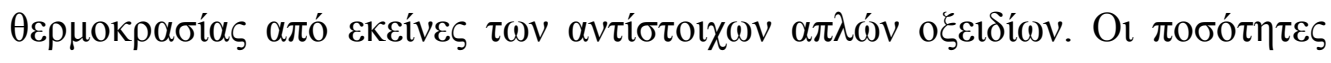

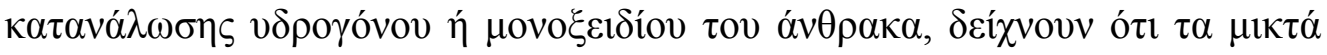

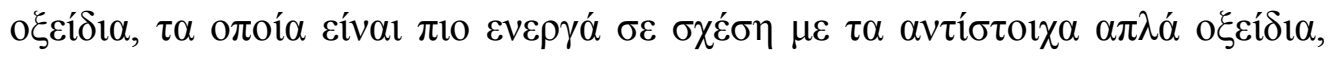

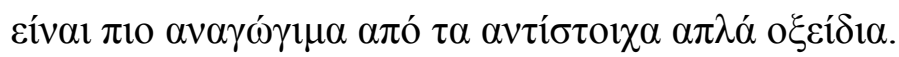

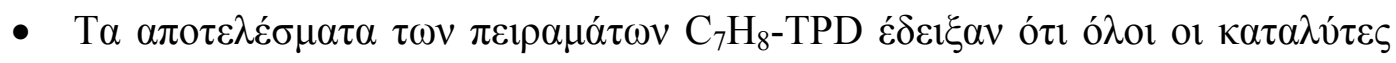

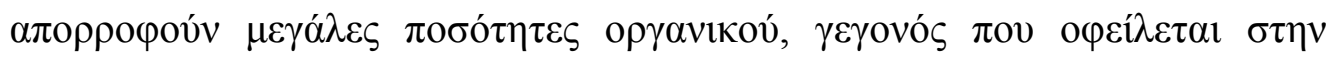

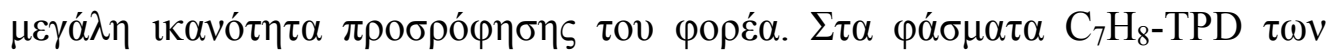

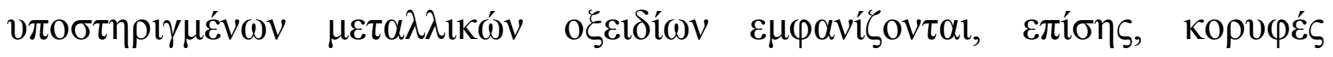

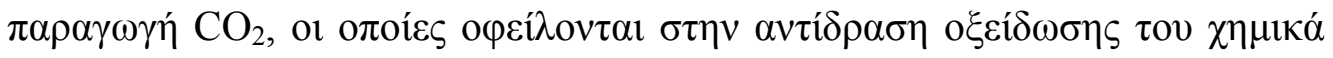

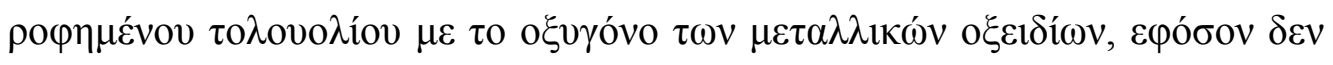

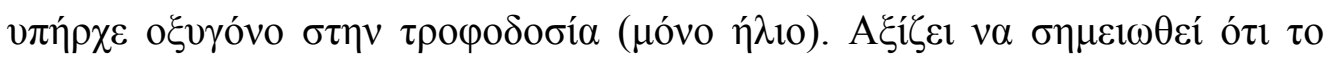

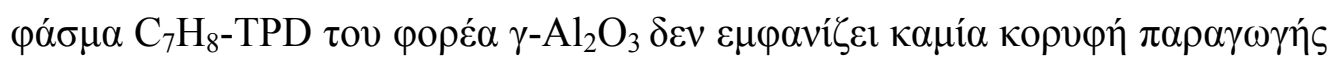

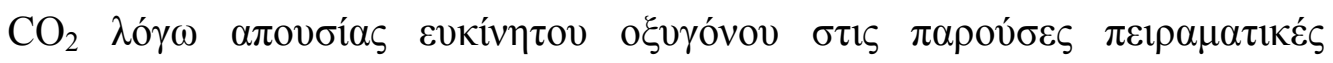

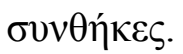

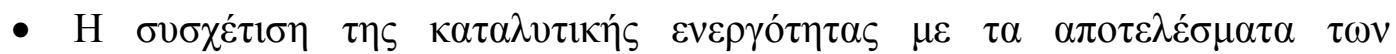

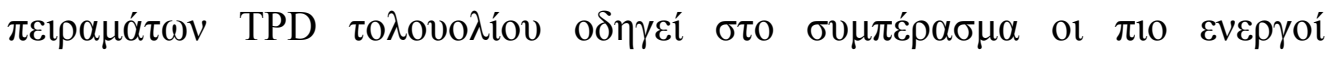

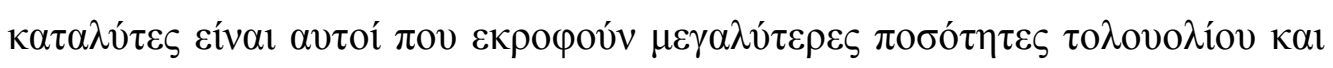

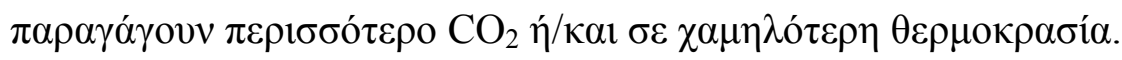

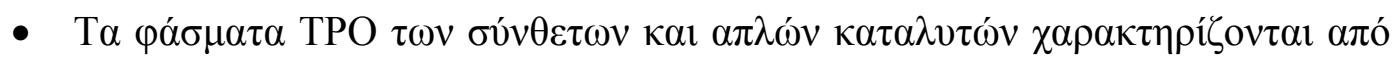

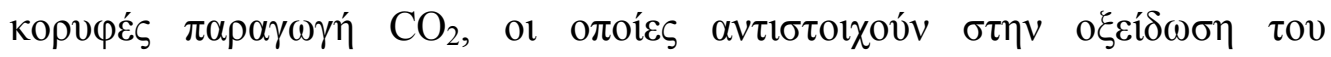

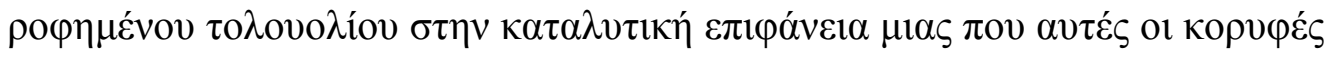

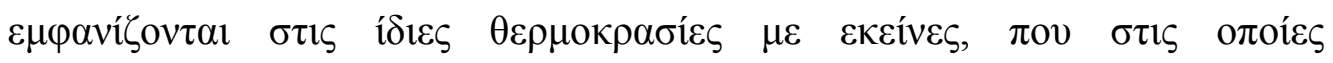

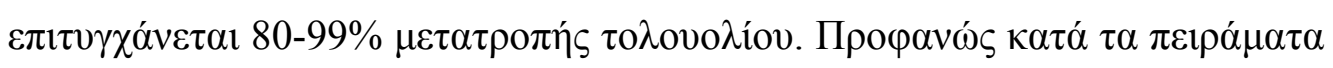

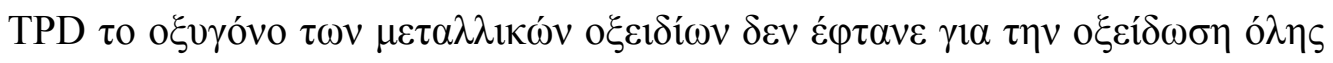

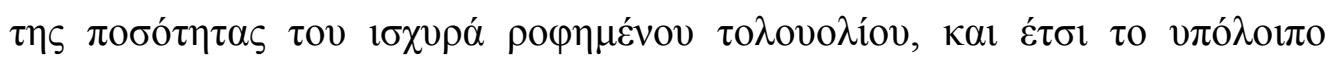

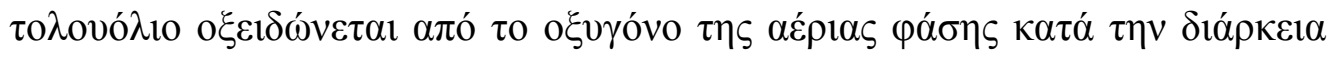

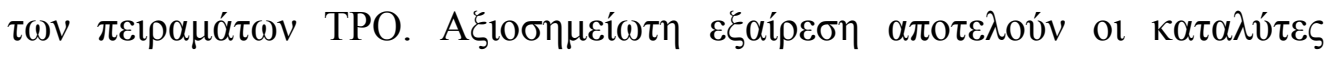

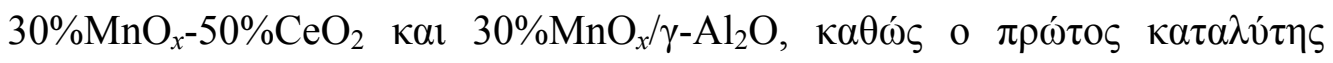

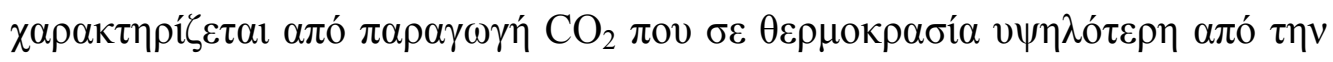




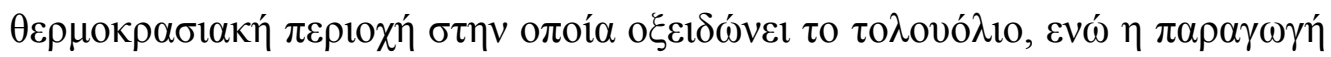

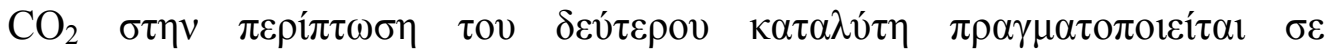

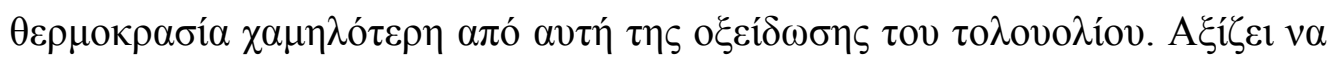

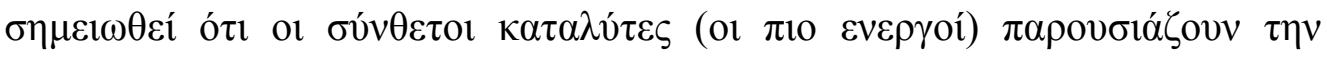

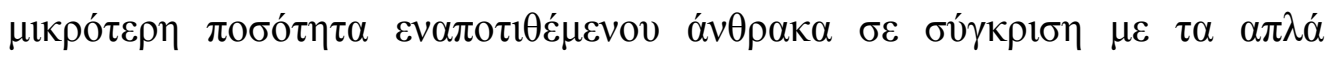
$\mu \varepsilon \tau \alpha \lambda \lambda 1 \kappa \alpha ́$ o $\xi \varepsilon i ́ \delta 1 \alpha$. 


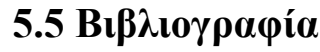

[1] C.-H.i Wang, S.-S. Lin, Appl. Catal. A: Gen. 268 (2004) 227.

[2] D. Delimaris, T. Ioannides, Appl. Catal. B: Environ 84 (2008) 303.

[3] C. Lahousse, A. Bernier, P. Grange, B. Delmon, P. Papaefthimiou, T. Ioannides, X. Verykios, J. Catal. 178 (1998) 214.

[4] W.B. Li, W.B. Chu, M. Zhuang, J. Hua, Catal. Today 93-95 (2004) 205.

[5] V. H. Vu, J. Belkouch, A. Ould-Dris, B. Taouk, J. of Hazardous Materials doi:10.1016/j.jhazmat.2009.04.010, Article in Press

[6] L.A. Palacio, J.M. Silva, F.R. Ribeiro, M. F. Ribeiro, Catal. Today 133135 (2008) 502.

[7] H. Chen, A. Sayari, A. Adont, F. Larachi, Appl. Catal. B: Environ 32 (2001) 195.

[8] D. Delimaris, “O

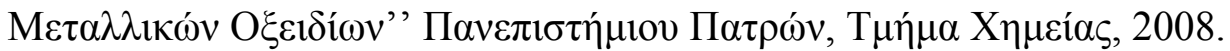

[9] C.-H. Wang, S.-S. Lin, C.-L. Chen, H.-S. Weng, Chemosphere 64 (2006) 503

[10] G. Picasso, M. Gutierrez, M.P. Pina, J. Herguido, Chem. Eng. J. 126 (2007) 119.

[11] G. Qi, R. T.Yang, R.Chang, Appl. Catal. B: Environ. 51,2, (2004) 93.

[12] M. Machida, M. Uto, D. Kurogi , T. Kijima. Chem. Mater. 12 (2000) 3158.

[13] B. Murugan, D. Srinivas, C.S. Gopinath, V. Ramaswamy, A.V. Ramaswamy, Chem. Mater. 17 (2005) 3983

[14] C.H.Y. Kang, H. Kusaba, H. Yahiro, K. Sasaki, Y. Teraoka, Solid State Ionics 177 (2006) 1799.

[15] S. Zhang, W. Huang, X. Qiu, B. Li, X. Zheng, S. Wu, Catal. Lett. 80 (2002) 41.

[16] P. Bera, S. T. Aruna, K. C. Patial, M.S Hegde, J. Catal. 186 (1999) 36.

[17] S. Hocevar, U. O. Krasovec, B. Orel, A. S. Arico, H. Kim, Appl. Catal. B: Env. 28 (2000) 113.

[18] X.L. Tang, B.C. Zhang, Y. Li, Y.D. Xu, Q. Xin, W.J Shen, Catal. Today 9395 (2004) 191.

[19] P. Bera, K. R. Priolkar, P.R. Sarode, M.S Hegde, S.Emura, R. Kumashiro. N.P. Lalla, Chem. Mater. 14 (2002) 3591 
[20] J. Xiaoyuan, Lu Guanglie, Zhou Renxian, Mao Jianxin, Chen Yu, Zheng Xiaoming, Appl. Surf. Sci. 173 (2001) 208

[21] S. Imamura, A. Dol, S. Ishido, Ind. Eng. Chem. Prod. Res. Dev. 24 (1985) 75.

[22] M. R. Morales, B.P Barbero, L.E. Cadus, Fuel 87 (2002) 1177.

[23] L. Lamaita, M.A. Peluso, J.E. Sambeth, H.J. Thomas, Appl. Catal. B: Environ. 61(2005) 128.

[24] L. Lamaita, M.A. Peluso, J.E. Sambeth, H.J. Thomas, G. Mineli, P. Porta, Catal. Today 107-108 (2005) 133.

[25] J. Trawczynski, B. Bielak, W. Mista, Appl. Catal. B: Env. 55(2005) 277.

[26] H.L. Tidahy, S. Siffert, F. Wyrwalski, J.-F. Lamonier, A. Aboukaïs, Catal. Today 119 (2007) 317

[27] S. C. Kim, W. G. Shim, Appl. Catal. B: Environ 79 (2008) 149.

[28] D. Döbber, D. Kießling, W. Schmitz, G. Wendt, Appl. Catal. B: Environ 52 (2004) 135.

[29] Jose I. Gutiérrez-Ortiz, B. de Rivas, R. López-Fonseca, S. Martín, J. R. González-Velasco, Chemosphere 68 (2007) 1004.

[30] B. de Rivas, Jose I. Gutiérrez-Ortiz, R. López-Fonseca, J. R. GonzálezVelasco, Appl. Catal. A: 314 (2006) 54.

[31] M. Boaro, M. Vicario, C. de Leitenburg, G. Dolcetti, A. Trovarelli, Catal. Today 77 (2003), p. 407.

[32] S. Bernal, C. Blanco, J.J. Calvino, J.M. Gatica, J.A. Pérez-Omil and J.M. Pintado, Top. Catal. 28 (2004) 31.

[33] F. Arena, G. Trunfio, J. Negro, B. Fazio, L. Spadaro, Chem. Mater. 19 (2007) 2269 .

[34] J. B. Wang, D. H. Tsai, T. J Huang, Journal of Catalysis 208 (2002) 370.

[35] Gong-Xin Qi, Xiao-Ming Zheng, Jin-Hua Fei, Zhao-Yin Hou, J.Molec. Catal. A: Chem. 176 (2001) 195.

[36] W. Liu, A.F, Sarofim, M. Flytzani-Stephanouplos, Chem. Eng. Sci. 49 (1995) 4871 .

[37] A. Martínez-Arias, J. Soria, R. Cataluña, J.C. Conesa, V. Cortés Corberán, Stud. Surf. Sci. Cat. 116 (1998) 591.

[38] A. Martínez-Arias, M. Fernández-García, J. Soria, J. C. Conesa, J. Catal. 182 (1999) 367.

[39] M. Machida, M. Uto, D. Kurogi and T. Kijima. Chem. Mater. 12 (2000) 3158 
[40] G. Qi, R. T.Yang, R.Chang, Appl. Catal. B: Environ. 51,2, (2004) 93.

[41] B. Murugan, D. Srinivas, C.S. Gopinath, V. Ramaswamy, A.V. Ramaswamy, Chem. Mater. 17 (2005) 3983.

[42] A. Wollner, F. Lange, Appl. Catal. 94 (1993) 181.

[43] P. Porta. G. Moretti, M. Musicanti and A. Nardella, Sol. Sta. Ion. 63-65 (1993) 257.

[44] Sukriti B. Kanungo, J. Catal. 58 (1978) 419.

[45] M. Paulis, L. M. Gandía, A. Gil, J. Sambeth, J. A. Odriozola, M. Montes, Appl. Catal. B: Env. 26 (2000) 37.

[46] J.A. Gonzalez-Marcos, J.I. Alvarez-Uriarte, J.I. Gutierrez-Ortiz, A.T. Aguayo, J.R. Gonzalez-Velasco, Appl. Catal. 60 (1990) 1.

[47] C.-H. Wang, S.-S. Lin, C.-L. Chen, Appl. Catal. A: Gen. 286 (2004) 227.

[48] F. Benseradj, F. Sadi, M. Chater Appl. Catal. A: Gen. 228 (2002) 135.

[49] J. Tsoua, P. Magnouxb, M. Guisnetb, J.J.M. O ' rfa oa, J.L. Figueiredo, App. Catal. B: Environ. 57 (2005) 117

[50] S. Jongpatiwut, P. Sackamduang, T. Rirksomboon, S. Osuwan, D. E. Resasco, J. Catalysis 218 (2003) 1.

[51] J. Tsou, P. Magnoux, M. Guisnet, J.J.M. Órfão, and J.L. Figueiredo, J. of Catal. 225 (2004) 147

[52] P. Papaefthimiou, T. Ioannides, X. E. Verykios, Catal. Today 54 (1999) 81.

[53] P. Papaefthimiou, T. Ioannides, X. E. Verykios, Appl. Catal. B: Environ. 15 (1998) 75 .

[54] H. Schulz, G. B. De Melo, F. Ousmanov, Combus. And Flame 118 (1999) 179.

[55] C. Freitag, S. Besselmann, E. Löffler, W. Grünert, F. Rosowski, M. Muhler, Catalysis Today 91-92 (2004) 143-147

[56] S. Esselmann, C. Freitag, O. Hinrichsen, M. Muhler, Phys. Chem. Chem. Phys. 3 (2001) 4633-4638.

[57] A.P. Antunes, M.F. Ribeiro, J.M. Silva b, F.R. Ribeiro, P. Magnoux, M. Guisnet, Appl. Catal. B: Environ. 33 (2001) 149.

[58] J.M. Padilla, G. Del Angel, J. Navarrete, Catalysis Today 133-135 (2008) 541. 
[59] G. Del Angel, J.M. Padilla, I. Cuauht'emoc, J. Navarrete, J. of Molecular Catal. A: Chem. 281 (2008) 173.

[60] D. A. Bulushev, S. I. Reshetnikov, L. Kiwi-Minsker, A. Renken, Appl. Catal. A: Gen. 220 (2001) 31. 


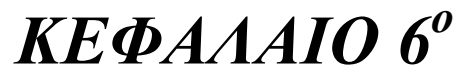

\section{KINHTIKH ME $\Lambda$ TH KAI ФAINOMENA ПAPEMПO $\Delta \mathrm{I} \Sigma \mathrm{H} \Sigma$}

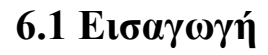

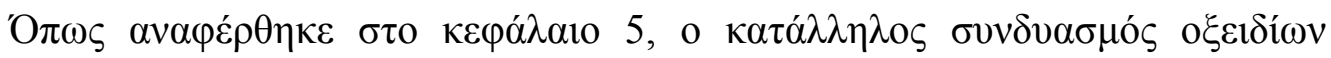

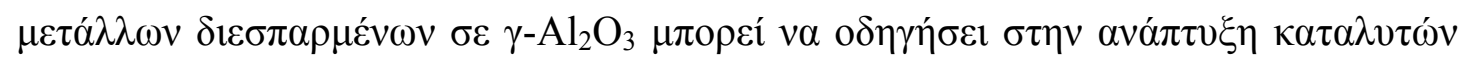

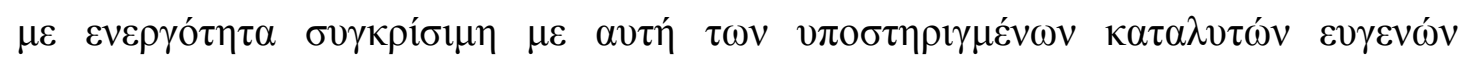
$\mu \varepsilon \tau \alpha \dot{\lambda} \lambda \lambda \omega v$.

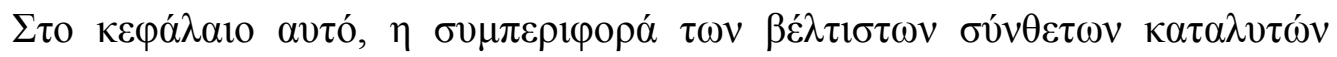

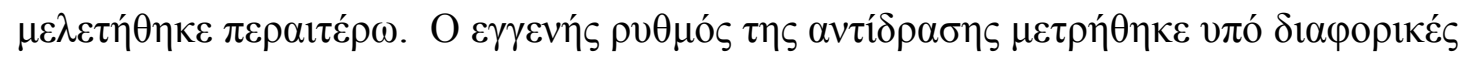

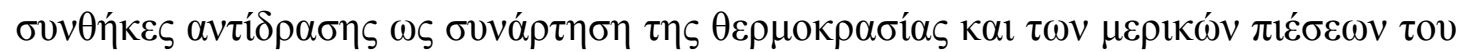

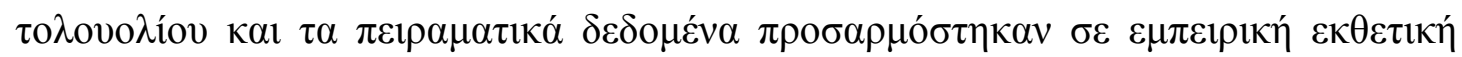

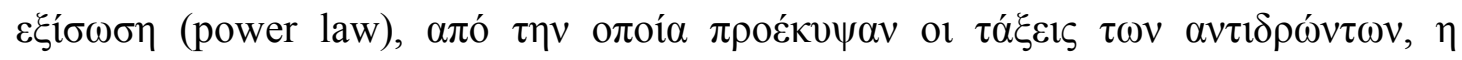

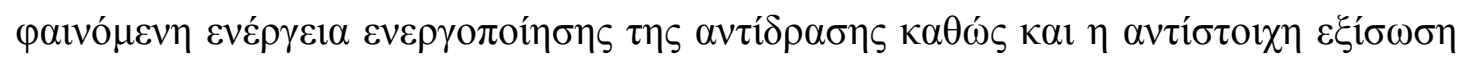

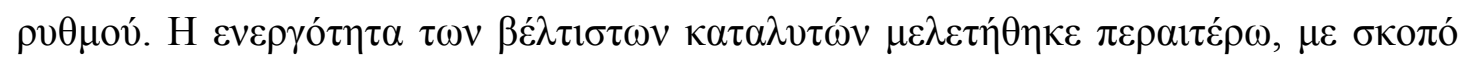

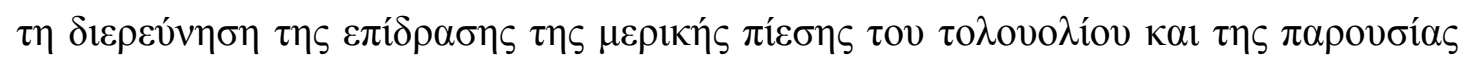

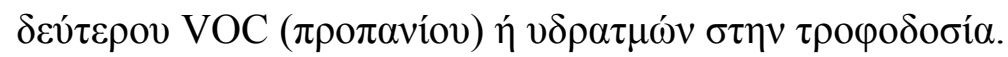




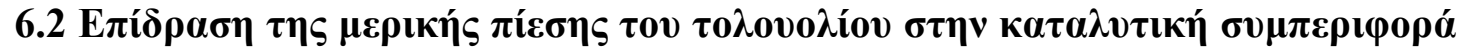

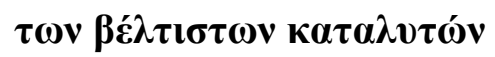

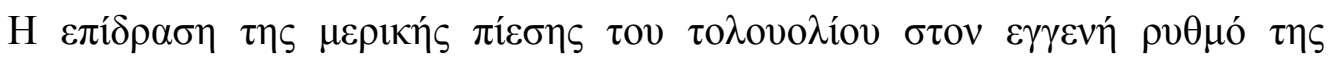

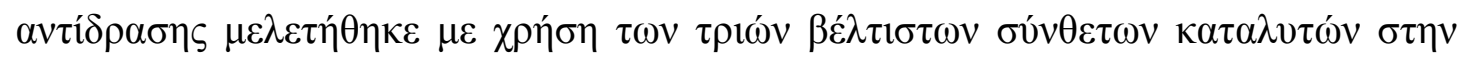

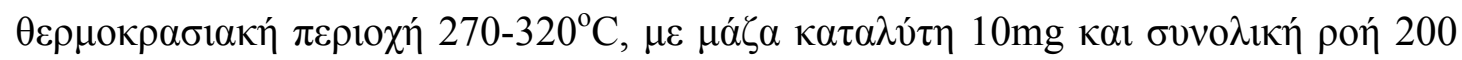

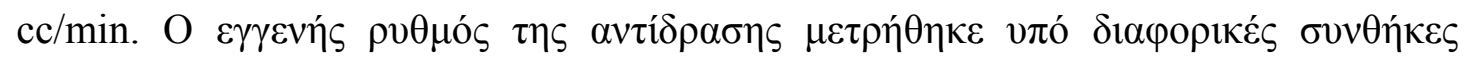

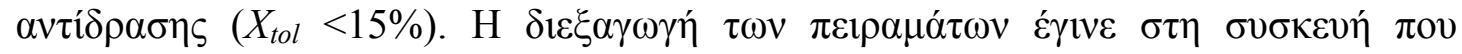

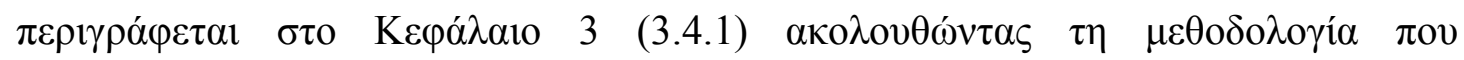

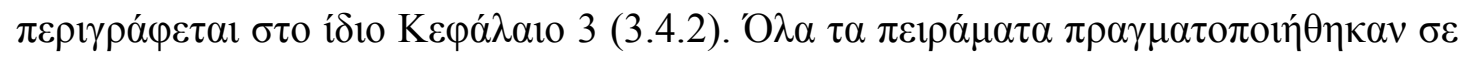

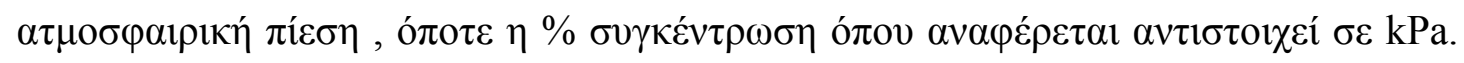

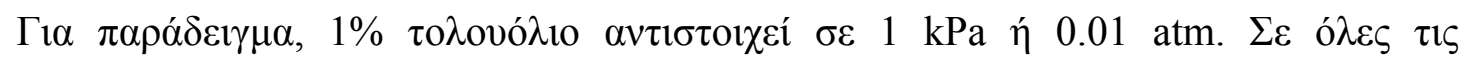

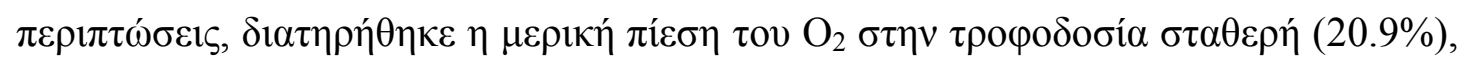

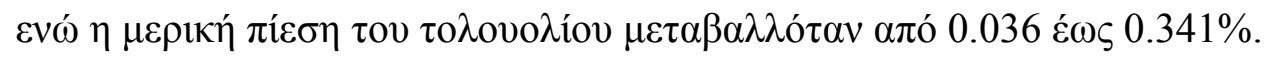

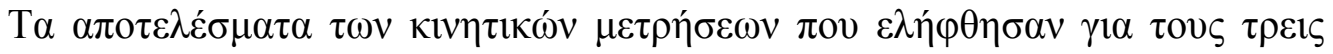

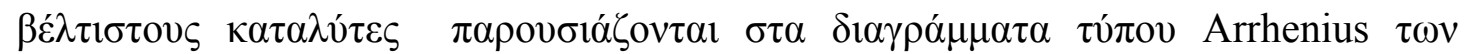

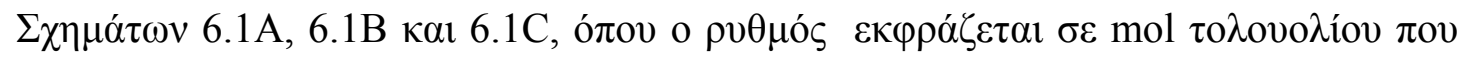

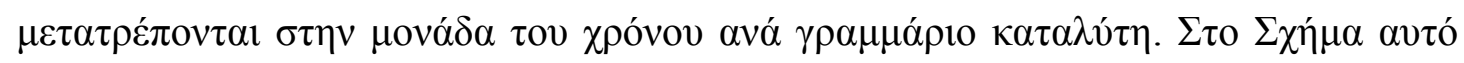

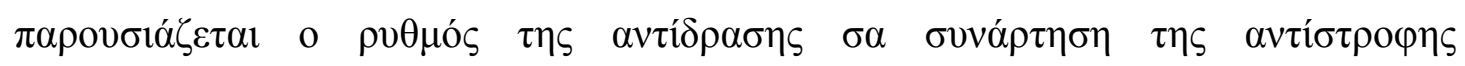

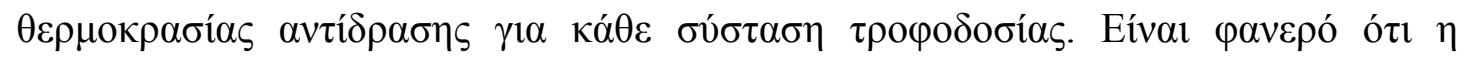

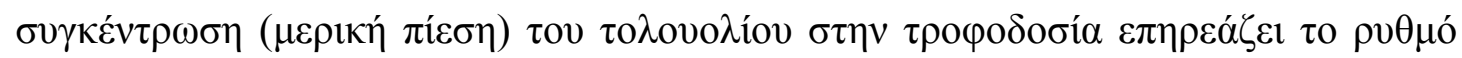

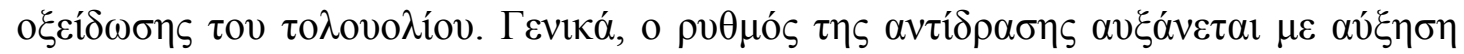

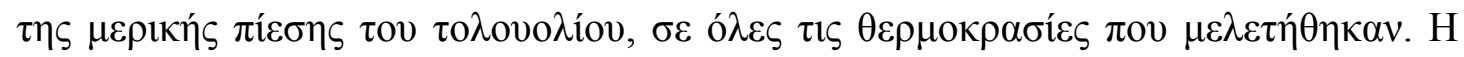

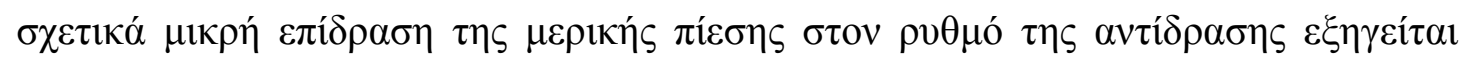

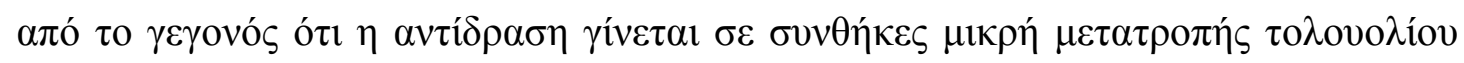

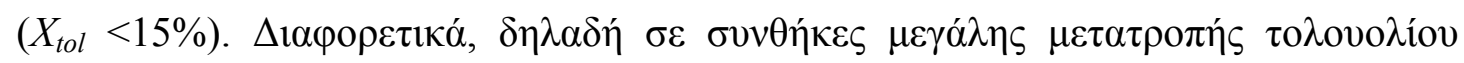

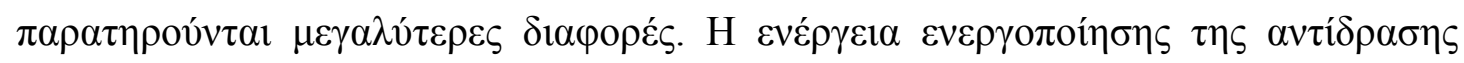

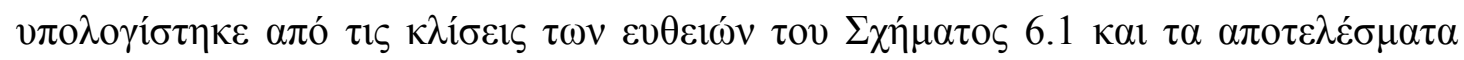

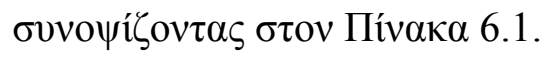



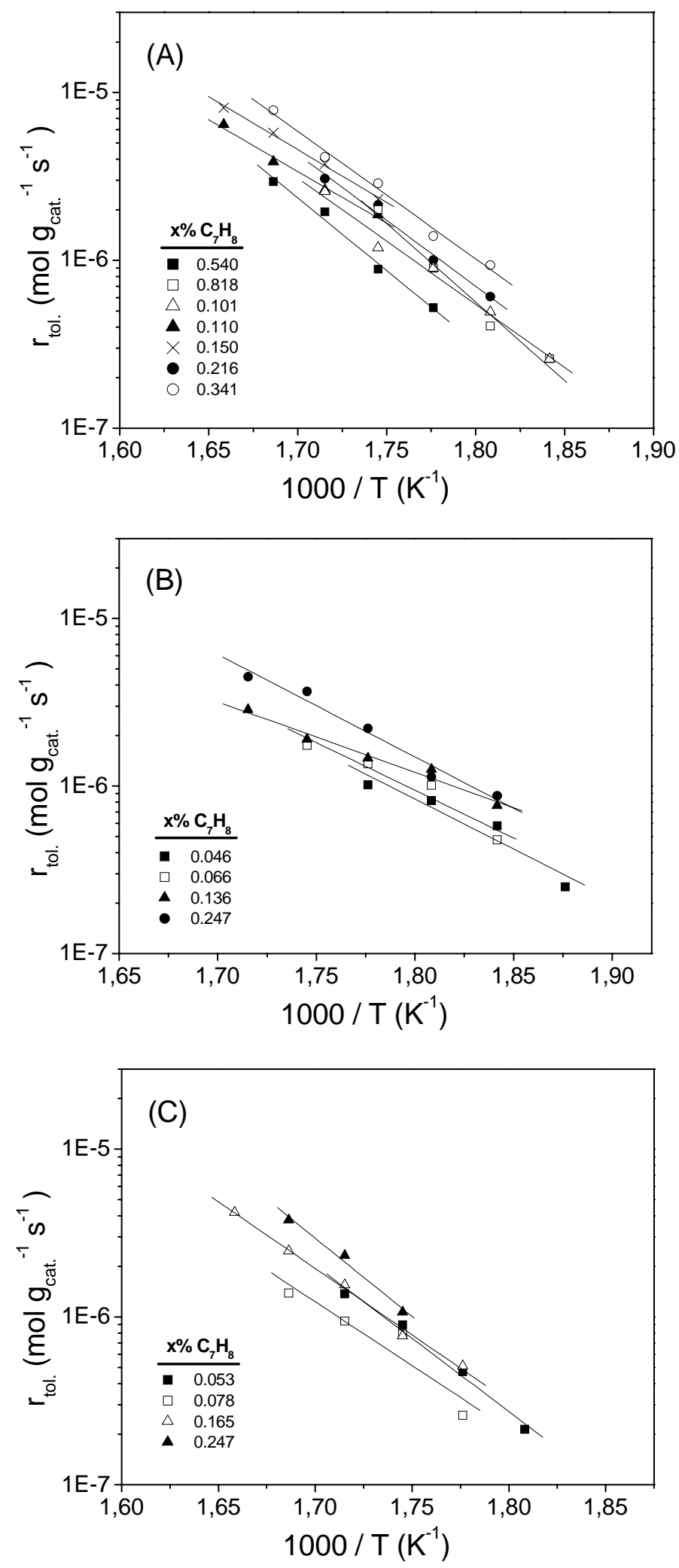

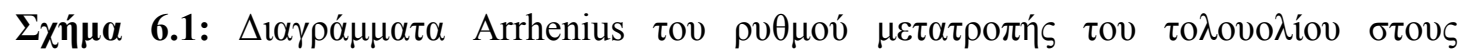

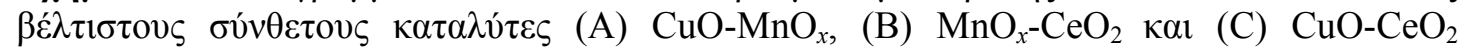

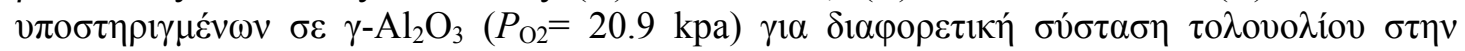

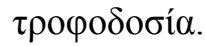




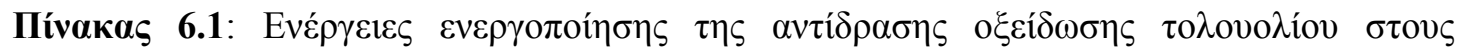

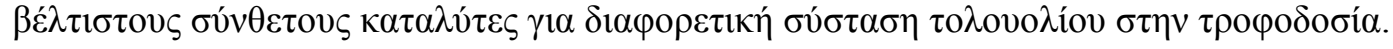

\begin{tabular}{|c|c|c|}
\hline 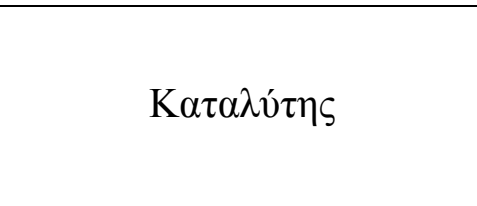 & $\% \mathrm{C}_{7} \mathrm{H}_{8}$ & 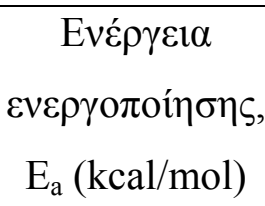 \\
\hline \multirow{7}{*}{$10 \% \mathrm{CuO}-60 \% \mathrm{MnO}_{x} / \mathrm{Al}_{2} \mathrm{O}_{3}$} & 0.0540 & 39.7 \\
\hline & 0.0818 & 42.2 \\
\hline & 0.1012 & 34.4 \\
\hline & 0.1107 & 27.2 \\
\hline & 0.1505 & 27.9 \\
\hline & 0.2160 & 35.9 \\
\hline & 0.3410 & 34.7 \\
\hline \multirow{4}{*}{$30 \% \mathrm{MnO}_{x}-50 \% \mathrm{CeO}_{2} / \mathrm{Al}_{2} \mathrm{O}_{3}$} & 0.0459 & 27.3 \\
\hline & 0.0658 & 26 \\
\hline & 0.1336 & 19.3 \\
\hline & 0.2473 & 28 \\
\hline \multirow{4}{*}{$15 \% \mathrm{CuO}-75 \% \mathrm{CeO}_{2} / \mathrm{Al}_{2} \mathrm{O}_{3}$} & 0.0534 & 39.9 \\
\hline & 0.0776 & 34.7 \\
\hline & 0.1651 & 36.3 \\
\hline & 0.2468 & 42.6 \\
\hline
\end{tabular}

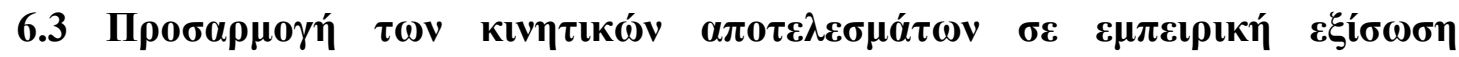
power-law

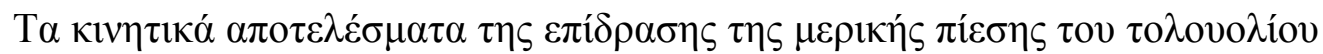

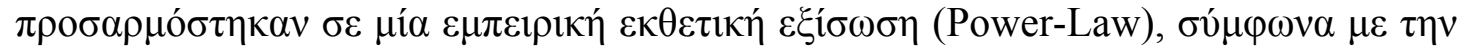

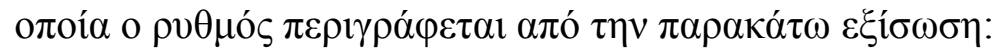

$$
r=k P_{t o l}^{a} . p_{O_{2}}^{b}
$$

$\mu \varepsilon$

$$
k=k_{0} \cdot e^{E_{a} / R \cdot T}
$$




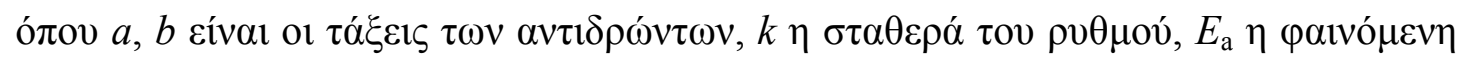

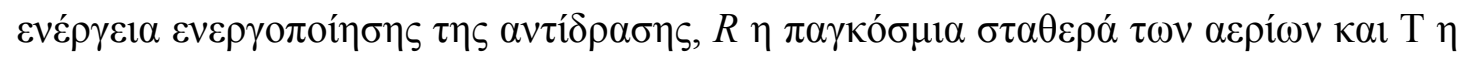

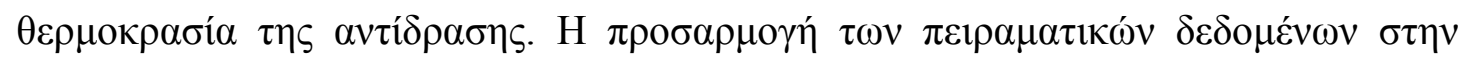

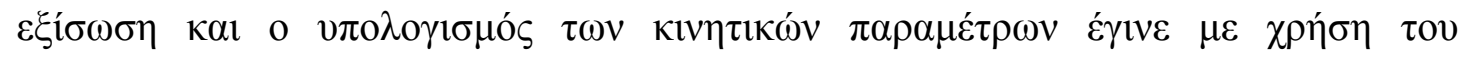

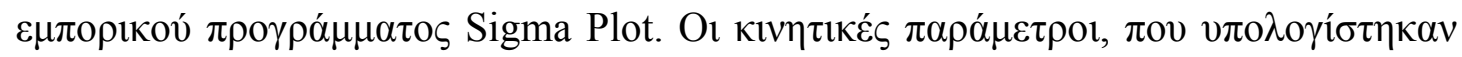

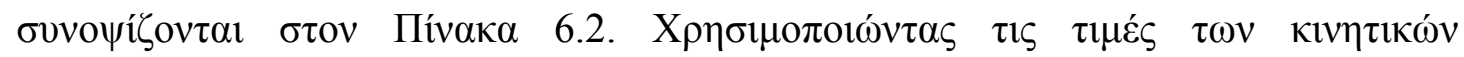

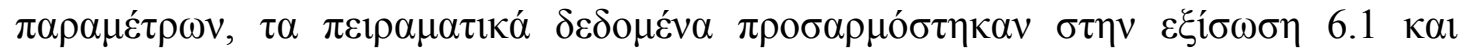

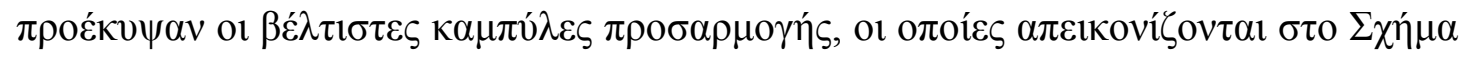

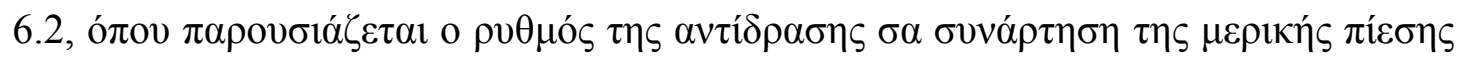

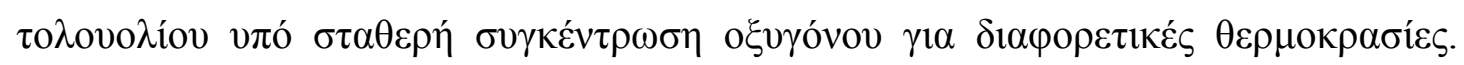

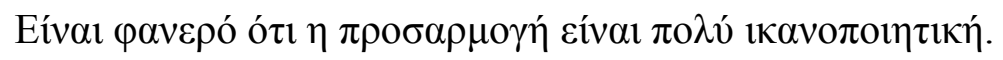

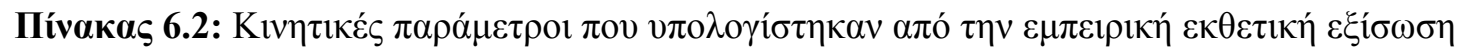

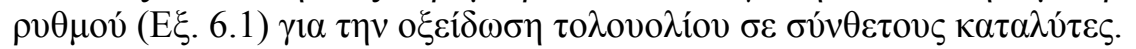

\begin{tabular}{|c|c|c|c|}
\hline \multirow[t]{2}{*}{ 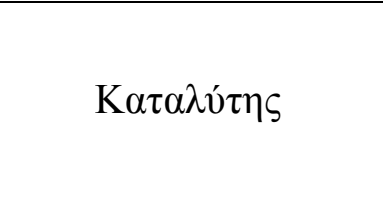 } & \multicolumn{3}{|c|}{ 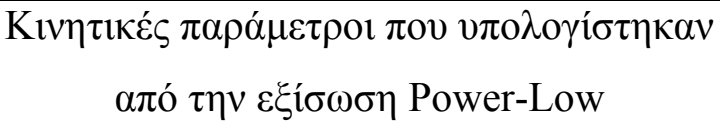 } \\
\hline & $a$ & $k P_{\mathrm{O} 2}^{b}$ & $E_{\mathrm{a}}\left(\mathrm{kcal} \mathrm{mol}^{-1}\right)$ \\
\hline $10 \% \mathrm{CuO}-60 \% \mathrm{MnO}_{x}$ & 0.38 & $3.52 \times 10^{8}$ & 36.5 \\
\hline $30 \% \mathrm{MnO}_{x}-50 \% \mathrm{CeO}_{2}$ & 0.35 & $3.36 \times 10^{3}$ & 23.2 \\
\hline $15 \% \mathrm{CuO}-75 \% \mathrm{CeO}_{2}$ & 0.34 & $3.36 \times 10^{7}$ & 34.9 \\
\hline
\end{tabular}

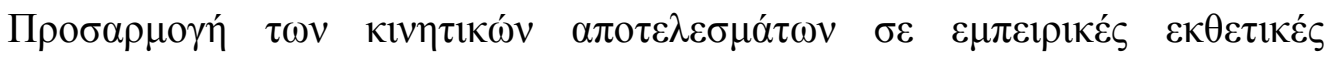

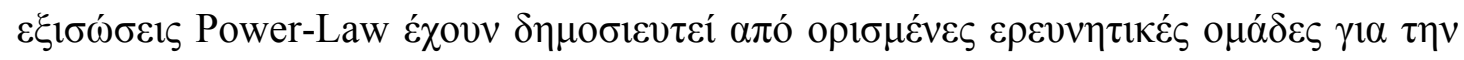

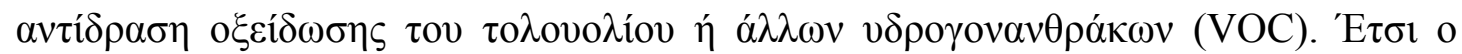

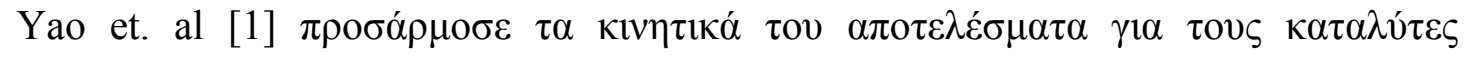

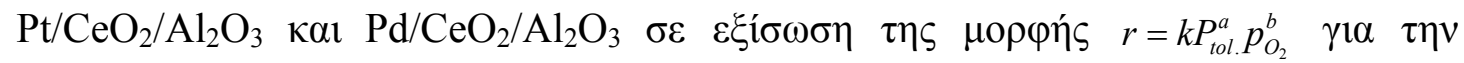

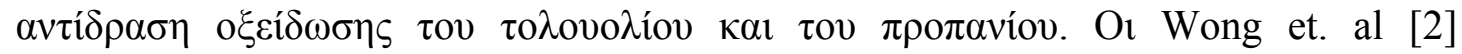

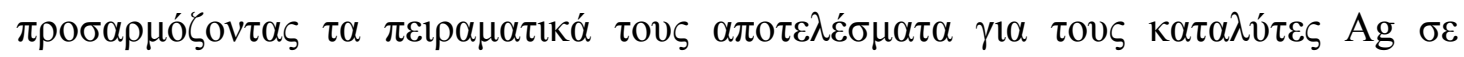

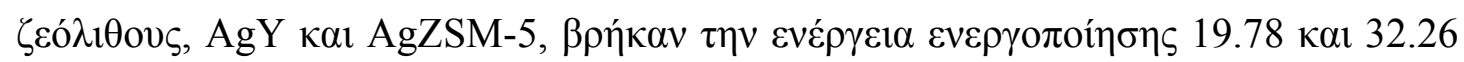

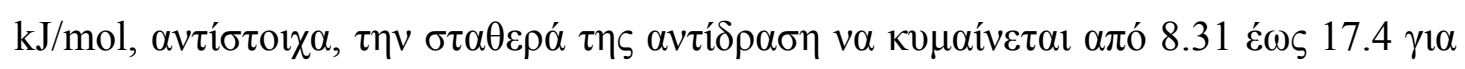

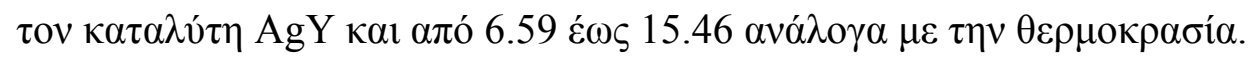



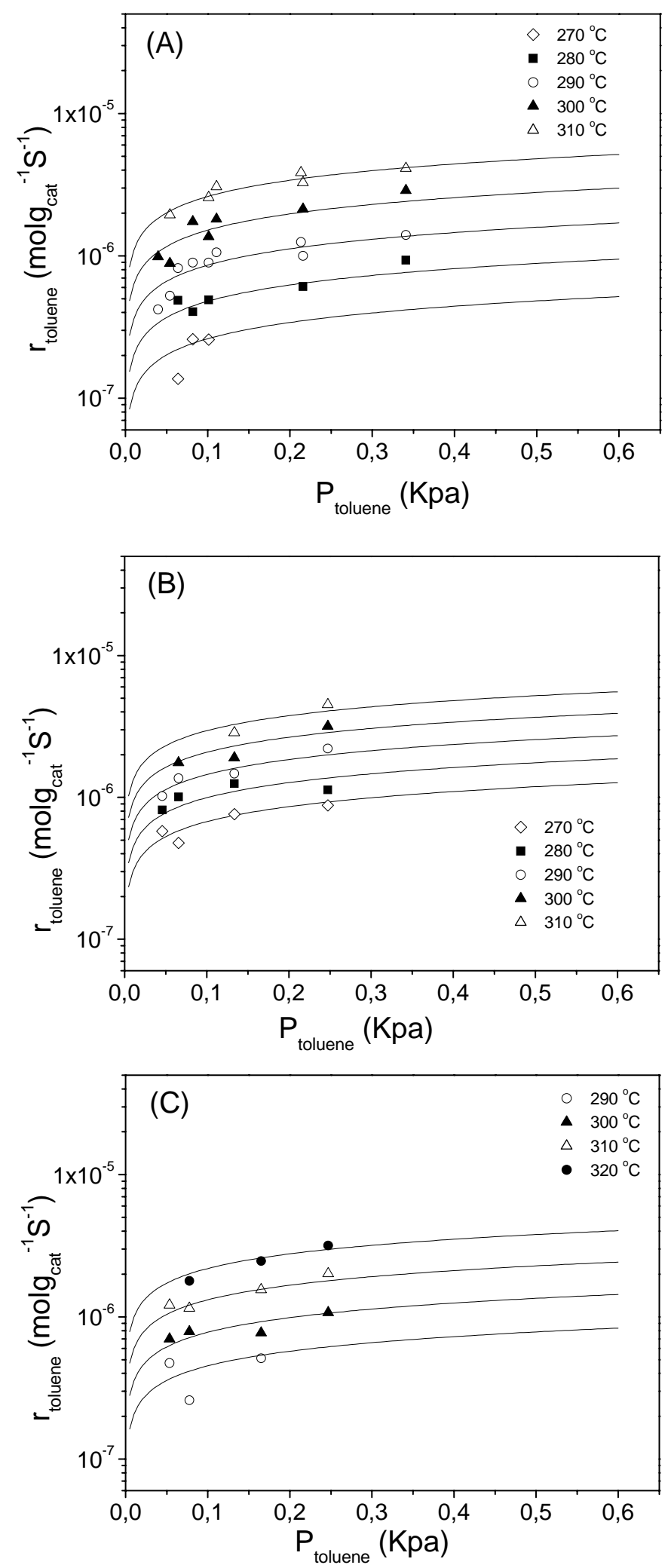

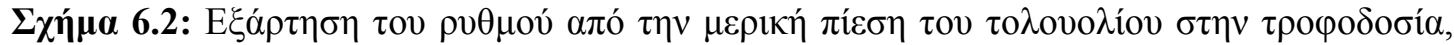

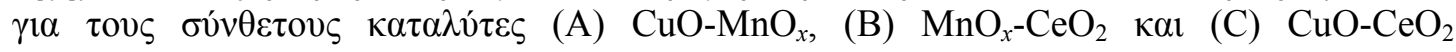

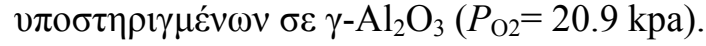




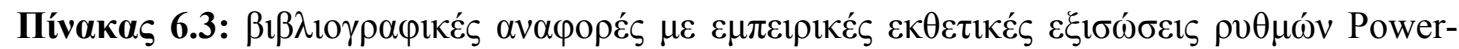
Law) $\gamma(\alpha \tau \eta v$ o $\xi \varepsilon i \delta \omega \sigma \eta \tau \omega v$ VOC.

\begin{tabular}{|c|c|c|c|c|c|c|}
\hline \multirow[t]{2}{*}{ Catalyst } & \multirow[t]{2}{*}{ VOC } & \multicolumn{3}{|c|}{$\begin{array}{c}\text { Power-Law } \\
\text { Expression Rate } \\
r=k P_{V O C .}^{a} p_{O_{2}}^{b}\end{array}$} & \multirow[t]{2}{*}{$\begin{array}{c}\text { Activation } \\
\text { Energy } \\
(\mathrm{kcal} / \mathrm{mol})\end{array}$} & \multirow[t]{2}{*}{ Reference } \\
\hline & & $\mathrm{a}$ & $\mathrm{b}$ & $\mathrm{k}$ & & \\
\hline \multirow{3}{*}{$\mathrm{Pt} / \mathrm{CeO}_{2} / \mathrm{Al}_{2} \mathrm{O}_{3}$} & Toluene & 0.2 & 0.7 & - & 22 & \multirow{6}{*}[1]{} \\
\hline & Propane & 0.1 & 0.8 & - & 19 & \\
\hline & 1-Hexane & 0.22 & 0.5 & - & 22 & \\
\hline \multirow{3}{*}{$\mathrm{Pd} / \mathrm{CeO}_{2} / \mathrm{Al}_{2} \mathrm{O}_{3}$} & Toluene & -0.3 & 0.6 & - & 18 & \\
\hline & Propane & -0.2 & 0.6 & - & 19 & \\
\hline & 1-Hexane & 0.2 & 2 & - & 18 & \\
\hline $\mathrm{AgY}$ & \multirow[b]{2}{*}{ butyl acetate } & 1.09 & 0 & 9.25 & 19.78 & \multirow[b]{2}{*}[2]{} \\
\hline AgZSM-5 & & 1.09 & 0 & $\begin{array}{c}14.5 \\
3\end{array}$ & 32.26 & \\
\hline \multirow{3}{*}{$\mathrm{Pt} / \mathrm{Al}_{2} \mathrm{O}_{3}$} & Benzene & 0.22 & 0.15 & - & 96 & \multirow{9}{*}[3]{} \\
\hline & Butanol & 0.84 & -0.61 & - & 100 & \\
\hline & Ethyl acetate & 0.41 & -0.12 & - & 121 & \\
\hline \multirow{3}{*}{$\mathrm{Pd} / \mathrm{Al}_{2} \mathrm{O}_{3}$} & Benzene & 0.53 & -0.15 & - & 92 & \\
\hline & Butanol & 0.94 & -0.73 & - & 71 & \\
\hline & Ethyl acetate & 0.19 & 0.02 & - & 121 & \\
\hline \multirow{3}{*}{$\mathrm{CO} / \mathrm{Al}_{2} \mathrm{O}_{3}$} & Benzene & 0.06 & 0.1 & - & 100 & \\
\hline & Butanol & 0.25 & 0.12 & - & 125 & \\
\hline & Ethyl acetate & -0.31 & 0.63 & - & 151 & \\
\hline \multirow{2}{*}{$\mathrm{Cu} / \mathrm{Al}_{2} \mathrm{O}_{3}$} & acetic acid & 0.12 & 0.48 & - & & \multirow{2}{*}{ [4] } \\
\hline & $i$-butyric acid & 0.46 & 0.5 & - & & \\
\hline $\mathrm{Pt} / \mathrm{TiO}_{2}$ & \multirow{2}{*}{ Ethyl acetate } & 0.35 & -0.13 & - & 114 & \multirow{2}{*}[5]{} \\
\hline $\mathrm{Pt} / \mathrm{TiO}_{2}\left(\mathrm{~W}^{6+}\right)$ & & 0.38 & -0.22 & - & 117 & \\
\hline
\end{tabular}




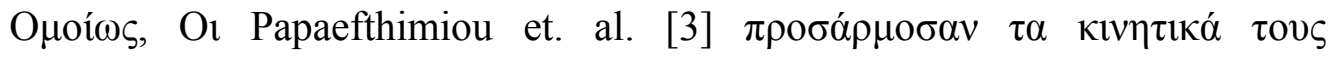

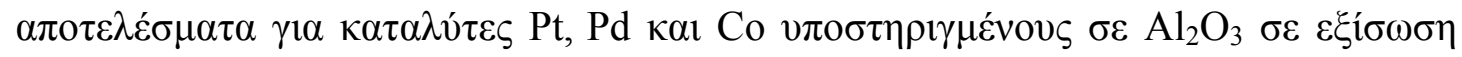

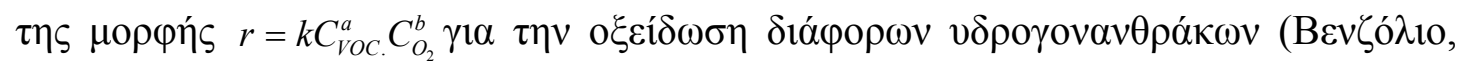

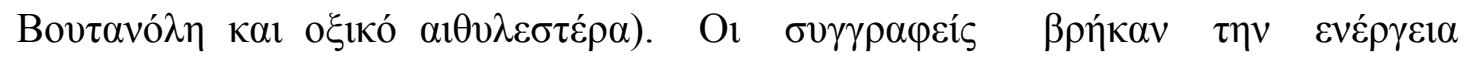

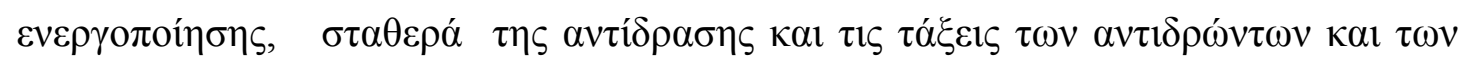

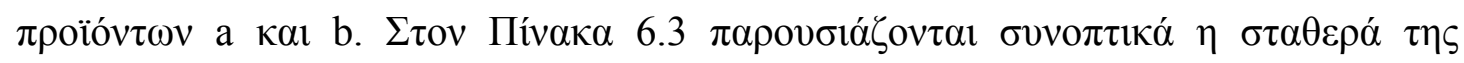

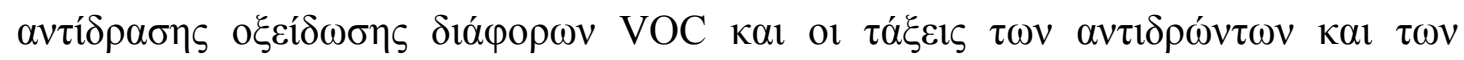

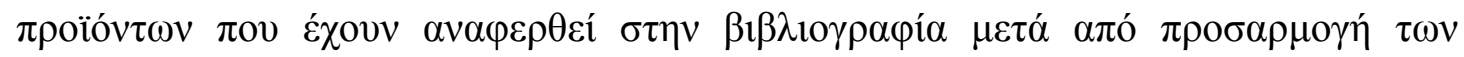

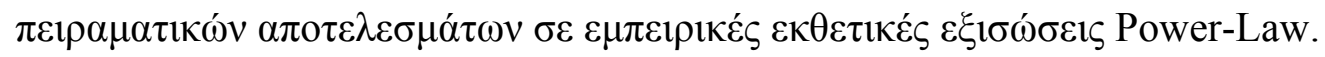

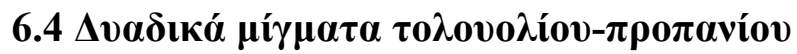

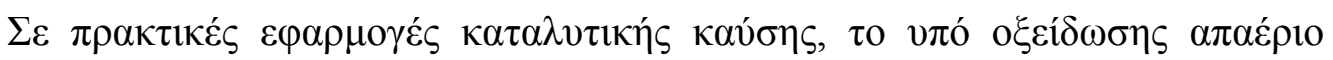

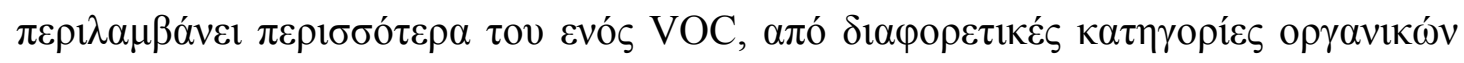

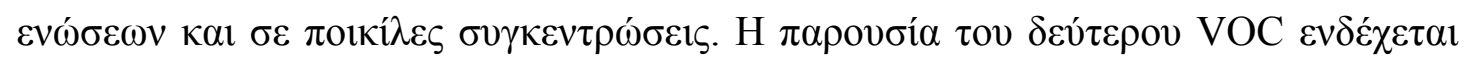

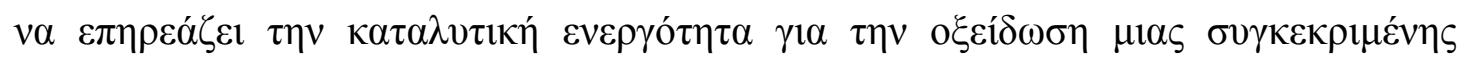

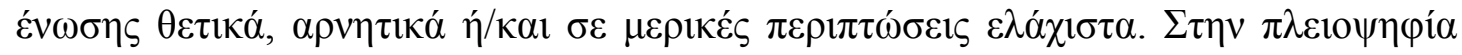

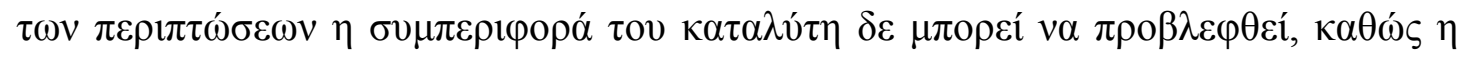

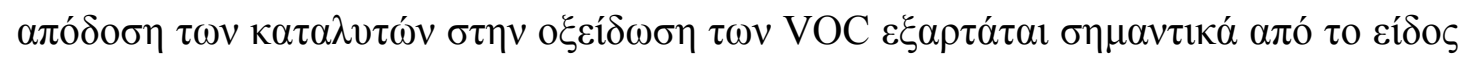

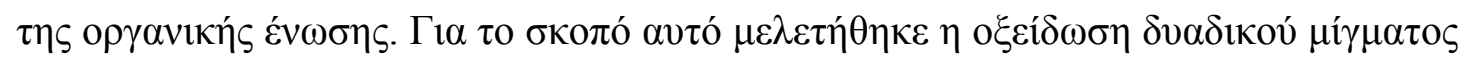

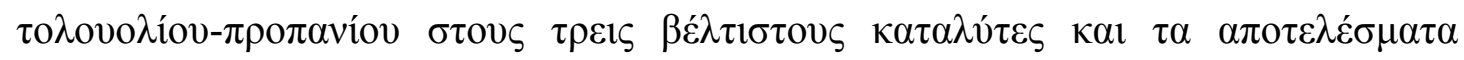

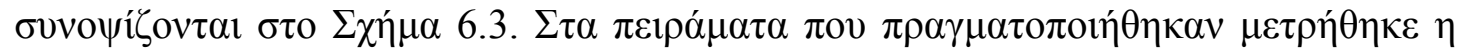

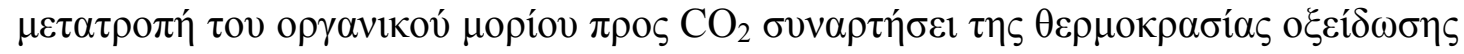

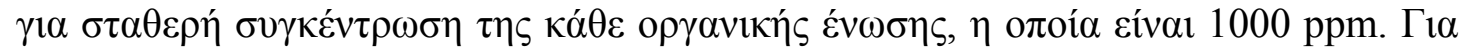
$\lambda$

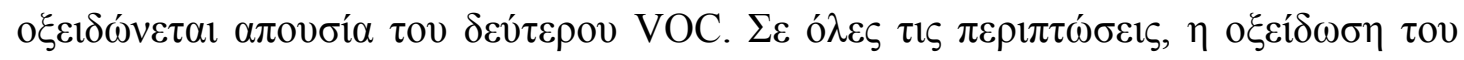

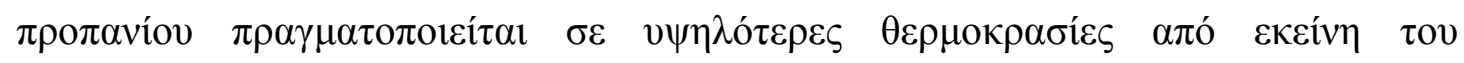

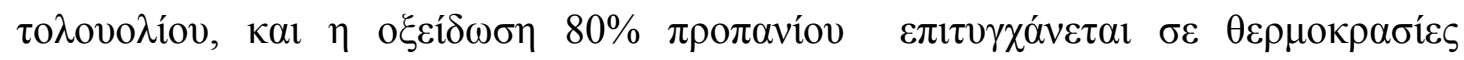

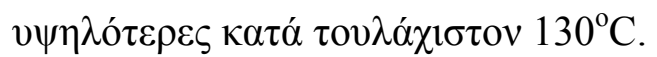

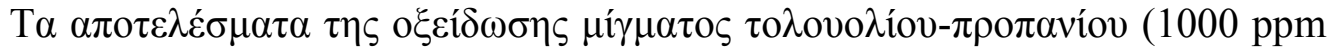

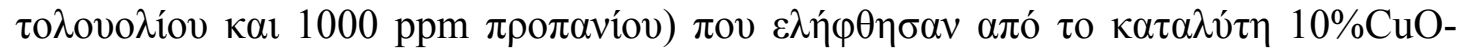

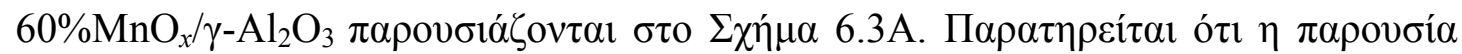

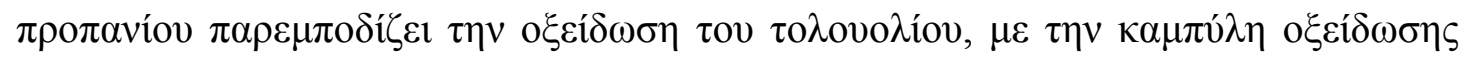

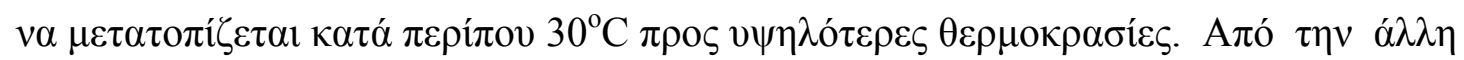



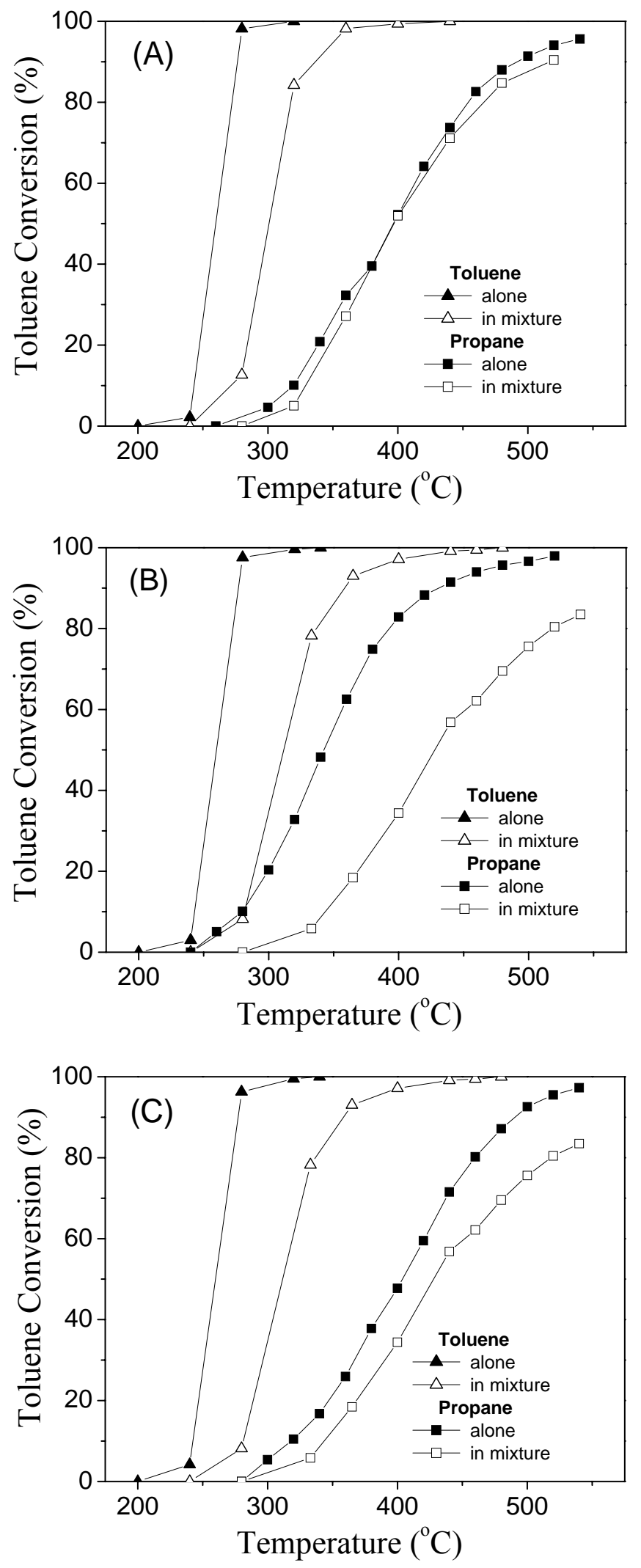

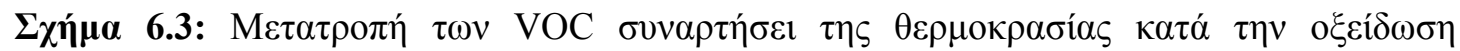

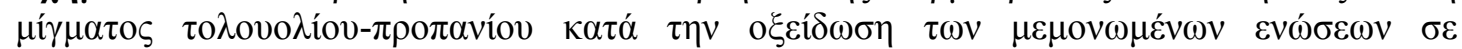

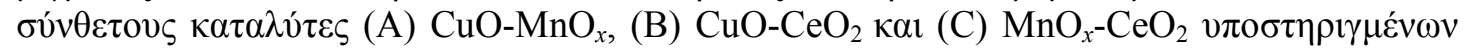
$\sigma \varepsilon \gamma-\mathrm{Al}_{2} \mathrm{O}_{3}$. 


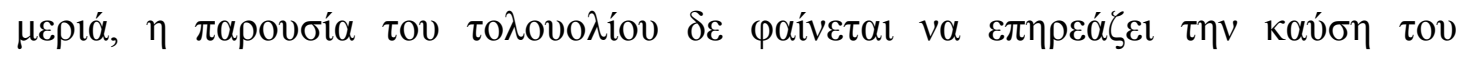

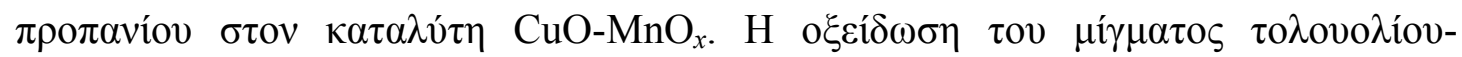

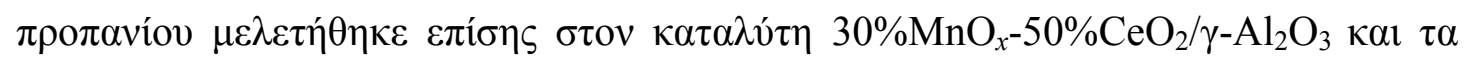

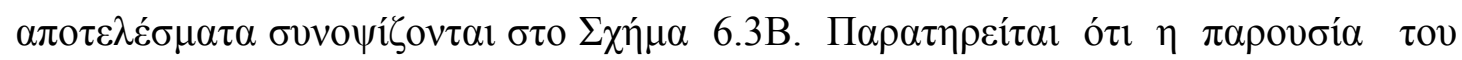

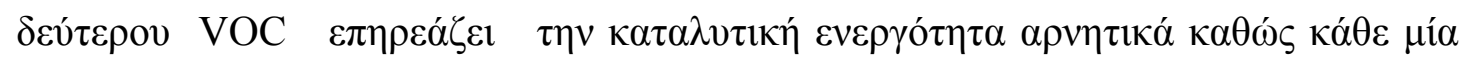

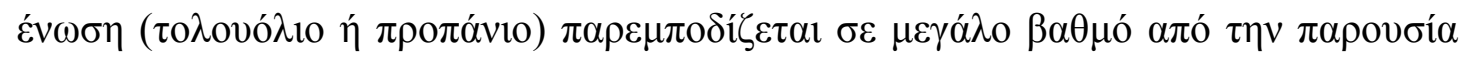

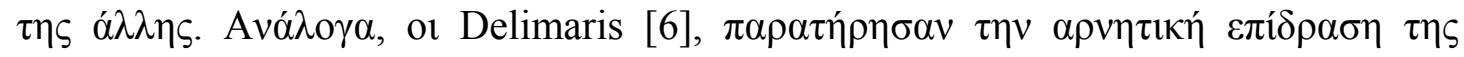

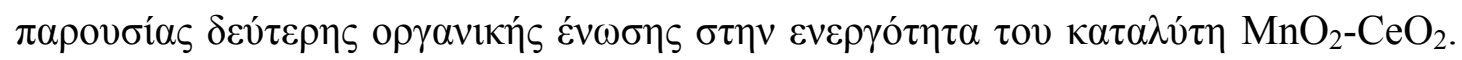

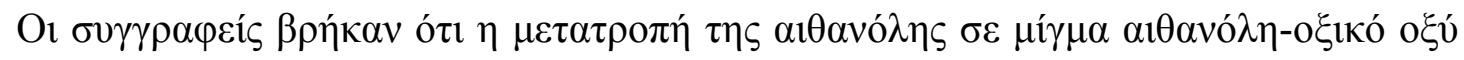

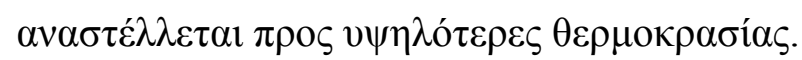

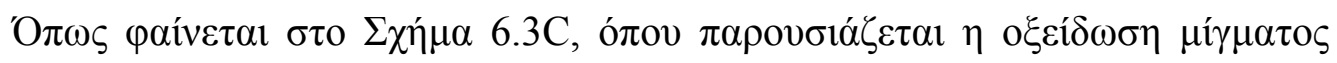

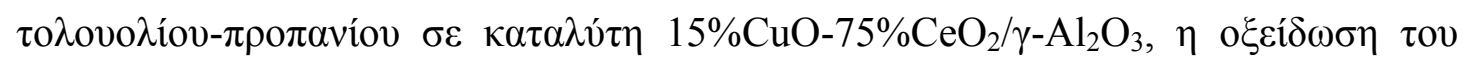

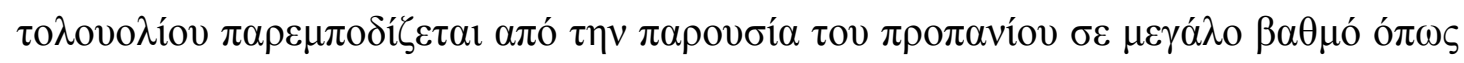

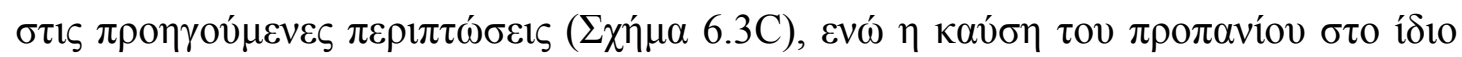

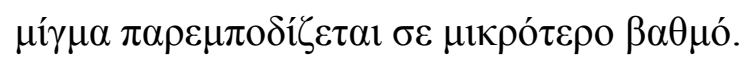

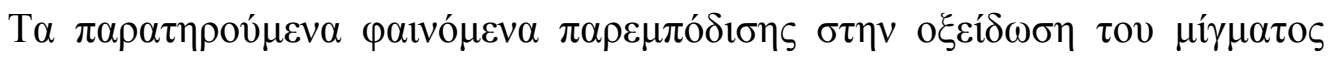

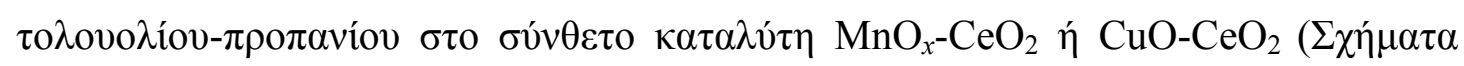

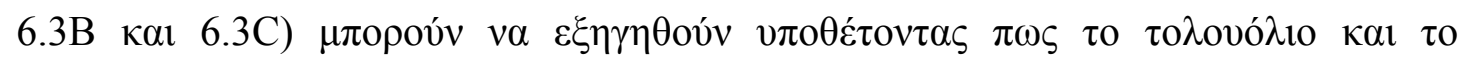

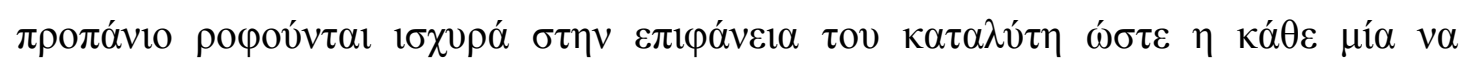

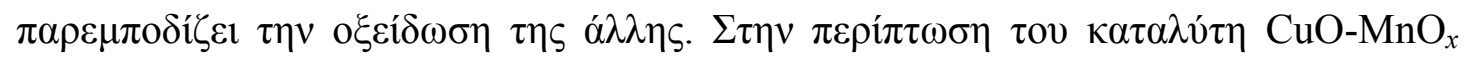

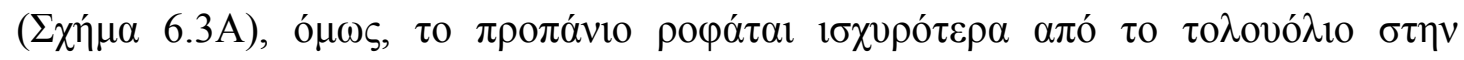

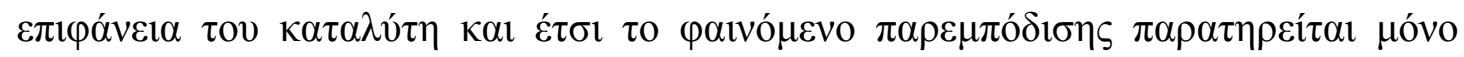

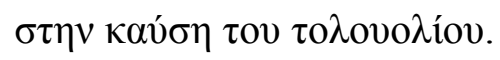

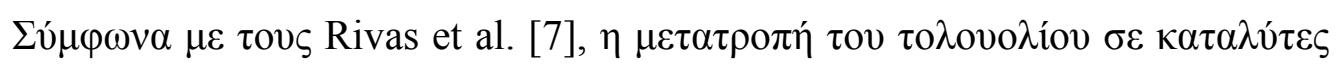

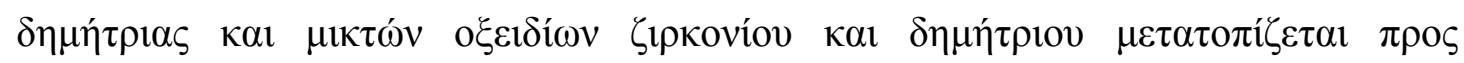

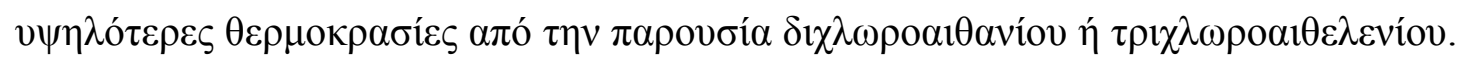

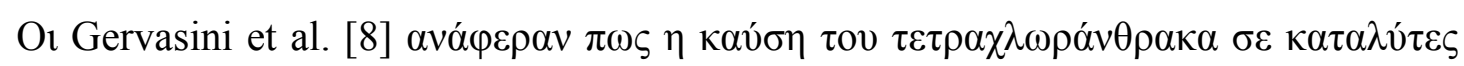

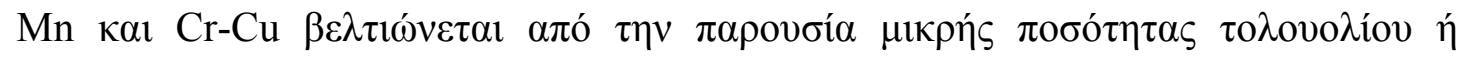

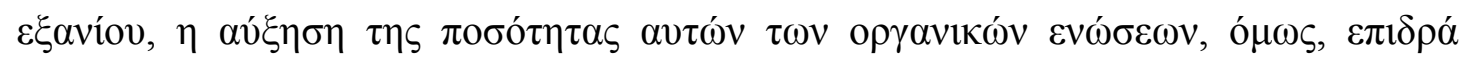

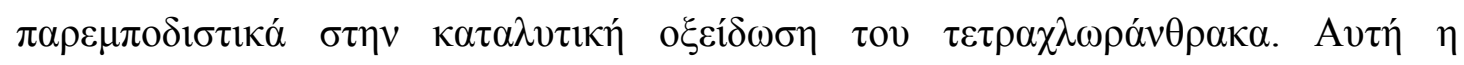

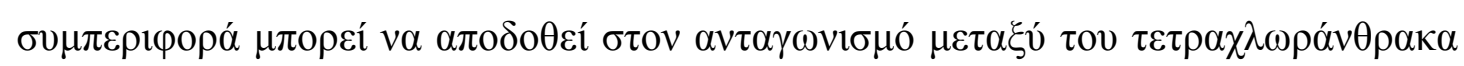

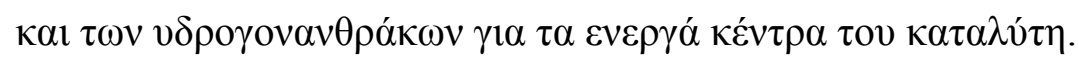



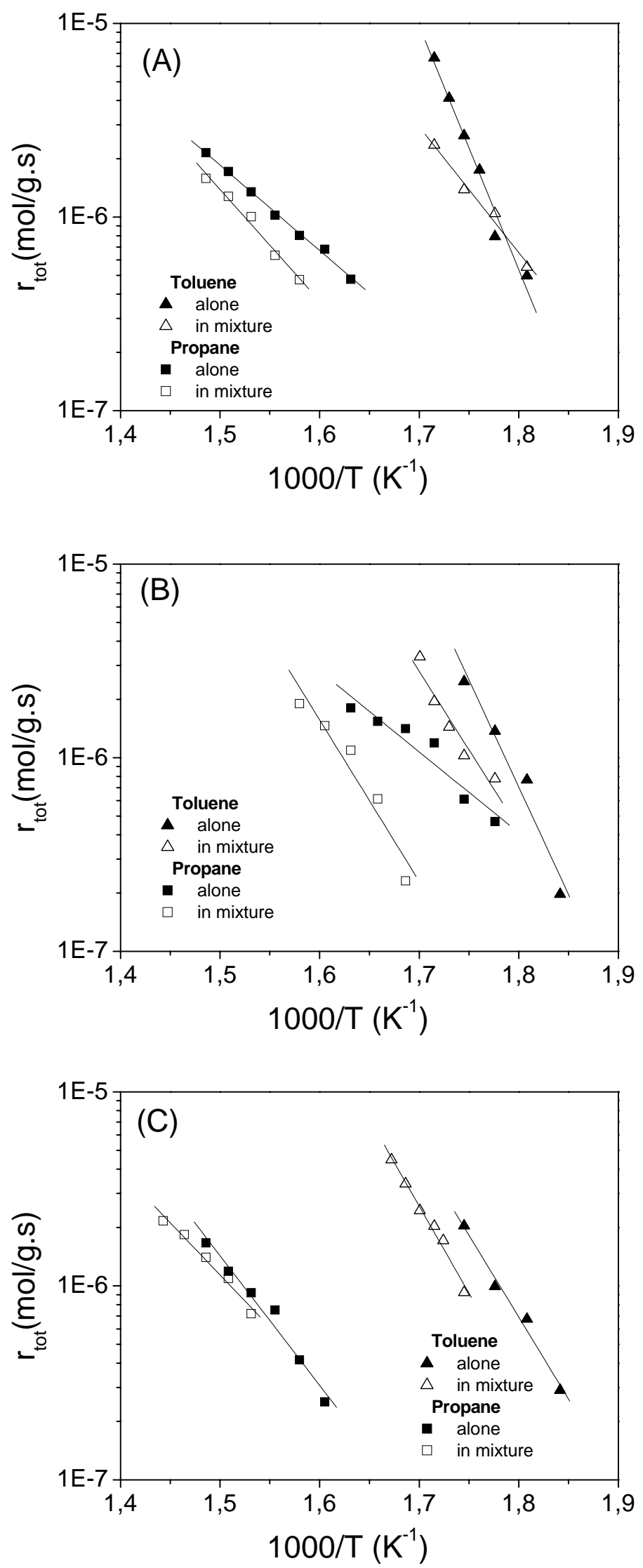

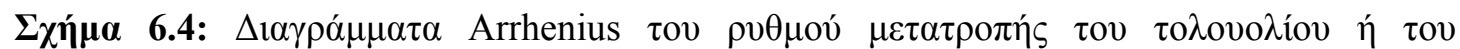

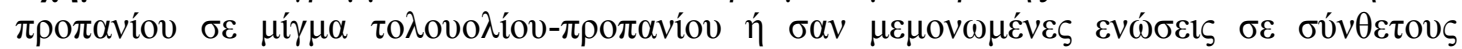

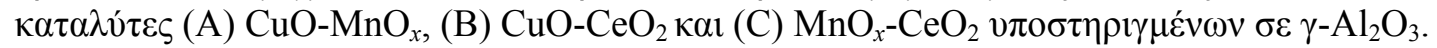




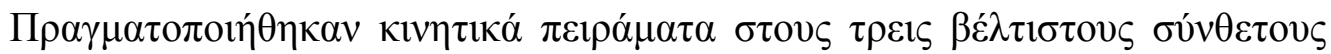

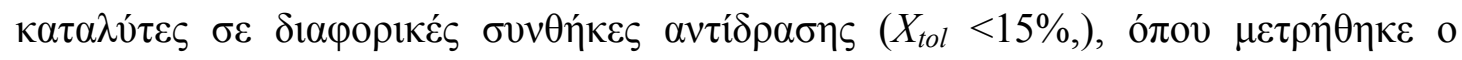

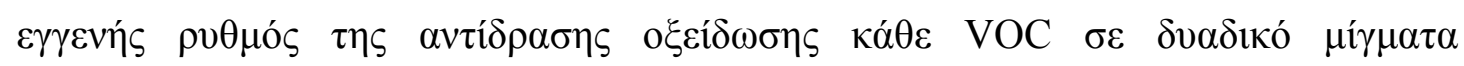

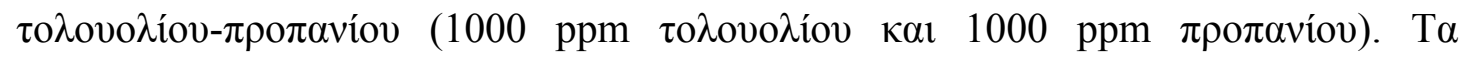

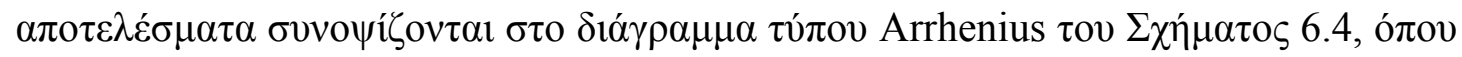

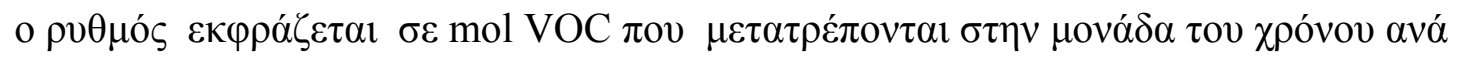

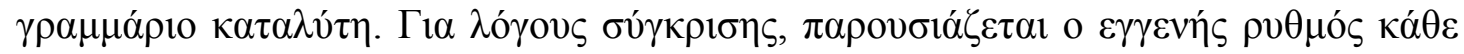

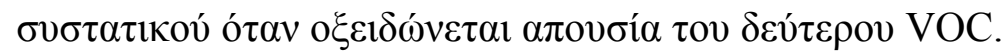

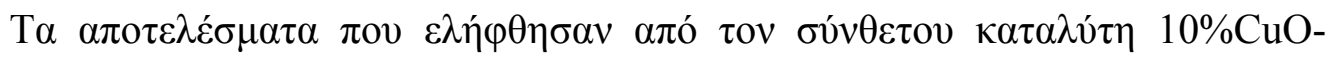

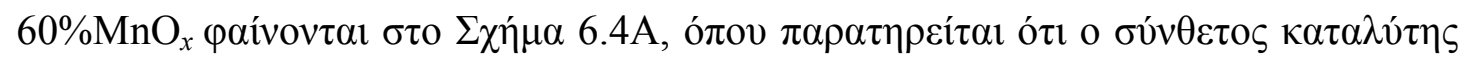

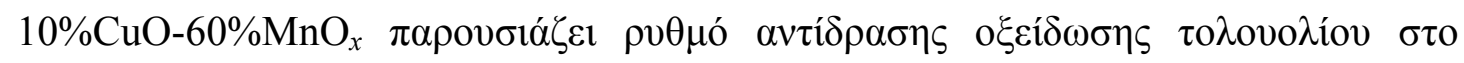

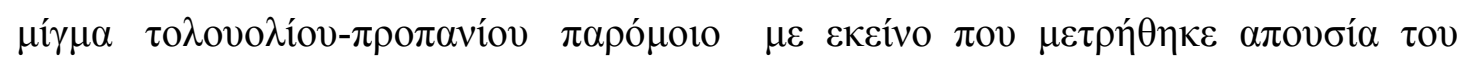

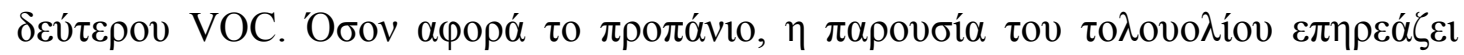

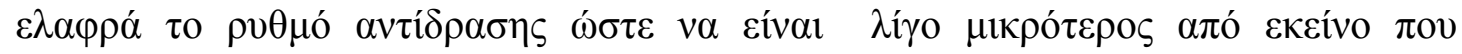

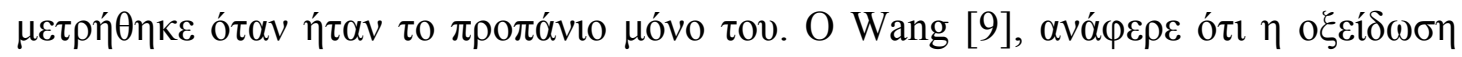

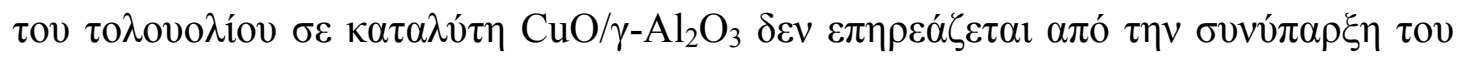

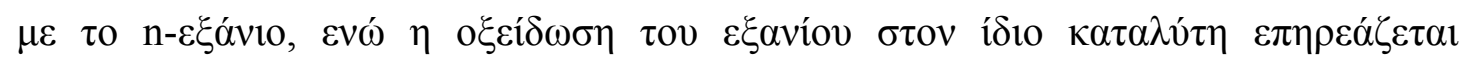

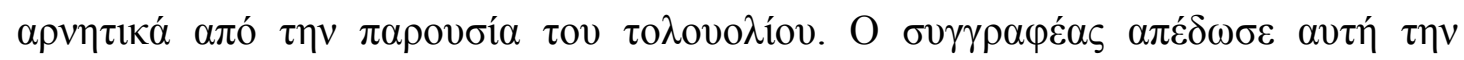

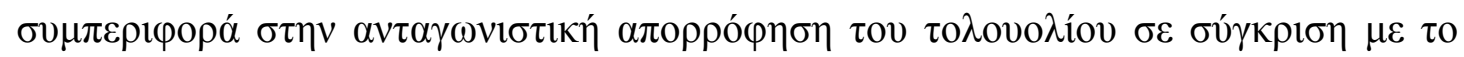
$\varepsilon \xi \alpha ́ v 10$.

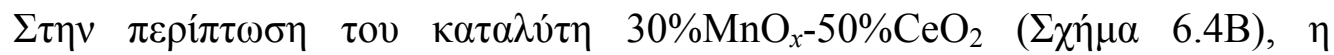

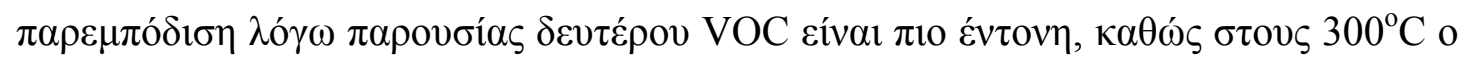

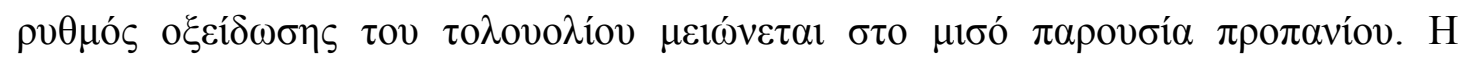

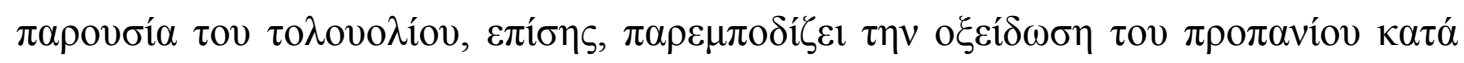

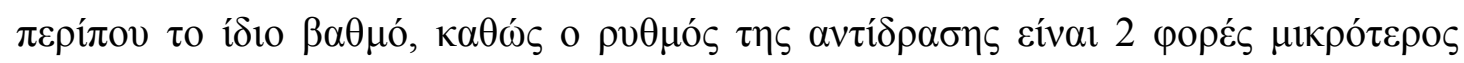

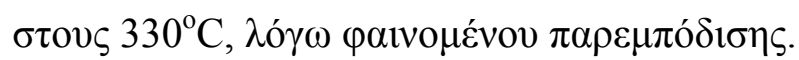

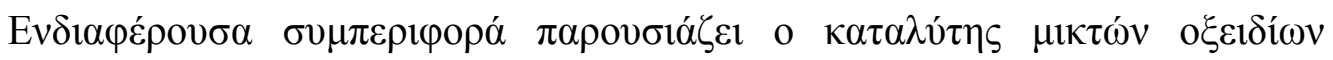

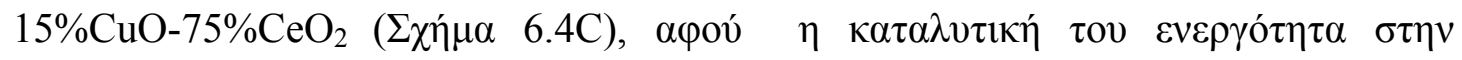

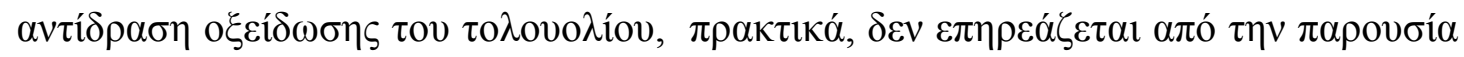

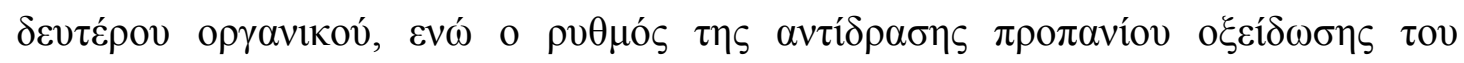

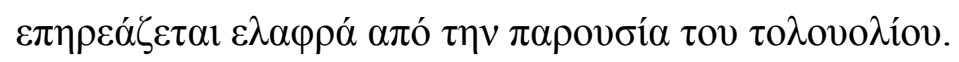




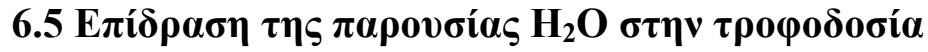

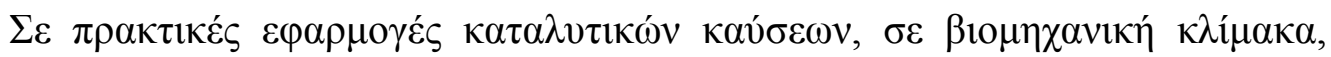

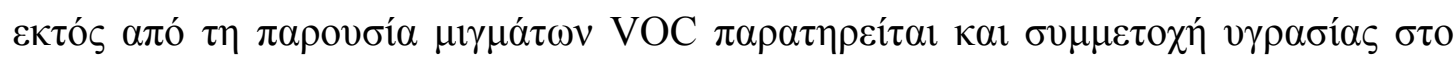

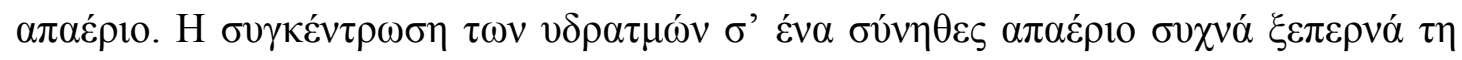

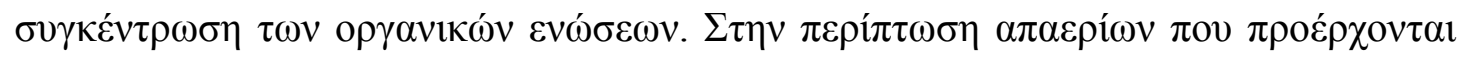

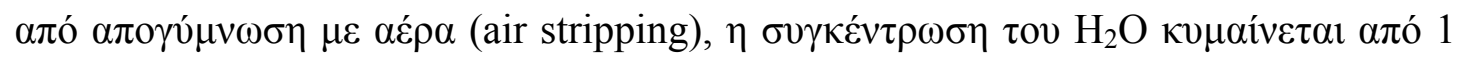

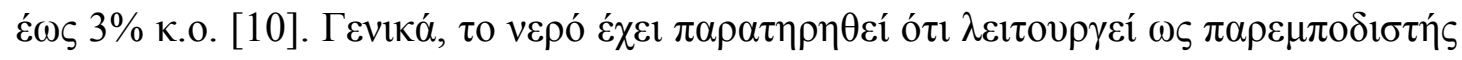

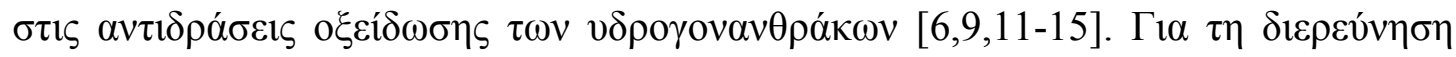

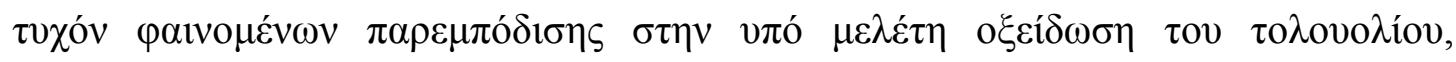

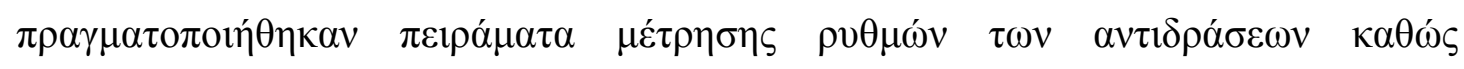

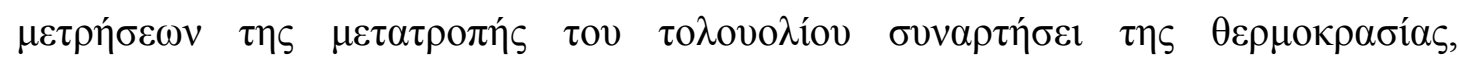

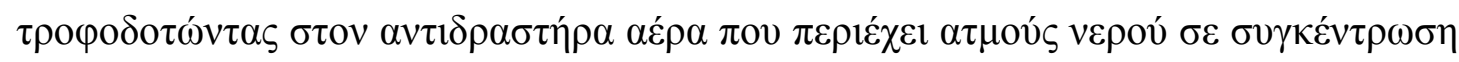
$1-5 \%$ к.о. $(10,000-50,000 \mathrm{ppm})$.

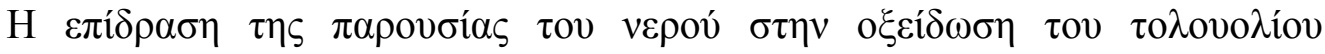

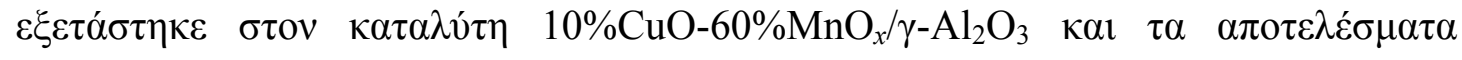

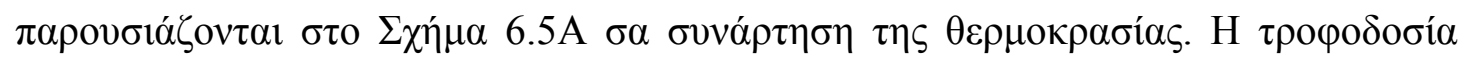

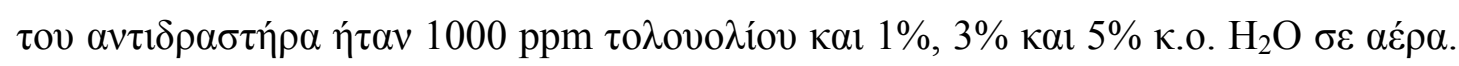

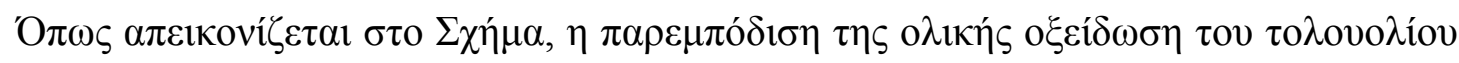

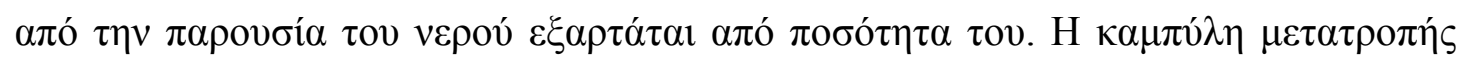

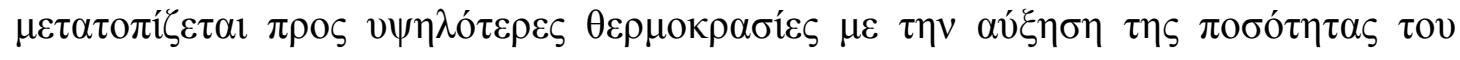

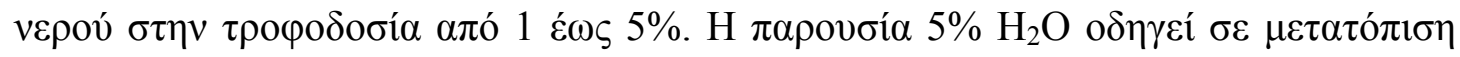

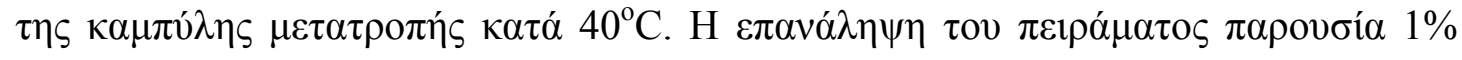

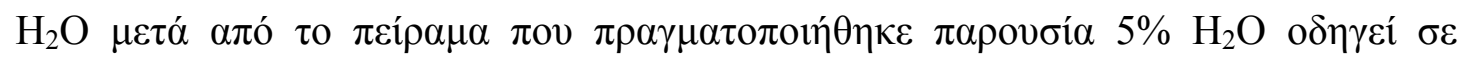

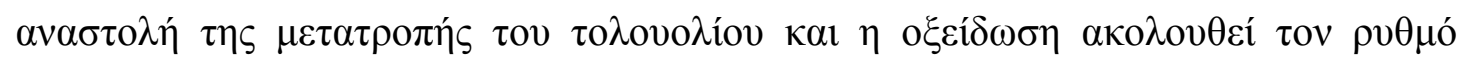

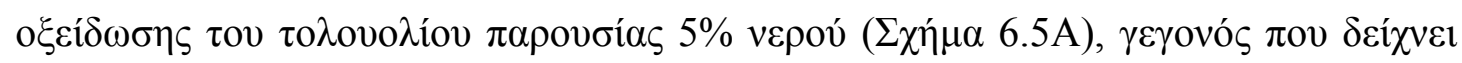

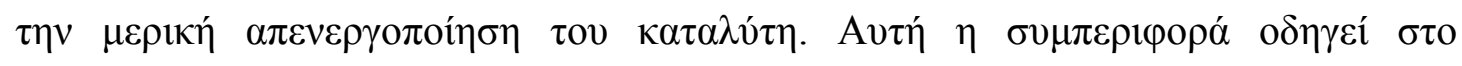

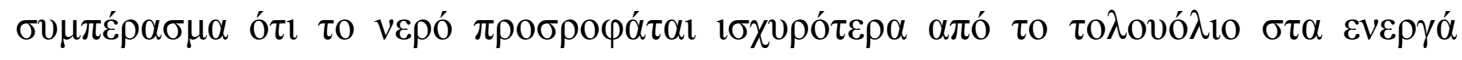

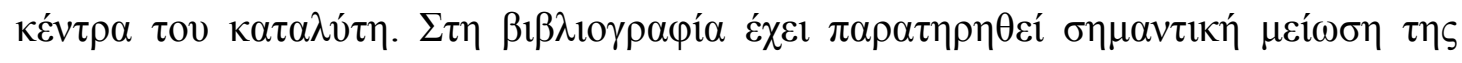

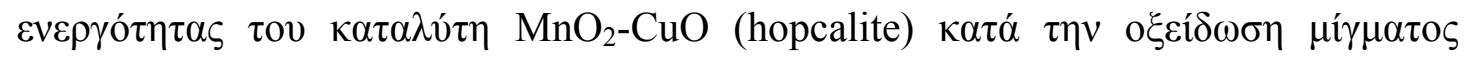

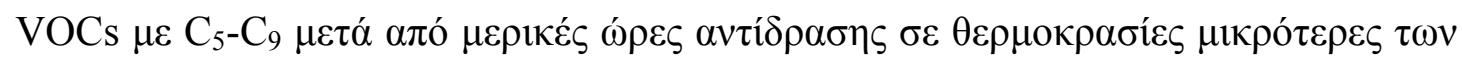
$300^{\circ} \mathrm{C}[14]$.

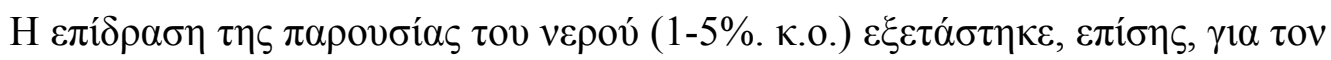

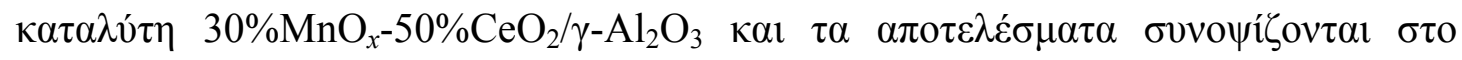

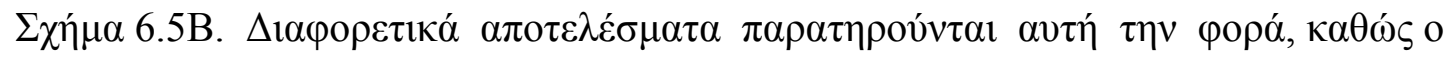



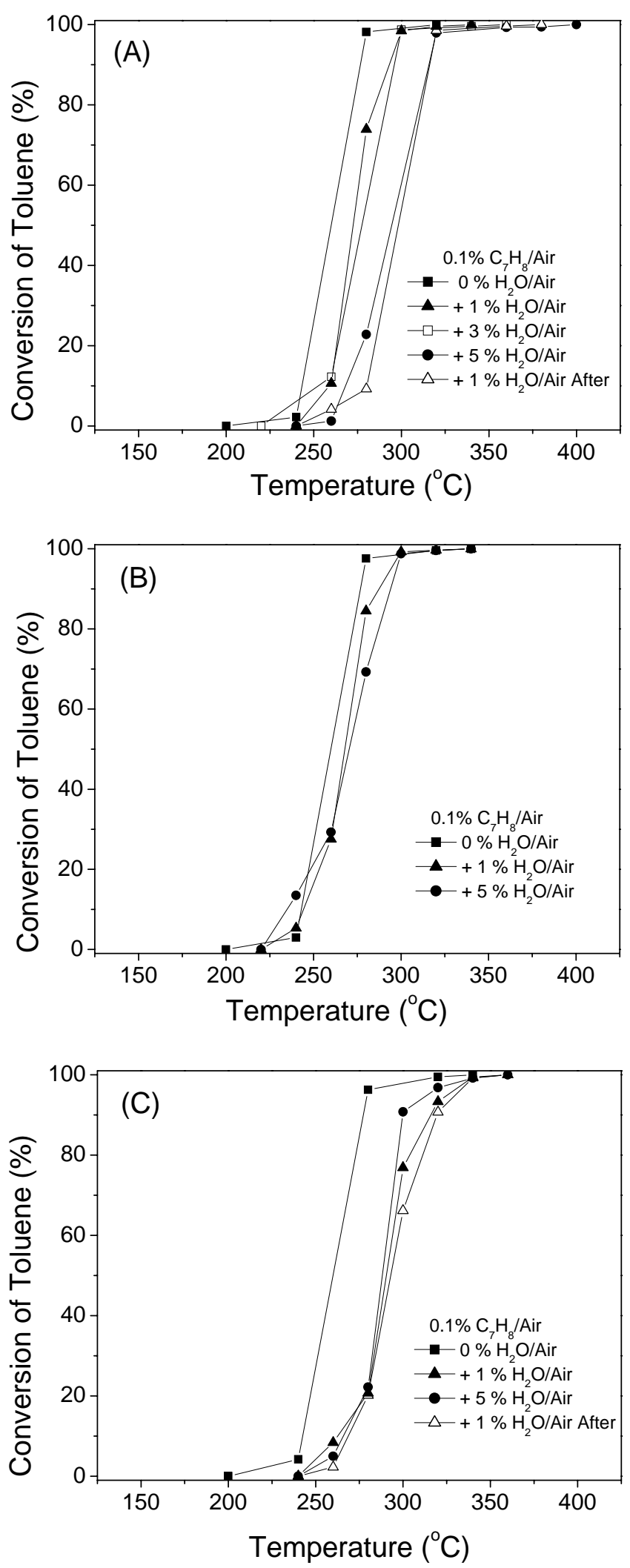

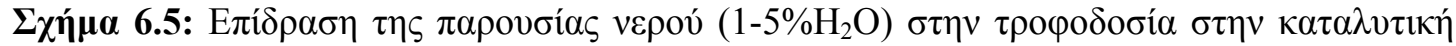

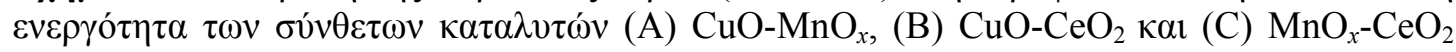
$v \pi \circ \sigma \tau \eta \rho \gamma \mu \varepsilon ́ v \omega v \sigma \varepsilon \gamma-\mathrm{Al}_{2} \mathrm{O}_{3}$. 


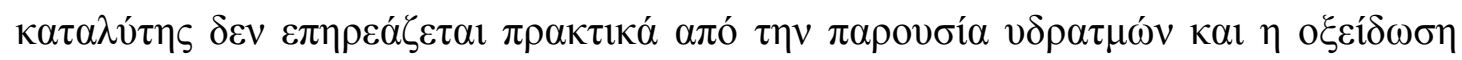

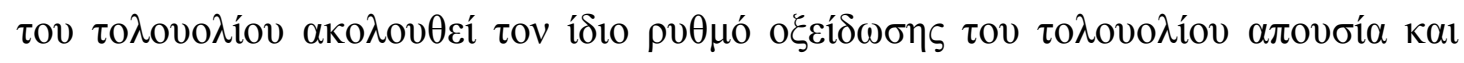

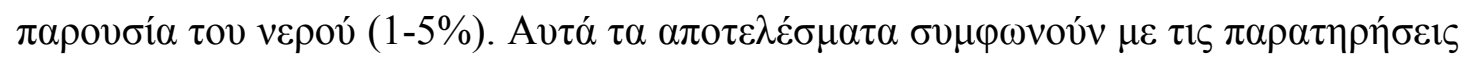

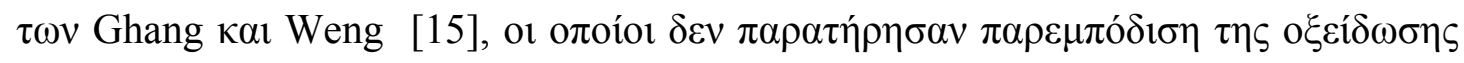

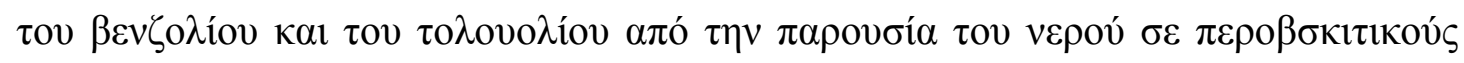

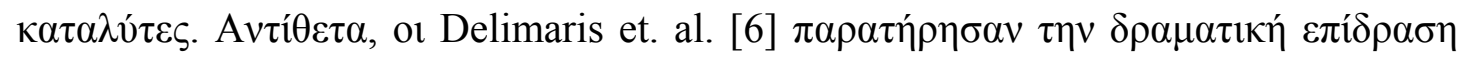

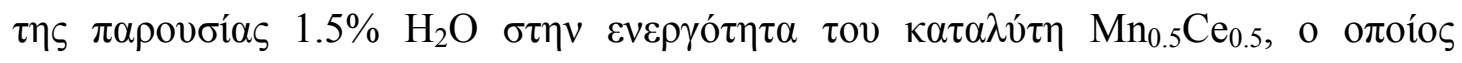

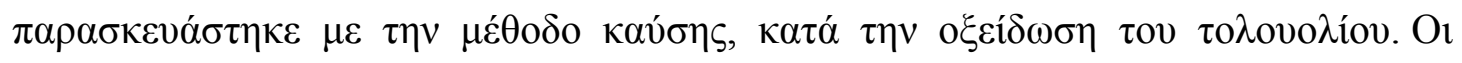

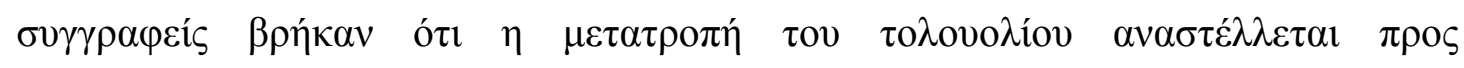

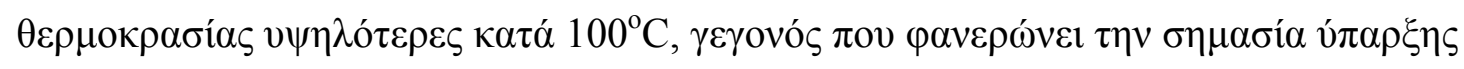

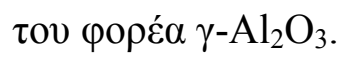

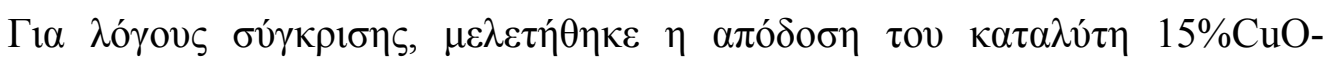

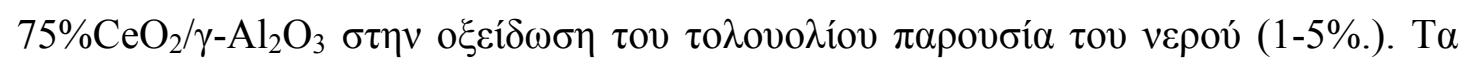

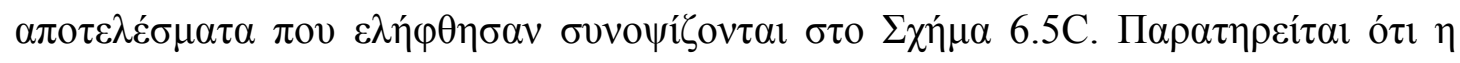

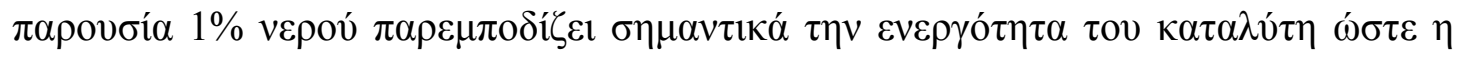

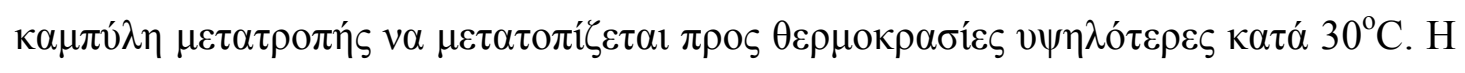

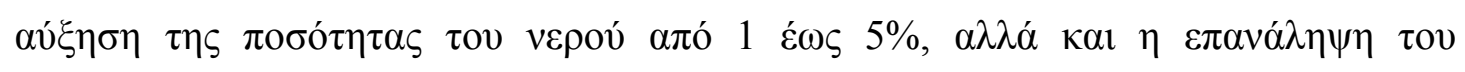

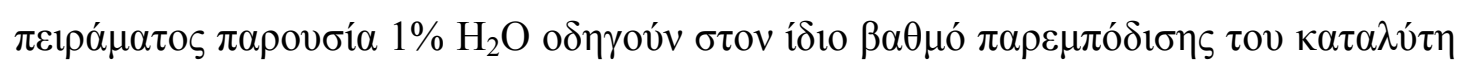

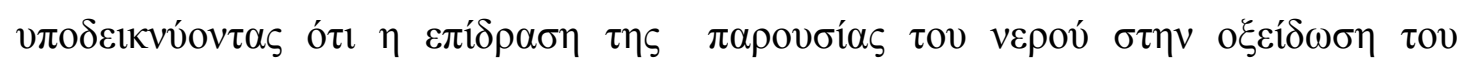

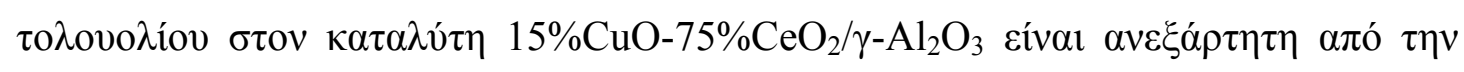

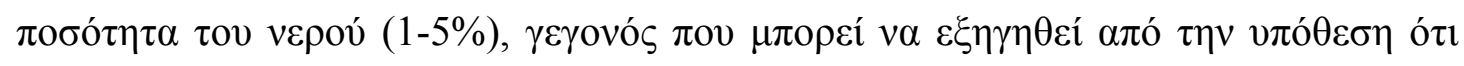

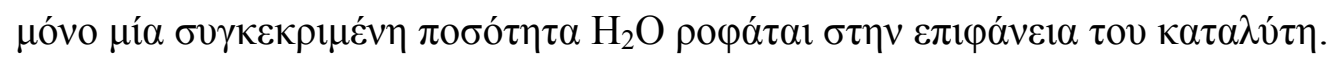

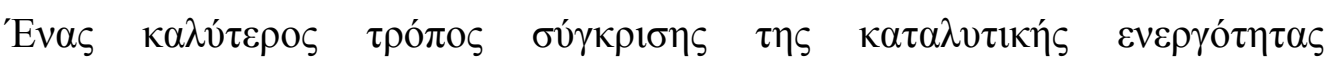

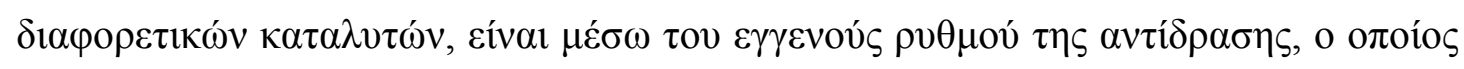

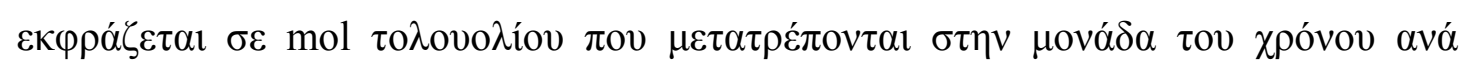

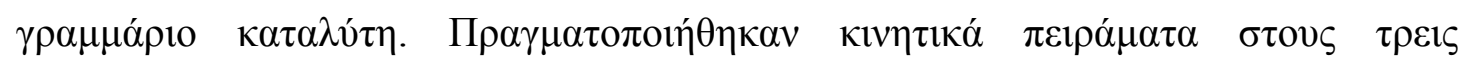

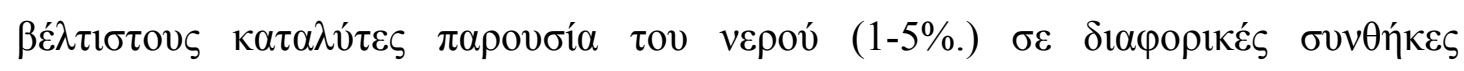

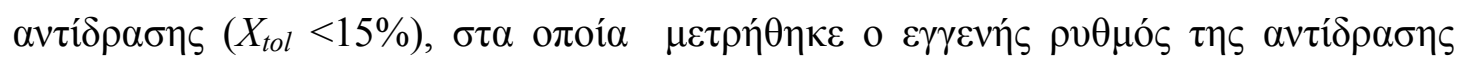

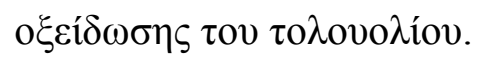

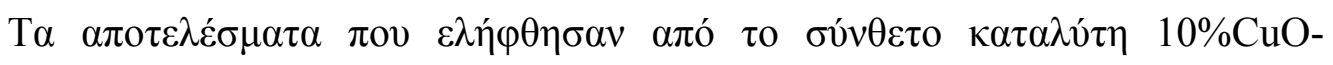

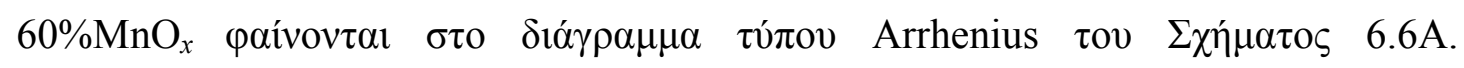

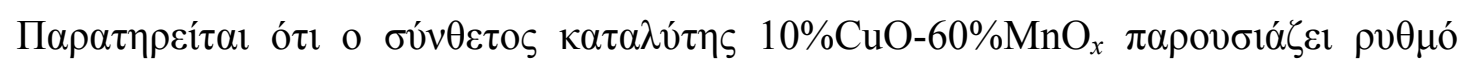

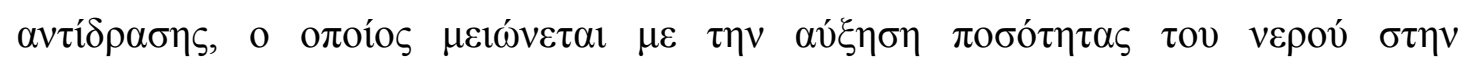

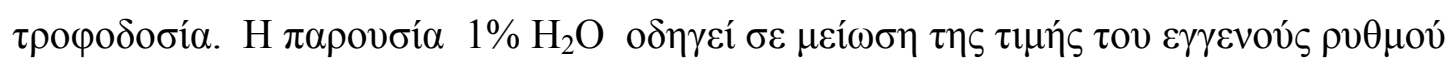



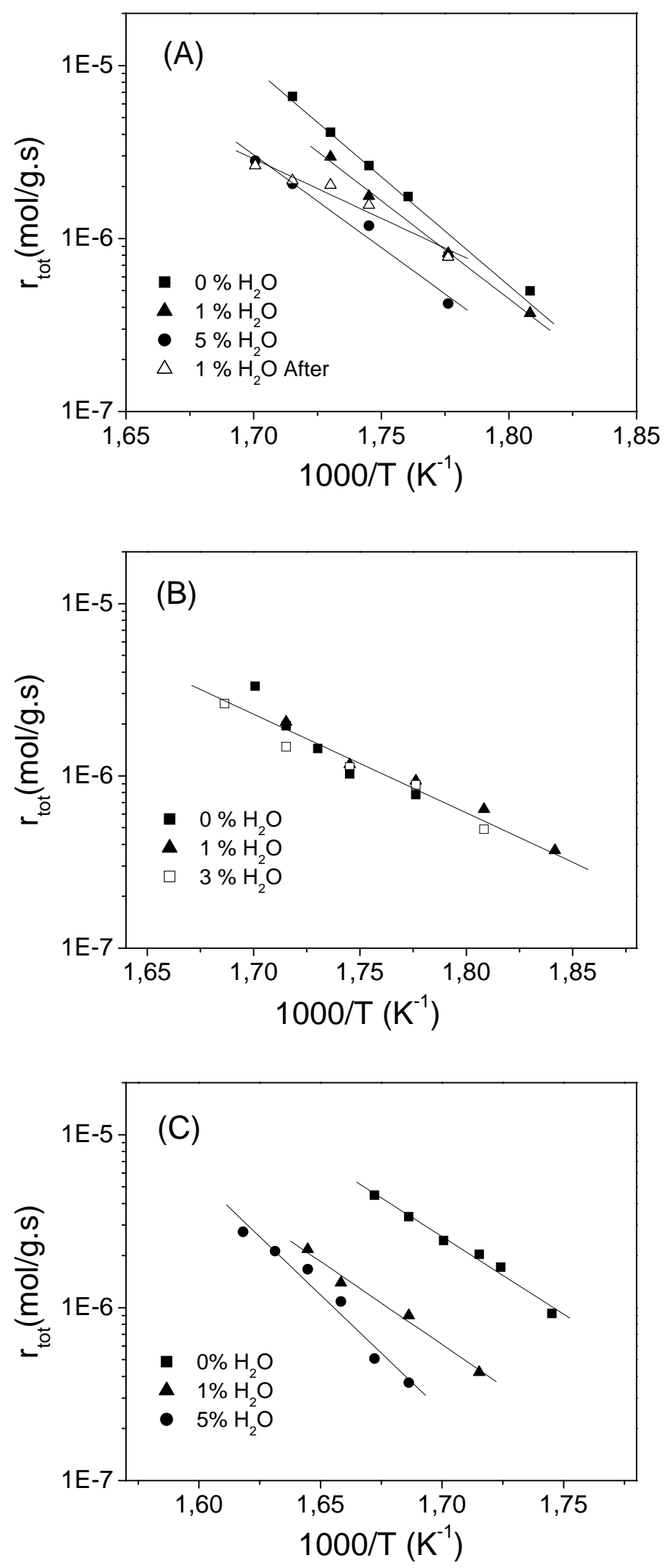

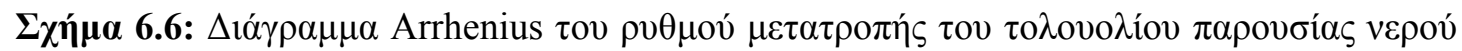

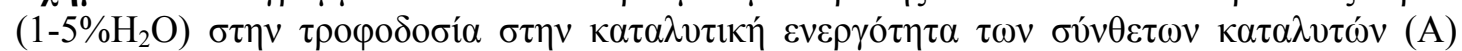

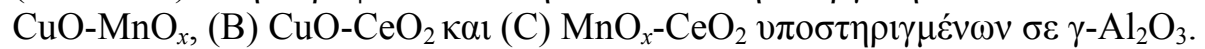




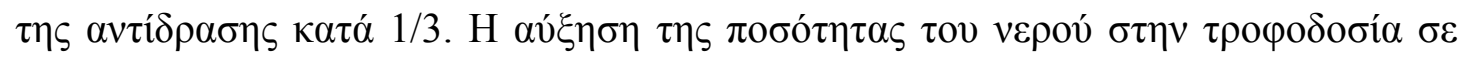

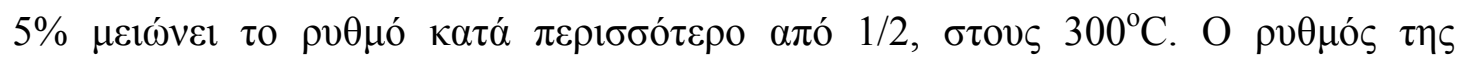

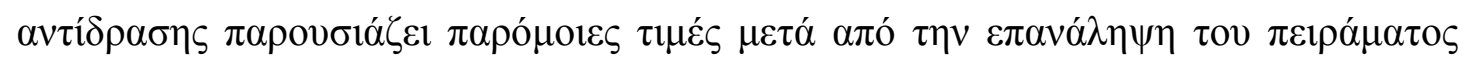

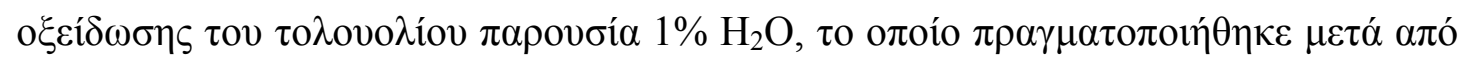

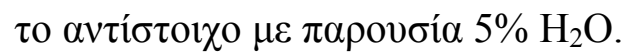

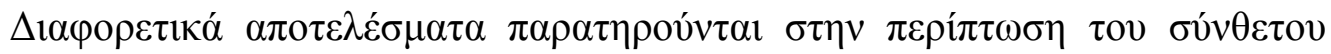

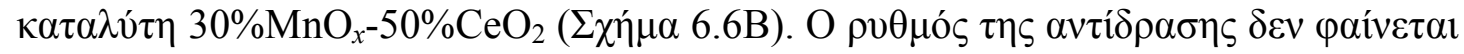

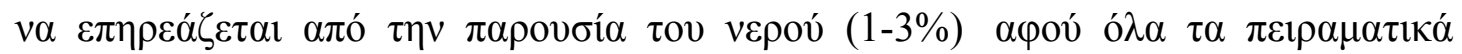

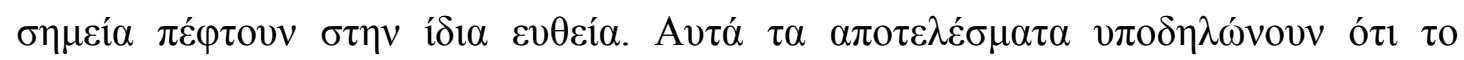

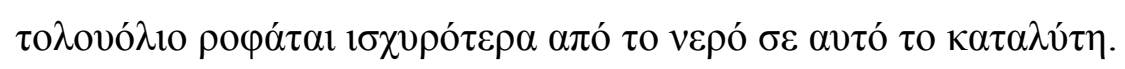

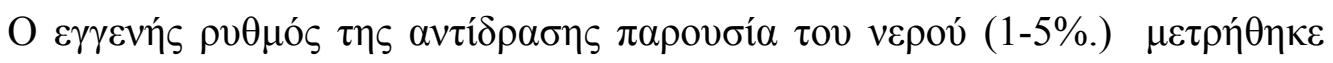

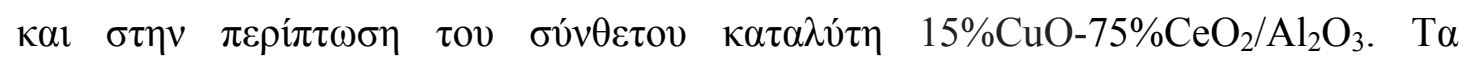

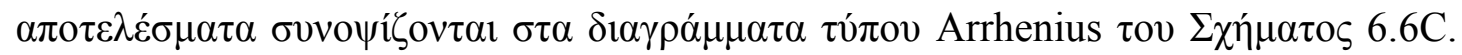

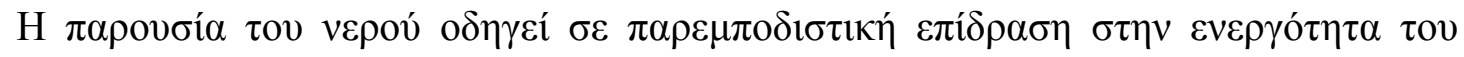

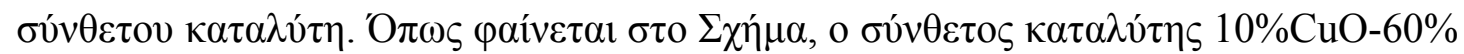

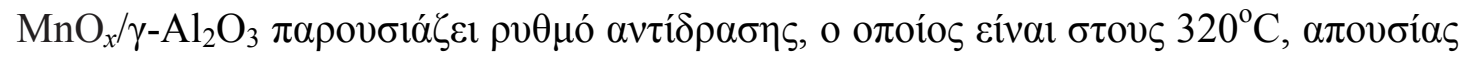

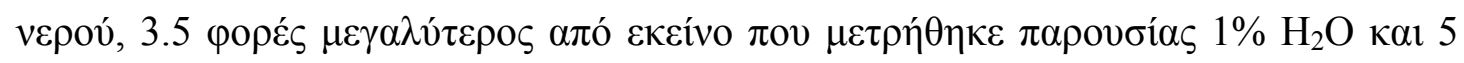

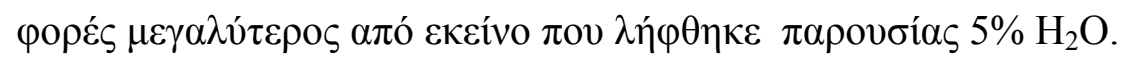

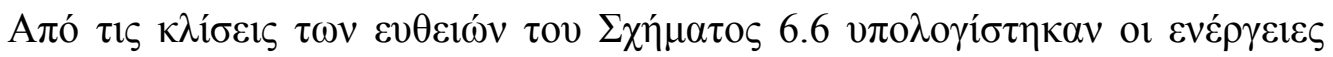

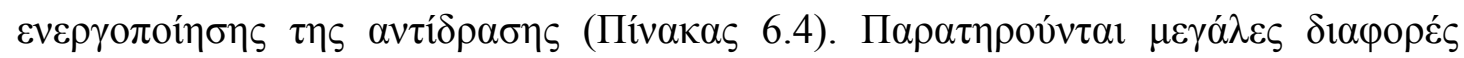

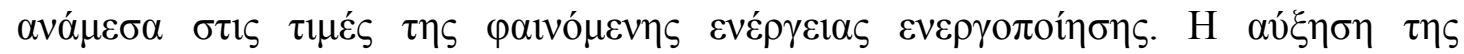

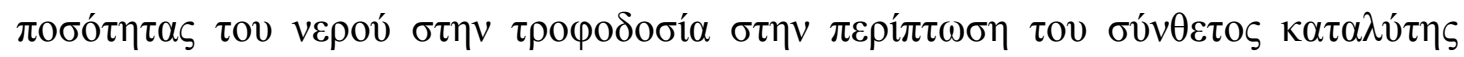

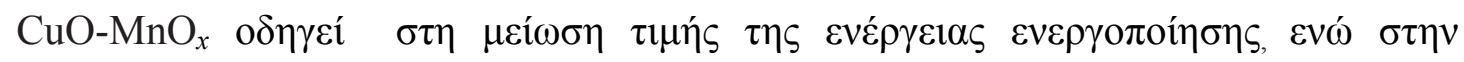

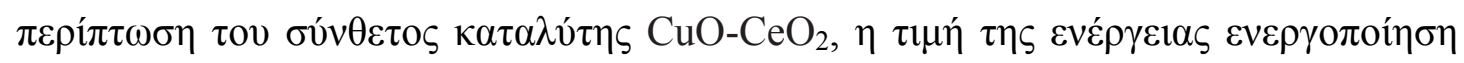
$\alpha v \xi \tilde{\alpha} v \varepsilon \tau \alpha 1$.

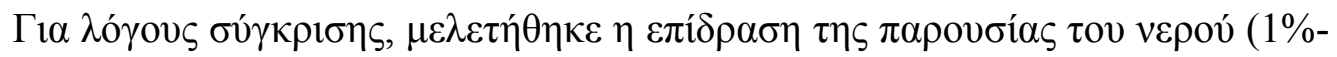

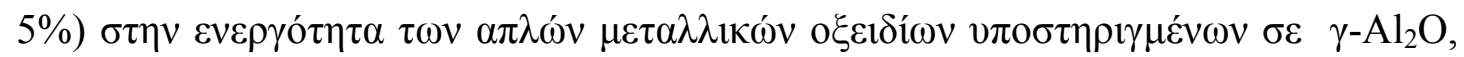

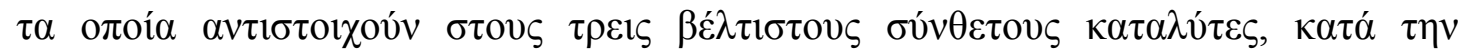

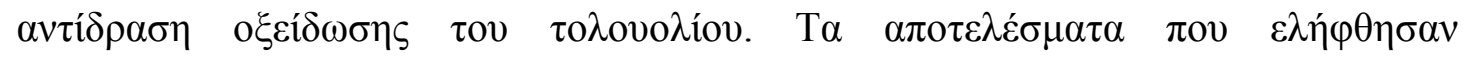

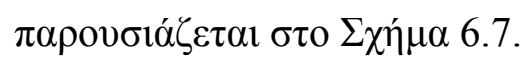




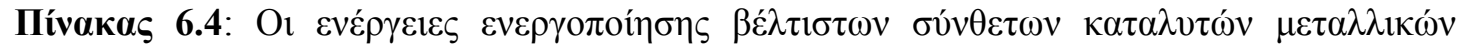

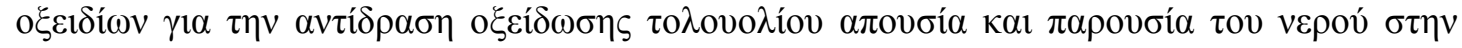

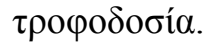

\begin{tabular}{|c|c|c|}
\hline 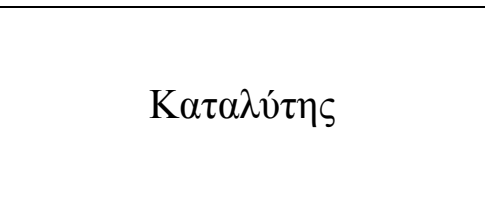 & $\% \mathrm{H}_{2} \mathrm{O}$ & 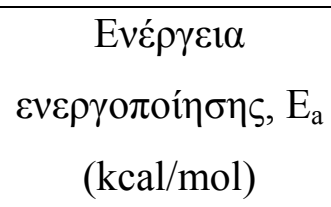 \\
\hline \multirow{3}{*}{$10 \% \mathrm{CuO}-60 \% \mathrm{MnO}_{x} / \mathrm{Al}_{2} \mathrm{O}_{3}$} & $0 \%$ & 57.5 \\
\hline & $1 \%$ & 42.3 \\
\hline & $5 \%$ & 36.7 \\
\hline $30 \% \mathrm{MnO}_{x}-50 \% \mathrm{CeO}_{2} / \mathrm{Al}_{2} \mathrm{O}_{3}$ & $0 \%-1 \%-3 \%$ & 26.1 \\
\hline \multirow{3}{*}{$15 \% \mathrm{CuO}-75 \% \mathrm{CeO}_{2} / \mathrm{Al}_{2} \mathrm{O}_{3}$} & $0 \%$ & 41 \\
\hline & $1 \%$ & 43.9 \\
\hline & $5 \%$ & 61.6 \\
\hline
\end{tabular}

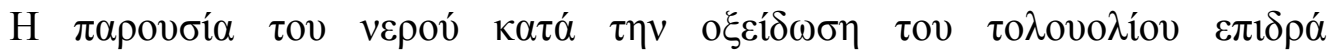

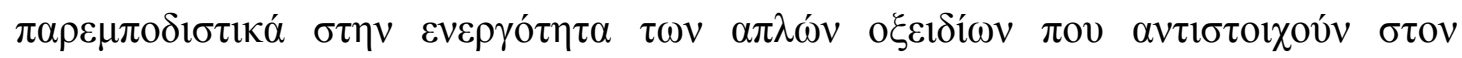

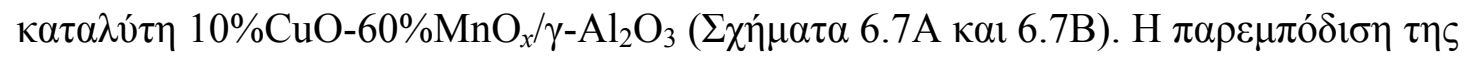

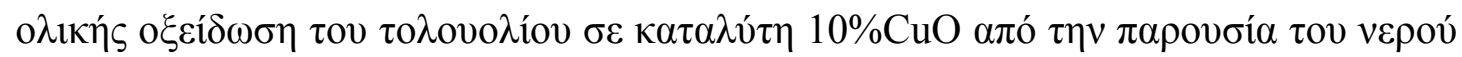

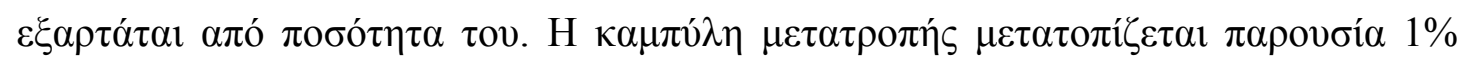

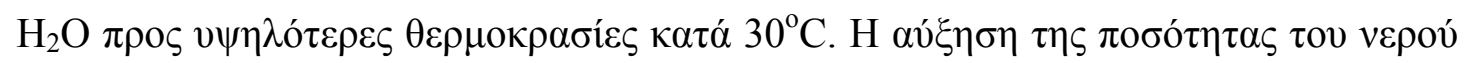

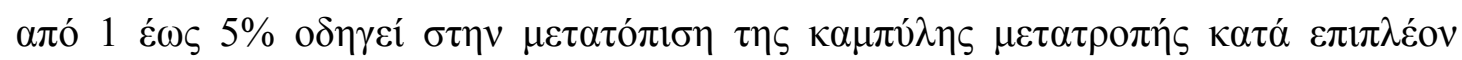

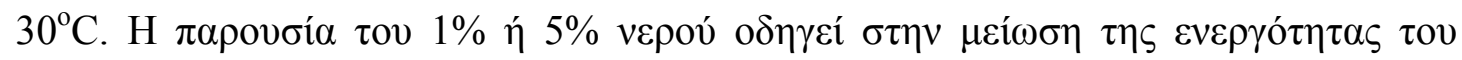

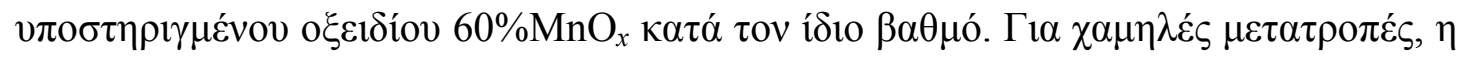

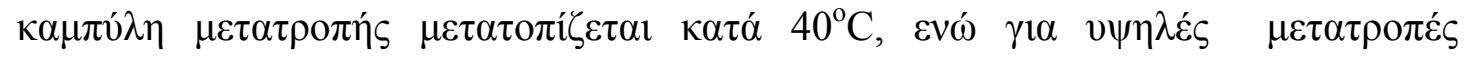

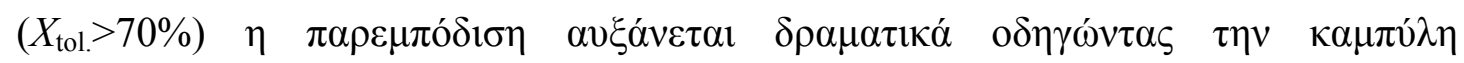

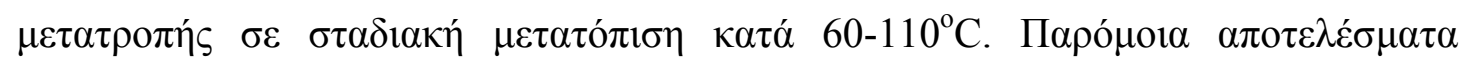

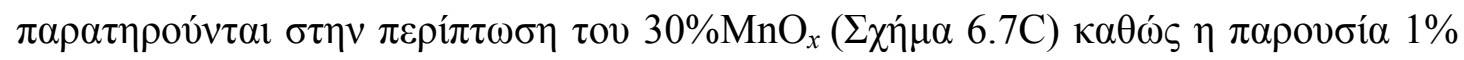

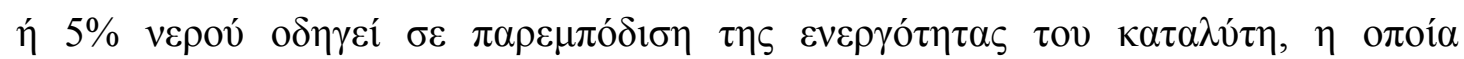

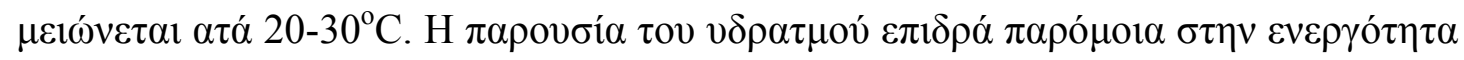

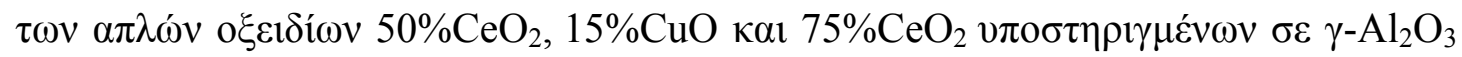

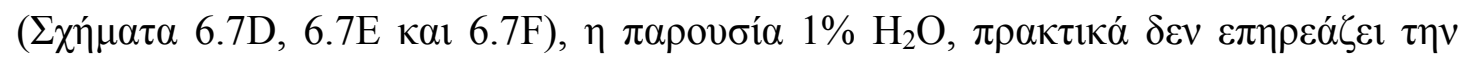

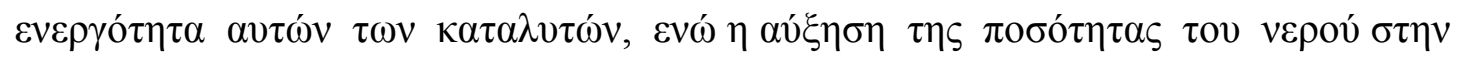



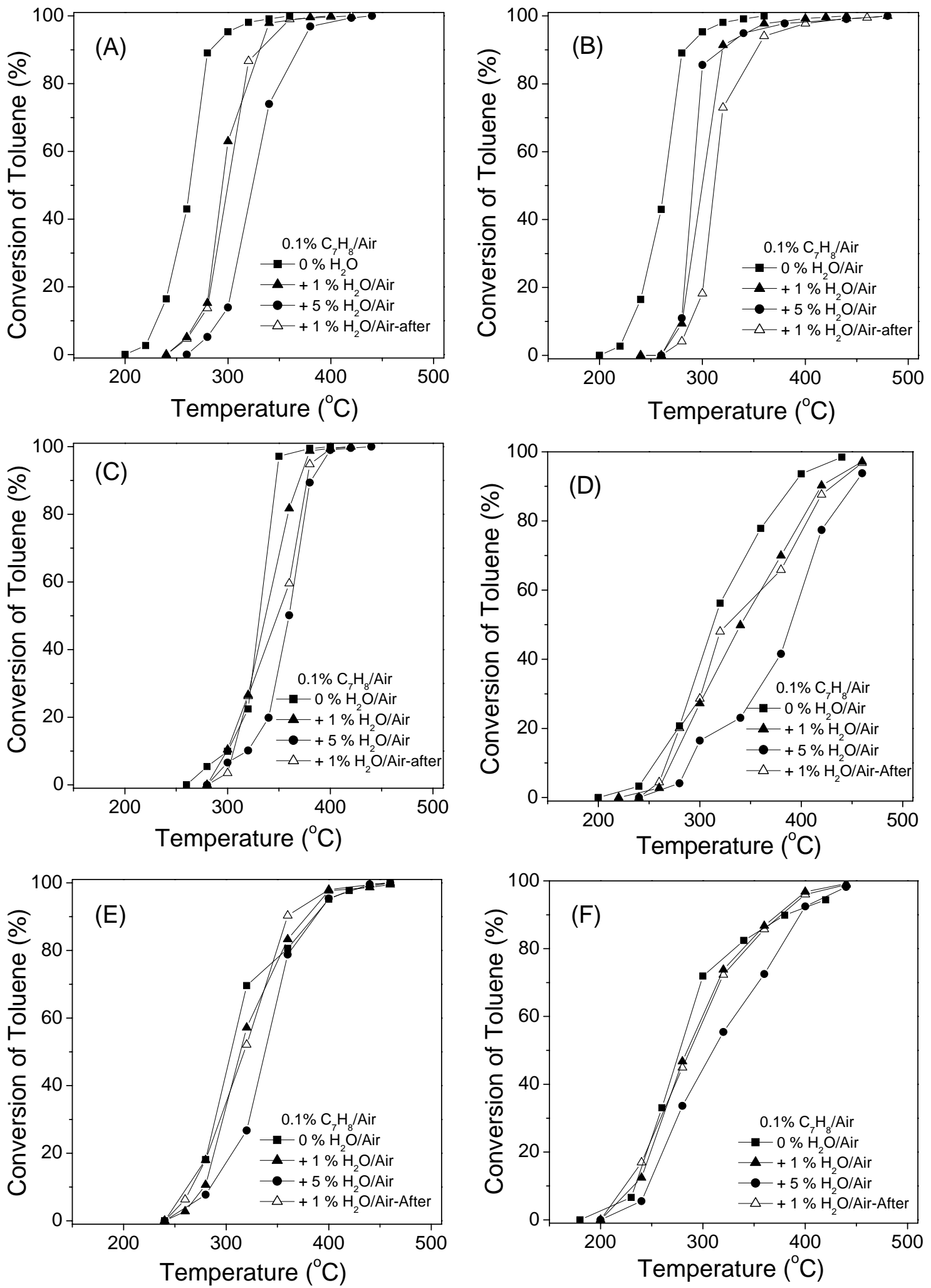

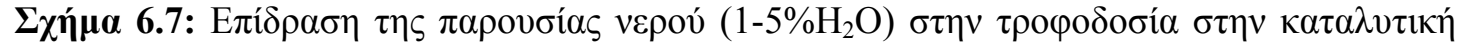

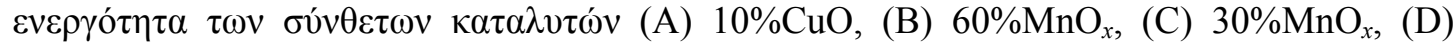

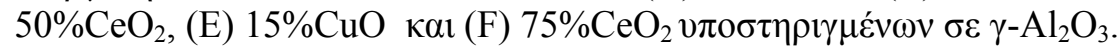




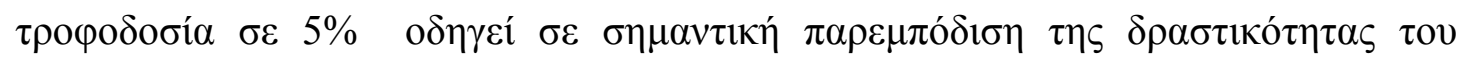

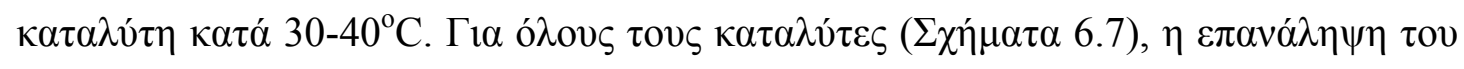

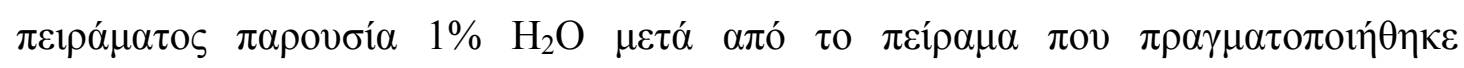

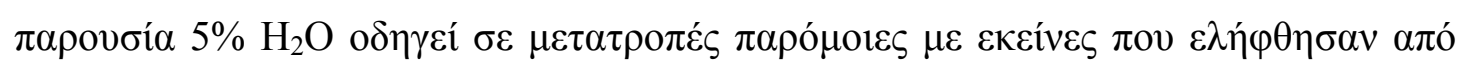

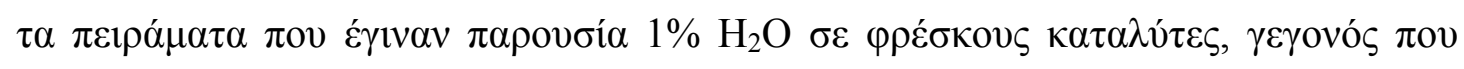


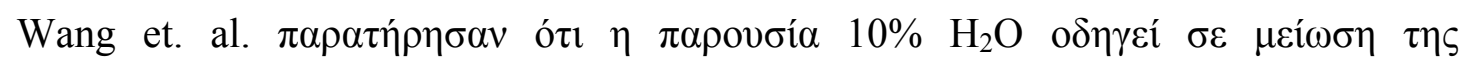

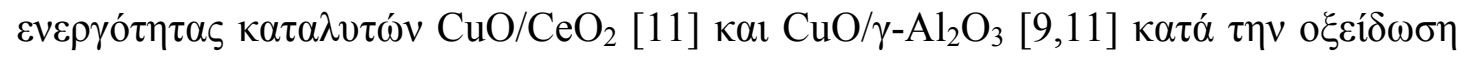

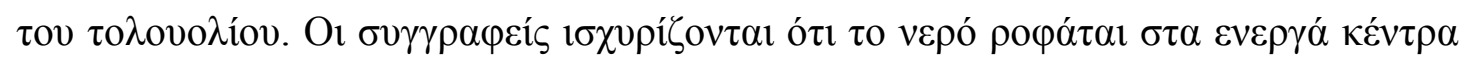

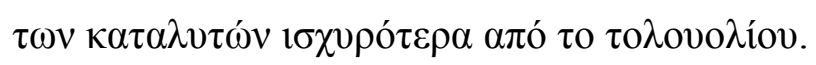

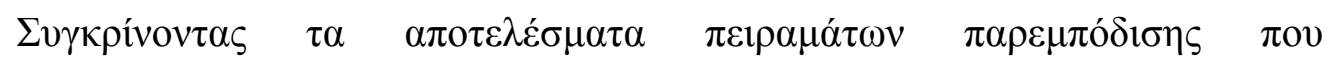

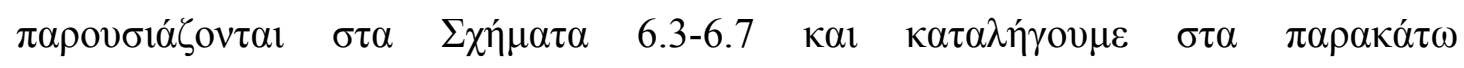
$\sigma v \mu \pi \varepsilon \rho \alpha ́ \sigma \mu \alpha \tau \alpha$ :

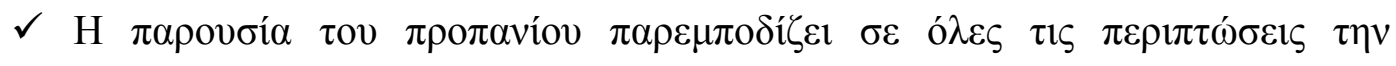

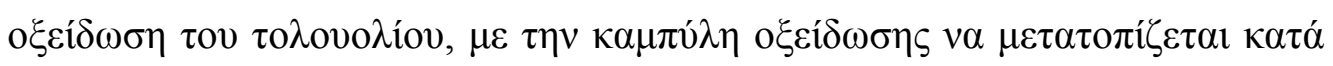

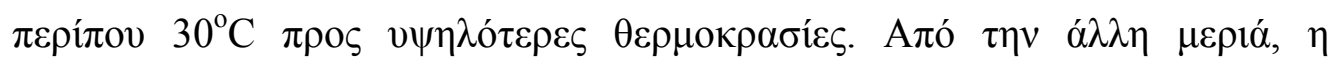

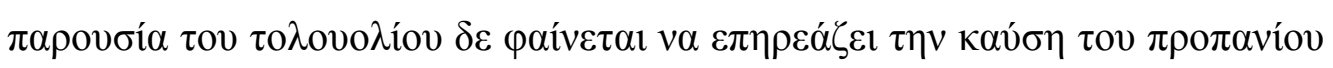

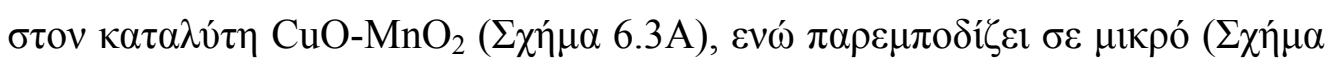

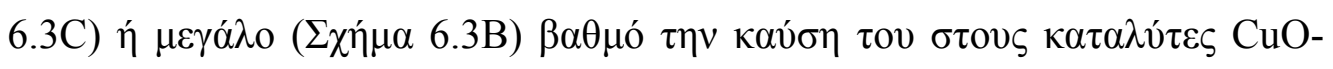
$\mathrm{CeO}_{2} \kappa \alpha \iota \mathrm{MnO}_{2}-\mathrm{CeO}_{2}$, $\alpha v \tau i ́ \sigma \tau o \imath \chi \alpha$.

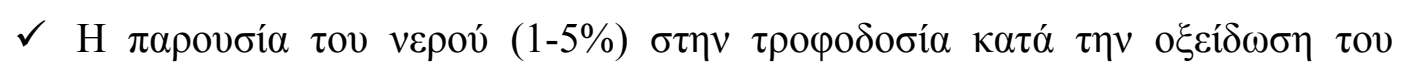

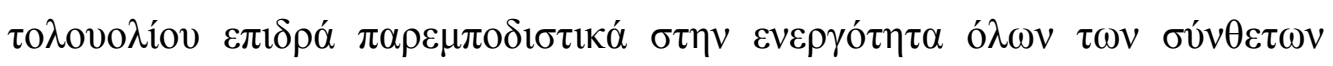

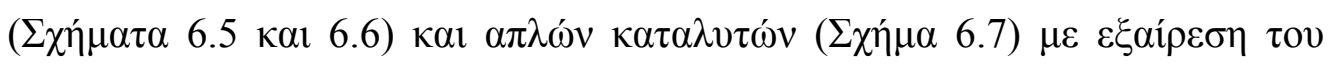

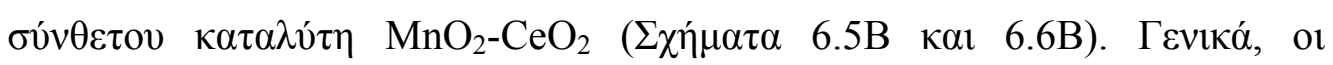

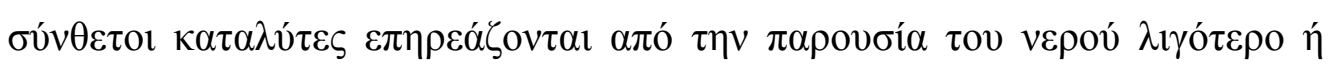

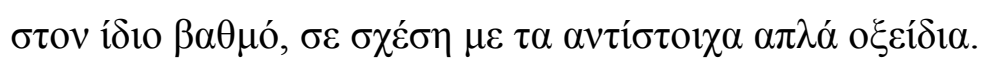




\section{$6.6 \Sigma v \mu \pi \varepsilon \rho \alpha ́ \sigma \mu \alpha \tau \alpha$}

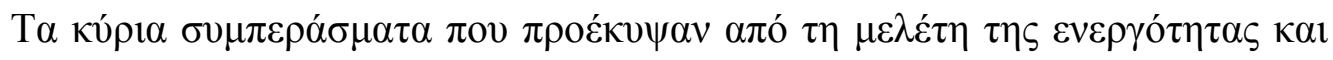

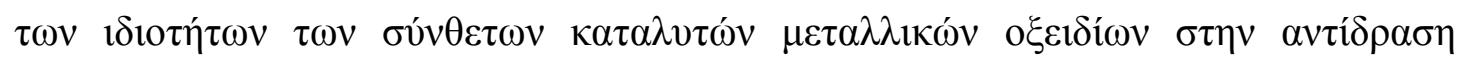

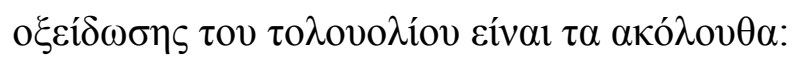

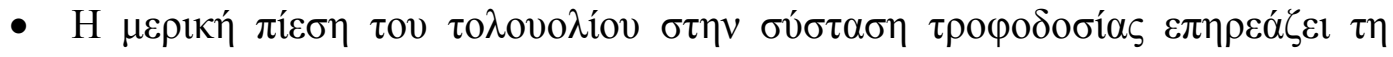

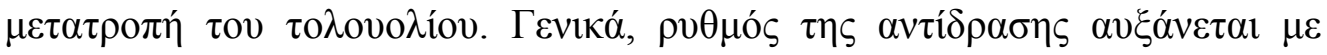

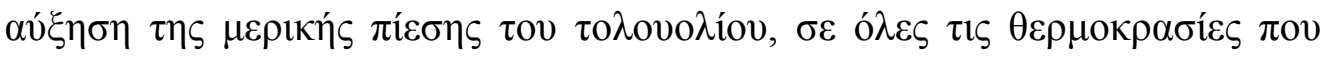

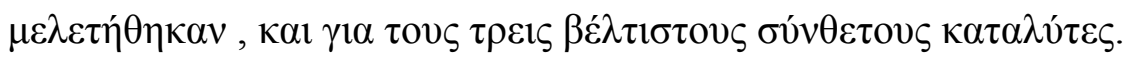

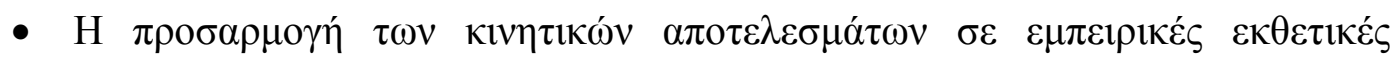

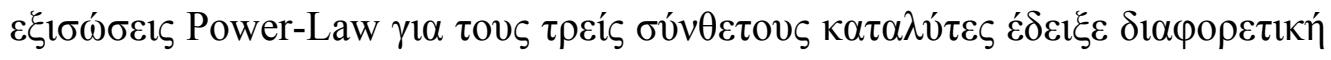

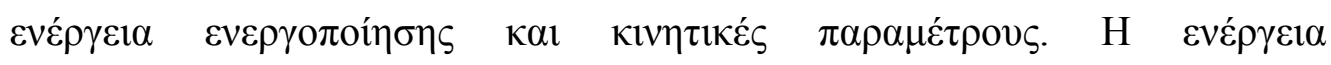

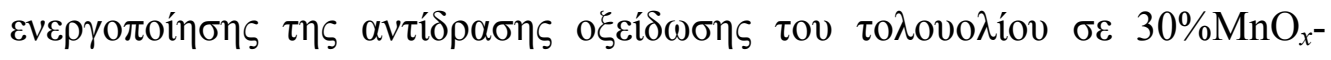

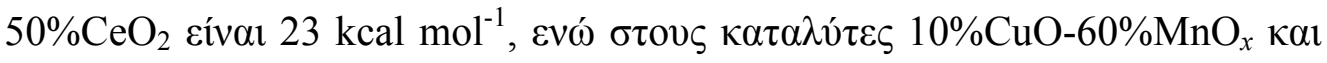

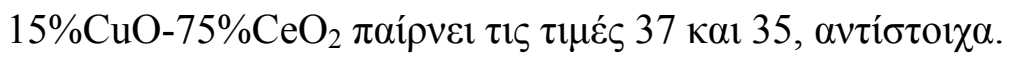

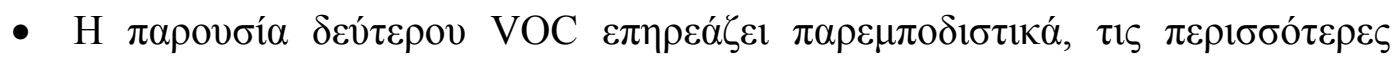

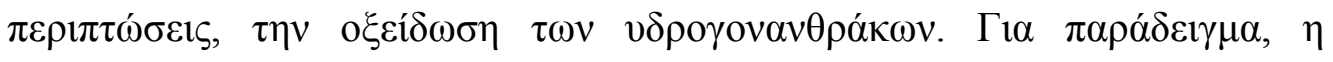

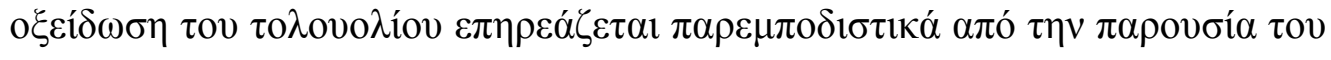

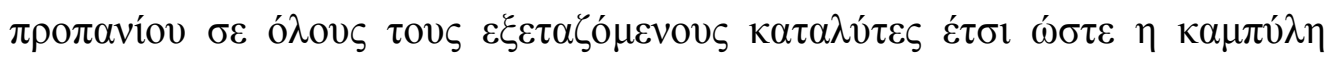

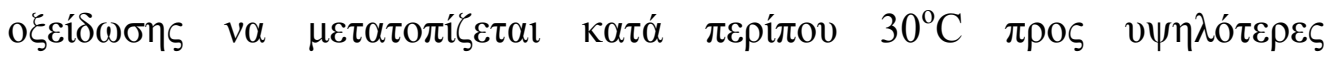

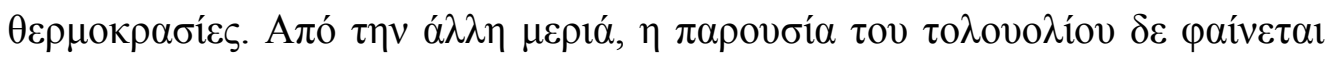

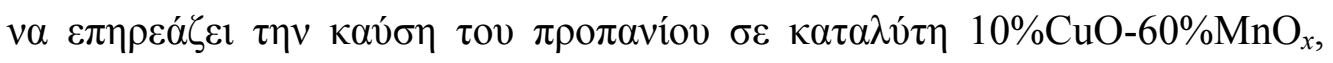

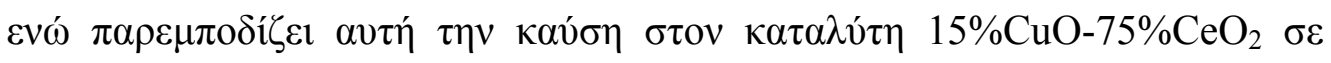

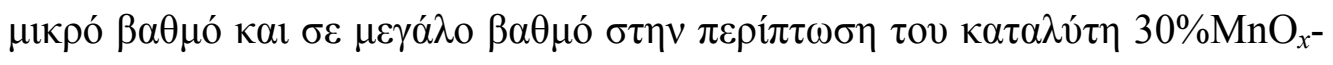
$50 \% \mathrm{CeO}_{2}$.

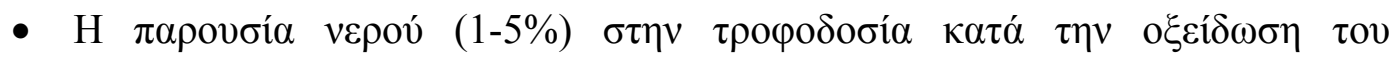

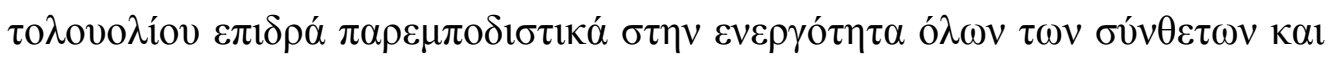

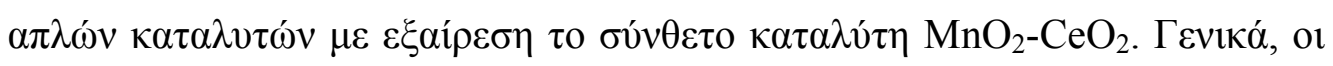

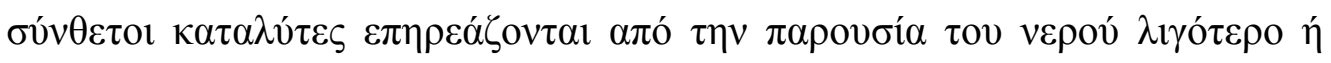

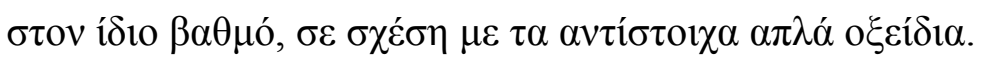




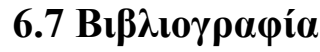

[1] Y.-F. Y. Yao, J. OF Catal. 87(1984) 152.

[2] C. T. Wong, A. Z. Abdullah, S. Bhatia, J. Hazard. Mat. 157 (2008) 480.

[3] P. Papaefthimiou, T. Ioannides, X.E. Verykios, Appl. Catal. B: Env. 13 (1997) 157.

[4] N. Ali, C. Lu, R. Masel, Catalysis Today 62 (2000) 347.

[5] P. Papaefthimiou , T. Ioannides , X. E. Verykios, Appl. Catal. B: Environ. 15 (1998) 75 .

[6] D. Delimaris, T. Ioannides, Appl. Catal. B: Environ 84 (2008) 303.

[7] B. de Rivas, Jose I. Gutiérrez-Ortiz, R. López-Fonseca, J. R. GonzálezVelasco, Appl. Catal. A: 314 (2006) 54.

[8] A. Gervasini, C. Pirola, V. Ragaini, Appl. Catal. B: Env. 38 (2002) 17.

[9] C.H. Wang, Chemosphere 55 (2004) 11.

[10] Horsley, J. A., Catalytica Environmental Report No. E4, Catalytica Studies Division, Mountain View, CA, USA, 1993.

[11] C.-H. Wang, S.-S. Lin, C.-L. Chen, H.-S. Weng, Chemosphere 64 (2006) 503

[12] C.F. Cullis and B.M. Willatt, J. Catal. 86 (1984)187.

[13] C.H. Wang and H.S. Weng, Ind. Eng. Chem. Res. 36 (1997) 2537.

[14] S.K. Agarwal, J.J. Spivey, J.B. But, Appl. Catal. A: Gen. 81 (1992) 239.

[15] C.C. Chang and H.S. Weng, Ind. Eng. Chem. Res., 32 (1993) 2930. 


\title{
$\operatorname{KE\Phi } A \Lambda A I O 7^{\circ}$
}

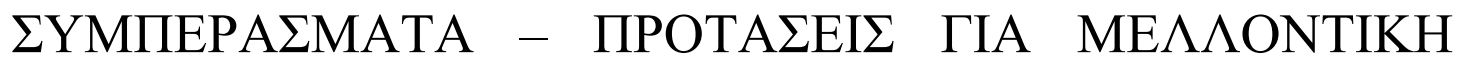

\author{
ME $\Lambda$ ETH
}

\section{$7.1 \Sigma v \mu \pi \varepsilon \rho \alpha ́ \sigma \mu \alpha \tau \alpha$}

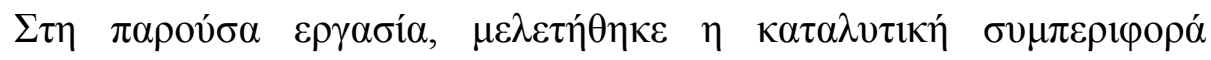

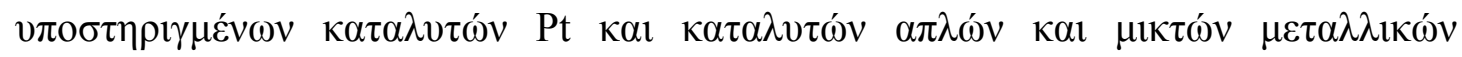

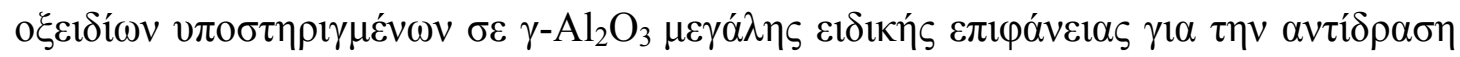

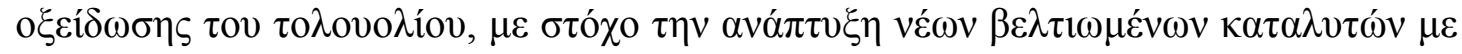

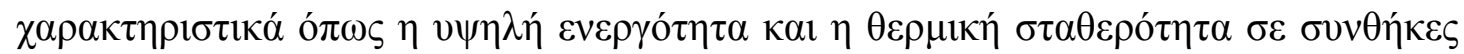

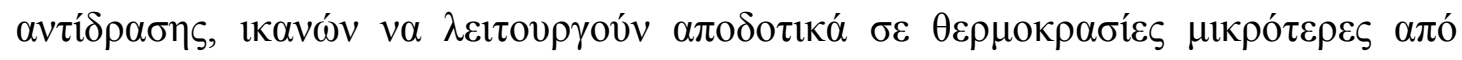

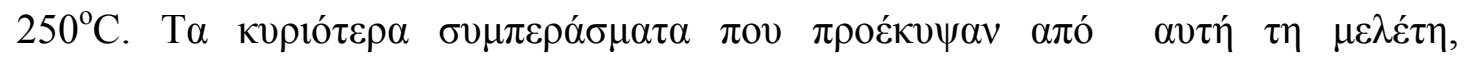

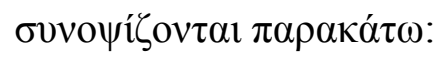




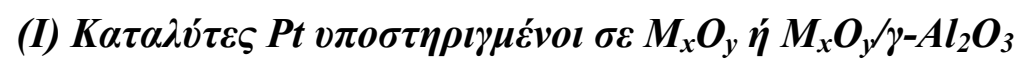

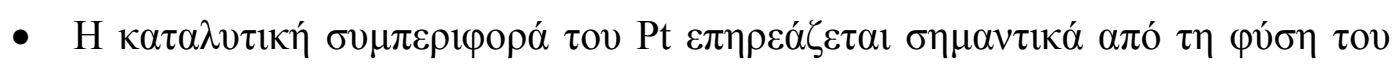

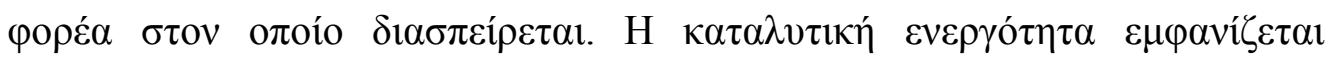

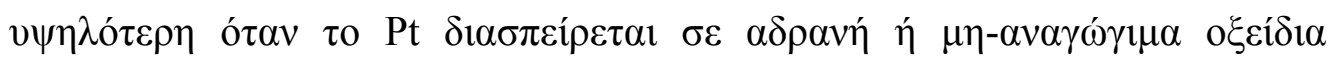
$\mu \varepsilon \tau \alpha \dot{\lambda} \lambda \lambda \omega v, \pi \cdot \chi . \mathrm{SiO}_{2}, \mathrm{Al}_{2} \mathrm{O}_{3}, \mathrm{ZrO}_{2}, \mathrm{MgO}, \sigma \varepsilon \sigma \chi \varepsilon \dot{\sigma} \eta \mu \varepsilon \tau \eta \nu \pi \varepsilon \rho i ́ \pi \tau \omega \sigma \eta \pi$

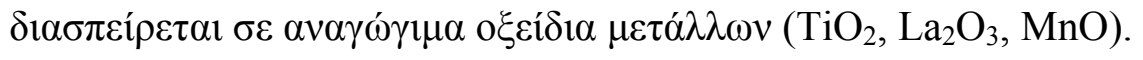

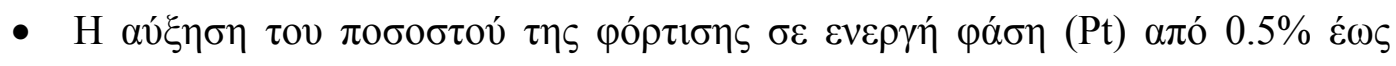

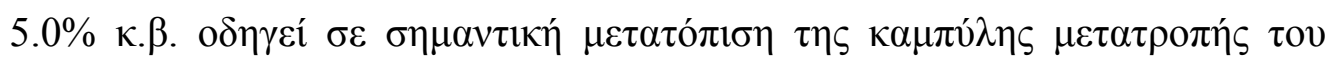

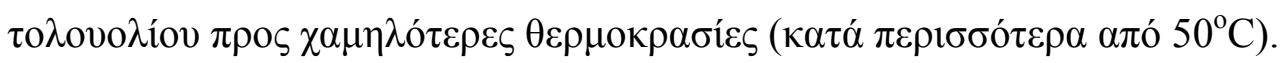

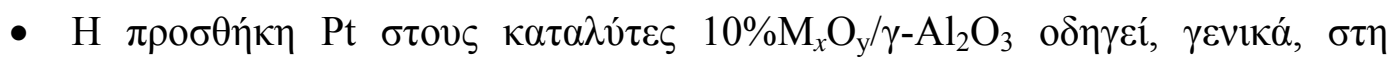

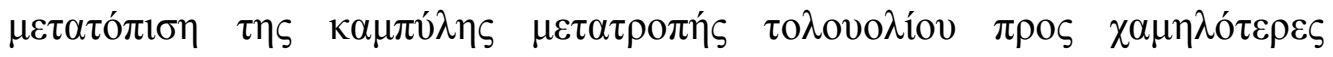

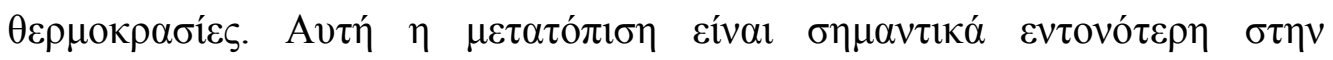
$\pi \varepsilon \rho i ́ \pi \tau \omega \sigma \eta \tau \omega \nu \mu \eta-\alpha v \alpha \gamma \omega ́ \gamma 1 \mu \omega \nu \mathrm{M}_{x} \mathrm{O}_{y} \kappa \alpha \tau \alpha \lambda \nu \tau \omega ́ v(\pi \cdot \chi . \mathrm{M}=\mathrm{Zr}, \mathrm{Mg}, \mathrm{Cs})$.

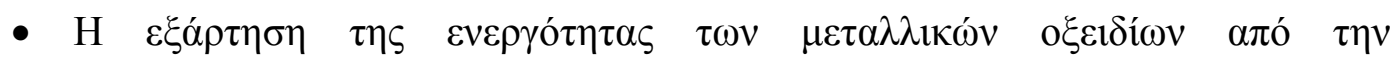

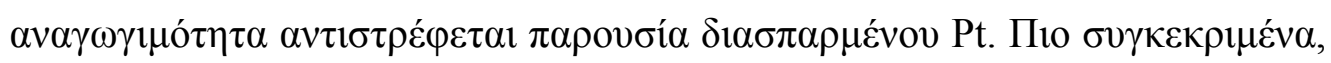

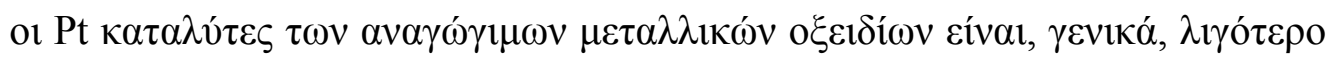

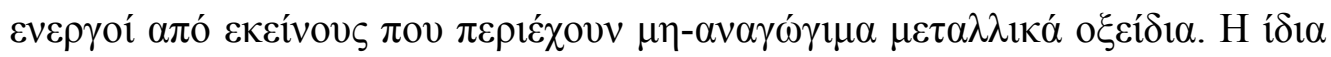
$\tau \alpha ́ \sigma \eta \pi \alpha \rho \alpha \tau \rho \eta ́ \theta \eta \kappa \varepsilon ~ \kappa \alpha \iota ~ \sigma \tau \eta v ~ \pi \varepsilon \rho i ́ \pi \tau \omega \sigma \eta ~ \tau \omega \nu ~ \kappa \alpha \tau \alpha \lambda \nu \tau \omega ́ v ~ P t / M_{x} \mathrm{O}_{y}$.

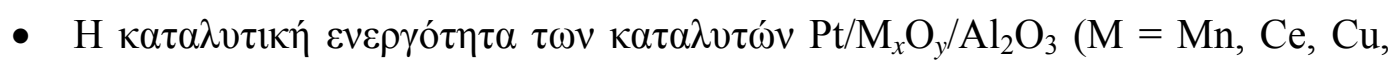

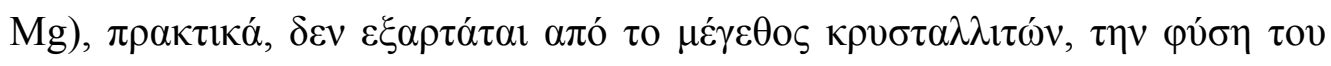

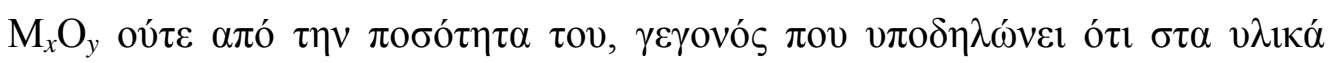

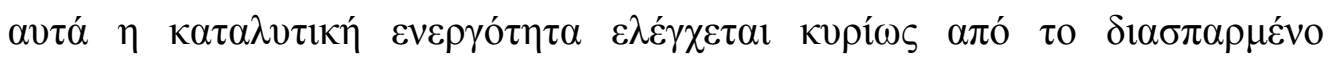
$\mu \varepsilon ́ \tau \alpha \lambda \lambda \mathrm{o}, \mathrm{Pt}$.

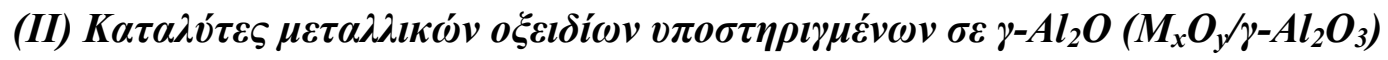

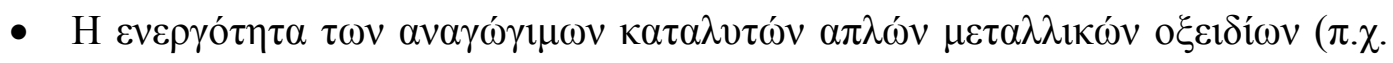

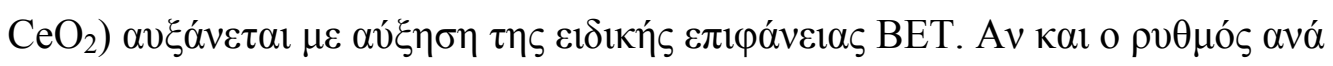

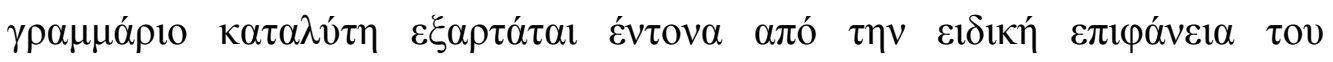

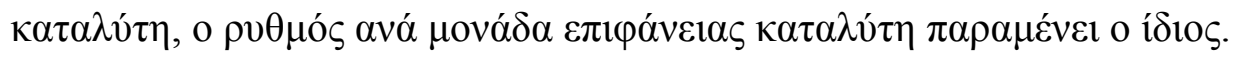

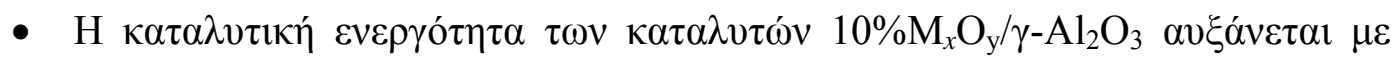

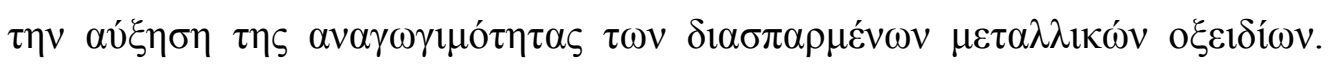

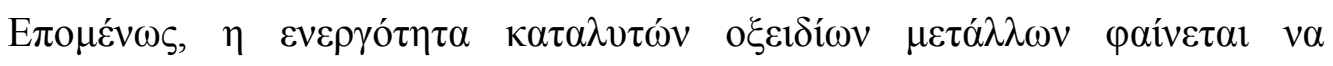

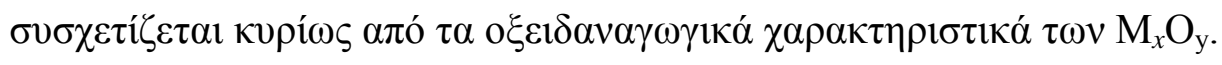




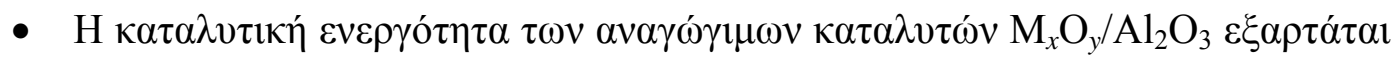

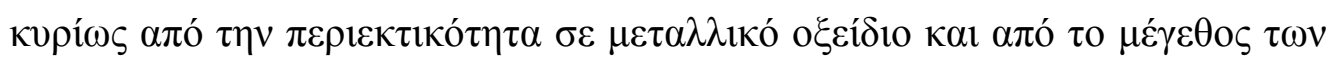

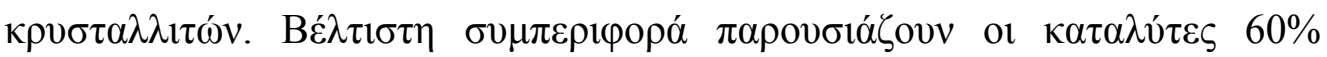

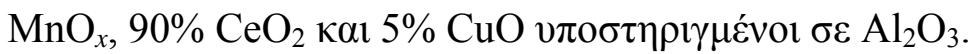

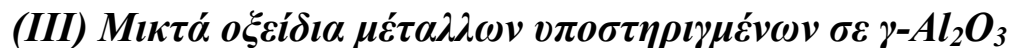

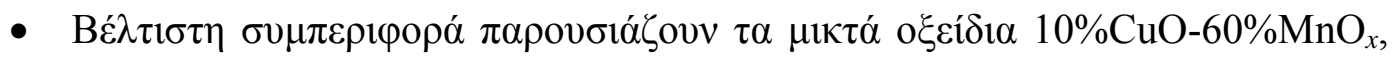

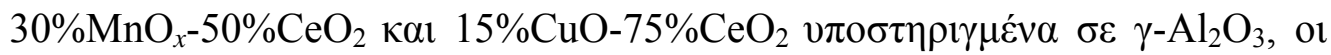

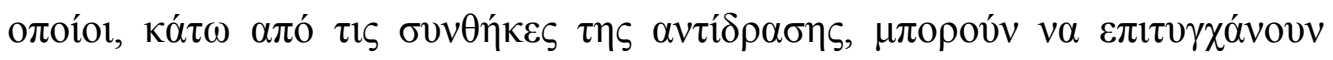

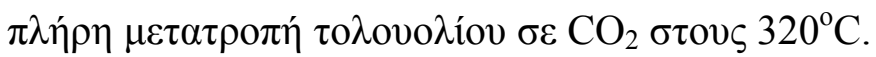

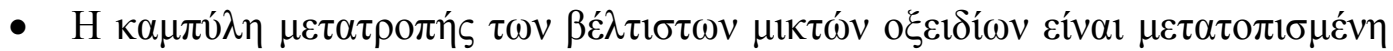

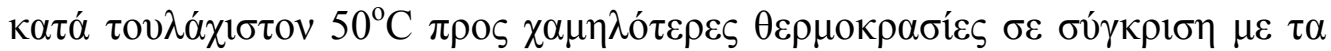

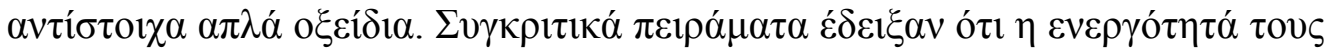

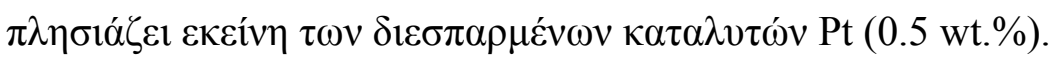

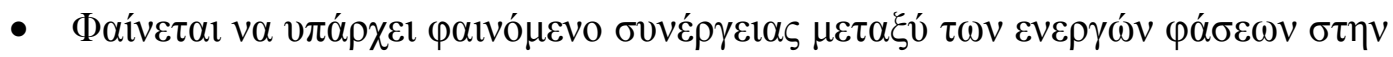

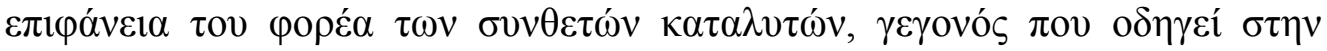

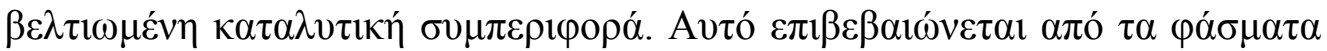

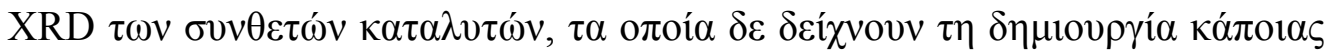

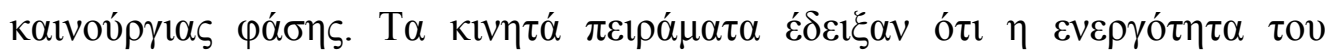

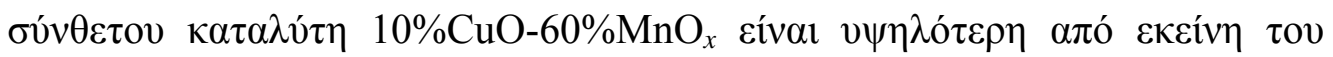

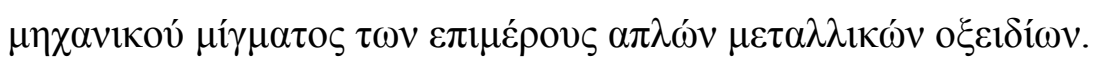

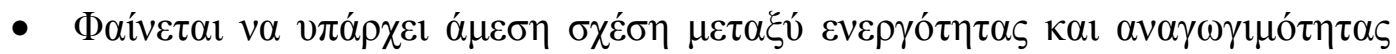

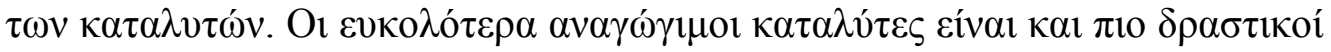

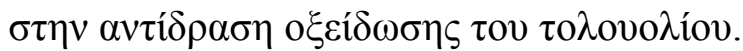

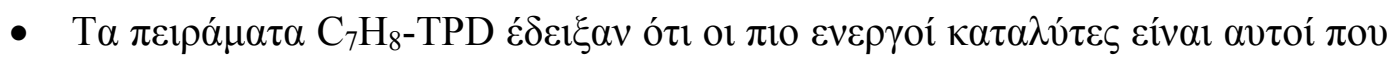

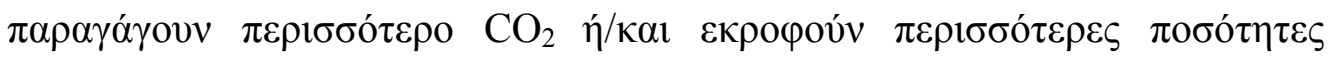

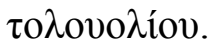

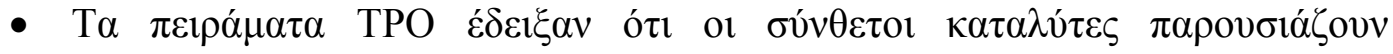

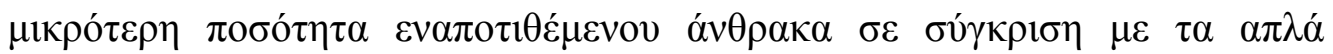
$\mu \varepsilon \tau \alpha \lambda \lambda \iota \kappa \alpha ́$ o $\xi \varepsilon i \delta 1 \alpha$.

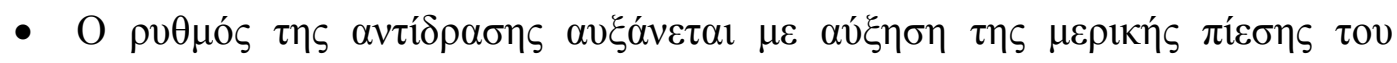

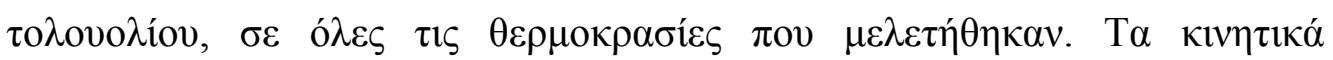




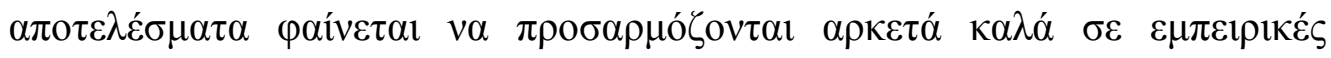

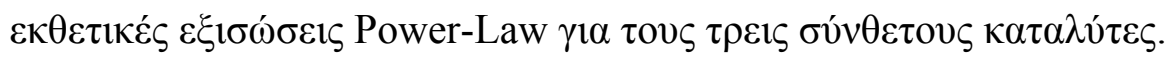

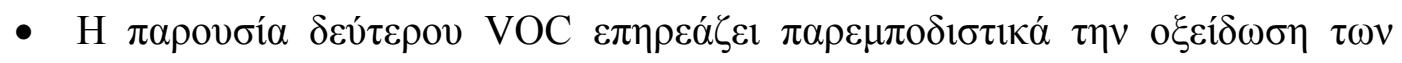

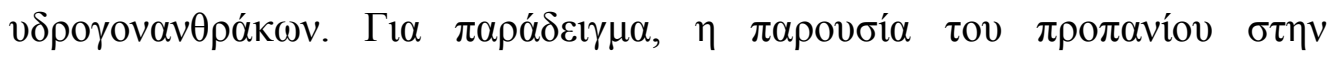

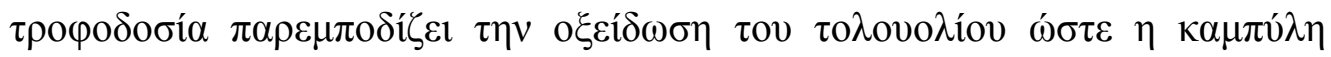

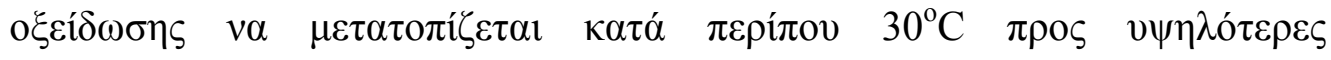

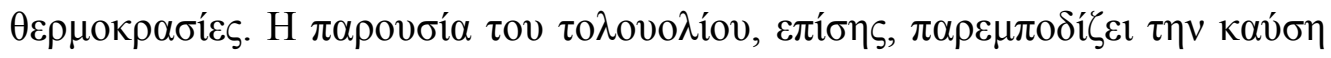

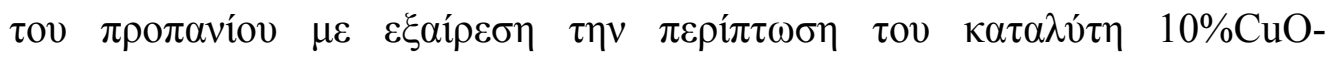
$60 \% \mathrm{MnO}_{x}$.

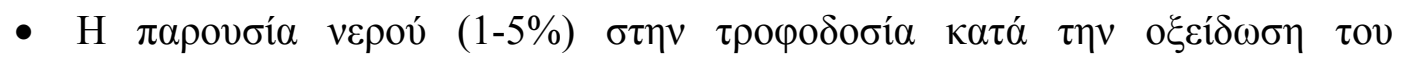

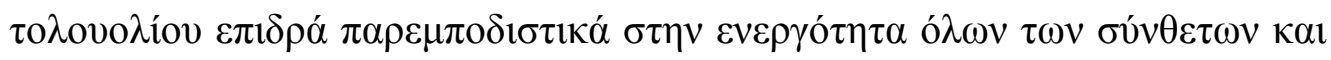

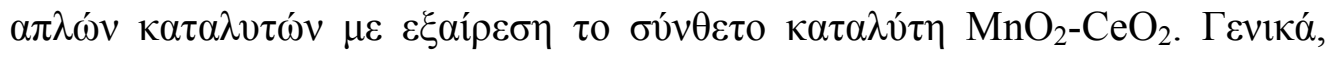

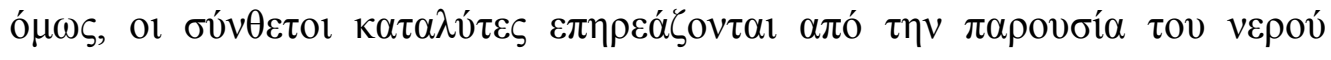

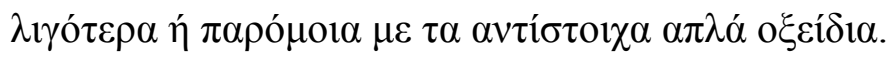

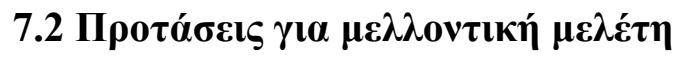

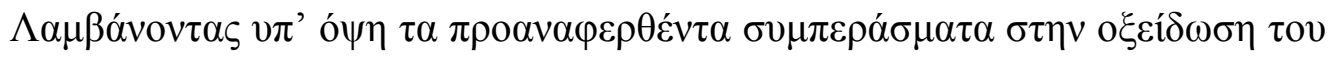

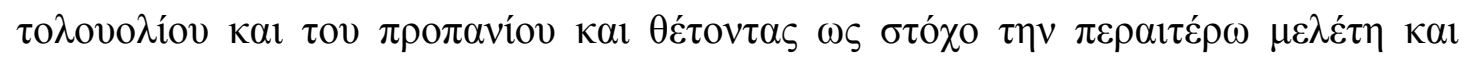

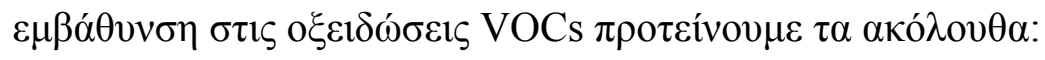

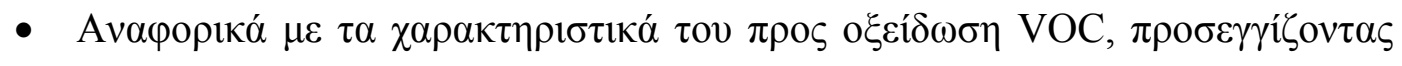

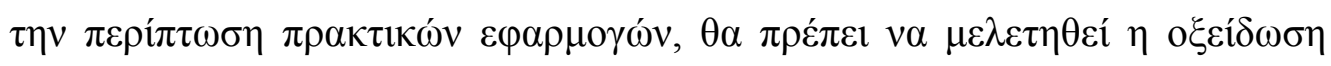

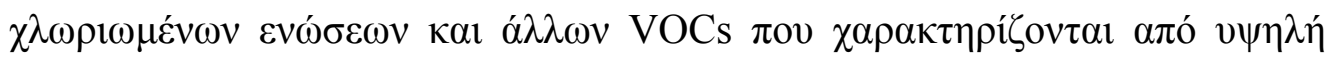

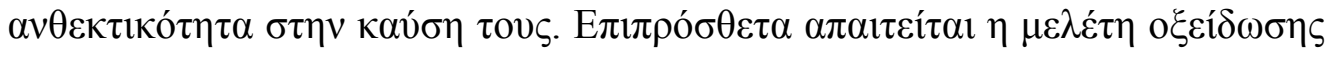

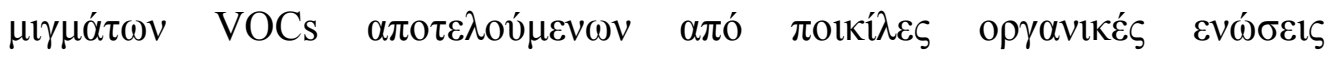

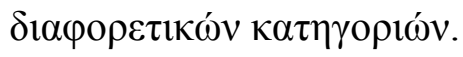

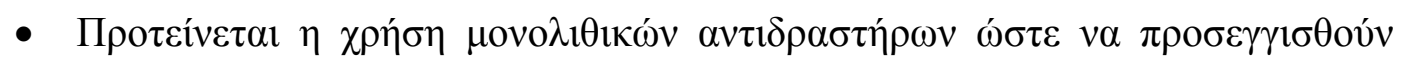

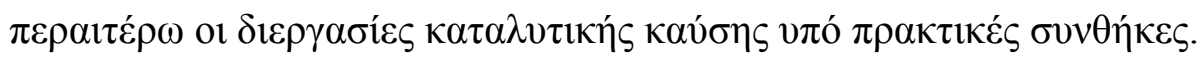

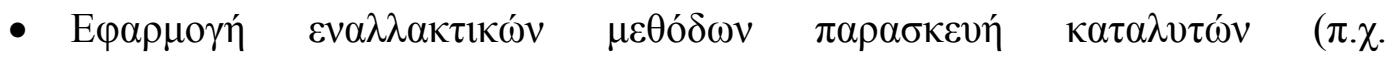

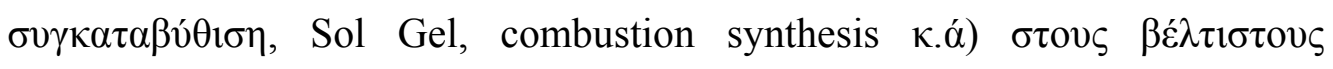

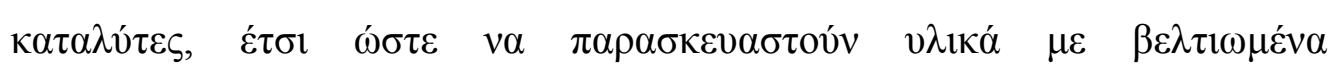




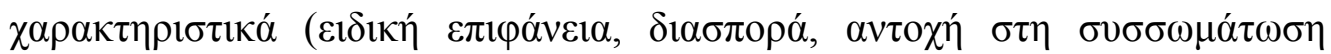

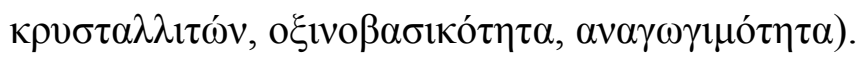

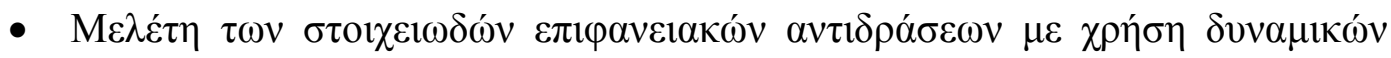

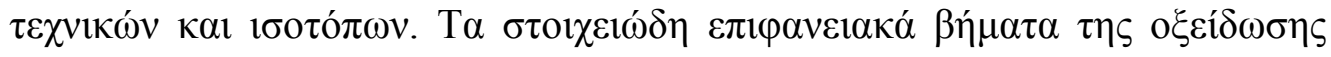

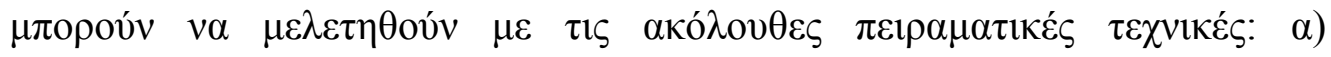

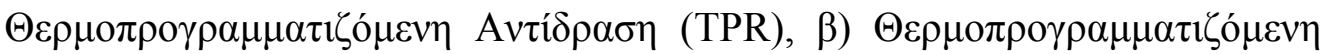

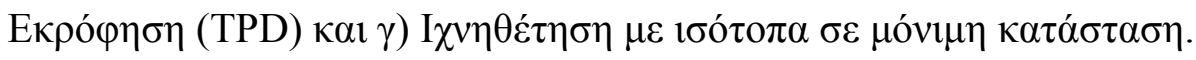

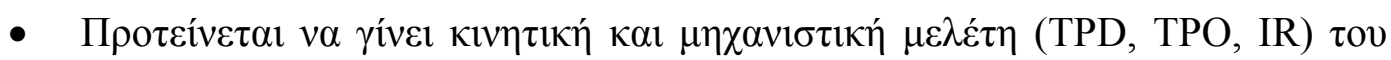

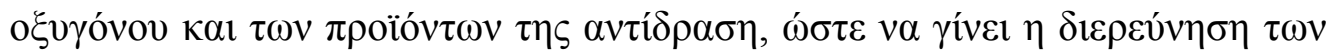

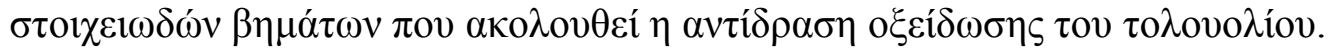

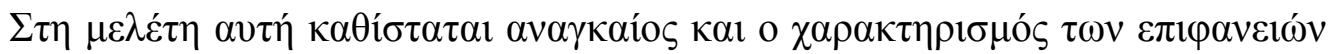

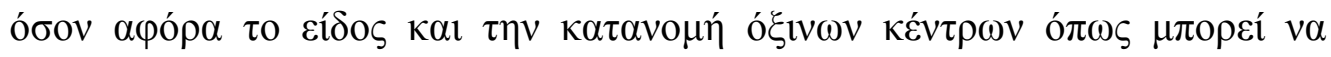

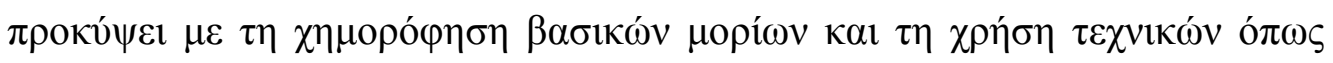

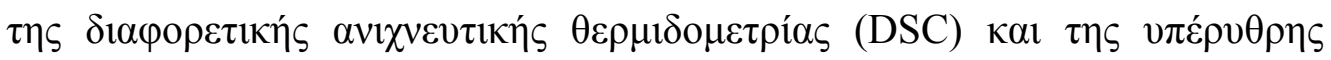

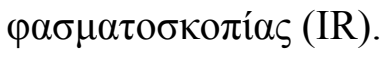

